\title{
DiE STRUKTURABHÄNGIGKEIT DER FESTKÖRPERFLUORESZENZ BEI PhOSPhanYLANTHRACENEN
}

\author{
Dissertation zur Erlangung des \\ mathematisch-naturwissenschaftlichen Doktorgrades \\ der Georg-August-Universität Göttingen
}

vorgelegt von

Gerald Harald Schwab

aus Würzburg

Göttingen 2008 

Eingereicht am:

Referent:

Co-Referent:

Datum der mündlichen Prüfung: 30.04.2008

Prüfer Anorganische Chemie: Prof. Dr. D. Stalke

Prüfer Organische Chemie: $\quad$ Prof. Dr. Dr. h. c. L. F. Tietze

Prüfer Kristallographie: $\quad$ Prof. Dr. W. Kuhs

Erweiterte Prüfungskommission: Prof. Dr. L. Ackermann

Prof. Dr. U. Klingebiel 

"Nur wer weiß wo er herkommt, der weiß auch wo er war." 



\section{DANKSAGUNG}

Die vorliegende Arbeit wurde in der Zeit von Oktober 2004 bis März 2008 im Arbeitskreis von Prof. Dr. Dietmar Stalke an den Instituten für Anorganische Chemie der Universitäten Würzburg und Göttingen angefertigt.

An erster Stelle gilt mein Dank Herrn Prof. Dr. Dietmar Stalke für die interessante Themenstellung mit allen mir gewährten wissenschaftlichen Freiheiten und die Möglichkeit, meine Promotion im niedersächsischen Ausland anzufertigen.

Ein ganz besonderer Dank geht an Herrn Dr. Dirk Leußer für die vielen Einführungsstunden in die Kristallographie, für zahlreiche Geschichten aus 1001 Urlaub und dafür, dass er aus einem Sportmuffel einen begeisterten Läufer gemacht hat.

Bedanken möchte ich mich bei Herrn Dipl.-Chem. Daniel Stern für ein erfolgreiches F-Praktikum und die nachfolgende glückliche Anthracen-Zusammenarbeit. Ein großer Dank auch für die zahlreichen Spätschichten zum Messen von NMRs!

Ganz herzlich möchte ich mich bei Herrn Dr. Stephan Deuerlein bedanken, der auf alle Computer- und Laborfragen eine passende Antwort wusste oder besorgen konnte. Außerdem danke ich Herrn Daniel Kratzert für die nahtlose Übernahme von Stephans Admin-Amt und der Computerfragen-Hotline.

Ein großes Dankeschön geht an meine Mit-Heimatvertriebenen: Herrn Dipl.-Chem. Holger Ott für kompetente Hilfe am Refraktometer und außeruniversitäre Entspannung sowie Frau Ulrike Flierler für zahlreiche halb viers auf der richtigen Seite.

Bei Herrn Dipl.-Chem. Andreas Oechsner sowie Herrn Dipl.-Chem. Thomas Schulz und Frau Dipl.-Chem. Ina Objartel möchte ich mich für die stets angenehme und entspannte Laboratmosphäre bedanken.

Ich danke Herrn Dipl.-Chem. Christian Kling für aufmunternde "Moin"s und "Loift"s sowie für den Shuttle-Service zum Zug. Der gleiche Dank gilt Frau Dipl.-Chem. Margret Meinholz für ein sehr ertragreiches F-Praktikum und dafür, dass sie sich zum AK Stalke bekehren ließ. 
Meinen weiteren Abteilungs-Praktikanten vom AC-Fortgeschrittenen-Praktikum möchte ich für die angenehme Zusammenarbeit danken.

Unseren beiden Füchsen Nils Finkelmeier und Markus Granitzka danke ich dafür, dass ich die Anthracenbiester bzw. das LöMi-Amt in gute Hände weitergeben kann.

Bei unserem wandelnden Gmelin Herrn Dr. Michael "Guschdl" Witt bedanke ich mich für hilfreiche Ratschläge im Laboralltag. Außerdem danke ich ihm sowie Daniel* und Frau Dipl.-Chem. Katrin Gruß für das Korrekturlesen dieser Arbeit.

Bedanken möchte ich mich bei Herrn Maj. d. R. Hans-Georg Schmidt für ServiceMontagen und IPDS-Messungen während den obligatorischen Bruker-Stillstandzeiten und bei Herrn Martin Schlote für die lückenlose Versorgung mit Chemikalien sowie bei beiden für wertvolle Urlaubstipps im und um den Harz.

Dem gesamten Werkstatt-Team unter Leitung von Herrn Hans-Joachim Heymel danke ich für diverse Reparaturen und Neubauten sowie für das Organisieren unvergesslicher Wandertage. Des Weiteren bedanke ich mich bei den Glasbläsern Herrn Berthold Fertig und Herrn Hans-Joachim Schlette für das Flicken und Umbauen von Glasgeräten sowie für die Diskussionen über die aktuelle Lage.

Ich danke Herrn Dr. Rüdiger Bertermann, Herrn Wolfgang Zolke und Herrn Dr. Michael John für die Messung der NMR-Spektren. Der gleiche Dank gilt auch den Damen und Herren aus den Analytik-Abteilungen sowie den Hausmeistern und Sekretärinnen. Allen voran danke ich Frau Heike Tappe dafür, dass sie mir in den letzten Monaten eine Oase des Asyls geboten hat.

Ein ganz besonderer Dank gebührt Herrn Dipl.-Chem. Marco Kellert für unsere wunderbare Freundschaft und dafür, dass er unser gemeinsames Studium durch Kaffee und Späßchen sehr viel erträglicher gemacht hat.

Meiner Freundin Katrin danke ich dafür, dass ich mich immer auf sie verlassen kann und dass unsere Auslandsaufenthalte unserer Liebe kein bisschen geschadet haben. Und schließlich möchte ich mich bei meinen Eltern bedanken, die immer für mich da sind und ohne deren Unterstützung es nie zu dieser Arbeit gekommen wäre. 


\section{INHALTSVERZEICHNIS}

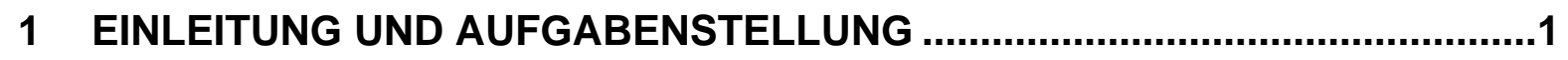

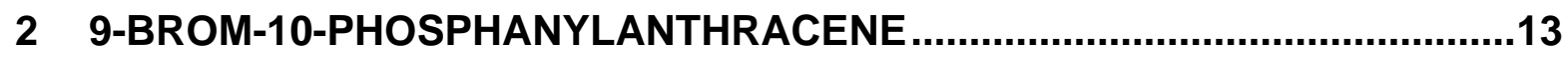

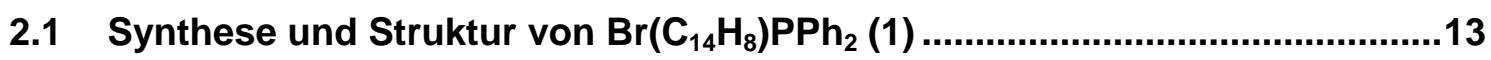

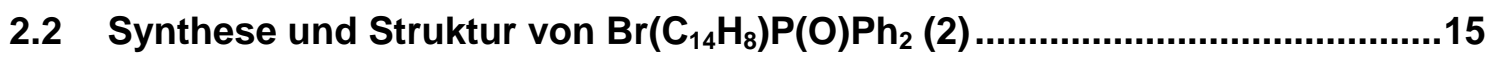

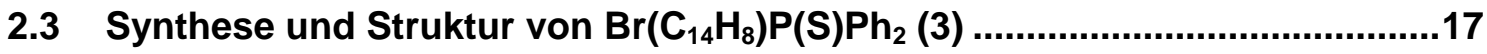

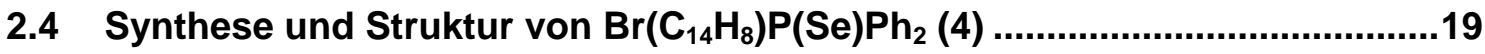

2.5 Synthese, Tieftemperatur-Verhalten und Struktur von $\operatorname{Br}\left(\mathrm{C}_{14} \mathrm{H}_{8}\right) \mathrm{P}^{i} \operatorname{Pr}_{2}(5) \ldots . .21$

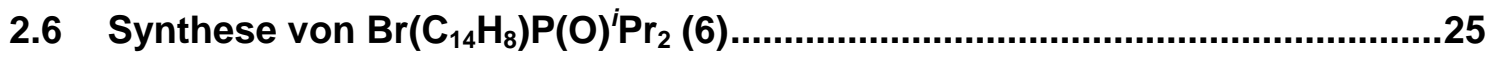

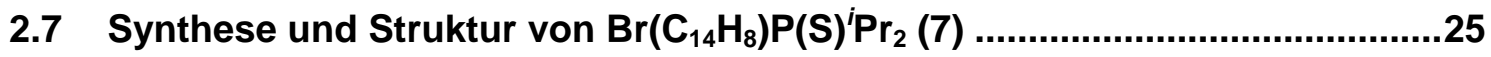

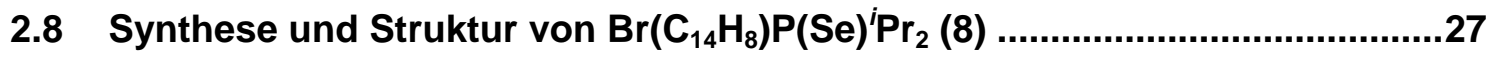

3 SYMMETRISCH SUBSTITUIERTE 9,10-DIPHOSPHANYLANTHRACENE....29

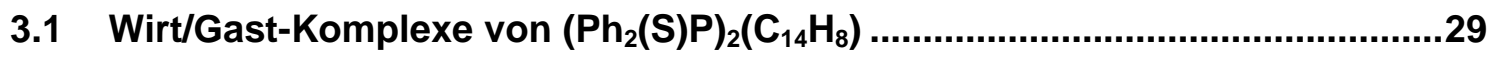

3.2 Synthese, Tieftemperatur-Verhalten und Struktur von $\left({ }^{i} \operatorname{Pr}_{2} P\right)_{2}\left(C_{14} H_{8}\right)(13) \ldots 38$

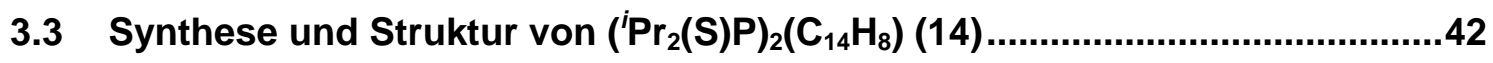

4 ASYMMETRISCH SUBSTITUIERTE 9,10-DIPHOSPHANYLANTHRACENE .45

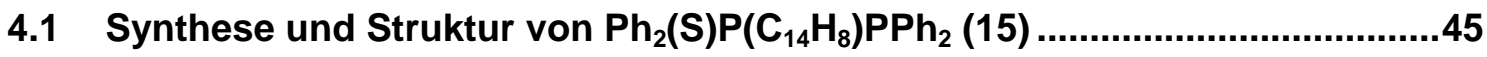

4.2 Synthese und Struktur von $\left[2 \mathrm{C}_{7} \mathrm{H}_{8} @\left\{\mathrm{Ph}_{2}(\mathrm{Se}) \mathrm{P}\left(\mathrm{C}_{14} \mathrm{H}_{8}\right) \mathrm{P}(\mathrm{S}) \mathrm{Ph}_{2}\right\}\right](16) \ldots . . . . .47$

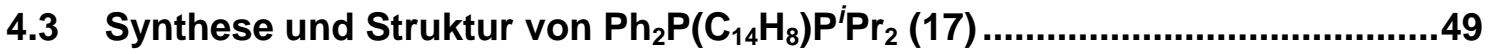

4.4 Synthesen und Strukturen der Oxidationsprodukte $\mathrm{Ph}_{2}(\mathrm{E}) \mathrm{P}\left(\mathrm{C}_{14} \mathrm{H}_{8}\right) \mathrm{P}(\mathrm{E})^{i} \mathrm{Pr}_{2}, \mathrm{E}=\mathrm{O}(18), \mathrm{S}(19)$ und Se (20)................................51

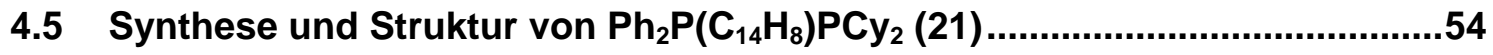

4.6 Synthese und Struktur von $\left[\mathrm{Ph}_{2}(\mathrm{~S}) \mathrm{P}\left(\mathrm{C}_{14} \mathrm{H}_{8}\right) \mathrm{P}(\mathrm{S}) \mathrm{Cy}_{2} \cdot \mathrm{C}_{7} \mathrm{H}_{8}\right](22) \ldots \ldots \ldots \ldots \ldots . . . . . . . . .55$ 
5.1 Synthese und Struktur von $\mathrm{Ph}_{2}(\mathrm{~S}) \mathrm{P}\left(\mathrm{C}_{14} \mathrm{H}_{8}\right) \mathrm{NH}_{2}(24)$ und $\left[\mathrm{Ph}_{2}(\mathrm{~S}) \mathrm{P}\left(\mathrm{C}_{14} \mathrm{H}_{8}\right)\left\{\mathrm{HNLi}(\mathrm{thf})_{3}\right\}\right](23)$ 58

5.2 Synthese und Struktur von $\operatorname{Br}\left(\mathrm{C}_{14} \mathrm{H}_{8}\right) \mathrm{P}\left(\mathrm{N}^{i} \mathrm{Pr}_{2}\right)_{2}(25)$. 61

5.3 Synthese und Struktur von $\mathrm{Py}_{2} \mathrm{P}\left(\mathrm{C}_{14} \mathrm{H}_{9}\right)(28)$ 63

6 UNERWARTETE REAKTIONEN .66

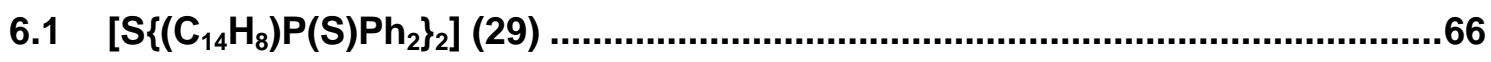

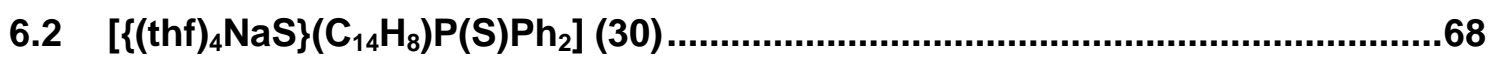

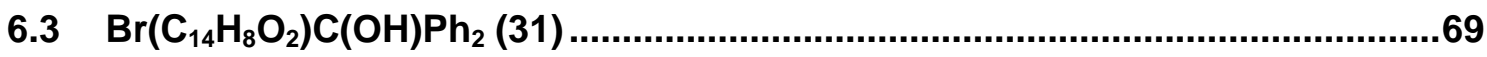

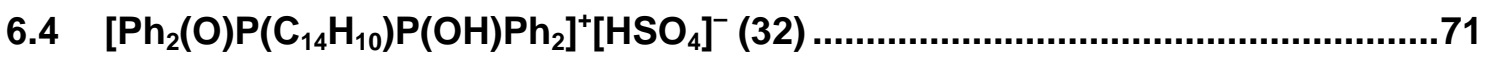

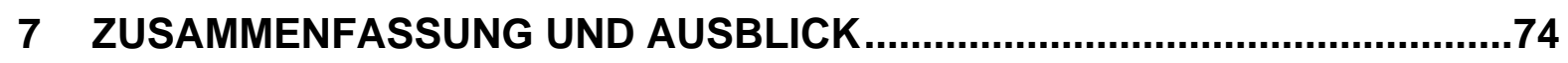

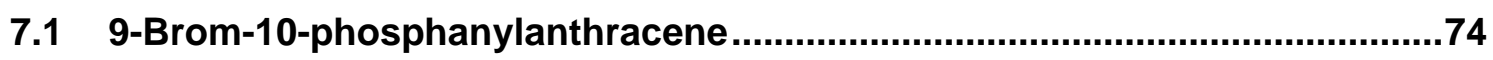

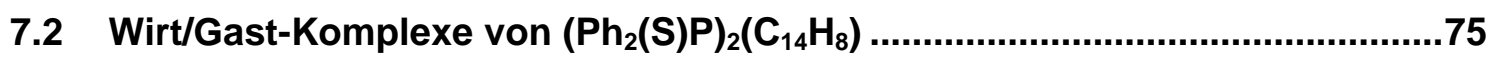

7.3 Asymmetrische 9,10-Diphosphanylanthracene ........................................77

7.4 Stickstoff-funktionalisierte Phosphanylanthracene ...................................78

7.5 Substituentenhängigkeit der Struktur des Anthracengerüstes....................79

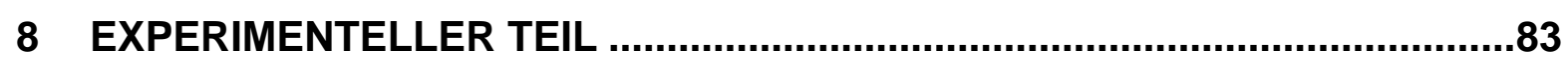

8.1 Allgemeine Arbeitstechniken und Analytische Methoden ..........................83

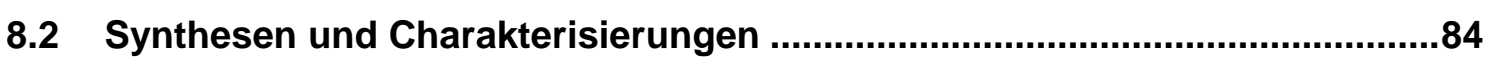

9 KRISTALLOGRAPHISCHER TEIL.....................................................106

9.1 Kristallapplikation und Datensammlung ...............................................106

9.2 Strukturlösung und Verfeinerung...........................................................108

9.3 Verfeinerung der Verbindungen 2 - 5, 7 - 8, 10 - 25, 28 - $32 \ldots \ldots \ldots \ldots \ldots \ldots \ldots . . . . . . . . . .110$

9.4 Kristallographische Servicemessungen .................................................137 


\section{ABKÜRZUNGSVERZEICHNIS}

\begin{tabular}{|c|c|c|c|}
\hline a & $\operatorname{Jahr}(\mathrm{e})$ & GoF & goodness of fit \\
\hline Abb. & Abbildung & $\mathrm{h}$ & Stunde(n) \\
\hline Abs. korr. & Absorptionskorrektur & i. Vak. & im Vakuum \\
\hline ADP & anisotropic displacement parameter & M & molar \\
\hline An & Anthryl / 9,10-Anthracendiyl & $\mathrm{Me}$ & Methyl \\
\hline $\mathrm{Ar}$ & Aromat(isch) & $\min$ & Minute(n) \\
\hline ber. & berechnet & MS & Massenspektrometrie \\
\hline bipy & 4,4'-Bipyridyl & Naph & Naphthalin / Naphthyl \\
\hline${ }^{n} \mathrm{Bu}$ & $n$-Butyl & n. b. & nicht bestimmt \\
\hline${ }^{t} \mathrm{Bu}$ & tert-Butyl & NMR & Nucular Magnetic Resonance \\
\hline CCD & charge coupled device & NOESY & Nuclear Overhauser Effect \\
\hline \multirow[t]{2}{*}{ CCDC } & Cambridge Crystallographic & & Spectroscopy \\
\hline & Data Centre & OTf $^{-}$ & Triflat $\left(\mathrm{CF}_{3} \mathrm{SO}_{3}^{-}\right)$ \\
\hline cosy & correlated spectroscopy & PAnP & 9,10-Bis(diphenylphosphanyl)- \\
\hline Cp & Cyclopentadienyl & & anthracen \\
\hline $\mathrm{Cp}^{*}$ & Pentamethylcyclopentadienyl & $\mathrm{Ph}$ & Phenyl \\
\hline Сy & Cyclohexyl & Pic & Picolyl \\
\hline d & $\operatorname{Tag}(e)$ & ppm & parts per million \\
\hline dest. & destilliert & ${ }^{i} \mathrm{Pr}$ & iso-Propyl \\
\hline Dipp & 2,6-Diisopropylphenyl & Py & 2-Pyridyl \\
\hline dppm & Bis(diphenylphosphanyl)methan & RT & Raumtemperatur \\
\hline El & Elektronenstoßionisation & SPAnPS & 9,10-Bis(diphenylthiophosphoryl)- \\
\hline Et & Ethyl & & anthracen \\
\hline ex & Anregung ("excitation") & Tab. & Tabelle \\
\hline$F_{\text {calc }}$ & berechneter Strukturfaktor & THF / thf & Tetrahydrofuran \\
\hline$F_{o b s}$ & beobachteter Strukturfaktor & Tripp & 2,4,6-Triisopropylphenyl \\
\hline g & gasförmig & UV & ultraviolett \\
\hline gef. & gefunden & w & Wichtungsfaktor \\
\hline Gl. & Gleichung & & \\
\hline
\end{tabular}




\section{IN DIESER ARBEIT DARGESTELLTE VeRBINDUNGEN:}<smiles>Brc1c2ccccc2c(-c2ccccc2)c2ccccc12</smiles>

1<smiles>FP=Pc1c2ccccc2c(Br)c2ccccc12</smiles>

$2 / 3$ / 4

$(E=0 / S / S e)$<smiles>CCCc1c2ccccc2c(Br)c2ccccc12</smiles>

5<smiles></smiles>

$6 / 7 / 8$ $(\mathrm{E}=\mathrm{O} / \mathrm{S} / \mathrm{Se})$<smiles>[X]c1ccccc1CCc1c2ccccc2c([PH](=S)c2ccccc2)c2ccccc12</smiles>

9 / 10 / 11 $(\mathrm{X}=\mathrm{Me} / \mathrm{H} / \mathrm{C} \equiv \mathrm{CH})$<smiles>CCCCc1c2ccccc2c(CCC)c2ccccc12</smiles>

13

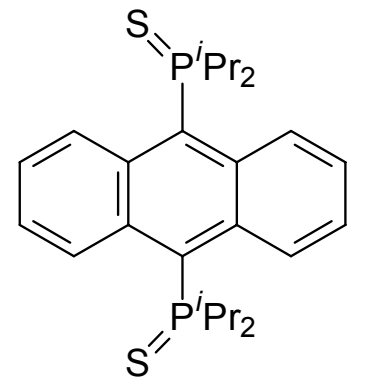

14<smiles>S=Pc1c2ccccc2c(-c2ccccc2)c2ccccc12</smiles>

15<smiles>S=[Pb]c1c2ccccc2c(P(c2ccccc2)c2ccccc2)c2ccccc12</smiles>

16<smiles>[X]c1cccc(C)c1</smiles><smiles>CCCCc1c2ccccc2c(-c2ccccc2)c2ccccc12</smiles>

17<smiles>FP=[PH+](P)c1c2ccccc2c(P=P)c2ccccc12</smiles>

$18 / 19 / 20$ $(E=0 / S / S e)$ 
<smiles>CCCCc1c2ccccc2c(-c2ccccc2)c2ccccc12</smiles>

21<smiles>S=Pc1c2ccccc2c(P(#[SH])c2ccccc2)c2ccccc12</smiles>

22<smiles>S=[PH](c1ccccc1)c1c2ccccc2c(NC2CCCCC2)c2ccccc12</smiles>

23<smiles>CCNCc1c2ccccc2cc2ccccc12</smiles>

26<smiles>Nc1c2ccccc2c([PH](=S)c2ccccc2)c2ccccc12</smiles>

24

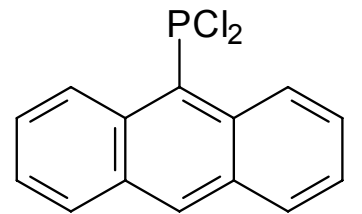

27<smiles>O=[PH](Nc1ccccc1)c1c2ccccc2c(Br)c2ccccc12</smiles>

25<smiles>CCCCCc1c2ccccc2cc2ccccc12</smiles>

28

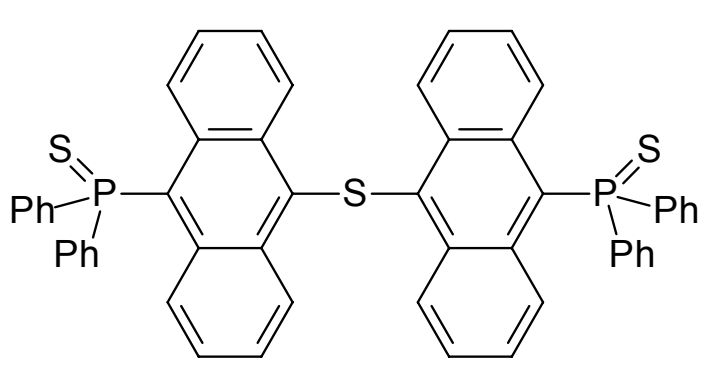

29<smiles>CCCCCCCCCCCNSc1c2ccccc2c([PH](=S)c2ccccc2)c2ccccc12</smiles>

30<smiles>OC(c1ccccc1)(c1ccccc1)C1(c2ccccc2)OOC2(Br)c3ccccc3C21O</smiles>

31

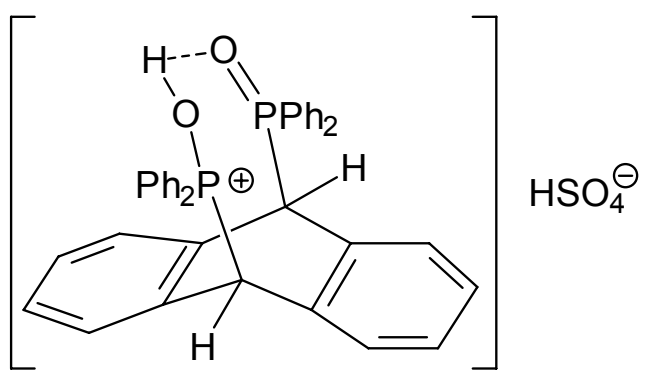

32 



\section{EINLEITUNG UND AUfGABENSTELLUNG}

\section{Entdeckung und Verwendung des Phosphors}

Im 17. Jahrhundert stieß der Hamburger Alchimist Hennig Brand auf der Suche nach dem Stein der Weisen auf eine Versuchsvorschrift, nach der angeblich Silber in Gold verwandelt werden konnte. ${ }^{[1]}$ Als er zu diesem Zwecke Urin faulen ließ, bis zur Trockene eindampfte und den Rückstand unter Luftabschluss glühte, erhielt er ein "im Dunkeln leuchtendes Produkt" (Abb. 1-1).

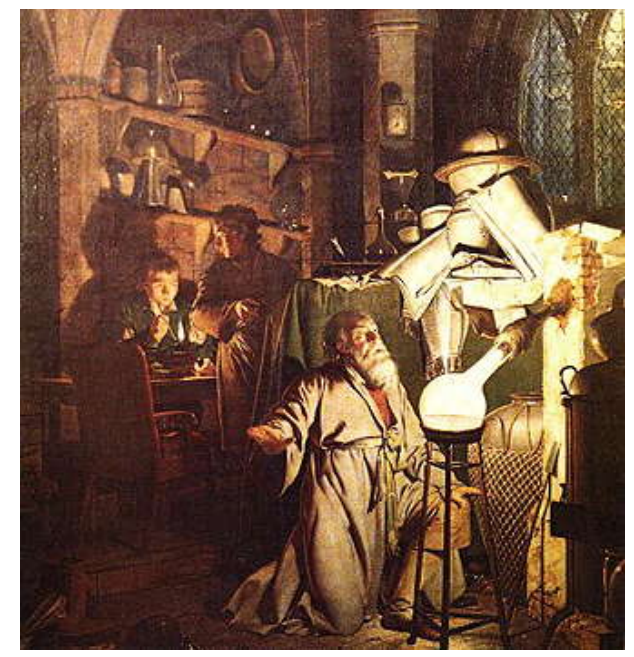

Abb. 1-1: Entdeckung des Phosphors durch Hennig Brand (Öl auf Leinwand, Joseph Wright, 1771).

Brand nannte den von inm entdeckten Stoff "kaltes Feuer" oder "mein Feuer" und verwendete in seinen nachfolgenden Briefen dafür häufig das Zeichen $\Delta$, das in der alchimistischen Literatur für das Element Feuer üblich war. 1676 wurde die Brand'sche Erfindung von Elsholz ${ }^{[2]}$ erstmalig als Phosphor bezeichnet (griech.:

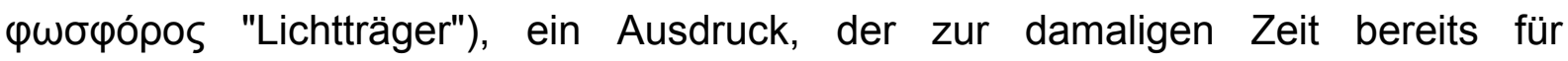
verschiedenartigste Leuchtstoffe wie etwa den Bologneser Leuchtstein (BaS) gebräuchlich war. Die von Brand beschriebene Methode, bei der das im Harn enthaltene Phosphorsalz $\mathrm{NaNH}_{4} \mathrm{HPO}_{4}$ beim Glühen von dem durch Verkohlung organischer Substanzen entstandenem Kohlenstoff zu weißem $\mathrm{P}_{4}$ reduziert wurde, blieb für lange Zeit der einzige Darstellungsweg für Phosphor. 1867 gelang es schließlich Aubertin und Boblique das Element durch Erhitzen von Phosphatmineralien mit Sand und Koks im Ofen zu erzeugen. ${ }^{[3]}$ Dieses Verfahren wird auch heute noch unter Verwendung von elektrischen Lichtbogenöfen zur großindustriellen Reduktion von Apatiten $\mathrm{Ca}_{5}\left(\mathrm{PO}_{4}\right)_{3}(\mathrm{OH}, \mathrm{F}, \mathrm{Cl})$ zu weißem Phosphor angewendet, welcher anschließend noch durch Destillation gereinigt und in Stangenform 
gegossen wird. Mehr als $90 \%$ des erzeugten elementaren Phosphors werden zu Phosphorpentaoxid verbrannt, das als Ausgangsmaterial für die Phosphorsäureherstellung sowie für die Darstellung verschiedener Phosphate verwendet wird. Der weitaus kleinere Teil findet in der Produktion von Phosphorchloriden und phosphororganischen Verbindungen sowie in der Zündholzfabrikation Verwendung. ${ }^{[4-6]}$

\section{Entdeckung und Verwendung des Anthracens}

Im Jahr 1832 isolierten Dumas und Laurent aus Steinkohleteer durch Destillation einen bis dahin unbekannten Kohlenwasserstoff und bezeichneten diesen zunächst als "Paranaphthalin", da sie inn für isomer mit Naphthalin hielten. ${ }^{[7,8]}$ Nach weiteren Untersuchungen sah sich Laurent fünf Jahre später gezwungen, den Namen in "Anthracen" (griech.: anthrax "Kohle") zu ändern. ${ }^{[9,10]}$

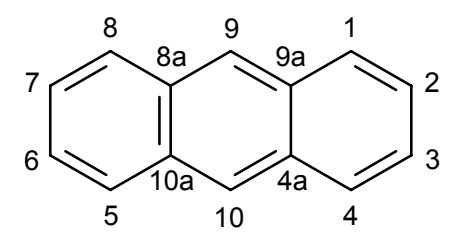

Abb. 1-2: Nummerierung des Anthracenkohlenstoffgerüstes.

1840 stellte Laurent durch Umsetzungen von Anthracen mit Salpetersäure einige Derivate wie beispielsweise Anthrachinon dar, ${ }^{[11]}$ dennoch geriet die Entdeckung zunächst wieder in Vergessenheit. In den 1860er Jahren wurde die gleiche Substanz von Fritzsche erneut entdeckt und als eine Mischung zweier neuer Kohlenwasserstoffe beschrieben, die er als "Photen" und "Phosen" bezeichnete. ${ }^{[12-14]}$ Mit diesen Namen wollte er deren "Verhalten gegen das Licht" andeuten und bereits 1892 konnte Linebarger das Dimer von Anthracen als das Belichtungsprodukt identifizieren. ${ }^{[15]}$ Die ausführlichsten Untersuchungen des neuen Kohlenwasserstoffes im 19. Jahrhundert gehen auf Anderson zurück, der sich unter anderem mit der Nitrierung, Bromierung und Chlorierung des Aromaten beschäftigte. ${ }^{[16]}$ Die erste chemische Anthracensynthese wurde 1866 von Limpricht beschrieben, wobei Benzylchlorid und Wasser für acht Stunden auf $190^{\circ} \mathrm{C}$ erhitzt wurden. ${ }^{[17]} 1871$ veröffentlichten Graebe und Liebermann einen Artikel über Anthracenderivate, in dem sie unter anderem die Darstellung von Anthracen aus dem Farbstoff Alizarin und Zinkstaub beschrieben. ${ }^{[18]}$ Anschütz und Eltzbacher stellten 1883 "eine neue Synthese des Anthracens" vor, bei der Benzol unter $\mathrm{AlCl}_{3}$-Katalyse mit Tetrabromethan umgesetzt wurde. ${ }^{[19]}$ 
Heute wird Anthracen industriell aus Steinkohleteer gewonnen und fast ausschließlich zu Anthrachinon weiterverarbeitet, das zur Synthese von Wasserstoffperoxid ("Anthrachinonverfahren" der BASF) sowie als Edukt zur Synthese von Farbstoffen wie z. B. Alizarinrot verwendet wird. Eine weitere Verwendung findet Anthracen bei der Herstellung von Schädlingsbekämpfungsmitteln und Gerbstoffen. ${ }^{[20,21]}$ Mittlerweile wurde eine große Zahl an Anthracenderivaten synthetisiert und die Effekte unterschiedlicher Substituenten, vor allem Halogen-, Alkyl-, Aryl-, Cyano-, Oxy- und Silylgruppen, untersucht. ${ }^{[22]}$ Dabei zeigte sich, dass vor allem Substituenten in den Positionen 9 und 10 den größten sterischen, elektronischen und elektrostatischen Einfluss haben. ${ }^{[23]}$

\section{Phosphanylanthracene}

Bereits 1948 untersuchte Mikhailov Reaktionen zur Metallierung von Bromanthracenen. ${ }^{[24]}$ Dabei zeigte sich, dass die Lithiierung von 9,10-Dibromanthracen mit elementarem Lithium je nach eingesetztem Stoffmengenverhältnis zum Austausch eines oder beider Bromatome durch Li führt (GI. 1-1).

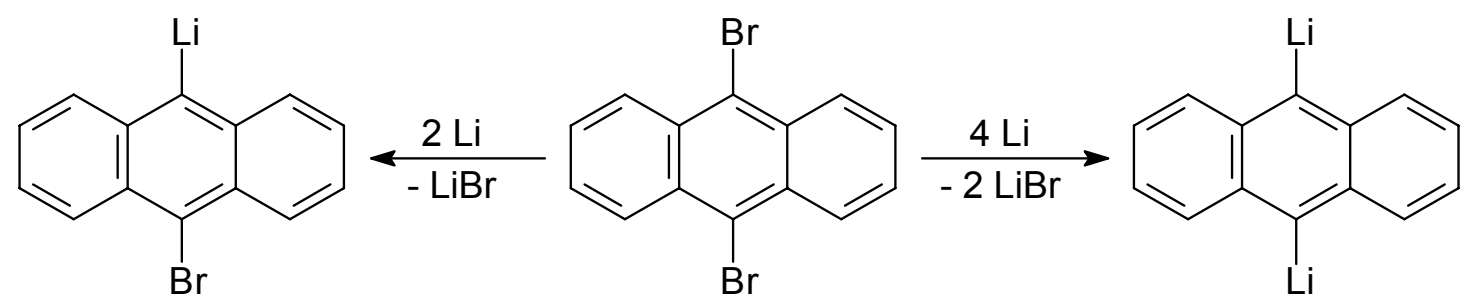

GI. 1-1: Lithiierungsreaktionen von 9,10-Dibromanthracen nach Mikhailov. ${ }^{[2]}$

Die so erhaltenen lithiierten Anthracene wurden anschließend mit Trockeneis umgesetzt und so die entsprechenden Anthrylcarbonsäuren erhalten. Es stellte sich heraus, dass die Verwendung von $n$-Butyllithium als Lithiierungsreagenz zu besseren Selektivitäten und höheren Ausbeuten führte (GI. 1-2).<smiles>Clc1c2ccccc2c(Br)c2ccc(Br)cc12</smiles>

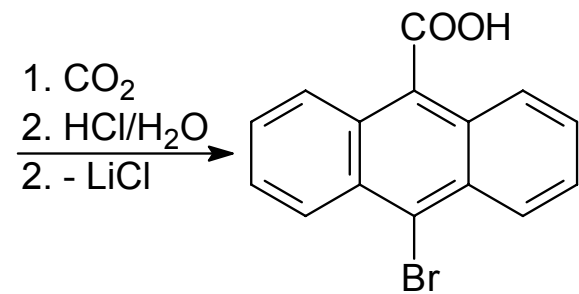

GI. 1-2: Mono-Lithiierung von 9,10-Dibromanthracen und anschließende Umsetzung mit $\mathrm{CO}_{2}$.

Die ersten Anthracenderivate mit Phosphorsubstituenten in Position 9 wurden 1950 von Mikhailov und Kucherova beschrieben. ${ }^{[25,26]}$ Dabei wurden 9-Lithiumanthracenide 
mit Trichlorphosphan umgesetzt und unter Lithiumchlorid-Eliminierung die entsprechenden Tris(anthryl)phosphane erhalten (GI. 1-3).

3

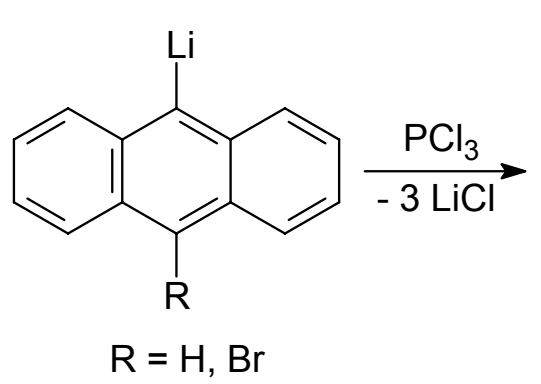

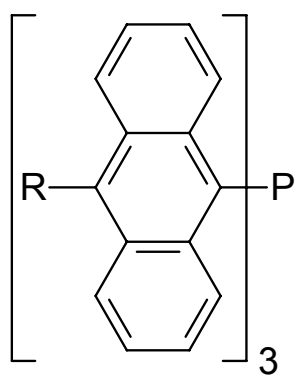

GI. 1-3: Darstellung der ersten Phosphanylanthracene. ${ }^{[25]}$

Auf der Suche nach einem Chemosensor für Hydroperoxide synthetisierte die Arbeitsgruppe Akasaka im Jahr 1987 aus Triphenylphosphan, tert-Butyllithium und 9-Bromanthracen das entsprechende 9-Phosphanylanthracenderivat in einer Ausbeute von $9 \%$ (GI. 1-4). ${ }^{[27]}$ Während das Anthrylphosphan keinerlei Fluoreszenz bei Bestrahlung mit UV-Licht zeigte, führte die Oxidation durch Wasserstoffperoxid zum Auftreten von blauer Lumineszenz in Lösung.

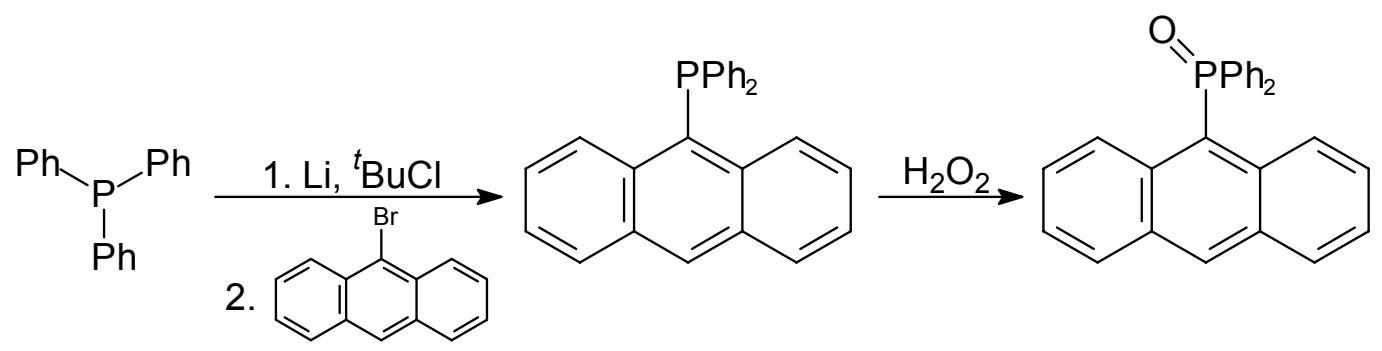

GI. 1-4: Synthese von 9-Diphenylphosphorylanthracen nach Akasaka et al. ${ }^{[27]}$

Ab 1989 beschäftigte sich die Arbeitsgruppe Schmutzler mit phosphanylsubstituierten Naphthalin- und Anthracenderivaten. So wurde beispielsweise 9-Difluorphosphanylanthracen synthetisiert (GI. 1-5), ein Organodifluorphosphan, das stabil gegenüber Redox-Disproportionierung ist und durch Sublimation bei $160{ }^{\circ} \mathrm{C}$ $\left(10^{-3}\right.$ Torr) gereinigt werden konnte. Durch photochemische Anregung wurde anschließend das entsprechende Kopf-Schwanz-Dimer erhalten. ${ }^{[28]}$

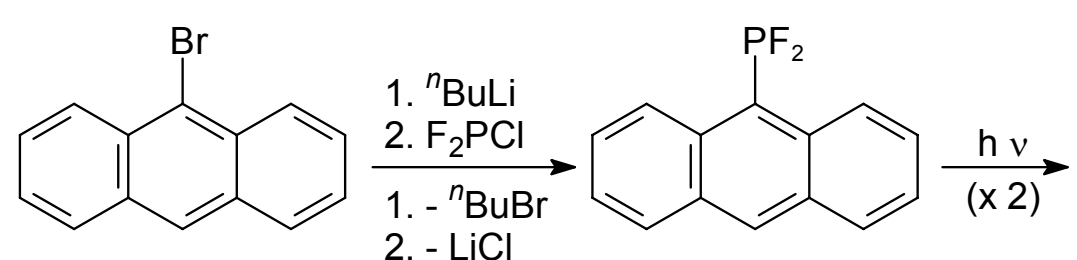

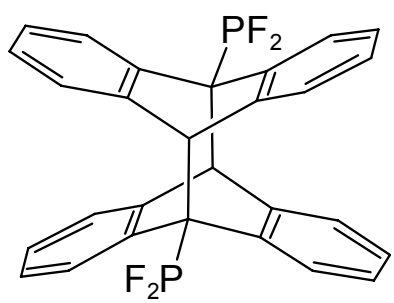

GI. 1-5: Darstellung von 9-Difluorphosphanylanthracen mit anschließender Dimerisierung. ${ }^{[28]}$ 
Analog stellten Schmutzler et al. kurze Zeit später das bereits von Akasaka beschriebene 9-Diphenylphosphanylanthracen aus Lithiumanthracenid und Chlordiphenylphosphan dar und steigerten die Ausbeute der Synthese damit auf $71 \%$. Durch Bestrahlung der Verbindung in Gegenwart von ( $\eta^{2}-1$-Cycloocten)pentacarbonylwolfram wurde der entsprechende $(\mathrm{OC})_{5} \mathrm{~W}$-Komplex erhalten und strukturell charakterisiert. ${ }^{[29]}$ In den folgenden Jahren wurden von der Arbeitsgruppe Schmutzler noch zahlreiche weitere mono-, bis- und trisanthrylsubstituierte Phosphane sowie entsprechende Naphthalinderivate synthetisiert. ${ }^{[30-35]}$

Kubiak et al. gelang 1999 die Synthese von 1-(9-Anthryl)phosphiran durch Umsetzung eines dilithiierten Phosphanylanthracens mit 1,2-Dichlorethan (GI. 1-6). Durch Reaktion des Phosphirans mit ( $\eta^{2}-1,5$-Dicyclooctadien)dichloroplatin wurde außerdem ein quadratisch-planarer Pt(II)-Komplex erhalten, in dem das Metall cisständig von zwei Phosphiranliganden koordiniert ist, die eine koplanare Anordung der Anthracengerüste mit einem Abstand von 340 pm aufweisen. ${ }^{[36,37]}$

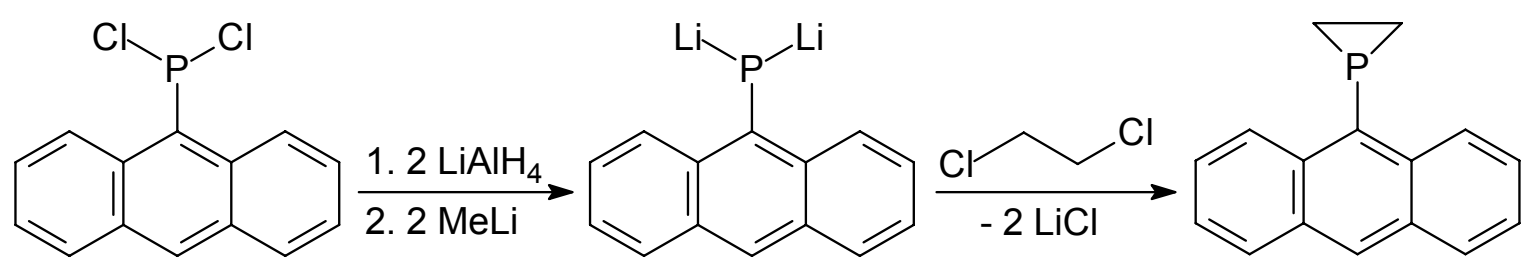

GI. 1-6: Synthese von 1-(9-Anthryl)phosphiran nach Kubiak. ${ }^{[36]}$

2001 berichteten Yip und Prabhavathy über die Synthese von 9,10-Bis(diphenylphosphanyl)anthracen (PAnP), das durch die Umsetzung von 9,10-Dibromanthracen mit jeweils zwei Äquivalenten ${ }^{n} \mathrm{BuLi}$ und $\mathrm{Ph}_{2} \mathrm{PCl}$ erhalten wurde. Bei der Reaktion von PAnP mit $\left(\mathrm{Me}_{2} \mathrm{~S}\right) \mathrm{AuCl}$ wurde ein dreikerniger Gold(I)-Komplex der Zusammensetzung $\left[\mathrm{Au}_{3}(\mathrm{PAnP})_{3}\right]\left[\mathrm{ClO}_{4}\right]_{3}$ isoliert (GI. 1-7) und durch NMR- und Röntgenstrukturuntersuchungen charakterisiert. ${ }^{[38]}$

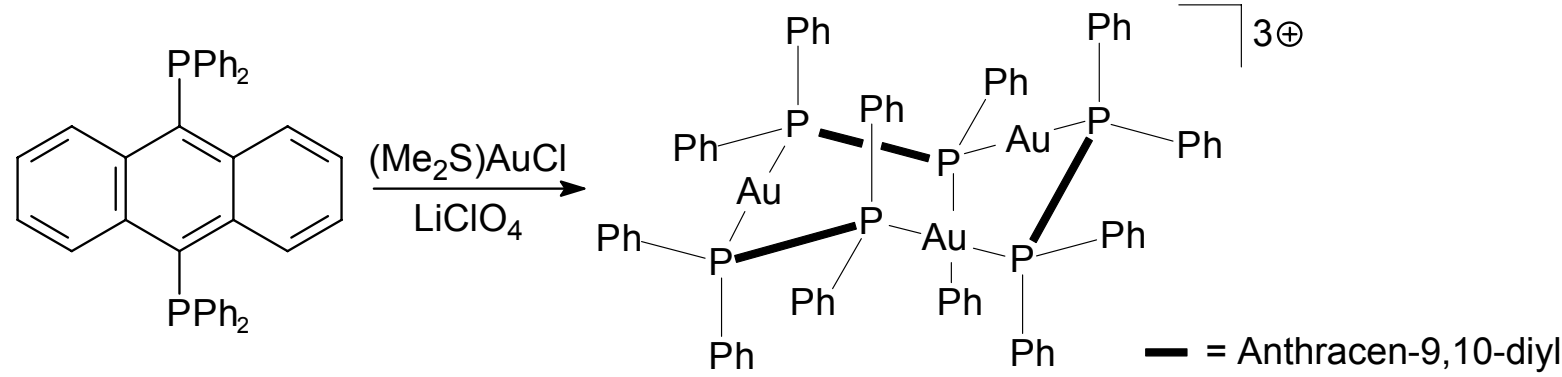

GI. 1-7: Umsetzung von PAnP und $\left(\mathrm{Me}_{2} \mathrm{~S}\right) \mathrm{AuCl}$ zu einem Gold(I)-Komplex. ${ }^{[38]}$ 
Demnach liegt das Kation als ein Heterozyklus vor, der aus drei $\mathrm{Au}(\mathrm{l})$-lonen und drei verbrückenden PAnP-Liganden mit nahezu linearen P-Au-P-Anordnungen besteht. Das Molekül liegt in einer cyclohexanartigen Sesselkonformation vor und im ${ }^{1} \mathrm{H}$ NMR-Spektrum werden bei $228 \mathrm{~K}$ zwei unterschiedliche Signalsätze für die axial und äquatorial angeordneten Phenylgruppen beobachtet.

In den folgenden Jahren wurden noch zwei weitere Gold- und Rutheniumkomplexe von PAnP untersucht. ${ }^{[39,40]}$ Darauf basierend synthetisierten Yip et al. 2004 den bipyridylverbrückten Komplex $\left[\mathrm{Au}_{4}(\mu \text {-PAnP })_{2}(\mu \text {-bipy })_{2}\right]^{4+} \quad($ Abb. 1-3), der eine rechteckige Molekülstruktur aufweist. Ferner wurde eine ganze Reihe von Einschluss-Verbindungen dieses Moleküls mit aromatischen Gästen wie Naphthalin, Phenanthren und unterschiedlich substituierten Anthracenen erhalten. Die Wirt/GastKomplexe zeichnen sich dadurch aus, dass die Gastmoleküle zwischen den beiden parallel angeordneten Bipyridyl-Liganden eingelagert werden und dass die Fluoreszenzeigenschaften der Verbindung in Lösung durch die Komplexierung beeinflusst werden. ${ }^{[41]}$

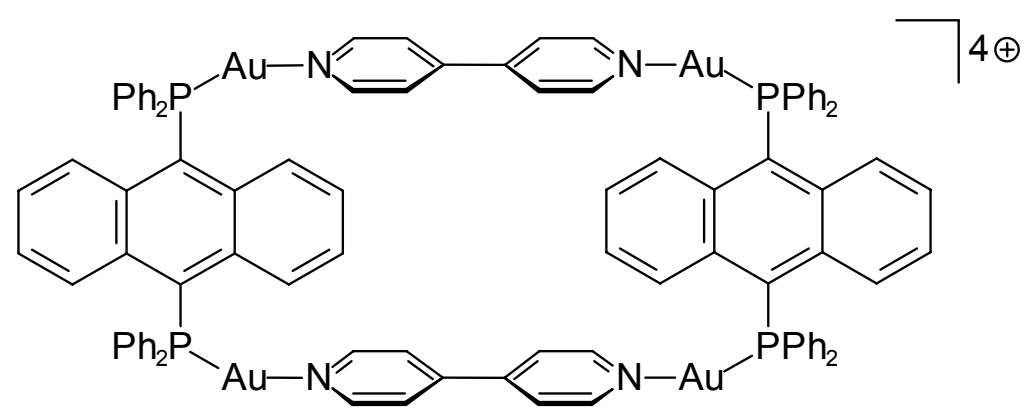

Abb. 1-3: $\left[\mathrm{Au}_{4}(\mu-\mathrm{PAnP})_{2}(\mu \text {-bipy })_{2}\right]^{4+}$ nach Yip et al. ${ }^{[41]}$

2006 und 2007 berichteten Yip et al. von weiteren Silber(I)- und Platin(II)-Komplexen mit dem PAnP-Liganden, die eine große strukturelle Vielfalt zeigen. So konnten durch Umsetzungen von PAnP mit AgX-Salzen ( $\mathrm{X}=\mathrm{OTf}^{-}, \mathrm{ClO}_{4}^{-}, \mathrm{PF}_{6}^{-}$und $\mathrm{BF}_{4}^{-}$) sowohl polymere als auch bi-, tri- und tetranukleare Metallacyclen erhalten werden, die aus Gleichgewichten der verschiedenen Komplexe in Lösung hervorgehen. ${ }^{[42]}$ Durch Metallierung von PAnP mit zwei Äquivalenten $\left[\mathrm{Pt}(\mathrm{dppm})(\mathrm{OTf})_{2}\right]$ wurde ein Gemisch aus den anti- und syn-Isomeren erhalten (Metallierung in den Positionen 1, 5 bzw. 1,4), die durch fraktionierte Kristallisation getrennt und röntgenkristallographisch untersucht werden konnten. ${ }^{[43]}$ 
Weniger etabliert als die Chemie der 9-Phosphanylanthracene ist die der 1,8disubstituierten Derivate, die seit 1991 untersucht wurden. ${ }^{[44,45]}$ In Abb. 1-4 sind drei Beispiele von bisher bekannten 1,8-Diphosphanylanthracenen dargestellt.<smiles></smiles><smiles></smiles><smiles></smiles>

Abb. 1-4: Beispiele für 1,8-di- und 1,8,9-triphosphanylsubstituierte Anthracene..$^{[46-48]}$

Bisherige Untersuchungen zeigten, dass die Verbindungen als PCP-Pincer-Liganden Metallfragmente in Position 9 stablisieren können. Derartige Iridium-, Nickel- und Palladiumkomplexe wurden bereits erfolgreich als Katalysatoren für die Dehydrogenierung von Alkanen ${ }^{[46]}$ sowie für die Allylierung von Aldehyden und Iminen untersucht. ${ }^{[49]} 2007$ berichteten Kilian et al. über interessante Molekülstrukturen von 1,8,9-triphosphanylsubstituierten Anthracenen. ${ }^{[48]}$

Alkylsubstituierte Phosphane werden üblicherweise durch Umsetzung von Chlorphosphanen mit metallorganischen Reagenzien darstellt. Dabei kommen insbesondere Phenylsubstituenten zum Einsatz, da Triphenylphosphan leicht aus Chlorbenzol, Natrium und $\mathrm{PCl}_{3}$ erhalten werden kann. Die thermische Synproportionierung von $\mathrm{PPh}_{3}$ und $\mathrm{PCl}_{3}$ führt zu $\mathrm{Ph}_{2} \mathrm{PCl}$ und $\mathrm{PhPCl}_{2}$ und wird industriell im großen Maßstab genutzt. ${ }^{[50]}$ Der Einsatz von Chlorphosphanen ist allerdings häufig mühsam, da sich diese oft durch unangenehmen Geruch, niedrige Schmelzpunkte und Toxizität auszeichnen und hydrolyseunbeständig sind. Bei der Suche nach alternativen Phosphansynthesen gelang es der Arbeitsgruppe Straub im Jahr 2006 tertiäre Phosphane mit jeweils drei unterschiedlichen Resten ausgehend vom preisgünstigen und leicht handhabbaren $\mathrm{P}(\mathrm{OPh})_{3}$ ("Triphenylphosphit") darzustellen. ${ }^{[51]}$ Voraussetzung dabei ist jedoch, dass im ersten Substitutionsschritt ein sterisch anspruchvolles Metallorganyl verwendet wird, um Mehrfachsubstitution zu vermeiden. GI. 1-8 zeigt den schrittweisen Aufbau von 9-Anthryl-1-naphthylphenylphosphan. 


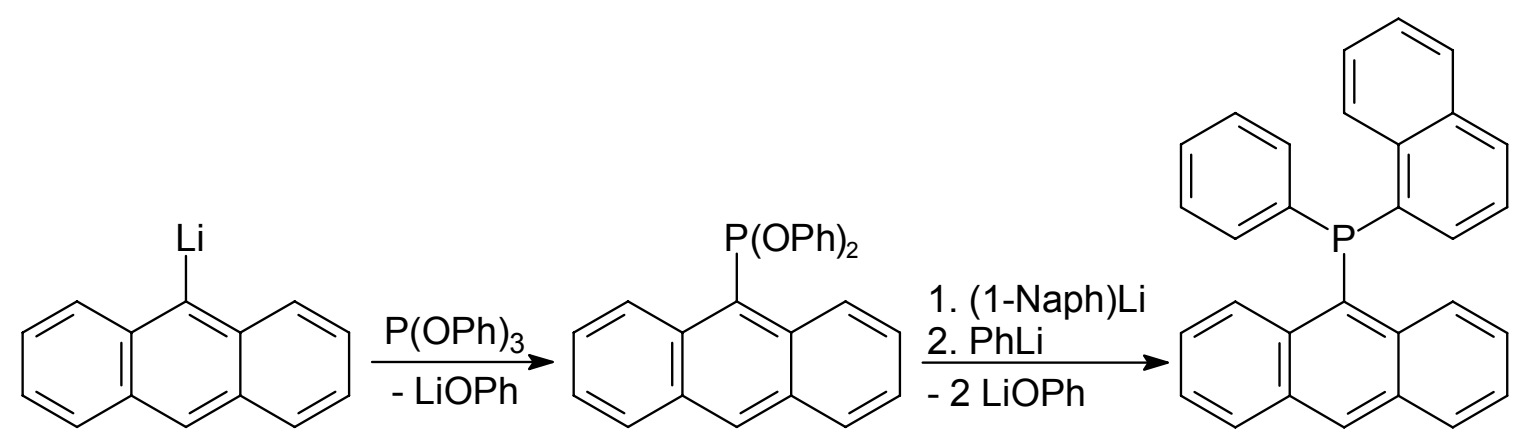

GI. 1-8: Synthese eines tertiären Phosphans ausgehend von Triphenylphosphit. ${ }^{[51]}$

\section{C-N-Bindungsknüpfungen}

Die Synthese von Arylaminen über $\mathrm{C}-\mathrm{N}$-Bindungsknüpfungsreaktionen hat in den letzten Jahren auch im Bereich der mehrkernigen aromatischen Kohlenwasserstoffen zunehmend an Bedeutung gewonnen. Bereits 1967 wurde die Umsetzung von 9-Bromphenanthren mit Natriumamid in flüssigem Ammoniak beschrieben, bei der das entsprechende 9-Phenanthrylamin erhalten wurde. ${ }^{[52]}$ In der Folgezeit wurde an verschiedenen Arylhalogeniden gezeigt, dass die Ausbeuten und Selektivitäten der Aminierungsreaktionen durch Zugabe katalytischer Mengen Eisen- oder Kupfersalze erhöht werden können. ${ }^{[53-55]}$

Buchwald et al. entwickelten 1996 ein verbessertes katalytisches System für die Knüpfung von aromatischen $\mathrm{C}-\mathrm{N}$-Bindungen, bei dem der Katalysator aus einer Mischung von $\mathrm{Pd}(0)$ und BINAP [2,2'-Bis(diphenylphosphanyl)-1,1'-binaphthyl] besteht. ${ }^{[56]}$ Im Katalysezyklus kommt es zu einer oxidativen Addition des Arylbromids an den gebildeten BINAP-Pd-Komplex mit anschließender Bildung eines AminAdduktes. Nach Salzeliminierung kommt es zu einer Umlagerung und schließlich unter reduktiver Eliminierung des Arylamins zur Rückbildung der aktiven BINAP-PdSpezies. Diese Methode hat sich seitdem an einer Vielzahl von Substraten bewährt und ist mittlerweile fester Bestandteil der präparativen Chemie. ${ }^{[57,58]}$ Auch sekundäre 9-Anthrylamine konnten so unter milden Bedingungen synthetisiert werden. ${ }^{[59-61]}$

Bis vor Kurzem war die Palladium-katalysierte Umsetzung von Ammoniak zu primären aromatischen Aminen noch unbekannt, diese Lücke konnte jedoch 2006 von Shen und Hartwig durch die Verwendung eines Diphosphanliganden mit Ferrocenylgerüst ("Josiphos-Ligand") ${ }^{[62]}$ geschlossen werden. ${ }^{[63]}$ Auch Lithiumamid kann mit Arylhalogeniden unter Zusatz dieses Katalysators zu Arylaminen gekuppelt werden. 


\section{Leuchtstoffe und Chemosensoren}

Anorganische Feststoffe lassen sich allgemein in Licht absorbierende Pigmente und Licht emittierende Leuchtstoffe unterteilen. Dabei besteht ein typischer anorganischer Leuchtstoff aus einem Wirt und einem sogenannten Aktivator, welcher den Wirt zum Leuchten aktiviert. Der wichtigste Vorteil dieser Kombination ist, dass die Art und die Menge des Aktivators genau eingestellt werden kann. Da konjugierte organische Moleküle wie Anthracene, Pyrene und Perylene sehr hohe Quantenausbeuten aufweisen, werden häufig anorganische und organische Bestandteile in einem Leuchtstoff vereint. ${ }^{[64]}$ In den letzten Jahren wurde eine Vielzahl von phosphorhaltigen Chromophoren synthetisiert, ${ }^{[6]}$ speziell funktionalisierte Anthracenderivate können für Anwendungen im Bereich der Feldeffekttransistoren sowie Leuchtdioden (LED = light emitting diode) eingesetzt werden. ${ }^{[66]}$ Zur Lichtemission kommt es, wenn ein Elektronen-Übergang von einem angeregten zu einem energieärmeren Zustand unter Freiwerden von Energie im sichtbaren Spektralbereich abläuft. Dieser Prozess wird allgemein als Lumineszenz bezeichnet und abhängig von der Art der Anregung wird zwischen Photolumineszenz, Radiolumineszenz, Chemilumineszenz, Elektrolumineszenz, Tribolumineszenz usw. unterschieden. ${ }^{[67]}$ Unter Fluoreszenz versteht man eine Lumineszenz im sichtbaren Emissionsbereich, wobei der Übergang zwischen Elektronenzuständen gleicher Spinmultiplizität und somit sehr schnell (Lebensdauer: $10^{-9}$ bis $10^{-3} \mathrm{~s}$ ) erfolgt. Phosphoreszenz erfolgt ebenfalls im sichtbaren Spektralbereich, allerdings zwischen elektronischen Zuständen unterschiedlicher Spinmultiplizität $\left(T_{1} \rightarrow S_{0}\right)$. Da dieser Übergang spinverboten ist und nur durch die Spin-Bahn-Kopplung ermöglicht wird, erfolgt er sehr langsam (Lebensdauer: $10^{-3}$ bis $100 \mathrm{~s}$ ), sodass die Phosphoreszenz auch noch nach dem Abschalten der Strahlungsquelle zu beobachten ist. ${ }^{[68,69]}$

Unter einem Sensor versteht man eine Einheit, die mit Materie oder Energie unter Erzeugung eines messbaren Signals wechselwirkt. In diesem Zusammenhang unterscheidet man zwischen Chemosensoren als Moleküle abiotischer Herkunft und Biosensoren als Verbindungen biotischen Ursprungs. ${ }^{[70]}$ Ein wichtiges Prinzip beim Design neuartiger Chemosensoren ist, dass die Erkennung des Zielmoleküls mit einem leicht zu messenden Signal verbunden sein muss. Beispielsweise wurden Wirtsmoleküle synthetisiert, die die Anwesenheit eines bestimmten Gastes durch Änderungen der Farbe oder der spektroskopischen (UV, NMR) bzw. elektrochemischen Eigenschaften (Redoxpotential) anzeigen. Ändern sich bei der Target- 
Erkennung die Fluoreszenzeigenschaften des Sensors spricht man von fluoreszierenden Chemosensoren. ${ }^{[71]}$

In den letzten Jahren wurden zahlreiche derartige Chemosensoren untersucht, die hauptsächlich der Detektion von Alkali-, Erdalkali- ${ }^{[72,73]}$ und Übergangsmetallkationen des $\mathrm{d}^{\left[{ }^{[7,75]}\right.}$ und f-Blocks dienen. ${ }^{[76]}$ Mit neueren Entwicklungen können auch Anionen wie Halogenide, Acetat oder Dihydrogenphosphat ${ }^{[77]}$ sowie neutrale Moleküle wie Schwefeldioxid nachgewiesen werden. ${ }^{[78]}$ Viele der untersuchten Erkennungsreaktionen erfolgen in Lösung und basieren auf dem sogenannten PET-Effekt (photoinduzierter Elektronentransfer). ${ }^{[79]}$ Ein fluoreszierender Chemosensor muss einerseits über einen Rezeptor verfügen, der mit dem Gastmolekül chemisch reagieren kann, und andererseits über einen Fluorophor, welcher mit Photonen geeigneter Wellenlänge wechselwirkt. Diese beiden Elemente sind überlicherweise über einen Spacer miteinander verbunden. Durch die Anlagerung des Gastmoleküls an den Rezeptor ändert sich die elektronische Situation des Komplexes, was zum Verschieben der Fluoreszenzwellenlänge in den sichtbaren Bereich führt. ${ }^{[80]}$

Es ist sehr wenig bekannt über den Einfluss der Kristallpackung von Fluorophoren auf deren Fluoreszenzeigenschaften im Festkörper ${ }^{[81]}$ Ein wesentlicher Faktor dabei sind intermolekulare Desaktivierungsmechanismen, bei denen die Anregungsenergie von einem Molekül auf ein anderes, den sog. Quencher oder Löscher, übertragen wird. Dieser Vorgang wird allgemein als Quenching (Anregungslöschung) bezeichnet, wobei speziell die Unterdrückung der Lichtemission durch Energieübertragungsprozesse als Strahlungs- bzw. Fluoreszenzlöschung bezeichnet wird. ${ }^{[69]}$ Häufig werden Wasserstoffbrückenbindungen zwischen benachbarten Fluorophoren ${ }^{[82]}$ oder intermolekulare $\pi-\pi$-Wechselwirkungen als Ursachen des Quenchings im Festkörper genannt. ${ }^{[83-85]}$ Durch den Einbau von Gastmolekülen in ein Kristallwirtsgitter kann die An- und Abwesenheit dieser zwischenmolekularen Wechselwirkungen und die daraus resultierenden Lumineszenzeigenschaften beeinflusst werden. Eine andere Möglichkeit ist das Auftreten von mehreren Kristallmodifikationen einer Verbindung, bei denen eine unterschiedliche räumliche Anordnung der Fluorophore eine Änderung des Fluoreszenzverhaltens bewirkt. ${ }^{[86-89]}$ So stellten Tohnai, Miyata et al. bei der Synthese primärer Ammoniumsalze der Anthracen-2,6-disulfonsäure fest, dass die Fluoreszenzeigenschaften im Festkörper von der unterschiedlichen Packung der Anthraceneinheiten im Kristallgitter 
abhängen. ${ }^{[90]}$ Bei einer zweidimensionalen, Fischgrät-Anordnung der Fluorophore (Abb. 1-5 links) tritt eine intensive Festkörperfluoreszenz auf, während Kristalle mit einer koplanaren Molekülpackung keinerlei Fluoreszenz bei Bestrahlung mit UV-Licht zeigen.
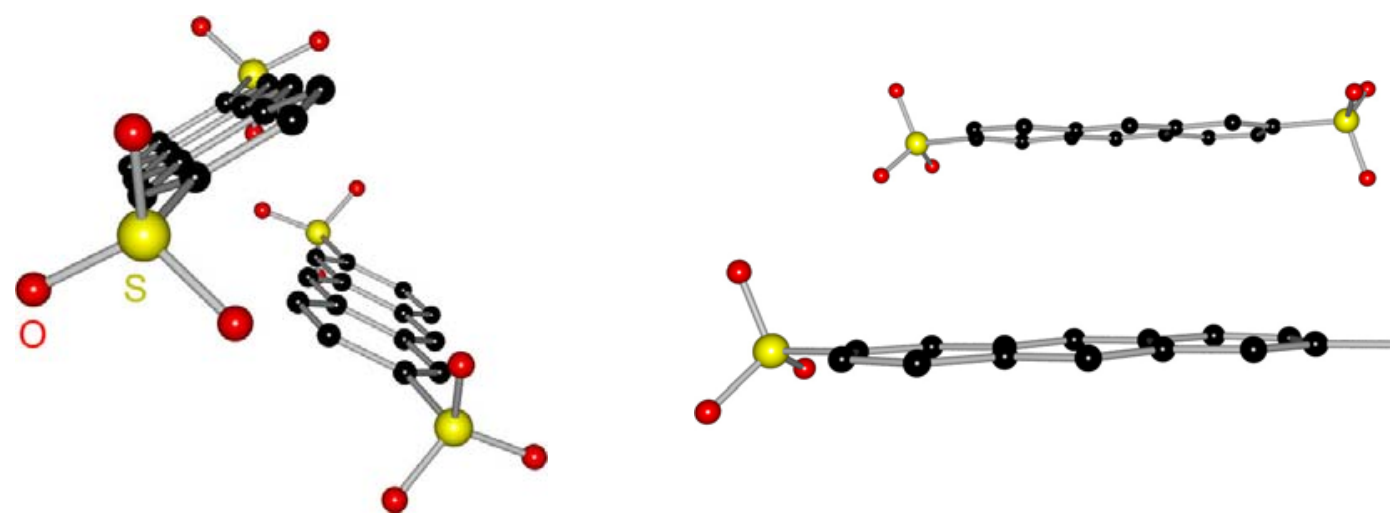

Abb. 1-5: Unterschiedliche Kristallpackungen von Anthracen-2,6-disulfonaten. ${ }^{[90]}$

Die strukturellen Veränderungen in Kristallgittern beim Einschluss von Gastmolekülen und die daraus resultierenden Änderungen der Festkörperfluoreszenz wurden in den letzten Jahren an etlichen Beispielen gezeigt. ${ }^{[1-98]}$ Beispielsweise bewirkt der Einbau von Toluolmolekülen in das polymere [Cul(4-pic)] $]_{\infty}$ eine Stabilisierung des tetrameren Komplexes [\{Cul(4-pic) $\}_{4} \cdot 2$ Toluol], was mit einer Änderung der Fluoreszenzwellenlänge verbunden ist. ${ }^{[99,100]}$ Ein weiteres Beispiel stammt von Che, Wong et al., die Wirt/Gast-Komplexe von quadratisch-planaren Platin(II)-bipyridyl-dialkinylkomplexen untersuchten. ${ }^{[101]}$ Dabei zeigte sich, dass die Festkörperfluoreszenz erheblich von der Kristallinität sowie vom Lösungsmittel abhängt, aus dem die Verbindungen kristallisiert wurden.

Ein in den letzten Jahren zunehmend geprägter Begriff in diesem Zusammenhang ist der sogenannte Vapochromismus. Darunter versteht man die Fähigkeit eines Stoffes, Moleküle aus der Gasphase reversibel unter Änderung der Absorptions- und Emissionswellenlänge zu sorbieren. ${ }^{[102]}$ Beispielsweise wurden einige Gold(I)-., ${ }^{[103-105]}$ Platin(II) ${ }_{-}^{[102,106,107]}$ und Zink(II)-Komplexe ${ }^{[97]}$ untersucht, die vapochromes Verhalten gegenüber leichtflüchtigen organischen Verbindungen (VOC $=$ volatile organic compound) zeigten. Eine mögliche Anwendung ist die Entwicklung von Sensoren zum Aufspüren von Nitroaromaten wie TNT (2,4,6-Trinitrotoluol). Diese sind Bestandteil von weltweit ca. 120 Millionen nicht explodierten Landminen und die bisher einzige praktikable Alternative zum Auffinden ist der Einsatz von Spürhunden, die jedoch erst kostenintensiv trainiert werden müssen und schnell ermüden. ${ }^{\text {108-111] }}$ 


\section{Zielsetzung der vorliegenden Arbeit}

Stalke et al. berichteten 2003 von der Oxidation von PAnP mit zwei Äquivalenten Schwefel zu 9,10-Bis(diphenylthiophosphoryl)anthracen (SPAnPS). Die Einlagerung von Toluolmolekülen in das Kristallgitter war mit einer intensiven Festkörperfluoreszenz verbunden, die Verbindung zeigte somit vapochromes Verhalten. ${ }^{[12]}$

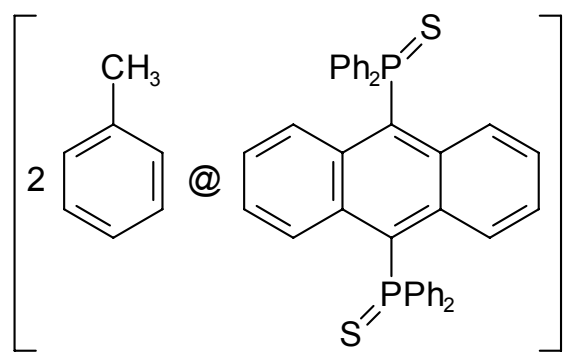

Abb. 1-6: [2 Toluol @ SPAnPS]

In der dieser Arbeit vorangegangenen Diplomarbeit ${ }^{[113]}$ wurden bereits erste Hinweise dafür gefunden, dass SPAnPS mit einer Vielzahl weiterer aromatischer Lösungsmittelmoleküle Einlagerungsverbindungen ausbildet, von denen ein Großteil ebenfalls Lumineszenz im Festkörper aufweist. Ein Ziel war es daher, weitere fluoreszierende und nichtfluoreszierende Wirt/Gast-Komplexe von SPAnPS strukturell zu charakterisieren, um somit mögliche Packungseffekte auf die Festkörperfluoreszenz zu untersuchen.<smiles>Brc1c2ccccc2c(-c2ccccc2)c2ccccc12</smiles>

1

Dabei gelang es auch, das erste 9-Brom-10-phosphanylanthracen (Abb. 1-7) zu synthetisieren und die Struktur mittels Einkristallröntgenstrukturanalyse $\mathrm{zu}$ bestimmen. $\mathrm{Br}\left(\mathrm{C}_{14} \mathrm{H}_{8}\right) \mathrm{PPh}_{2}$ ist durch Umsetzung von 9,10-Dibromanthracen mit jeweils einem Äquivalent $n$-Butyllithium und Abb. 1-7: $\mathrm{Br}\left(\mathrm{C}_{14} \mathrm{H}_{8}\right) \mathrm{PPh}_{2}$ Chlordiphenylphosphan in fast quantitativer Ausbeute zugänglich. ${ }^{[14]}$

In der nun vorliegenden Arbeit sollten die Oxidationsreaktionen von 1 mit Chalkogenen untersucht und mit den 9,10-symmetrisch substituierten Diphosphorylanthracenen verglichen werden. Darüber hinaus sollte überprüft werden, ob durch Lithiierung der 9-Brom-10-phosphanylanthracene und anschließender Umsetzung mit Chlorphosphanen gemischt substituierte 9,10-Diphosphanylanthracene zugänglich sind.

Ein weiteres Ziel war die spektroskopische und strukturelle Charakterisierung von diisopropylsubstituierten Phosphanylanthracenen, deren Naphthalinanaloga bereits erfolgreich als Katalysatoren eingesetzt werden. ${ }^{[115,116]}$ Desweiteren sollten Möglichkeiten untersucht werden, um Phosphanylanthracene zusätzlich mit stickstoffhaltigen Gruppen zu substituieren, die als Donoratome in Metallkomplexen fungieren könnten. 


\section{9-BROM-10-PHOSPHANYLANTHRACENE}

\subsection{Synthese und Struktur von $\operatorname{Br}\left(\mathrm{C}_{14} \mathrm{H}_{8}\right) \mathrm{PPh}_{2}(1)$}

Die Umsetzung von 9,10-Dibromanthracen mit einem Äquivalent ${ }^{n} \mathrm{BuLi}$ ergab das einfach lithiierte Bromanthracen, ${ }^{[117]}$ welches in situ mit einer stöchiometrischen Menge Chlordiphenylphosphan zu 9-Brom-10-(diphenylphosphanyl)anthracen (1) umgesetzt werden kann (GI. 2-1).

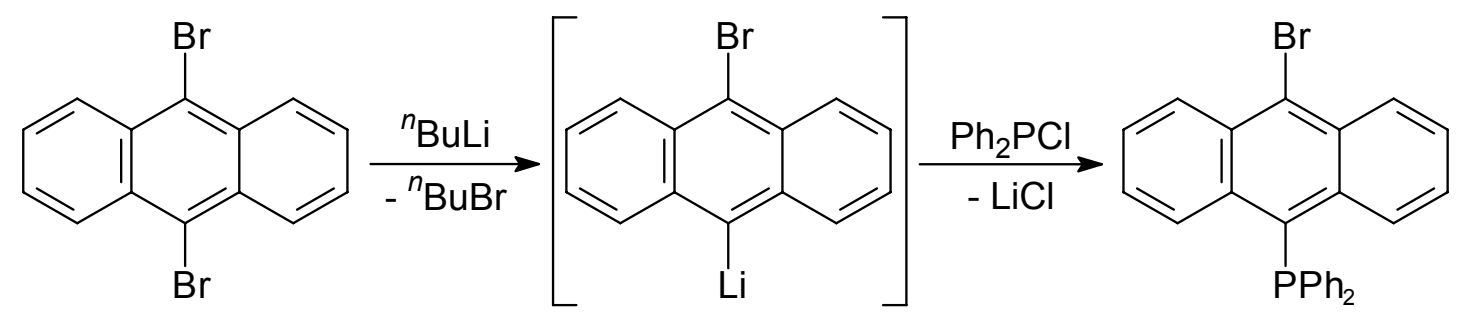

1

GI. 2-1: Synthese von $\operatorname{Br}\left(\mathrm{C}_{14} \mathrm{H}_{8}\right) \mathrm{PPh}_{2}$ (1) über die Zwischenstufe 9-Brom-10-lithiumanthracenid.

Die Lithiierung sowie die nachfolgende Reaktion mit $\mathrm{Ph}_{2} \mathrm{PCl}$ wurden in Diethylether bei $-15{ }^{\circ} \mathrm{C}$ durchgeführt. Im Gegensatz zum symmetrisch substituierten 9,10diphosphanylsubstituierten Analogon ${ }^{[38]}$ ist die Löslichkeit von 1 in Diethylether sehr gut, sodass unlösliche Nebenprodukte durch Filtration abgetrennt werden konnten. Nach Entfernen des Lösungsmittels i. Vak. und Umkristallisation des verbleibenden Rückstandes aus Diethylether wurde 1 in Form gelber Kristalle erhalten.

Die Synthese sowie die Molekülstruktur von 1 wurden bereits in der vorangegangenen Diplomarbeit beschrieben und mittlerweile publiziert. ${ }^{[113,114]}$ In der vorliegenden Arbeit wurde die Synthese optimiert (Erhöhung der Ausbeute von 79 auf 89 \%) sowie die mit Sauerstoff, Schwefel und Selen oxidierten Derivate 2, 3 und 4 dargestellt und charakterisiert. Zur besseren Vergleichbarkeit ist die Molekülstruktur von 1 in Abb. 2-1 erneut dargestellt, Tab. 2-1 enthält ausgewählte Bindungslängen und -winkel. 1 kristallisiert in der monoklinen, zentrosymmetrischen Raumgruppe $P 2_{1} / n$ und die asymmetrische Einheit enthält ein Molekül. Das zentrale Anthracengerüst ist planar (Faltungswinkel zwischen den idealisierten Ebenen durch C1 - C4 und C5 - C8: 177.4 ) und entspricht in Bindungslängen und -winkeln dem unsubstituierten Anthracen. ${ }^{[118]}$ Die Brom- und Phosphoratome sind näherungsweise in der Anthracen-Ebene angeordnet, ihr Abstand zur idealisierten Aromatenebene beträgt $6.12(\mathrm{Br} 1)$ bzw. $13.0 \mathrm{pm}(\mathrm{P} 1)$. 

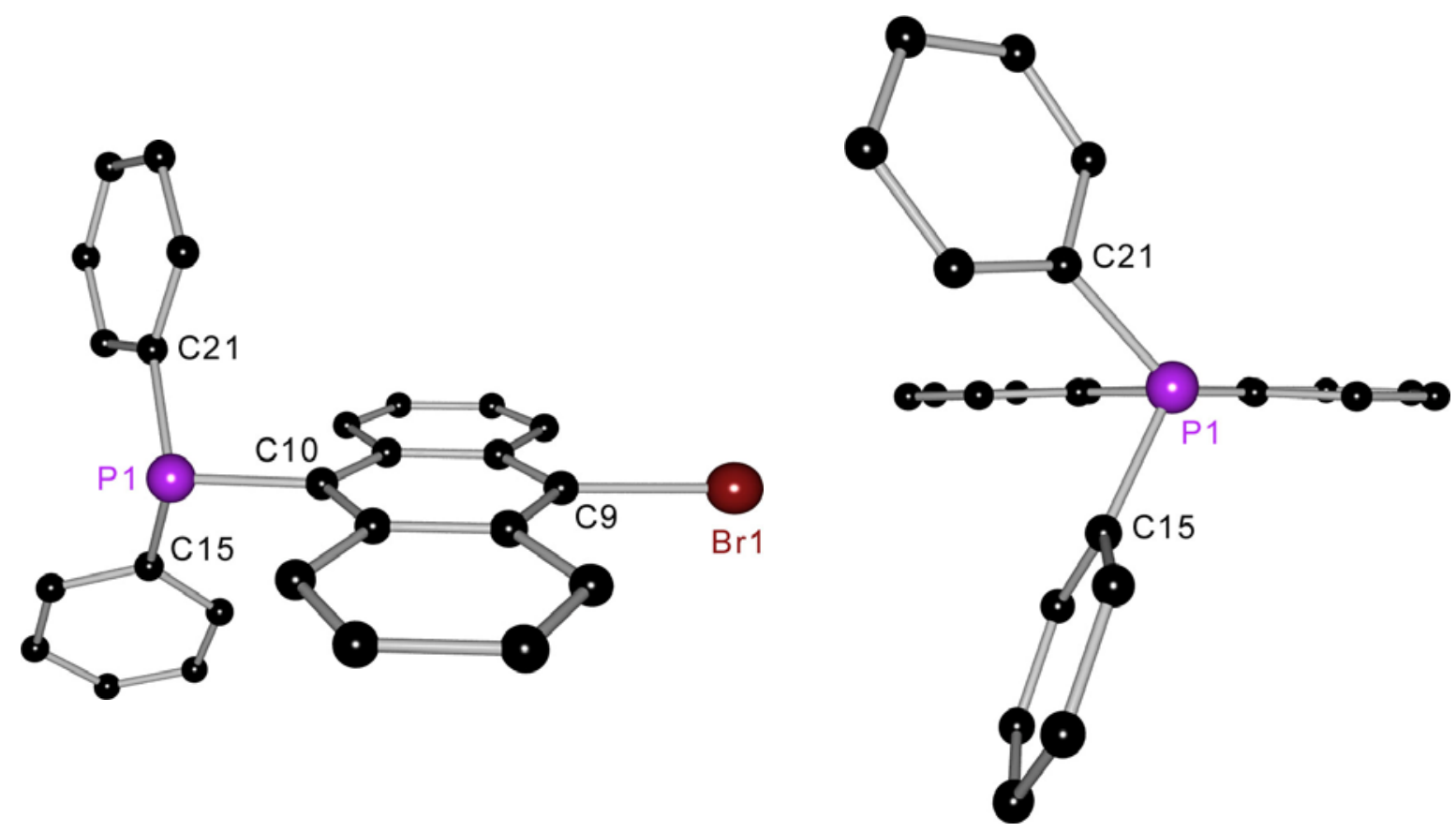

Abb. 2-1: Molekülstruktur von $\operatorname{Br}\left(\mathrm{C}_{14} \mathrm{H}_{8}\right) \mathrm{PPh}_{2}$ (1) (rechts: Blick entlang der $\left.\mathrm{P} 1-\mathrm{C} 10-B i n d u n g\right)$.

Tab. 2-1: Ausgewählte Bindungslängen / pm und -winkel $/{ }^{\circ}$ in 1.

\begin{tabular}{ll}
\hline C9-Br1 & $190.40(17)$ \\
$\mathrm{P} 1-\mathrm{C} 10$ & $184.50(17)$ \\
$\mathrm{P} 1-\mathrm{C} 15$ & $182.73(18)$ \\
$\mathrm{P} 1-\mathrm{C} 21$ & $183.20(18)$ \\
\hline
\end{tabular}

\begin{tabular}{ll}
\hline C10-P1-C15 & $106.07(8)$ \\
C10-P1-C21 & $103.76(8)$ \\
C15-P1-C21 & $106.34(8)$ \\
P1-C10 ‥C9 & $175.02(10)$ \\
\hline
\end{tabular}

Das $\mathrm{sp}^{3}$-hybridisierte Phosphoratom $\mathrm{P} 1$ ist pyramidal koordiniert und die Bindungswinkel sind aufgrund des erhöhten Raumbedarfs des freien Elektronenpaars kleiner als der ideale Tetraederwinkel. Der Blick entlang der P1-C10-Bindung (Abb. 2-1, rechts) zeigt die gestaffelte Anordnung der drei aromatischen Substituenten, das Anthracengerüst halbiert den C15-P1-C21Bindungswinkel. Dies entspricht auch den geometrischen Verhältnissen der Molekülstruktur des disubstituierten 9,10-Bis(diphenylphosphanyl)anthracens.

Die Längen der C9-Br1-Bindung und der drei P-C-Bindungen liegen im Bereich der Bindungslängen vergleichbarer Verbindungen wie 9,10-Dibromanthracen, ${ }^{[119]}$ 1,4-Dibromnaphthalin ${ }^{[120]}$ sowie weiterer Phosphanylanthracene. ${ }^{[29,121,122]}$ 


\subsection{Synthese und Struktur von $\operatorname{Br}\left(\mathrm{C}_{14} \mathrm{H}_{8}\right) \mathrm{P}(\mathrm{O}) \mathrm{Ph}_{2}(2)$}

Die Oxidationen von 1 mit Sauerstoff, Schwefel bzw. Selen wurden analog literaturbekannter Methoden durchgeführt. ${ }^{[123]}$ 9-Brom-10-(diphenylphosphoryl)anthracen (2) konnte mittels $\mathrm{H}_{2} \mathrm{O}_{2} \cdot\left(\mathrm{H}_{2} \mathrm{~N}\right)_{2} \mathrm{C}=\mathrm{O}$ als relativ mildem Oxidationsmittel (GI. 2-2) erhalten werden.

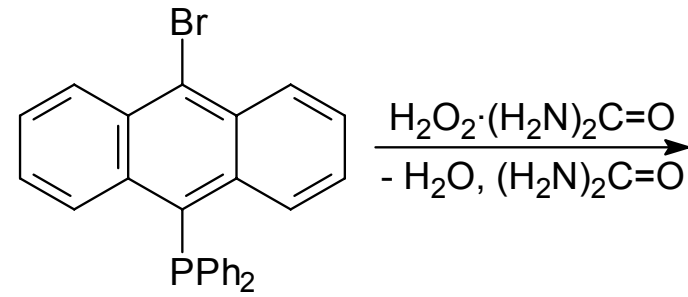

1<smiles>O=Pc1c2ccccc2c(Br)c2ccccc12</smiles>

2

GI. 2-2: Darstellung von $\operatorname{Br}\left(\mathrm{C}_{14} \mathrm{H}_{8}\right) \mathrm{P}(\mathrm{O}) \mathrm{Ph}_{2}$ (2).

Die Umsetzung wurde bei Raumtemperatur in Dichlormethan durchgeführt. Nach $1 \mathrm{~h}$ wurde die Reaktionsmischung mit Wasser extrahiert und die organische Phase i. Vak. eingeengt. Durch Umkristallisation des Rückstandes aus Toluol wurde 2 in einer Ausbeute von $91 \%$ als gelbe Kristalle erhalten. Die Molekülstruktur ist in Abb. 2-2 dargestellt, ausgewählte Bindungslängen und -winkel sind in Tab. 2-2 aufgeführt.
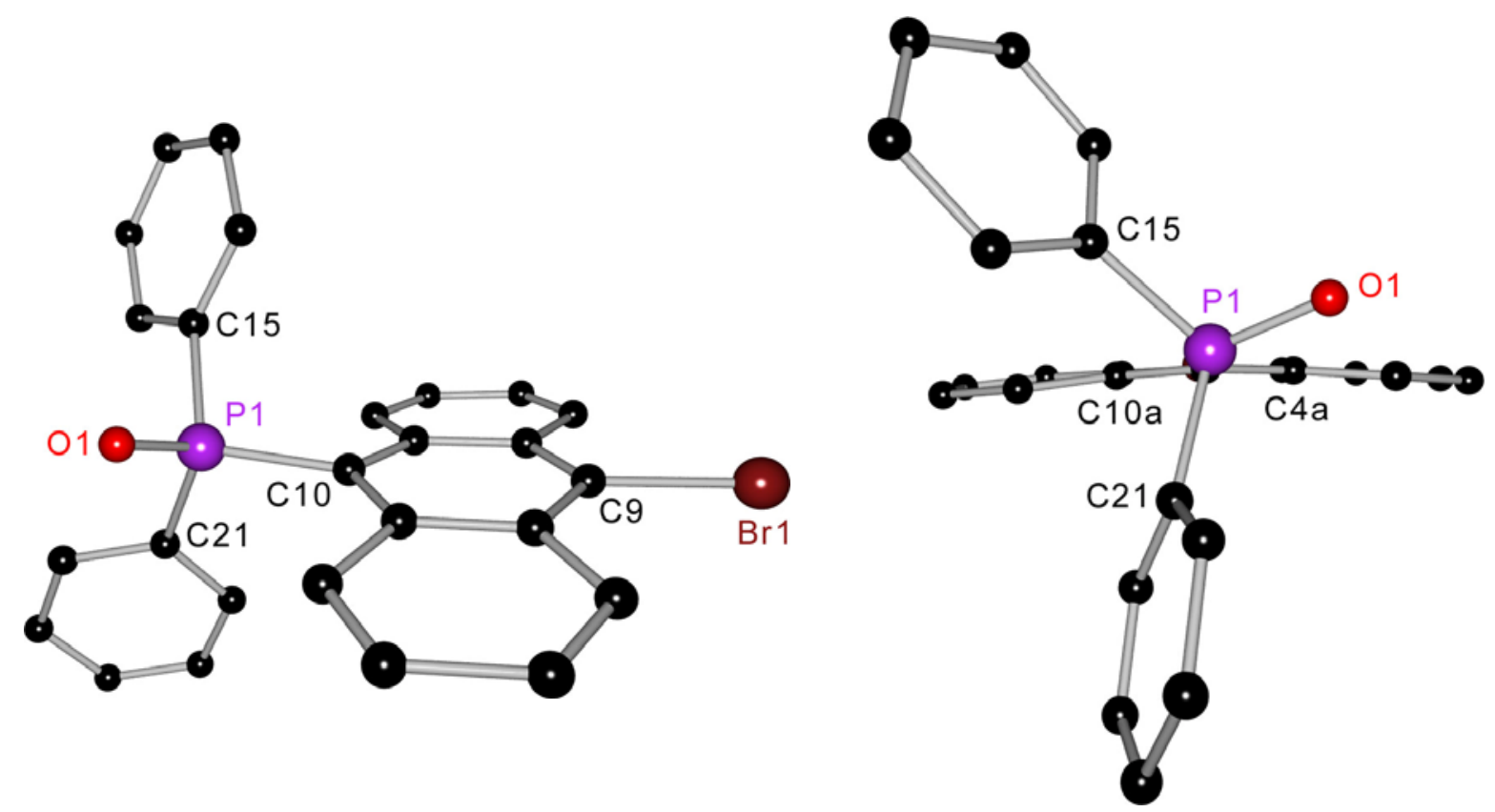

Abb. 2-2: Molekülstruktur von $\operatorname{Br}\left(\mathrm{C}_{14} \mathrm{H}_{8}\right) \mathrm{P}(\mathrm{O}) \mathrm{Ph}_{2}$ (2) (rechts: Blick entlang $\left.\mathrm{C} 9 \cdots \mathrm{C} 10\right)$. 
Tab. 2-2: Ausgewählte Bindungslängen / pm und -winkel $/^{\circ}$ in 2.

\begin{tabular}{ll}
\hline C9-Br1 & $189.9(2)$ \\
$\mathrm{P} 1-01$ & $148.93(18)$ \\
$\mathrm{P} 1-\mathrm{C} 10$ & $183.8(2)$ \\
$\mathrm{P} 1-\mathrm{C} 15$ & $181.1(3)$ \\
$\mathrm{P} 1-\mathrm{C} 21$ & $181.0(3)$ \\
\hline
\end{tabular}

\begin{tabular}{ll}
\hline O1-P1-C10 & $115.27(11)$ \\
C10-P1-C15 & $106.09(11)$ \\
C10-P1-C21 & $106.89(11)$ \\
C15-P1-C21 & $109.90(12)$ \\
P1-C10 ‥C9 & $173.59(13)$ \\
\hline
\end{tabular}

2 kristallisiert in der monoklinen, zentrosymmetrischen Raumgruppe $P 2_{1} / n$. Im Vergleich zu 1 ist das Anthracengerüst nicht mehr völlig planar, der Faltungswinkel zwischen den idealisierten Ebenen durch C1 - C4 und C5 - C8 beträgt $170.1^{\circ}$. Beim Blick entlang der C9 ‥C10-Achse (Abb. 2-2, rechts) ist zu erkennen, dass P1 nicht in der Ebene des Anthracen-Perimeters angeordnet ist. Der Abstand zu der durch C4a, C8a, C9a und C10a aufgespannten Ebene beträgt 29.1 pm (Br1: 8.59 pm).

Die Längen der P1-O1- sowie der drei P-C-Bindungen entsprechen den Bindungslängen in vergleichbaren Verbindungen wie z. B. Triphenylphosphanoxid. ${ }^{[124]}$ Die drei aromatischen Substituenten sind analog zu $\mathbf{1}$ derart um das Phosphoratom angeordnet, dass sich jeweils ein Phenylring ober- und unterhalb der Anthracen-Ebene befindet (transoid). Der Torsionswinkel P1-O1-C10-C4a beträgt 18. $1^{\circ}$, der Diederwinkel zwischen den Bindungen P1-C15 und C10-C10a 41.3 ${ }^{\circ}$.

Die Anordnung der Phenylgruppen in 1 und 2 entspricht den Orientierungen der Substituenten in analogen 1-Phosphanylnaphthalinen (Abb. 2-3). Während die $\mathrm{P}-\mathrm{C}_{i p s 0^{-}}$ Bindung bei unsubstituierter Position 2 in der Ebene des Naphthalin-Gerüstes angeordnet ist, ${ }^{[125]}$ bewirkt eine 2-Substitution die gleiche Orientierung wie in 1 und 2. $^{[126]}$
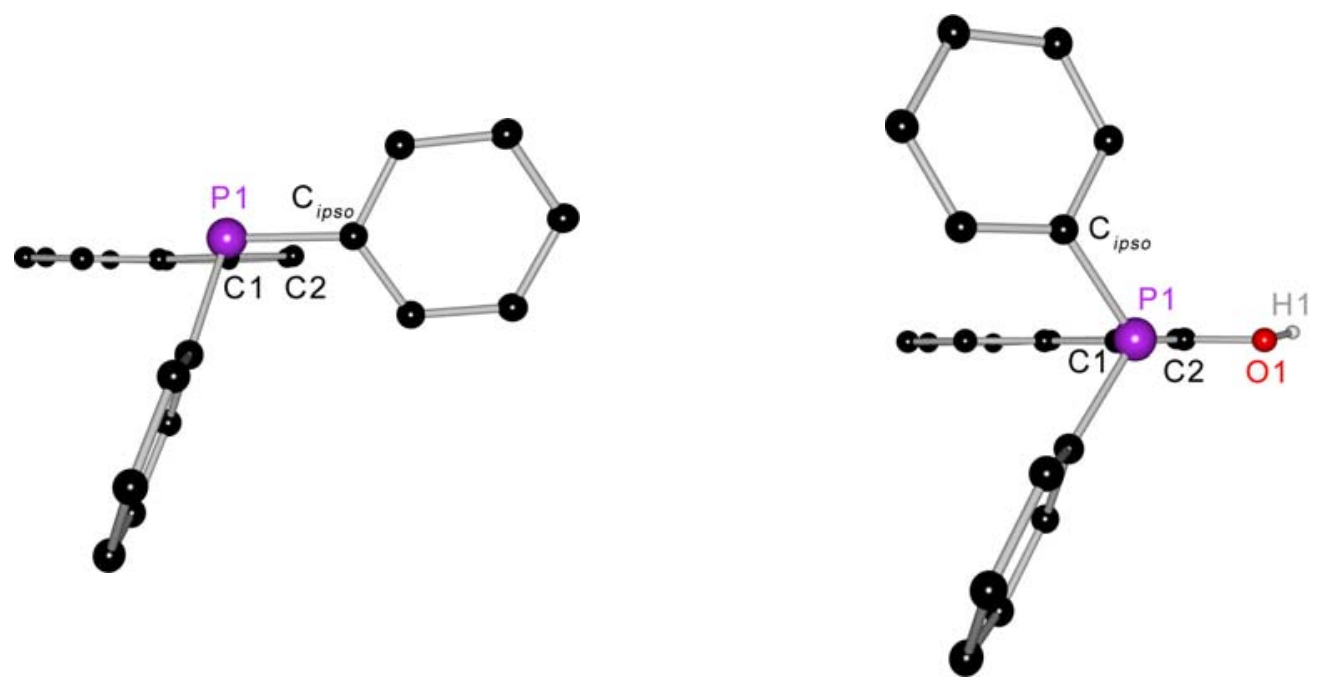

Abb. 2-3: Molekülstrukturen von 1-(Diphenylphosphanyl)naphthalin ${ }^{[125]}$ (links) und 1 -(Diphenylphosphanyl)naphth-2-ol ${ }^{[126]}$ (rechts); Blick jeweils entlang C1 $\cdots \mathrm{C} 4$. 


\subsection{Synthese und Struktur von $\operatorname{Br}\left(\mathrm{C}_{14} \mathrm{H}_{8}\right) \mathrm{P}(\mathrm{S}) \mathrm{Ph}_{2}(3)$}

Die Oxidation von 1 mit elementarem Schwefel lieferte 9-Brom-10-(diphenylthiophosphoryl)anthracen (3) in annähernd quantitativer Ausbeute (GI. 2-3).<smiles>S=Pc1c2ccccc2c(Br)c2ccc([AsH2+])c([AsH2+])c12</smiles>

1

3

GI. 2-3: Darstellung von $\operatorname{Br}\left(\mathrm{C}_{14} \mathrm{H}_{8}\right) \mathrm{P}(\mathrm{S}) \mathrm{Ph}_{2}(3)$.

Die Umsetzung erfolgte in Toluol in der Siedehitze. Kristalle, die für eine röntgenkristallographische Untersuchung geeignet sind, wurden aus einer gesättigten Toluollösung erhalten. Abb. 2-4 zeigt die Molekülstruktur von 3, ausgewählte Bindungslängen und -winkel sind in Tab. 2-3 aufgeführt.
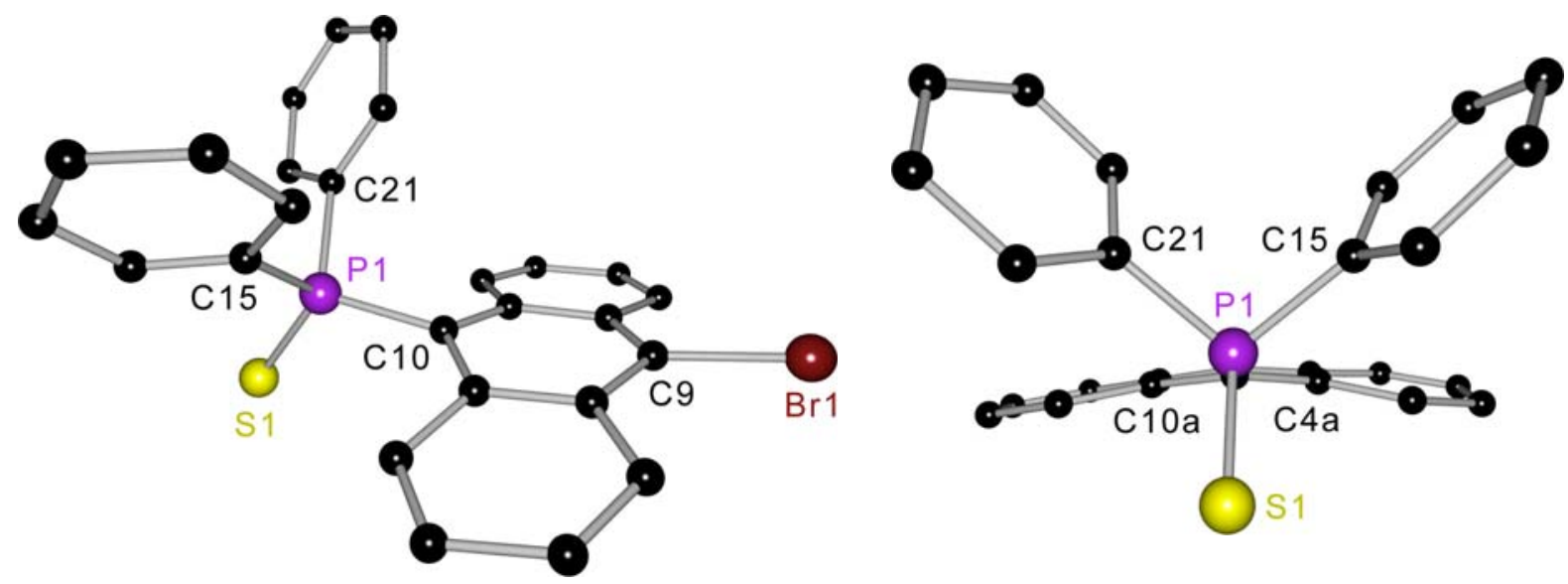

Abb. 2-4: Molekülstruktur von $\operatorname{Br}\left(\mathrm{C}_{14} \mathrm{H}_{8}\right) \mathrm{P}(\mathrm{S}) \mathrm{Ph}_{2}$ (3) (rechts: Blick entlang $\left.\mathrm{C} 9 \cdots \mathrm{C} 10\right)$.

Tab. 2-3: Ausgewählte Bindungslängen / pm und -winkel $/^{\circ}$ in 3.

\begin{tabular}{ll}
\hline C9-Br1 & $190.1(2)$ \\
P1-S1 & $195.46(9)$ \\
P1-C10 & $182.7(2)$ \\
P1-C15 & $182.7(2)$ \\
P1-C21 & $183.1(2)$ \\
\hline
\end{tabular}

\begin{tabular}{lr}
\hline S1-P1-C10 & $114.51(8)$ \\
C10-P1-C15 & $108.02(11)$ \\
C10-P1-C21 & $105.95(11)$ \\
C15-P1-C21 & $99.19(11)$ \\
P1-C10 $\cdots$ C9 & $169.56(13)$ \\
\hline
\end{tabular}


Wie auch 1 und 2 kristallisiert 3 in der Raumgruppe $P 2_{1} / n$, allerdings sind in der asymmetrischen Einheit zwei Moleküle. Alle Bindungslängen liegen im Bereich vergleichbarer Verbindungen wie beispielsweise Triphenylphosphansulfid (P-S: $195.0 \mathrm{pm}){ }^{[127]}$

Die Anordnung der beiden Phenylsubstituenten relativ zur Anthracen-Einheit unterscheidet sich deutlich von 1 und 2. Zur besseren Verdeutlichung zeigt Abb. 2-5 eine Superposition der beiden mit Sauerstoff (2) und Schwefel oxidierten (3) Derivate aus zwei unterschiedlichen Ansichten.
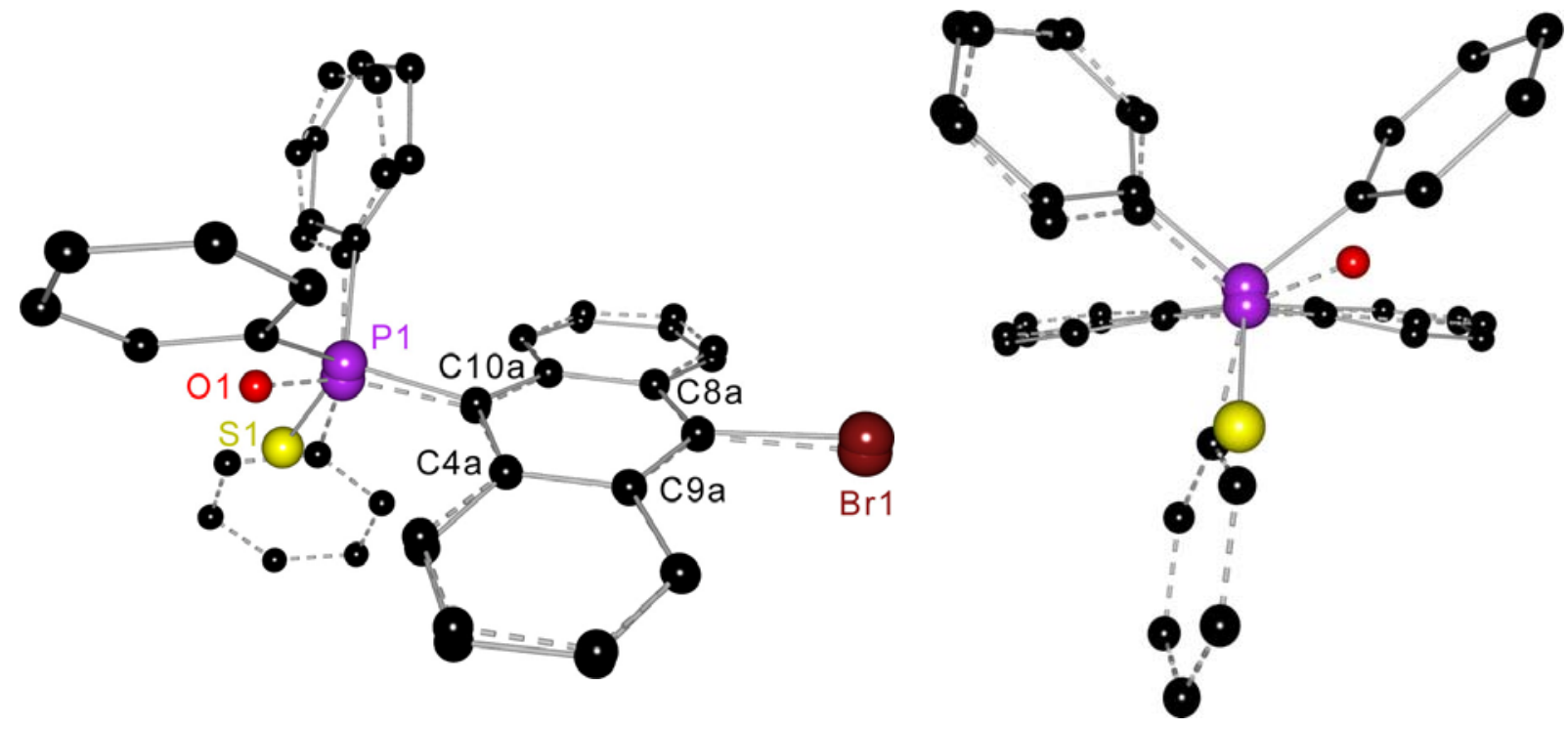

Abb. 2-5: Superposition von 2 (gestrichelte Bindungen) und 3 (durchgezogene Bindungen), erzeugt durch Überlagerung von C4a, C8a, C9a und C10a (rechts: Blick entlang C9 $\cdots \mathrm{C} 10$ ).

Die beiden Phenylringe sind in $\mathbf{3}$ zur gleichen Seite des Anthracen-Perimeters orientiert (cisoid), während die $\mathrm{P}=\mathrm{S}-$ Bindung fast rechtwinklig dazu angeordnet ist (Torsionswinkel zwischen den idealisierten Ebenen durch S1, P1, C10 und C4a, C8a, C9a, C10a: 86.4\%). Der Übergang von der transoiden (2) zur cisoiden Konfiguration (3) bewirkt eine noch stärkere Abwinkelung des Anthracengerüstes, der Faltungswinkel zwischen den beiden durch C1 - C4 und C5 - C8 aufgespannten Ebenen beträgt $162.1^{\circ}$. Des Weiteren vergrößert sich dadurch der Abstand des Phosphoratoms von der durch $\mathrm{C} 4 \mathrm{a}, \mathrm{C} 8 \mathrm{a}, \mathrm{C} 9 \mathrm{a}$ und $\mathrm{C} 10 \mathrm{a}$ aufgespannten Ebene um 24 pm auf 53.1 pm, das Bromatom ist 26.8 pm von dieser entfernt (8.59 pm in 2).

Eine analoge Orientierung der $\mathrm{P}(\mathrm{S}) \mathrm{Ph}_{2}$-Substituenten bezüglich der AnthracenEinheit findet sich auch in dem von Stalke et al. beschriebenen symmetrischen 9,10-Bis(diphenylthiophosphoryl)anthracen. ${ }^{[12]}$ 


\subsection{Synthese und Struktur von $\operatorname{Br}\left(\mathrm{C}_{14} \mathrm{H}_{8}\right) \mathrm{P}(\mathrm{Se}) \mathrm{Ph}_{2}(4)$}

Analog der Oxidation mit Schwefel ließ sich 9-Brom-10-(diphenylselenophosphoryl)anthracen (4) durch Umsetzung von 1 mit grauem Selen darstellen (GI. 2-4).<smiles>Brc1c2ccccc2c(-c2ccccc2)c2ccccc12</smiles>

1

4

GI. 2-4: Synthese von $\operatorname{Br}\left(\mathrm{C}_{14} \mathrm{H}_{8}\right) \mathrm{P}(\mathrm{Se}) \mathrm{Ph}_{2}$ (4).

Die Reaktion erfolgte in siedendem Toluol mit einem Überschuss an grauem Selen. Nach der Umsetzung wurde das überschüssige Selen abgetrennt und das Lösungsmittel i. Vak. entfernt. Aus dem Rückstand konnten aus Toluol Kristalle in einer Ausbeute von $81 \%$ erhalten werden, die für eine Strukturbestimmung geeignet waren (Abb. 2-6 und Tab. 2-4).
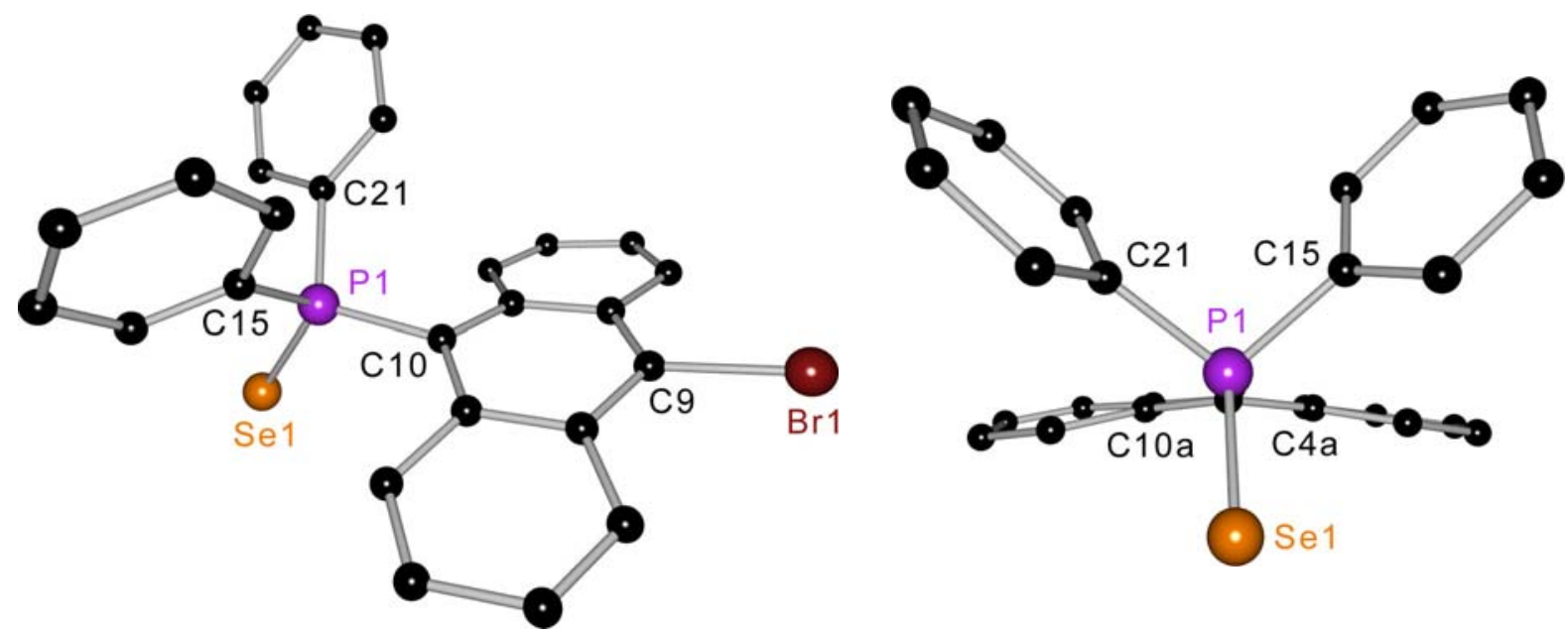

Abb. 2-6: Molekülstruktur von $\operatorname{Br}\left(\mathrm{C}_{14} \mathrm{H}_{8}\right) \mathrm{P}(\mathrm{Se}) \mathrm{Ph}_{2}$ (4) (rechts: Blick entlang $\left.\mathrm{C} 9 \cdots \mathrm{C} 10\right)$.

Tab. 2-4: Ausgewählte Bindungslängen / pm und -winkel / ${ }^{\circ}$ in 4.

\begin{tabular}{ll}
\hline C9-Br1 & $190.2(4)$ \\
P1-Se1 & $211.23(11)$ \\
P1-C10 & $183.3(3)$ \\
P1-C15 & $183.0(4)$ \\
P1-C21 & $183.0(4)$ \\
\hline
\end{tabular}

\begin{tabular}{lr}
\hline Se1-P1-C10 & $113.95(12)$ \\
C10-P1-C15 & $106.47(16)$ \\
C10-P1-C21 & $108.16(17)$ \\
C15-P1-C21 & $99.12(16)$ \\
P1-C10 ‥C9 & $169.65(19)$ \\
\hline
\end{tabular}


4 kristallisiert isostrukturell zu dem mit Schwefel oxidiertem Derivat 3 in der monoklinen Raumgruppe $P 2_{1} / n$. Auch hier entsprechen alle Bindungslängen und -winkel den erwarteten Werten, beispielsweise beträgt der P-Se-Abstand in Triphenylphosphanselenid 210.6 pm. ${ }^{\text {[128] }}$

Die Orientierung des $\mathrm{P}(\mathrm{Se}) \mathrm{Ph}_{2}$-Substituenten bezüglich des Anthracengerüstes ist analog dem räumlichen Aufbau von 3. Der Torsionswinkel zwischen den idealisierten Ebenen durch Se1, P1, C10 und C4a, C8a, C9a, C10a beträgt in $487.3^{\circ}$, die Phosphor- und Bromatome sind 50.3 bzw. 29.3 pm von letzterer entfernt. Auch der Faltungswinkel zwischen den durch C1 - C4 und C5 - C8 aufgespannten Ebenen hat trotz des größeren sterischen Anspruchs des Selenatoms den gleichen Wert $\left(162.3^{\circ}\right)$ wie in 3.

Im ${ }^{77}$ Se-NMR-Spektrum wird für 4 in $\mathrm{CDCl}_{3}$ ein Dublett bei $-281.1 \mathrm{ppm}$ mit einer ${ }^{1} \mathrm{~J}_{\mathrm{SeP}}$-Kopplungskonstante von $734.9 \mathrm{~Hz}$ beobachtet. Die chemischen ${ }^{31} \mathrm{P}-\mathrm{NMR}$ Verschiebungen von 1 - 4 sind in Tab. 2-5 aufgelistet; alle Signale erscheinen als scharfe Singuletts.

Tab. 2-5: Vergleich der chemischen ${ }^{31} \mathrm{P}-\mathrm{NMR}-$ Verschiebungen von 1 - 4 in $\mathrm{CDCl}_{3}$.

\begin{tabular}{c|cccc} 
& $\operatorname{Br}\left(\mathrm{C}_{14} \mathrm{H}_{8}\right) \mathrm{PPh}_{2}$ & $\operatorname{Br}\left(\mathrm{C}_{14} \mathrm{H}_{8}\right) \mathrm{P}(\mathrm{O}) \mathrm{Ph}_{2}$ & $\mathrm{Br}\left(\mathrm{C}_{14} \mathrm{H}_{8}\right) \mathrm{P}(\mathrm{S}) \mathrm{Ph}_{2}$ & $\mathrm{Br}\left(\mathrm{C}_{14} \mathrm{H}_{8}\right) \mathrm{P}(\mathrm{Se}) \mathrm{Ph}_{2}$ \\
& $(\mathbf{1})$ & $(\mathbf{2})$ & $(\mathbf{3})$ & $(\mathbf{4})$ \\
\hline$\delta / \mathrm{ppm}$ & -22.7 & 31.6 & 34.7 & 25.9
\end{tabular}

Die Molekülstrukturen und NMR-spektroskopischen Eigenschaften der beschriebenen 9-Brom-10-(diphenylphoshanyl)anthracene 1 - 4 stimmen weitgehend mit den symmetrischen 9,10-substituierten Diphosphanylanaloga überein, ${ }^{[112]}$ allerdings kristallisieren sie nicht als Wirt-GastSysteme, bei denen Toluol-Moleküle im Kristallgitter eingebaut sind, und zeigen keinerlei Festkörperlumineszenz im sichtbaren Bereich. Lösungen des mit Sauerstoff oxidierten Derivats $\mathbf{2}$ in gängigen organischen Lösungsmitteln zeigen starke Fluoreszenz bei Bestrahlung mit UV-Licht $\left(\lambda_{\mathrm{ex}}=366 \mathrm{~nm}\right)$, was auch bereits 1987 von Akasaka für 9-(Diphenylphosphanyl)anthracen beobachtet wurde. ${ }^{[27]}$

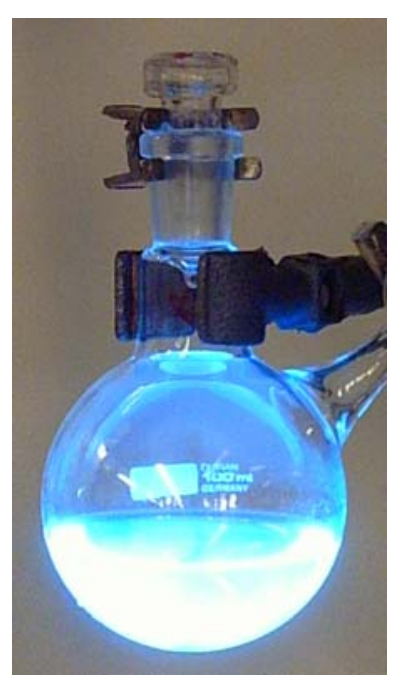

Abb. 2-7: Lösung von 2 in $\mathrm{CH}_{2} \mathrm{Cl}_{2}$ unter Bestrahlung mit UV-Licht $(\lambda=366 \mathrm{~nm})$ 


\subsection{Synthese, Tieftemperatur-Verhalten und Struktur von $\operatorname{Br}\left(\mathrm{C}_{14} \mathrm{H}_{8}\right) \mathrm{P}^{i} \mathrm{Pr}_{2}(5)$}

Wie bereits beschrieben kann durch Reaktion von 9,10-Dibromanthracen mit einem Äquivalent ${ }^{n} \mathrm{BuLi}$ das monolithiierte 9-Brom-10-lithiumanthracenid erhalten werden, welches wie in Kap. 2.1 dargestellt mit Chlordiphenylphosphan in situ umgesetzt wurde. Um die Übertragbarkeit der Reaktion auf andere phosphorhaltige Elektrophile zu überprüfen, wurde Chlordiisopropylphosphan verwendet und damit das entsprechende 9-Brom-10-(diisopropylphosphanyl)anthracen (5) dargestellt (GI. 2-5). ${ }^{[113,129]}$

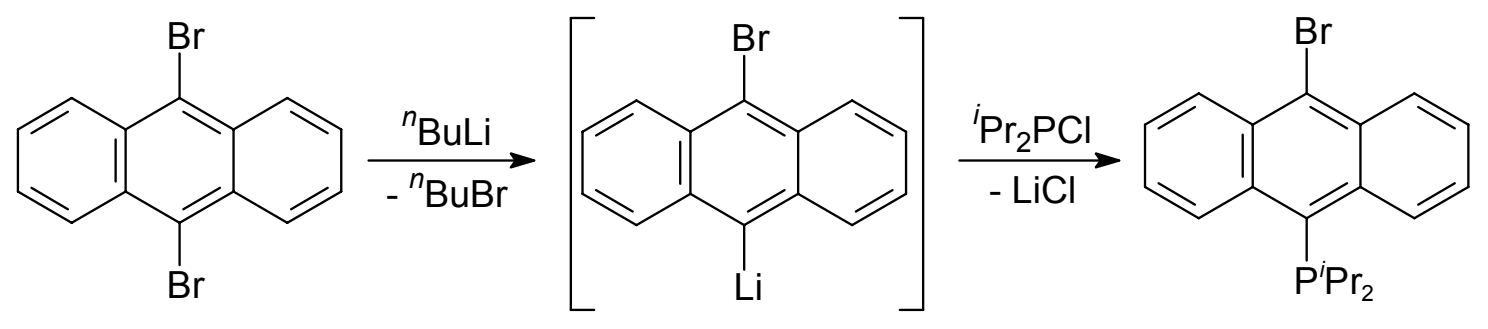

5

GI. 2-5: Synthese von $\operatorname{Br}\left(\mathrm{C}_{14} \mathrm{H}_{8}\right) \mathrm{P}^{i} \mathrm{Pr}_{2}$ (5) über die Zwischenstufe 9-Brom-10-lithiumanthracenid.

Analog zur Darstellung von 1 wurden die Lithiierung sowie die unmittelbar folgende Umsetzung mit Chlordiisopropylphosphan bei $-15^{\circ} \mathrm{C}$ in Diethylether durchgeführt. Nach Abtrennen von unlöslichen Nebenprodukten wurde das Lösungsmittel i. Vak. entfernt und $\mathbf{5}$ in einer Ausbeute von $86 \%$ erhalten.

Das ${ }^{1} \mathrm{H}$-NMR-Spektrum von 5 in $\mathrm{CDCl}_{3}$ zeigt im Alkylbereich gut aufgelöste Signale. Für die CH-Protonen der 'Pr-Substituenten wird ein Dublett von Septetts bei $2.92 \mathrm{ppm}$ beobachtet, das aufgrund identischer ${ }^{2} \mathrm{JHP}_{\mathrm{HP}}$ und ${ }^{3} \mathrm{JHH}_{\mathrm{H}}$-Kopplungskonstanten $(6.90 \mathrm{~Hz})$ als Pseudo-Oktett erscheint. Die $\mathrm{CH}_{3}$-Gruppen eines ${ }^{i} \mathrm{Pr}$-Substituenten sind diastereotop, was in Abb. 2-8 verdeutlicht werden soll.

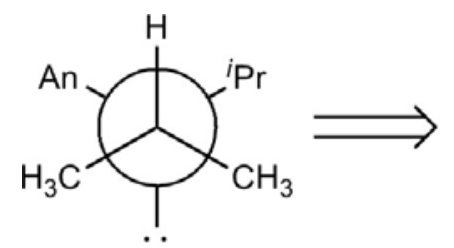

A

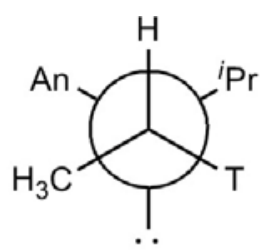

B

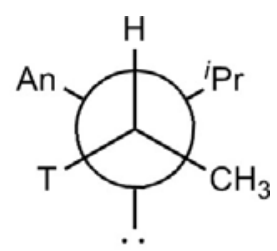

C

Abb. 2-8: Feststellung der Diastereotopie der Methylgruppen in $\mathbf{5}$.

Zur Feststellung der Diastereotopie der Methylgruppen wird jeweils ein $\mathrm{CH}_{3}$ Substituent durch eine achirale Testgruppe $T$ ersetzt und dann die beiden neu entstandenen Moleküle verglichen. ${ }^{[130]} \mathbf{A}$ in Abb. 2-8 zeigt die Newman-Projektion von 5 bei Blick entlang einer C-P-Bindung, in $\mathbf{B}$ und $\mathbf{C}$ ist jeweils eine der beiden 
Methylgruppen durch eine Testgruppe $\mathbf{T}$ ersetzt. Bei $\mathbf{B}$ und $\mathbf{C}$ handelt es sich um Diastereomere, somit sind die beiden $\mathrm{CH}_{3}$-Substituenten diastereotop und damit chemisch nicht äquivalent. Im ${ }^{1} \mathrm{H}-\mathrm{NMR}$-Spektrum werden entsprechend zwei Dubletts von Dubletts bei 1.44 und 0.70 ppm beobachtet, die jeweils durch ${ }^{3} J_{\mathrm{HP}}$ und ${ }^{3} \mathrm{JHH}_{\mathrm{H}-\text { Kopplungen zustande kommen. }}$

Die Integration der Signale im aromatischen Bereich des ${ }^{1} \mathrm{H}$-NMR-Spektrums bei Raumtemperatur führt jedoch nur zu sechs Protonen anstatt der erwarteten acht des Anthracengerüstes. Die Verbreiterung von Arylsignalen legt die Vermutung nahe, dass es sich um einen dynamischen Effekt handelt, der daher durch die Aufnahme von NMR-Spektren bei unterschiedlichen Temperaturen untersucht wurde. Abb. 2-9 zeigt ${ }^{1} \mathrm{H}-\mathrm{NMR}$-Spektren von 5 bei Temperaturen zwischen 243 und $323 \mathrm{~K}$.

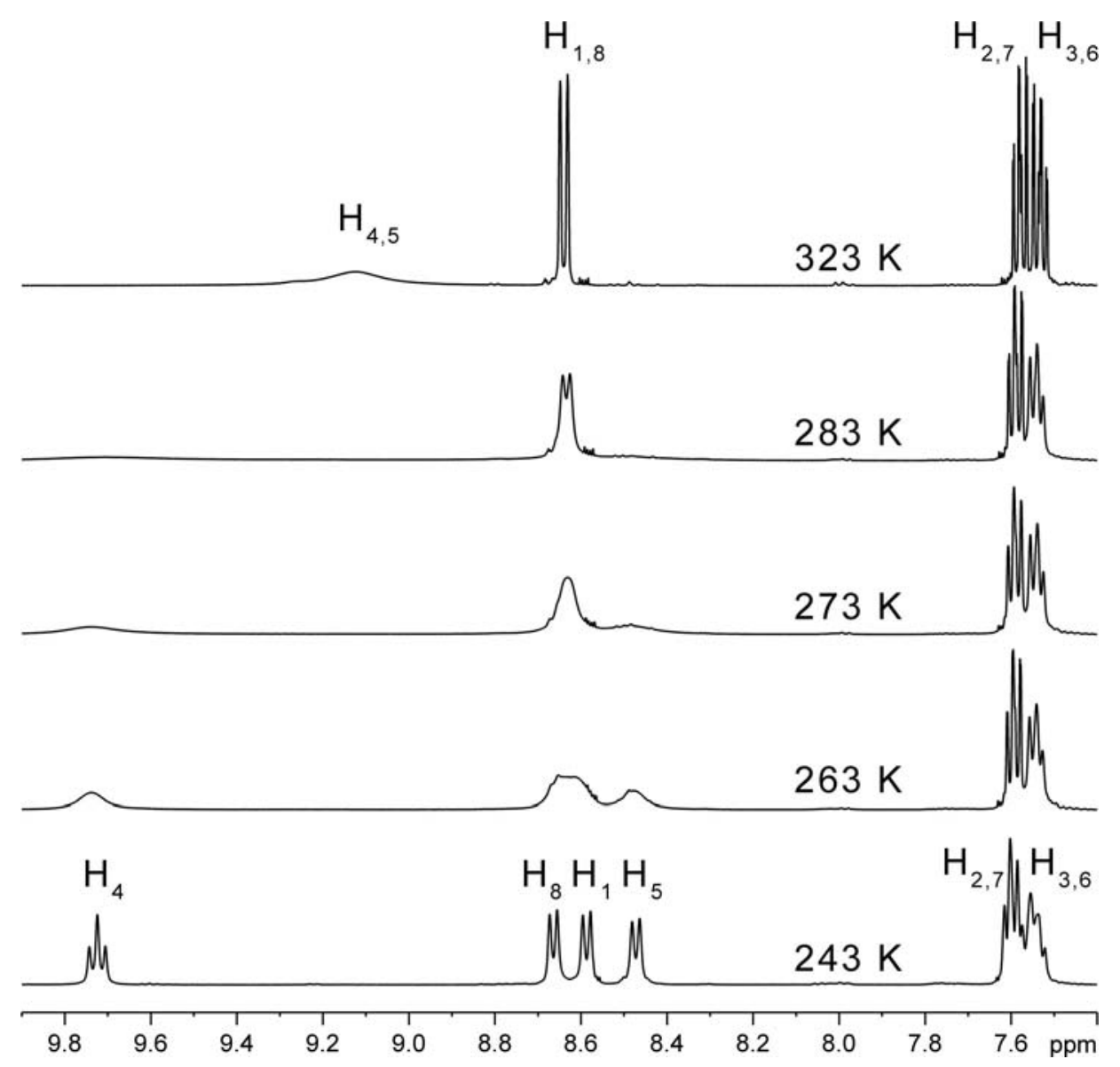

Abb. 2-9: ${ }^{1} \mathrm{H}-\mathrm{NMR}-S p e k t r e n$ von 5 in $\mathrm{CDCl}_{3}$ bei unterschiedlichen Temperaturen.

Beim Erwärmen auf $323 \mathrm{~K}$ wird das Aufkommen eines breiten Singuletts bei etwa $9.1 \mathrm{ppm}$ beobachtet, das durch $\mathrm{HH}$-COSY-Experimente $\mathrm{H}_{4}$ und $\mathrm{H}_{5}$ zugeordnet werden kann. Im Gegensatz dazu erscheint dieses Signal bei $283 \mathrm{~K}$ stark verbreitert und wird bei noch weiterer Abkühlung in zwei neue Resonanzen bei 9.73 und $8.48 \mathrm{ppm}$ aufgespaltet, die bei $243 \mathrm{~K}$ gut aufgelöst sind. Bei $\mathrm{H}_{5}$ resultiert die 
beobachtete Multiplizität aus der ${ }^{3} \mathrm{~J}_{\mathrm{HH}}$-Kopplung $(8.66 \mathrm{~Hz})$ mit dem benachbarten $\mathrm{H}_{6}$, die zu einem Dublett führt. Das NMR-Signal von $\mathrm{H}_{4}$ erscheint als Dublett von Dubletts, somit muss neben der ${ }^{3} \mathrm{~J}_{\mathrm{HH}}$-Kopplung mit $\mathrm{H}_{3}$ noch eine weitere Spin-SpinWechselwirkung berücksichtigt werden. Hierbei handelt es sich um eine Fernkopplung mit dem Phosphoratom, dessen nichtbindendes Elektronenpaar in die Richtung von $\mathrm{H}_{4}$ orientiert ist. Abb. 2-10 zeigt die bevorzugten Konformationen von 5 bei 323 und $243 \mathrm{~K}$.

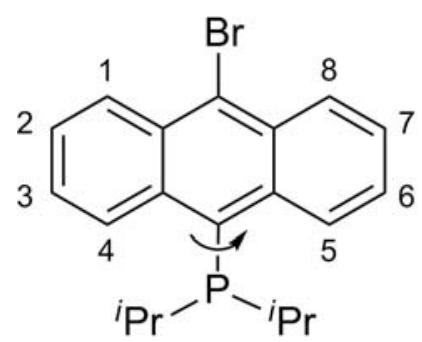

$323 \mathrm{~K}$

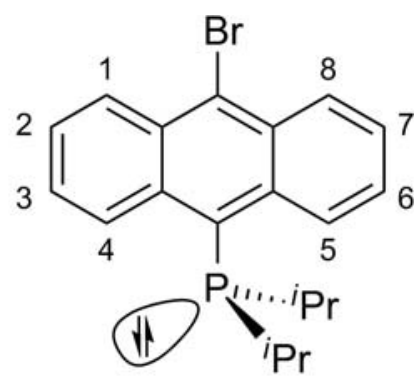

$243 \mathrm{~K}$

Abb. 2-10: Temperaturabhängige Konformationen von 5.

Durch die schnelle Rotation des ${ }^{i} \mathrm{Pr}_{2} \mathrm{P}$-Substituenten um die $\mathrm{P}-\mathrm{C}_{\mathrm{An}}$-Bindung sind bei $323 \mathrm{~K}$ die Wasserstoffpaare $\mathrm{H}_{1} / \mathrm{H}_{8}, \mathrm{H}_{2} / \mathrm{H}_{7}, \mathrm{H}_{3} / \mathrm{H}_{6}$ und $\mathrm{H}_{4} / \mathrm{H}_{5}$ jeweils chemisch äquivalent und führen zum Auftreten von vier Signalsätzen im ${ }^{1} \mathrm{H}-\mathrm{NMR}$-Spektrum. Durch das Abkühlen auf $243 \mathrm{~K}$ wird der dynamische Prozess verlangsamt und die in Abb. 2-10 auf der rechten Seite dargestellte gestaffelte Konformation liegt bevorzugt vor. Dies erklärt die Nicht-Äquivalenz der aromatischen Wasserstoffatome bei tiefer Temperatur.

Auch für $\mathrm{H}_{1}$ und $\mathrm{H}_{8}$ ist die Auswirkung der Temperaturänderung gut beobachtbar: Während die beiden Atome bei RT isochrone Resonanz bei $8.68 \mathrm{ppm}$ mit einer ${ }^{3} \mathrm{JHH}_{\mathrm{HH}}$ Kopplung von $8.53 \mathrm{~Hz}$ zeigen, verlieren sie beim Abkühlen ihre magnetische Äquivalenz und das Signal wird in zwei separate Dubletts bei 8.67 und 8.59 ppm aufgespaltet.

Um die Konformation von 5 im Festkörper zu untersuchen wurde die Molekülstruktur bestimmt. Das Ergebnis der Röntgenstrukturuntersuchung ist in Abb. 2-11 dargestellt, Tab. 2-6 enthält ausgewählte Bindungslängen und -winkel.

5 kristallisiert in der monoklinen, zentrosymmetrischen Raumgruppe $P 2_{1} / n$ mit zwei Molekülen in der asymmetrischen Einheit. Das zentrale Anthracengerüst ist planar (Faltungswinkel zwischen den Ebenen durch C1 - C4 und C5 - C8: 174.7), P1 ist näherungsweise in der idealisierten Anthracen-Ebene angeordnet (Abstand: $4.89 \mathrm{pm}), \mathrm{Br} 1$ ist $18.1 \mathrm{pm}$ von dieser entfernt. 

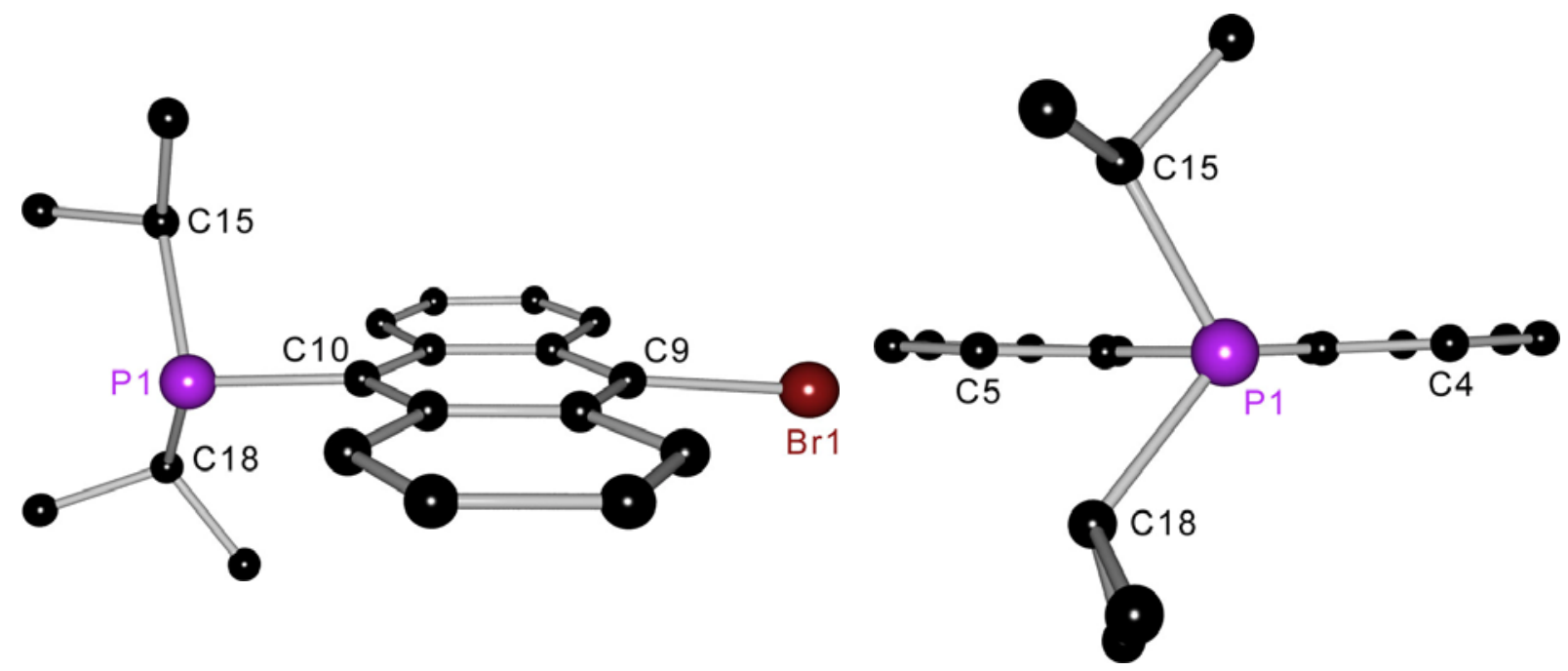

Abb. 2-11: Molekülstruktur von $\operatorname{Br}\left(\mathrm{C}_{14} \mathrm{H}_{8}\right) \mathrm{P}^{\prime} \mathrm{Pr}_{2}$ (5) (rechts: Blick entlang der $\left.\mathrm{P} 1-\mathrm{C} 10-\mathrm{Bindung}\right)$.

Tab. 2-6: Ausgewählte Bindungslängen / pm und -winkel $/^{\circ}$ in 5.

\begin{tabular}{ll}
\hline C9-Br1 & $191.3(2)$ \\
$\mathrm{P} 1-\mathrm{C} 10$ & $186.3(3)$ \\
$\mathrm{P} 1-\mathrm{C} 15$ & $187.2(3)$ \\
$\mathrm{P} 1-\mathrm{C} 18$ & $187.2(3)$ \\
\hline
\end{tabular}

\begin{tabular}{ll}
\hline C10-P1-C15 & $100.38(12)$ \\
C10-P1-C18 & $106.31(12)$ \\
C15-P1-C18 & $107.43(12)$ \\
P1-C10 ‥C9 & $175.70(14)$ \\
\hline
\end{tabular}

Beim Blick entlang der P1-C10-Bindung ist zu erkennen, dass die 'Pr-Substituenten in einer gestaffelten Anordnung relativ zum Anthracengerüst orientiert sind und dass der C15-P1-C18-Winkel durch die Aromatenebene halbiert wird. Somit befindet sich das nichtbindende Elektronenpaar des Phosphoratoms in der Anthracenebene und ist in Richtung von $\mathrm{H}_{4}$ angeordnet, während $\mathrm{H}_{5}$ zwischen den beiden Isopropylgruppen "eingeklemmt" ist. Die im Festkörper beobachtete Konformation von 5 entspricht exakt den Ergebnissen der NMR-spektroskopischen Untersuchungen bei tiefer Temperatur. Durch die räumliche Nähe des freien Elektronenpaars zu $\mathrm{H}_{4}$ lässt sich die zusätzlich beobachtete Kopplung im eindimensionalen ${ }^{1} \mathrm{H}-\mathrm{NMR}$-Spektrum erklären. Außerdem würde im 2D-NOESYSpektrum ein Kreuzsignal von $\mathrm{H}_{4}$ und den ${ }^{i} \mathrm{Pr}$-Gruppen auftreten, das die räumliche Nachbarschaft dieser Gruppen anzeigt. Für die 9,10-symmetrisch substituierte Verbindung $\left({ }^{i} \mathrm{Pr}_{2} \mathrm{P}\right)_{2}\left(\mathrm{C}_{14} \mathrm{H}_{8}\right)$ (13) wurde das 2D-NOESY-Spektrum auch tatsächlich gemessen (Abb. 3-12, Seite 40). 


\subsection{Synthese von $\operatorname{Br}\left(\mathrm{C}_{14} \mathrm{H}_{8}\right) \mathrm{P}(\mathrm{O})^{i} \mathrm{Pr}_{2}(6)$}

Die Oxidation von 5 mit einem Überschuss $\mathrm{H}_{2} \mathrm{O}_{2} \cdot\left(\mathrm{H}_{2} \mathrm{~N}\right)_{2} \mathrm{C}=\mathrm{O}$ lieferte 9-Brom10-(diisopropylphosphoryl)anthracen (6) in einer Ausbeute von 85 \% (GI. 2-6).<smiles>CCCc1c2ccccc2c(Br)c2ccccc12</smiles>

5

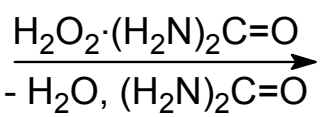<smiles>C#[PH](=O)c1c2ccccc2c(Br)c2ccccc12</smiles>

6

GI. 2-6: Darstellung von $\operatorname{Br}\left(\mathrm{C}_{14} \mathrm{H}_{8}\right) \mathrm{P}(\mathrm{O})^{i} \operatorname{Pr}_{2}$ (6).

Analog zur Synthese des Phenylanalogons 2 erfolgte die Reaktion in Dichlormethan. Nach beendeter Umsetzung wurde die Reaktionsmischung mit Wasser gewaschen, die organische Phase über Natriumsulfat getrocknet und das Lösungsmittel i. Vak. entfernt. Leider waren bislang alle Kristallisationsversuche erfolglos, sodass noch keine Molekülstruktur der Verbindung vorliegt.

Im ${ }^{31}$ P-NMR-Spektrum erfolgt während der Reaktion ein Tieffeldshift von $\delta 1.37 \mathrm{ppm}$ (5) nach $59.4 \mathrm{ppm}$ (6). Die Wasserstoffatome $\mathrm{H}_{4}$ und $\mathrm{H}_{5}$, die sich in räumlicher Nähe der Isopropylgruppen befinden, zeigen im ${ }^{1} \mathrm{H}-\mathrm{NMR}$-Spektrum ein breites Singulett bei $\delta 9.2 \mathrm{ppm}$. Dies lässt auf ein ähnliches Tieftemperaturverhalten schließen wie bereits für 5 beschrieben.

\subsection{Synthese und Struktur von $\operatorname{Br}\left(\mathrm{C}_{14} \mathrm{H}_{8}\right) \mathrm{P}(\mathrm{S})^{i} \mathrm{Pr}_{2}(7)$}

Gl. 2-7 zeigt die Oxidation von 5 mit elementarem Schwefel, die zu 9-Brom-10(diisopropylthiophosphoryl)anthracen (7) führte.

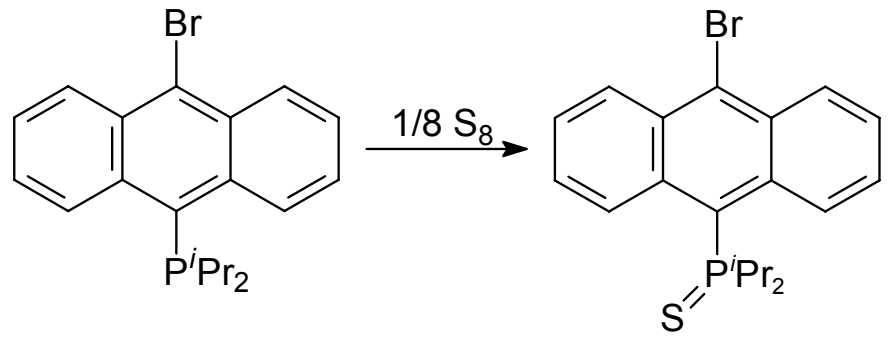

5

GI. 2-7: Darstellung von $\operatorname{Br}\left(\mathrm{C}_{14} \mathrm{H}_{8}\right) \mathrm{P}(\mathrm{S})^{i} \mathrm{Pr}_{2}$ (7). 
Analog zur Darstellung von 3 erfolgte die Synthese in Toluol in der Siedehitze. Nach Entfernen des Lösungsmittels i. Vak. wurde 7 in einer Ausbeute von $91 \%$ erhalten. Abb. 2-12 zeigt die Molekülstruktur aus zwei verschiedenen Ansichten, in Tab. 2-7 sind ausgewählte Bindungslängen und -winkel aufgeführt.
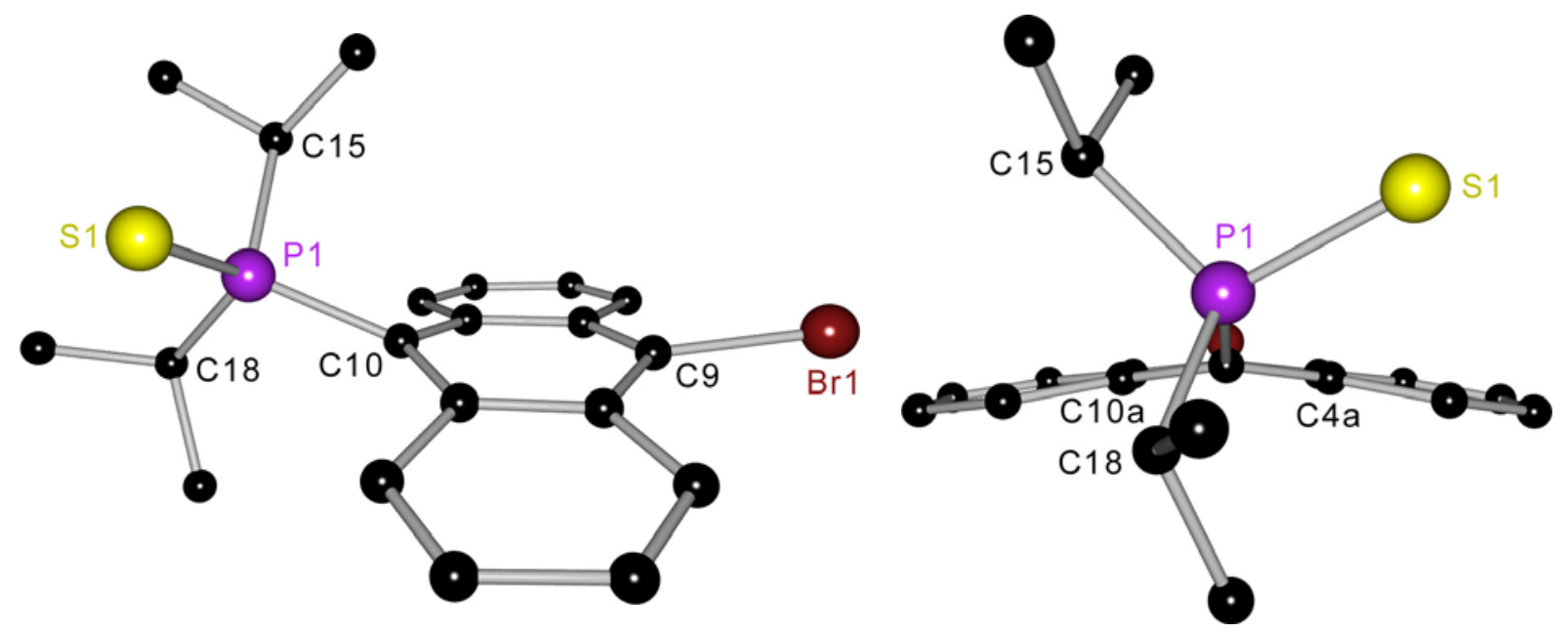

Abb. 2-12: Molekülstruktur von $\operatorname{Br}\left(\mathrm{C}_{14} \mathrm{H}_{8}\right) \mathrm{P}(\mathrm{S})^{i} \mathrm{Pr}_{2}$ (7) (rechts: Blick entlang C9 $\cdots \mathrm{C} 10$ ).

Tab. 2-7: Ausgewählte Bindungslängen / pm und -winkel / ${ }^{\circ}$ in 7.

\begin{tabular}{ll}
\hline C9-Br1 & $190.00(16)$ \\
P1-S1 & $196.89(6)$ \\
P1-C10 & $185.42(16)$ \\
P1-C15 & $184.69(16)$ \\
P1-C18 & $184.96(16)$ \\
\hline
\end{tabular}

\begin{tabular}{ll}
\hline S1-P1-C10 & $115.30(5)$ \\
C10-P1-C15 & $103.65(7)$ \\
C10-P1-C18 & $107.70(7)$ \\
C15-P1-C18 & $109.09(7)$ \\
P1-C10 ‥C9 & $158.22(8)$ \\
\hline
\end{tabular}

7 kristallisiert in der monoklinen, zentrosymmetrischen Raumgruppe $P 2_{1} / c$ mit zwei Molekülen in der asymmetrischen Einheit. Die Einführung des Chalkogensubstituenten bringt eine deutliche Änderung in der Molekülstruktur im Vergleich zur nichtoxidierten Stammverbindung $\mathbf{5}$ mit sich. So ist das Anthracengerüst nicht mehr planar, sondern weist einen Faltungswinkel zwischen den durch C1 - C4 und C5 - C8 aufgespannten Ebenen von $162.7^{\circ}$ auf. Die beiden Isopropylgruppen sind transoid bezüglich der Aromatenebene angeordnet und die $\mathrm{P}=\mathrm{S}$-Bindung bildet mit der C10a $\cdots C 4 a-V e r b i n d u n g s l i n i e ~ e i n e n ~ T o r s i o n s w i n k e l ~ v o n ~ 32.0^{\circ}$. Das Bromatom ist $42.1 \mathrm{pm}$ und das Phosphoratom sogar $89.6 \mathrm{pm}$ von der idealisierten Ebene durch C4a, C8a, C9a und C10a entfernt. 


\subsection{Synthese und Struktur von $\operatorname{Br}\left(\mathrm{C}_{14} \mathrm{H}_{8}\right) \mathrm{P}(\mathrm{Se})^{i} \mathrm{Pr}_{2}(8)$}

Analog zur Oxidation mit Schwefel lieferte die Umsetzung von $\mathbf{5}$ mit grauem Selen 9-Brom-10-(diisopropylselenophosphoryl)anthracen (8) in einer Ausbeute von $91 \%$ (GI. 2-8).<smiles>CCCCc1c2ccccc2c(Br)c2ccccc12</smiles>

5

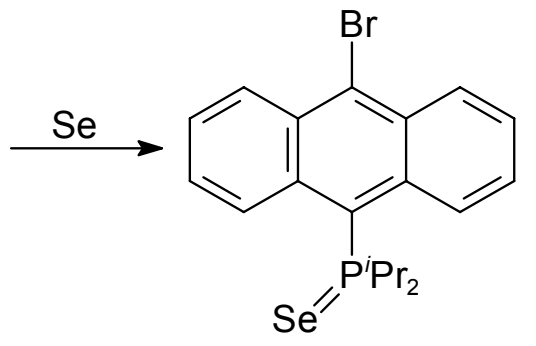

8

GI. 2-8: Darstellung von $\operatorname{Br}\left(\mathrm{C}_{14} \mathrm{H}_{8}\right) \mathrm{P}(\mathrm{Se})^{j} \mathrm{Pr}_{2}$ (8).

Durch Kristallisation aus heißem Toluol werden Kristalle erhalten, die für eine röntgenkristallographische Strukturbestimmung geeignet sind. In Abb. 2-13 ist die Molekülstruktur dargestellt, Tab. 2-8 enthält ausgewählte Bindungslängen und -winkel.
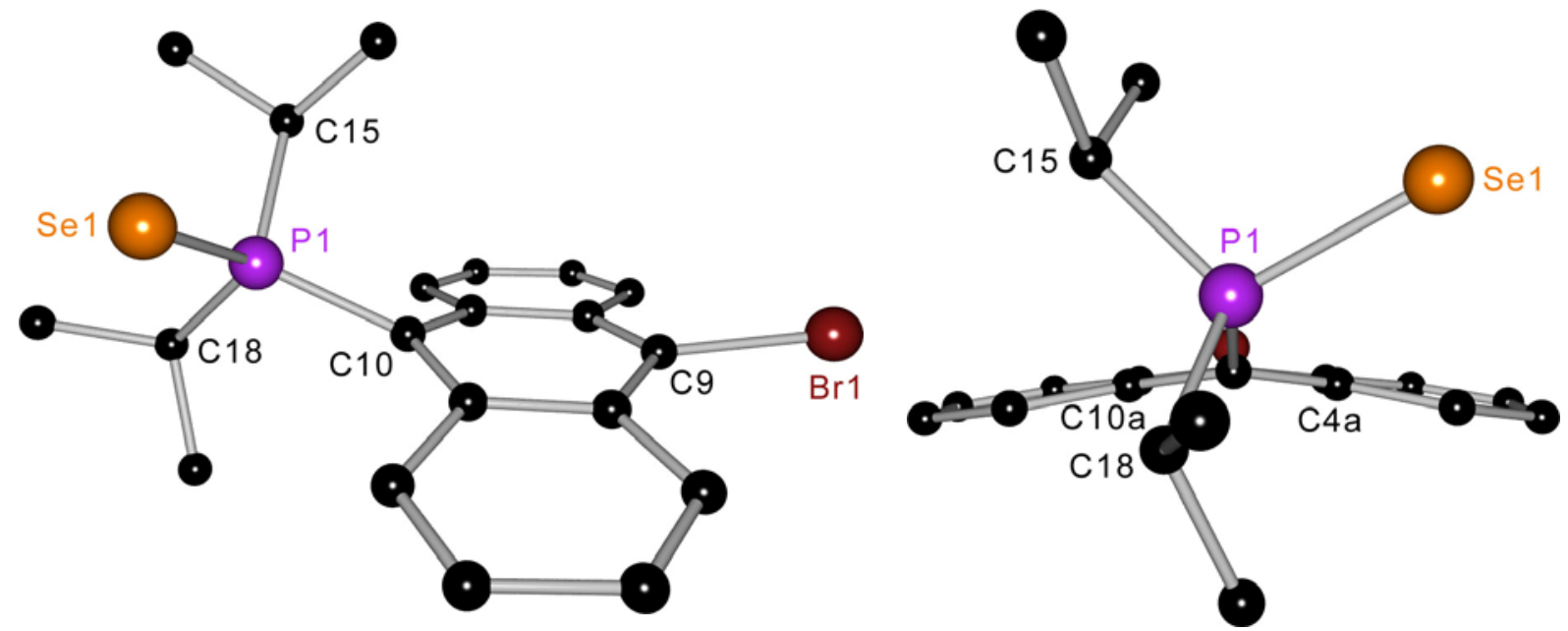

Abb. 2-13: Molekülstruktur von $\operatorname{Br}\left(\mathrm{C}_{14} \mathrm{H}_{8}\right) \mathrm{P}(\mathrm{Se})^{j} \mathrm{Pr}_{2}$ (8) (rechts: Blick entlang $\mathrm{C} 9 \cdots \mathrm{C} 10$ ).

Tab. 2-8: Ausgewählte Bindungslängen / pm und -winkel / ${ }^{\circ}$ in 8.

\begin{tabular}{ll}
\hline C9-Br1 & $190.04(19)$ \\
P1-Se1 & $212.26(5)$ \\
P1-C10 & $185.76(19)$ \\
P1-C15 & $185.6(2)$ \\
P1-C18 & $185.6(2)$ \\
\hline
\end{tabular}

\begin{tabular}{ll}
\hline Se1-P1-C10 & $116.82(6)$ \\
C10-P1-C15 & $107.37(9)$ \\
C10-P1-C18 & $103.17(9)$ \\
C15-P1-C18 & $109.62(9)$ \\
P1-C10 ‥C9 & $160.57(10)$ \\
\hline
\end{tabular}


8 kristallisiert isostrukturell zu dem mit Schwefel oxidierten Derivat 7 in der Raumgruppe $P 2_{1} / C$ und zeigt auch einen analogen Aufbau der Molekülstruktur. Die beiden Isopropylgruppen sind relativ zum Anthracengerüst in transoider Weise orientiert. Das Bromatom ist $42.2 \mathrm{pm}$ von der durch C4a, C8a, C9a und C10a aufgespannten Ebene entfernt, der Abstand des Phosphoratoms von dieser Ebene beträgt 93.3 pm und ist somit durch das größere Selenatom um fast $4 \mathrm{pm}$ größer als in 7. Der Faltungswinkel zwischen den idealisierten Ebenen durch $\mathrm{C} 1, \mathrm{C} 2, \mathrm{C} 3, \mathrm{C} 4$ und $\mathrm{C} 5, \mathrm{C} 6$, C7, C8 beträgt wie in $7162.7^{\circ}$.

Um die strukturellen Auswirkungen der Chalkogen-Oxidationen zu verdeutlichen, zeigt Abb. 2-14 eine Überlagerung der Molekülstruktur von 8 mit der nicht-oxidierten Stammverbindung 5.
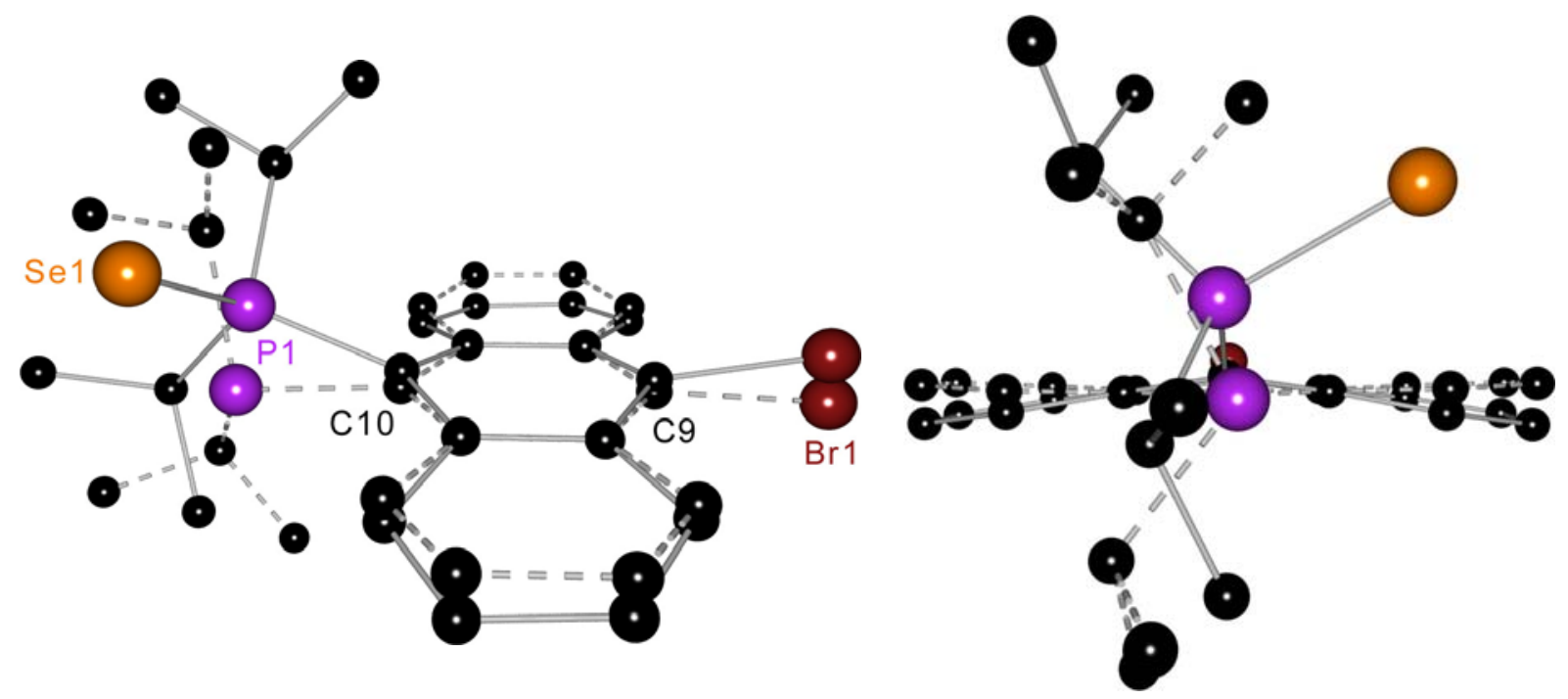

Abb. 2-14: Superposition von 5 (gestrichelte Bindungen) und 8 (durchgezogene Bindungen), erzeugt durch Überlagerung von $\mathrm{C} 4 \mathrm{a}, \mathrm{C} 8 \mathrm{a}, \mathrm{C} 9 \mathrm{a}$ und $\mathrm{C} 10 \mathrm{a}$ (rechts: Blick entlang C9 $\cdots \mathrm{C} 10$ ).

Interessanterweise bleibt bei der Umsetzung mit Schwefel bzw. Selen die transoide Anordnung der beiden Isopropylsubstituenten relativ zum Anthracengerüst erhalten, während bei den phenylsubstituierten Analoga $\mathbf{3}$ und $\mathbf{4}$ durch die Oxidation ein Übergang zur cisoiden Konfiguration erfolgt (Kap. 2.3 und 2.4). Dies ist höchstwahrscheinlich auf den erhöhten Raumbedarf der Isopropylgruppen zurückzuführen, sodass bei $\mathbf{7}$ und $\mathbf{8}$ eine cisoide Anordnung sterisch nicht möglich ist. 


\section{SYMMETRISCH SUBSTITUIERTE 9,10-DIPHOS- PHANYLANTHRACENE}

\subsection{Wirt/Gast-Komplexe von $\left(\mathrm{Ph}_{2}(\mathrm{~S}) \mathrm{P}\right)_{2}\left(\mathrm{C}_{14} \mathrm{H}_{8}\right)$}

Durch zweifache Lithiierung von 9,10-Dibromanthracen und anschließender Reaktion mit zwei Äquivalenten Chlordiphenylphosphan wird 9,10-Bis(diphenylphosphanyl)anthracen erhalten. ${ }^{[38]}$ Stalke et al. berichteten 2003 von der nachfolgenden Umsetzung der Verbindung mit elementarem Schwefel, die in 88\%iger Ausbeute das Oxidationsprodukt 9,10-Bis(diphenylthiophosphoryl)anthracen (SPAnPS) liefert (GI. 3-1). ${ }^{[12]}$

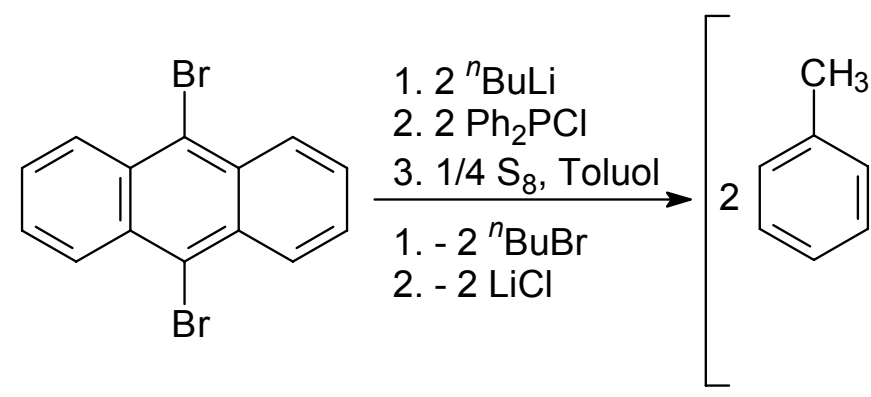

@<smiles>S=[PH](c1ccccc1)c1c2ccccc2c([PH](=S)c2ccccc2)c2ccccc12</smiles>

9

GI. 3-1: Synthese des Wirt/Gast-Komplexes $\left[2 \mathrm{C}_{7} \mathrm{H}_{8} @\left\{\left(\mathrm{Ph}_{2}(\mathrm{~S}) \mathrm{P}\right)_{2}\left(\mathrm{C}_{14} \mathrm{H}_{8}\right)\right\}\right](9) .{ }^{[112]}$

Bei der Reaktion entsteht das Produkt in Form gelber luftstabiler Kristalle, das Ergebnis der Röntgenstrukturuntersuchung ist in Abb. 3-1 dargestellt, Tab. 3-1 enthält ausgewählte Bindungslängen und -winkel.

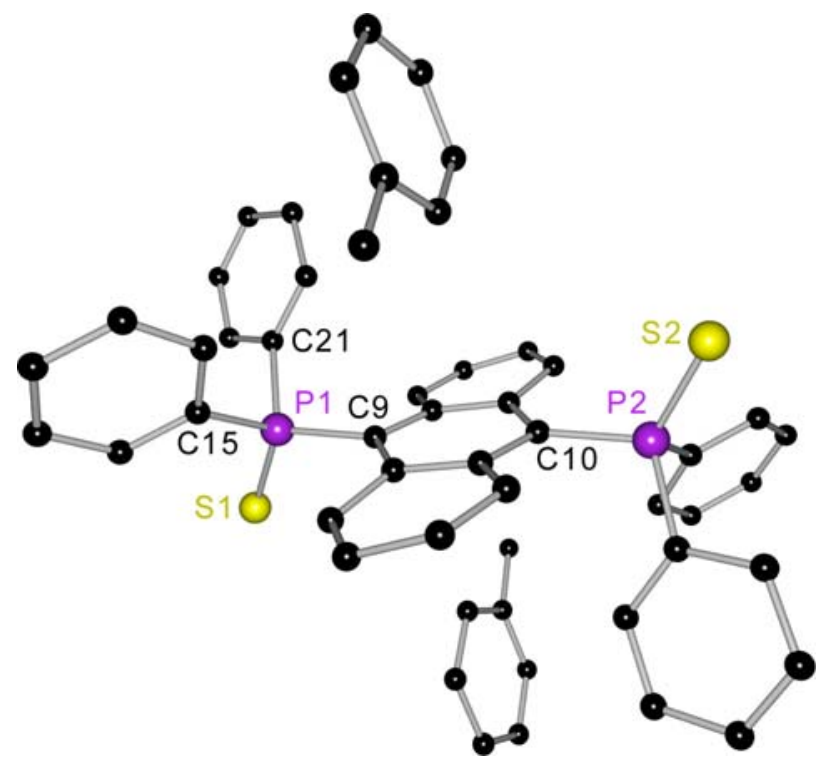

Tab. 3-1: Ausgewählte Bindungslängen / pm und -winkel $/{ }^{\circ}$ in 9.

Abb. 3-1: Molekülstruktur von 9

\begin{tabular}{ll}
\hline P1-S1 & $193.78(8)$ \\
P1-C9 & $183.39(19)$ \\
P1-C15 & $182.5(2)$ \\
P1-C21 & $182.0(2)$ \\
& \\
S1-P1-C9 & $113.44(7)$ \\
C15-P1-C21 & $97.91(9)$ \\
\hline
\end{tabular}


9 kristallisiert in der monoklinen, zentrosymmetrischen Raumgruppe $P 2_{1} / n$. Die beiden $\mathrm{P}=\mathrm{S}$-Bindungen sind transoid orientiert und befinden sich in einer Ebene, die senkrecht zum Anthracengerüst angeordnet ist. Während die durch C1 - C4 und C5 C8 aufgespannten Ebenen koplanar sind, betragen die Abstände von C9 und P1 zu diesen Ebenen 24.3 bzw. 75.8 pm.

Wie aus der Molekülstruktur ersichtlich ist, sind Lösungsmittelmoleküle in das Kristallgitter eingebaut, das Verhältnis Wirt (SPAnPS) : Gast (Toluol) beträgt 1:2. Die toluolhaltigen Kristalle weisen bei Raumtemperatur unter UV-Bestrahlung eine intensive Festkörperfluoreszenz auf. Wird das Toluol bei etwa $100^{\circ} \mathrm{C}$ im Vakuum aus dem Kristallgitter entfernt, so verliert SPAnPS diese Fähigkeit der Lumineszenz im Festkörper. Versetzt man hingegen das so erhaltene Pulver erneut mit wenig Toluol, so stellt sich kurz darauf die Festkörperfluoreszenz wieder ein. Dieser Prozess ist beliebig oft wiederholbar. Aus diesen Beobachtungen wurde zunächst gefolgert, dass SPAnPS selektiv und reversibel auf Toluol durch Änderung der Fluoreszenzeigenschaften reagiert.

\subsubsection{Fluoreszierende Wirt/Gast-Komplexe von SPAnPS}

Im Verlauf weiterer Untersuchungen wurde jedoch gezeigt, dass SPAnPS auch mit anderen Lösungsmitteln Wirt/Gast-Komplexe bildet. ${ }^{[113]}$ Beispielsweise werden durch Umkristallisation von toluolfreiem SPAnPS aus Brombenzol, $p$-Chlortoluol, Styrol oder den drei Xylolisomeren Kristalle erhalten, die intensive Festkörperfluoreszenz mit annähernd identischen Emissionsmaxima um $510 \mathrm{~nm}\left(\lambda_{\mathrm{ex}}=380 \mathrm{~nm}\right)$ aufweisen. Alle fluoreszierenden Verbindungen kristallisieren isostrukturell in der Raumgruppe $P 2_{1} / n$ und enthalten jeweils pro Wirtsmolekül SPAnPS zwei Lösungsmittelmoleküle. Die Gastmoleküle können in der Hitze i. Vak. entfernt werden, was stets zum Erlöschen der Festkörperfluoreszenz führt.

SPAnPS reagiert somit neben Toluol noch auf eine Vielzahl anderer aromatischer Gäste durch Ausbildung von Wirt/Gast-Systemen mit gleichen Fluoreszenzeigenschaften. Daher sollte neben möglichen Wirt/Gast-Wechselwirkungen auch die Anordnung der Wirtsmoleküle im Kristallgitter untersucht werden. Abb. 3-2 zeigt einen Ausschnitt aus der Kristallstruktur des SPAnPS/Toluol-Komplexes (9), wobei die Toluolmoleküle sowie die Kohlenstoffatome der Phenylgruppen (außer $\mathrm{C}_{i p s o}$ ) aus Gründen der Übersichtlichkeit nicht dargestellt sind. Es ist deutlich zu erkennen, dass die Anthracengerüste zickzackartig in zwei unterschiedlichen Ebenen angeordnet 
sind, die einen Winkel von $70.5^{\circ}$ miteinander einschließen. Da alle bisher erhaltenen fluoreszierenden Wirt/Gast-Systeme von SPAnPS isostrukturell kristallisieren, ist diese molekulare Anordnung möglicherweise essentiell für die Festkörperlumineszenz.
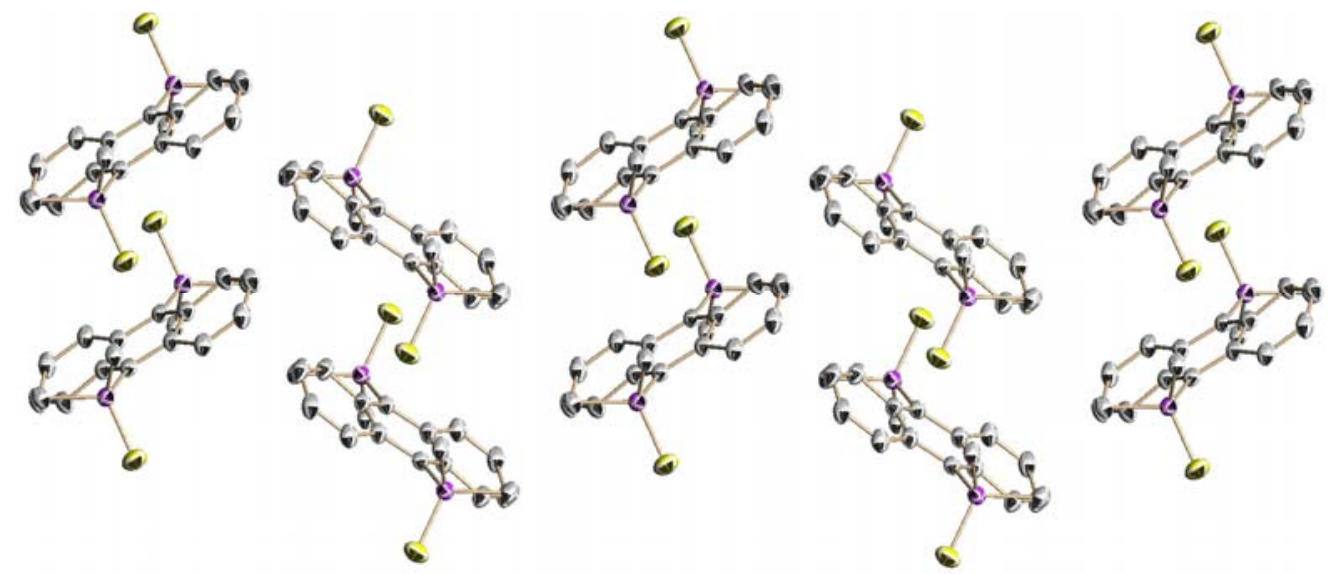

Abb. 3-2: Ausschnitt aus der Kristallstruktur von 9; Blickrichtung entlang $\vec{c}$ (die Toluolmoleküle sind aus Gründen der Übersichtlichkeit nicht dargestellt).

Im Rahmen dieser Arbeit wurden zwei weitere fluoreszierende Komplexe von SPAnPS untersucht, die in einem anderen Kristallsystem als die bisherigen kristallisieren und eine unterschiedliche Anordnung der Moleküle im Kristallgitter zeigen. Durch Umkristallisation aus Benzol bzw. Phenylacetylen werden die Wirt/Gast-Systeme $\quad\left[2 \mathrm{C}_{6} \mathrm{H}_{6} @\left\{\left(\mathrm{Ph}_{2}(\mathrm{~S}) \mathrm{P}\right)_{2}\left(\mathrm{C}_{14} \mathrm{H}_{8}\right)\right\}\right] \quad$ (10) bzw. $\quad\left[2 \mathrm{C}_{8} \mathrm{H}_{6} @\right.$ $\left.\left\{\left(\mathrm{P}(\mathrm{S}) \mathrm{Ph}_{2}\right)_{2}\left(\mathrm{C}_{14} \mathrm{H}_{8}\right)\right\}\right](\mathbf{1 1})$ erhalten. Abb. 3-3 zeigt die beiden Molekülstrukturen und in Tab. 3-2 sind ausgewählte Bindungslängen und -winkel aufgeführt.
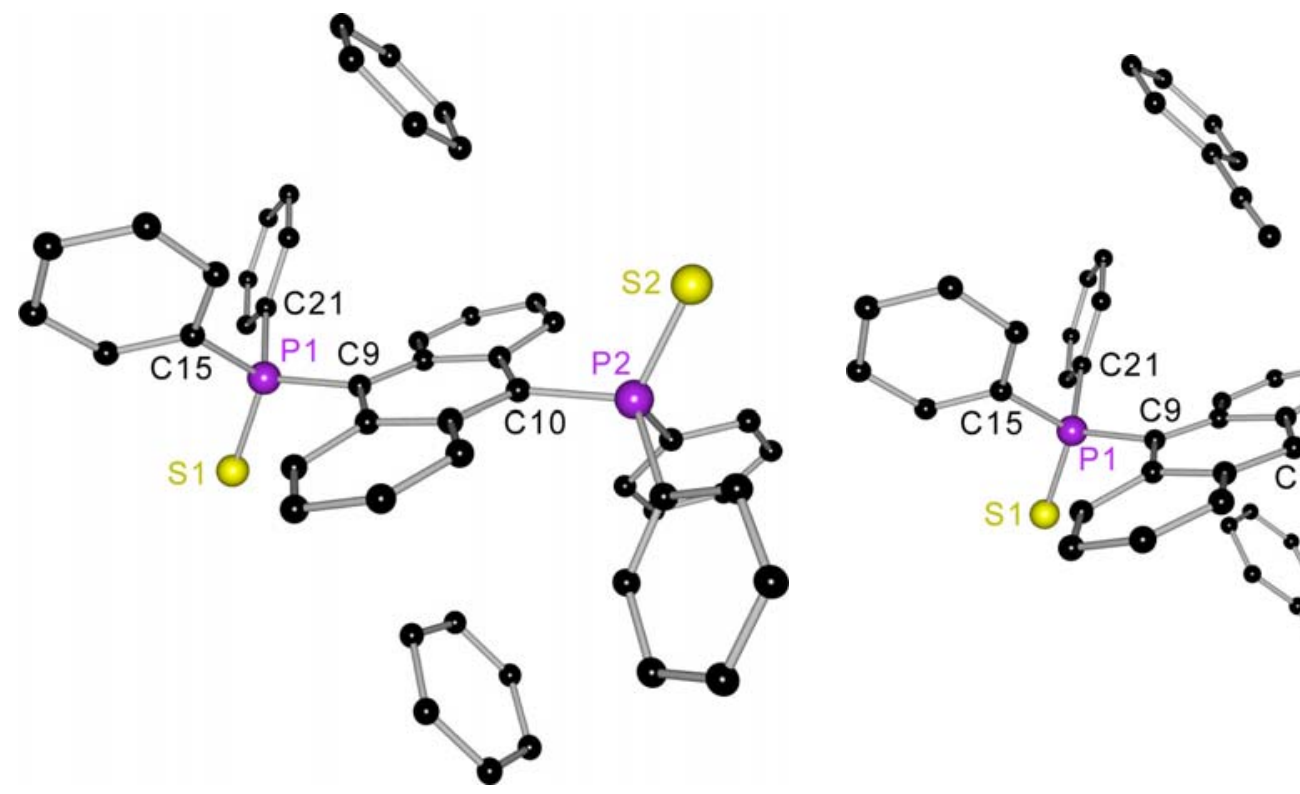

Abb. 3-3: Molekülstrukturen von $\left[2 \mathrm{C}_{6} \mathrm{H}_{6} @\left\{\left(\mathrm{Ph}_{2}(\mathrm{~S}) \mathrm{P}\right)_{2}\left(\mathrm{C}_{14} \mathrm{H}_{8}\right)\right\}\right]$ (10, links) und $\left[2 \mathrm{C}_{8} \mathrm{H}_{6} @\left\{\left(\mathrm{Ph}_{2}(\mathrm{~S}) \mathrm{P}\right)_{2}\left(\mathrm{C}_{14} \mathrm{H}_{8}\right)\right\}\right]$ (11, rechts). 
Tab. 3-2: Ausgewählte Bindungslängen / pm und -winkel / ${ }^{\circ}$ in 10 und 11.

\begin{tabular}{lcc} 
& 10 & 11 \\
\hline P1-S1 & $196.24(7)$ & $197.02(8)$ \\
P2-S2 & $196.05(7)$ & $197.02(8)$ \\
P1-C9 & $184.07(17)$ & $184.44(18)$ \\
P2-C10 & $184.02(18)$ & $184.44(18)$ \\
P1-C15 & $181.96(19)$ & $183.06(18)$ \\
P1-C21 & $181.70(18)$ & $183.77(18)$ \\
& & \\
S1-P1-C9 & $112.47(7)$ & $112.92(6)$ \\
S2-P2-C10 & $112.71(6)$ & $112.92(6)$ \\
C15-P1-C21 & $100.20(8)$ & $100.05(8)$ \\
\hline
\end{tabular}

Genau wie bei dem Toluol-Komplex von SPAnPS (9) sind auch in den Molekülstrukturen von $\mathbf{1 0}$ und $\mathbf{1 1}$ die beiden $\mathrm{P}=\mathrm{S}-B$ Bindungen jeweils transoid orientiert und befinden sich in einer Ebene, die senkrecht zum Anthracengerüst angeordnet ist. Auch die Bindungslängen und -winkel der Wirtsmoleküle in den drei Komplexen stimmen weitgehend überein und die Kristalle zeichnen sich durch intensive Festkörperfluoreszenz aus. Im Gegensatz zu den bisher untersuchten Wirt/GastKomplexen kristallisieren die beiden neuen Verbindungen jedoch in der triklinen Raumgruppe $P \overline{1}$, die asymmetrische Einheit von 10 umfasst neben einem kompletten Molekül SPAnPS noch zwei Benzolmoleküle, in der asymmetrischen Einheit von 11 sind ein halbes Wirtsmolekül und ein Molekül Phenylacetylen enthalten. Insgesamt beträgt das Verhältnis Wirt : Gast in beiden Kristallstrukturen somit $1: 2$ und entspricht damit dem der Stammverbindung $\mathbf{9}$.

Zum Vergleich der Wirt/Gast-Komplexe 9 - 11 ist in Abb. 3-4 eine Überlagerung der drei Molekülstrukturen aus zwei unterschiedlichen Ansichten dargestellt. Es fällt auf, dass sich sämtliche Anthracen-Kohlenstoffatome der drei Strukturen exakt überlagern und die Abweichung von der idealen Planarität folglich gleichermaßen ausgeprägt ist. Während die Thiophosphorylsubstituenten der triklin kristallisierenden Verbindungen $\mathbf{1 0}$ und $\mathbf{1 1}$ die gleiche Orientierung aufweisen, weicht das monokline Derivat 9 leicht von dieser $a b$ und die jeweiligen $P=S$-Bindungen schließen einen Winkel von $17.3^{\circ}$ ein. Am auffälligsten sind die unterschiedlichen Positionen der Lösungsmittelmoleküle relativ zum Wirtsmolekül SPAnPS. Jedes Toluolmolekül bildet mit der idealisierten Ebene durch C4a, C8a, C9a und C10a einen Winkel von 
$74.5^{\circ}$ und der Abstand des jeweils näheren meta-Kohlenstoffatoms zu dieser Ebene beträgt 304.4 pm. Diese Wirt/Gast-Anordnung liegt auch in allen bisher untersuchten monoklin kristallisierenden Solvat-Komplexen von SPAnPS vor. Im Gegensatz dazu beträgt der Winkel zwischen besagter Ebene und den Lösungsmittelmolekülen 54.3 in 10 und $54.2^{\circ}$ in 11. Auch die Positionen der Toluol-, Benzol- und Phenylacetylenmoleküle variieren beträchtlich, wie aus Abb. 3-4 ersichtlich ist. Somit scheint der molekulare Aufbau des SPAnPS-Wirtes (transoide Konformation, Verdrillung des Anthracengerüstes) für die resultierenden Fluoreszenzeigenschaften wichtiger zu sein als die relative Anordnung der aromatischen Gäste.
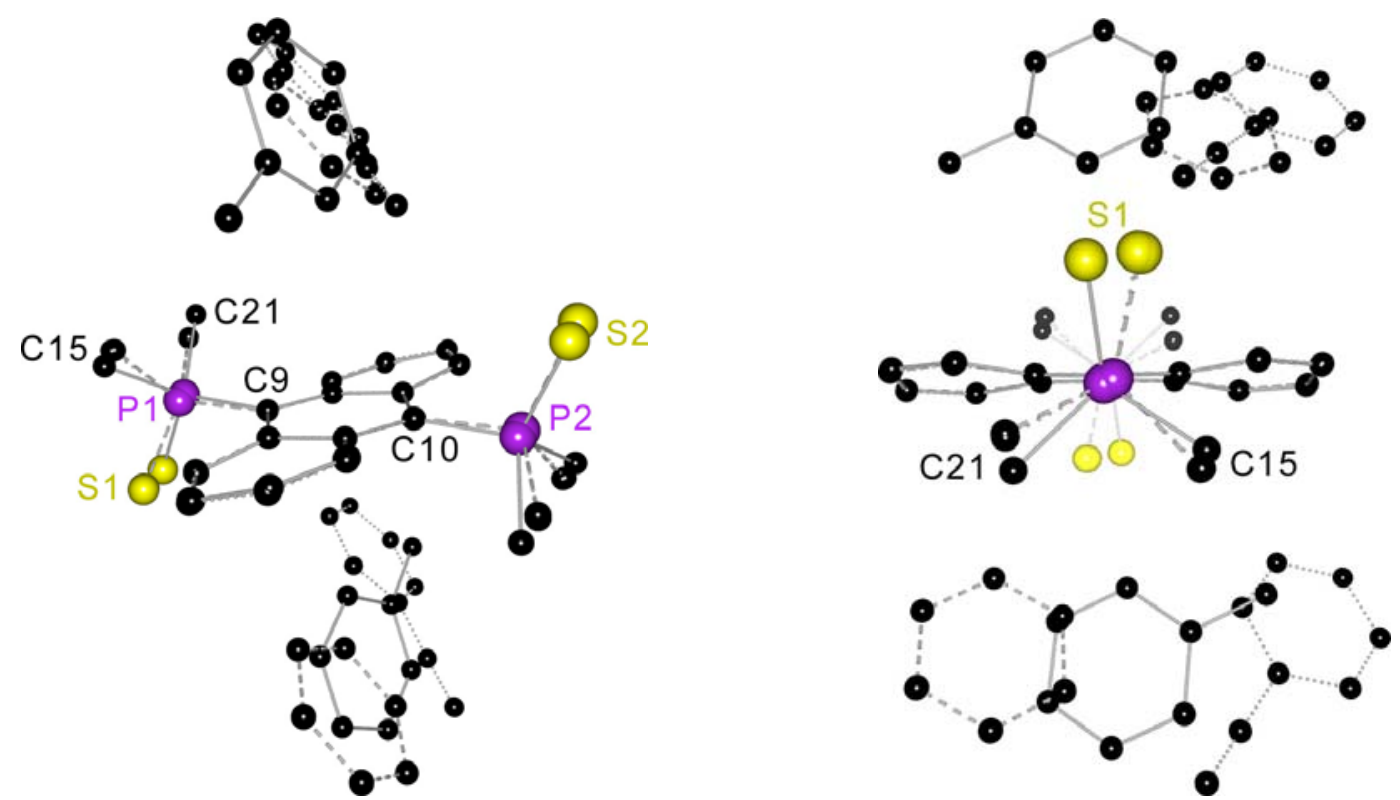

Abb. 3-4: Superposition von 9 (durchgezogene Bindungen), 10 (gestrichelte Bindungen) und 11 (gepunktete Bindungen), erzeugt durch Überlagerung von C4a, C8a, C9a und C10a (Phenylgruppen außer $\mathrm{C}_{i p s o}$ nicht dargestellt; rechts: Blick entlang P1‥C9).

Abb. 3-5 und Abb. 3-6 zeigen Ausschnitte aus den Kristallstrukturen von 10 und 11, um die Molekülpackungen der SPAnPS-Wirtsmoleküle zu verdeutlichen. Die Lösungsmittelmoleküle sowie die Kohlenstoffatome der Phenylgruppen (außer $\mathrm{C}_{i p s o}$ ) sind aus Gründen der Übersichtlichkeit nicht dargestellt. In beiden Strukturen sind die Anthracengrundgerüste ausschließlich in koplanaren Ebenen angeordnet, es liegt keine Zickzack-Orientierung wie in den monoklin kristallisierenden Wirt/GastKomplexen vor. Im Fall der Benzol-Einlagerungsverbindung 10 beträgt der Abstand zwischen zwei direkt übereinander angeordneten Fluorophor-Gerüsten 886 pm, bei der Struktur mit dem Gast Phenylacetylen (11) sind die Einheiten 1014 pm voneinander entfernt. 


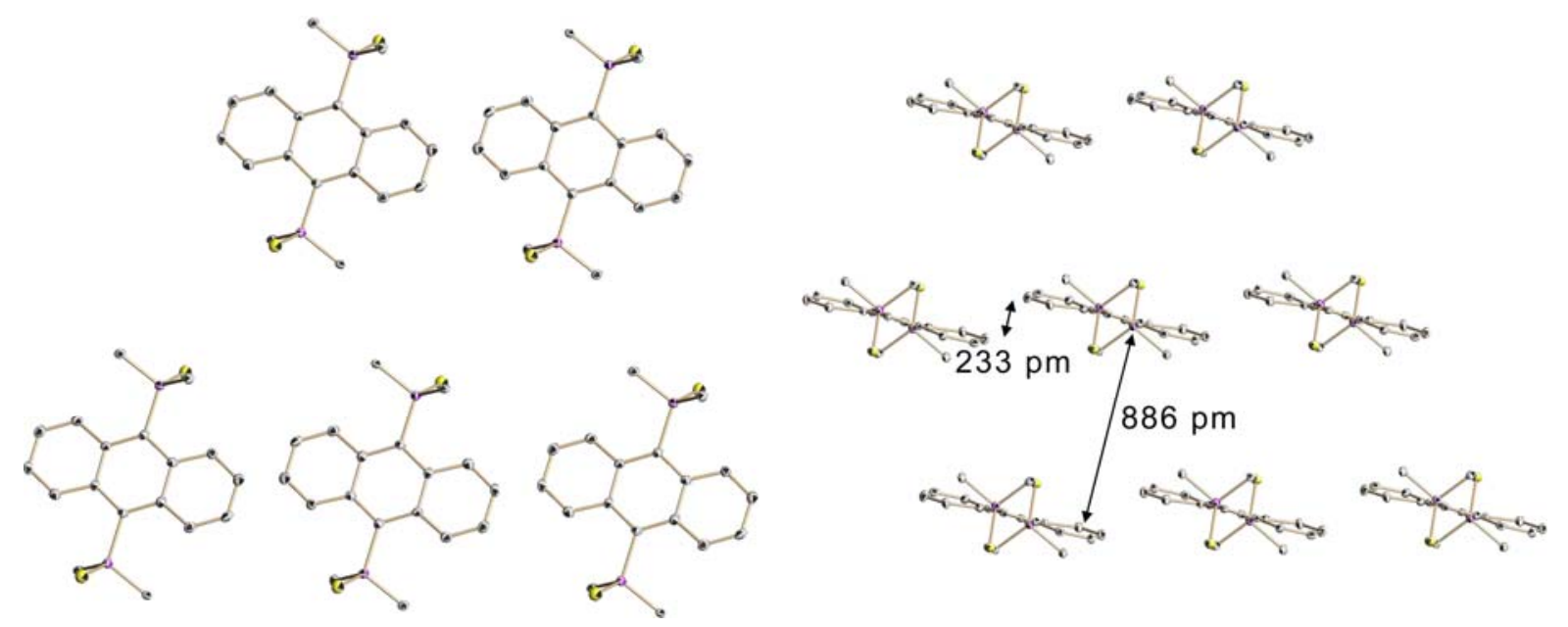

Abb. 3-5: Ausschnitt aus der Kristallstruktur von 10; Blickrichtung entlang $\vec{a}$ (links) bzw. $\vec{b}$ (rechts) (die Benzolmoleküle sind aus Gründen der Übersichtlichkeit nicht dargestellt).

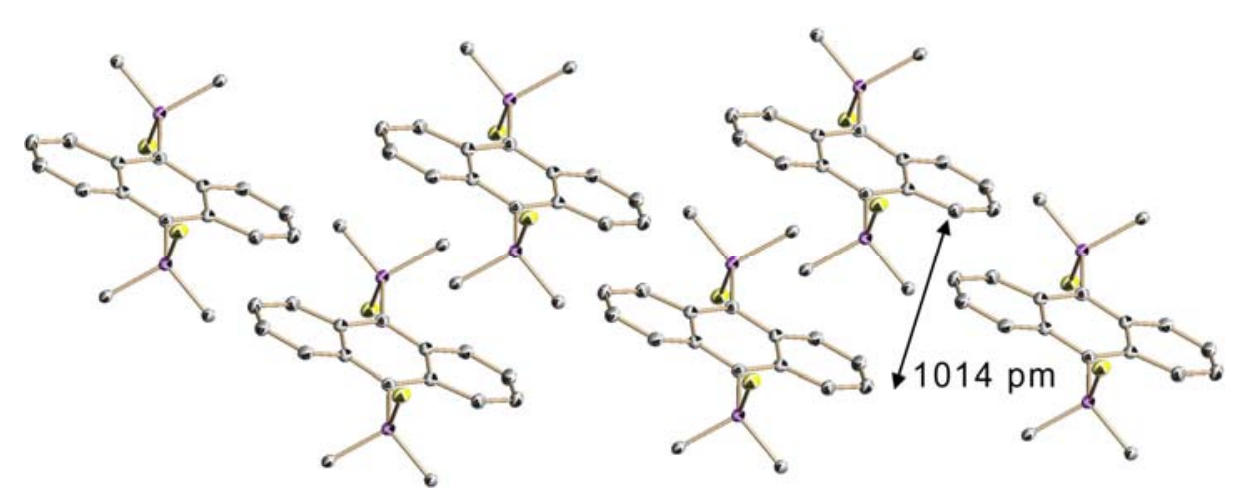

Abb. 3-6: Ausschnitt aus der Kristallstruktur von 11; Blickrichtung entlang $\overrightarrow{\mathrm{C}}$ (die Phenylacetylenmoleküle sind aus Gründen der Übersichtlichkeit nicht dargestellt).

Zusammenfassend lässt sich festhalten, dass alle fluoreszierenden SPAnPS/GastKomplexe die gleiche Konformation des Anthracengerüstes sowie dasselbe Verhältnis von Wirt zu Gast (1:2) aufweisen. Der Großteil der bisher untersuchten Verbindungen kristallisiert in der monoklinen Raumgruppe $P 2_{1} / n$, mit 10 und 11 liegen allerdings auch triklin kristallisierende Systeme $(P \overline{1})$ vor. Somit kann die isostrukturelle Anordnung der Moleküle im Festkörper als Voraussetzung für die Festkörperfluoreszenz ausgeschlossen werden. Da als Gäste sowohl polare (z. B. Halogenbenzole) als auch unpolare Aromaten (Benzol) eingelagert wurden und deren relative Orientierungen zum Wirtsmolekül deutlich variieren, deutet alles darauf hin, dass ausschließlich die transoide Anordnung der $\mathrm{P}=\mathrm{S}-$ Bindungen sowie die Verdrillung des Anthracengrundgerüstes für die Lumineszenz im Festkörper verantwortlich sind. 


\subsubsection{Nichtfluoreszierende Wirt/Gast-Komplexe von SPAnPS}

Neben den bisher gezeigten fluoreszierenden SPAnPS/Gast-Komplexen existieren auch Einlagerungsverbindungen von aromatischen Gästen und SPAnPS, die keine Lumineszenz im Festkörper zeigen. So konnten bereits während der dieser Arbeit vorangegangenen Diplomarbeit durch Kristallisation von SPAnPS mit Anisol, Benzylalkohol bzw. Benzonitril Kristalle erhalten werden, die keine Festkörperfluoreszenz im sichtbaren Bereich aufwiesen. Durch Röntgenstrukturuntersuchung konnte gezeigt werden, dass den drei Verbindungen eine cisoide Anordnung der jeweiligen $\mathrm{P}=\mathrm{S}$-Bindungen sowie eine wannenartige Faltung des Anthracengerüstes, bei der die beiden äußeren sechsgliedrigen Ringe Faltungswinkel zwischen $152^{\circ}$ und $157^{\circ}$ miteinander bilden, gemeinsam ist. Das Verhältnis SPAnPS : Gast beträgt $2: 1$ (Anisol, Benzylalkohol) bzw. 2:3 (Benzonitril) und unterscheidet sich somit vom $1: 2$-Verhältnis der untersuchten fluoreszierenden Komplexe. ${ }^{[113]}$

Im Laufe der vorliegenden Arbeit konnte durch Kristallisation von SPAnPS in $\mathrm{CDCl}_{3}$ ein weiterer Wirt/Gast-Komplex (12) erhalten werden, der keine Fluoreszenz im Festkörper zeigt. Das Ergebnis der Röntgenstrukturuntersuchung ist in Abb. 3-7 aus zwei unterschiedlichen Ansichten dargestellt, Tab. 3-3 enthält ausgewählte Bindungslängen und -winkel.

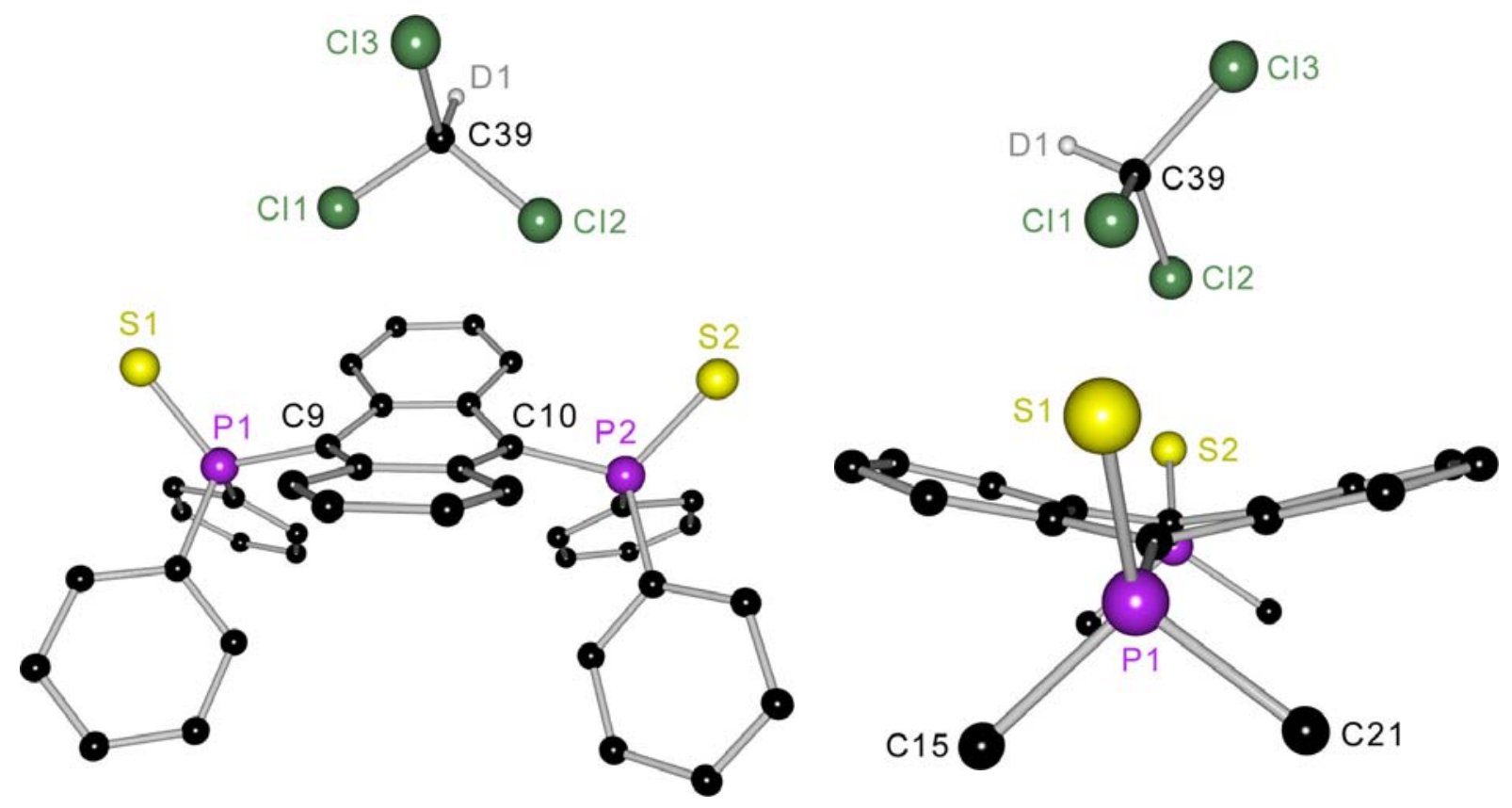

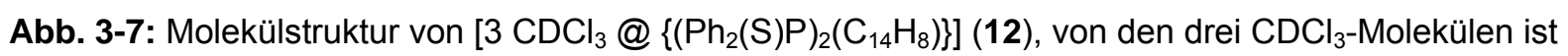
nur dasjenige dargestellt, welches sich in räumlicher Nähe des SPAnPS-Moleküls befindet (rechts: Blick entlang C9 $\cdots$ C10, von den Phenylkohlenstoffatomen ist jeweils nur $\mathrm{C}_{i p s o}$ dargestellt). 
Tab. 3-3: Ausgewählte Bindungslängen / pm und -winkel / ${ }^{\circ}$ in 12.

\begin{tabular}{|c|c|}
\hline P1-S1 & $195.35(6)$ \\
\hline P2-S2 & $196.36(6)$ \\
\hline P1-C9 & $181.78(17)$ \\
\hline P2-C10 & $182.22(17)$ \\
\hline P1-C15 & $182.23(18)$ \\
\hline P1-C21 & $182.01(17)$ \\
\hline $\mathrm{Cl} 1 \cdots \mathrm{S} 1$ & $395.78(8)$ \\
\hline $\mathrm{Cl} 2 \cdots \mathrm{S} 2$ & $354.68(7)$ \\
\hline
\end{tabular}

\begin{tabular}{ll}
\hline S1-P1-C9 & $112.34(6)$ \\
S2-P2-C10 & $114.67(6)$ \\
C9-P1-C15 & $107.44(8)$ \\
C9-P1-C21 & $107.59(8)$ \\
P1-C9 ‥C10 & $167.95(9)$ \\
Cl1-C39-Cl2 & $110.87(10)$ \\
Cl1-C39-Cl3 & $110.82(10)$ \\
Cl2-C39-Cl3 & $109.45(11)$ \\
\hline
\end{tabular}

12 kristallisiert in der triklinen, zentrosymmetrischen Raumgruppe $P \overline{1}$, die asymmetrische Einheit enthält neben einem Molekül SPAnPS noch drei $\mathrm{CDCl}_{3}$ Moleküle, von denen sich eines in räumlicher Nähe des Wirtsmoleküls befindet. Die beiden $\mathrm{P}=\mathrm{S}$-Bindungen zeigen eine cisoide Orientierung, dies entspricht auch den Molekülkonformationen der bereits untersuchten nichtfluoreszierenden Wirt/GastKomplexe. Der Faltungswinkel zwischen den durch C1-C4 und C5-C8 aufgespannten Ebenen beträgt $149.2^{\circ}$, was aus der cisoiden Anordnung der sterisch

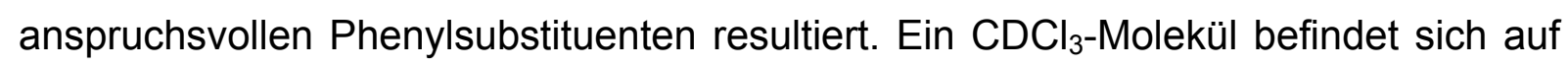
der konkaven Seite des Anthracengerüstes, die Abstände zwischen den jeweils nächsten Chlor- und Schwefelatomen betragen 395.8 bzw. 354.7 pm, letzterer Wert entspricht genau der Summe der beiden van-der-Waals-Radien (355 pm) ${ }^{[131]} \mathrm{C} 39$ ist 427.9 pm vom Mittelpunkt des mittleren sechsgliedrigen Ringes entfernt.

Um die Molekülstruktur von 12 mit der eines anderen nicht-fluoreszierenden SPAnPS-Komplexes zu vergleichen, ist in Abb. 3-8 eine Überlagerung mit der Benzonitril-Einschlussverbindung $\left[1.5 \mathrm{C}_{7} \mathrm{H}_{5} \mathrm{~N} @\left\{\left(\mathrm{Ph}_{2}(\mathrm{~S}) \mathrm{P}\right)_{2}\left(\mathrm{C}_{14} \mathrm{H}_{8}\right)\right\}\right]^{[113]}$ dargestellt. Dabei ist zu erkennen, dass in den beiden Wirtsmolekülen die Anthracengerüste sowie die Phosphoratome exakt übereinander liegen. Die $\mathrm{P}=\mathrm{S}-$ Bindungen sind in beiden Molekülstrukturen jeweils cisoid orientiert, allerdings sind die $\mathrm{P}(\mathrm{S}) \mathrm{Ph}_{2^{-}}$ Substituenten um die P1-C9- bzw. P2-C10-Bindungen leicht gedreht, sodass der Winkel zwischen den beiden P1-S1- bzw. P2-S2-Bindungen 10.0 bzw. $9.9^{\circ}$ beträgt. Auch die jeweils entsprechenden Phenylgruppen sind nicht koplanar angeordnet, sondern schließen Winkel zwischen 0.4 und $20.5^{\circ}$ ein. 


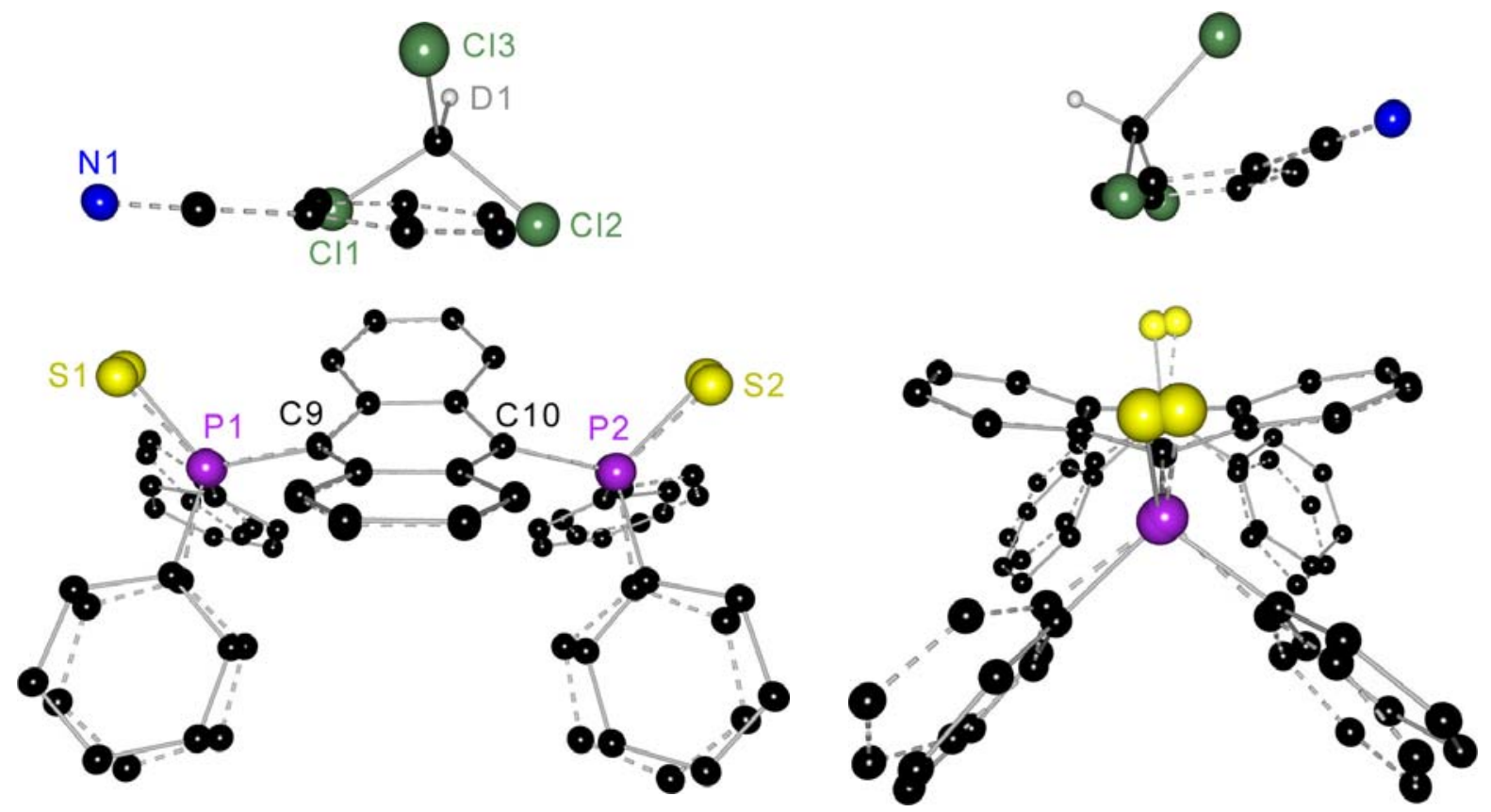

Abb. 3-8: Superposition von 12 mit [1.5 $\left.\mathrm{C}_{7} \mathrm{H}_{5} \mathrm{~N} @\left\{\left(\mathrm{Ph}_{2}(\mathrm{~S}) \mathrm{P}\right)_{2}\left(\mathrm{C}_{14} \mathrm{H}_{8}\right)\right\}\right]$ (gestrichelte Bindungen), erzeugt durch Überlagerung von C4a, C8a, C9a und C10a.

Zum direkten Vergleich der trans- und cis-Konformationen der fluoreszierenden bzw. nicht-fluoreszierenden SPAnPS-Komplexe ist in Abb. 3-9 eine Überlagerung der Wirtsmoleküle von 10 und $\mathbf{1 2}$ abgebildet, dabei sind von den Phenylsubstituenten nur die $\mathrm{C}_{\text {ipso-Atome dargestellt. }}$
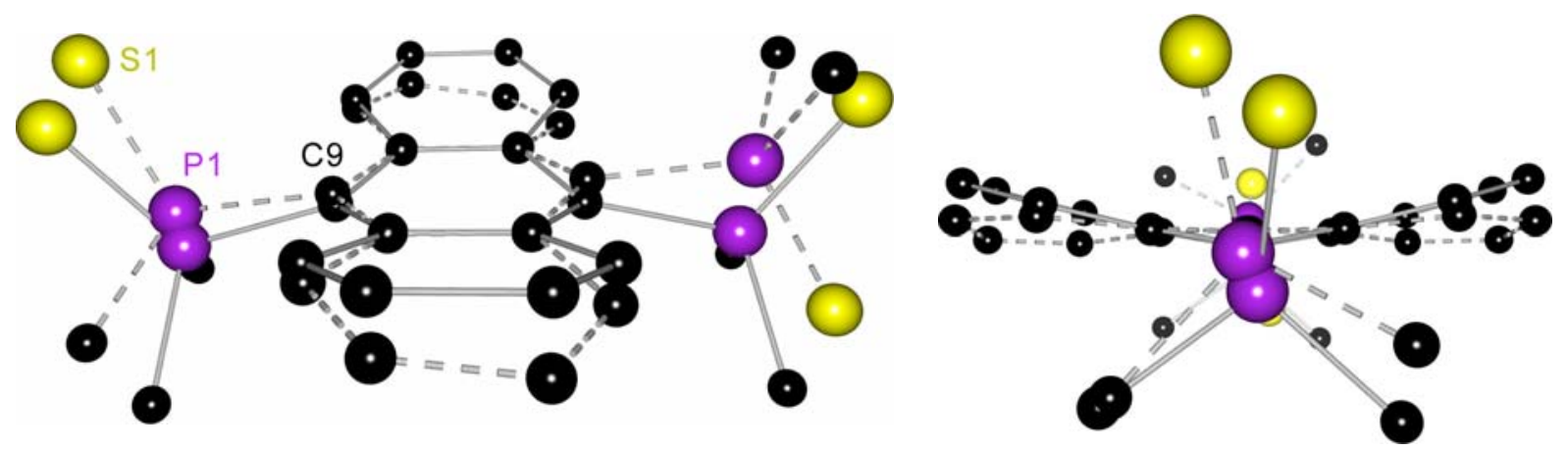

Abb. 3-9: Superposition von 10 (gestrichelte Bindungen) und 12 (durchgezogene Bindungen),

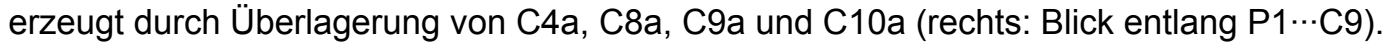




\subsection{Synthese, Tieftemperatur-Verhalten und Struktur von $\left({ }^{i} \mathrm{Pr}_{2} \mathrm{P}\right)_{2}\left(\mathrm{C}_{14} \mathrm{H}_{8}\right)(13)$}

Die Umsetzung von 9,10-Dibromanthracen mit jeweils zwei Äquivalenten ${ }^{n} \mathrm{BuLi}$ und Chlordiisopropylphosphan lieferte das symmetrisch 9,10-substituierte Bis(diisopropylphosphanyl)anthracen (13). Die zweifache Lithiierung wurde in Diethylether bei $-15{ }^{\circ} \mathrm{C}$ durchgeführt. Während die Löslichkeit von 9,10-Dibromanthracen in diesem Lösungsmittel sehr gering ist, lag die Reaktionsmischung nach der Zugabe einer äquimolaren Menge ${ }^{n} \mathrm{BuLi}$ als orange Lösung vor. Beim Zutropfen des zweiten Äquivalents an Lithiierungsreagenz fiel die Zwischenstufe 9,10-Dilithiumanthracendiid als orange-gelber Niederschlag aus und wurde direkt in situ mit ${ }^{1} \mathrm{Pr}_{2} \mathrm{PCl}$ weiter umgesetzt (GI. 3-2).

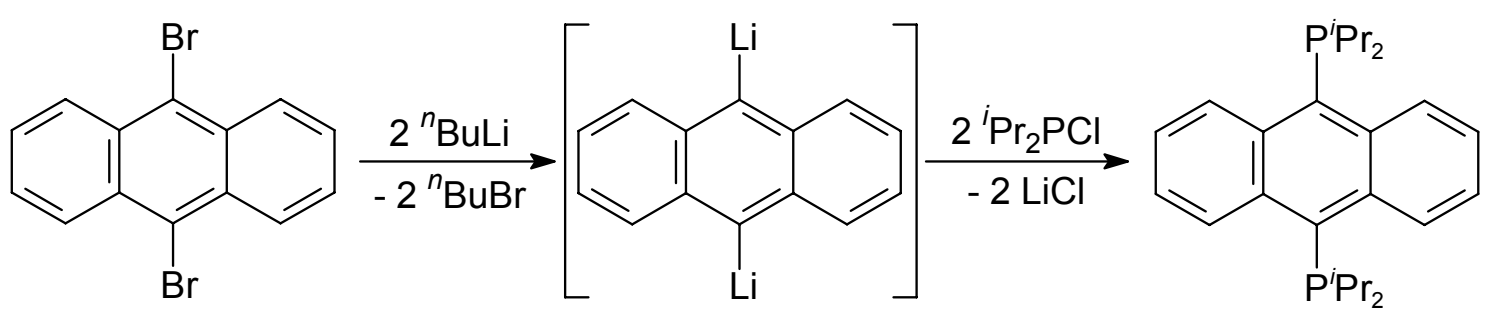

13

Gl. 3-2: Synthese von ( $\left.{ }^{(} \mathrm{Pr}_{2} \mathrm{P}\right)_{2}\left(\mathrm{C}_{14} \mathrm{H}_{8}\right)(13)$ über die Zwischenstufe 9,10-Dilithiumanthracendiid.

Die Darstellung von 13 wurde bereits in der vorangegangenen Diplomarbeit beschrieben. ${ }^{[113]}$ Im Rahmen der nun vorliegenden Arbeit konnte die Synthese optimiert (Steigerung der Ausbeute von 82 auf $95 \%$ ) sowie die Produktverbindung spektroskopisch und strukturell charakterisiert werden.

Das ${ }^{1} \mathrm{H}$-NMR-Spektrum von 13 in Toluol- $\mathrm{D}_{8}$ zeigt scharfe Signale im Alkylbereich. Die Methinprotonen der 'Pr-Substituenten ergeben ein Dublett von Septetts bei 2.77 ppm, das aufgrund identischer ${ }^{2} \mathrm{JP}_{\mathrm{HP}}$ und ${ }^{3} \mathrm{~J}_{\mathrm{HH}}$-Kopplungskonstanten $(7.04 \mathrm{~Hz})$ als PseudoOktett erscheint. Wie schon für 9-Brom-10-(diisopropylphosphanyl)anthracen (5) beschrieben, stehen die beiden Methylgruppen eines Isopropylsubstituenten in diastereotoper Beziehung zueinander und sind damit chemisch und magnetisch nicht äquivalent. Dementsprechend werden im ${ }^{1} \mathrm{H}$-Spektrum zwei Dubletts von Dubletts bei 1.32 und $0.65 \mathrm{ppm}$ mit jeweils charakteristischen ${ }^{3} \mathrm{JHP}_{\mathrm{HP}}$ und ${ }^{3} \mathrm{~J}_{\mathrm{HH}}$-Kopplungskonstanten beobachtet.

Die Integration der NMR-Signale im aromatischen Bereich bei Raumtemperatur führt zu lediglich vier Protonen, die $\mathrm{H}_{2}, \mathrm{H}_{3}, \mathrm{H}_{6}$ und $\mathrm{H}_{7}$ zugeordnet werden können. Somit sind die Signale für die Protonen $\mathrm{H}_{1}, \mathrm{H}_{4}, \mathrm{H}_{5}$ und $\mathrm{H}_{8}$, die sich in räumlicher Nähe zu 
den ${ }^{i} \operatorname{Pr}$-Gruppen befinden, nicht aufgelöst. Um mögliche dynamische Effekte zu untersuchen, wurden ${ }^{1} \mathrm{H}$-NMR-Spektren von 13 in Toluol- $\mathrm{D}_{8}$ bei Temperaturen zwischen 183 und $353 \mathrm{~K}$ aufgenommen (Abb. 3-10).

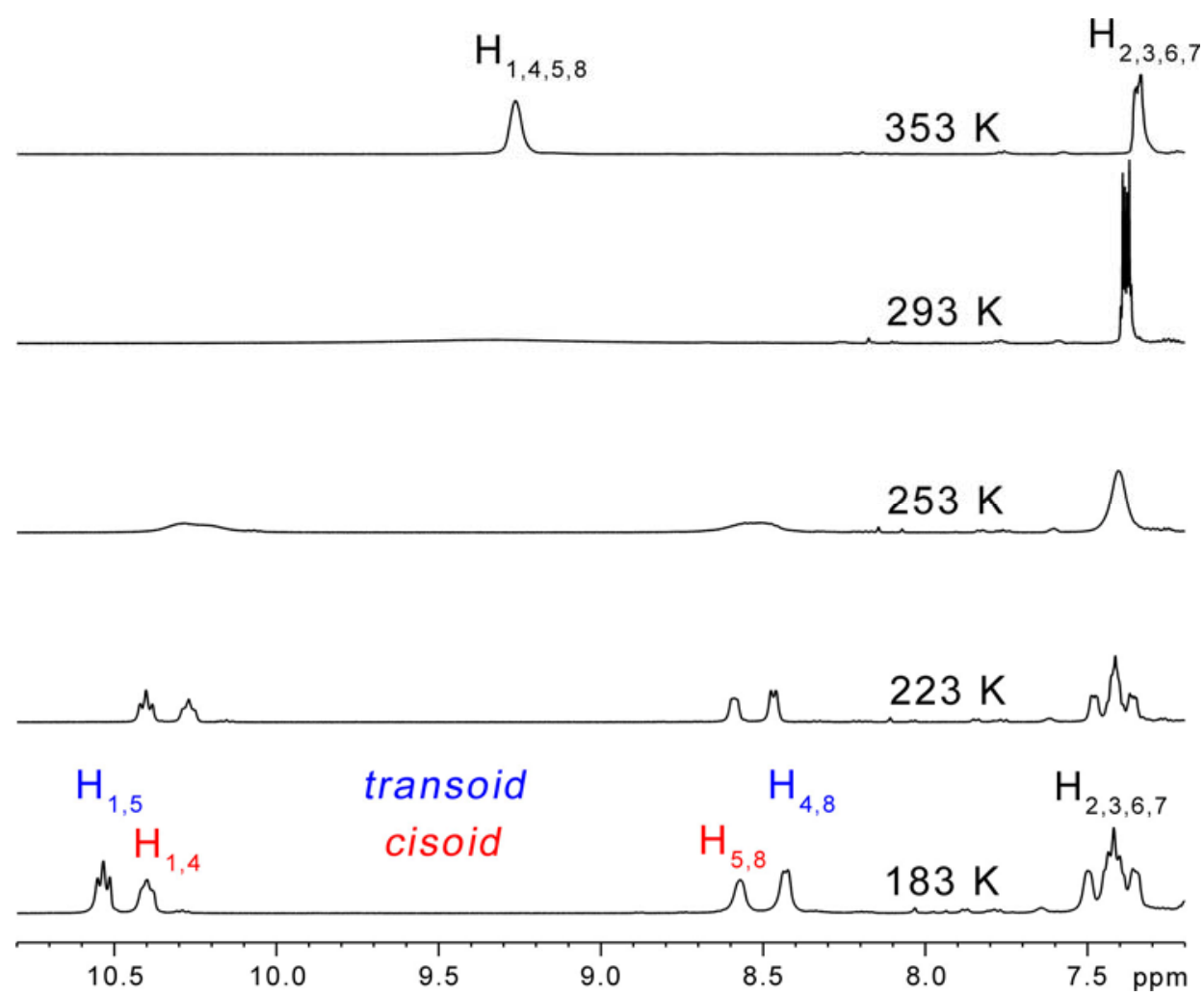

Abb. 3-10: ${ }^{1} \mathrm{H}-\mathrm{NMR}-S p e k t r e n$ von 13 in Toluol- $\mathrm{D}_{8}$ bei unterschiedlichen Temperaturen.

Beim Erwärmen auf $353 \mathrm{~K}$ ist das Auftreten eines neuen Singuletts bei $9.3 \mathrm{ppm}$ zu beobachten. Wie für $\mathbf{5}$ beschrieben, führt bei Erhöhung der Temperatur die schnelle Rotation der ${ }^{i} \mathrm{Pr}_{2} \mathrm{P}$-Substituenten um die $\mathrm{P}-\mathrm{C}_{\mathrm{An}}$-Bindungen dazu, dass $\mathrm{H}_{1}, \mathrm{H}_{4}, \mathrm{H}_{5}$ und $\mathrm{H}_{8}$ chemisch äquivalent sind, was im Auftreten eines breiten Singuletts im ${ }^{1} \mathrm{H}-\mathrm{NMR}$ Spektrum resultiert.

Die bei tiefer Temperatur gemessenen NMR-Spektren von 13 unterscheiden sich allerdings deutlich von dem monophosphanylsubstituierten Derivat 5. Das zu $\mathrm{H}_{1}, \mathrm{H}_{4}$, $\mathrm{H}_{5}$ und $\mathrm{H}_{8}$ gehörige Signal wird in vier neue Resonanzen bei 10.5, 10.4, 8.6 und $8.4 \mathrm{ppm}$ aufgespaltet, die sich auch in der Höhe der entsprechenden Integrale unterscheiden. Trotz schlechter Auflösung lassen sich zwei der vier Signale als Dubletts und die beiden anderen als Dubletts von Dubletts interpretieren. Um die beobachteten Multiplizitäten zu spezifizieren wurde neben dem regulären ${ }^{1} \mathrm{H}-\mathrm{NMR}$ Spektrum ein phosphorentkoppeltes ${ }^{1} \mathrm{H}$-Spektrum bei $213 \mathrm{~K}$ gemessen (Abb. 3-11). Wie deutlich zu erkennen ist, werden die Pseudo-Triplett-Aufspaltungen der beiden Signale bei 10.5 und $10.4 \mathrm{ppm}$ im ${ }^{1} \mathrm{H}\left\{{ }^{31} \mathrm{P}\right\}-N M R-S p e k t r u m$ zu zwei Dubletts reduziert, 
somit basieren die Multiplizitäten unter anderem auf Spin-Spin-Wechselwirkungen mit den Phosphoratomen. Die Aufspaltungen der Signale bei 8.6 und 8.4 ppm bleiben dagegen von der Phosphorentkopplung unberührt.

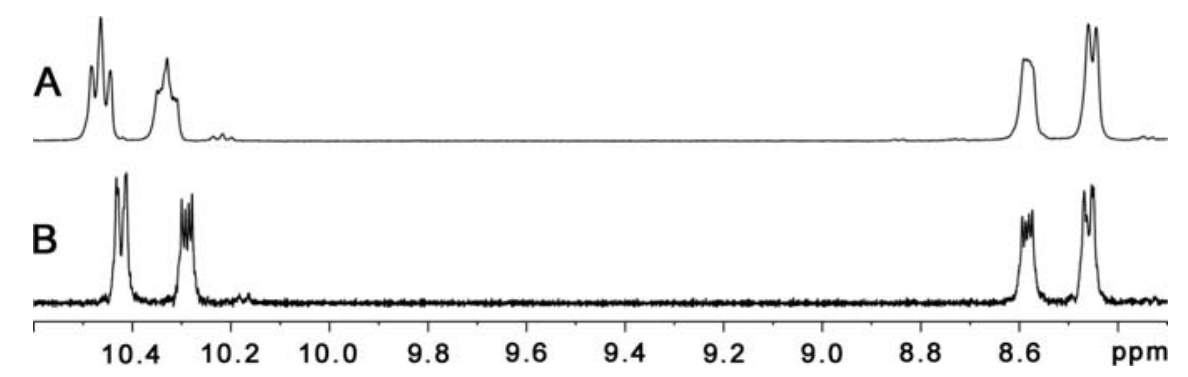

Abb. 3-11: Ausschnitte aus den ${ }^{1} \mathrm{H}-(\mathbf{A})$ und ${ }^{1} \mathrm{H}\left\{{ }^{31} \mathrm{P}\right\}-N M R-S p e k t r e n(B)$ von 13 in Toluol-D8 bei $213 \mathrm{~K}$.

Um mögliche H-H-Fernkopplungen zu untersuchen, wurde zusätzlich ein NOESYExperiment bei $213 \mathrm{~K}$ durchgeführt (Abb. 3-12). Während bei herkömmlichen $\mathrm{HH}$ COSY-Experimenten die Korrelation von Signalen auf Konnektivitäten durch Bindungen beruht, signalisieren Kreuzpeaks im NOESY-Spektrum die räumliche Nachbarschaft von Kernen. ${ }^{[130]}$ Abb. 3-12 zeigt solche Korrelationspeaks zwischen den $\mathrm{CH}$-Protonen der 'Pr-Gruppen und den aromatischen Signalen bei 8.6 und 8.4 ppm, was auf die räumliche Nähe der entsprechenden Kerne hinweist.

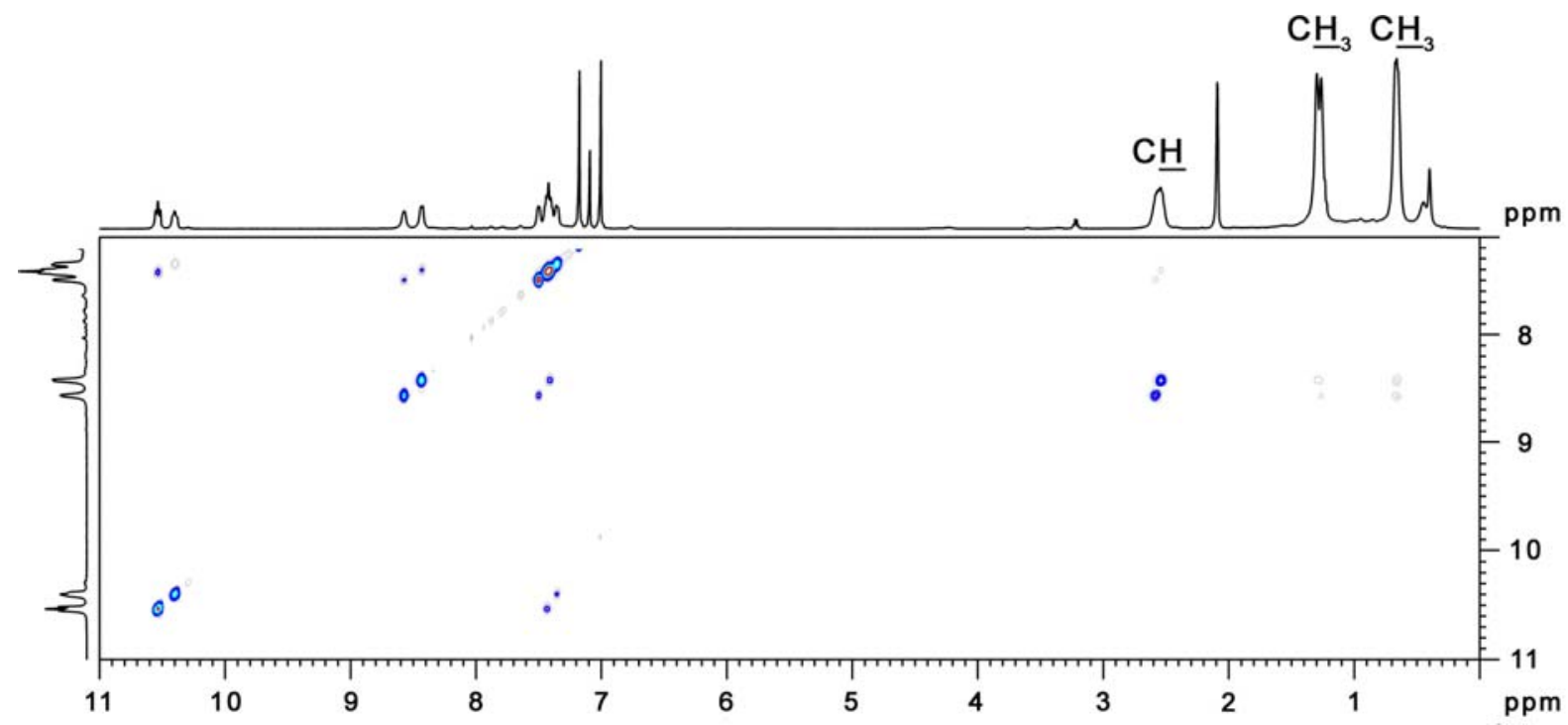

Abb. 3-12: Ausschnitt aus dem 2D-NOESY-Experiment von 13 in Toluol- $\mathrm{D}_{8}$ bei $183 \mathrm{~K}$.

Die vorangegangenen Beobachtungen lassen sich verstehen, indem man die möglichen Konformationen betrachtet, die 13 einnehmen kann. Bei höherer Temperatur sind die Wasserstoffatome $\mathrm{H}_{1}, \mathrm{H}_{4}, \mathrm{H}_{5}, \mathrm{H}_{8}$ sowie $\mathrm{H}_{2}, \mathrm{H}_{3}, \mathrm{H}_{6}, \mathrm{H}_{7}$ durch schnelle Rotation der ${ }^{i} \operatorname{Pr}_{2} \mathrm{P}$-Gruppen jeweils chemisch äquivalent (A in Abb. 3-13) und im ${ }^{1} \mathrm{H}-\mathrm{NMR}$-Spektrum werden zwei Signale beobachtet. 


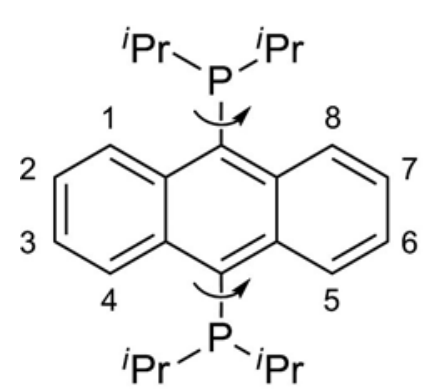

A

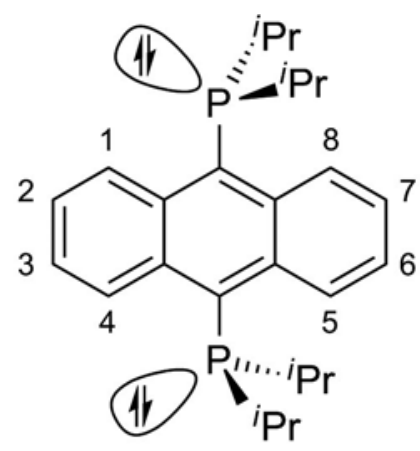

B

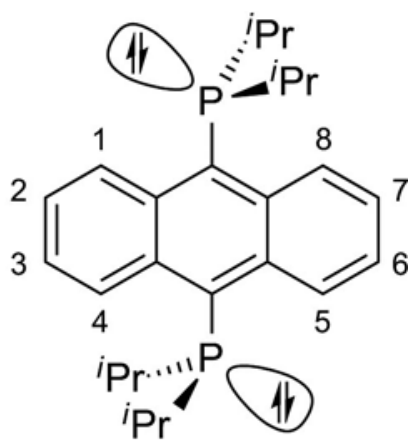

C

Abb. 3-13: Mögliche Konformationen von 13.

Durch Abkühlen auf $183 \mathrm{~K}$ wird der dynamische Prozess verlangsamt und eine gestaffelte Anordnung der Isopropylsubstituenten bezüglich des Anthracengerüsts bevorzugt. Die nichtbindenden Elektronenpaare der beiden Phosphoratome können dabei cisoid (B) oder transoid (C) zueinander angeordnet sein. Nun lassen sich alle ${ }^{1} \mathrm{H}-N M R-S i g n a l e$ den beiden unterschiedlichen Tieftemperatur-Konformeren zuordnen (Abb. 3-10): magnetische Äquivalenz liegt jeweils für die Wasserstoffpaare $\mathrm{H}_{1} / \mathrm{H}_{4}$ und $\mathrm{H}_{5} / \mathrm{H}_{8}$ des cisoiden Isomers $(B)$ sowie für $\mathrm{H}_{1} / \mathrm{H}_{5}$ und $\mathrm{H}_{4} / \mathrm{H}_{8}$ der transoiden Konformation (C) vor, sodass vier Signalsätze im entsprechenden Bereich resultieren.

Die Integration der ${ }^{1} \mathrm{H}-\mathrm{NMR}$-Signale ergibt, dass das Verhältnis der beiden Konformationsisomere 57:43 zugunsten des transoiden Rotamers beträgt, was auch aus Gründen der Sterik und der Symmetrie plausibel erscheint.

Um die Konformation von 13 im Festkörper zu untersuchen, wurde die Molekülstruktur bestimmt. Das Ergebnis der Röntgenstrukturanalyse ist in Abb. 3-14 dargestellt, Tab. 3-4 enthält ausgewählte Bindungslängen und -winkel.

13 kristallisiert in der monoklinen, zentrosymmetrischen Raumgruppe $P 2_{1} / c$ mit einem halben Molekül in der asymmetrischen Einheit. Durch Anwendung eines Inversionszentrums wird der Rest des Moleküls erzeugt, dadurch sind entsprechende Bindungsparameter in den beiden ${ }^{i} \mathrm{Pr}_{2} \mathrm{P}$-Substituenten identisch. Das zentrale Anthracengerüst ist exakt planar und die beiden Phosphoratome befinden sich genau in der Aromatenebene. 

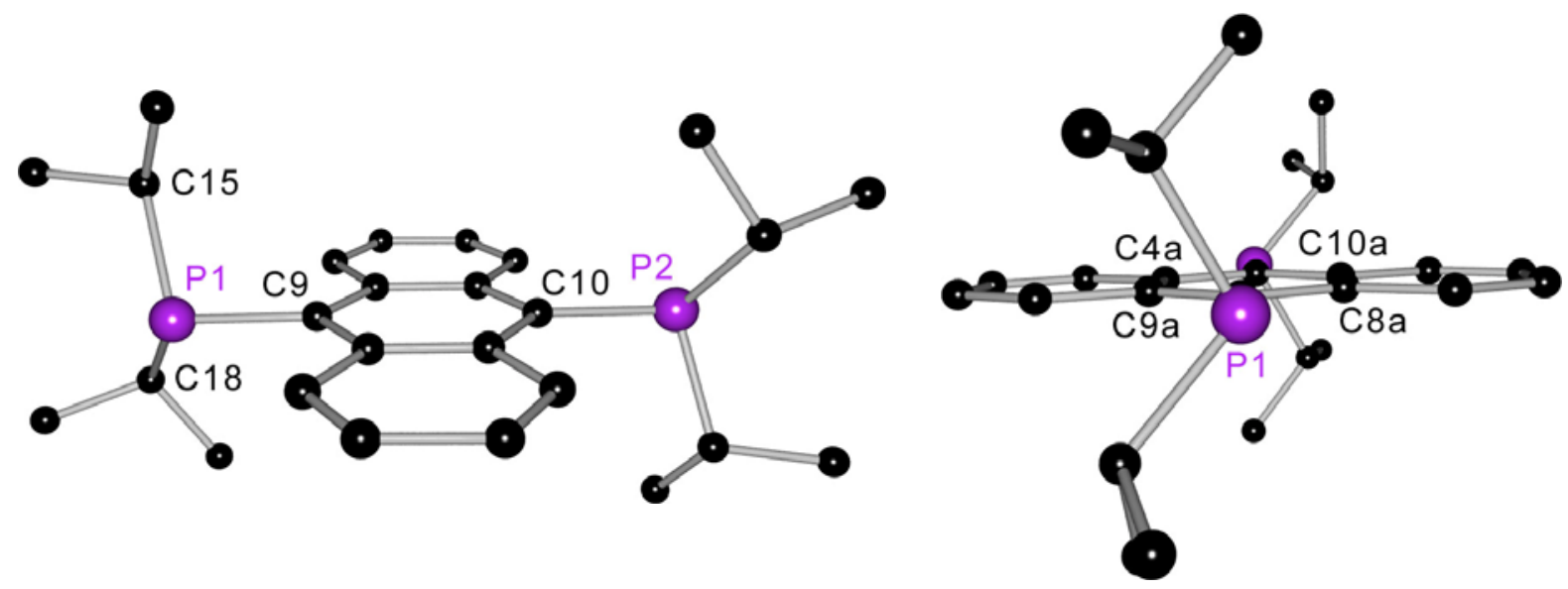

Abb. 3-14: Molekülstruktur von $\left({ }^{i} \mathrm{Pr}_{2} \mathrm{P}\right)_{2}\left(\mathrm{C}_{14} \mathrm{H}_{8}\right)$ (13) (rechts: Blick entlang $\left.\mathrm{P} 1-\mathrm{C} 9\right)$.

Tab. 3-4: Ausgewählte Bindungslängen / pm und -winkel $/^{\circ}$ in 13.

\begin{tabular}{ll}
\hline P1-C9 & $186.41(14)$ \\
P1-C15 & $187.55(15)$ \\
P1-C18 & $186.57(15)$ \\
\hline
\end{tabular}

\begin{tabular}{ll}
\hline C9-P1-C15 & $107.71(7)$ \\
C9-P1-C18 & $101.38(6)$ \\
C15-P1-C18 & $105.41(7)$ \\
\hline
\end{tabular}

Analog zur monophosphanylsubstituierten Verbindung 5 sind die ${ }^{i}$ Pr-Substituenten in einer gestaffelten Anordnung relativ zum Anthracengerüst orientiert. Beim Blick entlang der P1-C9-Bindung ist zu erkennen, dass das Molekül in der transoiden Konformation vorliegt ( $\mathbf{C}$ in Abb. 3-13), was somit auch die Ergebnisse der Tieftemperatur-NMR-Untersuchungen widerspiegelt.

\subsection{Synthese und Struktur von ( $\left.{ }^{i} \mathrm{Pr}_{2}(\mathrm{~S}) \mathrm{P}\right)_{2}\left(\mathrm{C}_{14} \mathrm{H}_{8}\right)(14)$}

Die Oxidation von 13 mit zwei Äquivalenten elementarem Schwefel lieferte 9,10-Bis(diisopropylthiophosphoryl)anthracen (14) in einer Ausbeute von 87 \% (GI. 3-3). Bei 14 handelt es sich um das Isopropylanalogon von SPAnPS, welches mit Toluol den im Festkörper fluoreszierenden Wirt/Gast-Komplex [2 $\mathrm{C}_{7} \mathrm{H}_{8} @$ SPAnPS] ausbildet. Somit sind die strukturellen und spektroskopischen Eigenschaften von 14 von besonderem Interesse. 


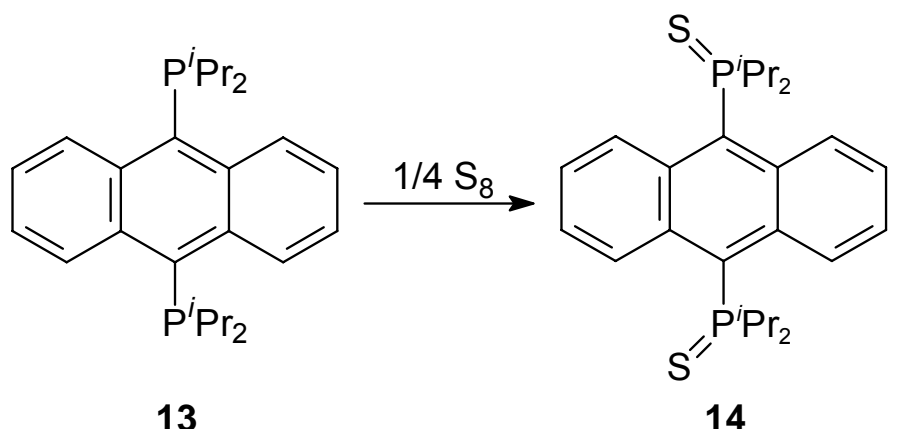

GI. 3-3: Oxidation von 13 zu ( $\left.{ }^{i} \mathrm{Pr}_{2}(\mathrm{~S}) \mathrm{P}\right)_{2}\left(\mathrm{C}_{14} \mathrm{H}_{8}\right) 14$.

Durch Lagerung einer gesättigten Lösung in Toluol und THF (1:1) wurden nach einiger Zeit Kristalle erhalten, die für eine röntgenkristallographische Strukturbestimmung geeignet waren. Abb. 3-15 zeigt die Molekülstruktur von 14, in Tab. 3-5 sind ausgewählte Bindungslängen und -winkel aufgeführt.
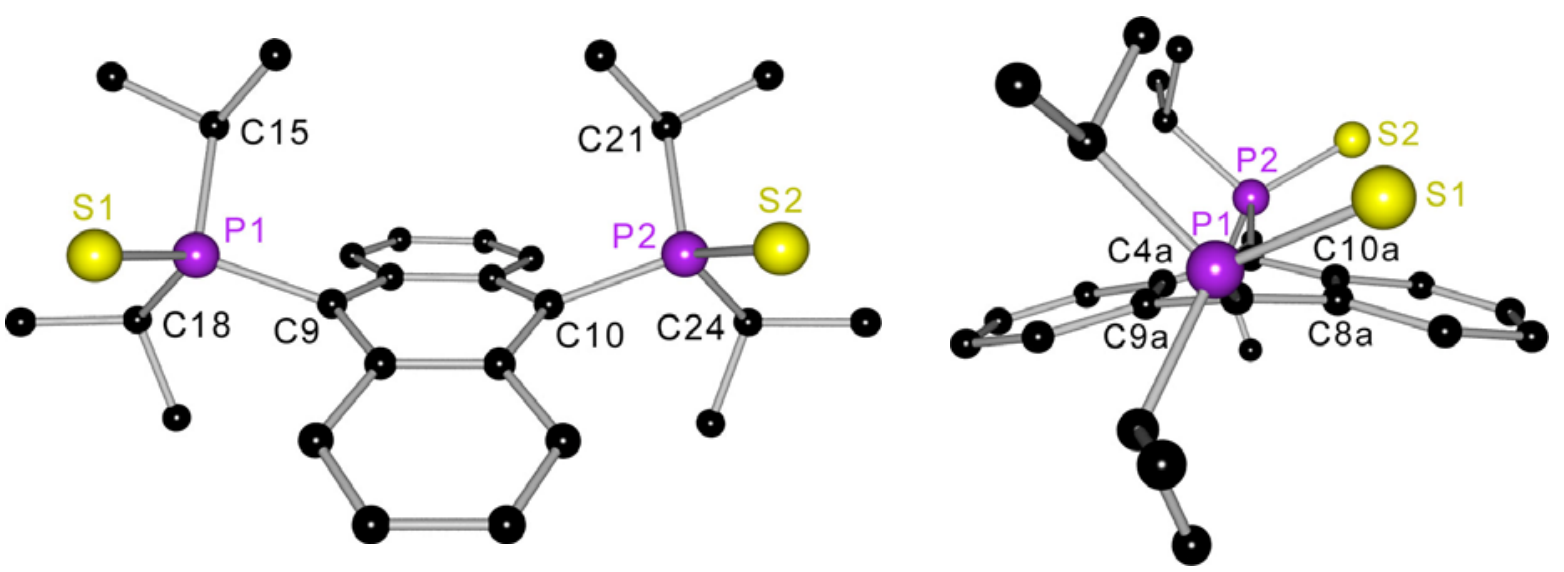

Abb. 3-15: Molekülstruktur von $\left({ }^{i} \mathrm{Pr}_{2}(\mathrm{~S}) \mathrm{P}\right)_{2}\left(\mathrm{C}_{14} \mathrm{H}_{8}\right)$ (14) (rechts: Blick entlang $\left.\mathrm{P} 1-\mathrm{C} 9\right)$.

Tab. 3-5: Ausgewählte Bindungslängen / pm und -winkel $/{ }^{\circ}$ in 14.

\begin{tabular}{ll}
\hline P1-S1 & $196.53(6)$ \\
P1-C9 & $186.42(16)$ \\
P1-C15 & $184.74(16)$ \\
P1-C18 & $185.90(16)$ \\
P2-S2 & $196.63(6)$ \\
P2-C10 & $186.66(15)$ \\
\hline
\end{tabular}

\begin{tabular}{|c|c|}
\hline C9-P1-S1 & $116.14(5)$ \\
\hline C9-P1-C15 & $103.30(7)$ \\
\hline C9-P1-C18 & $108.53(7)$ \\
\hline C15-P1-C18 & $107.29(8)$ \\
\hline $\mathrm{P} 1-\mathrm{C} 9 \cdots \mathrm{C} 10$ & 158.93(8) \\
\hline $\mathrm{P} 2-\mathrm{C} 10 \cdots \mathrm{C} 9$ & $157.97(8)$ \\
\hline
\end{tabular}

14 kristallisiert in der orthorhombischen, chiralen Raumgruppe $P 2_{1} 2_{1} 2_{1}$ mit einem Molekül in der asymmetrischen Einheit. Das Anthracengerüst ist stark abgewinkelt, der Faltungswinkel zwischen den durch C1 - C4 und C5 - C8 aufgespannten Ebenen beträgt 155.9 . Die beiden Phosphoratome sind 90.9 (P1) bzw. 85.5 pm (P2) von der 
idealisierten Ebene durch C4a, C8a, C9a und C10a entfernt. Somit sind die Abweichungen von der exakten Planarität noch ausgeprägter als in 9-Brom-10(diisopropylthiophosphoryl)anthracen (7, Kap. 2.7), was durch die Einführung des zweiten sperrigen Phosphorylsubstituenten bedingt ist. Die beiden Isopropylgruppen einer $\mathrm{P}(\mathrm{S})^{i} \mathrm{Pr}_{2}$-Einheit sind wie in 7 transoid bezüglich des Anthracengerüstes orientiert und die $\mathrm{S} 1=\mathrm{P} 1-$ Bindung bildet mit der $\mathrm{C} 8 \mathrm{a}-\mathrm{C} 9 \mathrm{a}-$ Verbindungslinie einen Torsionswinkel von $31.8^{\circ}$.

Bemerkenswerterweise zeigen die S1=P1- und S2=P2-Bindungen in die gleiche Richtung, die beiden $\mathrm{P}(\mathrm{S})^{i} \mathrm{Pr}_{2}$-Substituenten sind somit cisoid zueinander angeordnet. Diese Konformation unterscheidet sich deutlich von der der Stammverbindung 13, die eine transoide Molekülstruktur aufweist (Kap. 3.2). Um alle Unterschiede $\mathrm{zu}$ verdeutlichen ist in Abb. 3-16 eine Überlagerung der Molekülstrukturen von 13 und 14 dargestellt.

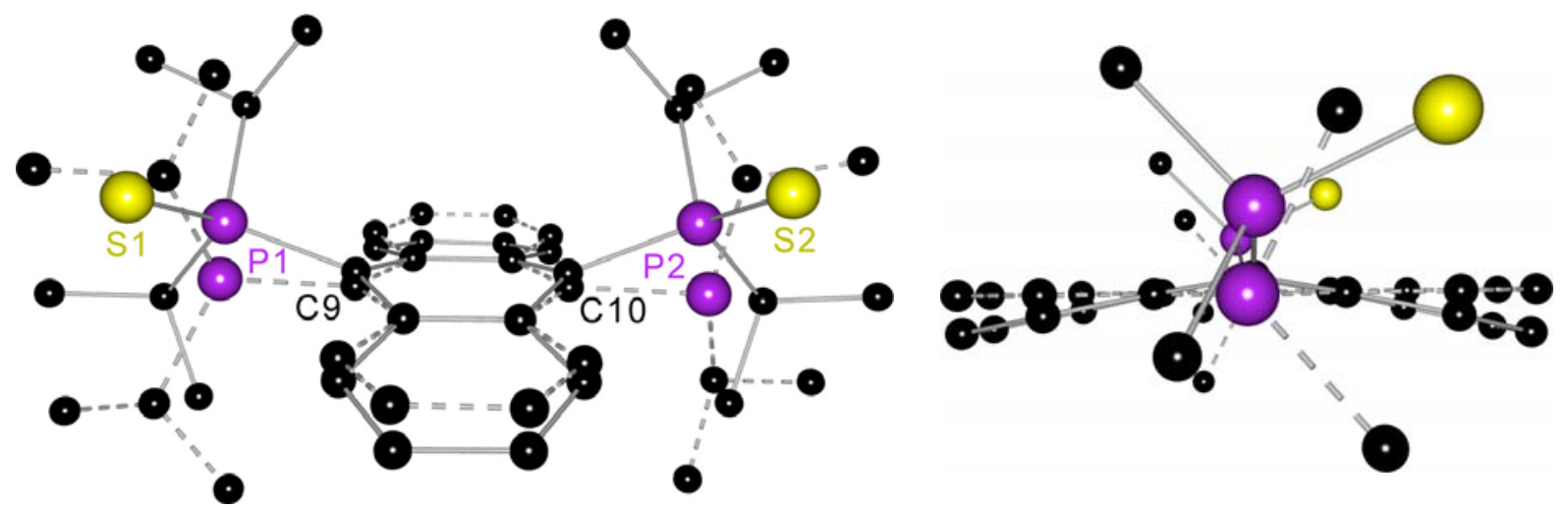

Abb. 3-16: Superposition von 13 (gestrichelte Bindungen) und 14 (durchgezogene Bindungen), erzeugt durch Überlagerung von C4a, C8a, C9a und C10a (rechts: Methylgruppen nicht dargestellt; Blick entlang C9 ‥C10).

Im Gegensatz zum Wirt/Gast-Komplex [2 $\mathrm{C}_{7} \mathrm{H}_{8} @$ SPAnPS] (9) sind im Kristallgitter von 14 keine Lösungsmittelmoleküle eingebaut, obwohl die Verbindung ebenfalls aus Toluol kristallisiert wurde. Außerdem zeigt das Isopropylderivat beim Bestrahlen mit UV-Licht keine Festkörperfluoreszenz im sichtbaren Bereich. 


\section{ASYMMETRISCH SUBSTITUIERTE 9,10-DIPHOS- PHANYLANTHRACENE}

\subsection{Synthese und Struktur von $\mathrm{Ph}_{2}(\mathrm{~S}) \mathrm{P}\left(\mathrm{C}_{14} \mathrm{H}_{8}\right) \mathrm{PPh}_{2}(15)$}

Bei der Synthese des fluoreszierenden Wirt/Gast-Komplexes [2 $\mathrm{C}_{7} \mathrm{H}_{8} @$ SPAnPS] (9) wird bei der Umsetzung von $\left(\mathrm{Ph}_{2} \mathrm{P}\right)_{2}\left(\mathrm{C}_{14} \mathrm{H}_{8}\right)$ mit elementarem Schwefel ausschließlich die Bildung des zweifach oxidierten Derivates SPAnPS beobachtet. Die mögliche Zwischenstufe $\mathrm{Ph}_{2}(\mathrm{~S}) \mathrm{P}\left(\mathrm{C}_{14} \mathrm{H}_{8}\right) \mathrm{PPh}_{2}(\mathbf{1 5})$, bei der im gleichen Molekül ein Phosphor(III)- sowie ein Phosphor(V)atom vorliegt, konnte bisher im Verlauf der Reaktion NMR-spektroskopisch nicht nachgewiesen werden. ${ }^{[112]}$

Mögliche Wege zur Darstellung von 15 sind auf der einen Seite die Oxidation von $\left(\mathrm{Ph}_{2} \mathrm{P}\right)_{2}\left(\mathrm{C}_{14} \mathrm{H}_{8}\right)$ mit der stöchiometrischen Menge Schwefel und auf der anderen Seite die Lithiierung von 9-Brom-10-(diphenylthiophosphoryl)anthracen (3) mit nachfolgender Umsetzung mit Chlordiphenylphosphan (GI. 4-1). Da für die vorliegende Arbeit unter anderem Lithiierungsreaktionen von Bromanthracenen untersucht werden sollten, wurde der zweite Weg gewählt.<smiles>PC1=CC=CC(c2ccccc2)=c2ccccc2=C1c1ccccc1</smiles>

PAnP

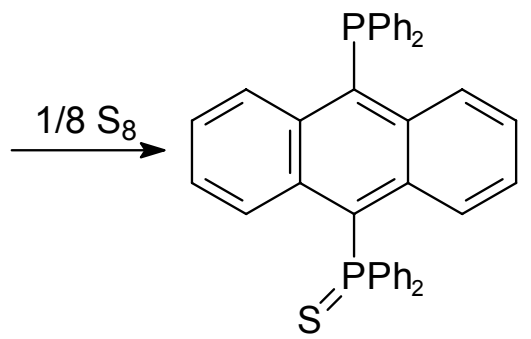

15

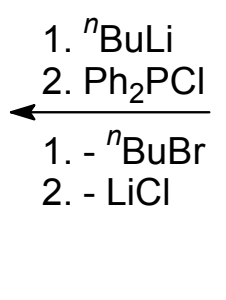

GI. 4-1: Denkbare Synthesen von $\mathrm{Ph}_{2}(\mathrm{~S}) \mathrm{P}\left(\mathrm{C}_{14} \mathrm{H}_{8}\right) \mathrm{PPh}_{2}$<smiles>S=Pc1c2ccccc2c(Br)c2ccccc12</smiles>

3

Die Umsetzungen von 3 mit ${ }^{n} \mathrm{BuLi}$ und $\mathrm{Ph}_{2} \mathrm{PCl}$ wurden in Diethylether bei $-10^{\circ} \mathrm{C}$ durchgeführt. Nach Abtrennen von unlöslichen Nebenprodukten wurde das Lösungsmittel i. Vak. entfernt und $\mathbf{1 5}$ als gelbes Pulver in einer Ausbeute von $64 \%$ erhalten. Lagerung einer gesättigten Lösung in Toluol lieferte nach einigen Tagen Kristalle, die für eine röntgenkristallographische Strukturbestimmung geeignet waren. Abb. 4-1 zeigt die Molekülstruktur der Verbindung, in Tab. 4-1 sind ausgewählte Bindungslängen und -winkel aufgeführt. 15 kristallisiert in der orthorhombischen, zentrosymmetrischen Raumgruppe Pbca, die asymmetrische Einheit umfasst ein Molekül des Phosphanylanthracens, es sind keine Toluol-Lösungsmittelmoleküle enthalten. 

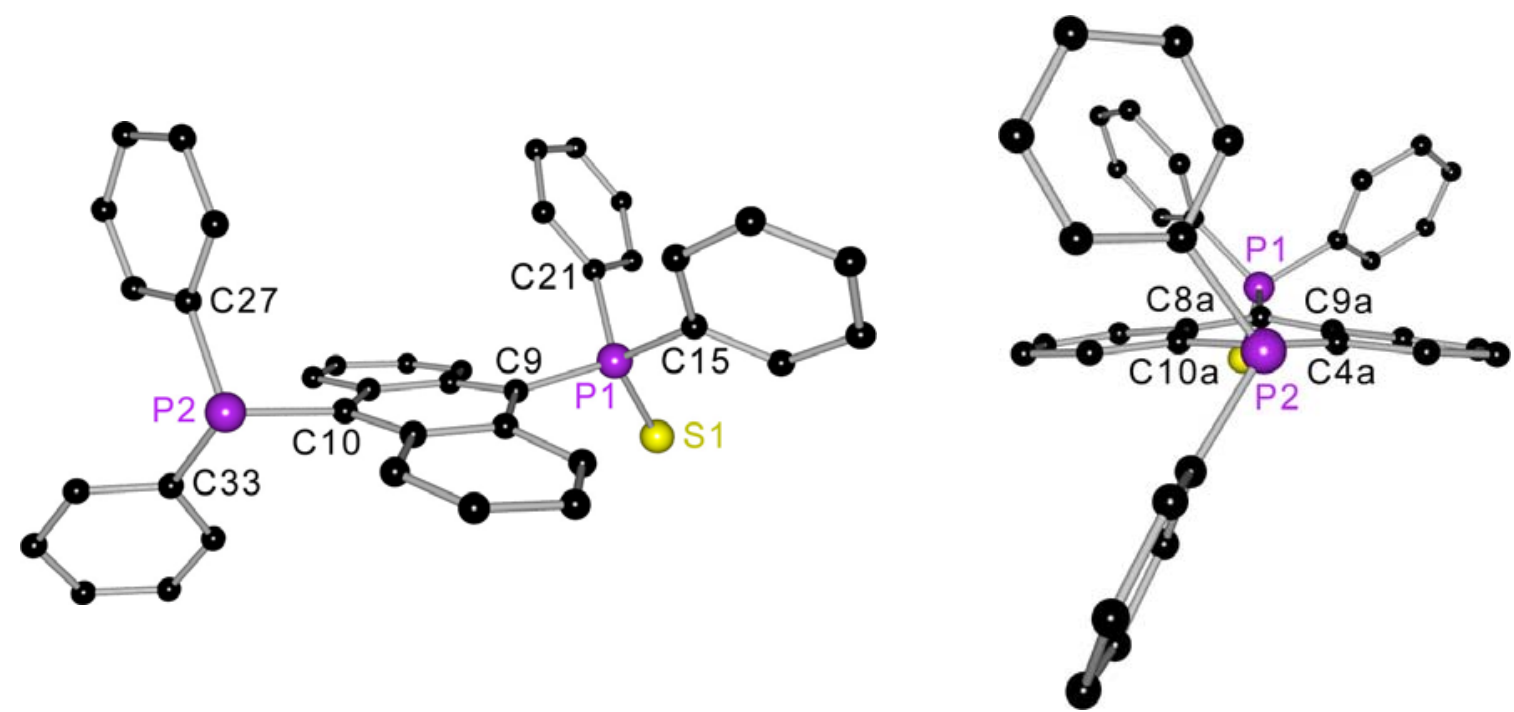

Abb. 4-1: Molekülstruktur von $\mathrm{Ph}_{2}(\mathrm{~S}) \mathrm{P}\left(\mathrm{C}_{14} \mathrm{H}_{8}\right) \mathrm{PPh}_{2}(15)$ (rechts: Blick entlang $\left.\mathrm{P} 2 \cdots \mathrm{C} 10\right)$.

Tab. 4-1: Ausgewählte Bindungslängen / pm und -winkel / ${ }^{\circ}$ in 15.

\begin{tabular}{ll}
\hline P1-C9 & $183.9(2)$ \\
P2-C10 & $185.4(2)$ \\
P1-S1 & $195.49(8)$ \\
P1-C15 & $182.3(2)$ \\
P1-C21 & $182.9(2)$ \\
P2-C27 & $184.1(2)$ \\
P2-C33 & $182.9(2)$ \\
\hline
\end{tabular}

\begin{tabular}{ll}
\hline C9-P1-S1 & $114.54(7)$ \\
C9-P1-C15 & $109.47(10)$ \\
C9-P1-C21 & $105.41(9)$ \\
C15-P1-C21 & $101.11(10)$ \\
C10-P2-C27 & $103.60(9)$ \\
C10-P2-C33 & $107.76(10)$ \\
C27-P2-C33 & $105.28(10)$ \\
\hline
\end{tabular}

Die beiden Phenylgruppen des $\mathrm{P}(\mathrm{S}) \mathrm{Ph}_{2}$-Substituenten sind relativ zum AnthracenGerüst cisoid angeordnet und die Ebene durch C9, P1 und S1 bildet mit der durch C4a, C8a, C9a und C10a aufgespannten Ebene einen Winkel von 79.9. Der Abstand von letzterer Ebene zu P1 beträgt 49.8 pm, während P2 nur 23.8 pm davon entfernt ist. Im Fall des $\mathrm{PPh}_{2}$-Substituenten liegt eine transoide Orientierung der beiden Phenylgruppen hinsichtlich der Anthracen-Einheit vor. Somit sind die beiden phosphorhaltigen Substituenten genauso angeordnet wie in den entsprechenden 9-Brom-10-phosphanylanthracenen 1 und 3. Durch den sterischen Einfluss des Schwefelatoms ist der Winkel C15-P1-C21 etwa $4^{\circ}$ kleiner als C27-P2-C33. Der Faltungswinkel zwischen den durch C1-C4 und C5 - C8 aufgespannten Ebenen beträgt $168.6^{\circ}$ und liegt damit zwischen den entsprechenden Werten in $1\left(177.4^{\circ}\right)$ und $3\left(162.1^{\circ}\right)$. 


\subsection{Synthese und Struktur von [2 $\mathrm{C}_{7} \mathrm{H}_{8} @$ $\left.\left\{\mathrm{Ph}_{2}(\mathrm{Se}) \mathrm{P}\left(\mathrm{C}_{14} \mathrm{H}_{8}\right) \mathrm{P}(\mathrm{S}) \mathrm{Ph}_{2}\right\}\right](16)$}

Die Oxidation von 15 mit grauem Selen in Toluol lieferte 9-(Diphenylselenophosphoryl)-10-(diphenylthiophosphoryl)anthracen (16) in einer Ausbeute von $90 \%$ (GI. 4-2).

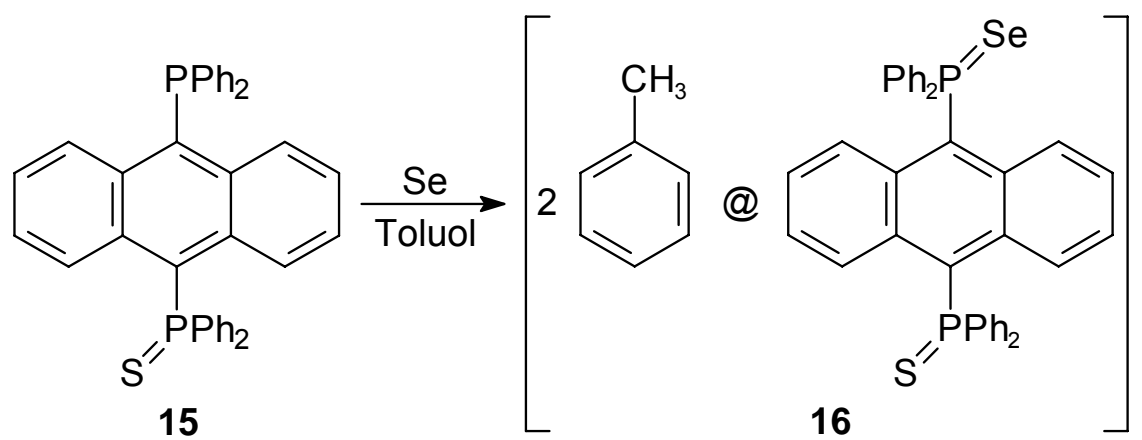

GI. 4-2: Darstellung von $\left[2 \mathrm{C}_{7} \mathrm{H}_{8} @\left\{\mathrm{Ph}_{2}(\mathrm{Se}) \mathrm{P}\left(\mathrm{C}_{14} \mathrm{H}_{8}\right) \mathrm{P}(\mathrm{S}) \mathrm{Ph}_{2}\right\}\right]$ (16).

Bei der Reaktion entstand das Produkt in Form gelber, luftstabiler Kristalle. Das Ergebnis der Röntgenstrukturuntersuchung ist in Abb. 4-2 aus zwei unterschiedlichen Blickrichtungen dargestellt, Tab. 4-2 enthält ausgewählte Bindungslängen und -winkel.
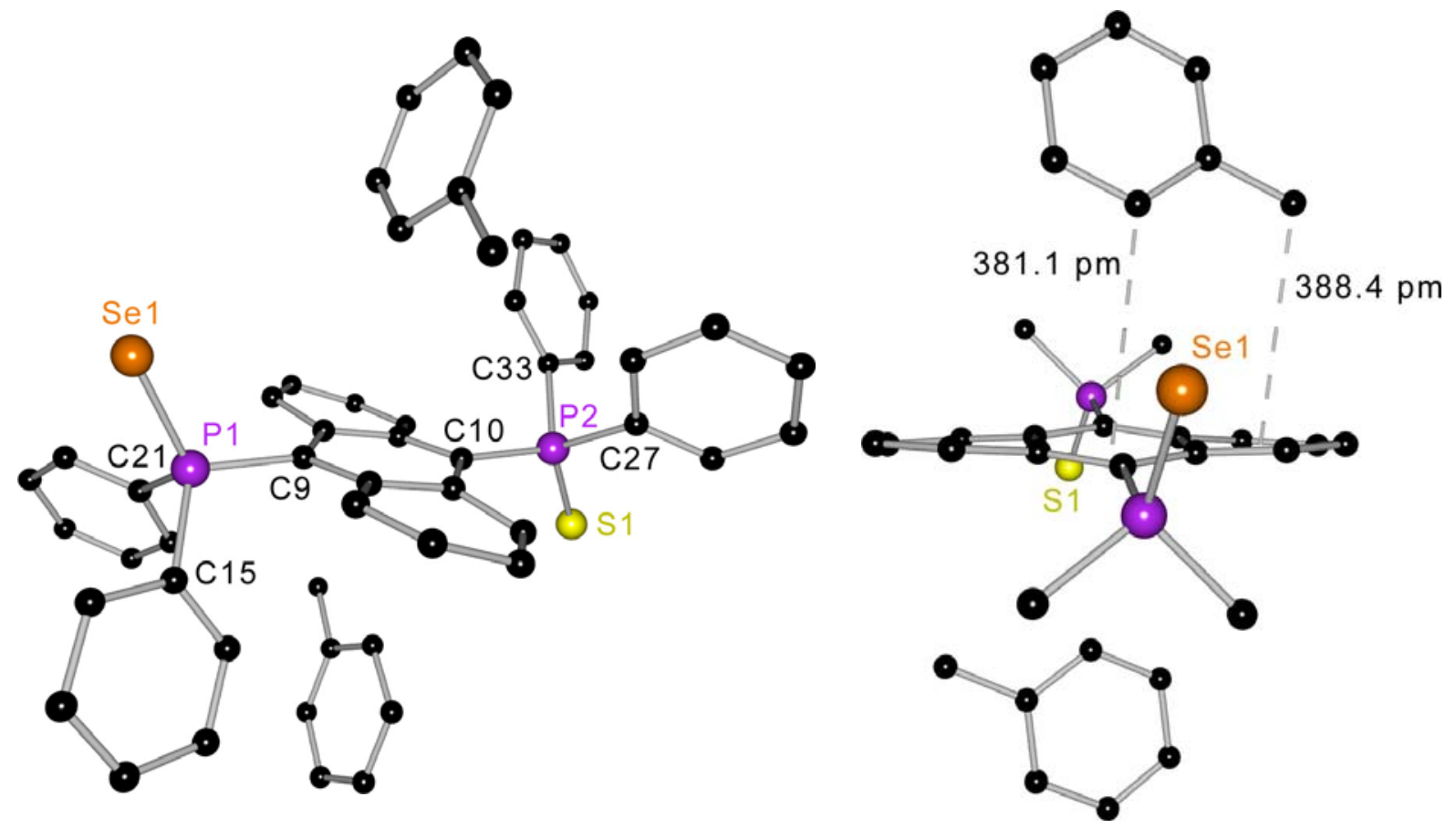

Abb. 4-2: Molekülstruktur von $\left[2 \mathrm{C}_{7} \mathrm{H}_{8} @\left\{\mathrm{Ph}_{2}(\mathrm{Se}) \mathrm{P}\left(\mathrm{C}_{14} \mathrm{H}_{8}\right) \mathrm{P}(\mathrm{S}) \mathrm{Ph}_{2}\right\}\right]$ (16)

(Se/S-Fehlordnung nicht dargestellt). 
Tab. 4-2: Ausgewählte Bindungslängen / pm und -winkel / ${ }^{\circ}$ in 16.

\begin{tabular}{ll}
\hline P1-C9 & $183.3(2)$ \\
P1-Se1 & $208.9(4)$ \\
P1-C15 & $182.4(2)$ \\
P1-C21 & $182.8(2)$ \\
P2-S1 & $196.2(9)$ \\
\hline
\end{tabular}

\begin{tabular}{lc}
\hline C9-P1-Se1 & $112.6(3)$ \\
C9-P1-C15 & $109.37(11)$ \\
C9-P1-C21 & $106.71(11)$ \\
C15-P1-C21 & $97.78(10)$ \\
C10-P2-S1 & $114.5(7)$ \\
\hline
\end{tabular}

16 kristallisiert in der monoklinen, zentrosymmetrischen Raumgruppe $P 2_{1} / n$ als Wirt/Gast-Komplex mit Toluol-Lösungsmittelmolekülen im Kristallgitter. Die Kristallstruktur ist isostrukturell zu der von $\left[2 \mathrm{C}_{7} \mathrm{H}_{8} @\left\{\left(\mathrm{Ph}_{2}(\mathrm{~S}) \mathrm{P}\right)_{2}\left(\mathrm{C}_{14} \mathrm{H}_{8}\right)\right\}\right]$ (9) und die asymmetrische Einheit enthält ein halbes Wirts- sowie ein komplettes Toluolmolekül. Die Position des Chalkogensubstituenten umfasst daher sowohl ein Selen- als auch ein Schwefelatom mit je 50\%iger Besetzung. Durch Anwendung eines Inversionszentrums im Mittelpunkt des Anthracengerüstes wird die zweite Hälfte des Wirtsmoleküls erhalten.

Die $\mathrm{P}=\mathrm{Se}-$ und $\mathrm{P}=\mathrm{S}-$ Bindungen sind transoid orientiert und das Verhältnis Wirt : Gast beträgt $1: 2$. Die Anthracen-Kohlenstoffatome $\mathrm{C} 1$ - $\mathrm{C} 8$ befinden sich in einer Ebene, während sich C9 und C10 25.2 bzw. -26.7 pm außerhalb dieser Ebene befinden. Die Abstände der beiden Phosphoratome von dieser Ebene betragen 77.0 (P1) bzw. 78.6 pm (P2). Auch die Anordnung der beiden Toluolmoleküle ist analog zu der in 9. Jedes Lösungsmittelmolekül bildet mit der idealisierten Ebene durch C4a, C8a, C9a und $\mathrm{C} 10 \mathrm{a}$ einen Winkel von $76.1^{\circ}$ (74.5 in 9) und das Methylkohlenstoffatom des Toluolmoleküls ist vom Mittelpunkt des äußeren sechsgliedrigen Ringes des Anthracengerüstes 388.4 pm entfernt (387.3 pm in 9).

Die Molekülstrukturen von $\mathbf{9}$ und $\mathbf{1 6}$ unterscheiden sich somit lediglich durch den Austausch eines Schwefelatoms durch ein Selenatom. Ansonsten sind beide Verbindungen isostrukturell und weisen dasselbe Wirt: Gast-Verhältnis auf. Trotzdem zeigen nur die Kristalle von 9 intensive Festkörperfluoreszenz, bei Bestrahlung von 16 mit UV-Licht $\left(\lambda_{\mathrm{ex}}=366 \mathrm{~nm}\right)$ ist keinerlei Lumineszenz im sichtbaren Bereich beobachtbar. Somit ist der elektronische Einfluss beider Schwefelatome sowie die transoide Anordnung der $\mathrm{P}=\mathrm{S}$-Bindungen eine unabdingbare Voraussetzung für das Auftreten der Fluoreszenz im Festkörper. 


\subsection{Synthese und Struktur von $\mathrm{Ph}_{2} \mathrm{P}\left(\mathrm{C}_{14} \mathrm{H}_{8}\right) \mathrm{P}^{i} \mathrm{Pr}_{2}(17)$}

In Kap. 4.1 wurde die Synthese eines asymmetrisch substituierten 9,10-Diphosphanylanthracens beschrieben, das ausgehend von 9-Brom-10-(diphenylthiophosphoryl)anthracen (3) erhalten wurde. Allerdings erweist sich die Lithiierung von 3 mit ${ }^{n} \mathrm{BuLi}$ als problematisch, da die Reaktion weder in Diethylether noch in THF quantitativ erfolgt. Auch unterschiedliche Reaktionstemperaturen sowie die Verwendung von ${ }^{t} \mathrm{BuLi}$ als Lithiierungsreagenz liefern ähnliche Ergebnisse und bei den nachfolgenden Umsetzungen mit Chlorphosphanen wird stets die Bildung unerwünschter Nebenprodukte beobachtet, die im ${ }^{31}$ P-NMR-Spektrum nicht eindeutig zugeordnet werden können.

Eine alternative Darstellungsmöglichkeit für Anthracenderivate, die in den Positionen 9 und 10 zwei unterschiedliche Phosphanylsubstituenten aufweisen, geht von 9-Brom-10-(diphenylphosphanyl)anthracen (1) aus (GI. 4-3). Nach Lithiierung und nachfolgender Reaktion mit Chlordiisopropylphosphan wurde 9-Diphenylphosphanyl10-(diisopropylphosphanyl)anthracen (17) erhalten.

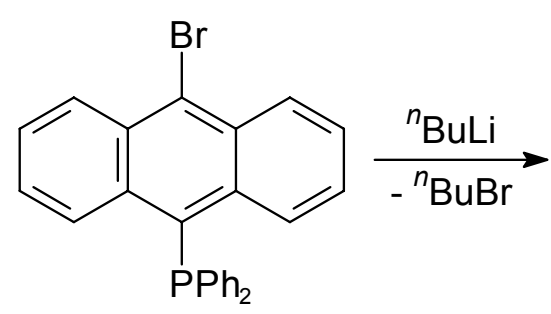

1<smiles>Clc1c2ccccc2c(-c2ccccc2)c2ccccc12</smiles>

GI. 4-3: Darstellung von 17 über die Zwischenstufe 9-Diphenylphosphanyl-10-lithiumanthracenid.

Der Brom/Lithium-Austausch von 1 erfolgte bei $-15^{\circ} \mathrm{C}$ in Diethylether mit ${ }^{n}$ BuLi. Nach der anschließenden Umsetzung mit ${ }^{i} \mathrm{Pr}_{2} \mathrm{PCl}$ wurden unlösliche Nebenprodukte abgetrennt und das Lösungsmittel i. Vak. entfernt. Dabei wurde 17 in einer Ausbeute von $66 \%$ und weitestgehend frei von Nebenprodukten erhalten. Die Lithiierung von 1 verläuft somit selektiver als die von 3 und ist für die Synthese von asymmetrisch substituierten 9,10-Diphosphanylanthracenen besser geeignet.

Durch Lagerung einer gesättigten Lösung von 17 in einer Mischung von THF und $n$-Pentan (1:1) wurden nach einigen Tagen Kristalle erhalten, die für eine Röntgenstrukturanalyse geeignet waren. Die Molekülstruktur ist in Abb.4-3 dargestellt, Tab. 4-3 enthält ausgewählte Bindungslängen und -winkel. 

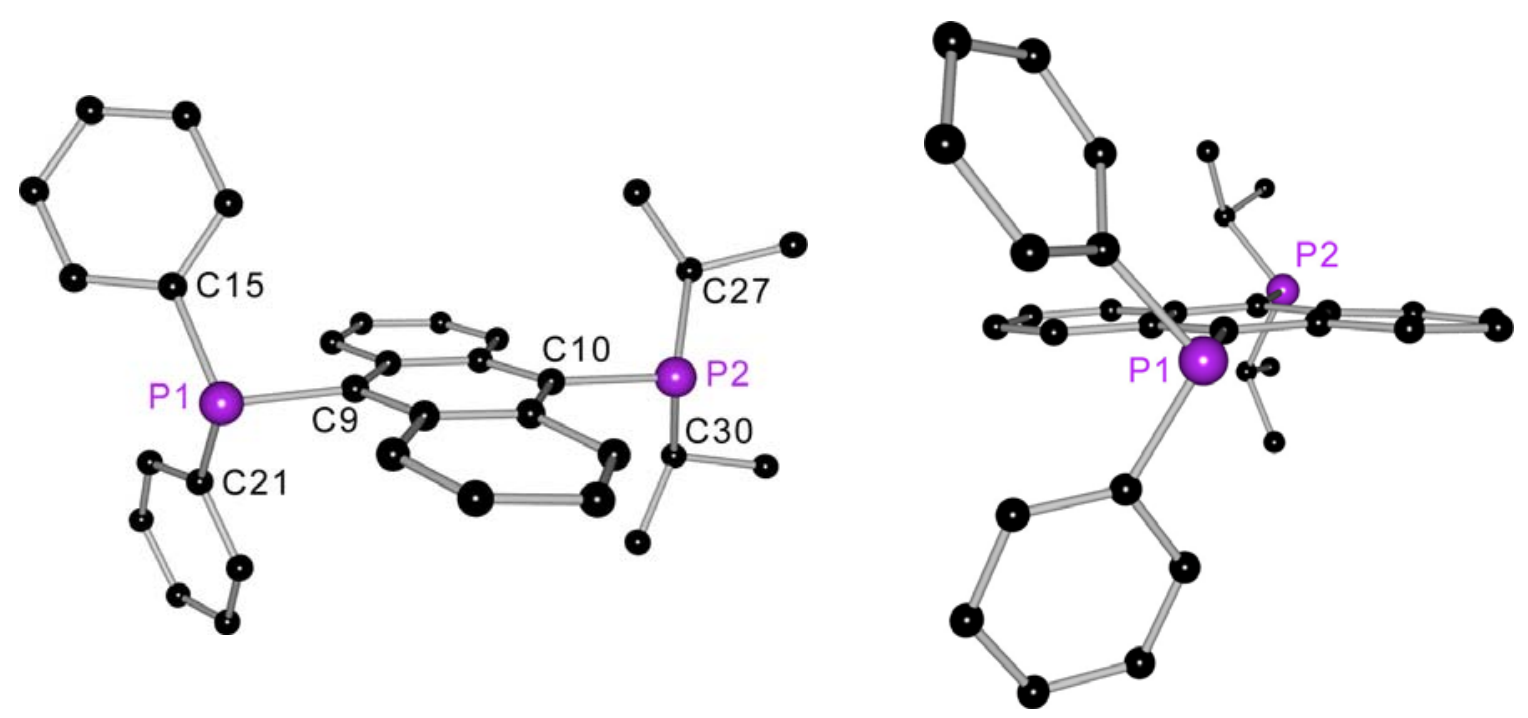

Abb. 4-3: Molekülstruktur von $\mathrm{Ph}_{2} \mathrm{P}\left(\mathrm{C}_{14} \mathrm{H}_{8}\right) \mathrm{P}^{\prime} \mathrm{Pr}_{2}$ (17) aus zwei unterschiedlichen Ansichten.

Tab. 4-3: Ausgewählte Bindungslängen / pm und -winkel / ${ }^{\circ}$ in 17.

\begin{tabular}{ll}
\hline P1-C9 & $185.08(14)$ \\
P2-C10 & $186.57(14)$ \\
P1-C15 & $182.67(14)$ \\
P1-C21 & $183.72(14)$ \\
P2-C27 & $187.65(15)$ \\
P2-C30 & $186.58(15)$ \\
\hline
\end{tabular}

\begin{tabular}{ll}
\hline C9-P1-C15 & $104.51(6)$ \\
C9-P1-C21 & $100.82(6)$ \\
C15-P1-C21 & $107.46(6)$ \\
C10-P2-C27 & $107.49(6)$ \\
C10-P2-C30 & $103.39(6)$ \\
C27-P2-C30 & $104.40(7)$ \\
\hline
\end{tabular}

17 kristallisiert in der monoklinen, zentrosymmetrischen Raumgruppe $P 2_{1} / C$ mit einem Molekül in der asymmetrischen Einheit. Das zentrale Anthracengerüst ist planar und der Faltungswinkel zwischen den beiden äußeren sechsgliedrigen Ringen beträgt $176.6^{\circ}$. Die Abstände zwischen den beiden Phosphoratomen und der durch C4a, C8a, C9a und C10a aufgespannten Ebene betragen 12.3 (P1) bzw. $-10.2 \mathrm{pm}(\mathrm{P} 2)$.

Die $\mathrm{Ph}_{2} \mathrm{P}$ - und ${ }^{i} \mathrm{Pr}_{2} \mathrm{P}$-Substituenten sind bezüglich der Anthracen-Einheit ebenso orientiert wie die Phosphanylsubstituenten in den entsprechenden 9-Brom-10phosphanylderivaten $\operatorname{Br}\left(\mathrm{C}_{14} \mathrm{H}_{8}\right) \mathrm{PPh}_{2}$ (1) und $\mathrm{Br}\left(\mathrm{C}_{14} \mathrm{H}_{8}\right) \mathrm{P}^{\prime} \mathrm{Pr}_{2}$ (5) und es liegt jeweils eine gestaffelte Konformation vor. Anders als in den symmetrischen Verbindungen $\left(\mathrm{Ph}_{2} \mathrm{P}\right)_{2}\left(\mathrm{C}_{14} \mathrm{H}_{8}\right)$ und $\left({ }^{i} \mathrm{Pr}_{2} \mathrm{P}\right)_{2}\left(\mathrm{C}_{14} \mathrm{H}_{8}\right)$ (13) sind die Phosphanylsubstituenten in 17 jedoch ekliptisch zueinander angeordnet (Abb. 4-3 rechts). 


\subsection{Synthesen und Strukturen der Oxidationsprodukte $\mathrm{Ph}_{2}(\mathrm{E}) \mathrm{P}\left(\mathrm{C}_{14} \mathrm{H}_{8}\right) \mathrm{P}(\mathrm{E})^{i} \mathrm{Pr}_{2}, \mathrm{E}=\mathrm{O}(18), \mathrm{S}$ (19) und Se (20)}

Um die strukturellen Auswirkungen der Oxidation der beiden Phosphoratome in $\mathrm{Ph}_{2} \mathrm{P}\left(\mathrm{C}_{14} \mathrm{H}_{8}\right) \mathrm{P}^{\prime} \mathrm{Pr}_{2}$ (17) zu untersuchen, wurden durch Umsetzung von $17 \mathrm{mit}$ $\mathrm{H}_{2} \mathrm{O}_{2} \cdot\left(\mathrm{H}_{2} \mathrm{~N}\right)_{2} \mathrm{C}=\mathrm{O}$, elementarem Schwefel bzw. grauem Selen die Oxidationsprodukte 18 - 20 synthetisiert (Gl. 4-4).<smiles>FP=Pc1c2ccccc2c(-c2ccccc2)c2ccc(N(c3ccccc3)c3ccccc3)c(P(F)F)c12</smiles>

18, $\mathrm{E}=\mathrm{O},[\mathrm{Ox}]=\mathrm{H}_{2} \mathrm{O}_{2} \cdot\left(\mathrm{H}_{2} \mathrm{~N}\right)_{2} \mathrm{C}=\mathrm{O}$

$19, E=S,[O x]=1 / 8 S_{8}$

20, $\mathrm{E}=\mathrm{Se},[\mathrm{Ox}]=$ graues $\mathrm{Se}$

GI. 4-4: Oxidation von $17 \mathrm{zu} \mathrm{Ph}_{2}(\mathrm{E}) \mathrm{P}\left(\mathrm{C}_{14} \mathrm{H}_{8}\right) \mathrm{P}(\mathrm{E})^{i} \mathrm{Pr}_{2}, \mathrm{E}=\mathrm{O}(\mathbf{1 8}), \mathrm{S}(19)$, Se (20).

Die Molekülstruktur des mit Sauerstoff oxidierten Derivates 18 ist in Abb. 4-4 dargestellt, Abb. 4-5 zeigt die Strukturen der entsprechenden Schwefel- und SelenOxidationsprodukte. Tab. 4-4 enthält ausgewählte Bindungslängen und -winkel.
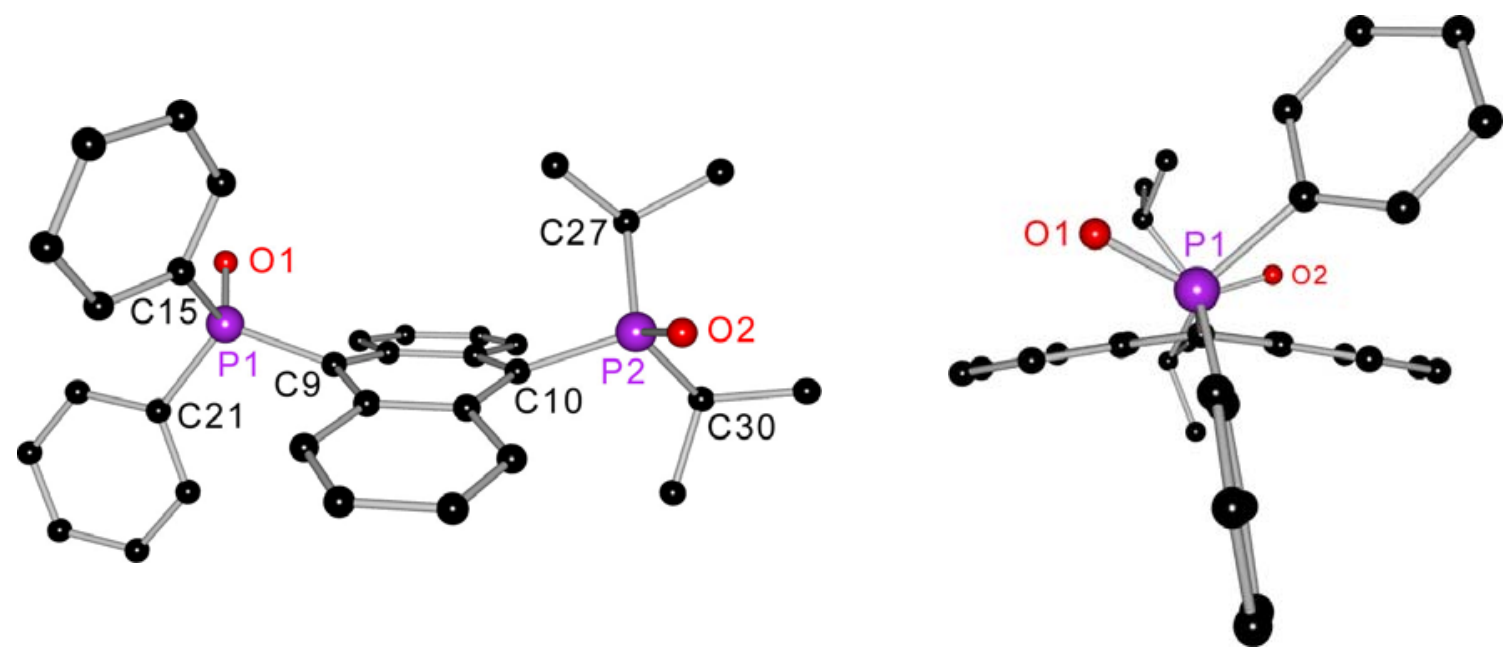

Abb. 4-4: Molekülstruktur von $\mathrm{Ph}_{2}(\mathrm{O}) \mathrm{P}\left(\mathrm{C}_{14} \mathrm{H}_{8}\right) \mathrm{P}(\mathrm{O})^{j} \mathrm{Pr}_{2}$ (18) (rechts: Blick entlang $\left.\mathrm{C} 9 \cdots \mathrm{C} 10\right)$.

18 kristallisiert in der orthorhombischen, chiralen Raumgruppe $P 2{ }_{1} 2{ }_{1}{ }_{1}$, was auf das Vorhandensein von Wasserstoffbrückenbindungen zurückzuführen ist. So weist die Kristallstruktur beispielsweise $\mathrm{H}$-Brücken zwischen 01 -Atomen unterschiedlicher 
Moleküle und $\mathrm{C}-\mathrm{H}_{\text {meta }}(\mathrm{Ph})$ - sowie $\mathrm{C}-\mathrm{H}\left({ }^{i} \mathrm{Pr}\right)$-Wasserstoffatomen von benachbarten Molekülen mit Bindungslängen von 239.1 bzw. 242.4 pm auf.
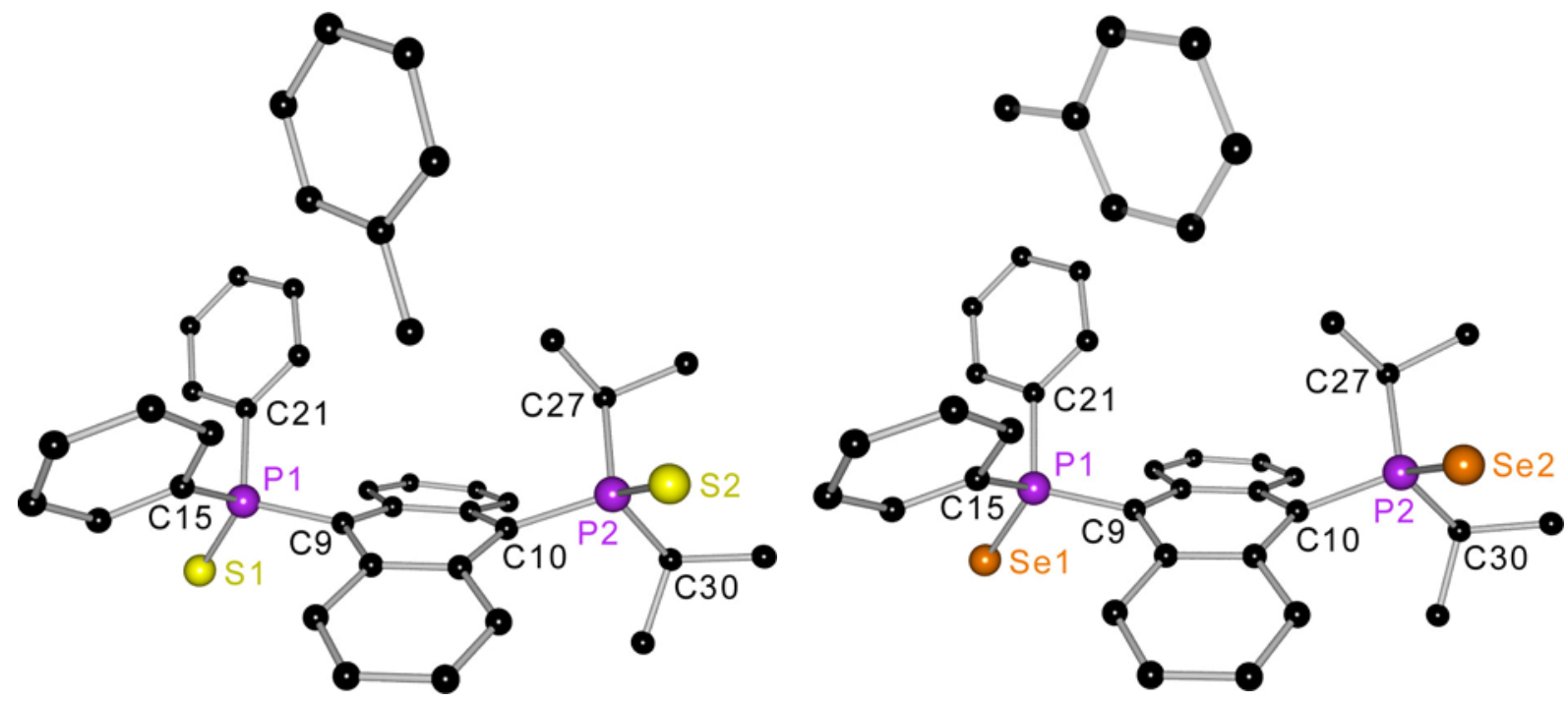

Abb. 4-5: Molekülstrukturen von $\left[\mathrm{Ph}_{2}(\mathrm{~S}) \mathrm{P}\left(\mathrm{C}_{14} \mathrm{H}_{8}\right) \mathrm{P}(\mathrm{S})^{i} \mathrm{Pr}_{2} \cdot 1.5 \mathrm{C}_{7} \mathrm{H}_{8}\right]$ (19) und $\left[\mathrm{Ph}_{2}(\mathrm{Se}) \mathrm{P}\left(\mathrm{C}_{14} \mathrm{H}_{8}\right) \mathrm{P}(\mathrm{Se})^{i} \mathrm{Pr}_{2} \cdot 1.5 \mathrm{C}_{7} \mathrm{H}_{8}\right](20)$.

Tab. 4-4: Ausgewählte Bindungslängen / pm und -winkel / ${ }^{\circ}$ in 18 - 20.

\begin{tabular}{|c|c|c|c|}
\hline & $18(E=O)$ & $19(E=S)$ & $20(E=S e)$ \\
\hline P1-E1 & $148.72(15)$ & $195.59(6)$ & $211.34(6)$ \\
\hline P2-E2 & $149.28(14)$ & $196.47(6)$ & $212.00(6)$ \\
\hline P1-C9 & $183.6(2)$ & $182.77(16)$ & $182.6(2)$ \\
\hline P1-C15 & $181.9(2)$ & $181.67(18)$ & $181.9(2)$ \\
\hline P1-C21 & $180.1(2)$ & $182.51(17)$ & $182.6(2)$ \\
\hline P2-C10 & $184.94(19)$ & $185.80(16)$ & $186.2(2)$ \\
\hline P2-C27 & $182.7(2)$ & $184.67(17)$ & $185.4(2)$ \\
\hline P2-C30 & $183.9(2)$ & $185.31(17)$ & $185.8(2)$ \\
\hline out-of-plane ${ }^{a} \mathrm{P}$ & $75.90 / 85.44$ & $49.95 / 85.37$ & $55.99 / 92.70$ \\
\hline E1-P1-C9 & $111.90(9)$ & $113.73(5)$ & $113.34(7)$ \\
\hline E2-P2-C10 & $112.98(9)$ & $116.03(5)$ & $116.23(7)$ \\
\hline C15-P1-C21 & $108.11(9)$ & $100.30(8)$ & $100.03(10)$ \\
\hline C27-P2-C30 & $111.58(9)$ & $110.65(8)$ & $110.48(11)$ \\
\hline Faltungswinkel $^{b}$ & 159.3 & 153.2 & 152.7 \\
\hline
\end{tabular}


Die beiden mit Schwefel und Selen oxidierten Derivate 19 und 20 kristallisieren isostrukturell in der monoklinen, zentrosymmetrischen Raumgruppe $P 2_{1} / c$. Beide enthalten in der asymmetrischen Einheit jeweils neben einem Molekül Diphosphanylanthracen noch 1.5 Lösungsmittelmoleküle Toluol. Wie aus Abb. 4-5 erkennbar ist, befindet sich jeweils ein Toluolmolekül in räumlicher Nähe des Wirtsmoleküls, die Orientierung der Methylgruppen ist jedoch unterschiedlich. So beträgt der Abstand zwischen dem Methylkohlenstoffatom und C4a in 19405.6 pm, während die $\mathrm{C}_{i p s o}-\mathrm{C}_{M e}$-Bindung in 20 nicht in Richtung des Anthracengerüstes weist. Die Entfernungen zwischen $\mathrm{C} 4 \mathrm{a}$ und $\mathrm{C}_{\text {ipso }}(\mathbf{1 9})$ bzw. $\mathrm{C}_{\text {meta }}(\mathbf{2 0})$ sind mit 550.6 bzw. 551.3 pm identisch.

In Abb. 4-6 ist eine Überlagerung der Molekülstrukturen von 19 und dem ToluolKomplex von SPAnPS (9) dargestellt, um die unterschiedliche Anordnung der Toluolmoleküle zu verdeutlichen. Im Gegensatz zu 9 zeigen 19 und 20 keine Festkörperfluoreszenz im sichtbaren Bereich.
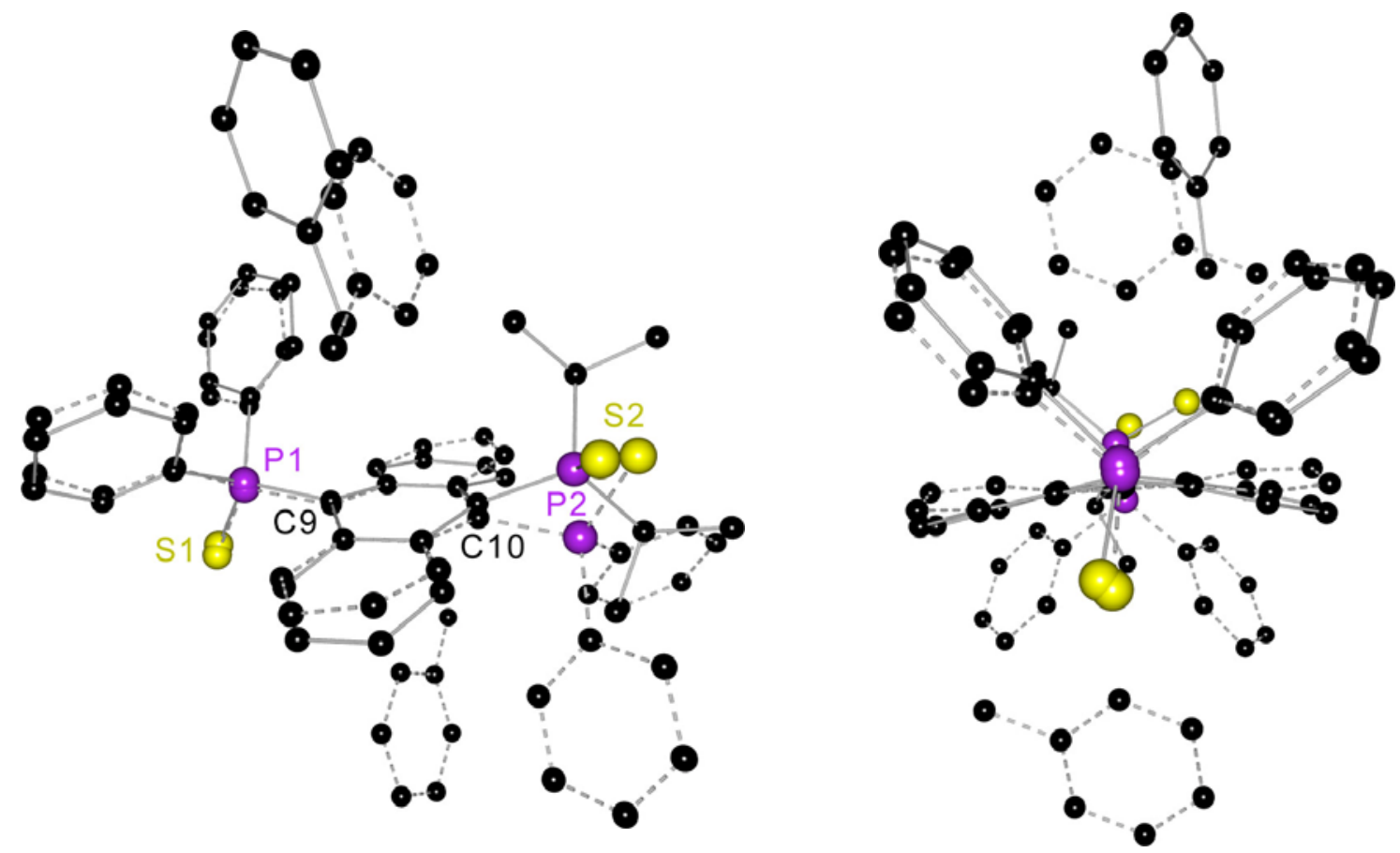

Abb. 4-6: Superposition von 9 (gestrichelte Bindungen) und 19 (durchgezogene Bindungen), erzeugt durch Überlagerung von C4a, C8a, C9a und C10a (rechts: Blick entlang P1‥C9).

Die Orientierungen der $P(E) R_{2}$-Substituenten von $18-20$ in Bezug auf das Anthracengerüst sind analog denen der bereits beschriebenen 9-Brom-10phosphorylderivate 2 - 4, 7 und 8 und sollen daher nicht erneut diskutiert werden. Die beiden Isopropylgruppen in $\mathbf{1 8}$ sind relativ zur Anthracen-Einheit transoid angeordnet und die Ebene durch C10, P2 und O2 bildet mit der durch C4a, C8a, C9a und C10a aufgespannten Ebene einen Winkel von 23.6 . 


\subsection{Synthese und Struktur von $\mathrm{Ph}_{2} \mathrm{P}\left(\mathrm{C}_{14} \mathrm{H}_{8}\right) \mathrm{PCy}_{2}(21)$}

In Kap. 4.3 wurde beschrieben, dass aus $\operatorname{Br}\left(\mathrm{C}_{14} \mathrm{H}_{8}\right) \mathrm{PPh}_{2}$ (1) durch Umsetzung mit ${ }^{n} \mathrm{BuLi}$ und ${ }^{i} \mathrm{Pr}_{2} \mathrm{PCl}$ das asymmetrisch substituierte 9,10-Diphosphanylanthracen 17 zugänglich ist. Um die Übertragbarkeit der Synthese auf andere phosphorhaltige Elektrophile zu überprüfen, wurde das sterisch ebenfalls anspruchsvolle Chlordicyclohexylphosphan gewählt. Nach Lithiierung von 1 und nachfolgender Reaktion mit $\mathrm{Cy}_{2} \mathrm{PCl}$ wurde 9-Diphenylphosphanyl-10-(dicyclohexylphosphanyl)anthracen (21) in einer Ausbeute von 40 \% erhalten (GI. 4-5).<smiles>Brc1c2ccccc2c(-c2ccccc2)c2ccccc12</smiles>

1
1. ${ }^{n} \mathrm{BuLi}$

2. $\mathrm{Cy}_{2} \mathrm{PCl}$

1. $-{ }^{n} \mathrm{BuBr}$

2. $-\mathrm{LiCl}$<smiles>CCCCc1c2ccccc2c(-c2ccccc2)c2ccccc12</smiles>

21

GI. 4-5: Synthese von $\mathrm{Ph}_{2} \mathrm{P}\left(\mathrm{C}_{14} \mathrm{H}_{8}\right) \mathrm{PCy}_{2}$ (21).

Abb. 4-7 zeigt die Molekülstruktur von 21, in Tab. 4-5 sind ausgewählte Bindungslängen und -winkel aufgeführt.
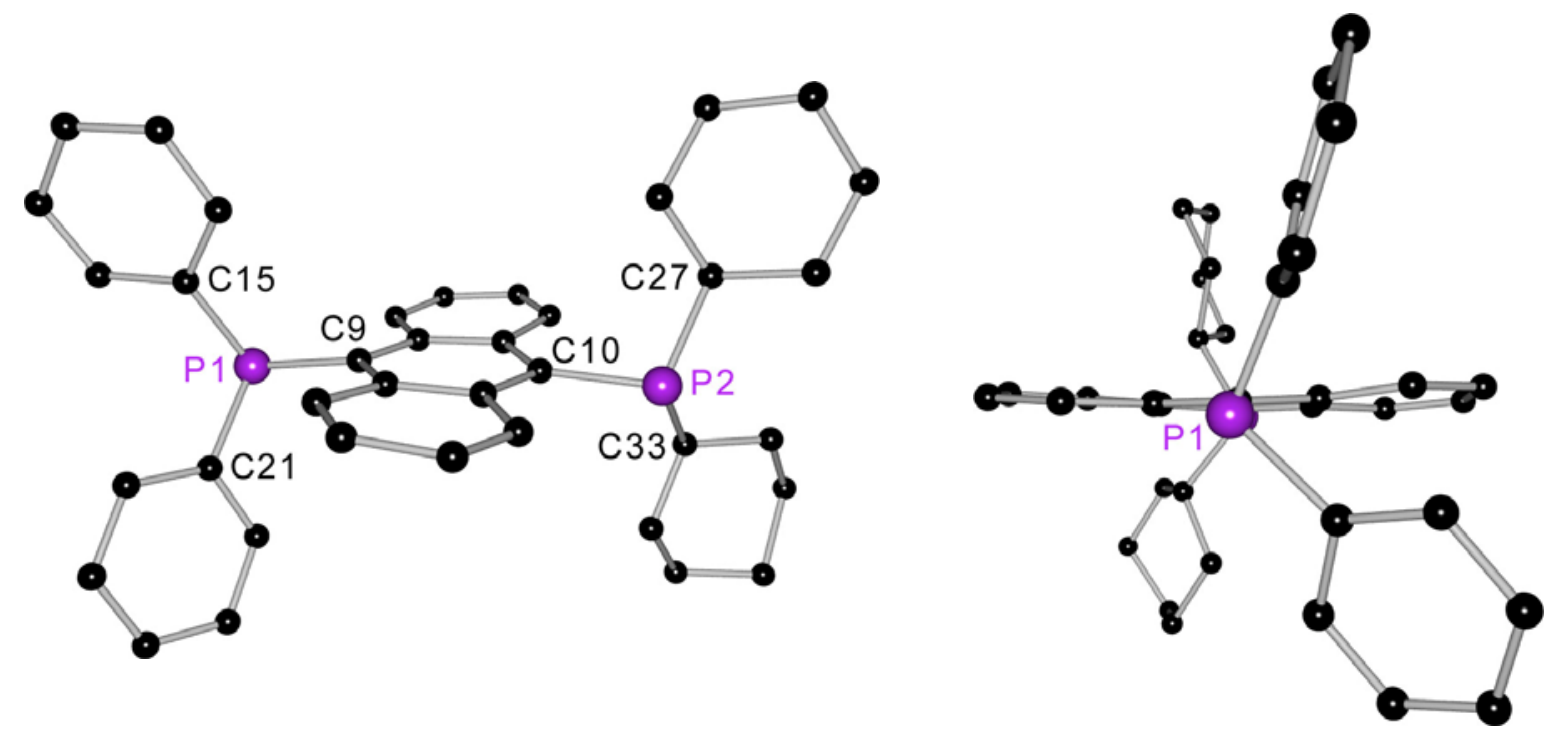

Abb. 4-7: Molekülstruktur von $\mathrm{Ph}_{2} \mathrm{P}\left(\mathrm{C}_{14} \mathrm{H}_{8}\right) \mathrm{PCy}_{2}$ (21) (rechts: Blick entlang $\mathrm{P} 1-\mathrm{C} 9$ ). 
Tab. 4-5: Ausgewählte Bindungslängen / pm und -winkel / ${ }^{\circ}$ in 21.

\begin{tabular}{ll}
\hline P1-C9 & $185.1(3)$ \\
P2-C10 & $186.7(3)$ \\
P1-C15 & $182.3(3)$ \\
P1-C21 & $183.4(3)$ \\
P2-C27 & $185.4(3)$ \\
P2-C33 & $185.0(4)$ \\
\hline
\end{tabular}

\begin{tabular}{ll}
\hline C9-P1-C15 & $106.06(13)$ \\
C9-P1-C21 & $102.68(14)$ \\
C15-P1-C21 & $106.27(14)$ \\
C10-P2-C27 & $106.16(15)$ \\
C10-P2-C33 & $104.45(16)$ \\
C27-P2-C33 & $105.05(16)$ \\
\hline
\end{tabular}

21 kristallisiert in der monoklinen, zentrosymmetrischen Raumgruppe $P 2_{1} / n$ mit einem Molekül in der asymmetrischen Einheit. Jeweils ein Phenyl- und Cyclohexylsubstituent befindet sich in einer gestaffelten Anordnung ober- und unterhalb des Anthracengerüstes, was auch der Orientierung der Phenylgruppen im symmetrisch 9,10-substituierten $\left(\mathrm{Ph}_{2} \mathrm{P}\right)_{2}\left(\mathrm{C}_{14} \mathrm{H}_{8}\right)$ entspricht. Ebenfalls analog ist die transoide Ausrichtung der nichtbindenden Elektronenpaare der beiden Phosphoratome. Die Anthracen-Einheit in $\left(\mathrm{Ph}_{2} \mathrm{P}\right)_{2}\left(\mathrm{C}_{14} \mathrm{H}_{8}\right)$ ist exakt planar, während der Faltungswinkel zwischen den beiden Ebenen durch C1 - C4 und C5 - C8 in 21 169.4 beträgt, was den größeren Raumbedarf der Cyclohexylgruppen widerspiegelt.

\subsection{Synthese und Struktur von $\left[\mathrm{Ph}_{2}(\mathrm{~S}) \mathrm{P}\left(\mathrm{C}_{14} \mathrm{H}_{8}\right) \mathrm{P}(\mathrm{S}) \mathrm{Cy}_{2}\right.$. $\left.\mathrm{C}_{7} \mathrm{H}_{8}\right](22)$}

Die Umsetzung von $\mathrm{Ph}_{2} \mathrm{P}\left(\mathrm{C}_{14} \mathrm{H}_{8}\right) \mathrm{PCy}_{2}$ (21) mit zwei Äquivalenten Schwefel in Toluol ergab das Oxidationsprodukt 9-Diphenylthiophosphoryl-10-(dicyclohexylthiophosphoryl)anthracen (22) in einer Ausbeute von 71 \% (GI. 4-6).

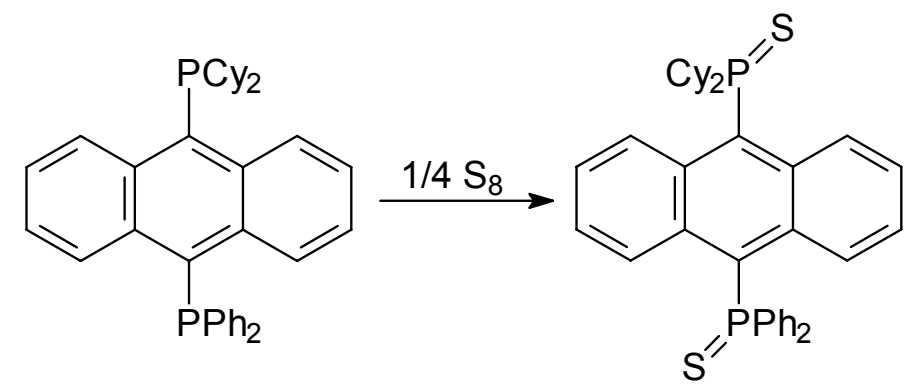

21
22

GI. 4-6: Oxidation von 21 zu $\mathrm{Ph}_{2}(\mathrm{~S}) \mathrm{P}\left(\mathrm{C}_{14} \mathrm{H}_{8}\right) \mathrm{P}(\mathrm{S}) \mathrm{Cy}_{2} 22$. 
Durch Lagerung einer gesättigten Lösung in Toluol bei RT wurden nach einigen Tagen Kristalle erhalten, die für eine Röntgenstrukturuntersuchung geeignet waren. In Abb. 4-8 ist die Molekülstruktur dargestellt, in Tab. 4-6 sind ausgewählte Bindungslängen und -winkel aufgeführt.
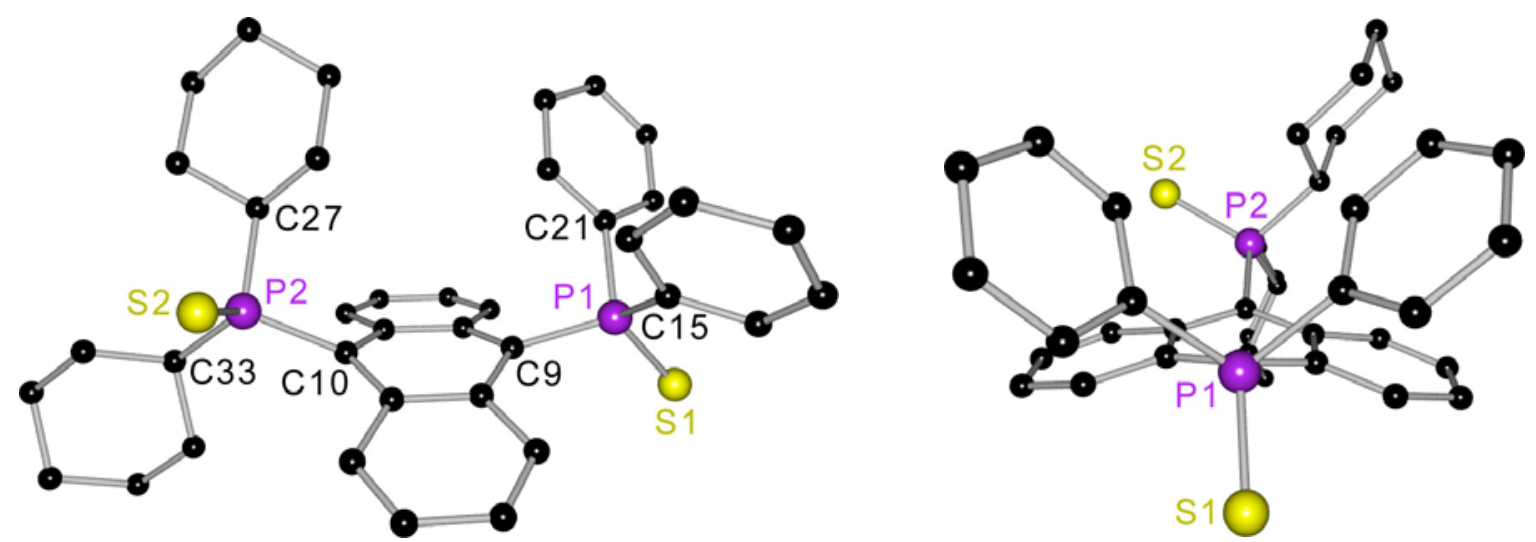

Abb. 4-8: Molekülstruktur von $\left[\mathrm{Ph}_{2}(\mathrm{~S}) \mathrm{P}\left(\mathrm{C}_{14} \mathrm{H}_{8}\right) \mathrm{P}(\mathrm{S}) \mathrm{Cy}_{2} \cdot \mathrm{C}_{7} \mathrm{H}_{8}\right]$ (22) (rechts: Blick entlang P1-C9), die Toluolmoleküle sind aus Gründen der Übersichtlichkeit nicht dargestellt.

Tab. 4-6: Ausgewählte Bindungslängen / pm und -winkel $/{ }^{\circ}$ in 22.

\begin{tabular}{ll}
\hline P1-S1 & $194.70(7)$ \\
P2-S2 & $196.15(7)$ \\
P2-C27 & $184.55(18)$ \\
P2-C33 & $185.22(18)$ \\
\hline
\end{tabular}

\begin{tabular}{ll}
\hline C9-P1-S1 & $115.42(6)$ \\
C15-P1-C21 & $100.35(8)$ \\
C10-P2-S2 & $113.97(6)$ \\
C27-P2-C33 & $109.33(8)$ \\
\hline
\end{tabular}

22 kristallisiert in der triklinen, zentrosymmetrischen Raumgruppe $P \overline{1}$. Die asymmetrische Einheit enthält je ein Diphosphanylanthracen- und ein Toluolmolekül. 22 unterscheidet sich von dem in Kap. 3.1 gezeigten Wirtsmolekül SPAnPS lediglich durch den Austausch von zwei Phenyl- durch zwei Cyclohexylgruppen. Dennoch weisen beide Verbindungen deutliche Unterschiede in den Molekülstrukturen auf. So ist die P1-S1-Bindung in 22 zwar auch senkrecht zum Anthracengerüst angeordnet, die $\mathrm{P}=\mathrm{S}$-Bindung des $\mathrm{P}(\mathrm{S}) \mathrm{Cy}_{2}$-Substituenten ist allerdings nicht transoid zu dieser orientiert, sondern es befindet sich jeweils eine Cyclohexylgruppe ober- und unterhalb der Anthracen-Einheit. Unter der Annahme einer Verbindungslinie zwischen $\mathrm{P} 1$ und $\mathrm{P} 2$ beträgt der Newman-Winkel zwischen den beiden $\mathrm{P}=\mathrm{S}$ Bindungen $121.7^{\circ}$. Das zentrale Anthracengerüst ist stark abgewinkelt und der Faltungswinkel zwischen den Ebenen durch C1 - C4 und C5 - C8 beträgt $151.7^{\circ}$. Die 
beiden Phosphoratome sind mit 69.0 (P1) bzw. 105.1 pm (P2) deutlich von der idealisierten Ebene durch C4a, C8a, C9a und C10a entfernt.

Nach der Kristallisation aus Toluol sind Gitterlösungsmittelmoleküle in das Kristallgitter von 22 eingebaut. Im Gegensatz zu den fluoreszierenden Komplexen von SPAnPS (9-11) befinden sich die Toluolmoleküle jedoch nicht in direkter räumlicher Nähe des Phosphanylanthracens (Abb. 4-9). Das Wirt/Gast-Verhältnis in 22 beträgt $1: 1$ und es ist keine Festkörperfluoreszenz im sichtbaren Bereich beobachtbar.

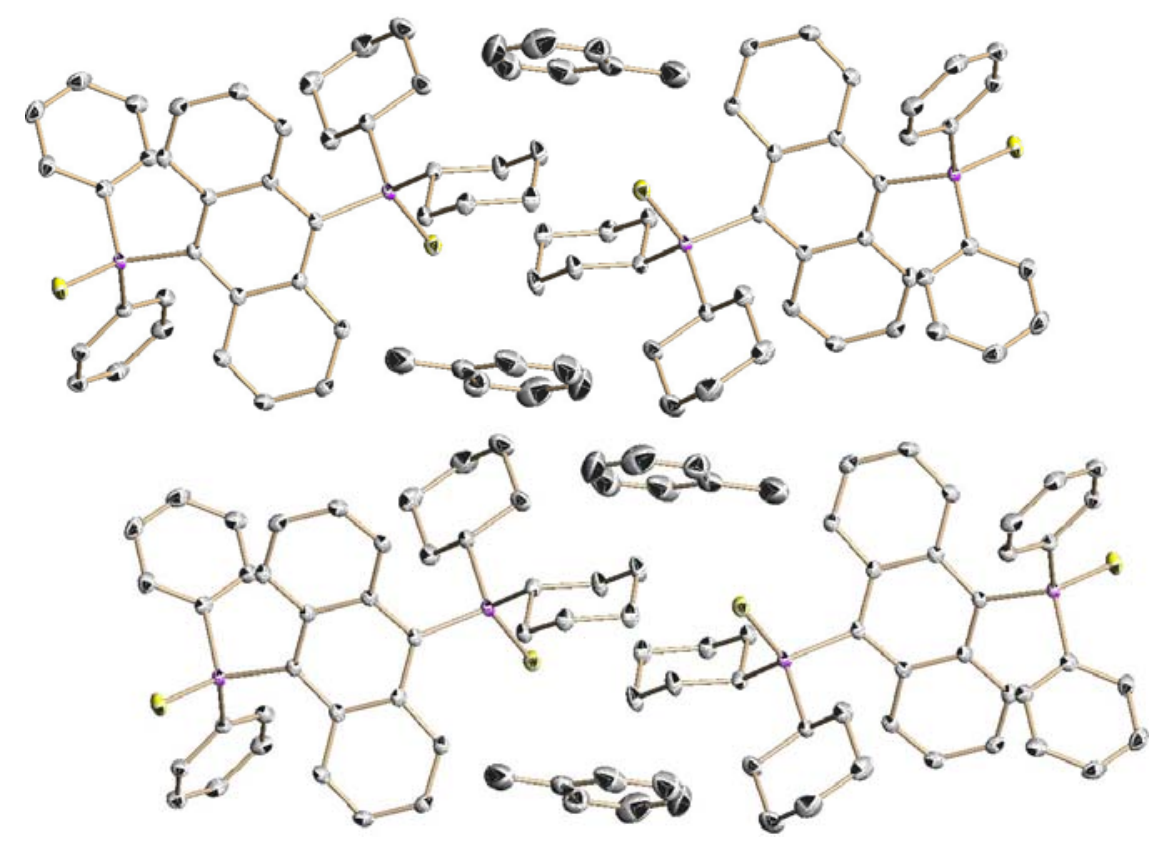

Abb. 4-9: Ausschnitt aus der Kristallstruktur von 22, Blickrichtung entlang $\vec{b}$. 


\section{Stickstoff-Funktionalisierte Phosphanyl- ANTHRACENE}

\subsection{Synthese und Struktur von $\mathrm{Ph}_{2}(\mathrm{~S}) \mathrm{P}\left(\mathrm{C}_{14} \mathrm{H}_{8}\right) \mathrm{NH}_{2}$ (24) und $\left[\mathrm{Ph}_{2}(\mathrm{~S}) \mathrm{P}\left(\mathrm{C}_{14} \mathrm{H}_{8}\right)\left\{\mathrm{HNLi}(\text { thf })_{3}\right\}\right](23)$}

Der Einbau eines Stickstoffatoms in ein Phosphanylanthracen ist von besonderem Interesse, da hierdurch neben einem eher weichen $(P)$ noch ein hartes Donoratom (N) zur Komplexierung von Metallen zur Verfügung steht. Somit wäre auch die Verwendung als Januskopf-Liganden ${ }^{[132,133]}$ durch Koordination von zwei unterschiedlichen Metallfragmenten denkbar. Im vorliegenden Kapitel soll die direkte Funktionalisierung von $\mathrm{C} 10(\mathrm{An})$ mit einem Aminsubstituenten beschrieben werden.

Gl. 5-1 zeigt die Reaktion von 9-Brom-10-(diphenylthiophosphoryl)anthracen (3) mit Lithiumamid, die nach anschließender Hydrolyse das primäre Amin 24 lieferte.<smiles>S=Pc1c2ccccc2c(Br)c2ccccc12</smiles>

3

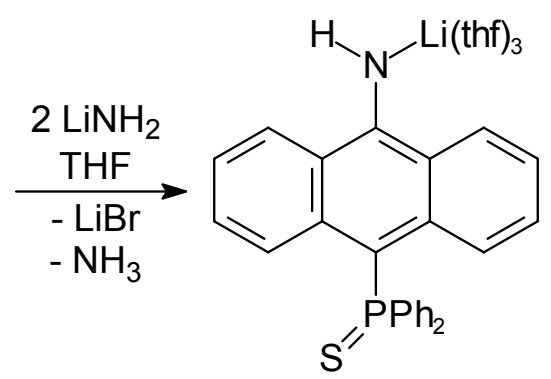

23<smiles>Nc1c2ccccc2c([PH](=S)Pc2ccccc2)c2ccccc12</smiles>

24

GI. 5-1: Umsetzung von 3 mit $\mathrm{LiNH}_{2}$ zu 23 und anschließende Hydrolyse zu 24.

Die Umsetzung von 3 mit einem etwa sechsfachen Überschuss $\mathrm{LiNH}_{2}$ wurde in THF ohne Zusatz eines Katalysators durchgeführt. Nach neun Tagen Rühren bei Raumtemperatur lag eine tiefrote Reaktionsmischung vor. Bisherige Versuche, die Reaktionsdauer durch Erhöhung der Temperatur zu verkürzen, zeigten keinen Erfolg. Nach Abtrennen von ausgefallenem $\mathrm{LiBr}$ wurde das Filtrat aufkonzentriert und mit $n$-Hexan überschichtet. Durch Lagerung bei $6^{\circ} \mathrm{C}$ wurden nach einigen Tagen Kristalle des Lithiumamids $\mathbf{2 3}$ erhalten, die für eine Röntgenstrukturanalyse geeignet waren. In Abb. 5-1 ist die Molekülstruktur dargestellt, Tab. 5-1 enthält ausgewählte Bindungslängen und -winkel. 

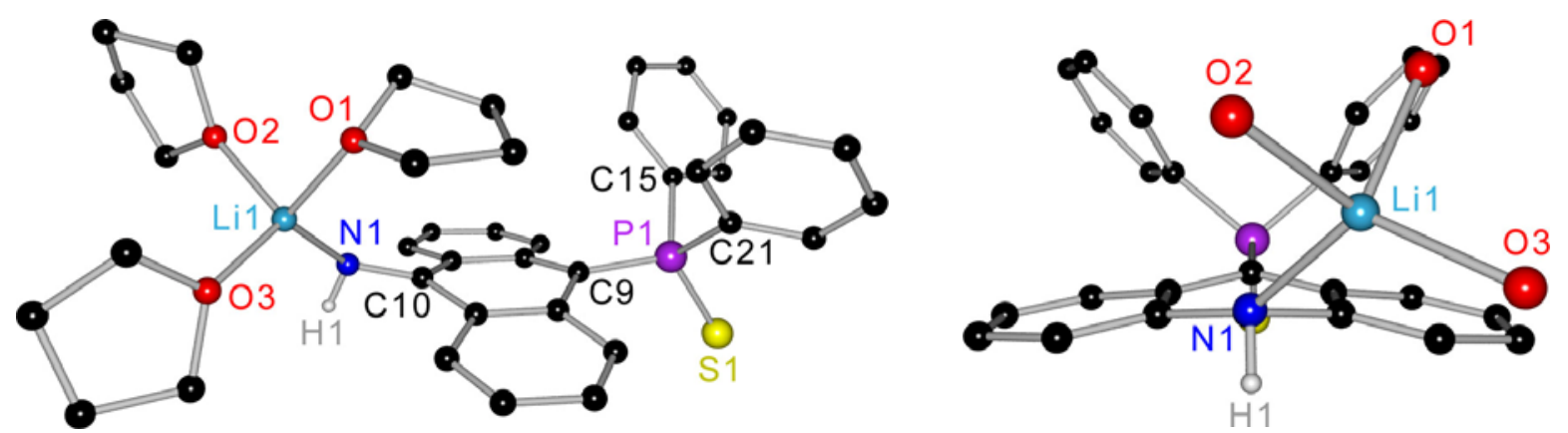

Abb. 5-1: Molekülstruktur von $\left[\mathrm{Ph}_{2}(\mathrm{~S}) \mathrm{P}\left(\mathrm{C}_{14} \mathrm{H}_{8}\right)\left\{\mathrm{HNLi}(\text { thf })_{3}\right\}\right](23)$

(rechts: Blick entlang N1-C10; THF-Kohlenstoffatome nicht dargestellt).

Tab. 5-1: Ausgewählte Bindungslängen / pm und -winkel $/^{\circ}$ in 23.

\begin{tabular}{|c|c|c|c|}
\hline C10-N1 & $135.4(3)$ & C10-N1-Li1 & $132.9(2)$ \\
\hline N1-Li1 & $201.8(5)$ & C10-N1-H1 & 100.17 \\
\hline Li1-O1 & 195.3(4) & N1-Li1-O1 & $114.4(2)$ \\
\hline Li1-O2 & $195.9(4)$ & N1-Li1-O2 & $104.0(2)$ \\
\hline Li1-O3 & $190.5(4)$ & N1-Li1-O3 & $123.7(2)$ \\
\hline
\end{tabular}

23 kristallisiert in der monoklinen, zentrosymmetrischen Raumgruppe $P 2_{1} / c$ mit einem Molekül in der asymmetrischen Einheit. Die Position des amidischen

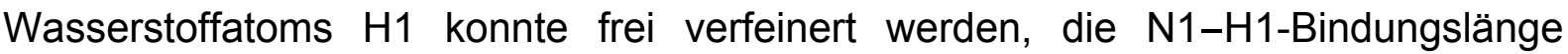
beträgt $83.1 \mathrm{pm}$. Zur Vervollständigung seiner Koordinationssphäre wird Li1 neben N1 noch von drei THF-Molekülen verzerrt tetraedrisch koordiniert. Während die $\mathrm{N} 1-\mathrm{H} 1-B i n d u n g ~ s e n k r e c h t$ zum Anthracengerüst angeordnet ist, bildet die Ebene durch C10, N1 und Li1 einen Winkel von $47.3^{\circ}$ mit der idealisierten Ebene durch C4a, C8a, C9a und C10a. Der Abstand von N1 zu letzterer Ebene beträgt 52.0 pm und $\mathrm{P} 1$ ist $39.8 \mathrm{pm}$ davon entfernt. Die Orientierung der Phosphorylgruppe entspricht der Anordnung der $\mathrm{P}(\mathrm{S}) \mathrm{Ph}_{2}$-Substituenten in den bisher diskutierten Verbindungen, die beiden Phenylringe befinden sich auf der gleichen Seite der Anthracen-Einheit wie die (thf) $)_{3}$ Li-Gruppe. Der Faltungswinkel zwischen den durch C1 - C4 und C5 - C8 aufgespannten Ebenen beträgt $165.7^{\circ}$.

Bislang ist nur eine vergleichbare Molekülstruktur mit einer (thf) ${ }_{3} \mathrm{Li}-\mathrm{N}(\mathrm{H})-A r-E i n h e i t$ bekannt, bei dieser bildet das freie Elektronenpaar des Stickstoffatoms allerdings zusätzlich eine dative Bindung zu einem Aluminiumfragment aus. Bei der Verbindung handelt es sich um $\left[\mathrm{Me}_{2}{ }^{{ } \mathrm{BuAl}} \mathrm{H}-\mathrm{N}(\mathrm{H})(\mathrm{Dipp})-\mathrm{Li}(\text { thf })_{3}\right]$ (Dipp = 2,6-Diisopropylphenyl), das von der Arbeitsgruppe Atwood durch Alkylierung von $\left[\mathrm{Me}_{2} \mathrm{AlNHDipp}\right]_{2}$ mit ${ }^{\mathrm{t}} \mathrm{BuLi}$ 
erhalten wurde. ${ }^{[134,135]}$ Mit $217.1 \mathrm{bzw} .139 .6 \mathrm{pm}$ sind die Li-N- bzw. Li- $\mathrm{C}_{\mathrm{Ar}}-\mathrm{Bindungen}$ in der Vergleichsstruktur deutlich länger als in 23 (201.9 bzw. 135.4), was auf die zusätzliche Wechselwirkung mit dem Aluminiumatom zurückzuführen ist. Der $\mathrm{C}_{\mathrm{Ar}}-\mathrm{N}-\mathrm{Li}-$ Winkel in $\mathbf{2 3}$ ist mit $132.9^{\circ}$ erheblich größer als in [Me $\left.{ }_{2}{ }^{t} \mathrm{BuAl}-\mathrm{N}(\mathrm{H})(\mathrm{Dipp})-\mathrm{Li}(\text { thf })_{3}\right]\left(113.4^{\circ}\right)$, was durch den sterisch anspruchsvollen Anthrylsubstituenten sowie durch das Fehlen der $\mathrm{AlR}_{3}$-Einheit begründet ist.

Nach der Hydrolyse des Lithiumamids $\mathbf{2 3}$ mit anschließender Aufarbeitung wurde das Amin 24 erhalten. Abb. 5-2 zeigt die Molekülstruktur der Verbindung, in Tab. 5-2 sind ausgewählte Bindungslängen und -winkel aufgeführt.

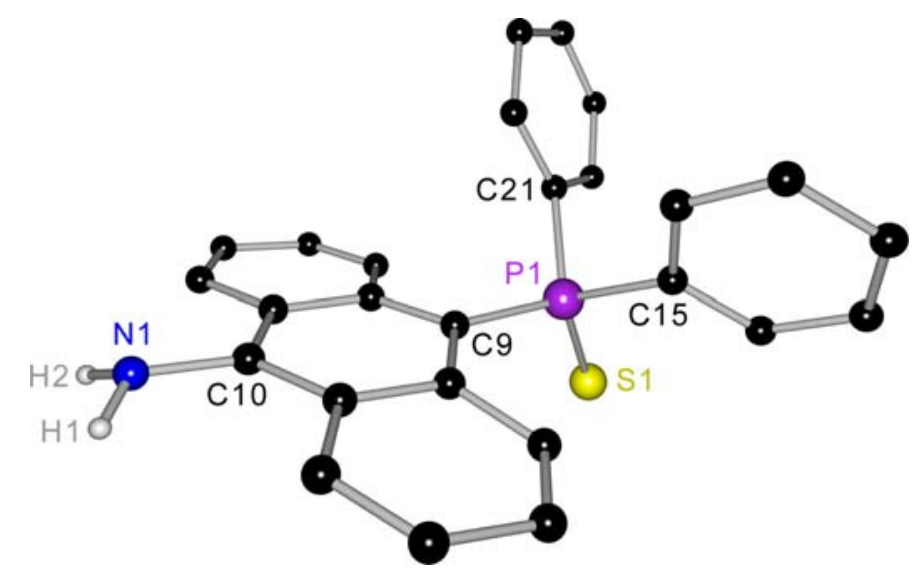

Tab. 5-2: Ausgewählte Bindungslängen / pm und -winkel $/{ }^{\circ}$ in 24.

\begin{tabular}{ll}
\hline P1-C9 & $181.0(2)$ \\
C10-N1 & $136.8(3)$ \\
& \\
H1-N1-H2 & 112.02 \\
N1-C10 $\cdots$ C9 & $177.60(18)$ \\
\hline
\end{tabular}

Abb. 5-2: Molekülstruktur von $\mathrm{Ph}_{2}(\mathrm{~S}) \mathrm{P}\left(\mathrm{C}_{14} \mathrm{H}_{8}\right) \mathrm{NH}_{2}$ (24).

24 kristallisiert ebenfalls in der monoklinen Raumgruppe $P 2_{1} / C$ und die asymmetrische Einheit enthält neben einem Aminmolekül noch ein Gitterlösungsmittelmolekül THF. Die Positionen der beiden aminischen Wasserstoffatome wurden auch hier frei gefunden, die Bindungen zu N1 haben Längen von 90.3 (H1) bzw. 89.9 pm (H2). Der Faltungswinkel des Anthracengerüstes beträgt $172^{\circ}, \mathrm{N} 1$ befindet sich in der durch C4a, C8a, C9a und C10a aufgespannten Ebene, während P1 25.3 pm davon entfernt ist.

Bislang ist nur die Molekülstruktur eines weiteren 9-Anthrylamins bekannt (siehe rechts). Die Verbindung trägt in Position 10 noch einen 2,4,6Trinitrophenylsubstituenten und wurde von Struchkov et al. strukturell charakterisiert. ${ }^{[136]}$ Die C-N-Bindung zeigt mit 138.1 pm gute Übereinstimmung mit der entsprechenden Bindungslänge in $\mathbf{2 4}$ und auch die beiden Amin-Wasser-

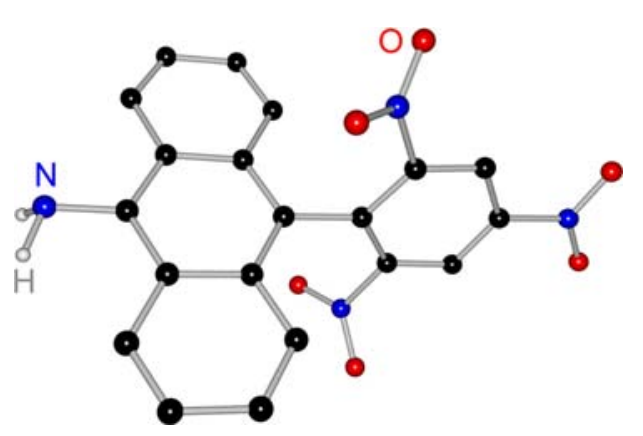
stoffatome sind in ähnlicher Weise orientiert. 


\subsection{Synthese und Struktur von $\operatorname{Br}\left(\mathrm{C}_{14} \mathrm{H}_{8}\right) \mathrm{P}\left(\mathrm{N}^{i} \mathrm{Pr}_{2}\right)_{2}(25)$}

Neben der $\mathrm{NH}_{2}$-Funktionalisierung eines 9-Phosphanylanthracens ist der Austausch der Alkyl- oder Arylsubstituenten am Phosphoratom durch $\mathrm{NR}_{2}$-Einheiten denkbar, um zusätzliche Donoratome im Molekül zu erhalten.

Ein Beispiel für einen $\mathrm{P}\left(\mathrm{NR}_{2}\right)_{2}$-Januskopf-Liganden stammt von der Arbeitsgruppe Nakazawa, die einen Halbsandwich-Komplex mit einer $\mathrm{Fe}=\mathrm{Sn}$-Bindung synthetisierte, bei dem ein Ligand mit $\mathrm{P}\left(\mathrm{NR}_{2}\right)_{2}$-Gerüst sowohl das Eisen- als auch das Zinnatom über

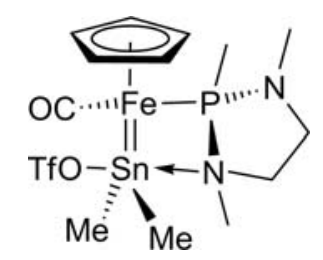
zwei unterschiedliche Donoratome koordiniert (siehe rechts). ${ }^{[137]}$

GI. 5-2 zeigt die Monolithiierung von 9,10-Dibromanthracen mit anschließender Umsetzung mit einem Bis(amino)chlorphosphan, die zu 9-Brom-10-[bis(diisopropylamino)phosphanyl]anthracen (25) in 90\%iger Ausbeute führte. Durch Umkristallisation aus Toluol wurden Kristalle für eine Röntgenstrukturanalyse erhalten, die Molekülstruktur ist in Abb. 5-3 dargestellt, Tab. 5-3 enthält ausgewählte Bindungslängen und -winkel.<smiles>Brc1c2ccccc2c(Br)c2ccccc12</smiles>

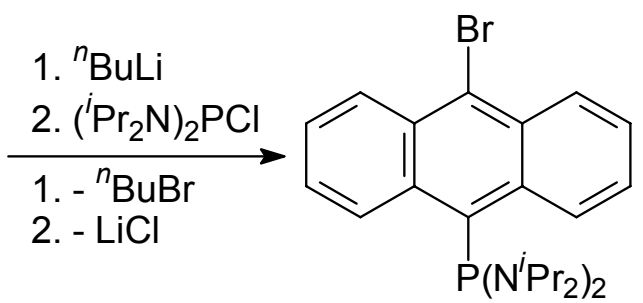

25

GI. 5-2: Synthese von $\operatorname{Br}\left(\mathrm{C}_{14} \mathrm{H}_{8}\right) \mathrm{P}\left(\mathrm{N}^{j} \mathrm{Pr}_{2}\right)_{2}$ (25).
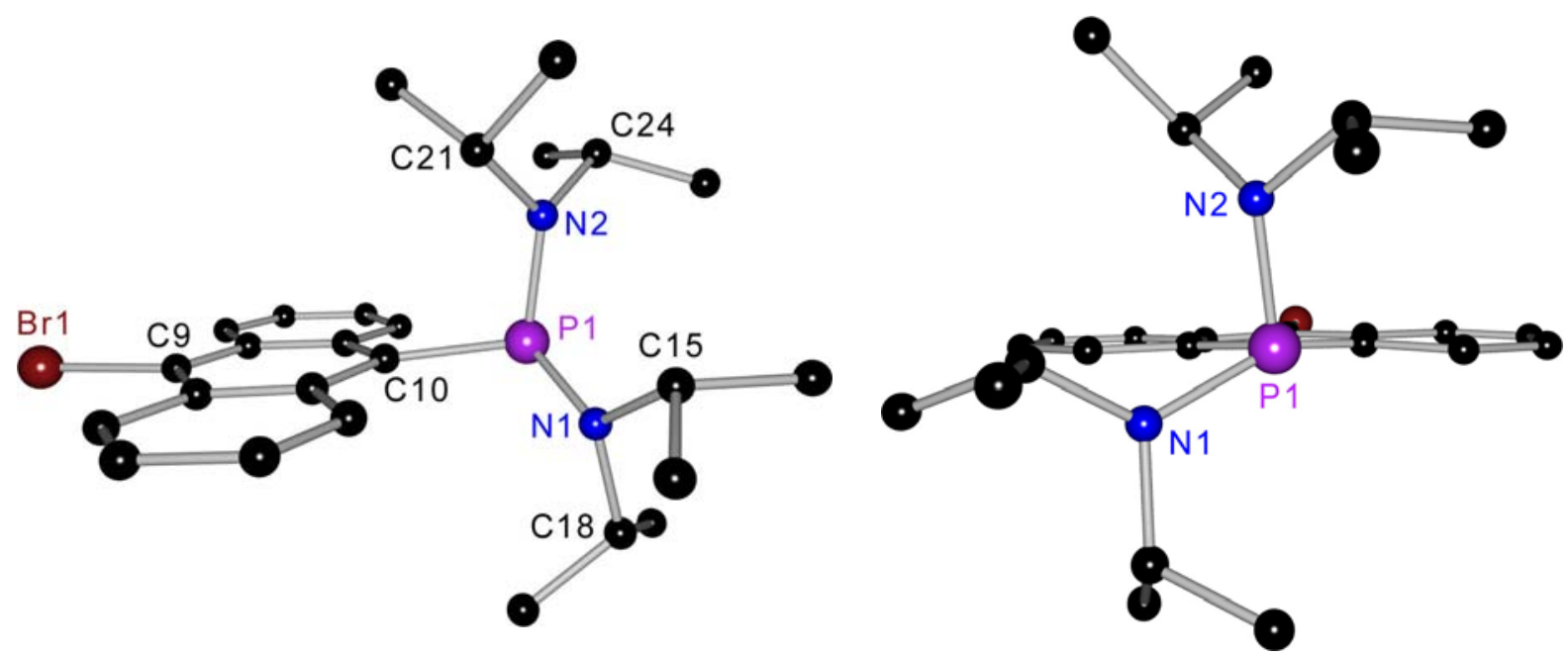

Abb. 5-3: Molekülstruktur von $\operatorname{Br}\left(\mathrm{C}_{14} \mathrm{H}_{8}\right) \mathrm{P}\left(\mathrm{N}^{\prime} \mathrm{Pr}{ }_{2}\right)_{2}$ (25) (rechts: Blick entlang $\left.\mathrm{P} 1-\mathrm{C} 10\right)$. 
Tab. 5-3: Ausgewählte Bindungslängen / pm und -winkel $/{ }^{\circ}$ in 25.

\begin{tabular}{ll}
\hline P1-C10 & $189.55(18)$ \\
P1-N1 & $171.77(13)$ \\
P1-N2 & $169.60(13)$ \\
N1-C15 & $147.7(2)$ \\
N1-C18 & $148.4(2)$ \\
N2-C21 & $148.2(2)$ \\
N2-C24 & $148.8(2)$ \\
\hline
\end{tabular}

\begin{tabular}{ll}
\hline C10-P1-N1 & $108.88(7)$ \\
C10-P1-N2 & $101.06(7)$ \\
N1-P1-N2 & $106.73(6)$ \\
P1-N1-C15 & $124.49(10)$ \\
P1-N1-C18 & $117.27(11)$ \\
C15-N1-C18 & $115.08(12)$ \\
P1-C10‥C9 & $172.24(9)$ \\
\hline
\end{tabular}

25 kristallisiert in der monoklinen, polaren Raumgruppe Cc. Die asymmetrische Einheit enthält ein Molekül. Das Anthracengerüst ist planar (Faltungswinkel: $178.3^{\circ}$ ) während Br1 und P1 10.4 bzw. 14.7 pm von der durch C4a, C8a, C9a und C10a aufgespannten Ebene entfernt sind. Diese schließt mit den Ebenen durch C10, P1, $\mathrm{N} 1$ und $\mathrm{C} 10, \mathrm{P} 1, \mathrm{~N} 2$ Winkel von 27.9 bzw. $-84.4^{\circ}$ ein. Eine ähnliche Anordnung der $\mathrm{P}\left(\mathrm{N}^{i} \mathrm{Pr}_{2}\right)_{2}$-Substituenten findet sich in 1-[Bis(diisopropylamino)phosphanyl]naphthalin (Abb. 5-4). ${ }^{[34]}$ Durch den geringeren sterischen Anspruch des Naphthylrestes sind die entsprechenden Winkel dort allerdings auf $7.0 \mathrm{bzw}$. $-72.9^{\circ}$ verkleinert. Die P-N-Bindungslängen stimmen mit 170.6 (N1) und 169.7 pm (N2) gut mit den in 25 gefundenen Werten überein, die P-C-Bindung der Vergleichsverbindung ist mit 185.7 pm etwas kürzer. In den zwei Verbindungen sind die beiden Stickstoff-

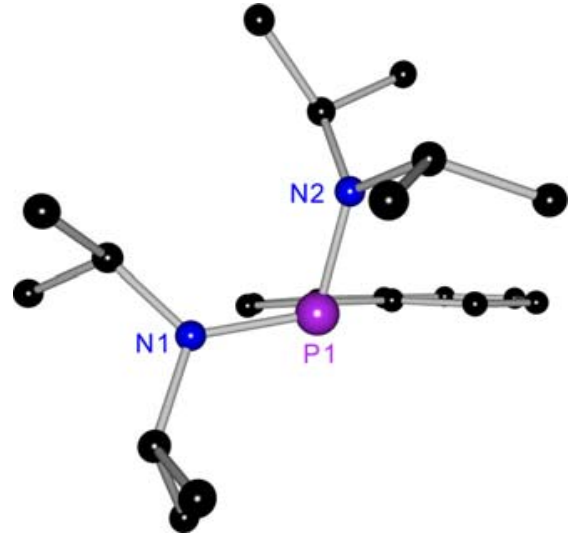

Abb. 5-4: Molekülstruktur von $\left({ }^{i} \mathrm{Pr}_{2} \mathrm{~N}\right)_{2} \mathrm{P}(1-\mathrm{Naph}) \cdot{ }^{[34]}$ atome jeweils trigonal planar koordiniert.

Mit der Synthese von 25 wurde gezeigt, dass die Umsetzung von Lithiumanthraceniden mit Amino-funktionalisierten Chlorphosphanen eine Möglichkeit darstellt, um Phosphanylanthracene mit zusätzlichen Stickstoffatomen zu synthetisieren, die als Donoren für die Koordination von Metallen fungieren könnten. Ziel weiterer Studien wird unter anderem die Verwendung solcher AnthracenDerivate als Januskopf-Liganden sein. 


\subsection{Synthese und Struktur von $\mathrm{Py}_{2} \mathrm{P}\left(\mathrm{C}_{14} \mathrm{H}_{9}\right)(28)$}

9-(Dichlorphosphanyl)anthracen (27) stellt ein mögliches Edukt für die Synthese von Stickstoff-funktionalisierten Phosphanylanthracenen dar, da sich die beiden Chloratome durch eine Vielzahl stickstoffhaltiger Substituenten wie etwa Pyridyl- oder Picolylgruppen austauschen lassen sollte. 1992 gelang es der Arbeitsgruppe Schmutzler, 27 zu synthetisieren und spektroskopisch zu charakterisieren. ${ }^{[29]}$ Dazu wurde 9-Lithiumanthracenid langsam zu einem großen Überschuss von Trichlorphosphan in Diethylether getropft (Gl.5-3). Trotz kontrollierter Reaktionsführung entstand bei der Umsetzung neben dem gewünschten Dichlorphosphan 27 $\left(\delta_{\mathrm{P}} 159 \mathrm{ppm}\right)$ auch stets Chlorbis(9-anthryl)phosphan ( $\left.\delta_{\mathrm{P}} 80 \mathrm{ppm}\right)$ als Nebenprodukt, das sich nur mühsam durch Sublimation abtrennen lässt. Für eine einfache und gezielte Darstellung von 27 erschien dieser Weg somit eher ungeeignet zu sein und deshalb wurde nach einer alternativen Methode gesucht.<smiles>Clc1c2ccccc2cc2ccccc12</smiles>

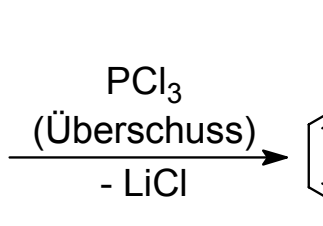<smiles>CCCc1c2ccccc2cc2ccccc12</smiles>

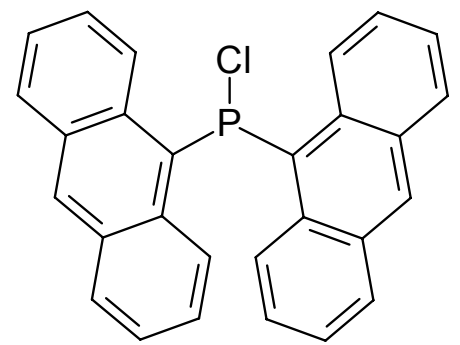

GI. 5-3: Synthese von $\mathrm{Cl}_{2} \mathrm{P}\left(\mathrm{C}_{14} \mathrm{H}_{9}\right)(27)$ nach Schmutzler et al. ${ }^{[29]}$

Duff und Shaw berichteten bereits 1972 von der Synthese des Naphthalinderivats $\mathrm{Cl}_{2} \mathrm{P}$ (1-Naph). ${ }^{[138]}$ Dabei wurde zunächst aus der entsprechenden 1-NaphthylGrignardverbindung und $\left(\mathrm{Et}_{2} \mathrm{~N}\right)_{2} \mathrm{PCl}$ ein Diaminophosphanylnaphthalin dargestellt. Durch Umsetzung mit etherischer $\mathrm{HCl}$-Lösung wurden anschließend die beiden $\mathrm{P}-\mathrm{N}$ Bindungen unter Abspaltung von Diethylammoniumchlorid chloriert und somit Dichlor(1-naphthyl)phosphan in einer Ausbeute von $50 \%$ erhalten. Schmidbaur et al. konnten 2004 die Ausbeute der Chlorierungsreaktion durch Verwendung von gasförmigem $\mathrm{HCl}$ auf $83 \%$ erhöhen. ${ }^{[139]}$

GI. 5-4 zeigt die in der vorliegenden Arbeit durchgeführten Umsetzungen, um aus 9-Bromanthracen selektiv 9-(Dichlorphosphanyl)anthracen (27) zu erhalten. Im Jahr 2006 wurde eine fast identische Synthesevorschrift bereits von der Arbeitsgruppe Tokitoh veröffentlicht, ${ }^{[140]}$ die 27 allerdings direkt weiter zu einem Diphosphen umsetzten, ohne auf das hohe synthetische Potential als Ausgangsstoff für verschiedenste Phosphanylanthracene hinzuweisen. 


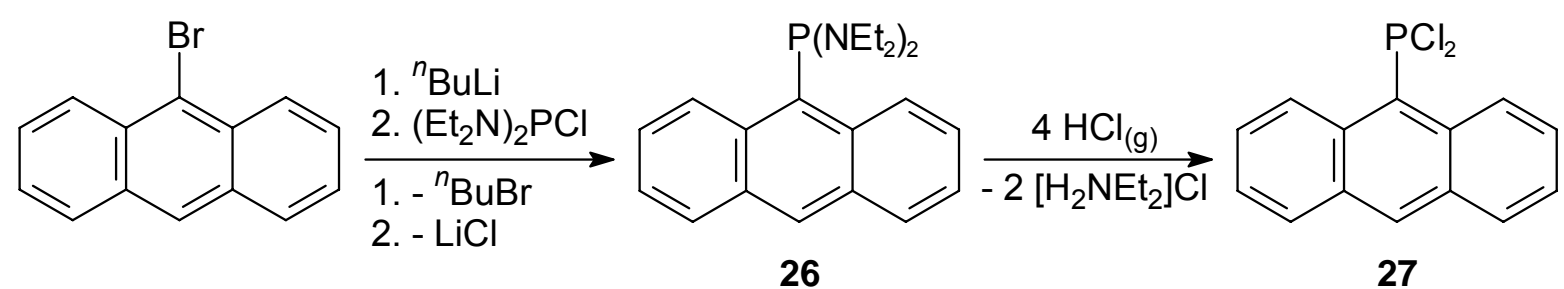

GI. 5-4: Darstellung von $\mathrm{Cl}_{2} \mathrm{P}\left(\mathrm{C}_{14} \mathrm{H}_{9}\right)$ (27) über $\left(\mathrm{Et}_{2} \mathrm{~N}\right)_{2} \mathrm{P}\left(\mathrm{C}_{14} \mathrm{H}_{9}\right)(26)$.

Die Zwischenstufe 26 wurde aus 9-Bromanthracen, ${ }^{n} \mathrm{BuLi}$ und Chlorbis(diethylamino)phosphan in einer Ausbeute von $93 \%$ erhalten. Das Produkt fiel als rotes ÖI an, bisherige Kristallisationsversuche blieben bisher erfolglos. Zur weiteren Umsetzung wurde 26 in $n$-Hexan gelöst und $\mathrm{HCl}$-Gas durch die Reaktionsmischung geleitet. Dabei fiel 27 zusammen mit Diethylammoniumchlorid als gelber Niederschlag aus und konnte abfiltriert werden. Da die Löslichkeit von 27 in Toluol sehr gut ist, wurde der Niederschlag mit Toluol gewaschen und das Dichlorphosphan 27 somit in sehr reiner Form erhalten. Leider konnten auch von der Produktverbindung bislang keine Kristalle zur Bestimmung der Molekülstruktur gewonnen werden.

Die Synthese von 9-[Di(2-pyridyl)phosphanyl]anthracen (28) wurde analog literaturbekannter Darstellungsmethoden von Dipyridylphosphanen des Typs $\mathrm{Py}_{2} \mathrm{PR}(\mathrm{R}=\mathrm{Ph}$, p-Tolyl, $p$-Anisyl) ${ }^{[141,142]}$ durchgeführt (GI. 5-5).<smiles>Clc1ccccn1</smiles>

27<smiles>CCCCCc1c2ccccc2cc2ccc(Cl)cc12</smiles>

28

GI. 5-5: Darstellung von $\mathrm{Py}_{2} \mathrm{P}\left(\mathrm{C}_{14} \mathrm{H}_{9}\right)$ (28).

Die Lithiierung von 2-Brompyridin mit ${ }^{n} \mathrm{BuLi}$ erfolgte in Diethylether bei $-78^{\circ} \mathrm{C}$. Anschließend wurde eine Lösung des Dichlorphosphans 27 in Toluol langsam zu der 2-Lithiopyridinlösung getropft. Nach saurer Aufarbeitung wurde die Reaktionsmischung mit Natronlauge auf einen alkalischen pH-Wert gebracht, wobei 28 als brauner Feststoff ausfiel. Durch Lagerung einer gesättigten Lösung des Produktes in THF / $n$-Hexan wurden nach einigen Tagen bei RT Kristalle erhalten, die für eine röntgenkristallographische Strukturuntersuchung geeignet waren. In Abb. 5-5 (links) ist die Molekülstruktur von 28 dargestellt, Tab. 5-4 enthält ausgewählte Bindungslängen und -winkel. 

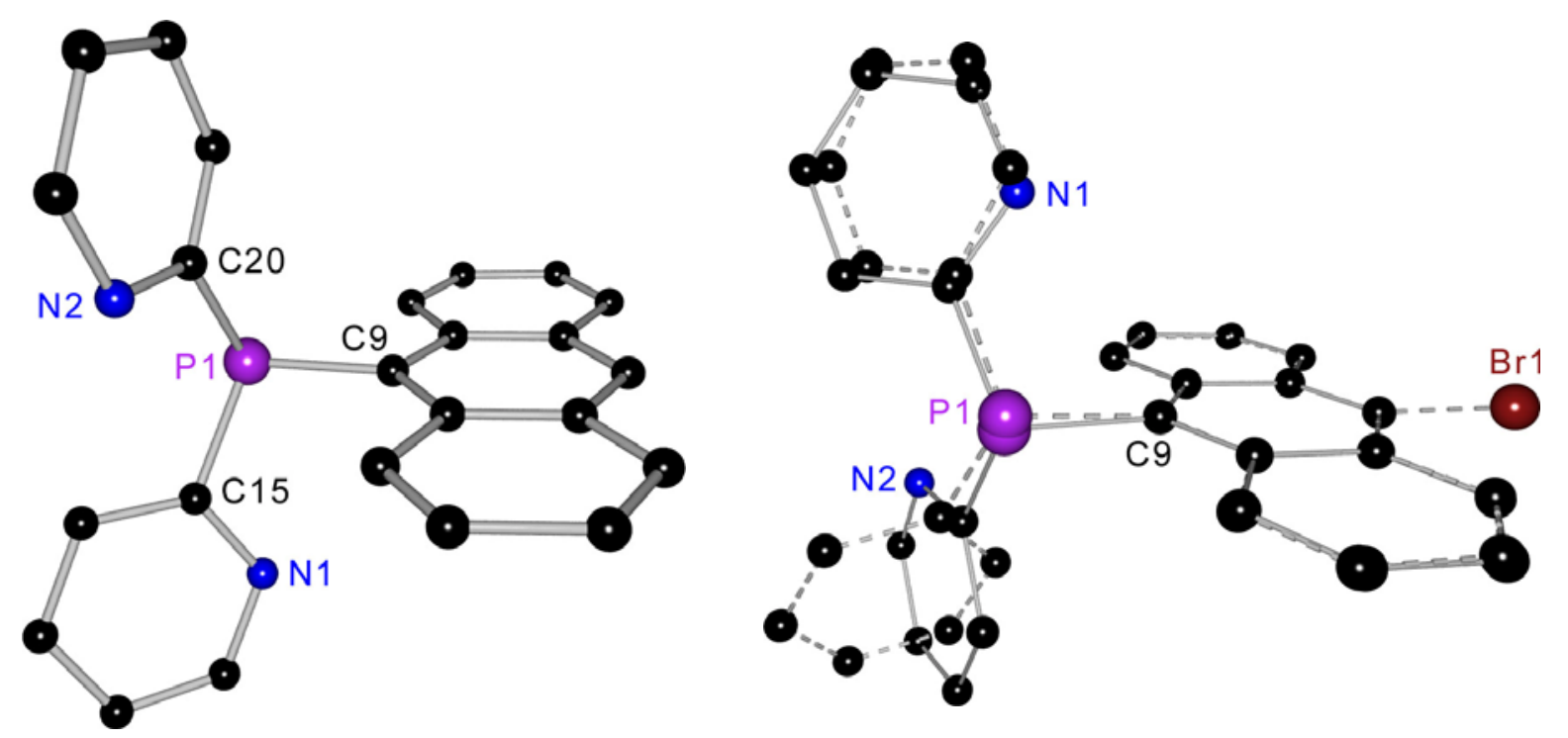

Abb. 5-5: links: Molekülstruktur von $\mathrm{Py}_{2} \mathrm{P}\left(\mathrm{C}_{14} \mathrm{H}_{9}\right)(\mathbf{2 8})$, rechts: Superposition von $\mathbf{2 8}$ und $\mathbf{1}$ (gestrichtelte Bindungen), erzeugt durch Überlagerung von C4a, C8a, C9a und C10a.

Tab. 5-4: Ausgewählte Bindungslängen / pm und -winkel / ${ }^{\circ}$ in 28.

\begin{tabular}{ll}
\hline $\mathrm{P} 1-\mathrm{C} 9$ & $184.29(19)$ \\
$\mathrm{P} 1-\mathrm{C} 15$ & $182.81(19)$ \\
$\mathrm{P} 1-\mathrm{C} 20$ & $184.7(2)$ \\
$\mathrm{C} 15-\mathrm{N} 1$ & $135.3(2)$ \\
$\mathrm{C} 20-\mathrm{N} 2$ & $135.0(2)$ \\
\hline
\end{tabular}

\begin{tabular}{ll}
\hline C9-P1-C15 & $103.48(8)$ \\
C9-P1-C20 & $101.12(8)$ \\
C15-P1-C20 & $106.84(9)$ \\
P1-C9 ‥C10 & $176.33(10)$ \\
\hline
\end{tabular}

28 kristallisiert in der monoklinen, zentrosymmetrischen Raumgruppe $P 2_{1} / n$. Die asymmetrische Einheit enthält ein Molekül. Das Anthracengerüst ist planar, während der Abstand von P1 zu der durch C4a, C8a, C9a und C10a aufgespannten Ebene $7.38 \mathrm{pm}$ beträgt. Die Bindungslängen und -winkel des $\mathrm{Py}_{2} \mathrm{P}$-Substituenten entsprechen denen bereits bekannter Aryldi(2-pyridyl)phosphanen. ${ }^{[141]}$

In Abb. 5-5 (rechts) ist eine Überlagerung der Molekülstrukturen von 28 und 9-Brom10-(diphenylphosphanyl)anthracen (1) dargestellt. Die PPy $y_{2}$ bzw. $\mathrm{PPh}_{2}-\mathrm{Substituen-}$ ten sind jeweils in einer gestaffelten Anordnung relativ zur Anthracen-Einheit orientiert. Während jedoch ein Phenyl- und Pyridylring fast koplanar angeordnet sind, schließen die beiden anderen einen Winkel von $85.7^{\circ}$ miteinander ein. 


\section{UNERWARTETE REAKTIONEN}

In diesem Kapitel werden die vier Verbindungen 29 - 32 diskutiert, deren Synthesen nicht unmittelbar beabsichtigt waren und die größtenteils lediglich über Röntgenstrukturuntersuchungen charakterisiert wurden. Da 29-32 sehr ungewöhnliche Molekülstrukturen aufweisen, wurden diese Verbindungen in die vorliegende Arbeit mit aufgenommen.

\section{$6.1\left[\mathrm{~S}\left\{\left(\mathrm{C}_{14} \mathrm{H}_{8}\right) \mathrm{P}(\mathrm{S}) \mathrm{Ph}_{2}\right\}_{2}\right](29)$}

Gl. 6-1 zeigt die geplante Umsetzung von 3 mit Lithiumdi(2-pyridyl)amid, die analog zur Synthese von 24 zur Bildung eines Anthracenylamins führen sollte.<smiles>CCCCN(C(=S)N(CCCC)c1c2ccccc2c([PH](=S)c2ccccc2)c2ccccc12)c1ccccc1</smiles>

3

GI. 6-1: Geplante Umsetzung von 3 mit LiNPy.

Dazu wurde eine Lösung von 3 in THF mit einem zweifachen Überschuss LiNPy 2 versetzt und für $20 \mathrm{~d}$ bei RT gerührt. Nach Abtrennen von unlöslichen Bestandteilen wurde die Reaktionsmischung auf etwa die Hälfte aufkonzentriert und bei $4{ }^{\circ} \mathrm{C}$ gelagert. Nach einigen Tagen bildeten sich nadelförmige Kristalle, die für eine Röntgenstrukturuntersuchung geeignet waren (Abb. 6-1 und Tab. 6-1).

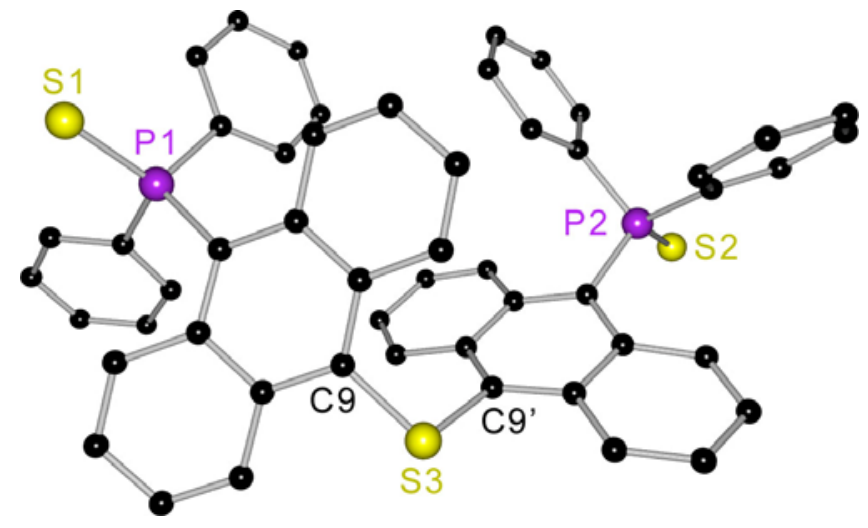

Abb. 6-1: Molekülstruktur von 29.
Tab. 6-1: Ausgewählte Bindungslängen / pm und -winkel $/{ }^{\circ}$ in 29.

\begin{tabular}{ll}
\hline S3-C9 & $178.0(3)$ \\
S3-C9' & $178.2(3)$ \\
& \\
C9-S3-C9' & $102.26(15)$ \\
C9 $\cdots C 10-P 1$ & $165.07(17)$ \\
C10 ‥C9-S3 & $169.31(17)$ \\
\hline
\end{tabular}


Das Ergebnis der Strukturuntersuchung zeigt, dass nicht das gewünschte Anthracenylamin entstanden ist, sondern ein Bis(9-anthracenyl)sulfid (29). Die Verbindung kristallisiert in der triklinen, zentrosymmetrischen Raumgruppe $P \overline{1}$. Die asymmetrische Einheit enthält neben einem Sulfidmolekül noch drei nichtkoordinierende THF-Moleküle. Der Faltungswinkel der beiden Anthracengerüste beträgt etwa $162^{\circ}$ und die beiden Phosphoratome sind 64.7 (P1) bzw. 68.6 pm (P2) von der durch C4a, C8a, C9a und C10a aufgespannten Ebene entfernt. Die Bindungslängen und -winkel um das verbrückende Schwefelatom S3 entsprechen denen bekannter Bis(1,1'-naphthyl)sulfide. ${ }^{\text {143] }}$

Die Entstehung von 29 lässt sich nur dadurch erklären, dass das Edukt 3 noch mit elementarem Schwefel aus der Oxidationsreaktion verunreinigt war. In Gl. 6-2 ist eine mögliche Reaktionsfolge dargestellt, die zur Bildung von 29 führt.

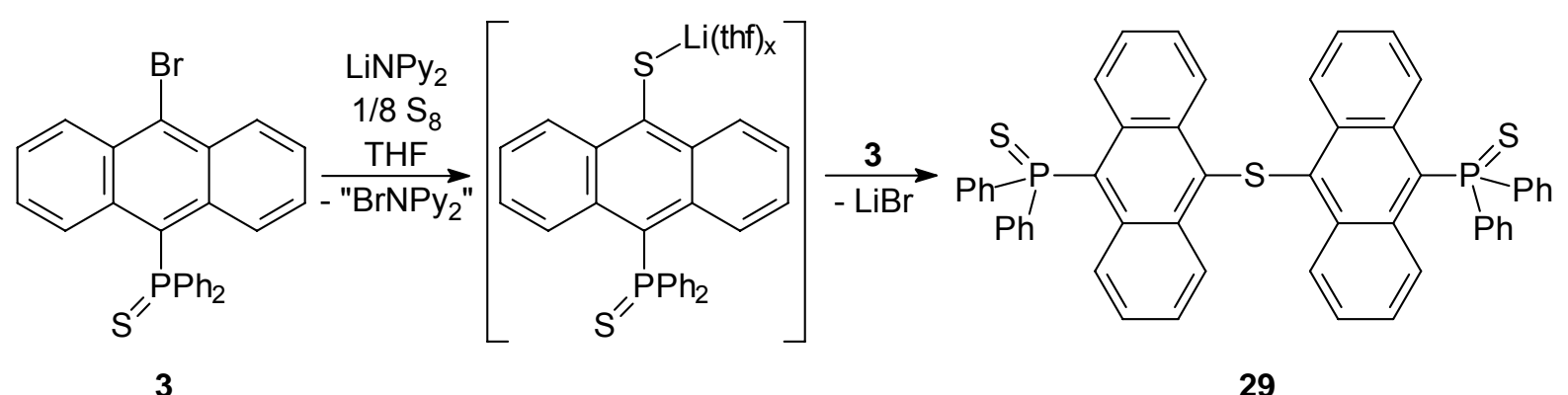

3

29

Gl. 6-2: Reaktion von 3 mit $\mathrm{LiNPy}_{2}$ und $\mathrm{S}_{8}$ unter Bildung von 29.

Im ersten Schritt erfolgt durch das verwendete Lithiumamid eine Lithiierung des Anthracengerüstes unter Bromidabspaltung. Anschließend wird ein Schwefelatom in die $\mathrm{C}_{\mathrm{An}}$-Li-Bindung insertiert und es kommt zur Bildung der in GI. 6-2 dargestellten Zwischenstufe. Derartige Insertionsreaktionen von Chalkogenatomen in $\mathrm{C}_{\mathrm{Ar}}-\mathrm{Li}$ Bindungen wurden bereits für elementaren Schwefel sowie für rotes Selen beobachtet. ${ }^{[144,145]}$ Die Stabilisierung des dabei entstehenden Lithiumthiolats erfolgt durch die Koordination von (vermutlich drei) THF-Molekülen.

Durch die nachfolgende Reaktion mit einem weiteren Eduktmolekül 3 kommt es schließlich unter Abspaltung von Lithiumbromid zur Bildung von 29. Bei der Verbindung handelt es sich um das erste bekannte Bis(9-anthracenyl)sulfid. 


\section{$6.2\left[\left\{(\text { thf })_{4} \mathrm{NaS}\right\}\left(\mathrm{C}_{14} \mathrm{H}_{8}\right) \mathrm{P}(\mathrm{S}) \mathrm{Ph}_{2}\right](30)$}

In Kap. 5.1 wurde gezeigt, dass durch die Reaktion von 3 mit $\mathrm{LiNH}_{2}$ das Lithiumanthracenylamid $\mathbf{2 3}$ erhalten werden kann. Um das entsprechende Natriumamid zu synthetisieren, wurde 3 unter analogen Reaktionsbedingungen mit $\mathrm{NaNH}_{2}$ in THF umgesetzt. Nach $5 \mathrm{~d}$ Rühren bei RT wurden unlösliche Bestandteile abgetrennt und die Reaktionsmischung mit $n$-Hexan überschichtet. Einige Tage später hatten sich rote Kristalle gebildet, die zur Bestimmung der Molekülstruktur geeignet waren. Das Ergebnis der Röntgenstrukturanalyse zeigt allerdings, dass bei der Reaktion nicht das erwartete Natriumamid, sondern ein Natrium(anthracenyl)thiolat (30) entstand (GI. 6-3). Dies lässt sich analog Kap. 6.1 dadurch erklären, dass das Edukt 3 noch mit elementarem Schwefel verunreinigt war, welcher in die $\mathrm{C}_{\mathrm{An}}-\mathrm{Na}-\mathrm{Bindung}$ der metallierten Zwischenstufe insertiert wurde.

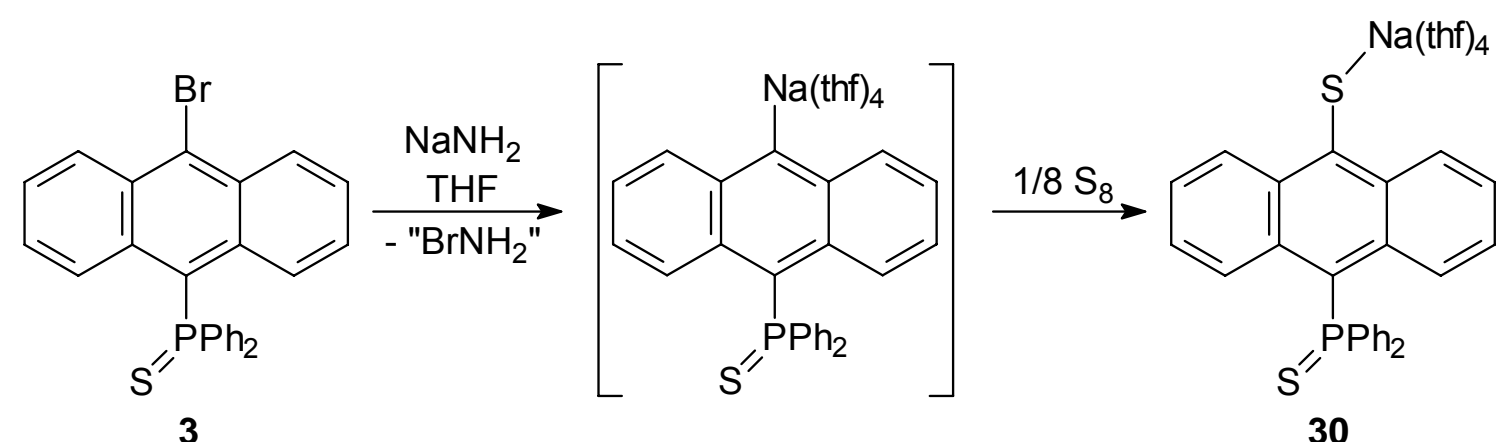

GI. 6-3: Reaktion von 3 mit $\mathrm{NaNH}_{2}$ und $\mathrm{S}_{8}$ unter Bildung von 30.

Die Molekülstruktur von 30 ist in Abb. 6-2 dargestellt, Tab. 6-2 enthält ausgewählte Bindungslängen und -winkel.

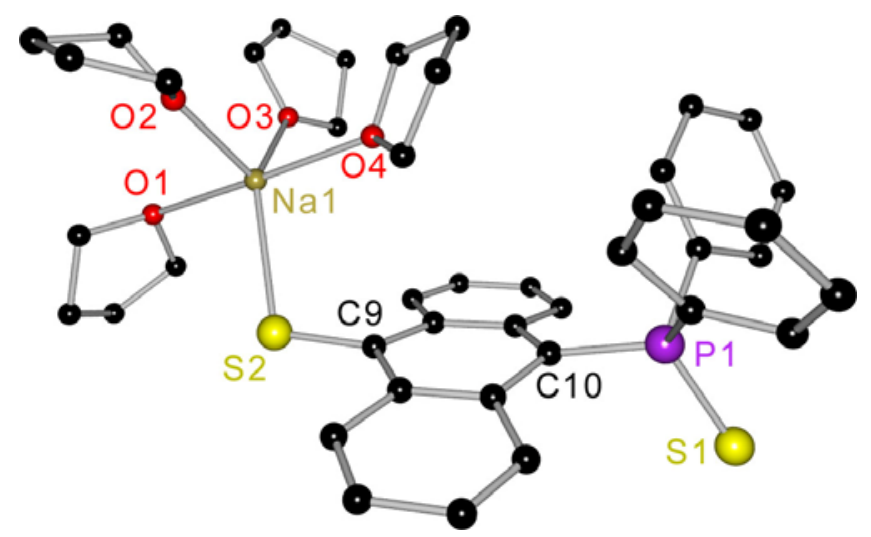

Abb. 6-2: Molekülstruktur von 30 (Fehlordnungen der THF-Moleküle nicht dargestellt).
Tab. 6-2: Ausgewählte Bindungslängen / pm und -winkel $/^{\circ}$ in $\mathbf{3 0}$.

\begin{tabular}{ll}
\hline S2-C9 & $172.8(3)$ \\
S2-Na1 & $275.76(15)$ \\
& \\
C9-S2-Na1 & $108.63(10)$ \\
C9‥C10-P1 & $171.68(14)$ \\
C10‥C9-S2 & $173.40(16)$ \\
\hline
\end{tabular}


30 kristallisiert in der orthorhombischen, polaren Raumgruppe Pna2 ${ }_{1}$ mit einem Molekül in der asymmetrischen Einheit. Na1 wird neben dem sulfidischen S2 noch von vier THF-Molekülen koordiniert, sodass eine trigonal-bipyramidale Atomanordnung vorliegt. Dieses Koordinationsmuster ist äußerst ungewöhnlich, da sich Alkalimetallarylthiolate bei Anwesenheit einzähniger Liganden meist durch Aggregation stabilisieren. Die Bindungslängen und -winkel um S2 liegen im Bereich derartiger dimerer oder polymerer Verbindungen. ${ }^{[146,147]}$ Der Faltungswinkel des Anthracengerüstes in 30 beträgt $164.9^{\circ}$ und der $\mathrm{Ph}_{2}(\mathrm{~S}) \mathrm{P}$-Substituent weist die gleiche Orientierung auf wie in allen bisher beschriebenen Verbindungen, die diese Thiophosphoryl-Gruppe beinhalten.

\section{3 $\operatorname{Br}\left(\mathrm{C}_{14} \mathrm{H}_{8} \mathrm{O}_{2}\right) \mathrm{C}(\mathrm{OH}) \mathrm{Ph}_{2}(31)$}

Um die Reaktivität von 9-Brom-10-lithiumanthracenid gegenüber unterschliedlichen Elektrophilen zu untersuchen, wurde die Verbindung mit verschiedenen Aldehyden und Ketonen umgesetzt, um nach anschließender Hydrolyse die entsprechenden sekundären bzw. tertiären Alkohole zu erhalten. Gl.6-4 zeigt die geplante Umsetzung von 9-Brom-10-lithiumanthracenid mit Benzophenon, die das dargestellte 9-Anthryldiphenylmethanolderivat liefern sollte.

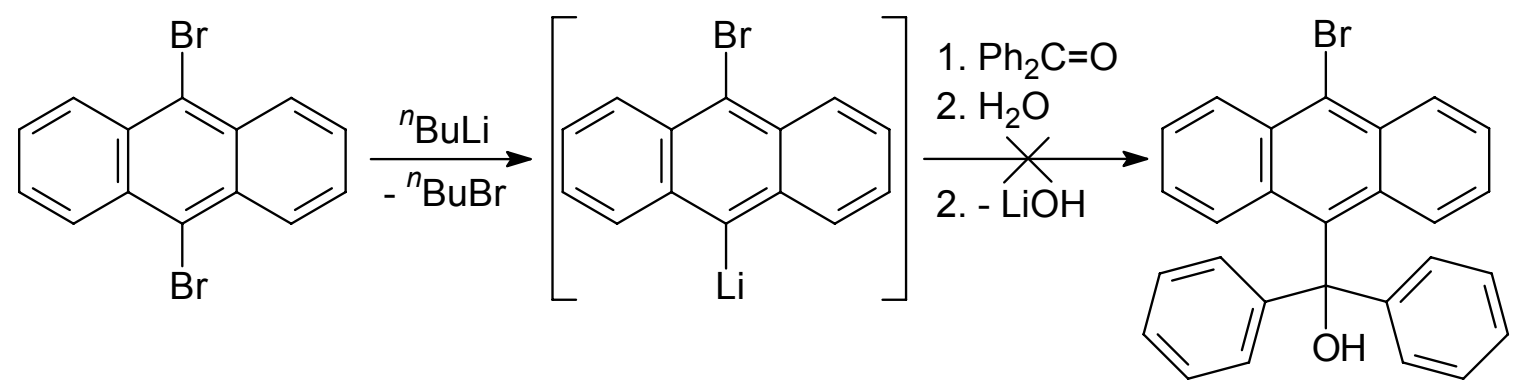

GI. 6-4: Lithiierung von 9,10-Dibromanthracen und in situ geplante Umsetzung mit Benzophenon.

Nach der wässrigen Aufarbeitung wurde das entstandene Produkt mit Diethylether extrahiert und anschließend das Lösungsmittel i. Vak. entfernt. Der verbleibende gelbe Rückstand erwies sich unerwartet als sehr luft- und feuchtigkeitsempfindlich. Durch Lagerung einer gesättigten Lösung in Diethylether wurden nach einigen Tagen Kristalle erhalten, die zur Bestimmung der Molekülstruktur geeignet waren. Abb. 6-3 zeigt das Ergebnis der Röntgenstrukturanalyse, ausgewählte Bindungslängen und -winkel der entstandenen Verbindung sind in Tab. 6-3 aufgeführt. 


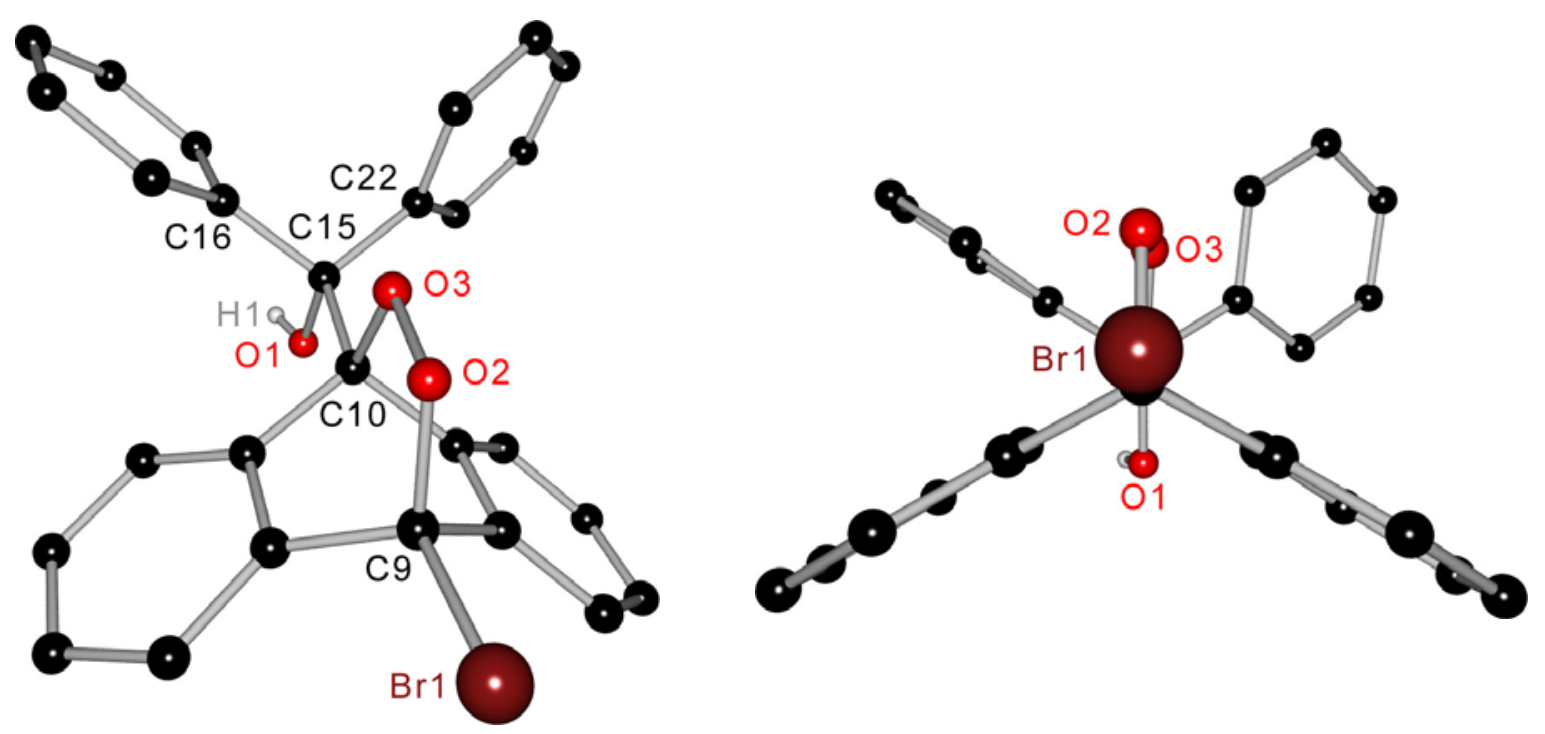

Abb. 6-3: Molekülstruktur von 31 (rechts: Blick entlang C9 ‥C10).

Tab. 6-3: Ausgewählte Bindungslängen / pm und -winkel $/{ }^{\circ}$ in 31.

\begin{tabular}{lllll}
\hline C9-Br1 & $192.85(15)$ & & $\mathrm{Br} 1-\mathrm{C} 9-\mathrm{O} 2$ & $102.20(9)$ \\
$\mathrm{C} 9-\mathrm{O} 2$ & $145.93(19)$ & & $\mathrm{C} 15-\mathrm{C} 10-\mathrm{O} 3$ & $103.53(11)$ \\
$\mathrm{O} 2-\mathrm{O} 3$ & $149.42(15)$ & $\mathrm{C} 9-\mathrm{O} 2-\mathrm{O} 3$ & $109.24(10)$ \\
$\mathrm{C} 10-\mathrm{O} 3$ & $147.76(17)$ & $\mathrm{C} 10-\mathrm{O} 3-\mathrm{O} 2$ & $112.22(10)$ \\
$\mathrm{C} 10-\mathrm{C} 15$ & $156.4(2)$ & $\mathrm{C} 10-\mathrm{C} 15-\mathrm{O} 1$ & $101.74(11)$ \\
$\mathrm{C} 15-\mathrm{O} 1$ & $143.48(17)$ & $\mathrm{C} 16-\mathrm{C} 15-\mathrm{C} 22$ & $106.07(12)$ \\
\hline
\end{tabular}

Die Molekülstruktur zeigt, dass der $\mathrm{Ph}_{2}(\mathrm{OH}) \mathrm{C}$-Substituent wie beabsichtigt in Position 10 eingeführt wurde. Darüber hinaus sind allerdings C9 und $\mathrm{C} 10$ durch ein Sauerstoffmolekül verbrückt; es hat sich das endo-Peroxid $\mathbf{3 1}$ gebildet.<smiles>OC(c1ccccc1)(c1ccccc1)C1(c2ccccc2)OOC2(Br)c3ccccc3C21c1ccccc1</smiles>

31

31 kristallisiert in der monoklinen, zentrosymmetrischen Raumgrupp $\mathrm{P} 2{ }_{1} / \mathrm{c}$ mit einem Molekül in der asymmetrischen Einheit. Durch die $\mathrm{O}_{2}$-Addition sind $\mathrm{C} 9$ und $\mathrm{C} 10$ jetzt $\mathrm{sp}^{3}$-hybridisiert und weisen jeweils eine verzerrt tetraedrische Koordination auf. 
Dadurch ist die Aromatizität des mittleren sechsgliedrigen Ringes aufgehoben und das 9,10-Epidioxyanthracengerüst ist stark gefaltet (Faltungswinkel zwischen den beiden äußeren Phenylenringen: $116.9^{\circ}$ ). Die Bindungslängen und -winkel von 31 stimmen mit den entsprechenden Werten ähnlicher Verbindungen wie 9,10-Dihydro9,10-epidioxyanthracen ${ }^{[148]}$ oder 9,10-Diphenyl-9,10-epidioxyanthracen ${ }^{[149]}$ überein.

Die Sauerstoffbrücke muss durch eine Diels-Alder-Reaktion des Anthracengerüstes mit Singulett-Sauerstoff $\left({ }^{1} \mathrm{O}_{2}\right)$ entstanden sein. ${ }^{[22,150]}$ Derartige Insertionsreaktionen wurden bereits für zahlreiche Acene beschrieben. Die Bildung von SingulettSauerstoff (Termsymbol: ${ }^{1} \Delta_{\mathrm{g}}$ ) ist sowohl chemisch aus anderen Sauerstoffverbindungen als auch photochemisch aus Triplett-Sauerstoff möglich. So kann aus Wasserstoffperoxid mit Hilfe geeigneter Metallkatalysatoren (z. B. $\left.\mathrm{Na}_{2} \mathrm{MoO}_{4}\right){ }^{1} \mathrm{O}_{2}$ freigesetzt werden. ${ }^{[151,152]}$ Eine direkte Gewinnung aus dem Triplett-Sauerstoff der Luft durch Bestrahlung ist aus quantenmechanischen Gründen verboten, es muss zusätzlich ein Photokatalysator vorhanden sein. ${ }^{[153-155]}$

$\mathrm{Da}$ bei oben beschriebener Reaktionsführung die Anwesenheit von $\mathrm{H}_{2} \mathrm{O}_{2}$ ausgeschlossen werden kann, muss der insertierte Sauerstoff aus den nicht entgasten Lösungsmitteln stammen. Allerdings wird es wohl für immer ein Rätsel bleiben, woher die Energie für die Anregung des Triplett-Grundzustandes von $\mathrm{O}_{2}$ kam und welche Verbindung als Photokatalysator reagierte. Möglicherweise fungierte die Zielverbindung selbst als intermolekularer Photokatalysator.

\section{4 $\left[\mathrm{Ph}_{2}(\mathrm{O}) \mathrm{P}\left(\mathrm{C}_{14} \mathrm{H}_{10}\right) \mathrm{P}(\mathrm{OH}) \mathrm{Ph}_{2}\right]^{+}\left[\mathrm{HSO}_{4}\right]^{-}$(32)}

GI. 6-5 zeigt die geplante Oxidation von 15, die das dargestellte SPAnPS-ähnliche Molekül liefern sollte.

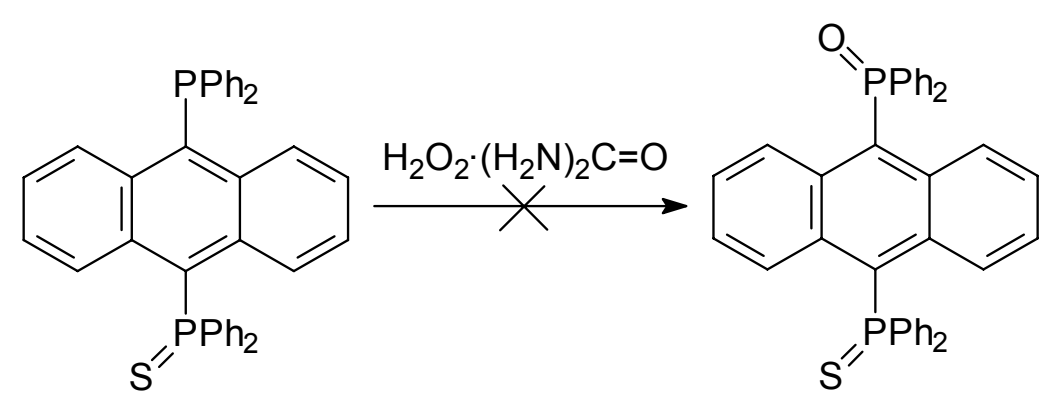

15

GI. 6-5: Geplante Oxidation von 15. 
Analog zu den Synthesen der mit Sauerstoff oxidierten Derivate 2, 6 und 18 wurde dazu eine Lösung von 15 in Dichlormethan mit $\mathrm{H}_{2} \mathrm{O}_{2} \cdot\left(\mathrm{H}_{2} \mathrm{~N}\right)_{2} \mathrm{C}=\mathrm{O}$ versetzt und die Reaktionsmischung nach beendeter Reaktion hydrolysiert und aufgearbeitet. Durch Umkristallisation des erhaltenen Produktes aus Toluol wurden Kristalle erhalten, die zur Bestimmung der Molekülstruktur geeignet waren. Abb. 6-4 zeigt das Ergebnis der Röntgenstrukturanalyse, in Tab. 6-4 sind ausgewählte Bindungslängen und -winkel dargestellt.
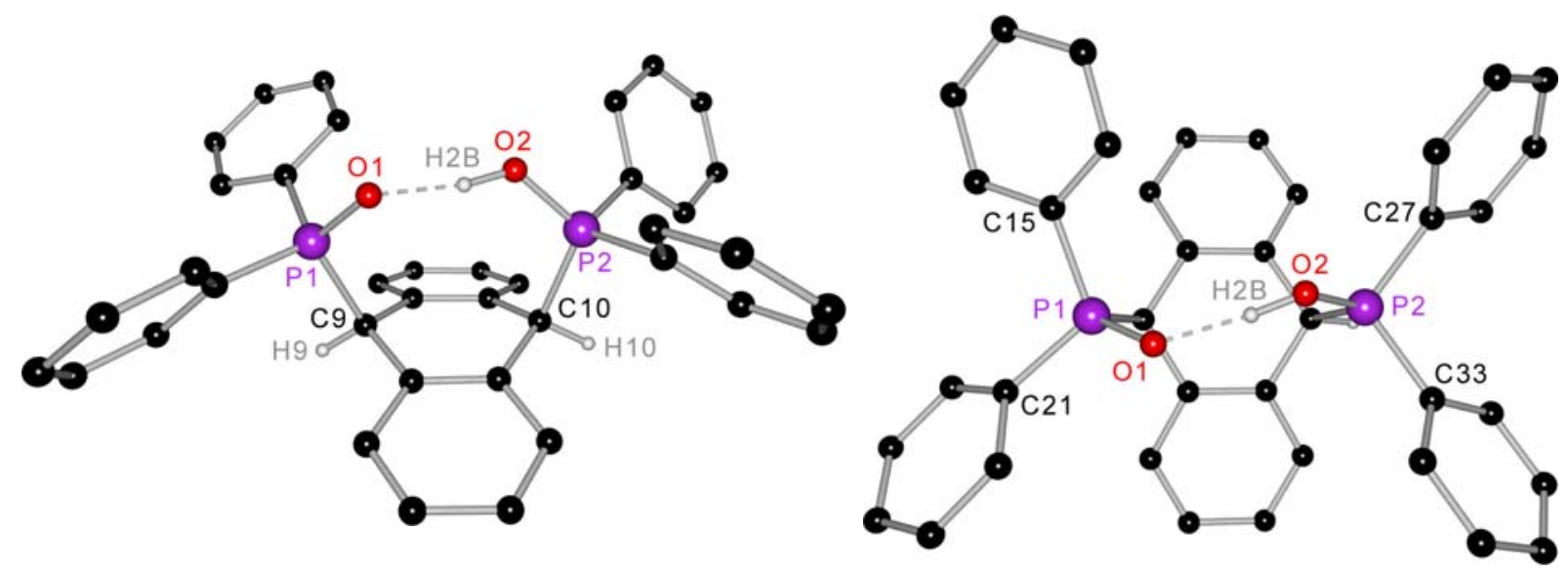

Abb. 6-4: Molekülstruktur von 32 aus zwei unterschiedlichen Ansichten, das Hydrogensulfat-Gegenion ist nicht dargestellt.

Tab. 6-4: Ausgewählte Bindungslängen / pm und -winkel / ${ }^{\circ}$ in 32.

\begin{tabular}{|c|c|}
\hline P1-C9 & $184.9(2)$ \\
\hline P2-C10 & $184.2(2)$ \\
\hline P1-01 & $152.51(16)$ \\
\hline P2-O2 & $151.25(16)$ \\
\hline $\mathrm{O} 2-\mathrm{H} 2 \mathrm{~B}$ & 87.4 \\
\hline $\mathrm{O} 1 \cdots \mathrm{H} 2 \mathrm{~B}$ & 151.9 \\
\hline $\mathrm{O} 1 \cdots \mathrm{O} 2$ & 238.6 \\
\hline
\end{tabular}

\begin{tabular}{ll}
\hline C9-P1-O1 & $111.44(9)$ \\
C10-P2-O2 & $113.14(9)$ \\
P2-O2-H2B & 125.43 \\
P1-C9-H9 & 100.42 \\
P2-C10-H10 & 99.27 \\
C15-P1-C21 & $111.98(10)$ \\
C27-P2-C33 & $107.93(10)$ \\
\hline
\end{tabular}

Die Molekülstruktur zeigt, dass beide Phosphoratome der entstandenen Verbindung mit Sauerstoff oxidiert sind, es ist kein Schwefel im Molekül enthalten. Dies lässt sich nur dadurch erklären, dass das Edukt (15) noch mit nicht-oxidiertem $\left(\mathrm{Ph}_{2} \mathrm{P}\right)_{2}\left(\mathrm{C}_{14} \mathrm{H}_{8}\right)$ (PAnP) verunreinigt war, welches mit dem $\mathrm{H}_{2} \mathrm{O}_{2}$-Derivat unter Bildung von Verbindung 32 reagierte. Überraschenderweise fand zusätzlich am AnthracenKohlenstoffgerüst eine Reaktion statt, bei dem gebildeten Produkt handelt es sich um ein 9,10-Dihydroanthracen. Desweiteren wurde eine $\mathrm{P}=\mathrm{O}$-Bindung protoniert und die 
Verbindung trägt insgesamt eine positive Ladung. Möglicherweise war an der Bildung ein Phosphadioxiran als Zwischenstufe beteiligt, das durch eine Umlagerungsreaktion schließlich 32 lieferte. $^{[156]}$

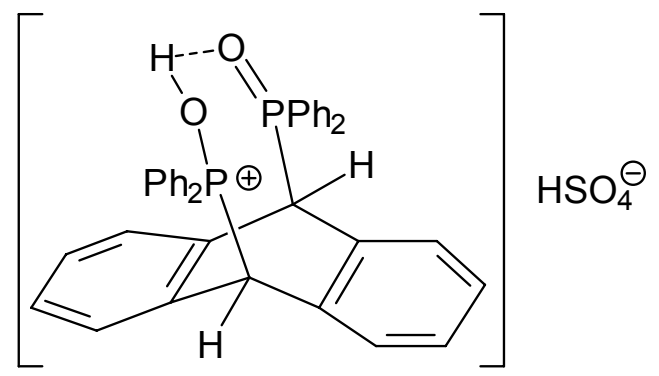

32

32 kristallisiert in der monoklinen, zentrosymmetrischen Raumgruppe $P 2_{1} / c$. Die asymmetrische Einheit enthält neben einem Molekül Dihydroanthracen noch ein Hydrogensulfatanion als Gegenion. Dieses wurde in die Reaktionsmischung gebracht, als die etherische Phase mit Magnesiumsulfat getrocknet wurde. Das Anthracengerüst ist stark abgewinkelt, der Faltungswinkel zwischen den beiden äußeren Phenylenringen beträgt $150.9^{\circ}$. Das Wasserstoffatom H1B ist durch Wasserstoffbrückenbindungen an beide Sauerstoffatome gebunden. Sämtliche P-Ound P-C-Bindungslängen entsprechen denen der bisher diskutierten Molekülstrukturen. Die Bindungslängen und -winkel des Dihydroanthracengerüstes stimmen mit denen vergleichbarer Verbindungen überein. ${ }^{[157-159]}$ 


\section{ZUSAMMENFASSUNG UND AUSBLICK}

Im Rahmen dieser Dissertation wurden im Wesentlichen vier Ziele verfolgt:

1. Synthese und Charakterisierung von 9-Brom-10-phosphanylanthracenen

2. Untersuchung des Zusammenhangs zwischen Kristallstruktur und Fluoreszenzverhalten von SPAnPS/Gast-Komplexen

3. Möglichkeiten zur Lithiierung von 9-Brom-10-phosphanylanthracenen und nachfolgende Umsetzung mit Chlorphosphanen

4. Darstellung von phosphanylsubstituierten Anthracenderivaten, die außerdem über Stickstoffatome als potentielle Donoren für Metall-Komplexe verfügen

\subsection{9-Brom-10-phosphanylanthracene}

Im Rahmen der dieser Arbeit vorangegangenen Diplomarbeit wurde bereits das 9-Brom-10-phosphanylanthracen 1 dargestellt und strukturell charakterisiert. ${ }^{[113]}$ Da es sich hierbei um ein potentielles Edukt für die Synthese von asymmetrisch 9,10-substituierten Anthracenderivaten handelt, die interessante Fluoreszenzeigenschaften aufweisen könnten, wurde die Verbindung im Verlauf dieser Dissertation intensiver untersucht. So konnten die Chalkogen-Oxidationsprodukte 2 - 4 in hohen Ausbeuten dargestellt und charakterisiert werden. ${ }^{[114]}$

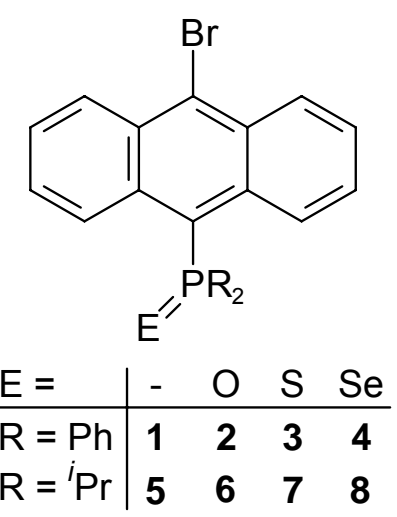

Abb. 7-1: 9-Brom-10phosphanylanthracene.

Die Molekülstrukturen von 2 - 4 entsprechen denen der von Stalke et al. beschriebenen symmetrisch 9,10-substituierten Diphosphanylanaloga. ${ }^{[112]}$ Allerdings kristallisieren 2 - 4 nicht als Wirt/Gast-Komplexe mit Lösungsmittelmolekülen im Kristallgitter und das mit Schwefel oxidierte Derivat 3 zeigt keine Fluoreszenz im Festkörper.

Die Synthese von 1 erfolgte durch Monolithiierung von 9,10-Dibromanthracen und nachfolgende Umsetzung mit Chlordiphenylphosphan. Um die Übertragbarkeit der Reaktion auf andere phosphorhaltige Elektrophile zu überprüfen, wurde Chlordiisopropylphosphan eingesetzt und das entsprechende 9-Brom-10-(diisopropylphosphanyl)anthracen (5) dargestellt. Durch Oxidation mit $\mathrm{H}_{2} \mathrm{O}_{2} \cdot\left(\mathrm{H}_{2} \mathrm{~N}\right)_{2} \mathrm{C}=\mathrm{O}$, elemetarem Schwefel bzw. grauem Selen wurden außerdem die entsprechenden Oxidationsprodukte 6 - 8 erhalten (Abb. 7-1). 


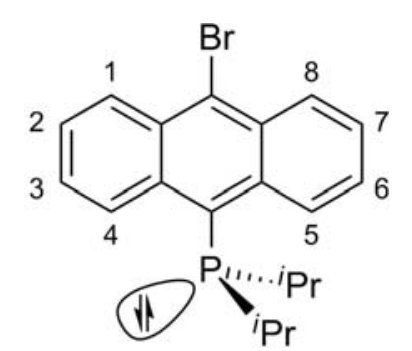

Abb. 7-2: Konformation von 5 bei $243 \mathrm{~K}$.

Das ${ }^{1} \mathrm{H}-\mathrm{NMR}$-Spektrum von $\mathbf{5}$ zeigt bei Raumtemperatur eine deutlich Verbreiterung der Signale im Arylbereich, was auf ein dynamisches Verhalten hindeutet. Durch Messungen von NMR-Spektren bei unterschiedlichen Temperaturen konnte gezeigt werden, dass das Molekül bei tiefen Temperaturen die in Abb. 7-2 gezeigte Konformation einnimmt, die auch bei der Bestimmung der Molekülstruktur gefunden wurde.

\subsection{Wirt/Gast-Komplexe von $\left(\mathrm{Ph}_{2}(\mathrm{~S}) \mathrm{P}\right)_{2}\left(\mathrm{C}_{14} \mathrm{H}_{8}\right)$}

Stalke et al. berichteten 2003 über die Synthese von 9,10-Bis(diphenylthiophosphoryl)anthracen (SPAnPS), das sich durch eine intensive Festkörperfluoreszenz auszeichnet. Die Röntgenstrukturanalyse ergab, dass die Verbindung in Form eines Wirt/Gast-Komplexes vorliegt, wobei jedes Wirtsmolekül von zwei Toluolmolekülen umgeben ist. Durch Entfernen der Lösungsmittelmoleküle i. Vak. bei etwa $100^{\circ} \mathrm{C}$ geht auch die Fähigkeit zur Festkörperfluoreszenz verloren. ${ }^{[112]}$<smiles>S=Pc1c2ccccc2c([PH](=S)c2ccccc2)c2ccccc12</smiles>

Abb. 7-3: SPAnPS In der vorangegangenen Diplomarbeit wurde beschrieben, dass SPAnPS noch mit einer Vielzahl anderer aromatischen Lösungsmitteln sowohl im Festkörper fluoreszierende als auch nicht-fluoreszierende Wirt/Gast-Komplexe ausbildet. ${ }^{[13]}$ Allen fluoreszierenden Verbindungen ist gemeinsam, dass die beiden $\mathrm{P}=\mathrm{S}-$ Bindungen transoid angeordnet sind und dass die Komplexe isostrukturell in der monoklinen Raumgruppe $P 2_{1} / n$ mit jeweils zwei Lösungsmittel-Gastmolekülen pro Molekül SPAnPS kristallisieren. Dabei sind die Anthracengerüste zickzack-artig in zwei unterschiedlichen Ebenen angeordnet, die einen Winkel von $70.5^{\circ}$ miteinander einschließen. Bei den nicht-fluoreszierenden Wirt/Gast-Komplexen sind dagegen die beiden $\mathrm{P}=\mathrm{S}$-Bindungen cisoid auf einer Seite des Anthracengerüstes angeordnet und das Verhältnis Wirt: Gast beträgt 2:1 (Gäste: Anisol, Benzylalkohol) bzw. 2:3 (Benzonitril).

Im Verlauf dieser Dissertation konnten weitere SPAnPS/Gast-Komplexe mit den Gästen Benzol (10), Phenylacetylen (11) sowie Deuterochloroform (12) erhalten und röntgenkristallographisch untersucht werden. Dabei zeigen Kristalle von $\mathbf{1 0}$ und $\mathbf{1 1}$ eine intensive Festkörperfluoreszenz, während sich 12 als nicht-fluoreszierend 
erwies. In Abb. 7-4 sind die Molekülstrukturen von 10 und 12 dargestellt, die Konformation des SPAnPS-Wirtsmoleküls in $\mathbf{1 1}$ sowie die Anordnung der aromatischen Gäste entsprechen weitestgehend der Struktur von 10.
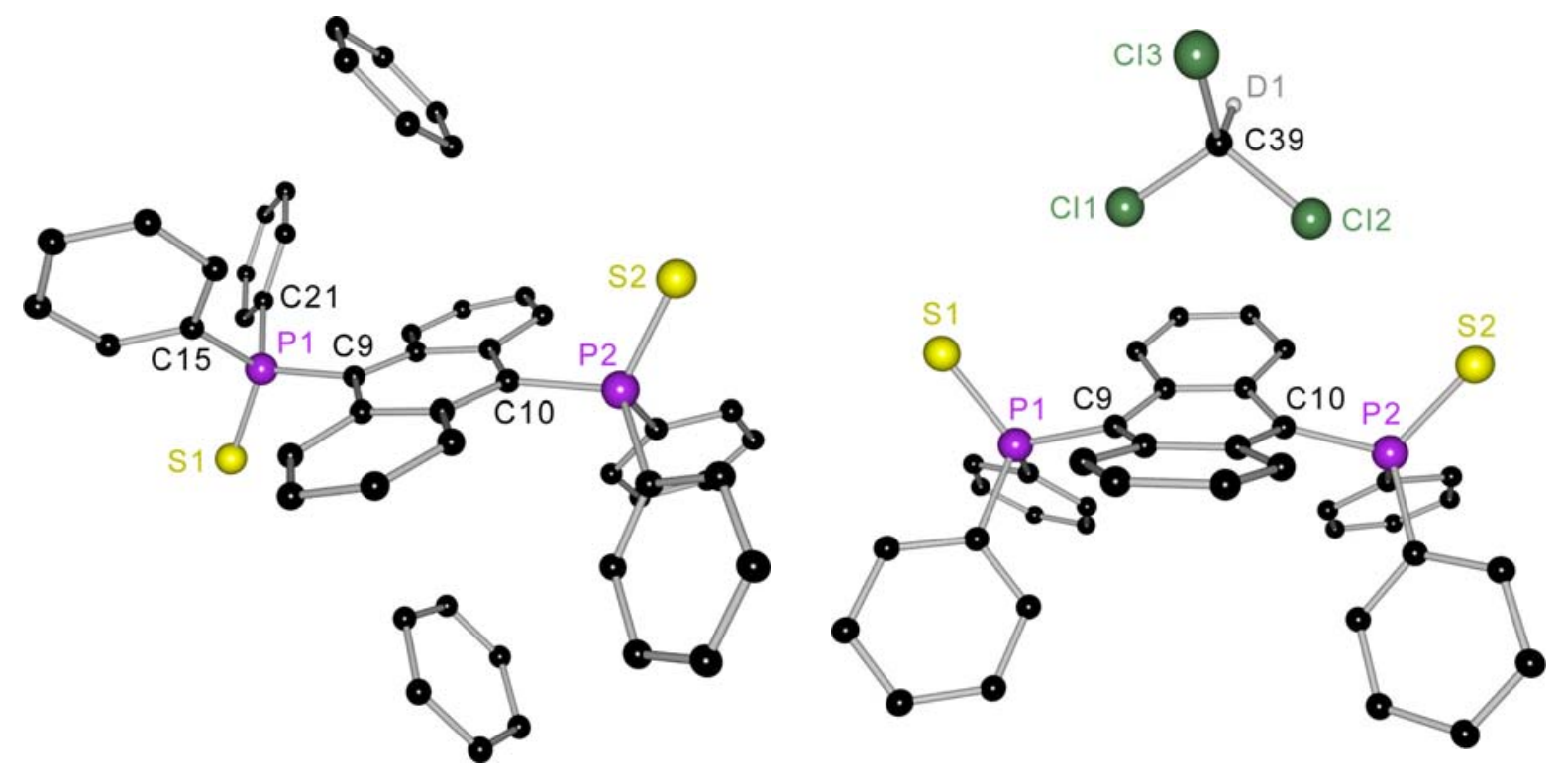

Abb. 7-4: Molekülstrukturen von [2 $\mathrm{C}_{6} \mathrm{H}_{6} @$ SPAnPS] (10, links) und [3 $\mathrm{CDCl}_{3} @$ SPAnPS] (12, rechts).

Wie bei allen bisher untersuchten fluoreszierenden SPAnPS/Gast-Komplexen weisen die $\mathrm{P}=\mathrm{S}$-Bindungen in $\mathbf{1 0}$ und $\mathbf{1 1}$ eine transoide Orientierung auf und das Wirt : GastVerhältnis beträgt $1: 2$. Allerdings kristallisieren die beiden neuen Verbindungen in der triklinen Raumgruppe $P \overline{1}$ und alle Anthracengerüste sind in koplanaren Ebenen angeordnet. Somit kann die isostrukturelle Anordnung der Moleküle im Festkörper als Voraussetzung für die Festkörperfluoreszenz ausgeschlossen werden. Da als Gäste sowohl polare (z. B. Halogenbenzole) als auch unpolare Aromaten (Benzol) beobachtet wurden und deren relative Orientierungen zum Wirtsmolekül deutlich variieren, deutet alles darauf hin, dass ausschließlich die transoide Anordnung der $\mathrm{P}=\mathrm{S}$-Bindungen sowie die Verdrillung des Anthracengrundgerüstes für die Lumineszenz im Festkörper verantwortlich sind.

Die beiden $\mathrm{P}=\mathrm{S}$-Bindungen des nicht-fluoreszierenden SPAnPS/CDCl $3-$ Komplexes 12 sind bezüglich des Anthracengerüstes cisoid orientiert und die beiden äußeren sechsgliedrigen Ringe schließen einen Faltungswinkel von $149.2^{\circ}$ ein. Eines der drei Lösungsmittelmoleküle befindet sich in räumlicher Nähe des Fluorophors, die beiden anderen sind weiter entfernt im Kristallgitter angeordnet. 
Um den Einfluss der Phenylsubstituenten auf das Fluoreszenzverhalten zu untersuchen, wurden das Bis(diisopropylphosphanyl)substituierte Anthracenderivat 13 sowie das entsprechende Oxidationsprodukt mit Schwefel (14) synthetisiert. Durch Messungen von NMR-Spektren bei unterschiedlichen Temperaturen konnte gezeigt werden, dass 13 bei tiefen Temperaturen die in Abb. 7-5 gezeigte Konformation einnimmt, die auch bei der Bestimmung der

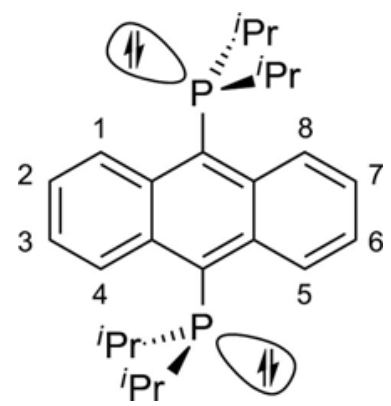

Abb. 7-5: Konformation von 13 bei $183 \mathrm{~K}$. Molekülstruktur gefunden wurde.

Im Gegensatz zum Phenylanalogon SPAnPS kristallisiert 14 nicht als Wirt/GastKomplex mit Lösungsmittelmolekülen im Kristallgitter und zeigt auch keinerlei Festkörperfluoreszenz.

\subsection{Asymmetrische 9,10-Diphosphanylanthracene}

Um Möglichkeiten zur Lithiierung von 10-phosphanylsubstituierten 9-Bromanthracenen zu untersuchen, wurden die in Abb.7-6 dargestellten asymmetrisch substituierten 9,10-Diphosphanylanthracene 15 - 22 synthetisiert.<smiles>[R2]P=[R7]c1c2ccccc2c(P=[R2])c2ccccc12</smiles>

\begin{tabular}{l|cccccccc} 
& $\mathbf{1 5}$ & $\mathbf{1 6}$ & $\mathbf{1 7}$ & $\mathbf{1 8}$ & $\mathbf{1 9}$ & $\mathbf{2 0}$ & $\mathbf{2 1}$ & $\mathbf{2 2}$ \\
\hline $\mathrm{R}$ & $\mathrm{Ph}$ & $\mathrm{Ph}$ & $\mathrm{Ph}$ & $\mathrm{Ph}$ & $\mathrm{Ph}$ & $\mathrm{Ph}$ & $\mathrm{Ph}$ & $\mathrm{Ph}$ \\
$\mathrm{R}^{\prime}$ & $\mathrm{Ph}$ & $\mathrm{Ph}$ & ${ }^{i} \mathrm{Pr}$ & ${ }^{i} \mathrm{Pr}$ & ${ }^{i} \mathrm{Pr}$ & ${ }^{i} \mathrm{Pr}$ & $\mathrm{Cy}$ & $\mathrm{Cy}$ \\
$\mathrm{E}$ & $\mathrm{S}$ & $\mathrm{Se}$ & - & $\mathrm{O}$ & $\mathrm{S}$ & $\mathrm{Se}$ & - & $\mathrm{S}$ \\
$\mathrm{E}^{\prime}$ & - & $\mathrm{S}$ & - & $\mathrm{O}$ & $\mathrm{S}$ & $\mathrm{Se}$ & - & $\mathrm{S}$
\end{tabular}

Abb. 7-6: Asymmetrisch substituierte 9,10-Diphosphanylanthracene.

Dabei erfolgte die Darstellung von $\mathbf{1 5}$ und $\mathbf{1 6}$ durch Lithiierung der mit Schwefel oxidierten Verbindung $\mathrm{Br}\left(\mathrm{C}_{14} \mathrm{H}_{8}\right) \mathrm{P}(\mathrm{S}) \mathrm{Ph}_{2}$ (3) und anschließende Umsetzung mit Chlordiphenylphosphan, während $\mathbf{1 7}$ - 22 ausgehend von $\operatorname{Br}\left(\mathrm{C}_{14} \mathrm{H}_{8}\right) \mathrm{PPh}_{2}(\mathbf{1})$ erhalten wurden. Allerdings erwies sich die Lithiierung von 3 mit ${ }^{n} \mathrm{BuLi}$ als problematisch, da 
die Reaktion weder in Diethylether noch in THF quantitativ erfolgt. Die Lithiierung von 1 verläuft deutlich selektiver als die von $\mathbf{3}$ und ist für die Synthese von asymmetrisch substituierten 9,10-Diphosphanylanthracenen besser geeignet.

\subsection{Stickstoff-funktionalisierte Phosphanylanthracene}

Der Einbau eines Stickstoffatoms in ein Phosphanylanthracen ist von besonderem Interesse, da hierdurch neben einem eher weichen $(P)$ noch ein hartes Donoratom (N) zur Komplexierung von Metallen zur Verfügung steht. Im Verlauf dieser Doktorarbeit wurden die in Abb.7-7 dargestellten Stickstoff-funktionalisierten Phosphanylanthracene synthetisiert und charakterisiert.<smiles>[R]Nc1c2ccccc2c([PH]([PH])=S)c2ccccc12</smiles>

23: $R=L i(\text { thf })_{3}$

24: $\mathrm{R}=\mathrm{H}$<smiles>[R]c1c2ccccc2c(P([R12])N([R2])[R12])c2ccccc12</smiles>

25: $\mathrm{R}={ }^{i} \mathrm{Pr}, \mathrm{R}^{\prime}=\mathrm{Br}$ 26: $R=E t, R^{\prime}=H$<smiles>P#P([Pb])c1c2ccccc2cc2ccccc12</smiles>

28

Abb. 7-7: Stickstoff-funktionalisierte Phosphanylanthracene.

Das Lithiumamid 23 wurde durch Umsetzung von $\operatorname{Br}\left(\mathrm{C}_{14} \mathrm{H}_{8}\right) \mathrm{P}(\mathrm{S}) \mathrm{Ph}_{2}$ (3) mit einem sechsfachen Überschuss $\mathrm{LiNH}_{2}$ in THF erhalten. In zukünftigen Untersuchungen sollte versucht werden, die lange Reaktionszeit von $9 \mathrm{~d}$ zu verkürzen. Dies könnte beispielsweise durch die Verwendung von flüssigem Ammoniak als Lösungsmittel oder durch Zugabe eines BINAP-Pd-Katalysators erfolgen (vgl. Kap. 1). Ist ein alternativer Syntheseweg gefunden, könnten durch anschließende Ummetallierungsreaktionen verschiedene Metall-Amid-Komplexe erhalten werden.

Die beiden Bis(dialkylamino)phosphanylanthracene 25 und 26 wurden durch Umsetzungen der entsprechenden Lithiumanthracenide mit $\left(R_{2} N\right)_{2} P C l$ erhalten. Durch Reaktion von 26 mit $\mathrm{HCl}-\mathrm{Gas}$ erfolgte unter Ammoniumchlorid-Abspaltung eine Chlorierung der beiden $\mathrm{P}-\mathrm{N}$-Bindungen unter Bildung von $\mathrm{Cl}_{2} \mathrm{P}\left(\mathrm{C}_{14} \mathrm{H}_{9}\right)$ (27), das ansonsten nur schwierig quantitativ zugänglich ist. Schließlich konnte durch 
Umsetzung von 27 mit lithiiertem 2-Brompyridin 9-[Di(2-pyridyl)phosphanyl]anthracen (28) dargestellt und röntgenkristallographisch untersucht werden.

Ein Ziel zukünftiger Studien sollte es sein, das Koordinationsverhalten der Stickstofffunktionalisierten Phosphanylanthracene 25, 26 und 28 in Metall-Komplexen zu untersuchen. Erst kürzlich berichteten Dyer und Kemmitt über die Koordinationschemie von Dialkylamino- und Bis(dialkylamino)phosphanen sowie deren AlkoxyAnaloga, die durch saure Umsetzung der Aminophosphane in alkoholischen Lösungen zugänglich sind. ${ }^{[160]}$

Besonders vielversprechend und interessant erscheint die Synthese von homosowie bimetallischen Koordinationsverbindungen des pyridylsubstituierten Phosphanylanthracens 28. In Abb. 7-8 - Abb. 7-10 sind vergleichbare d-Metall-Komplexe aus der Arbeitsgruppe Espinet dargestellt, die die unterschiedlichen Koordinationsmodi dieser Ligandklasse zeigen.

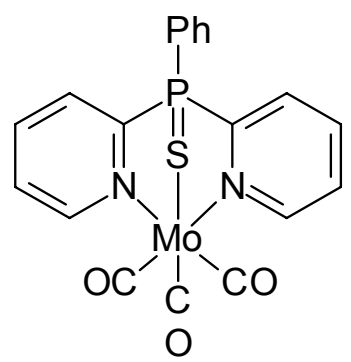

Abb. 7-8: $\mathrm{Mo}(\mathrm{CO})_{3}$-Komplex von Espinet et al. ${ }^{[161]}$<smiles></smiles>

Abb. 7-9: $\mathrm{PdBr}\left(\mathrm{C}_{6} \mathrm{~F}_{5}\right)-$ Komplex von Espinet et al. ${ }^{[162]}$<smiles></smiles>

Abb. 7-10: Januskopf-Komplex von Espinet et al..$^{[163]}$

\subsection{Substituentenhängigkeit der Struktur des Anthracen- gerüstes}

Die in dieser Arbeit beschriebenen Molekülstrukturen lassen sich abhängig vom Aufbau ihrer Anthracengrundgerüste in drei Kategorien unterteilen:

- Verbindungen mit Anthracengerüst in Twistkonformation

- Verbindungen mit planarem Anthracengerüst

- Verbindungen mit gefaltetem Anthracengerüst 


\section{a) Twistform des Anthracengerüstes}

Bei dieser Form sind die beiden äußeren sechsgliedrigen Ringe des Anthracengerüstes koplanar orientiert und die entsprechenden Kohlenstoffatome befinden sich mit einer mittleren Abweichung von $4.54 \mathrm{pm}$ in einer Ebene. C9 und C10 sind deutlich außerhalb der idealisierten Ebene durch die übrigen Kohlenstoffatome angeordnet (siehe Abb. 7-11). Darüber hinaus weist die Twistform im Zentrum des mittleren Ringes ein Inversionszentrum auf.
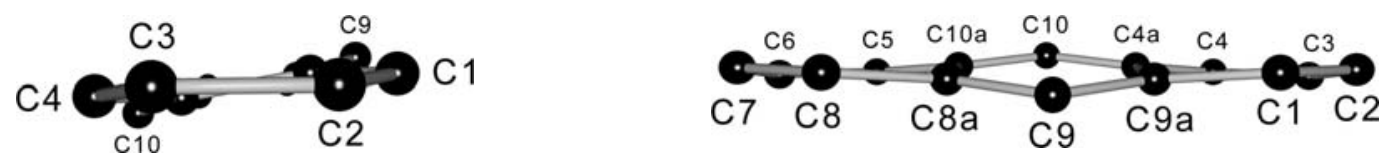

Abb. 7-11: Twistform des Anthracengerüstes.

Die Twistform tritt immer dann auf, wenn das Anthracengerüst mit zwei $\mathrm{Ph}_{2}(\mathrm{E}) \mathrm{P}$ Gruppen $(\mathrm{E}=\mathrm{S}$ oder $\mathrm{Se}$ ) substituiert ist und die beiden $\mathrm{P}=\mathrm{E}-$-Bindungen transoid angeordnet sind. Diese Voraussetzung erfüllen die fluoreszierenden Wirt/GastKomplexe von $\left(\mathrm{Ph}_{2}(\mathrm{~S}) \mathrm{P}\right)_{2}\left(\mathrm{C}_{14} \mathrm{H}_{8}\right)(\mathbf{9}$ - 11) sowie die Toluol-Einschlussverbindung des 9,10-asymmetrisch substituierten $\mathrm{Ph}_{2}(\mathrm{Se}) \mathrm{P}\left(\mathrm{C}_{14} \mathrm{H}_{8}\right) \mathrm{P}(\mathrm{S}) \mathrm{Ph}_{2}$ (16). Der out-of-planeAbstand von C9 und C10 liegt im Bereich von 20.62 (11) und 28.23 pm (9), die beiden Phosphoratome sind zwischen 64.85 (10) und $79.74 \mathrm{pm}$ (9) von den idealisierten Ebenen durch C1 - C4 und C5 - C8 entfernt. Die C9-C8a- und C9-C9aBindungslängen betragen 141.3 bzw. 141.4 pm.

Die Twistform scheint zusammen mit zwei transoid angeordneten $\mathrm{P}=\mathrm{S}$-Bindungen eine unabdingbare Voraussetzung für das Auftreten von Festkörperfluoreszenz im sichtbaren Bereich zu sein.

\section{b) Planare und gefaltete Anthracengerüste}

Beim unsubstituierten Anthracen befinden sich alle Kohlenstoffatome in einer Ebene, die C9-C8a- und C9-C9a-Bindungslängen betragen 140.2 bzw. 141.1 pm. ${ }^{[118]}$ Wie in Abb. 7-12 dargestellt führt die Faltung des Anthracengerüstes entlang der C9 ‥C10Verbindungslinie dazu, dass der Faltungwinkel $\varphi$ kleiner als $180^{\circ}$ wird.

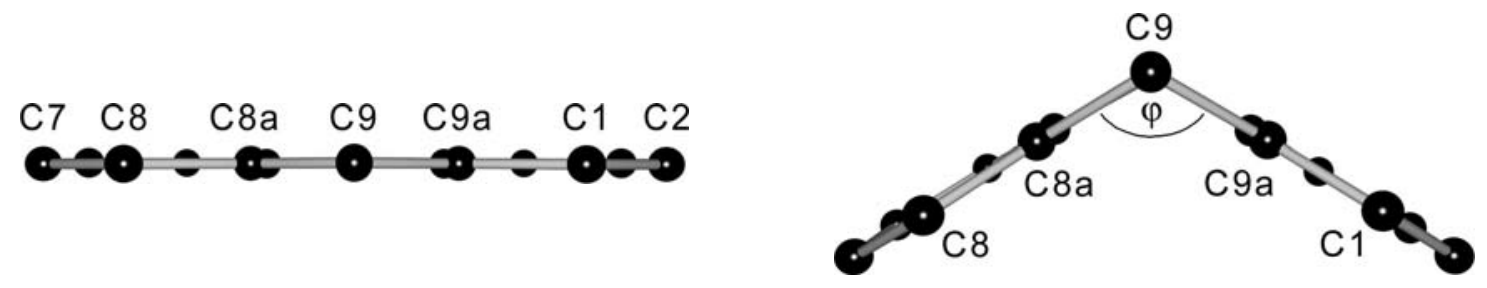

Abb. 7-12: Planares (links) und gefaltetes Anthracengerüst (rechts). 
Den Extremfall eines gefalteten Anthracengerüstes stellt das Epidioxyanthracen 31 $\operatorname{dar}\left(\varphi=116.9^{\circ}\right)$. Durch die $\mathrm{O}_{2}$-Insertion ist $\mathrm{C} 9 \mathrm{sp}^{3}$-hybridisiert, was sich auch in einer Verlängerung der Bindungen zu C8a und C9a auf 153 pm widerspiegelt.

Die restlichen in dieser Arbeit beschriebenen Verbindungen liegen zwischen den beiden Grenzfällen des unsubstituierten Anthracens und des Epidioxyanthracens 31. Dabei lassen sich abhängig vom Substitutionsmuster folgende Trends feststellen:

- Faltungswinkel $\varphi$ zwischen 180 und $175^{\circ}$ :

Ein derartiges fast planares Grundgerüst liegt immer dann vor, wenn die Phosphoratome der Phosphanylsubstituenten nicht oxidiert sind, Phosphor also die Oxidationsstufe +III aufweist $(1,5,13,17,25,28)$. Dabei kann das Anthracengerüst in den Positionen 9 und 10 sowohl symmetrisch als auch asymmetrisch substituiert sein. Beispiele sind $\operatorname{Br}\left(\mathrm{C}_{14} \mathrm{H}_{8}\right) \mathrm{PPh}_{2}(\mathbf{1})$ und $\left({ }^{i} \mathrm{Pr}_{2} \mathrm{P}\right)_{2}\left(\mathrm{C}_{14} \mathrm{H}_{8}\right)(\mathbf{1 3})$ mit Faltungswinkeln von 178.3 bzw. $180^{\circ}$. Die out-of-plane-Abstände der Phosphoratome sind bei diesem Strukturmotiv mit Werten zwischen 0.16 (13) und $14.7 \mathrm{pm}$ (25) sehr klein.

Die einzige Ausnahme stellt $\mathrm{Ph}_{2} \mathrm{P}\left(\mathrm{C}_{14} \mathrm{H}_{8}\right) \mathrm{PCy}_{2}$ (21) dar, bei dem $\varphi$ auf $169.4^{\circ}$ verkleinert ist und sich eines der beiden Phosphoratome 29.08 pm von der Anthracenebene entfernt befindet. Diese Abweichung beruht vermutlich auf dem Vorliegen von Besetzungsfehlordnungen der Phenyl- und Cyclohexylgruppen sowie des Anthracengerüstes, die in der Strukturverfeinerung nur unzureichend beschrieben werden konnten.

- Faltungswinkel $\varphi$ zwischen 172 und $162^{\circ}$ :

Die Verbindungen 2 - 4, 7, 8, 15, 23, 24, 29 und 30 weisen jeweils einen oxidierten Phosphorylsubstituenten pro Anthracengerüst auf, was eine Verkleinerung von $\varphi$ mit sich bringt. So liegen die Faltungswinkel der entsprechenden Molekülstrukturen zwischen $172^{\circ}$ in $\mathrm{Ph}_{2}(\mathrm{~S}) \mathrm{P}\left(\mathrm{C}_{14} \mathrm{H}_{8}\right) \mathrm{NH}_{2}$ (24) und $162^{\circ}$ in $\mathrm{S}\left\{\left(\mathrm{C}_{14} \mathrm{H}_{8}\right) \mathrm{P}(\mathrm{S}) \mathrm{Ph}_{2}\right\}_{2}$ (29). Die Abstände der Phosphoratome von den idealisierten Anthracenebenen sind dabei erheblich von den Substituenten abhängig: So schwanken die Werte für $\mathrm{Ph}_{2}(\mathrm{E}) \mathrm{P}$ Gruppen zwischen 25.3 (24) und 64.7 pm (29), bei Isopropylsubstituenten werden Abstände bis 93.3 pm (8) erreicht.

- Faltungswinkel $\varphi$ zwischen 159 und $149^{\circ}$ :

Die Substitution des Anthracengerüstes mit zwei oxidierten Phosphorylgruppen in den Positionen 9 und 10 (12, 14, 18, 19, 20 und 22) bewirkt eine weitere Verklei- 
nerung des Faltungswinkels. So liegt $\varphi$ in den entsprechenden Molekülstrukturen im Bereich zwischen $159.3^{\circ}$ bei $\mathrm{Ph}_{2}(\mathrm{O}) \mathrm{P}\left(\mathrm{C}_{14} \mathrm{H}_{8}\right) \mathrm{P}(\mathrm{O})^{i} \mathrm{Pr}_{2}$ (18) und $149.2^{\circ}$ bei $\left[3 \mathrm{CDCl}_{3} @\left\{\left(\mathrm{Ph}_{2}(\mathrm{~S}) \mathrm{P}\right)_{2}\left(\mathrm{C}_{14} \mathrm{H}_{8}\right)\right\}\right](12)$.

Während die C9-C8a- und C9-C9a-Bindungslängen bei allen Verbindungen mit Ausnahme des Epidioxyanthracens 31 zwischen 140 und 143 pm liegen, zeigt sich bei $\left[\mathrm{Ph}_{2}(\mathrm{O}) \mathrm{P}\left(\mathrm{C}_{14} \mathrm{H}_{10}\right)\left\{(\mathrm{HO}) \mathrm{PPh}_{2}\right\}\right]^{+}$(32) die $\mathrm{sp}^{3}$-Hybridisierung von $\mathrm{C} 9$ und $\mathrm{C} 10$ in einer Verlängerung der C9-C8a-, C9-C9a-, C10-C4a- und C10-C10a-Bindungen auf durchschnittlich 151.8 pm. Der Faltungswinkel $\varphi$ beträgt hier $150.9^{\circ}$.

Zusammenfassend lässt sich festhalten, dass durch die Ausbildung der Twistform eine geringe Störung der Konjugation im Anthracen-m-Gerüst erfolgt. Diese führt dazu, dass die Festkörperfluoreszenz in den sichtbaren Bereich verschoben wird. Zusätzlich müssen dazu allerdings $z$ wei $\mathrm{Ph}_{2}(\mathrm{~S}) \mathrm{P}$-Substituenten mit transoid angeordneten $\mathrm{P}=\mathrm{S}$-Bindungen vorhanden sein.

Die Abweichung von der idealen Planarität in Form einer Faltung der beiden äußeren

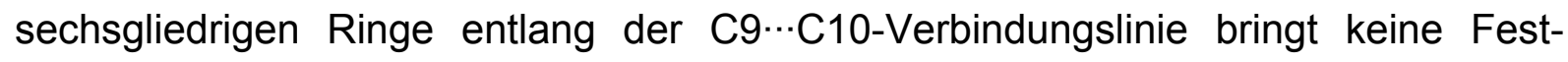
körperlumineszenz im sichtbaren Bereich mit sich. Wenn die Aromatizität des mittleren sechsgliedrigen Ringes des Anthracengerüstes durch $\mathrm{sp}^{3}$-Hybridisierung von $\mathrm{C} 9$ und $\mathrm{C} 10$ zerstört wird, verringert sich der Faltungswinkel $\varphi$ auf bis zu $116.9^{\circ}$ und die C9-C8a- und C9-C9a-Bindungen verlängern sich auf bis zu 153 pm. 


\section{EXPERIMENTELLER TEIL}

\subsection{Allgemeine Arbeitstechniken und Analytische Methoden}

Alle Umsetzungen wurden unter Luft- und Feuchtigkeitsausschluss in trockener, nachgereinigter Stickstoffatmosphäre durchgeführt. Die Glasgeräte wurden vor Gebrauch mehrere Stunden bei $120^{\circ} \mathrm{C}$ ausgeheizt, heiß zusammengesetzt und unter Hochvakuum abgekühlt. Luft- und feuchtigkeitsempfindliche Substanzen wurden mittels Schlenk-Technik ${ }^{[164-166]}$ oder in einer Argontrockenbox gehandhabt. Die Lösungsmittel wurden über Natrium-Kalium-Legierung ( $\mathrm{Et}_{2} \mathrm{O}, n$-Pentan), Kalium (THF, $n$-Hexan) oder Natrium (Toluol) getrocknet und frisch destilliert eingesetzt. Die verwendeten Reagenzien waren käuflich erhältlich oder wurden gemäß publizierter Synthesen hergestellt $\left(\left[\left(\mathrm{Et}_{2} \mathrm{~N}\right)_{2} \mathrm{PCl}\right]^{[167]}\right)$.

\subsubsection{Schmelz- und Zersetzungspunkte}

Die Schmelz- und Zersetzungspunkte wurden mit einem Büchi Melting Point B-540 in abgeschmolzenen Glaskapillaren $\left(\varnothing=1.4 \mathrm{~mm}\right.$ ) mit Heizraten von $5{ }^{\circ} \mathrm{C} / \mathrm{min}$ bestimmt.

\subsubsection{Massenspektrometrie}

Die Massenspektren wurden mittels Elektronenstoßionisation (El-MS: $70 \mathrm{eV}$ ) mit einem Finnigan MAT 95 Spektrometer aufgenommen. Den $\mathrm{m} / \mathrm{z}$-Werten der Molekülionen und den angegebenen Fragmentionen liegen jeweils die Massenzahlen der Isotope mit den größten natürlichen Häufigkeiten zugrunde.

\subsubsection{NMR-Spektroskopie}

Die Aufnahme der Spektren erfolgte mit den Bruker Avance Spektrometern DPX 300 und DRX 500, die Messfrequenzen sind bei den jeweiligen Spektren angegeben. Wenn nicht anders angegeben wurden die Messungen bei Raumtemperatur mit 5-10\%igen Lösungen durchgeführt. 
Die chemischen Verschiebungen $\delta$ sind in ppm und die Kopplungskonstanten $J$ in $\mathrm{Hz}$ angegeben. Als externe Standards dienten Tetramethylsilan $\left({ }^{1} \mathrm{H},{ }^{13} \mathrm{C}\right), 85 \%$ ige Phosphorsäure $\left({ }^{31} \mathrm{P}\right)$, Dimethylselenid $\left({ }^{77} \mathrm{Se}\right)$, Nitromethan $\left({ }^{15} \mathrm{~N}\right)$ und Lithiumchlorid $\left({ }^{7} \mathrm{Li}\right)$. Als interner Standard wurden die Restprotonensignale des deuterierten Lösungsmittels verwendet.

Die Multiplizitäten werden wie folgt abgekürzt: (br) $s=$ (breites) Singulett, $d=$ Dublett, $\mathrm{dd}=$ Dublett von Dubletts, $\mathrm{ddd}=$ Dublett von Dublett von Dubletts, $\mathrm{dq}=$ Dublett von Quartetts, $\mathrm{dsept}=$ Dublett von Septetts, $\mathrm{t}=$ Triplett, $\mathrm{m}=$ Multiplett.

Zur Signalzuordnung der Anthracengerüste sowie der Phenyl-, 2-Pyridyl- und isoPropylsubstituenten werden folgende Bezeichnungen verwendet:
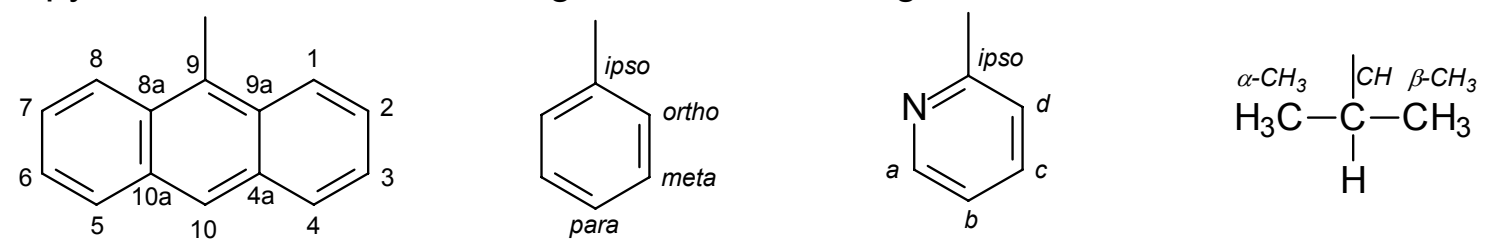

\subsubsection{Elementaranalysen}

Elementaranalysen wurden durch das Mikroanalytische Labor des Instituts für Anorganische Chemie der Universität Göttingen mit einem Vario EL3 Gerät durchgeführt.

\subsection{Synthesen und Charakterisierungen}

\subsubsection{9-Brom-10-(diphenylphosphanyl)anthracen (1)}

Eine Suspension von $1.00 \mathrm{~g}$ (2.98 mmol) 9,10-Dibromanthracen in $25 \mathrm{~mL}$ Diethylether wurde bei $-15^{\circ} \mathrm{C}$ mit $1.37 \mathrm{~mL}$ (3.04 mmol) ${ }^{n} \mathrm{BuLi}$ in $n$-Hexan (2.22 M) versetzt. Nach 30 min wurden $0.660 \mathrm{~g}(2.99 \mathrm{mmol})$ Chlordiphenylphosphan zugegeben, die Reaktionsmischung für weitere 30 min gerührt und unlösliche Bestandteile über Celite abgetrennt. Durch Einengen des Filtrats i. Vak. wurde 1 als gelbes Pulver erhalten. Lagerung einer gesättigten Lösung in Diethylether lieferte nach einigen Tagen Kristalle, die zur röntgenkristallographischen Untersuchung geeignet waren.

Summenformel: $\quad \mathrm{C}_{26} \mathrm{H}_{18} \mathrm{BrP}$

Ausbeute: 
${ }^{1} \mathrm{H}-N M R\left(500.130 \mathrm{MHz}, \mathrm{CDCl}_{3}\right): \delta 8.84\left(\mathrm{dd},{ }^{3} \mathrm{~J}_{\mathrm{HH}}=9.00 \mathrm{~Hz},{ }^{4} J_{\mathrm{HP}}=5.33 \mathrm{~Hz}, 2 \mathrm{H}, \mathrm{H}_{4,5}\right.$, An), 8.66 (d, $\left.{ }^{3} J_{H H}=8.85 \mathrm{~Hz}, 2 \mathrm{H}, \mathrm{H}_{1,8}, A n\right), 7.54$ (ddd, ${ }^{3} J_{\mathrm{HH}}=8.88 \mathrm{~Hz},{ }^{3} J_{\mathrm{HH}}=8.88 \mathrm{~Hz}$, $\left.{ }^{3} J_{\mathrm{HH}}=6.50 \mathrm{~Hz},{ }^{4} J_{\mathrm{HH}}=1.09 \mathrm{~Hz}, 2 \mathrm{H}, \mathrm{H}_{2,7}, \mathrm{An}\right), 7.41-7.37\left(\mathrm{~m}, 4 \mathrm{H}, \mathrm{H}_{\text {ortho }}, \mathrm{Ph}\right), 7.33$ $\left(\mathrm{ddd},{ }^{3} \mathrm{~J}_{\mathrm{HH}}=9.00 \mathrm{~Hz},{ }^{3} \mathrm{~J}_{\mathrm{HH}}=6.50 \mathrm{~Hz},{ }^{4} \mathrm{~J}_{\mathrm{HH}}=1.20 \mathrm{~Hz}, 2 \mathrm{H}, \mathrm{H}_{3,6}\right.$, An), $7.27-7.22 \mathrm{ppm}$ (m, $\left.6 \mathrm{H}, \mathrm{H}_{\text {meta/para }}, \mathrm{Ph}\right)$.

${ }^{13} \mathrm{C}\left\{{ }^{1} \mathrm{H}\right\}-N M R\left(125.756 \mathrm{MHz}, \mathrm{CDCl}_{3}\right): \delta 136.7\left(\mathrm{~d},{ }^{1} \mathrm{~J}_{\mathrm{CP}}=102.3 \mathrm{~Hz}, 2 \mathrm{C}, \mathrm{C}_{i p s o}, \mathrm{Ph}\right)$, $131.4\left(\mathrm{~d},{ }^{2} J_{\mathrm{CP}}=18.4 \mathrm{~Hz}, 4 \mathrm{C}, \mathrm{C}_{\text {ortho }}, \mathrm{Ph}\right), 130.9$ (d, $\left.{ }^{3} J_{\mathrm{CP}}=4.21 \mathrm{~Hz}, 2 \mathrm{C}, \mathrm{C}_{8 \mathrm{a}, 9 a}, A n\right)$, $130.7\left(\mathrm{~d},{ }^{2} J_{\mathrm{CP}}=11.5 \mathrm{~Hz}, 2 \mathrm{C}, \mathrm{C}_{4 \mathrm{a}, 10 \mathrm{a}}, \mathrm{An}\right), 129.7$ (d, $\left.{ }^{4} J_{\mathrm{CP}}=21.7 \mathrm{~Hz}, 1 \mathrm{C}, \mathrm{C}_{9}, \mathrm{An}\right)$, $128.9\left(\mathrm{~d},{ }^{1} J_{\mathrm{CP}}=25.5 \mathrm{~Hz}, 1 \mathrm{C}, \mathrm{C}_{10}, \mathrm{An}\right) 129.2\left(\mathrm{~d},{ }^{3} J_{\mathrm{CP}}=1.85 \mathrm{~Hz}, 2 \mathrm{C}, \mathrm{C}_{4,5}, \mathrm{An}\right), 128.8$ (s, $\left.2 \mathrm{C}, \mathrm{C}_{1,8}, \mathrm{An}\right), 128.5$ (d, $\left.{ }^{3} \mathrm{~J}_{\mathrm{CP}}=5.51 \mathrm{~Hz}, 4 \mathrm{C}, \mathrm{C}_{\text {meta }}, \mathrm{Ph}\right), 127.7$ (s, $2 \mathrm{C}, \mathrm{C}_{\text {para }}, \mathrm{Ph}$ ), $126.9\left(\mathrm{~d},{ }^{5} J_{\mathrm{CP}}=1.53 \mathrm{~Hz}, 2 \mathrm{C}, \mathrm{C}_{2,7}, \mathrm{An}\right), 126.1 \mathrm{ppm}\left(\mathrm{d},{ }^{4} J_{\mathrm{CP}}=1.75 \mathrm{~Hz}, 2 \mathrm{C}, \mathrm{C}_{3,6}, A n\right)$. ${ }^{31} \mathrm{P}\left\{{ }^{1} \mathrm{H}\right\}-N M R\left(202.456 \mathrm{MHz}, \mathrm{CDCl}_{3}\right): \delta-22.7 \mathrm{ppm}(\mathrm{s})$.

El-MS (70 eV): m/z (\%): 442 (100) [M] $]^{+}, 361(35)[\mathrm{M}-\mathrm{Br}]^{+}, 207(10)\left[\mathrm{C}_{14} \mathrm{H}_{8} \mathrm{P}\right]^{+}, 176$ (24) $\left[\mathrm{C}_{14} \mathrm{H}_{8}\right]^{+}$.

Elementaranalyse (gef. (ber.) [\%]): C 70.71 (70.76), H 4.73 (4.11).

\subsubsection{9-Brom-10-(diphenylphosphoryl)anthracen (2)}

Eine Lösung von $0.330 \mathrm{~g}(0.748 \mathrm{mmol}) 1$ in $40 \mathrm{~mL}$ Dichlormethan wurde mit einem Überschuss von $0.300 \mathrm{~g}(3.19 \mathrm{mmol}) \mathrm{H}_{2} \mathrm{O}_{2} \cdot\left(\mathrm{H}_{2} \mathrm{~N}\right)_{2} \mathrm{C}=\mathrm{O}$ versetzt. Die Reaktionsmischung wurde für $1 \mathrm{~h}$ gerührt und gefiltert. Das Filtrat wurde zweimal mit je $10 \mathrm{~mL}$ Wasser gewaschen und über Nacht mit Magnesiumsulfat getrocknet. Nach Abtrennen des Trockenmittels wurde das Lösungsmittel i. Vak. entfernt. Umkristallisation des verbleibenden Rückstandes aus Toluol lieferte $\mathbf{2}$ als gelbe Kristalle, die zur röntgenkristallographischen Untersuchung geeignet waren.

Summenformel: $\quad \mathrm{C}_{26} \mathrm{H}_{18} \mathrm{BrOP}$

Ausbeute: $0.310 \mathrm{~g}(6.78 \mathrm{mmol}), 91 \%$
Molare Masse: $\quad 457.28 \mathrm{~g} / \mathrm{mol}$

Schmelzpunkt: $164^{\circ} \mathrm{C}$

${ }^{1} \mathrm{H}-N M R\left(500.130 \mathrm{MHz} \mathrm{CDCl}_{3}\right): \delta 8.64\left(\mathrm{~d},{ }^{3} \mathrm{~J}_{\mathrm{HH}}=8.93 \mathrm{~Hz}, 2 \mathrm{H}, \mathrm{H}_{1,8}, \mathrm{An}\right), 8.59$ (d, $\left.{ }^{3} J_{\mathrm{HH}}=8.92 \mathrm{~Hz}, 2 \mathrm{H}, \mathrm{H}_{4,5}, \mathrm{An}\right), 7.68-7.63\left(\mathrm{~m}, 4 \mathrm{H}, \mathrm{H}_{\text {ortho }}, \mathrm{Ph}\right), 7.52-7.46(\mathrm{~m}, 4 \mathrm{H}$, $\mathrm{H}_{2,7}, \mathrm{An} / \mathrm{H}_{\text {para }}, \mathrm{Ph}$ ), $7.40-7.36$ (m, $\left.4 \mathrm{H}, \mathrm{H}_{\text {meta }}, \mathrm{Ph}\right), 7.23 \mathrm{ppm}$ (ddd, ${ }^{3} J_{\mathrm{HH}}=8.92 \mathrm{~Hz}$, $\left.{ }^{3} J_{\mathrm{HH}}=6.66 \mathrm{~Hz},{ }^{4} \mathrm{~J}_{\mathrm{HH}}=1.20 \mathrm{~Hz}, 2 \mathrm{H}, \mathrm{H}_{3,6}, \mathrm{An}\right)$.

${ }^{13} \mathrm{C}\left\{{ }^{1} \mathrm{H}\right\}-N M R$ (125.756 MHz, $\mathrm{CDCl}_{3}$ ): $\delta 135.7$ (d, $\left.{ }^{1} \mathrm{~J}_{\mathrm{CP}}=74.0 \mathrm{~Hz}, 2 \mathrm{C}, \mathrm{C}_{i p s o}, \mathrm{Ph}\right)$, $131.7\left(\mathrm{~d},{ }^{4} J_{\mathrm{CP}}=2.79 \mathrm{~Hz}, 2 \mathrm{C}, \mathrm{C}_{\text {para }}, \mathrm{Ph}\right), 131.4\left(\mathrm{~d},{ }^{2} J_{\mathrm{CP}}=10.1 \mathrm{~Hz}, 4 \mathrm{C}, \mathrm{C}_{\text {ortho }}, \mathrm{Ph}\right)$, $131.2\left(\mathrm{~d},{ }^{3} \mathrm{~J}_{\mathrm{CP}}=10.5 \mathrm{~Hz}, 2 \mathrm{C}, \mathrm{C}_{8 \mathrm{a}, 9 \mathrm{a}}, \mathrm{An}\right), 130.7$ (d, $\left.{ }^{2} \mathrm{~J}_{\mathrm{CP}}=11.6 \mathrm{~Hz}, 2 \mathrm{C}, \mathrm{C}_{4 a}, 10 \mathrm{a}, \mathrm{An}\right)$, 
$130.4\left(\mathrm{~d},{ }^{4} J_{\mathrm{CP}}=10.9 \mathrm{~Hz}, 1 \mathrm{C}, \mathrm{C}_{9}, \mathrm{An}\right), 128.8\left(\mathrm{~d},{ }^{3} \mathrm{~J}_{\mathrm{CP}}=6.34 \mathrm{~Hz}, 4 \mathrm{C}, \mathrm{C}_{\text {meta }}, \mathrm{Ph}\right), 128.7$ (s, 2 C, C $1,8, A n$ ), 127.7 (d, ${ }^{3} J_{\mathrm{CP}}=7.43 \mathrm{~Hz}, 2 \mathrm{C}, \mathrm{C}_{4,5}, \mathrm{An}$ ), 126.8 (s, 2 C, C $2,7, A n$ ), 126.4 (s, $\left.2 \mathrm{C}, \mathrm{C}_{3,6}, \mathrm{An}\right), 122.7 \mathrm{ppm}\left(\mathrm{d},{ }^{1} \mathrm{~J}_{\mathrm{CP}}=99.0 \mathrm{~Hz}, 1 \mathrm{C}, \mathrm{C}_{10}, \mathrm{An}\right)$.

${ }^{31} \mathrm{P}\left\{{ }^{1} \mathrm{H}\right\}-N M R$ (202.456 MHz, $\left.\mathrm{CDCl}_{3}\right): \delta 31.6 \mathrm{ppm}(\mathrm{s})$.

El-MS (70 eV): m/z (\%): 457 (100) [M] $]^{+}, 377$ (26) [M - Br] $]^{+}, 201$ (8) $\left[\mathrm{Ph}_{2} \mathrm{PO}\right]^{+}, 185$ (21) $\left[\mathrm{PPh}_{2}\right]^{+}, 176(17)\left[\mathrm{C}_{14} \mathrm{H}_{8}\right]^{+}$.

Elementaranalyse (gef. (ber.) [\%]): C 68.04 (68.29), H 4.05 (3.97).

\subsubsection{9-Brom-10-(diphenylthiophosphoryl)anthracen (3)}

Eine Suspension von $1.10 \mathrm{~g}(2.49 \mathrm{mmol}) 1$ in $30 \mathrm{~mL}$ Toluol wurde mit $80 \mathrm{mg}$ (2.50 mmol) Schwefel versetzt und für $4 \mathrm{~h}$ unter Rückfluss erhitzt. Nach Abkühlung auf RT wurde das Lösungsmittel i. Vak. entfernt. Umkristallisation des Rückstandes aus Diethylether lieferte nach einigen Tagen $\mathbf{3}$ als gelbe Kristalle, die zur röntgenkristallographischen Untersuchung geeignet waren.

Summenformel: $\mathrm{C}_{26} \mathrm{H}_{18} \mathrm{BrPS} \quad$ Molare Masse: $\quad 473.34 \mathrm{~g} / \mathrm{mol}$

Ausbeute: $\quad 1.10 \mathrm{~g}(2.32 \mathrm{mmol}), 93 \% \quad$ Schmelzpunkt: $181{ }^{\circ} \mathrm{C}$

${ }^{1} \mathrm{H}-\mathrm{NMR}\left(\mathbf{5 0 0 . 1 3 0 ~ M H z}, \mathrm{CDCl}_{3}\right): \delta 8.55\left(\mathrm{~d},{ }^{3} \mathrm{~J}_{\mathrm{HH}}=8.90 \mathrm{~Hz}, 2 \mathrm{H}, \mathrm{H}_{1,8}, \mathrm{An}\right), 8.04(\mathrm{~d}$, ${ }^{3} J_{\mathrm{HH}}=8.84 \mathrm{~Hz}, 2 \mathrm{H}, \mathrm{H}_{4,5}, \mathrm{An}$ ), $7.72-7.67\left(\mathrm{~m}, 4 \mathrm{H}, \mathrm{H}_{\text {ortho }}, \mathrm{Ph}\right.$ ), 7.40 (ddd, ${ }^{3} \mathrm{~J}_{\mathrm{HH}}=$ $\left.8.90 \mathrm{~Hz},{ }^{3} \mathrm{~J}_{\mathrm{HH}}=6.54 \mathrm{~Hz},{ }^{4} \mathrm{~J}_{\mathrm{HH}}=0.70 \mathrm{~Hz}, 2 \mathrm{H}, \mathrm{H}_{2,7}, \mathrm{An}\right), 7.29-7.26\left(\mathrm{~m}, 2 \mathrm{H}, \mathrm{H}_{\text {para }}, \mathrm{Ph}\right)$, $7.23-7.20\left(\mathrm{~m}, 4 \mathrm{H}, \mathrm{H}_{\text {meta }}, \mathrm{Ph}\right), 7.01 \mathrm{ppm}\left(\mathrm{ddd},{ }^{3} \mathrm{~J}_{\mathrm{HH}}=8.84 \mathrm{~Hz},{ }^{3} \mathrm{~J}_{\mathrm{HH}}=6.54 \mathrm{~Hz},{ }^{4} \mathrm{~J}_{\mathrm{HH}}=\right.$ $\left.1.03 \mathrm{~Hz}, 2 \mathrm{H}, \mathrm{H}_{3,6}, \mathrm{An}\right)$.

${ }^{13} \mathrm{C}\left\{{ }^{1} \mathrm{H}\right\}$-NMR (125.756 MHz, CDCl 3$): \delta 136.9\left(\mathrm{~d},{ }^{1} \mathrm{~J}_{\mathrm{CP}}=83.2 \mathrm{~Hz}, 2 \mathrm{C}, \mathrm{C}_{\text {ipso }}, \mathrm{Ph}\right)$, $133.5\left(\mathrm{~d},{ }^{4} J_{\mathrm{CP}}=8.04 \mathrm{~Hz}, 1 \mathrm{C}, \mathrm{C}_{9}, \mathrm{An}\right), 131.1\left(\mathrm{~d},{ }^{2} \mathrm{~J}_{\mathrm{CP}}=11.2 \mathrm{~Hz}, 2 \mathrm{C}, \mathrm{C}_{4 \mathrm{a}, 10 \mathrm{a}}, \mathrm{An}\right)$, $130.8\left(\mathrm{~d},{ }^{3} J_{\mathrm{CP}}=10.3 \mathrm{~Hz}, 2 \mathrm{C}, \mathrm{C}_{8 \mathrm{a}, 9 \mathrm{a}}, \mathrm{An}\right), 130.7$ (d, ${ }^{2} \mathrm{~J}_{\mathrm{CP}}=10.3 \mathrm{~Hz}, 4 \mathrm{C}, \mathrm{C}_{\text {ortho }}, \mathrm{Ph}$ ), $130.6\left(\mathrm{~d},{ }^{4} \mathrm{~J}_{\mathrm{CP}}=2.43 \mathrm{~Hz}, 2 \mathrm{C}, \mathrm{C}_{\text {para }}, \mathrm{Ph}\right), 128.5\left(\mathrm{~d},{ }^{3} \mathrm{~J}_{\mathrm{CP}}=12.6 \mathrm{~Hz}, 4 \mathrm{C}, \mathrm{C}_{\text {meta, }}, \mathrm{Ph}\right)$, 128.4 (s, $2 \mathrm{C}, \mathrm{C}_{1,8}, \mathrm{An}$ ), 127.6 (d, ${ }^{3} \mathrm{~J}_{\mathrm{CP}}=10.4 \mathrm{~Hz}, 2 \mathrm{C}, \mathrm{C}_{4,5}, \mathrm{An}$ ), 126.9 (s, $2 \mathrm{C}, \mathrm{C}_{2,7}$, $\mathrm{An}), 125.7$ (s, $\left.2 \mathrm{C}, \mathrm{C}_{3,6}, \mathrm{An}\right), 123.7 \mathrm{ppm}\left(\mathrm{d},{ }^{1} \mathrm{~J}_{\mathrm{CP}}=88.8 \mathrm{~Hz}, 1 \mathrm{C}, \mathrm{C}_{10}, \mathrm{An}\right)$.

${ }^{31} \mathrm{P}\left\{{ }^{1} \mathrm{H}\right\}-N M R\left(202.456 \mathrm{MHz}, \mathrm{CDCl}_{3}\right): \delta 34.7 \mathrm{ppm}(\mathrm{s})$.

El-MS (70 eV): $m / z(\%): 474$ (100) [M] ${ }^{+}, 363$ (25) [M - SPh $]^{+}, 286(17)\left[\mathrm{M}-\mathrm{SPh}_{2}\right]^{+}$, $185(86)\left[\mathrm{PPh}_{2}\right]^{+}, 176(12)\left[\mathrm{C}_{14} \mathrm{H}_{8}\right]^{+}$.

Elementaranalyse (gef. (ber.) [\%]): C 64.54 (65.97), H 4.08 (3.83). 


\subsubsection{9-Brom-10-(diphenylselenophosphoryl)anthracen (4)}

Eine Suspension von $0.240 \mathrm{~g}(0.544 \mathrm{mmol}) 1$ in $30 \mathrm{~mL}$ Toluol wurde mit einem Überschuss von $0.120 \mathrm{~g}(1.52 \mathrm{mmol})$ grauem Selen versetzt und für $4 \mathrm{~h}$ unter Rückfluss erhitzt. Nach Abkühlen auf RT wurde über Celite filtriert und das Filtrat i. Vak. eingeengt. Umkristallisation des verbleibenden Rückstandes aus Toluol lieferte nach einigen Tagen 4 als gelbe Kristalle, die zur röntgenkristallographischen Strukturbestimmung geeignet waren.

Summenformel: $\quad \mathrm{C}_{26} \mathrm{H}_{18} \mathrm{BrPSe}$

Ausbeute: $\quad 0.230 \mathrm{~g}(0.442 \mathrm{mmol}), 81 \% \quad$ Schmelzpunkt: $\quad 235^{\circ} \mathrm{C}$

${ }^{1} \mathrm{H}-\mathrm{NMR}\left(\mathbf{5 0 0 . 1 3 0 ~ M H z}, \mathrm{CDCl}_{3}\right): \delta 8.59\left(\mathrm{~d},{ }^{3} \mathrm{~J}_{\mathrm{HH}}=8.92 \mathrm{~Hz}, 2 \mathrm{H}, \mathrm{H}_{1,8}, \mathrm{An}\right), 8.08(\mathrm{~d}$, ${ }^{3} J_{\mathrm{HH}}=8.92 \mathrm{~Hz}, 2 \mathrm{H}, \mathrm{H}_{4,5}, \mathrm{An}$ ), $7.79-7.74\left(\mathrm{~m}, 4 \mathrm{H}, \mathrm{H}_{\text {ortho }}, \mathrm{Ph}\right.$ ), 7.44 (ddd, ${ }^{3} \mathrm{JHH}_{\mathrm{HH}}=$ $\left.8.92 \mathrm{~Hz},{ }^{3} \mathrm{~J}_{\mathrm{HH}}=6.70 \mathrm{~Hz},{ }^{4} \mathrm{~J}_{\mathrm{HH}}=0.72 \mathrm{~Hz}, 2 \mathrm{H}, \mathrm{H}_{2,7}, \mathrm{An}\right), 7.33-7.29\left(\mathrm{~m}, 2 \mathrm{H}, \mathrm{H}_{\text {para }}, \mathrm{Ph}\right)$, $7.26-7.22\left(\mathrm{~m}, 4 \mathrm{H}, \mathrm{H}_{\text {meta }}, \mathrm{Ph}\right), 7.07 \mathrm{ppm}$ (ddd, ${ }^{3} \mathrm{JHH}_{\mathrm{HH}}=8.92 \mathrm{~Hz},{ }^{3} \mathrm{~J}_{\mathrm{HH}}=6.70 \mathrm{~Hz},{ }^{4} \mathrm{~J}_{\mathrm{HH}}=$ $\left.1.06 \mathrm{~Hz}, 2 \mathrm{H}, \mathrm{H}_{3,6}, \mathrm{An}\right)$.

${ }^{13} \mathrm{C}\left\{{ }^{1} \mathrm{H}\right\}-N M R\left(125.756 \mathrm{MHz}, \mathrm{CDCl}_{3}\right): \delta 135.2\left(\mathrm{~d},{ }^{1} \mathrm{~J}_{\mathrm{CP}}=74.7 \mathrm{~Hz}, 2 \mathrm{C}, \mathrm{C}_{\text {ipso }}, \mathrm{Ph}\right)$, $133.3\left(\mathrm{~d},{ }^{4} J_{\mathrm{CP}}=7.69 \mathrm{~Hz}, 1 \mathrm{C}, \mathrm{C}_{9}, \mathrm{An}\right), 131.3\left(\mathrm{~d},{ }^{2} \mathrm{~J}_{\mathrm{CP}}=10.7 \mathrm{~Hz}, 4 \mathrm{C}, \mathrm{C}_{\text {ortho }}, \mathrm{Ph}\right), 130.7$ $\left(\mathrm{d},{ }^{2} \mathrm{~J}_{\mathrm{CP}}=6.48 \mathrm{~Hz}, 2 \mathrm{C}, \mathrm{C}_{4 \mathrm{a}, 10 \mathrm{a}}, \mathrm{An}\right), 130.6\left(\mathrm{~s}, 2 \mathrm{C}, \mathrm{C}_{\text {para }}, \mathrm{Ph}\right), 130.3\left(\mathrm{~d},{ }^{3} J_{\mathrm{CP}}=\right.$ $\left.5.02 \mathrm{~Hz}, 2 \mathrm{C}, \mathrm{C}_{8 \mathrm{a}, 9 \mathrm{a}}, \mathrm{An}\right), 128.5$ (d, $\left.{ }^{3} \mathrm{~J}_{\mathrm{CP}}=12.7 \mathrm{~Hz}, 4 \mathrm{C}, \mathrm{C}_{\text {meta }}, \mathrm{Ph}\right), 128.4$ (s, $2 \mathrm{C}, \mathrm{C}_{1,8}$, An), 127.6 (d, ${ }^{3} J_{\mathrm{CP}}=7.43 \mathrm{~Hz}, 2 \mathrm{C}, \mathrm{C}_{4,5}, \mathrm{An}$ ), 127.0 (s, $2 \mathrm{C}, \mathrm{C}_{2,7}, \mathrm{An}$ ), 125.5 (s, $2 \mathrm{C}$, $\left.\mathrm{C}_{3,6}, \mathrm{An}\right), 122.4 \mathrm{ppm}\left(\mathrm{d},{ }^{1} \mathrm{~J}_{\mathrm{CP}}=80.8 \mathrm{~Hz}, 1 \mathrm{C}, \mathrm{C}_{10}, \mathrm{An}\right)$

${ }^{31} \mathrm{P}\left\{{ }^{1} \mathrm{H}\right\}-N M R\left(202.456 \mathrm{MHz}, \mathrm{CDCl}_{3}\right): \delta 25.9 \mathrm{ppm}(\mathrm{s})$.

${ }^{77} \mathrm{Se}\left\{{ }^{1} \mathrm{H}\right\}-N M R\left(95.382 \mathrm{MHz}, \mathrm{CDCl}_{3}\right): \delta-281.1\left(\mathrm{~d},{ }^{1} \mathrm{~J}_{\mathrm{SeP}}=734.9 \mathrm{~Hz}\right)$.

El-MS (70 eV): $\mathbf{m} / \mathbf{z}(\%): 520$ (59) [M] ${ }^{+}, 442$ (100) [M - Se] $]^{+}, 363$ (62) [M - SePh] $]^{+}, 185$ (57) $\left[\mathrm{PPh}_{2}\right]^{+}, 176(32)\left[\mathrm{C}_{14} \mathrm{H}_{8}\right]^{+}$.

Elementaranalyse (gef. (ber.) [\%]): C 60.19 (60.02), H 3.64 (3.49).

\subsubsection{9-Brom-10-(diisopropylphosphanyl)anthracen (5)}

Eine Suspension von $2.00 \mathrm{~g}(5.95 \mathrm{mmol})$ 9,10-Dibromanthracen in $50 \mathrm{~mL}$ Diethylether wurde bei $-15{ }^{\circ} \mathrm{C}$ mit $2.56 \mathrm{~mL}(5.96 \mathrm{mmol}){ }^{n} \mathrm{BuLi}$ in $n$-Hexan $(2.33 \mathrm{M})$ versetzt. Nach $15 \mathrm{~min}$ wurden $0.910 \mathrm{~g}$ (5.96 mmol) Chlordiisopropylphosphan zugegeben, die Reaktionsmischung für $1 \mathrm{~h}$ gerührt und anschließend unlösliche Bestandteile über Celite abgetrennt. Durch Einengen des Filtrats i. Vak. wurde 5 als gelbes Pulver erhalten. Lagerung einer gesättigten Lösung in $n$-Pentan bei RT lieferte nach einigen 
Tagen Kristalle, die zur röntgenkristallographischen Strukturbestimmung geeignet waren.

Summenformel: $\quad \mathrm{C}_{20} \mathrm{H}_{22} \mathrm{BrP}$

Ausbeute:
Molare Masse: $\quad 373.26 \mathrm{~g} / \mathrm{mol}$

Schmelzpunkt: $148^{\circ} \mathrm{C}$

${ }^{1} \mathrm{H}-N M R\left(500.130 \mathrm{MHz} \mathrm{CDCl}_{3}, \mathbf{R T}\right): \delta 9.60-8.90$ (br s, $\left.2 \mathrm{H}, \mathrm{H}_{4,5}, \mathrm{An}\right), 8.68\left(\mathrm{~d},{ }^{3} \mathrm{~J}_{\mathrm{HH}}=\right.$ $8.53 \mathrm{~Hz}, 2 \mathrm{H}, \mathrm{H}_{1,8}$, An), 7.58 (ddd, ${ }^{3} \mathrm{~J}_{\mathrm{HH}}=8.68 \mathrm{~Hz},{ }^{3} J_{\mathrm{HH}}=6.49 \mathrm{~Hz},{ }^{4} J_{\mathrm{HH}}=1.29 \mathrm{~Hz}$, $2 \mathrm{H}, \mathrm{H}_{2,7}, \mathrm{An}$ ), 7.54 (ddd, ${ }^{3} \mathrm{~J}_{\mathrm{HH}}=8.88 \mathrm{~Hz},{ }^{3} \mathrm{~J}_{\mathrm{HH}}=6.49 \mathrm{~Hz},{ }^{4} J_{\mathrm{HH}}=1.38 \mathrm{~Hz}, 2 \mathrm{H}, \mathrm{H}_{3,6}$, An), 2.92 (dsept, $\left.{ }^{2} J_{\mathrm{HP}}={ }^{3} J_{\mathrm{HH}}=6.90 \mathrm{~Hz}, 2 \mathrm{H}, \mathrm{CH},{ }^{i} \mathrm{Pr}\right), 1.44\left(\mathrm{dd},{ }^{3} J_{\mathrm{HP}}=17.8 \mathrm{~Hz},{ }^{3} J_{\mathrm{HH}}=\right.$ $\left.6.75 \mathrm{~Hz}, \quad 6 \mathrm{H}, \alpha-\underline{\mathrm{H}}_{3},{ }^{i} \mathrm{Pr}\right), 0.70 \mathrm{ppm}\left(\mathrm{dd},{ }^{3} \mathrm{~J}_{\mathrm{HP}}=13.8 \mathrm{~Hz},{ }^{3} \mathrm{~J}_{\mathrm{HH}}=6.98 \mathrm{~Hz}, 6 \mathrm{H}\right.$, $\left.\beta-\underline{\mathrm{H}}_{3},{ }^{i} \mathrm{Pr}\right)$.

${ }^{1} \mathrm{H}-N M R\left(500.130 \mathrm{MHz}, \mathrm{CDCl}_{3}, 248 \mathrm{~K}\right): \delta 9.73\left(\mathrm{dd},{ }^{0} \mathrm{~J}_{\mathrm{HP}}={ }^{3} \mathrm{~J}_{\mathrm{HH}}=9.34 \mathrm{~Hz}, 1 \mathrm{H}, \mathrm{H}_{4}\right.$, An), $8.67\left(\mathrm{~d},{ }^{3} \mathrm{~J}_{\mathrm{HH}}=8.58 \mathrm{~Hz}, 1 \mathrm{H}, \mathrm{H}_{8}, \mathrm{An}\right), 8.59\left(\mathrm{~d},{ }^{3} \mathrm{~J}_{\mathrm{HH}}=8.86 \mathrm{~Hz}, 1 \mathrm{H}, \mathrm{H}_{1}, \mathrm{An}\right), 8.48$ $\left(\mathrm{d},{ }^{3} \mathrm{~J}_{\mathrm{HH}}=8.66 \mathrm{~Hz}, 1 \mathrm{H}, \mathrm{H}_{5}, \mathrm{An}\right), 7.62-7.58\left(\mathrm{~m}, 2 \mathrm{H}, \mathrm{H}_{2,7}, \mathrm{An}\right), 7.57-7.52(\mathrm{~m}, 2 \mathrm{H}$, $\mathrm{H}_{3,6}, \mathrm{An}$ ), 2.88 (dsept, ${ }^{2} J_{\mathrm{HP}}={ }^{3} J_{\mathrm{HH}}=7.26 \mathrm{~Hz}, 2 \mathrm{H}, \mathrm{CH},{ }^{i} \mathrm{Pr}$ ), 1.39 (dd, ${ }^{3} J_{\mathrm{HP}}=18.1 \mathrm{~Hz}$, $\left.{ }^{3} J_{\mathrm{HH}}=6.72 \mathrm{~Hz}, 6 \mathrm{H}, \alpha-\underline{\mathrm{C}}_{3},{ }^{i} \mathrm{Pr}\right), 0.61 \mathrm{ppm}\left(\mathrm{dd},{ }^{3} J_{\mathrm{HP}}=14.0 \mathrm{~Hz},{ }^{3} J_{\mathrm{HH}}=6.95 \mathrm{~Hz}, 6 \mathrm{H}\right.$, $\left.\beta-\underline{\mathrm{H}}_{3},{ }^{i} \mathrm{Pr}\right)$.

${ }^{13} \mathrm{C}\left\{{ }^{1} \mathrm{H}\right\}-N M R\left(125.756 \mathrm{MHz}, \mathrm{CDCl}_{3}\right.$ ): $\delta 138$ - 136 (br s, $\left.2 \mathrm{C}, \mathrm{C}_{4 \mathrm{a}, 10 \mathrm{a}}, \mathrm{An}\right), 133.5$ (d, $\left.{ }^{3} J_{\mathrm{CP}}=30.6 \mathrm{~Hz}, 2 \mathrm{C}, \mathrm{C}_{8 \mathrm{a}, 9 a}, \mathrm{An}\right), 130.3$ (s, $\left.1 \mathrm{C}, \mathrm{C}_{10}, \mathrm{An}\right), 128.8$ (s, $\left.2 \mathrm{C}, \mathrm{C}_{1,8}, \mathrm{An}\right), 128.3$ (s, 2 C, $\left.\mathrm{C}_{4,5}, \mathrm{An}\right), 127.4$ (s, $\left.1 \mathrm{C}, \mathrm{C}_{9}, \mathrm{An}\right), 126.6$ (s, $2 \mathrm{C}, \mathrm{C}_{2,7}, \mathrm{An}$ ), 125.6 (s, 2 C, C , $_{3,6}$, An), $26.3\left(\mathrm{~d},{ }^{1} J_{\mathrm{CP}}=13.4 \mathrm{~Hz}, 2 \mathrm{C}, \underline{\mathrm{C}} \mathrm{H},{ }^{i} \mathrm{Pr}\right), 23.1$ (d, $\left.{ }^{2} J_{\mathrm{CP}}=29.2 \mathrm{~Hz}, 2 \mathrm{C}, \alpha-\underline{\mathrm{C}} \mathrm{H}_{3},{ }^{i} \operatorname{Pr}\right)$, $21.2 \mathrm{ppm}\left(\mathrm{d},{ }^{2} \mathrm{~J}_{\mathrm{CP}}=12.9 \mathrm{~Hz}, 2 \mathrm{C}, \beta-\underline{\mathrm{C}} \mathrm{H}_{3},{ }^{i} \mathrm{Pr}\right)$.

${ }^{31} \mathrm{P}\left\{{ }^{1} \mathrm{H}\right\}-N M R\left(202.456 \mathrm{MHz}, \mathrm{CDCl}_{3}\right): \delta 1.37 \mathrm{ppm}(\mathrm{s})$.

EI-MS (70 eV): $\boldsymbol{m} / \mathbf{z}(\%): 372$ (58) [M] ${ }^{+}, 330$ (9) [M - $\left.{ }^{i} \mathrm{Pr}\right]^{+}, 287$ (100) [M - $\left.2^{i} \mathrm{Pr}\right]^{+}, 207$ (60) $\left[\mathrm{M}-\mathrm{Br}-2^{i} \mathrm{Pr}\right]^{+}$.

Elementaranalyse (gef. (ber.) [\%]): C 63.91 (64.36), H 6.30 (5.94).

\subsubsection{9-Brom-10-(diisopropylphosphoryl)anthracen (6)}

Eine Lösung von $1.46 \mathrm{~g}$ (3.91 mmol) 5 in $40 \mathrm{~mL}$ Dichlormethan wurde mit einem Überschuss von $700 \mathrm{mg}(7.44 \mathrm{mmol}) \mathrm{H}_{2} \mathrm{O}_{2} \cdot\left(\mathrm{H}_{2} \mathrm{~N}\right)_{2} \mathrm{C}=\mathrm{O}$ versetzt. Die Reaktionsmischung wurde für $1 \mathrm{~h}$ gerührt und anschließend zweimal mit je $10 \mathrm{~mL}$ Wasser gewaschen. Die organische Phase wurde mit Natriumsulfat getrocknet und das Lösungsmittel i. Vak. entfernt; 6 wurde als gelbes Pulver erhalten.

Summenformel: $\mathrm{C}_{20} \mathrm{H}_{22} \mathrm{BrOP}$

Ausbeute:
Molare Masse: $\quad 389.27 \mathrm{~g} / \mathrm{mol}$

Schmelzpunkt: $170^{\circ} \mathrm{C}$ 
${ }^{1} \mathrm{H}-N M R\left(500.130 \mathrm{MHz}, \mathrm{CDCl}_{3}\right): \delta 9.30-9.10\left(\mathrm{br} \mathrm{s}, 2 \mathrm{H}, \mathrm{H}_{4,5}, \mathrm{An}\right), 8.64\left(\mathrm{~d},{ }^{3} \mathrm{JHH}_{\mathrm{HH}}=\right.$ $8.61 \mathrm{~Hz}, 2 \mathrm{H}, \mathrm{H}_{1,8}, \mathrm{An}$ ), 7.57 (ddd, ${ }^{3} \mathrm{~J}_{\mathrm{HH}}=8.61 \mathrm{~Hz},{ }^{3} \mathrm{~J}_{\mathrm{HH}}=6.68 \mathrm{~Hz},{ }^{4} J_{\mathrm{HH}}=1.36 \mathrm{~Hz}$, $2 \mathrm{H}, \mathrm{H}_{2,7}, \mathrm{An}$ ), 7.54 (ddd, ${ }^{3} \mathrm{~J}_{\mathrm{HH}}=8.86 \mathrm{~Hz},{ }^{3} \mathrm{~J}_{\mathrm{HH}}=6.68 \mathrm{~Hz},{ }^{4} J_{\mathrm{HH}}=1.88 \mathrm{~Hz}, 2 \mathrm{H}, \mathrm{H}_{3,6}$, An), 2.77 (dsept, ${ }^{2} J_{\mathrm{HP}}=1.35 \mathrm{~Hz},{ }^{3} \mathrm{~J}_{\mathrm{HH}}=7.05 \mathrm{~Hz}, 2 \mathrm{H}, \mathrm{C} \underline{\mathrm{H}},{ }^{i} \mathrm{Pr}$ ), 1.48 (dd, ${ }^{3} J_{\mathrm{HP}}=$ $\left.15.0 \mathrm{~Hz},{ }^{3} \mathrm{~J}_{\mathrm{HH}}=6.93 \mathrm{~Hz}, 6 \mathrm{H}, \alpha-\underline{\mathrm{H}}_{3},{ }^{i} \mathrm{Pr}\right), 0.88 \mathrm{ppm}\left(\mathrm{dd},{ }^{3} \mathrm{~J}_{\mathrm{HP}}=16.3 \mathrm{~Hz},{ }^{3} \mathrm{~J}_{\mathrm{HH}}=\right.$ $\left.7.20 \mathrm{~Hz}, 6 \mathrm{H}, \beta-\mathrm{CH}_{3},{ }^{i} \mathrm{Pr}\right)$.

${ }^{13} \mathrm{C}\left\{{ }^{1} \mathrm{H}\right\}$-NMR (125.756 MHz, CDCl $)$ ): $\delta 135.4$ (br s, $\left.2 \mathrm{C}, \mathrm{C}_{4 \mathrm{a}, 10 \mathrm{a}}, \mathrm{An}\right), 130.3\left(\mathrm{~d},{ }^{3} \mathrm{~J}_{\mathrm{CP}}=\right.$ $\left.4.11 \mathrm{~Hz}, 2 \mathrm{C}, \mathrm{C}_{8 \mathrm{a}, 9 \mathrm{a}}, \mathrm{An}\right), 130.1$ (d, $\left.{ }^{4} \mathrm{~J}_{\mathrm{CP}}=19.5 \mathrm{~Hz}, 1 \mathrm{C}, \mathrm{C}_{9}, \mathrm{An}\right), 128.9$ (s, $2 \mathrm{C}, \mathrm{C}_{4,5}$, An), 126.7 (s, 2 C, $C_{3,6}, A n$ ), 126.6 (s, 2 C, C $2,7, A n$ ), 126.3 (s, 2 C, $C_{1,8}, A n$ ), 124.8 $\left(\mathrm{d},{ }^{1} J_{\mathrm{CP}}=75.6 \mathrm{~Hz}, 1 \mathrm{C}, \mathrm{C}_{10}, \mathrm{An}\right), 31.2\left(\mathrm{~d},{ }^{1} \mathrm{~J}_{\mathrm{CP}}=64.3 \mathrm{~Hz}, 2 \mathrm{C}, \underline{\mathrm{C}} \mathrm{H},{ }^{1} \mathrm{Pr}\right), 17.1\left(\mathrm{~d},{ }^{2} J_{\mathrm{CP}}=\right.$ $\left.22.9 \mathrm{~Hz}, 2 \mathrm{C}, \alpha-\underline{\mathrm{C}} \mathrm{H}_{3},{ }^{i} \mathrm{Pr}\right), 17.0 \mathrm{ppm}\left(\mathrm{d},{ }^{2} J_{\mathrm{CP}}=22.9 \mathrm{~Hz}, 2 \mathrm{C}, \beta-\underline{\mathrm{C}} \mathrm{H}_{3},{ }^{i} \mathrm{Pr}\right)$.

${ }^{31} \mathrm{P}\left\{{ }^{1} \mathrm{H}\right\}-N M R\left(202.456 \mathrm{MHz}, \mathrm{CDCl}_{3}\right): \delta 59.4 \mathrm{ppm}(\mathrm{s})$.

EI-MS (70 eV): m/z (\%): 388 (100) [M] ${ }^{+}, 345$ (68) [M - $\left.{ }^{i} \mathrm{Pr}\right]^{+}, 303$ (89) [M - $\left.2^{i} \mathrm{Pr}\right]^{+}, 256$ (44) $\left[\mathrm{M}-{ }^{i} \mathrm{Pr}_{2} \mathrm{PO}\right]^{+}$.

Elementaranalyse (gef. (ber.) [\%]): C 62.82 (61.71), H 6.02 (5.70).

\subsubsection{9-Brom-10-(diisopropylthiophosphoryl)anthracen (7)}

Eine Lösung von $1.49 \mathrm{~g}(3.99 \mathrm{mmol}) \mathbf{5}$ in $30 \mathrm{~mL}$ Toluol wurde mit $150 \mathrm{mg}$ (4.68 mmol) Schwefel versetzt und für $2 \mathrm{~h}$ unter Rückfluss erhitzt. Nach Abkühlen auf RT wurde das Lösungsmittel i. Vak. entfernt. Durch Umkristallisation aus heißem Toluol wurden gelbe Kristalle erhalten, die zur röntgenkristallographischen Strukturbestimmung geeignet waren.

Summenformel: $\mathrm{C}_{20} \mathrm{H}_{22} \mathrm{BrPS}$

Ausbeute: $\quad 1.47 \mathrm{~g}(3.63 \mathrm{mmol}), 91 \% \quad$ Schmelzpunkt: $190{ }^{\circ} \mathrm{C}$

${ }^{1} \mathrm{H}-\mathrm{NMR}\left(\mathbf{5 0 0 . 1 3 0 ~ M H z}, \mathrm{CDCl}_{3}\right): \delta 9.30\left(\mathrm{~d},{ }^{3} \mathrm{~J}_{\mathrm{HH}}=8.58 \mathrm{~Hz}, 2 \mathrm{H}, \mathrm{H}_{4,5}, \mathrm{An}\right), 8.61(\mathrm{~d}$, $\left.{ }^{3} J_{\mathrm{HH}}=8.39 \mathrm{~Hz}, 2 \mathrm{H}, \mathrm{H}_{1,8}, \mathrm{An}\right), 7.55\left(\mathrm{ddd},{ }^{3} J_{\mathrm{HH}}=8.39 \mathrm{~Hz},{ }^{3} J_{\mathrm{HH}}=6.63 \mathrm{~Hz},{ }^{4} J_{\mathrm{HH}}=\right.$ $1.30 \mathrm{~Hz}, 2 \mathrm{H}, \mathrm{H}_{2,7}, \mathrm{An}$ ), 7.52 (ddd, ${ }^{3} \mathrm{~J}_{\mathrm{HH}}=8.58 \mathrm{~Hz},{ }^{3} \mathrm{~J}_{\mathrm{HH}}=6.63 \mathrm{~Hz},{ }^{4} \mathrm{~J}_{\mathrm{HH}}=1.65 \mathrm{~Hz}$, $2 \mathrm{H}, \mathrm{H}_{3,6}, \mathrm{An}$ ), 3.25 (dsept, ${ }^{2} \mathrm{~J}_{\mathrm{HP}}=3.92 \mathrm{~Hz},{ }^{3} \mathrm{~J}_{\mathrm{HH}}=6.80 \mathrm{~Hz}, 2 \mathrm{H}, \mathrm{CH},{ }^{i} \mathrm{Pr}$ ), 1.45 (dd, $\left.{ }^{3} J_{\mathrm{HP}}=17.9 \mathrm{~Hz},{ }^{3} \mathrm{~J}_{\mathrm{HH}}=6.80 \mathrm{~Hz}, 6 \mathrm{H}, \alpha-\mathrm{CH}_{3},{ }^{i} \mathrm{Pr}\right), 0.86 \mathrm{ppm}\left(\mathrm{dd},{ }^{3} J_{\mathrm{HP}}=18.6 \mathrm{~Hz},{ }^{3} J_{\mathrm{HH}}=\right.$ $\left.6.80 \mathrm{~Hz}, 6 \mathrm{H}, \beta-\mathrm{CH}_{3},{ }^{i} \mathrm{Pr}\right)$.

${ }^{13} \mathrm{C}\left\{{ }^{1} \mathrm{H}\right\}$-NMR $\left(125.756 \mathrm{MHz}, \mathrm{CDCl}_{3}\right): \delta 134.8\left(\mathrm{~d},{ }^{2} \mathrm{~J}_{\mathrm{CP}}=6.90 \mathrm{~Hz}, 2 \mathrm{C}, \mathrm{C}_{4 \mathrm{a}, 10 \mathrm{a}}, \mathrm{An}\right)$, $130.4\left(\mathrm{~d},{ }^{4} J_{\mathrm{CP}}=9.83 \mathrm{~Hz}, 1 \mathrm{C}, \mathrm{C}_{9}, \mathrm{An}\right), 130.2\left(\mathrm{~d},{ }^{3} \mathrm{~J}_{\mathrm{CP}}=4.55 \mathrm{~Hz}, 2 \mathrm{C}, \mathrm{C}_{8 \mathrm{a}, 9 a}, A n\right)$, 128.6 (s, $\left.2 \mathrm{C}, \mathrm{C}_{1,8}, \mathrm{An}\right), 126.8$ (d, $\left.{ }^{4} J_{\mathrm{CP}}=0.87 \mathrm{~Hz}, 2 \mathrm{C}, \mathrm{C}_{3,6}, \mathrm{An}\right), 126.5$ (d, ${ }^{3} J_{\mathrm{CP}}=$ $\left.5.61 \mathrm{~Hz}, 2 \mathrm{C}, \mathrm{C}_{4,5}, \mathrm{An}\right), 125.4\left(\mathrm{~d},{ }^{5} \mathrm{~J}_{\mathrm{CP}}=0.80 \mathrm{~Hz}, 2 \mathrm{C}, \mathrm{C}_{2,7}, \mathrm{An}\right), 122.9\left(\mathrm{~d},{ }^{1} \mathrm{~J}_{\mathrm{CP}}=\right.$ 
$\left.60.0 \mathrm{~Hz}, 1 \mathrm{C}, \mathrm{C}_{10}, \mathrm{An}\right), 32.3\left(\mathrm{~d},{ }^{1} \mathrm{~J}_{\mathrm{CP}}=48.0 \mathrm{~Hz}, 2 \mathrm{C}, \underline{\mathrm{CH}},{ }^{i} \mathrm{Pr}\right), 18.2\left(\mathrm{~d},{ }^{2} \mathrm{~J}_{\mathrm{CP}}=1.17 \mathrm{~Hz}\right.$, $\left.2 \mathrm{C}, \alpha-\underline{\mathrm{CH}}_{3},{ }^{i} \mathrm{Pr}\right), 17.9 \mathrm{ppm}\left(\mathrm{d},{ }^{2} \mathrm{~J}_{\mathrm{CP}}=1.76 \mathrm{~Hz}, 2 \mathrm{C}, \beta-\underline{\mathrm{C}} \mathrm{H}_{3},{ }^{i} \mathrm{Pr}\right)$.

${ }^{31} \mathrm{P}\left\{{ }^{1} \mathrm{H}\right\}-N M R\left(202.456 \mathrm{MHz}, \mathrm{CDCl}_{3}\right): \delta 72.6 \mathrm{ppm}(\mathrm{s})$.

EI-MS (70 eV): m/z (\%): 406 (64) [M] ${ }^{+}, 363$ (20) [M - ' $\left.\mathrm{Pr}\right]^{+}, 320$ (46) [M - $\left.2^{i} \mathrm{Pr}\right]^{+}, 256$ (21) [M - $\left.{ }^{i} \mathrm{Pr}_{2} \mathrm{PS}\right]^{+}, 239$ (100) $\left[\mathrm{M}-\mathrm{Br}-2{ }^{i} \mathrm{Pr}\right]^{+}$.

Elementaranalyse (gef. (ber.) [\%]): C 59.83 (59.26), H 5.63 (5.47).

\subsubsection{9-Brom-10-(diisopropylselenophosphoryl)anthracen (8)}

Die Synthese wurde analog zur Darstellung von 7 aus $580 \mathrm{mg}(1.55 \mathrm{mmol}) 5$ und $150 \mathrm{mg}(1.90 \mathrm{mmol})$ grauem Selen in $30 \mathrm{~mL}$ Toluol und anschließender Kristallisation aus heißem Toluol durchgeführt.

Summenformel: $\mathrm{C}_{20} \mathrm{H}_{22} \mathrm{BrPSe}$

Ausbeute:

$640 \mathrm{mg}(1.42 \mathrm{mmol}), 91 \%$

Molare Masse: $\quad 452.22 \mathrm{~g} / \mathrm{mol}$

${ }^{1} \mathrm{H}$-NMR (500.130 MHz, $\left.\mathrm{CDCl}_{3}\right): \delta 9.30\left(\mathrm{~d},{ }^{3} \mathrm{~J}_{\mathrm{HH}}=8.66 \mathrm{~Hz}, 2 \mathrm{H}, \mathrm{H}_{4,5}, \mathrm{An}\right), 8.61(\mathrm{~d}$, $\left.{ }^{3} J_{\mathrm{HH}}=8.14 \mathrm{~Hz}, 2 \mathrm{H}, \mathrm{H}_{1,8}, \mathrm{An}\right), 7.56\left(\mathrm{ddd},{ }^{3} \mathrm{~J}_{\mathrm{HH}}=8.14 \mathrm{~Hz},{ }^{3} \mathrm{~J}_{\mathrm{HH}}=6.58 \mathrm{~Hz},{ }^{4} J_{\mathrm{HH}}=\right.$ $1.30 \mathrm{~Hz}, 2 \mathrm{H}, \mathrm{H}_{2,7}, \mathrm{An}$ ), 7.53 (ddd, ${ }^{3} \mathrm{~J}_{\mathrm{HH}}=8.66 \mathrm{~Hz},{ }^{3} \mathrm{~J}_{\mathrm{HH}}=6.58 \mathrm{~Hz},{ }^{4} J_{\mathrm{HH}}=1.55 \mathrm{~Hz}$, $2 \mathrm{H}, \mathrm{H}_{3,6}, \mathrm{An}$ ), 3.31 (dsept, ${ }^{2} \mathrm{~J}_{\mathrm{HP}}=5.28 \mathrm{~Hz},{ }^{3} \mathrm{~J}_{\mathrm{HH}}=6.75 \mathrm{~Hz}, 2 \mathrm{H}, \mathrm{CH},{ }^{i} \mathrm{Pr}$ ), 1.43 (dd, $\left.{ }^{3} J_{\mathrm{HP}}=18.6 \mathrm{~Hz},{ }^{3} \mathrm{JHH}_{\mathrm{HH}}=6.63 \mathrm{~Hz}, 6 \mathrm{H}, \alpha-\mathrm{C}_{3},{ }^{i} \mathrm{Pr}\right), 0.86 \mathrm{ppm}\left(\mathrm{dd},{ }^{3} J_{\mathrm{HP}}=19.0 \mathrm{~Hz},{ }^{3} J_{\mathrm{HH}}=\right.$ $\left.6.93 \mathrm{~Hz}, 6 \mathrm{H}, \beta-\mathrm{CH}_{3},{ }^{\prime} \mathrm{Pr}\right)$.

${ }^{13} \mathrm{C}\left\{{ }^{1} \mathrm{H}\right\}-N M R\left(125.756 \mathrm{MHz}, \mathrm{CDCl}_{3}\right): \delta 134.7\left(\mathrm{~d},{ }^{2} \mathrm{~J}_{\mathrm{CP}}=6.48 \mathrm{~Hz}, 2 \mathrm{C}, \mathrm{C}_{4 \mathrm{a}, 10 \mathrm{a}}, \mathrm{An}\right)$, $130.5\left(\mathrm{~d},{ }^{4} J_{\mathrm{CP}}=9.65 \mathrm{~Hz}, 1 \mathrm{C}, \mathrm{C}_{9}, \mathrm{An}\right), 130.3\left(\mathrm{~d},{ }^{3} J_{\mathrm{CP}}=4.61 \mathrm{~Hz}, 2 \mathrm{C}, \mathrm{C}_{8 \mathrm{a}, 9 a}, A n\right)$, 128.5 (s, 2 C, $C_{1,8}, A n$ ), 127.0 (s, 2 C, C $\left.3,6, A n\right), 126.9$ (d, ${ }^{3} J_{\mathrm{CP}}=6.00 \mathrm{~Hz}, 2 \mathrm{C}, \mathrm{C}_{4,5}$, $\mathrm{An}), 125.2$ (s, $\left.2 \mathrm{C}, \mathrm{C}_{2,7}, \mathrm{An}\right), 120.5\left(\mathrm{~d},{ }^{1} \mathrm{~J}_{\mathrm{CP}}=52.5 \mathrm{~Hz}, 1 \mathrm{C}, \mathrm{C}_{10}, \mathrm{An}\right), 31.7\left(\mathrm{~d},{ }^{1} \mathrm{~J}_{\mathrm{CP}}=\right.$ $\left.40.5 \mathrm{~Hz}, 2 \mathrm{C}, \underline{\mathrm{C}} \mathrm{H},{ }^{\prime} \mathrm{Pr}\right), 19.5$ (s, $2 \mathrm{C}, \alpha-\underline{\mathrm{C}} \mathrm{H}_{3}$, 'Pr), $18.5 \mathrm{ppm}$ (s, $\left.2 \mathrm{C}, \beta-\underline{\mathrm{C}} \mathrm{H}_{3},{ }^{i} \mathrm{Pr}\right)$.

${ }^{31} \mathrm{P}\left\{{ }^{1} \mathrm{H}\right\}-N M R\left(202.456 \mathrm{MHz}, \mathrm{CDCl}_{3}\right): \delta 66.3 \mathrm{ppm}(\mathrm{s})$.

${ }^{77} \mathrm{Se}\left\{{ }^{1} \mathrm{H}\right\}-N M R\left(95.382 \mathrm{MHz}, \mathrm{CDCl}_{3}\right): \delta-354.2 \mathrm{ppm}\left(\mathrm{d},{ }^{1} \mathrm{~J}_{\mathrm{SeP}}=715.0 \mathrm{~Hz}\right)$.

El-MS (70 eV): m/z (\%): 452 (42) [M] ${ }^{+}, 409$ (12) [M - $\left.{ }^{i} \mathrm{Pr}\right]^{+}, 374$ (37) [M - Se)] ${ }^{+}, 287$ (100) $\left[\mathrm{M}-\mathrm{Br}-2{ }^{i} \mathrm{Pr}\right]^{+}, 256$ (32) [M - $\left.{ }^{i} \mathrm{Pr}_{2} \mathrm{PSe}\right]^{+}$.

Elementaranalyse (gef. (ber.) [\%]): C 53.58 (53.12), H 5.07 (4.90). 


\subsubsection{Wirt/Gast-Komplexe von 9,10-Bis(diphenylthiophosphoryl)- anthracen (9-12)}

Die Darstellung des Toluol-Komplexes [2 $\left.\mathrm{C}_{7} \mathrm{H}_{8} @\left\{\left(\mathrm{Ph}_{2}(\mathrm{~S}) \mathrm{P}\right)_{2}\left(\mathrm{C}_{14} \mathrm{H}_{8}\right)\right\}\right]$ (9) erfolgte gemäß den publizierten Vorschriften. ${ }^{[112,113]}$ Die toluolfreie Verbindung wurde durch mehrstündiges Erhitzen i. Vak. auf $100^{\circ} \mathrm{C}$ erhalten. Die anschließende Kristallisation aus Benzol, Phenylacetylen bzw. Deuterochloroform lieferte die Wirt/Gast-Komplexe 10-12, deren Charakterisierungen mittels Einkristall-Röntgenstrukturanalysen erfolgten.

Bisher nicht publizierte analytische Daten für $\left(\mathrm{Ph}_{2}(\mathrm{~S}) \mathrm{P}\right)_{2}\left(\mathrm{C}_{14} \mathrm{H}_{8}\right)$ :

Summenformel: $\mathrm{C}_{38} \mathrm{H}_{28} \mathrm{P}_{2} \mathrm{~S}_{2}$

Molare Masse: $\quad 610.70 \mathrm{~g} / \mathrm{mol}$ ${ }^{1} \mathrm{H}-\mathrm{NMR}\left(\mathbf{5 0 0 . 1 3 0 ~ M H z}, \mathrm{CDCl}_{3}\right): \delta 8.13-8.10\left(\mathrm{~m}, 4 \mathrm{H}, \mathrm{H}_{1,4,5,8}, \mathrm{An}\right), 7.76-7.71(\mathrm{~m}$, $\left.8 \mathrm{H}, \mathrm{H}_{\text {ortho }}, \mathrm{Ph}\right), 7.39$ - $7.34\left(\mathrm{~m}, 4 \mathrm{H}, \mathrm{H}_{\text {para }}, \mathrm{Ph}\right), 7.33$ - $7.24\left(\mathrm{~m}, 8 \mathrm{H}, \mathrm{H}_{\text {meta }}, \mathrm{Ph}\right), 6.91$ $6.87 \mathrm{ppm}\left(\mathrm{m}, 4 \mathrm{H}, \mathrm{H}_{1,4,5,8}, \mathrm{An}\right)$.

${ }^{13} \mathrm{C}\left\{{ }^{1} \mathrm{H}\right\}-N M R\left(125.756 \mathrm{MHz}, \mathrm{CDCl}_{3}\right): \delta 136.5\left(\mathrm{~d},{ }^{1} \mathrm{~J}_{\mathrm{CP}}=83.9 \mathrm{~Hz}, 4 \mathrm{C}, \mathrm{C}_{\text {ipso }}, \mathrm{Ph}\right)$,

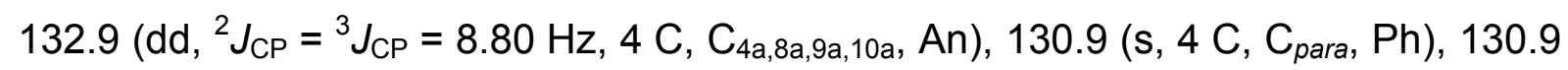
(d, ${ }^{2} J_{\mathrm{CP}}=10.4 \mathrm{~Hz}, 8 \mathrm{C}, \mathrm{C}_{\text {ortho }}, \mathrm{Ph}$ ), 129.7 (dd, ${ }^{1} \mathrm{~J}_{\mathrm{CP}}=86.0 \mathrm{~Hz},{ }^{4} \mathrm{~J}_{\mathrm{CP}}=4.71 \mathrm{~Hz}, 2 \mathrm{C}$, $\left.\mathrm{C}_{9,10}, \mathrm{An}\right), 128.5\left(\mathrm{~d},{ }^{3} \mathrm{~J}_{\mathrm{CP}}=12.7 \mathrm{~Hz}, 8 \mathrm{C}, \mathrm{C}_{\text {meta }}, \mathrm{Ph}\right), 127.3-127.1\left(\mathrm{~m}, 4 \mathrm{C}, \mathrm{C}_{1,4,5,8}\right.$, An), 125.2 ppm (s, 4 C, $\left.\mathrm{C}_{1,4,5,8}, \mathrm{An}\right)$.

\subsubsection{9,10-Bis(diisopropylphosphanyl)anthracen (13)}

Eine Suspension von $710 \mathrm{mg}$ (2.11 mmol) 9,10-Dibromanthracen in $40 \mathrm{~mL}$ Diethylether wurde bei $-15^{\circ} \mathrm{C}$ mit $2.52 \mathrm{~mL}(4.23 \mathrm{mmol}){ }^{n} \mathrm{BuLi}$ in $n$-Hexan $(1.68 \mathrm{M})$ versetzt. Nach Erwärmen auf RT (ca. $1 \mathrm{~h}$ ) wurde eine Lösung von $650 \mathrm{mg}(4.26 \mathrm{mmol})$ Chlordiisopropylphosphan in $10 \mathrm{~mL}$ Diethylether innerhalb von 30 min zugetropft. Nach $4 \mathrm{~h}$ wurde die Reaktionsmischung über Celite filtriert und das Filtrat i. Vak. eingeengt. Durch Lagerung einer gesättigten Lösung in Toluol bei RT wurden nach einigen Tagen Kristalle erhalten, die zur kristallographischen Strukturbestimmung geeignet waren.

Summenformel: $\mathrm{C}_{26} \mathrm{H}_{36} \mathrm{P}_{2}$

Ausbeute: $\quad 820 \mathrm{mg}(2.00 \mathrm{mmol}), 95 \%$

${ }^{1} \mathrm{H}-\mathrm{NMR}$ (500.130 MHz, $\mathrm{CDCl}_{3}$ ): $\delta 9.80-8.80$ (br s, $\left.4 \mathrm{H}, \mathrm{H}_{1,4,5,8}, \mathrm{An}\right), 7.39-7.36$ (m, $4 \mathrm{H}, \mathrm{H}_{2,3,6,7}, \mathrm{An}$ ), 2.77 (dsept, ${ }^{2} \mathrm{~J}_{\mathrm{HP}}={ }^{3} \mathrm{~J}_{\mathrm{HH}}=7.04 \mathrm{~Hz}, 4 \mathrm{H}, \mathrm{CH},{ }^{i} \mathrm{Pr}$ ), 1.32 (dd, ${ }^{3} \mathrm{~J}_{\mathrm{HP}}=$
Molare Masse: $\quad 410.49 \mathrm{~g} / \mathrm{mol}$

Schmelzpunkt: $152{ }^{\circ} \mathrm{C}$ 
$\left.17.6 \mathrm{~Hz},{ }^{3} \mathrm{~J}_{\mathrm{HH}}=6.73 \mathrm{~Hz}, 12 \mathrm{H}, \alpha-\underline{C}_{3},{ }^{i} \mathrm{Pr}\right), 0.65 \mathrm{ppm}\left(\mathrm{dd},{ }^{3} J_{\mathrm{HP}}=13.5 \mathrm{~Hz},{ }^{3} \mathrm{~J}_{\mathrm{HH}}=\right.$ $\left.6.98 \mathrm{~Hz}, 12 \mathrm{H}, \beta-\mathrm{C}_{3},{ }^{i} \mathrm{Pr}\right)$.

$\left.{ }^{13} \mathrm{C}^{1}{ }^{1} \mathrm{H}\right\}-N M R$ (125.756 MHz, $\left.\mathrm{CDCl}_{3}\right): \delta 136.2-135.9$ (m, 4 C, C4a,8a,9a,10a, An),

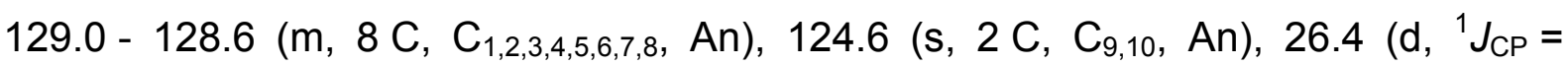
$\left.14.06 \mathrm{~Hz}, 4 \mathrm{C}, \underline{\mathrm{CH}},{ }^{i} \mathrm{Pr}\right), 23.1$ (d, $\left.{ }^{2} J_{\mathrm{CP}}=29.2 \mathrm{~Hz}, 4 \mathrm{C}, \alpha-\underline{\mathrm{CH}}_{3},{ }^{i} \mathrm{Pr}\right), 21.2 \mathrm{ppm}\left(\mathrm{d},{ }^{2} J_{\mathrm{CP}}=\right.$ $\left.13.6 \mathrm{~Hz}, 4 \mathrm{C}, \beta-\underline{\mathrm{CH}}_{3},{ }^{i} \mathrm{Pr}\right)$.

${ }^{31} \mathrm{P}\left\{{ }^{1} \mathrm{H}\right\}-N M R\left(202.456 \mathrm{MHz}, \mathrm{CDCl}_{3}\right): \delta 1.72 \mathrm{ppm}(\mathrm{s})$.

El-MS (70 eV): $\boldsymbol{m} / \mathbf{z}$ (\%): 410 (91) [M] ${ }^{+}, 367$ (49) [M - $\left.{ }^{i} \mathrm{Pr}\right]^{+}, 325$ (100) [M - $\left.2^{i} \mathrm{Pr}+\mathrm{H}\right]^{+}$.

Elementaranalyse (gef. (ber.) [\%]): C 75.80 (76.07), H 8.87 (8.84).

\subsubsection{9,10-Bis(diisopropylthiophosphoryl)anthracen (14)}

Eine Lösung von $200 \mathrm{mg}(0.487 \mathrm{mmol}) 13$ in $15 \mathrm{~mL}$ Toluol wurde mit $32 \mathrm{mg}$ $(1.00 \mathrm{mmol})$ Schwefel versetzt und für $2 \mathrm{~h}$ unter Rückfluss erhitzt. Nach Abkühlen auf RT wurde das Lösungsmittel i. Vak. entfernt. Durch Lagerung einer gesättigten Lösung in Toluol / THF (1:1) bei RT wurden nach 1.5 a Kristalle erhalten, die zur röntgenkristallographischen Strukturuntersuchung geeignet waren.

Summenformel: $\mathrm{C}_{26} \mathrm{H}_{36} \mathrm{P}_{2} \mathrm{~S}_{2} \quad$ Molare Masse: $474.61 \mathrm{~g} / \mathrm{mol}$ Ausbeute: $\quad 200 \mathrm{mg}(0.421 \mathrm{mmol}), 87 \% \quad$ Schmelzpunkt: $196{ }^{\circ} \mathrm{C}$ ${ }^{1} \mathrm{H}$-NMR (500.130 MHz, $\mathrm{CDCl}_{3}$ ): $\delta 9.05-8.98$ (br s, $\left.4 \mathrm{H}, \mathrm{H}_{1,4,5,8}, \mathrm{An}\right), 7.48-7.44$ (m, $4 \mathrm{H}, \mathrm{H}_{2,3,6,7}, \mathrm{An}$ ), 3.17 (dsept, ${ }^{2} J_{\mathrm{HP}}=4.04 \mathrm{~Hz},{ }^{3} J_{\mathrm{HH}}=6.75 \mathrm{~Hz}, 4 \mathrm{H}, \mathrm{C} \underline{\mathrm{H}},{ }^{i} \mathrm{Pr}$ ), 1.42 (dd, $\left.{ }^{3} J_{\mathrm{HP}}=17.9 \mathrm{~Hz},{ }^{3} J_{\mathrm{HH}}=6.65 \mathrm{~Hz}, 12 \mathrm{H}, \alpha-\underline{C}_{3},{ }^{i} \mathrm{Pr}\right), 0.82 \mathrm{ppm}\left(\mathrm{dd},{ }^{3} J_{\mathrm{HP}}=18.6 \mathrm{~Hz}\right.$, $\left.{ }^{3} \mathrm{~J}_{\mathrm{HH}}=6.96 \mathrm{~Hz}, 12 \mathrm{H}, \beta-\underline{\mathrm{H}}_{3},{ }^{i} \mathrm{Pr}\right)$.

${ }^{13} \mathrm{C}\left\{{ }^{1} \mathrm{H}\right\}-N M R\left(125.756 \mathrm{MHz}, \mathrm{CDCl}_{3}\right): \delta 134-124\left(\mathrm{~m}, \mathrm{C}_{\mathrm{An}}\right), 32.3\left(\mathrm{~d},{ }^{1} \mathrm{~J}_{\mathrm{CP}}=48.6 \mathrm{~Hz}\right.$, $\left.4 \mathrm{C}, \alpha-\underline{\mathrm{C}} \mathrm{H}_{3},{ }^{i} \mathrm{Pr}\right), 18.3$ (s, $\left.4 \mathrm{C}, \alpha-\underline{\mathrm{CH}}_{3},{ }^{i} \mathrm{Pr}\right), 17.9 \mathrm{ppm}\left(\mathrm{s}, 4 \mathrm{C}, \beta-\underline{\mathrm{CH}}_{3},{ }^{i} \mathrm{Pr}\right)$.

${ }^{31} \mathrm{P}\left\{{ }^{1} \mathrm{H}\right\}-N M R$ (202.456 MHz, $\left.\mathrm{CDCl}_{3}\right): \delta 73.0 \mathrm{ppm}(\mathrm{s})$.

El-MS (70 eV): $\mathbf{m} / \mathbf{z}(\%): 474$ (16) [M] ${ }^{+}, 431$ (100) [M - $\left.{ }^{i} \mathrm{Pr}\right]^{+}, 388$ (5) [M - $\left.2^{i} \mathrm{Pr}\right]^{+}, 239$ (29) $\left[\mathrm{M}-\mathrm{Br}-2^{i} \mathrm{Pr}\right]^{+}$.

Elementaranalyse (gef. (ber.) [\%]): C 63.49 (65.79), H 7.24 (7.64). 


\subsubsection{9-Diphenylthiophosphoryl-10-(diphenylphosphanyl)- anthracen (15)}

Eine Suspension von $410 \mathrm{mg}$ (0.866 mmol) 9-Brom-10-(diphenylthiophosphoryl)anthracen (3) in $30 \mathrm{~mL}$ Diethylether wurde bei $-10^{\circ} \mathrm{C}$ mit $0.60 \mathrm{~mL}(0.960 \mathrm{mmol}){ }^{n} \mathrm{BuLi}$ in $n$-Hexan $(1.60 \mathrm{M})$ versetzt. Nach $15 \mathrm{~min}$ wurden $200 \mathrm{mg}(0.906 \mathrm{mmol})$ Chlordiphenylphosphan zugegeben und anschließend die Reaktionsmischung auf RT erwärmt. Nach 30 min wurde über Celite filtriert und das Filtrat i. Vak. eingeengt. Durch Umkristallisation des verbleibenden Rückstandes aus Toluol wurde 15 in Form gelber Kristalle erhalten, die zur röntgenkristallographischen Untersuchung geeignet waren.

Es soll erwähnt werden, dass es trotz mehrerer Versuche nicht gelang, die Verbindung erneut zu synthetisieren, um fehlende Analytik nachzuholen.

Summenformel: $\mathrm{C}_{38} \mathrm{H}_{28} \mathrm{P}_{2} \mathrm{~S}$

Ausbeute:
Molare Masse: $\quad 578.60 \mathrm{~g} / \mathrm{mol}$ Schmelzpunkt: n. b.

${ }^{1} \mathrm{H}$-NMR (500.130 MHz, $\left.\mathbf{C D C l}_{3}\right)$ : $\delta 8.69-8.63\left(\mathrm{~m}, 2 \mathrm{H}, \mathrm{H}_{4,5}, \mathrm{An}\right), 8.07\left(\mathrm{~d},{ }^{3} \mathrm{JHH}_{\mathrm{HH}}=\right.$ $\left.8.99 \mathrm{~Hz}, 2 \mathrm{H}, \mathrm{H}_{1,8}, \mathrm{An}\right), 7.77$ - 7.71 (m, $\left.4 \mathrm{H}, \mathrm{H}_{\text {ortho }}, \mathrm{Ph}\right), 7.42$ - 7.37 (m, $4 \mathrm{H}, \mathrm{H}_{\text {ortho }}, \mathrm{Ph}$ ), $7.33-7.25\left(\mathrm{~m}, 12 \mathrm{H}, \mathrm{H}_{\text {meta/para }}, \mathrm{Ph}\right), 7.06$ (ddd, ${ }^{3} \mathrm{~J}_{\mathrm{HH}}=8.99 \mathrm{~Hz},{ }^{3} \mathrm{~J}_{\mathrm{HH}}=6.52 \mathrm{~Hz},{ }^{4} \mathrm{~J}_{\mathrm{HH}}=$ $\left.1.14 \mathrm{~Hz}, 2 \mathrm{H}, \mathrm{H}_{2,7}, \mathrm{An}\right), 6.91 \mathrm{ppm}\left(\mathrm{ddd},{ }^{3} \mathrm{~J}_{\mathrm{HH}}=8.95 \mathrm{~Hz},{ }^{3} \mathrm{~J}_{\mathrm{HH}}=6.58 \mathrm{~Hz},{ }^{4} \mathrm{~J}_{\mathrm{HH}}=\right.$ $\left.1.10 \mathrm{~Hz}, 2 \mathrm{H}, \mathrm{H}_{3,6}, \mathrm{An}\right)$.

${ }^{13} \mathrm{C}\left\{{ }^{1} \mathrm{H}\right\}-N M R\left(125.756 \mathrm{MHz}, \mathrm{CDCl}_{3}\right): \delta 137.0\left(\mathrm{~d},{ }^{1} \mathrm{~J}_{\mathrm{CP}}=103.4 \mathrm{~Hz}\right), 136.2-135.9(\mathrm{~m})$, $133.1-132.9(\mathrm{~m}), 131.8(\mathrm{~s}), 131.7(\mathrm{~s}), 131.6(\mathrm{~s}), 130.8(\mathrm{~s}), 130.6(\mathrm{~s}), 128.8$ - 128.4 (m), $128.3(\mathrm{~s}), 128.1-127.7(\mathrm{~m}), 125.4(\mathrm{~s}), 125.2 \mathrm{ppm}(\mathrm{s})$.

${ }^{31} \mathrm{P}\left\{{ }^{1} \mathrm{H}\right\}-N M R\left(202.456 \mathrm{MHz}, \mathrm{CDCl}_{3}\right): \delta 34.8\left(\mathrm{~s}, \mathrm{P}(\mathrm{S}) \mathrm{Ph}_{2}\right),-19.7 \mathrm{ppm}\left(\mathrm{s}, \mathrm{PPh}_{2}\right)$. EI-MS (70 eV): m/z (\%): 578 (71) [M] $]^{+}, 546$ (100) [M - S] , 393 (42) [M - PPh $]^{+}$.

\subsubsection{9-Diphenylselenophosphoryl-10-(diphenylthiophosphoryl)- anthracen (16)}

Eine Suspension von $110 \mathrm{mg}(0.190 \mathrm{mmol}) 15$ in $15 \mathrm{~mL}$ Toluol wurde mit $15 \mathrm{mg}$ grauem Selen versetzt und für $3 \mathrm{~h}$ unter Rückfluss erhitzt. Nach Abkühlen auf RT wurde die Suspension über Celite filtriert. Durch Einengen des Filtrats i. Vak. wurde 16 als gelbes Pulver erhalten. Lagerung einer gesättigten Lösung in Toluol bei RT lieferte nach einigen Tagen Kristalle, die zur Bestimmung der Molekülstruktur geeignet waren. 
Summenformel: $\quad \mathrm{C}_{38} \mathrm{H}_{28} \mathrm{P}_{2} \mathrm{SSe}$

Ausbeute:
Molare Masse: $\quad 657.60 \mathrm{~g} / \mathrm{mol}$

Schmelzpunkt: $228^{\circ} \mathrm{C}$

${ }^{1} \mathrm{H}$-NMR (500.130 MHz, $\left.\mathrm{CDCl}_{3}\right): \delta 8.17$ - $8.14\left(\mathrm{~m}, 4 \mathrm{H}, \mathrm{H}_{1,4,5,8}, \mathrm{An}\right), 7.83-7.74(\mathrm{~m}$, $\left.8 \mathrm{H}, \mathrm{H}_{\text {ortho }}, \mathrm{Ph}\right), 7.40$ - $7.32\left(\mathrm{~m}, 12 \mathrm{H}, \mathrm{H}_{\text {meta/para }} \mathrm{Ph}\right), 6.94$ - $6.91 \mathrm{ppm}\left(\mathrm{m}, 4 \mathrm{H}, \mathrm{H}_{2,3,6,7}\right.$, An).

${ }^{13} \mathrm{C}\left\{{ }^{1} \mathrm{H}\right\}-N M R\left(125.756 \mathrm{MHz}, \mathrm{CDCl}_{3}\right): \delta 136.6\left(\mathrm{~d},{ }^{1} \mathrm{~J}_{\mathrm{CP}}=83.6 \mathrm{~Hz}, 2 \mathrm{C}, \mathrm{C}_{\text {ipso }}, \mathrm{Ph}\right)$,

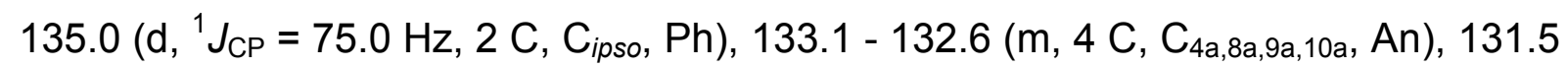
(d, $\left.{ }^{2} J_{\mathrm{CP}}=10.7 \mathrm{~Hz}, 8 \mathrm{C}, \mathrm{C}_{\text {ortho }}, \mathrm{Ph}\right), 131.0-130.9\left(\mathrm{~m}, 12 \mathrm{C}, \mathrm{C}_{\text {meta/para }}, \mathrm{Ph}\right), 128.6$ (d, $\left.{ }^{3} J_{\mathrm{CP}}=7.81 \mathrm{~Hz}, 4 \mathrm{C}, \mathrm{C}_{1,4,5,8}, \mathrm{An}\right), 127.4-127.2$ (m, 4 C, $\left.\mathrm{C}_{2,3,6,7}, \mathrm{An}\right), 125.2 \mathrm{ppm}(\mathrm{d}$, $\left.{ }^{1} J_{\mathrm{CP}}=31.6 \mathrm{~Hz}, 2 \mathrm{C}, \mathrm{C}_{9,10}, \mathrm{An}\right)$.

$\left.{ }^{31} \mathrm{P}^{1} \mathrm{H}\right\}-N M R\left(202.456 \mathrm{MHz}, \mathrm{CDCl}_{3}\right): \delta 35.7\left(\mathrm{~d},{ }^{5} \mathrm{JPP}_{\mathrm{PP}}=4.51 \mathrm{~Hz}, \mathrm{P}(\mathrm{S}) \mathrm{Ph}_{2}\right), 25.9 \mathrm{ppm}$ (d, ${ }^{5} \mathrm{JPP}_{\mathrm{PP}}=4.51 \mathrm{~Hz}, \mathrm{P}(\mathrm{Se}) \mathrm{Ph}_{2}$ ).

${ }^{77} \mathrm{Se}\left\{{ }^{1} \mathrm{H}\right\}-N M R\left(95.382 \mathrm{MHz}, \mathrm{CDCl}_{3}\right): \delta-260.1\left(\mathrm{~d},{ }^{1} \mathrm{~J}_{\mathrm{SeP}}=740.2 \mathrm{~Hz}\right)$.

El-MS (70 eV): $m / z(\%): 658(21)[M]^{+}, 626(8)[\mathrm{M}-\mathrm{S}]^{+}, 578$ (100) [M - Se] $]^{+}, 546$ (43) $[\mathrm{M}-\mathrm{S}-\mathrm{Se}]^{+}, 393(73)\left[\mathrm{M}-\mathrm{Ph}_{2} \mathrm{PSe}\right]^{+}, 185(87)\left[\mathrm{PPh}_{2}\right]^{+}$.

Elementaranalyse (gef. (ber.) [\%]): C 70.68 (69.41), H 5.04 (4.29).

\subsubsection{9-Diphenylphosphanyl-10-(diisopropylphosphanyl)- anthracen (17)}

Eine Suspension von $1.40 \mathrm{~g}$ (3.17 mmol) 9-Brom-10-(diphenylphosphanyl)anthracen (1) in $40 \mathrm{~mL}$ Diethylether wurde bei $-15^{\circ} \mathrm{C}$ mit $1.36 \mathrm{~mL}(3.17 \mathrm{mmol}){ }^{n} \mathrm{BuLi}$ in $n$-Hexan $(2.33 \mathrm{M})$ versetzt. Nach $20 \mathrm{~min}$ wurden $0.48 \mathrm{~g}(3.16 \mathrm{mmol})$ Chlordiisopropylphosphan zugetropft und anschließend die Reaktionsmischung für $1.5 \mathrm{~h}$ gerührt, dabei erwärmte sie sich auf RT. Nach Filtration über Celite und Entfernen des Lösungsmittels i. Vak. wurde 17 als gelbes Pulver erhalten. Durch Lagerung einer gesättigten Lösung in THF/n-Pentan (1:1) wurden nach einigen Tagen Kristalle erhalten, die für eine röntgenkristallographische Strukturbestimmung geeignet waren.

Summenformel: $\mathrm{C}_{32} \mathrm{H}_{32} \mathrm{P}_{2}$

Ausbeute:

$1.00 \mathrm{~g}(2.09 \mathrm{mmol}), 66 \%$
Molare Masse: $\quad 478.52 \mathrm{~g} / \mathrm{mol}$

Schmelzpunkt: $184^{\circ} \mathrm{C}$

${ }^{1} \mathrm{H}$-NMR (500.130 MHz, $\mathrm{CDCl}_{3}$ ): $\delta 8.90\left(\mathrm{br} \mathrm{s}, 2 \mathrm{H}, \mathrm{H}_{4,5}, \mathrm{An}\right), 8.90\left(\mathrm{dd},{ }^{3} \mathrm{~J}_{\mathrm{HH}}=8.49 \mathrm{~Hz}\right.$, $\left.{ }^{4} J_{\mathrm{HP}}=5.53 \mathrm{~Hz}, 2 \mathrm{H}, \mathrm{H}_{1,8}, \mathrm{An}\right), 7.49-7.46\left(\mathrm{~m}(\sim \mathrm{dd}), 2 \mathrm{H}, \mathrm{H}_{3,6}, \mathrm{An}\right), 7.45-7.41$ (m, $\left.4 \mathrm{H}, \mathrm{H}_{\text {ortho }}, \mathrm{Ph}\right), 7.30$ (dd, $\left.{ }^{3} \mathrm{~J}_{\mathrm{HH}}=8.26 \mathrm{~Hz},{ }^{3} \mathrm{~J}_{\mathrm{HH}}=7.01 \mathrm{~Hz}, 2 \mathrm{H}, \mathrm{H}_{2,7}, \mathrm{An}\right), 7.27-7.21$ (m, $6 \mathrm{H}, \mathrm{H}_{\text {meta/para }}, \mathrm{Ph}$ ), 2.97 (dsept, ${ }^{2} \mathrm{~J}_{\mathrm{HP}}={ }^{3} \mathrm{~J}_{\mathrm{HH}}=7.02 \mathrm{~Hz}, 2 \mathrm{H}, \mathrm{C} \underline{\mathrm{H}},{ }^{i} \mathrm{Pr}$ ), 1.47 (dd, 
$\left.{ }^{3} \mathrm{~J}_{\mathrm{HP}}=17.7 \mathrm{~Hz},{ }^{3} \mathrm{~J}_{\mathrm{HH}}=6.72 \mathrm{~Hz}, 6 \mathrm{H}, \alpha-\mathrm{CH}_{3},{ }^{i} \mathrm{Pr}\right), 0.76 \mathrm{ppm}\left(\mathrm{dd},{ }^{3} J_{\mathrm{HP}}=13.8 \mathrm{~Hz},{ }^{3} J_{\mathrm{HH}}=\right.$ $\left.6.94 \mathrm{~Hz}, 6 \mathrm{H}, \beta-\mathrm{C}_{3},{ }^{i} \mathrm{Pr}\right)$.

${ }^{13} \mathrm{C}\left\{{ }^{1} \mathrm{H}\right\}-N M R\left(125.756 \mathrm{MHz}, \mathrm{CDCl}_{3}\right): \delta 138.4\left(\mathrm{~d},{ }^{1} \mathrm{~J}_{\mathrm{CP}}=32.3 \mathrm{~Hz}, 2 \mathrm{C}, \mathrm{C}_{i p s o}, \mathrm{Ph}\right)$, 136.1 - 135.9 (m, 4 C, $\left.C_{4 a, 8 a, 9 a, 10 a}, A n\right), 132.0$ (d, ${ }^{3} J_{C P}=21.6 \mathrm{~Hz}, 2 \mathrm{C}, \mathrm{C}_{4,5}, \mathrm{An}$ ), 131.5 (d, $\left.{ }^{2} J_{\mathrm{CP}}=18.4 \mathrm{~Hz}, 4 \mathrm{C}, \mathrm{C}_{\text {ortho }}, \mathrm{Ph}\right), 129.4\left(\mathrm{~d},{ }^{3} \mathrm{~J}_{\mathrm{CP}}=26.1 \mathrm{~Hz}, 2 \mathrm{C}, \mathrm{H}_{1,8}, \mathrm{An}\right), 128.4$ (d, $\left.{ }^{3} J_{\mathrm{CP}}=5.50 \mathrm{~Hz}, 4 \mathrm{C}, \mathrm{C}_{\text {meta }}, \mathrm{Ph}\right), 127.5$ (s, 2 C, C para, $\left.\mathrm{Ph}\right), 125.5$ (d, ${ }^{1} J_{\mathrm{CP}}=63.2 \mathrm{~Hz}$, $\left.2 \mathrm{C}, \mathrm{C}_{9,10}, \mathrm{An}\right), 125.1$ (s, $\left.2 \mathrm{C}, \mathrm{C}_{2,7}, \mathrm{An}\right), 124.9$ (s, $\left.2 \mathrm{C}, \mathrm{C}_{3,6}, \mathrm{An}\right), 26.5$ (d, ${ }^{1} \mathrm{~J}_{\mathrm{CP}}=$ $\left.14.1 \mathrm{~Hz}, 2 \mathrm{C}, \underline{\mathrm{C}} \mathrm{H},{ }^{\mathrm{i}} \mathrm{Pr}\right), 23.2\left(\mathrm{~d},{ }^{2} J_{\mathrm{CP}}=29.2 \mathrm{~Hz}, 2 \mathrm{C}, \alpha-\underline{\mathrm{C}} \mathrm{H}_{3},{ }^{i} \mathrm{Pr}\right), 21.3 \mathrm{ppm}\left(\mathrm{d},{ }^{2} J_{\mathrm{CP}}=\right.$ $\left.13.6 \mathrm{~Hz}, 2 \mathrm{C}, \beta-\mathrm{CH}_{3},{ }^{i} \mathrm{Pr}\right)$.

$\left.{ }^{31} \mathrm{P}^{1}{ }^{1} \mathrm{H}\right\}-N M R\left(202.456 \mathrm{MHz}, \mathrm{CDCl}_{3}\right): \delta 2.65$ (s, $\left.\mathrm{P}^{\mathrm{i}} \mathrm{Pr}_{2}\right),-21.6 \mathrm{ppm}\left(\mathrm{s}, \mathrm{PPh}_{2}\right)$.

El-MS (70 eV): $\mathbf{m} / \mathbf{z}(\%): 478$ (100) $[\mathrm{M}]^{+}, 435$ (33) [M - $\left.{ }^{i} \mathrm{Pr}\right]^{+}, 393$ (72) $\left[\mathrm{M}-2^{i} \mathrm{Pr}+\mathrm{H}\right]^{+}$, 315 (33) $\left[\mathrm{M}-\mathrm{Ph}-2^{i} \mathrm{Pr}\right]^{+}$.

Elementaranalyse (gef. (ber.) [\%]): C 79.60 (80.32), H 6.63 (6.74).

\subsubsection{9-Diphenylphosphoryl-10-(diisopropylphosphoryl)anthracen} (18)

Eine Lösung von $1.00 \mathrm{~g}(2.09 \mathrm{mmol}) 17$ in $30 \mathrm{~mL}$ Dichlormethan wurde bei $-15{ }^{\circ} \mathrm{C}$ mit $0.50 \mathrm{~g}(5.32 \mathrm{mmol}) \mathrm{H}_{2} \mathrm{O}_{2} \cdot\left(\mathrm{H}_{2} \mathrm{~N}\right)_{2} \mathrm{C}=\mathrm{O}$ versetzt. Nach $1 \mathrm{~h}$ wurde die Reaktionsmischung mit Wasser gewaschen $(2 \times 10 \mathrm{~mL})$ und die organische Phase über Natriumsulfat getrocknet. Nach Abtrennen des Trockenmittels und Entfernen des Lösungsmittels i. Vak. wurde 18 als gelbes, schaumiger Rückstand erhalten. Beim langsamen Abkühlen einer in Toluol gesättigten Lösung bildeten sich Kristalle, die für eine Röntgenstrukturanalyse geeignet waren.

Summenformel: $\mathrm{C}_{32} \mathrm{H}_{32} \mathrm{O}_{2} \mathrm{P}_{2}$

Ausbeute: $\quad 940 \mathrm{mg}(1.84 \mathrm{mmol}), 88 \%$
Molare Masse: $\quad 510.52 \mathrm{~g} / \mathrm{mol}$

Schmelzpunkt: $226{ }^{\circ} \mathrm{C}$

${ }^{1} \mathrm{H}-\mathrm{NMR}\left(\mathbf{5 0 0 . 1 3 0 ~ M H z}, \mathrm{CDCl}_{3}\right): \delta 9.03\left(\mathrm{br} \mathrm{s}, 2 \mathrm{H}, \mathrm{H}_{4,5}, \mathrm{An}\right), 8.45\left(\mathrm{~d},{ }^{3} \mathrm{~J}_{\mathrm{HH}}=8.04 \mathrm{~Hz}\right.$, $\left.2 \mathrm{H}, \mathrm{H}_{1,8}, \mathrm{An}\right), 7.62$ - 7.58 (m, $\left.4 \mathrm{H}, \mathrm{H}_{\text {ortho }}, \mathrm{Ph}\right), 7.49$ - 7.45 (m, $\left.2 \mathrm{H}, \mathrm{H}_{\text {para }}, \mathrm{Ph}\right), 7.44$ 7.41 (m ( dd), $\left.2 \mathrm{H}, \mathrm{H}_{3,6}, \mathrm{An}\right), 7.39-7.35$ (m, $\left.4 \mathrm{H}, \mathrm{H}_{\text {meta }}, \mathrm{Ph}\right), 7.18\left(\mathrm{dd},{ }^{3} \mathrm{~J}_{\mathrm{HH}}=8.63 \mathrm{~Hz}\right.$, $\left.{ }^{3} J_{\mathrm{HH}}=6.79 \mathrm{~Hz}, 2 \mathrm{H}, \mathrm{H}_{2,7}, \mathrm{An}\right), 2.86-2.80\left(\mathrm{~m}, 2 \mathrm{H}, \mathrm{C} \underline{\mathrm{H}},{ }^{i} \mathrm{Pr}\right), 1.54\left(\mathrm{dd},{ }^{3} \mathrm{~J}_{\mathrm{HP}}=15.0 \mathrm{~Hz}\right.$, $\left.{ }^{3} \mathrm{~J}_{\mathrm{HH}}=6.90 \mathrm{~Hz}, 6 \mathrm{H}, \alpha-\underline{\mathrm{C}}_{3},{ }^{\mathrm{i}} \mathrm{Pr}\right), 0.97 \mathrm{ppm}\left(\mathrm{dd},{ }^{3} \mathrm{~J}_{\mathrm{HP}}=16.5 \mathrm{~Hz},{ }^{3} \mathrm{~J}_{\mathrm{HH}}=7.17 \mathrm{~Hz}, 6 \mathrm{H}\right.$, $\left.\beta-\underline{\mathrm{H}}_{3},{ }^{i} \mathrm{Pr}\right)$.

${ }^{13} \mathrm{C}\left\{{ }^{1} \mathrm{H}\right\}-N M R\left(125.756 \mathrm{MHz}, \mathrm{CDCl}_{3}\right): \delta 135.7\left(\mathrm{~d},{ }^{1} \mathrm{~J}_{\mathrm{CP}}=104.2 \mathrm{~Hz}, 2 \mathrm{C}, \mathrm{C}_{i p s o}, \mathrm{Ph}\right)$,

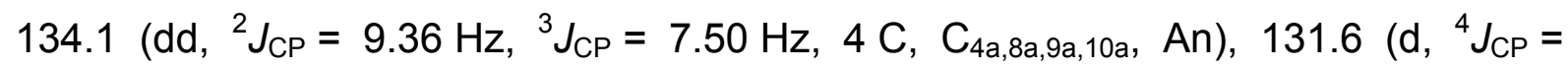
$\left.2.76 \mathrm{~Hz}, 2 \mathrm{C}, \mathrm{C}_{\text {para }}, \mathrm{Ph}\right), 131.3\left(\mathrm{~d},{ }^{2} \mathrm{~J}_{\mathrm{CP}}=9.98 \mathrm{~Hz}, 4 \mathrm{C}, \mathrm{C}_{\text {ortho }}, \mathrm{Ph}\right), 128.9\left(\mathrm{~d},{ }^{1} J_{\mathrm{CP}}=\right.$ 
$\left.97.9 \mathrm{~Hz}, 1 \mathrm{C}, \mathrm{C}_{9}, \mathrm{An}\right), 128.8\left(\mathrm{~d},{ }^{1} J_{\mathrm{CP}}=97.8 \mathrm{~Hz}, 1 \mathrm{C}, \mathrm{C}_{10}, \mathrm{An}\right), 128.7\left(\mathrm{~d},{ }^{3} J_{\mathrm{CP}}=\right.$ $12.4 \mathrm{~Hz}, 4 \mathrm{C}, \mathrm{C}_{\text {meta }}, \mathrm{Ph}$ ), 127.9 (d, ${ }^{3} \mathrm{~J}_{\mathrm{CP}}=7.99 \mathrm{~Hz}, 2 \mathrm{C}, \mathrm{C}_{1,8}, \mathrm{An}$ ), 126.3 (s, $2 \mathrm{C}, \mathrm{C}_{4,5}$, An), 125.9 (s, 2 C, $\left.C_{3,6}, A n\right), 125.5$ (s, 2 C, $\left.C_{2,7}, A n\right), 31.3\left(d,{ }^{1} J_{C P}=64.2 \mathrm{~Hz}, 2 \mathrm{C}, \underline{\mathrm{C}}\right.$, $\left.{ }^{i} \mathrm{Pr}\right), 17.4\left(\mathrm{~d},{ }^{2} J_{\mathrm{CP}}=3.26 \mathrm{~Hz}, 2 \mathrm{C}, \alpha-\underline{\mathrm{C}} \mathrm{H}_{3},{ }^{i} \mathrm{Pr}\right), 17.1 \mathrm{ppm}\left(\mathrm{d},{ }^{2} J_{\mathrm{CP}}=3.31 \mathrm{~Hz}, 2 \mathrm{C}\right.$, $\left.\beta-\underline{\mathrm{C}} \mathrm{H}_{3},{ }^{\mathrm{i}} \mathrm{Pr}\right)$.

$\left.{ }^{31} \mathrm{P}^{1} \mathrm{H}\right\}-N M R\left(202.456 \mathrm{MHz}, \mathrm{CDCl}_{3}\right): \delta 58.8\left(\mathrm{~d},{ }^{5} \mathrm{JPP}_{\mathrm{PP}}=3.98 \mathrm{~Hz}, \mathrm{P}(\mathrm{O}){ }^{i} \mathrm{Pr}_{2}\right), 30.3 \mathrm{ppm}$ (d, ${ }^{5} J_{\mathrm{PP}}=3.98 \mathrm{~Hz}, \mathrm{P}(\mathrm{O}) \mathrm{Ph}_{2}$ ).

EI-MS (70 eV): $\mathbf{m} / \mathbf{z}(\%): 510$ (100) [M] ${ }^{+}, 467$ (26) [M - $\left.{ }^{i} \mathrm{Pr}\right]^{+}, 377$ (90) [M - $\left.{ }^{i} \mathrm{Pr}_{2} \mathrm{PO}\right]^{+}$, 309 (7) [M - $\left.\mathrm{Ph}_{2} \mathrm{PO}\right]^{+}, 201(23)\left[\mathrm{Ph}_{2} \mathrm{PO}\right]^{+}$.

Elementaranalyse (gef. (ber.) [\%]): C 75.08 (75.28), H 6.31 (6.32).

\subsubsection{9-Diphenylthiophosphoryl-10-(diisopropylthiophosphoryl)- anthracen (19)}

Eine Suspension von $790 \mathrm{mg}(1.65 \mathrm{mmol}) 17$ in $30 \mathrm{~mL}$ Toluol wurde mit $110 \mathrm{mg}$ (3.43 mmol) Schwefel versetzt und für $2.5 \mathrm{~h}$ unter Rückfluss erhitzt. Nach Abkühlen auf RT wurde die Suspension über Celite filtriert und das Filtrat i. Vak. eingeengt. Durch Umkristallisation des Rückstandes aus Toluol wurden gelbe Kristalle erhalten, die zur Bestimmung der Molekülstruktur geeignet waren.

Summenformel: $\mathrm{C}_{32} \mathrm{H}_{32} \mathrm{P}_{2} \mathrm{~S}_{2}$

Molare Masse: $\quad 542.67 \mathrm{~g} / \mathrm{mol}$

Ausbeute: $\quad 590 \mathrm{mg}(1.09 \mathrm{mmol}), 66 \% \quad$ Schmelzpunkt: $253^{\circ} \mathrm{C}$

${ }^{1} \mathrm{H}-\mathrm{NMR}\left(\mathbf{5 0 0 . 1 3 0 ~ M H z}, \mathrm{CDCl}_{3}\right): \delta 9.06\left(\mathrm{br} \mathrm{d},{ }^{3} \mathrm{~J}_{\mathrm{HH}}=6.97 \mathrm{~Hz}, 2 \mathrm{H}, \mathrm{H}_{4,5}, \mathrm{An}\right), 8.18(\mathrm{~d}$, $\left.{ }^{3} J_{\mathrm{HH}}=8.87 \mathrm{~Hz}, 2 \mathrm{H}, \mathrm{H}_{1,8}, \mathrm{An}\right), 7.64\left(\mathrm{dd},{ }^{3} \mathrm{~J}_{\mathrm{HH}}=13.1 \mathrm{~Hz},{ }^{4} J_{\mathrm{HH}}=7.52 \mathrm{~Hz}, 4 \mathrm{H}, \mathrm{H}_{\text {ortho }}\right.$, $\mathrm{Ph}$ ), 7.35 - 7.29 (m, $4 \mathrm{H}, \mathrm{H}_{3,6}, \mathrm{An} / \mathrm{H}_{\text {para }}, \mathrm{Ph}$ ), 7.26 - 7.22 (m, $4 \mathrm{H}, \mathrm{H}_{\text {meta }}, \mathrm{Ph}$ ), 7.03 (dd, $\left.{ }^{3} J_{\mathrm{HH}}=8.69 \mathrm{~Hz},{ }^{3} \mathrm{~J}_{\mathrm{HH}}=6.71 \mathrm{~Hz}, 2 \mathrm{H}, \mathrm{H}_{2,7}, A n\right), 3.25$ (dsept, ${ }^{3} J_{\mathrm{HH}}=6.77 \mathrm{~Hz},{ }^{2} J_{\mathrm{HP}}=$ $4.00 \mathrm{~Hz}, 2 \mathrm{H}, \mathrm{CH},{ }^{i} \mathrm{Pr}$ ), 1.48 (dd, ${ }^{3} J_{\mathrm{HP}}=18.0 \mathrm{~Hz},{ }^{3} J_{\mathrm{HH}}=6.62 \mathrm{~Hz}, 6 \mathrm{H}, \alpha-\underline{\mathrm{C}}_{3},{ }^{i} \mathrm{Pr}$ ), $0.93 \mathrm{ppm}\left(\mathrm{dd},{ }^{3} \mathrm{~J}_{\mathrm{HP}}=18.6 \mathrm{~Hz},{ }^{3} \mathrm{JHH}_{\mathrm{HH}}=6.91 \mathrm{~Hz}, 6 \mathrm{H}, \beta-\underline{\mathrm{CH}}_{3},{ }^{i} \mathrm{Pr}\right)$.

${ }^{13} \mathrm{C}\left\{{ }^{1} \mathrm{H}\right\}$-NMR (125.756 MHz, CDCl 3$): \delta 136.3\left(\mathrm{~d},{ }^{1} J_{\mathrm{CP}}=83.7 \mathrm{~Hz}, 2 \mathrm{C}, \mathrm{C}_{\text {ipso }}, \mathrm{Ph}\right)$,

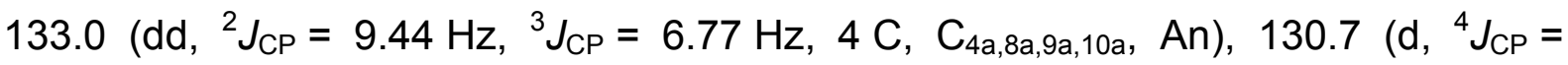
$3.02 \mathrm{~Hz}, 2 \mathrm{C}, \mathrm{C}_{\text {para }}, \mathrm{Ph}$ ), 130.6 (d, ${ }^{2} \mathrm{~J}_{\mathrm{CP}}=10.4 \mathrm{~Hz}, 4 \mathrm{C}, \mathrm{C}_{\text {ortho }}, \mathrm{Ph}$ ), 129.6 (dd, ${ }^{1} \mathrm{~J}_{\mathrm{CP}}=$ $\left.71.7 \mathrm{~Hz},{ }^{4} J_{\mathrm{CP}}=4.31 \mathrm{~Hz}, 1 \mathrm{C}, \mathrm{C}_{9}, \mathrm{An}\right), 129.4\left(\mathrm{dd},{ }^{1} \mathrm{~J}_{\mathrm{CP}}=73.6 \mathrm{~Hz},{ }^{4} J_{\mathrm{CP}}=4.31 \mathrm{~Hz}, 1 \mathrm{C}\right.$, $\left.\mathrm{C}_{10}, \mathrm{An}\right), 128.5\left(\mathrm{~d},{ }^{3} \mathrm{~J}_{\mathrm{CP}}=12.6 \mathrm{~Hz}, 4 \mathrm{C}, \mathrm{C}_{\text {meta }}, \mathrm{Ph}\right), 127.2\left(\mathrm{~d},{ }^{3} \mathrm{~J}_{\mathrm{CP}}=10.6 \mathrm{~Hz}, 2 \mathrm{C}, \mathrm{C}_{1,8}\right.$, An), 126.2 (d, $\left.{ }^{3} J_{\mathrm{CP}}=4.68 \mathrm{~Hz}, 2 \mathrm{C}, \mathrm{C}_{4,5}, \mathrm{An}\right), 125.3$ (s, $\left.2 \mathrm{C}, \mathrm{C}_{2,7}, \mathrm{An}\right), 125.1$ (s, $2 \mathrm{C}$, $\left.\mathrm{C}_{3,6}, \mathrm{An}\right), 32.4\left(\mathrm{~d},{ }^{1} \mathrm{~J}_{\mathrm{CP}}=48.2 \mathrm{~Hz}, 2 \mathrm{C}, \underline{\mathrm{C}} \mathrm{H},{ }^{i} \mathrm{Pr}\right), 18.3$ (br s, $\left.2 \mathrm{C}, \alpha-\underline{\mathrm{C}} \mathrm{H}_{3},{ }^{i} \mathrm{Pr}\right), 17.7 \mathrm{ppm}$ (br s, $2 \mathrm{C}, \beta-\underline{\mathrm{C}} \mathrm{H}_{3},{ }^{i} \mathrm{Pr}$ ). 
$\left.{ }^{31} \mathrm{P}^{1} \mathrm{H}\right\}-N M R\left(202.456 \mathrm{MHz}, \mathrm{CDCl}_{3}\right): \delta 73.0\left(\mathrm{~d},{ }^{5} \mathrm{JPP}_{\mathrm{PP}}=4.51 \mathrm{~Hz}, \mathrm{P}(\mathrm{S}){ }^{i} \mathrm{Pr}_{2}\right), 34.7 \mathrm{ppm}$ (d, ${ }^{5} J_{\mathrm{PP}}=4.51 \mathrm{~Hz}, \mathrm{P}(\mathrm{S}) \mathrm{Ph}_{2}$ ).

El-MS (70 eV): m/z (\%): 542 (100) [M] ${ }^{+}, 499$ (89) [M - $\left.{ }^{i} \mathrm{Pr}\right]^{+}, 393$ (13) [M - $\left.{ }^{i} \mathrm{Pr}_{2} \mathrm{PS}\right]^{+}$, $357(71)\left[\mathrm{PPh}_{2}\right]^{+}, 217(18)\left[\mathrm{Ph}_{2} \mathrm{PS}\right]^{+}$.

Elementaranalyse (gef. (ber.) [\%]): C 71.28 (70.83), H 6.07 (5.94).

\subsubsection{9-Diphenylselenophosphoryl-10-(diisopropylseleno- phosphoryl)anthracen (20)}

Die Darstellung erfolgte analog 19 (Kap. 8.2.16) aus $900 \mathrm{mg}(1.88 \mathrm{mmol}) 17$ und einem Überschuss von $400 \mathrm{mg}(5.07 \mathrm{mmol})$ grauem Selen in $30 \mathrm{~mL}$ Toluol. Orange Kristalle für eine Röntgenstrukturanalyse wurden durch langsames Abkühlen einer heißgesättigten Lösung in Toluol erhalten.

Summenformel: $\mathrm{C}_{32} \mathrm{H}_{32} \mathrm{P}_{2} \mathrm{Se}_{2}$

Molare Masse: $\quad 636.47 \mathrm{~g} / \mathrm{mol}$ Ausbeute: $1.06 \mathrm{mg}(1.67 \mathrm{mmol}), 89 \%$

Schmelzpunkt: $243^{\circ} \mathrm{C}$

${ }^{1} \mathrm{H}-\mathrm{NMR}\left(\mathbf{5 0 0 . 1 3 0} \mathrm{MHz}, \mathrm{CDCl}_{3}\right): \delta 9.11\left(\mathrm{~d},{ }^{3} \mathrm{JHH}_{\mathrm{HH}}=7.87 \mathrm{~Hz}, 2 \mathrm{H}, \mathrm{H}_{4,5}, \mathrm{An}\right), 8.19(\mathrm{~d}$, $\left.{ }^{3} J_{\mathrm{HH}}=8.63 \mathrm{~Hz}, 2 \mathrm{H}, \mathrm{H}_{1,8}, \mathrm{An}\right), 7.69\left(\mathrm{dd},{ }^{3} \mathrm{~J}_{\mathrm{HH}}=13.6 \mathrm{~Hz},{ }^{4} J_{\mathrm{HH}}=7.79 \mathrm{~Hz}, 4 \mathrm{H}, \mathrm{H}_{\text {ortho, }}\right.$, $\mathrm{Ph}), 7.35\left(\mathrm{dd},{ }^{3} \mathrm{~J}_{\mathrm{HH}}=7.87 \mathrm{~Hz},{ }^{3} \mathrm{JHH}_{\mathrm{HH}}=6.60 \mathrm{~Hz}, 2 \mathrm{H}, \mathrm{H}_{3,6}, \mathrm{An}\right), 7.26-7.22(\mathrm{~m}, 6 \mathrm{H}$, $\mathrm{H}_{\text {meta/para, }} \mathrm{Ph}$ ), 7.06 (dd, ${ }^{3} \mathrm{~J}_{\mathrm{HH}}=8.63 \mathrm{~Hz},{ }^{3} \mathrm{JHH}_{\mathrm{HH}}=6.60 \mathrm{~Hz}, 2 \mathrm{H}, \mathrm{H}_{2,7}, \mathrm{An}$ ), 3.32 (dsept, $\left.{ }^{2} J_{\mathrm{HP}}={ }^{3} J_{\mathrm{HH}}=5.40 \mathrm{~Hz}, 2 \mathrm{H}, \mathrm{CH},{ }^{i} \mathrm{Pr}\right), 1.47\left(\mathrm{dd},{ }^{3} J_{\mathrm{HP}}=18.8 \mathrm{~Hz},{ }^{3} J_{\mathrm{HH}}=6.58 \mathrm{~Hz}, 6 \mathrm{H}\right.$, $\left.\alpha-\underline{H}_{3},{ }^{i} \mathrm{Pr}\right), 0.93 \mathrm{ppm}\left(\mathrm{dd},{ }^{3} \mathrm{JHP}_{\mathrm{HP}}=19.0 \mathrm{~Hz},{ }^{3} \mathrm{JHH}_{\mathrm{HH}}=6.87 \mathrm{~Hz}, 6 \mathrm{H}, \beta-\underline{\mathrm{C}}_{3},{ }^{i} \mathrm{Pr}\right)$.

${ }^{13} \mathrm{C}\left\{{ }^{1} \mathrm{H}\right\}-N M R\left(125.756 \mathrm{MHz}, \mathrm{CDCl}_{3}\right): \delta 134.4\left(\mathrm{~d},{ }^{1} J_{\mathrm{CP}}=57.3 \mathrm{~Hz}, 2 \mathrm{C}, \mathrm{C}_{i p s o}, \mathrm{Ph}\right)$,

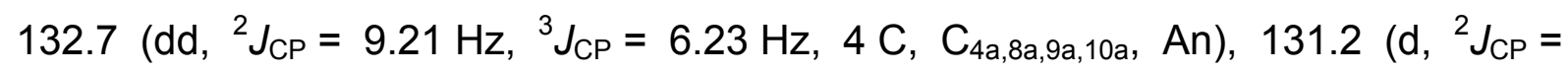
$\left.10.7 \mathrm{~Hz}, 4 \mathrm{C}, \mathrm{C}_{\text {ortho }}, \mathrm{Ph}\right), 130.7$ (d, $\left.{ }^{4} \mathrm{~J}_{\mathrm{CP}}=3.07 \mathrm{~Hz}, 2 \mathrm{C}, \mathrm{C}_{\text {para }}, \mathrm{Ph}\right), 128.5$ (d, ${ }^{3} \mathrm{JPP}_{\mathrm{CP}}=$ $\left.12.6 \mathrm{~Hz}, 4 \mathrm{C}, \mathrm{C}_{\text {meta }}, \mathrm{Ph}\right), 127.0\left(\mathrm{~d},{ }^{3} \mathrm{~J}_{\mathrm{CP}}=11.0 \mathrm{~Hz}, 2 \mathrm{C}, \mathrm{C}_{1,8}, \mathrm{An}\right), 126.6\left(\mathrm{~d},{ }^{1} \mathrm{~J}_{\mathrm{CP}}=\right.$ $\left.50.3 \mathrm{~Hz}, 1 \mathrm{C}, \mathrm{C}_{9}, \mathrm{An}\right), 126.5\left(\mathrm{~d},{ }^{1} J_{\mathrm{CP}}=50.2 \mathrm{~Hz}, 1 \mathrm{C}, \mathrm{C}_{10}, A n\right), 126.2\left(\mathrm{~d},{ }^{3} J_{\mathrm{CP}}=\right.$ $5.62 \mathrm{~Hz}, 2 \mathrm{C}, \mathrm{C}_{4,5}, \mathrm{An}$ ), 125.2 (s, $2 \mathrm{C}, \mathrm{C}_{2,7}, \mathrm{An}$ ), 124.8 (s, $2 \mathrm{C}, \mathrm{C}_{3,6}, \mathrm{An}$ ), 31.7 (d, $\left.{ }^{1} J_{\mathrm{CP}}=40.6 \mathrm{~Hz}, 2 \mathrm{C}, \underline{\mathrm{CH}},{ }^{i} \mathrm{Pr}\right), 19.5$ (s, $\left.2 \mathrm{C}, \alpha-\underline{\mathrm{C}} \mathrm{H}_{3},{ }^{i} \mathrm{Pr}\right), 18.2 \mathrm{ppm}\left(\mathrm{s}, 2 \mathrm{C}, \beta-\underline{\mathrm{C}} \mathrm{H}_{3},{ }^{i} \mathrm{Pr}\right.$ ).

$\left.{ }^{31} \mathrm{P}^{1} \mathrm{H}\right\}-N M R\left(202.456 \mathrm{MHz}, \mathrm{CDCl}_{3}\right): \delta 68.0\left(\mathrm{~d},{ }^{5} \mathrm{JPP}_{\mathrm{PP}}=4.61 \mathrm{~Hz}, \mathrm{P}(\mathrm{Se}){ }^{i} \mathrm{Pr}_{2}\right), 25.6 \mathrm{ppm}$ (d, ${ }^{5} J_{\mathrm{PP}}=4.61 \mathrm{~Hz}, \mathrm{P}(\mathrm{Se}) \mathrm{Ph}_{2}$ ).

${ }^{77} \mathrm{Se}\left\{{ }^{1} \mathrm{H}\right\}-N M R \quad\left(95.382 \mathrm{MHz}, \quad \mathrm{CDCl}_{3}\right): \delta-276.6\left(\mathrm{~d}, \quad{ }^{1} \mathrm{~J}_{\mathrm{SeP}}=736 \mathrm{~Hz}, \mathrm{P}(\mathrm{Se}) \mathrm{Ph}_{2}\right)$, $-367.1 \mathrm{ppm}\left(\mathrm{d},{ }^{1} \mathrm{~J}_{\mathrm{SeP}}=722 \mathrm{~Hz}, \mathrm{P}(\mathrm{Se}){ }^{i} \mathrm{Pr}_{2}\right)$.

El-MS (70 eV): $\boldsymbol{m} / \mathbf{z}(\%): 638(37)[\mathrm{M}]^{+}, 595$ (13) [M - $\left.{ }^{i} \mathrm{Pr}\right]^{+}, 558(91)\left[\mathrm{M}-\mathrm{Se}^{+}, 478\right.$ (92) $[\mathrm{M}-2 \mathrm{Se}]^{+}, 441$ (43) [M - $\left.{ }^{i} \mathrm{Pr}_{2} \mathrm{PSe}\right]^{+}, 393$ (67) [M - $\left.2 \mathrm{Se}-2^{i} \mathrm{Pr}\right]^{+}, 183$ (100).

Elementaranalyse (gef. (ber.) [\%]): C 61.72 (60.39), H 5.14 (5.07). 


\subsubsection{9-Diphenylphosphanyl-10-(dicyclohexylphosphanyl)- anthracen (21)}

Eine Suspension von $470 \mathrm{mg} \quad(1.07 \mathrm{mmol})$ 9-Brom-10-(diphenylphosphanyl)anthracen (1) in $20 \mathrm{~mL}$ Diethylether wurde bei $-15^{\circ} \mathrm{C}$ mit $0.82 \mathrm{~mL}(1.38 \mathrm{mmol}){ }^{n} \mathrm{BuLi}$ in $n$-Hexan (1.68 M) versetzt. Nach 15 min wurden 250 mg (1.07 mmol) Chlordicyclohexylphosphan zugetropft und die Reaktionsmischung für $2 \mathrm{~h}$ gerührt, dabei erwärmte sie sich auf RT. Der ausgefallene Feststoff wurde abgetrennt und mit Diethylether gewaschen $(2 \times 2 \mathrm{~mL})$. Nach Trocknen i. Vak. wurde 21 mit leichten Verunreinigungen erhalten, Umkristallisation aus $n$-Pentan lieferte gelbe Kristalle, die für eine Röntgenstrukturanalyse geeignet waren.

Summenformel: $\quad \mathrm{C}_{38} \mathrm{H}_{40} \mathrm{P}_{2}$

Ausbeute: $\quad 240 \mathrm{mg}(0.430 \mathrm{mmol}), 40 \% \quad$ Schmelzpunkt: $270{ }^{\circ} \mathrm{C}$

${ }^{1} \mathrm{H}$-NMR (500.130 MHz, $\mathbf{C D C l}_{3}$ ): $\delta 9.70\left(\mathrm{br} \mathrm{s}, 1 \mathrm{H}, \mathrm{H}_{4}, \mathrm{An}\right.$ ), $8.70\left(\mathrm{br} \mathrm{s}, 2 \mathrm{H}, \mathrm{H}_{1,8}, \mathrm{An}\right.$ ), 8.50 (br s, $\left.1 \mathrm{H}, \mathrm{H}_{5}, \mathrm{An}\right), 7.51$ - $7.40\left(\mathrm{~m}, 4 \mathrm{H}, \mathrm{H}_{\text {ortho }}, \mathrm{Ph}\right), 7.39$ - $7.33\left(\mathrm{~m}, 4 \mathrm{H}, \mathrm{H}_{2,3,6,7}\right.$, $\mathrm{An}), 7.27$ - 7.19 (m, $\left.6 \mathrm{H}, \mathrm{H}_{\text {meta/para }}, \mathrm{Ph}\right), 2.57$ - 2.44 (m, $\left.2 \mathrm{H}, \mathrm{C} \underline{\mathrm{H}}, \mathrm{Cy}\right), 2.07$ - 1.98 (m, $\left.2 \mathrm{H}, \underline{\mathrm{H}}_{2}, \mathrm{Cy}\right), 1.73$ - 1.65 (m, $\left.2 \mathrm{H}, \underline{\mathrm{C}}_{2}, \mathrm{Cy}\right), 1.48$ - 1.37 (m, $\left.4 \mathrm{H}, \underline{\mathrm{C}}_{2}, \mathrm{Cy}\right), 1.29$ $1.18\left(\mathrm{~m}, 4 \mathrm{H}, \underline{\mathrm{C}}_{2}, \mathrm{Cy}\right), 1.10-0.98\left(\mathrm{~m}, 2 \mathrm{H}, \mathrm{CH}_{2}, \mathrm{Cy}\right), 0.93-0.79 \mathrm{ppm}(\mathrm{m}, 6 \mathrm{H}$, $\left.\mathrm{C}_{2}, \mathrm{Cy}\right)$.

${ }^{13} \mathrm{C}\left\{{ }^{1} \mathrm{H}\right\}$-NMR (125.756 MHz, $\left.\mathrm{CDCl}_{3}\right): \delta 136.6(\mathrm{~d}, J=14.7 \mathrm{~Hz}), 131.6(\mathrm{~d}, J=18.5 \mathrm{~Hz})$, 128.4 (d, $J=5.38 \mathrm{~Hz}$ ), 127.6 (s), 125.1 (s), 124.9 (s), 36.7 (d, $J=14.9 \mathrm{~Hz}), 33.8$ (d, $J=25.9 \mathrm{~Hz}), 30.5(\mathrm{~d}, J=11.2 \mathrm{~Hz}), 30.3(\mathrm{~s}), 26.9(\mathrm{~d}, J=5.17 \mathrm{~Hz}), 26.8(\mathrm{~d}, J=$ $10.8 \mathrm{~Hz}$ ), 26.2 (d, $J=1.08 \mathrm{~Hz}$ ), $25.6 \mathrm{ppm}(\mathrm{s})$.

${ }^{31} \mathrm{P}\left\{{ }^{1} \mathrm{H}\right\}$-NMR (202.456 MHz, $\left.\mathrm{CDCl}_{3}\right): \delta-10.2(\mathrm{~s}, \mathrm{PCy}),-22.3 \mathrm{ppm}\left(\mathrm{s}, \mathrm{PPh}_{2}\right)$.

El-MS (70 eV): m/z (\%): 558.2 (100) [M] $]^{+}, 476$ (38) [M - Cy] $]^{+}, 393$ (35) [M - 2 Cy] $]^{+}$, $361(21)\left[\mathrm{M}-\mathrm{PCy}_{2}\right]^{+}$.

Elementaranalyse (gef. (ber.) [\%]): C 66.91 (81.70), H 6.05 (7.22).

\subsubsection{9-Diphenylthiophosphoryl-10-(dicyclohexylthiophosphoryl)- anthracen (22)}

Eine Suspension von $820 \mathrm{mg}(1.47 \mathrm{mmol}) 21$ in $50 \mathrm{~mL}$ Toluol wurde mit $100 \mathrm{mg}$ (3.12 mmol) Schwefel versetzt und $2.5 \mathrm{~h}$ unter Rückfluss erhitzt. Nach Abkühlen auf RT wurde der ausgefallene Feststoff abgetrennt und mit Toluol gewaschen $(2 \times 10 \mathrm{~mL})$. Nach Trocknen i. Vak. wurde 22 als gelbes Pulver erhalten, durch 
Lagerung einer gesättigten Lösung in Toluol bei RT wurden Kristalle gewonnen, die zur Bestimmung der Molekülstruktur geeignet waren.

Summenformel: $\quad \mathrm{C}_{38} \mathrm{H}_{40} \mathrm{P}_{2} \mathrm{~S}_{2}$

Molare Masse: $\quad 622.80 \mathrm{~g} / \mathrm{mol}$

Ausbeute: $\quad 650 \mathrm{mg}(1.04 \mathrm{mmol}), 71 \% \quad$ Schmelzpunkt: $285{ }^{\circ} \mathrm{C}$

${ }^{1} \mathrm{H}-N M R\left(500.130 \mathrm{MHz} \mathrm{CDCl}_{3}\right): \delta 8.98\left(\mathrm{br} \mathrm{s}, 2 \mathrm{H}, \mathrm{H}_{4,5}, \mathrm{An}\right), 8.15\left(\mathrm{~d},{ }^{3} \mathrm{~J}_{\mathrm{HH}}=8.61 \mathrm{~Hz}\right.$, $\left.2 \mathrm{H}, \mathrm{H}_{1,8}\right), 7.60\left(\mathrm{dd},{ }^{3} J_{\mathrm{HH}}=12.8 \mathrm{~Hz},{ }^{4} \mathrm{~J}_{\mathrm{HH}}=7.67 \mathrm{~Hz}, 4 \mathrm{H}, \mathrm{H}_{\text {ortho }}, \mathrm{Ph}\right), 7.36-7.33(\mathrm{~m}$, $\left.2 \mathrm{H}, \mathrm{H}_{3,6}, \mathrm{An}\right), 7.31$ - 7.28 (m, $\left.2 \mathrm{H}, \mathrm{H}_{\text {para }}, \mathrm{Ph}\right), 7.24$ - 7.21 (m, $4 \mathrm{H}, \mathrm{H}_{\text {meta }}, \mathrm{Ph}$ ), 7.03 (dd, $\left.{ }^{3} J_{\mathrm{HH}}=8.61 \mathrm{~Hz},{ }^{3} J_{\mathrm{HH}}=6.91 \mathrm{~Hz}, 2 \mathrm{H}, \mathrm{H}_{2,7}, \mathrm{An}\right), 2.95-2.90(\mathrm{~m}, 2 \mathrm{H}, \mathrm{CH}, \mathrm{Cy}), 2.05-$ 1.96 (m, $\left.4 \mathrm{H}, \mathrm{CH}_{2}, \mathrm{Cy}\right), 1.93$ - 1.91 (m, $\left.2 \mathrm{H}, \mathrm{CH}_{2}, \mathrm{Cy}\right), 1.66$ - 1.63 (m, $\left.6 \mathrm{H}, \mathrm{CH}_{2}, \mathrm{Cy}\right)$, 1.46 - 1.38 (m, $\left.2 \mathrm{H}, \underline{\mathrm{C}}_{2}, \mathrm{Cy}\right), 1.28-1.20$ (m, $\left.2 \mathrm{H}, \underline{\mathrm{C}}_{2}, \mathrm{Cy}\right), 1.14$ - 1.02 ppm (m, $4 \mathrm{H}$, $\left.\mathrm{C}_{2}, \mathrm{Cy}\right)$.

${ }^{13} \mathrm{C}\left\{{ }^{1} \mathrm{H}\right\}-N M R\left(125.756 \mathrm{MHz}, \mathrm{CDCl}_{3}\right): \delta 137.9-125.0(\mathrm{~m}), 42.7(\mathrm{~d}), 28.4-25.6 \mathrm{ppm}$ (m).

${ }^{31} \mathrm{P}\left\{{ }^{1} \mathrm{H}\right\}-N M R\left(202.456 \mathrm{MHz}, \mathrm{CDCl}_{3}\right): \delta 65.7\left(\mathrm{~d},{ }^{5} J_{\mathrm{PP}}=5.95 \mathrm{~Hz}, \mathrm{P}(\mathrm{S}) \mathrm{Cy} \mathrm{y}_{2}\right), 34.2 \mathrm{ppm}$ (d, ${ }^{5} J_{\mathrm{PP}}=5.95 \mathrm{~Hz}, \mathrm{P}(\mathrm{S}) \mathrm{Ph}_{2}$ ).

El-MS (70 eV): m/z (\%): 622 (100) [M] ${ }^{+}, 590(25)[\mathrm{M}-\mathrm{S}]^{+}, 558(3)[\mathrm{M}-2 \mathrm{~S}]^{+}, 405(24)$ $\left[\mathrm{M}-\mathrm{Ph}_{2} \mathrm{PS}\right]^{+}, 394$ (11) [M - $\left.{ }^{i} \mathrm{Pr}_{2} \mathrm{PS}\right]^{+}, 217$ (27) $\left[\mathrm{Ph}_{2} \mathrm{PS}\right]^{+}$.

Elementaranalyse (gef. (ber.) [\%]): C 62.02 (73.28), H 6.09 (6.47).

\subsubsection{0 [9-Diphenylthiophosphoryl-10-(lithioamino)anthracen · 3 thf]} (23)

Eine Suspension von $500 \mathrm{mg}$ (1.06 mmol) 9-Brom-10-(diphenylthiophosphoryl)anthracen (3) in $20 \mathrm{~mL}$ THF wurde mit einem Überschuss von $155 \mathrm{mg}$ (6.75 mmol) $\mathrm{LiNH}_{2}$ versetzt und für $9 \mathrm{~d}$ bei RT gerührt. Die tiefrote Reaktionsmischung wurde über Celite filtriert, das Filtrat auf etwa die Hälfte i. Vak. aufkonzentriert und mit $10 \mathrm{~mL}$ Hexan überschichtet. Nach einigen Tagen bei $6{ }^{\circ} \mathrm{C}$ bildeten sich Kristalle, die zur röntgenkristallographischen Untersuchung geeignet waren.

\section{Summenformel: $\quad \mathrm{C}_{38} \mathrm{H}_{43} \mathrm{LiNO}_{3} \mathrm{PS}$}

Ausbeute: $500 \mathrm{mg}(0.791 \mathrm{mmol}), 75 \%$
Molare Masse: $\quad 631.73 \mathrm{~g} / \mathrm{mol}$ Schmelzpunkt: $218^{\circ} \mathrm{C}$

${ }^{1} \mathrm{H}$-NMR (500.130 MHz, THF-D ${ }_{8}$ ): $\delta 8.19\left(\mathrm{~d},{ }^{3} \mathrm{~J}_{\mathrm{HH}}=8.71 \mathrm{~Hz}, 2 \mathrm{H}, \mathrm{H}_{4,5}, \mathrm{An}\right), 7.87(\mathrm{~d}$, $\left.{ }^{3} J_{\mathrm{HH}}=8.86 \mathrm{~Hz}, 2 \mathrm{H}, \mathrm{H}_{1,8}, \mathrm{An}\right), 7.82-7.77\left(\mathrm{~m}, 4 \mathrm{H}, \mathrm{H}_{\text {ortho }}, \mathrm{Ph}\right), 7.25-7.23(\mathrm{~m}, 2 \mathrm{H}$, $\left.\mathrm{H}_{\text {para }}, \mathrm{Ph}\right), 7.21-7.17\left(\mathrm{~m}, 4 \mathrm{H}, \mathrm{H}_{\text {meta }}, \mathrm{Ph}\right), 7.15\left(\mathrm{ddd},{ }^{3} \mathrm{~J}_{\mathrm{HH}}=8.71 \mathrm{~Hz},{ }^{3} \mathrm{~J}_{\mathrm{HH}}=6.51 \mathrm{~Hz}\right.$, $\left.{ }^{4} J_{\mathrm{HH}}=1.07 \mathrm{~Hz}, 2 \mathrm{H}, \mathrm{H}_{3,6}, \mathrm{An}\right), 6.91$ (ddd, ${ }^{3} \mathrm{~J}_{\mathrm{HH}}=8.86 \mathrm{~Hz},{ }^{3} \mathrm{~J}_{\mathrm{HH}}=6.51 \mathrm{~Hz},{ }^{4} J_{\mathrm{HH}}=$ $\left.1.22 \mathrm{~Hz}, 2 \mathrm{H}, \mathrm{H}_{2,7}, \mathrm{An}\right), 3.64$ - $3.58 \mathrm{ppm}$ (m, thf), 1.79 - 1.73 (m, thf). 
${ }^{13} \mathrm{C}\left\{{ }^{1} \mathrm{H}\right\}$-NMR (125.756 MHz, THF-D $): \delta 148.2\left(\mathrm{~d},{ }^{4} J_{\mathrm{CP}}=3.04 \mathrm{~Hz}, 1 \mathrm{C}, \mathrm{C}_{10}, A n\right)$, $140.1\left(\mathrm{~d},{ }^{1} \mathrm{~J}_{\mathrm{CP}}=82.3 \mathrm{~Hz}, 2 \mathrm{C}, \mathrm{C}_{\text {ipso }}, \mathrm{Ph}\right), 135.9\left(\mathrm{~d},{ }^{3} \mathrm{~J}_{\mathrm{CP}}=8.98 \mathrm{~Hz}, 2 \mathrm{C}, \mathrm{C}_{4 \mathrm{a}, 10 \mathrm{a}}, \mathrm{An}\right)$, $131.9\left(\mathrm{~d},{ }^{2} J_{\mathrm{CP}}=10.2 \mathrm{~Hz}, 4 \mathrm{C}, \mathrm{C}_{\text {ortho }}, \mathrm{Ph}\right), 130.5\left(\mathrm{~d},{ }^{4} \mathrm{~J}_{\mathrm{CP}}=2.87 \mathrm{~Hz}, 2 \mathrm{C}, \mathrm{C}_{\text {para }}, \mathrm{Ph}\right)$, $128.8\left(\mathrm{~d},{ }^{3} \mathrm{~J}_{\mathrm{CP}}=10.4 \mathrm{~Hz}, 2 \mathrm{C}, \mathrm{C}_{1,8}, \mathrm{An}\right), 128.7$ (d, ${ }^{3} \mathrm{~J}_{\mathrm{CP}}=12.2 \mathrm{~Hz}, 4 \mathrm{C}, \mathrm{C}_{\text {meta }}, \mathrm{Ph}$ ),

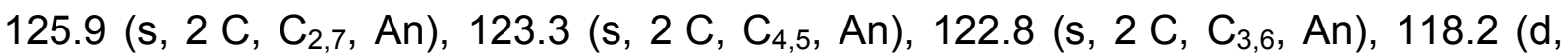
$\left.{ }^{2} J_{\mathrm{CP}}=11.5 \mathrm{~Hz}, 2 \mathrm{C}, \mathrm{C}_{8 \mathrm{a}, 9 \mathrm{a}}, \mathrm{An}\right), 106.2\left(\mathrm{~d},{ }^{1} J_{\mathrm{CP}}=101.5 \mathrm{~Hz}, 1 \mathrm{C}, \mathrm{C}_{9}, \mathrm{An}\right), 68.2\left(\mathrm{~s}, \underline{\mathrm{C}}_{2}\right.$, thf), $26.4 \mathrm{ppm}$ (s, $\underline{\mathrm{C}} \mathrm{H}_{2}$, thf).

${ }^{31} \mathrm{P}\left\{{ }^{1} \mathrm{H}\right\}-\mathrm{NMR}$ (121.495 MHz, $\mathrm{CDCl}_{3}$ ): $\delta 33.6 \mathrm{ppm}(\mathrm{s})$.

${ }^{7}$ Li-NMR (116.642 MHz, THF-D 8 ): $\delta 0.30 \mathrm{ppm}(\mathrm{s})$.

EI-MS (70 eV): $\mathbf{m} / \mathbf{z}(\%): 409$ (61) $\left[\mathrm{Ph}_{2}(\mathrm{~S}) \mathrm{P}\left(\mathrm{C}_{14} \mathrm{H}_{8}\right) \mathrm{NH}_{2}\right]^{+}, 217(100)\left[\mathrm{P}(\mathrm{S}) \mathrm{Ph}_{2}\right]$.

Elementaranalyse (gef. (ber.) [\%]): C 70.19 (72.25), H 6.13 (6.86), N 2.26 (2.22).

\subsubsection{9-Diphenylthiophosphoryl-10-(amino)anthracen (24)}

Eine Lösung von $200 \mathrm{mg}(0.317 \mathrm{mmol}) 23$ in $10 \mathrm{~mL}$ THF wurde mit $0.05 \mathrm{~mL}$ dest. Wasser versetzt und für $2 \mathrm{~d}$ bei RT gerührt. Die rote Reaktionsmischung wurde über Celite filtriert und das Filtrat i. Vak. eingeengt. Lagerung einer gesättigten Lösung des rotbraunen Rückstandes in THF lieferte nach einigen Tagen braune Kristalle, die zur Bestimmung der Molekülstruktur geeignet waren.

Summenformel: $\mathrm{C}_{26} \mathrm{H}_{20} \mathrm{NPS} \quad$ Molare Masse: $\quad 409.48 \mathrm{~g} / \mathrm{mol}$ Ausbeute: $\quad 110 \mathrm{mg}(0.269 \mathrm{mmol}), 85 \% \quad$ Schmelzpunkt: $\quad$ n. b.

${ }^{1} \mathrm{H}$-NMR (500.130 MHz, CDCl $\left.)_{3}\right): \delta 7.95\left(\mathrm{~d},{ }^{3} \mathrm{~J}_{\mathrm{HH}}=8.94 \mathrm{~Hz}, 2 \mathrm{H}, \mathrm{H}_{1,8}, \mathrm{An}\right), 7.89(\mathrm{~d}$, $\left.{ }^{3} J_{H H}=8.65 \mathrm{~Hz}, 2 \mathrm{H}, \mathrm{H}_{4,5}, \mathrm{An}\right), 7.79-7.75\left(\mathrm{~m}, 4 \mathrm{H}, \mathrm{H}_{\text {ortho }}, \mathrm{Ph}\right), 7.30-7.27(\mathrm{~m}, 2 \mathrm{H}$, $\mathrm{H}_{\text {para }}, \mathrm{Ph}$ ), $7.25-7.21\left(\mathrm{~m}, 6 \mathrm{H}, \mathrm{H}_{3,6}, \mathrm{An} / \mathrm{H}_{\text {meta }}, \mathrm{Ph}\right), 6.99$ (ddd, ${ }^{3} \mathrm{~J}_{\mathrm{HH}}=8.94 \mathrm{~Hz},{ }^{3} \mathrm{JHH}_{\mathrm{HH}}=$ $\left.6.51 \mathrm{~Hz},{ }^{4} J_{\mathrm{HH}}=1.11 \mathrm{~Hz}, 2 \mathrm{H}, \mathrm{H}_{2,7}, \mathrm{An}\right), 5.42 \mathrm{ppm}\left(\mathrm{br} \mathrm{s}, 2 \mathrm{H}, \mathrm{N}_{2}\right.$ ).

${ }^{13} \mathrm{C}\left\{{ }^{1} \mathrm{H}\right\}-N M R(125.756 \mathrm{MHz}$, THF-D $): \delta 144.5\left(\mathrm{~d},{ }^{4} \mathrm{~J}_{\mathrm{CP}}=3.43 \mathrm{~Hz}, 1 \mathrm{C}, \mathrm{C}_{10}, \mathrm{An}\right)$, $137.7\left(\mathrm{~d},{ }^{1} \mathrm{~J}_{\mathrm{CP}}=83.3 \mathrm{~Hz}, 2 \mathrm{C}, \mathrm{C}_{\text {ipso }}, \mathrm{Ph}\right), 134.5\left(\mathrm{~d},{ }^{3} \mathrm{~J}_{\mathrm{CP}}=8.84 \mathrm{~Hz}, 2 \mathrm{C}, \mathrm{C}_{4 \mathrm{a}, 10 \mathrm{a}}, \mathrm{An}\right)$, $131.0\left(\mathrm{~d},{ }^{2} J_{\mathrm{CP}}=10.4 \mathrm{~Hz}, 4 \mathrm{C}, \mathrm{C}_{\text {ortho }}, \mathrm{Ph}\right), 130.2\left(\mathrm{~d},{ }^{4} J_{\mathrm{CP}}=2.93 \mathrm{~Hz}, 2 \mathrm{C}, \mathrm{C}_{\text {para }}, \mathrm{Ph}\right)$, $128.3\left(\mathrm{~d},{ }^{3} \mathrm{~J}_{\mathrm{CP}}=12.5 \mathrm{~Hz}, 4 \mathrm{C}, \mathrm{C}_{\text {meta }}, \mathrm{Ph}\right), 128.1$ (d, ${ }^{3} \mathrm{~J}_{\mathrm{CP}}=10.3 \mathrm{~Hz}, 2 \mathrm{C}, \mathrm{C}_{1,8}, \mathrm{An}$ ), 125.7 (s, 2 C, $C_{2,7}, A n$ ), 123.4 (s, 2 C, $\left.C_{3,6}, A n\right), 121.2$ (s, 2 C, $C_{4,5}, A n$ ), 117.6 (d, $\left.{ }^{2} J_{\mathrm{CP}}=11.5 \mathrm{~Hz}, 2 \mathrm{C}, \mathrm{C}_{8 \mathrm{a}, 9 \mathrm{a}}, \mathrm{An}\right), 107.8 \mathrm{ppm}\left(\mathrm{d},{ }^{1} \mathrm{~J}_{\mathrm{CP}}=98.6 \mathrm{~Hz}, 1 \mathrm{C}, \mathrm{C}_{9}, A n\right)$.

${ }^{31} \mathrm{P}\left\{{ }^{1} \mathrm{H}\right\}-N M R\left(202.456 \mathrm{MHz}, \mathrm{CDCl}_{3}\right): \delta 34.7 \mathrm{ppm}(\mathrm{s})$.

EI-MS (70 eV): m/z (\%): 409 (63) [M] $]^{+}, 217$ (100) [P(S)Ph $]$. 


\subsubsection{9-Brom-10-[bis(diisopropylamino)phosphanyl]anthracen (25)}

Eine Suspension von $1.00 \mathrm{~g}$ (2.98 mmol) 9,10-Dibromanthracen in $25 \mathrm{~mL}$ Diethylether wurde bei $-15^{\circ} \mathrm{C}$ mit $1.30 \mathrm{~mL}(3.03 \mathrm{mmol}){ }^{n} \mathrm{BuLi}$ in $n$-Hexan $(2.33 \mathrm{M})$ versetzt. Nach $10 \mathrm{~min}$ wurde eine Lösung von $800 \mathrm{mg}(3.00 \mathrm{mmol})$ Chlorbis(diisopropylamino)phosphan in $10 \mathrm{~mL}$ THF zugetropft, dabei färbte sich die Reaktionsmischung rot-braun. Nach weiteren 30 min wurde die Suspension über Celite filtriert, das Lösungsmittel i. Vak. entfernt und 25 als rot-braunes Pulver erhalten. Geeignete Kristalle für eine Röntgenstrukturanalyse bildeten sich aus einer gesättigten Lösung in Toluol nach einigen Tagen bei RT.

Summenformel: $\mathrm{C}_{26} \mathrm{H}_{36} \mathrm{BrN}_{2} \mathrm{P}$

Ausbeute:
Molare Masse: $\quad 487.45 \mathrm{~g} / \mathrm{mol}$

Schmelzpunkt: $144^{\circ} \mathrm{C}$

${ }^{1}$ H-NMR (500.130 MHz, Toluol-D $)$ : $\delta 9.89-9.85$ (m, $\left.2 \mathrm{H}, \mathrm{H}_{4,5}, \mathrm{An}\right), 8.67-8.65$ (m, $2 \mathrm{H}, \mathrm{H}_{1,8}, \mathrm{An}$ ), $7.32-7.28\left(\mathrm{~m}, 4 \mathrm{H}, \mathrm{H}_{2,3,6,7}, \mathrm{An}\right.$ ), 3.61 (dsept, ${ }^{3} \mathrm{~J}_{\mathrm{HP}}=11.9 \mathrm{~Hz},{ }^{3} \mathrm{JHH}_{\mathrm{HH}}=$ $\left.6.70 \mathrm{~Hz}, 4 \mathrm{H}, \mathrm{CH},{ }^{i} \mathrm{Pr}\right), 1.27\left(\mathrm{~d},{ }^{3} \mathrm{H}_{\mathrm{HH}}=6.70 \mathrm{~Hz}, 12 \mathrm{H}, \alpha-\mathrm{C}_{3},{ }^{i} \mathrm{Pr}\right), 0.79 \mathrm{ppm}\left(\mathrm{d},{ }^{3} J_{\mathrm{HH}}=\right.$ $\left.6.70 \mathrm{~Hz}, 12 \mathrm{H}, \beta-\mathrm{C}_{3},{ }^{i} \mathrm{Pr}\right)$.

${ }^{13} \mathrm{C}\left\{{ }^{1} \mathrm{H}\right\}-N M R\left(125.756 \mathrm{MHz}\right.$, Toluol-D $\left.\mathrm{D}_{8}\right): \delta 140.3\left(\mathrm{~d},{ }^{1} \mathrm{~J}_{\mathrm{CP}}=60.9 \mathrm{~Hz}, 1 \mathrm{C}, \mathrm{C}_{10}, \mathrm{An}\right)$, $136.0\left(\mathrm{~d},{ }^{2} \mathrm{~J}_{\mathrm{CP}}=14.7 \mathrm{~Hz}, 2 \mathrm{C}, \mathrm{C}_{4 \mathrm{a}, 10 \mathrm{a}}, \mathrm{An}\right), 130.0\left(\mathrm{~d},{ }^{3} \mathrm{~J}_{\mathrm{CP}}=1.22 \mathrm{~Hz}, 2 \mathrm{C}, \mathrm{C}_{8 \mathrm{a}, 9 \mathrm{a}}, \mathrm{An}\right)$, 128.7 (s, 2 C, C ${ }_{1,8}, A n$ ), 128.5 (s, 2 C, C $4,5, A n$ ), 126.9 (s, 2 C, C $2,7, A n$ ), 126.0 (s, $\left.1 \mathrm{C}, \mathrm{C}_{9}, \mathrm{An}\right), 124.4$ (s, $\left.2 \mathrm{C}, \mathrm{C}_{3,6}, \mathrm{An}\right), 48.0$ (d, $\left.{ }^{2} \mathrm{~J}_{\mathrm{CP}}=15.2 \mathrm{~Hz}, 4 \mathrm{C}, \underline{\mathrm{C}} \mathrm{H},{ }^{\mathrm{i}} \mathrm{Pr}\right), 24.1$ (d, $\left.{ }^{3} J_{\mathrm{CP}}=6.15 \mathrm{~Hz}, 4 \mathrm{C}, \alpha-\underline{\mathrm{CH}}_{3},{ }^{\mathrm{i}} \mathrm{Pr}\right), 23.6 \mathrm{ppm}\left(\mathrm{d},{ }^{3} \mathrm{~J}_{\mathrm{CP}}=7.01 \mathrm{~Hz}, 4 \mathrm{C}, \beta-\underline{\mathrm{CH}}_{3},{ }^{i} \mathrm{Pr}\right)$.

${ }^{31} \mathrm{P}\left\{{ }^{1} \mathrm{H}\right\}-N M R$ (202.456 MHz, Toluol-D $)$ : $\delta 72.6 \mathrm{ppm}(\mathrm{s})$.

El-MS (70 eV): $\boldsymbol{m} / \mathbf{z}(\%): 488(20)[\mathrm{M}]^{+}, 386(85)\left[\mathrm{M}-\mathrm{N}^{i} \mathrm{Pr}_{2}\right]^{+}, 287(100)\left[\mathrm{M}-2 \mathrm{~N}^{\prime} \mathrm{Pr}_{2}\right]^{+}$, $100(19)\left[\mathrm{N}^{i} \mathrm{Pr}\right]^{+}$.

Elementaranalyse (gef. (ber.) [\%]): C 64.19 (64.06), H 7.38 (7.44), N 5.46 (5.75).

\subsubsection{9-[Bis(diethylamino)phosphanyl]anthracen (26)}

Eine Lösung von $2.00 \mathrm{~g}(7.78 \mathrm{mmol})$ 9-Bromanthracen in $40 \mathrm{~mL}$ Diethylether wurde bei $-15^{\circ} \mathrm{C}$ mit $3.85 \mathrm{~mL}(7.82 \mathrm{mmol}){ }^{n} \mathrm{BuLi}$ in $n$-Hexan $(2.03 \mathrm{M})$ versetzt. Nach $15 \mathrm{~min}$ wurden $1.64 \mathrm{~g}$ (7.78 mmol) Chlorbis(diethylamino)phosphan zu der Reaktionsmischung getropft. Nach 45 min Rühren wurde das Kältebad entfernt und nach weiteren 15 min wurde die orange Suspension über Celite filtriert. Durch Entfernen des Lösungsmittels i. Vak. wurde 26 als rotes Öl erhalten. 
Eine ähnliche Vorschrift zur Darstellung der Verbindung wurde 2006 von Tokitoh et al. publiziert, allerdings ohne Zuordnung der NMR-Signale. ${ }^{[140]}$

Summenformel: $\mathrm{C}_{22} \mathrm{H}_{29} \mathrm{~N}_{2} \mathrm{P}$

Molare Masse: $352.45 \mathrm{~g} / \mathrm{mol}$

Ausbeute: $\quad 2.54 \mathrm{~g}(7.21 \mathrm{mmol}), 93 \% \quad$ Schmelzpunkt: $\mathrm{n}$. b.

${ }^{1} \mathrm{H}-N M R\left(500.130 \mathrm{MHz}, \mathrm{C}_{6} \mathrm{D}_{6}\right): \delta 9.44\left(\mathrm{~d},{ }^{3} \mathrm{~J}_{\mathrm{HH}}=9.07 \mathrm{~Hz}, 2 \mathrm{H}, \mathrm{H}_{1,8}, \mathrm{An}\right), 8.17(\mathrm{~s}, 1 \mathrm{H}$, $\left.\mathrm{H}_{10}, A n\right), 7.80\left(\mathrm{~d},{ }^{3} J_{\mathrm{HH}}=8.43 \mathrm{~Hz}, 2 \mathrm{H}, \mathrm{H}_{4,5}, \mathrm{An}\right), 7.35$ (ddd, ${ }^{3} J_{\mathrm{HH}}=9.07 \mathrm{~Hz},{ }^{3} J_{\mathrm{HH}}=$ $\left.6.47 \mathrm{~Hz},{ }^{4} J_{\mathrm{HH}}=1.42 \mathrm{~Hz}, 2 \mathrm{H}, \mathrm{H}_{2,7}, \mathrm{An}\right), 7.27\left(\mathrm{dd},{ }^{3} J_{\mathrm{HH}}=8.43 \mathrm{~Hz},{ }^{3} J_{\mathrm{HH}}=6.47 \mathrm{~Hz}, 2 \mathrm{H}\right.$, $\left.\mathrm{H}_{3,6}, \mathrm{An}\right), 2.98\left(\mathrm{dq},{ }^{3} \mathrm{~J}_{\mathrm{HP}}=8.97 \mathrm{~Hz},{ }^{3} \mathrm{~J}_{\mathrm{HH}}=7.09 \mathrm{~Hz}, 8 \mathrm{H}, \mathrm{CH}_{2}, \mathrm{Et}\right), 0.92 \mathrm{ppm}\left(\mathrm{t},{ }^{3} J_{\mathrm{HH}}=\right.$ $\left.7.09 \mathrm{~Hz}, 12 \mathrm{H}, \underline{\mathrm{CH}}_{3}, \mathrm{Et}\right)$.

${ }^{13} \mathrm{C}\left\{{ }^{1} \mathrm{H}\right\}-N M R(125.756 \mathrm{MHz}$, Toluol-D $): \delta 135.0\left(\mathrm{~d},{ }^{1} \mathrm{~J}_{\mathrm{CP}}=40.1 \mathrm{~Hz}, 1 \mathrm{C}, \mathrm{C}_{9}, \mathrm{An}\right)$, $134.8\left(\mathrm{~d},{ }^{2} J_{\mathrm{CP}}=12.9 \mathrm{~Hz}, 2 \mathrm{C}, \mathrm{C}_{8 \mathrm{a}, 9 \mathrm{a}}, \mathrm{An}\right), 132.0$ (s, $\left.2 \mathrm{C}, \mathrm{C}_{4 \mathrm{a}, 10 \mathrm{a}}, \mathrm{An}\right), 129.5$ (d, ${ }^{4} \mathrm{~J}_{\mathrm{CP}}=$ $\left.1.91 \mathrm{~Hz}, 1 \mathrm{C}, \mathrm{C}_{10}, \mathrm{An}\right), 129.3\left(\mathrm{~d},{ }^{4} J_{\mathrm{CP}}=1.50 \mathrm{~Hz}, 2 \mathrm{C}, \mathrm{C}_{4,5}, \mathrm{An}\right), 127.5\left(\mathrm{~d},{ }^{3} J_{\mathrm{CP}}=\right.$ $\left.20.0 \mathrm{~Hz}, 2 \mathrm{C}, \mathrm{C}_{1,8}, \mathrm{An}\right), 124.9$ (s, $\left.2 \mathrm{C}, \mathrm{C}_{3,6}, \mathrm{An}\right), 124.7$ (d, ${ }^{4} \mathrm{~J}_{\mathrm{CP}}=2.95 \mathrm{~Hz}, 2 \mathrm{C}, \mathrm{C}_{2,7}$, An), $44.3\left(\mathrm{~d},{ }^{2} J_{\mathrm{CP}}=18.9 \mathrm{~Hz}, 4 \mathrm{C}, \underline{\mathrm{CH}}_{2}, A n\right), 14.3 \mathrm{ppm}\left(\mathrm{d},{ }^{3} \mathrm{~J}_{\mathrm{CP}}=3.88 \mathrm{~Hz}, 4 \mathrm{C}\right.$, $\left.\mathrm{C}_{3}, \mathrm{An}\right)$.

${ }^{15} \mathrm{~N}-\mathrm{NMR}\left(\mathbf{5 0 . 6 9 7} \mathrm{MHz}, \mathrm{C}_{6} \mathrm{D}_{6}\right): \delta-330.6 \mathrm{ppm}\left(\mathrm{d},{ }^{1} \mathrm{~J}_{\mathrm{NP}}=65.0 \mathrm{~Hz}\right)$.

${ }^{31} \mathrm{P}\left\{{ }^{1} \mathrm{H}\right\}-N M R\left(202.456 \mathrm{MHz}, \mathrm{CDCl}_{3}\right): \delta 103.2 \mathrm{ppm}(\mathrm{s})$.

El-MS (70 eV): m/z (\%): 352 (31) [M] $]^{+}, 280$ (77) [M - NEt $]^{+}, 209$ (100) [M - $\left.2 \mathrm{NEt}_{2}\right]^{+}$.

\subsubsection{9-(Dichlorphosphanyl)anthracen (27)}

Eine Lösung von $2.54 \mathrm{~g}(7.21 \mathrm{mmol}) 26$ in $100 \mathrm{~mL} n$-Hexan wurde auf $0{ }^{\circ} \mathrm{C}$ gekühlt und anschließend zweimal für jeweils ca. 5 min $\mathrm{HCl}-\mathrm{Gas}$ (durch konz. $\mathrm{H}_{2} \mathrm{SO}_{4}$ getrocknet) durch die Reaktionsmischung geleitet. Nach $1 \mathrm{~h}$ Rühren bei RT wurde die gelbe Suspension auf etwa die Hälfte aufkonzentriert und der produkthaltige gelbe Niederschlag abgetrennt. Anschließend wurde dieser portionsweise mit Toluol (insgesamt $50 \mathrm{~mL}$ ) in einen anderen Kolben gewaschen. Durch Entfernen des Lösungsmittels i. Vak. wurde 27 als gelbes Pulver erhalten.

Die Verbindung wurde erstmals 1992 von Schmutzler beschrieben, allerdings führte die damalige Synthese stets zum Auftreten unerwünschter Nebenprodukte. ${ }^{[29]}$

Summenformel: $\mathrm{C}_{14} \mathrm{H}_{9} \mathrm{Cl}_{2} \mathrm{P}$

Ausbeute:

$1.40 \mathrm{~g}(5.02 \mathrm{mmol}), 70 \%$
Molare Masse: $\quad 279.10 \mathrm{~g} / \mathrm{mol}$

Schmelzpunkt: $138^{\circ} \mathrm{C}$

${ }^{1} \mathrm{H}-N M R\left(500.130 \mathrm{MHz}, \mathrm{C}_{6} \mathrm{D}_{6}\right): \delta 9.16\left(\mathrm{~d},{ }^{3} \mathrm{~J}_{\mathrm{HH}}=9.03 \mathrm{~Hz}, 2 \mathrm{H}, \mathrm{H}_{1,8}, \mathrm{An}\right), 8.04(\mathrm{~s}, 1 \mathrm{H}$, $\left.\mathrm{C}_{10}, \mathrm{An}\right), 7.59\left(\mathrm{~d},{ }^{3} \mathrm{~J}_{\mathrm{HH}}=8.44 \mathrm{~Hz}, 2 \mathrm{H}, \mathrm{H}_{4,5}, \mathrm{An}\right), 7.23$ (ddd, ${ }^{3} J_{\mathrm{HH}}=9.03 \mathrm{~Hz},{ }^{3} J_{\mathrm{HH}}=$ 
$\left.6.58 \mathrm{~Hz},{ }^{4} J_{\mathrm{HH}}=1.43 \mathrm{~Hz}, 2 \mathrm{H}, \mathrm{H}_{2,7}, A n\right), 7.11 \mathrm{ppm}\left(\mathrm{ddd},{ }^{3} J_{\mathrm{HH}}=8.44 \mathrm{~Hz},{ }^{3} J_{\mathrm{HH}}=\right.$ $\left.6.58 \mathrm{~Hz},{ }^{4} J_{\mathrm{HH}}=0.99 \mathrm{~Hz}, 2 \mathrm{H}, \mathrm{H}_{3,6}, \mathrm{An}\right)$.

${ }^{13} \mathrm{C}\left\{{ }^{1} \mathrm{H}\right\}-N M R\left(125.756 \mathrm{MHz}, \mathrm{CDCl}_{3}\right): \delta 135.5\left(\mathrm{~d},{ }^{4} \mathrm{~J}_{\mathrm{CP}}=0.49 \mathrm{~Hz}, 1 \mathrm{C}, \mathrm{C}_{10}, \mathrm{An}\right), 134.5$ $\left(\mathrm{d},{ }^{2} J_{\mathrm{CP}}=21.6 \mathrm{~Hz}, 2 \mathrm{C}, \mathrm{C}_{8 \mathrm{a}, 9 a}, \mathrm{An}\right), 131.4$ (d, $\left.{ }^{3} J_{\mathrm{CP}}=3.80 \mathrm{~Hz}, 2 \mathrm{C}, \mathrm{C}_{4 \mathrm{a}, 10 \mathrm{a}}, \mathrm{An}\right), 129.9$ (d, $\left.{ }^{4} J_{\mathrm{CP}}=1.29 \mathrm{~Hz}, 2 \mathrm{C}, \mathrm{C}_{4,5}, \mathrm{An}\right), 129.3\left(\mathrm{~d},{ }^{1} J_{\mathrm{CP}}=73.9 \mathrm{~Hz}, 1 \mathrm{C}, \mathrm{C}_{9}, A n\right), 127.7$ (d, $\left.{ }^{4} J_{\mathrm{CP}}=3.68 \mathrm{~Hz}, 2 \mathrm{C}, \mathrm{C}_{2,7}, \mathrm{An}\right), 125.9\left(\mathrm{~d},{ }^{3} J_{\mathrm{CP}}=34.2 \mathrm{~Hz}, 2 \mathrm{C}, \mathrm{C}_{1,8}\right.$, An), $125.5 \mathrm{ppm}(\mathrm{d}$, $\left.{ }^{5} J_{\mathrm{CP}}=1.83 \mathrm{~Hz}, 2 \mathrm{C}, \mathrm{C}_{3,6}, \mathrm{An}\right)$.

${ }^{31} \mathrm{P}\left\{{ }^{1} \mathrm{H}\right\}-N M R\left(202.456 \mathrm{MHz}, \mathrm{CDCl}_{3}\right): \delta 159.3 \mathrm{ppm}(\mathrm{s})$.

El-MS (70 eV): m/z (\%): 278 (100) [M] ${ }^{+}, 243$ (78) [M - Cl] $]^{+}, 207$ (73) [M - 2 Cl] .

Elementaranalyse (gef. (ber.) [\%]): C 61.34 (60.25), H 3.77 (3.25), P 9.50 (11.10).

\subsubsection{9-[Di(2-pyridyl)phosphanyl]anthracen (28)}

$3.77 \mathrm{~mL}$ (7.65 mmol) ${ }^{n}$ BuLi in $n$-Hexan (2.03 M) wurden in $20 \mathrm{~mL}$ Diethylether gelöst und bei $-78^{\circ} \mathrm{C}$ langsam mit $1.21 \mathrm{~g}$ (7.66 mmol, ca. $\left.0.73 \mathrm{~mL}\right)$ 2-Brompyridin versetzt. Nach $1 \mathrm{~h}$ Rühren bei $-78{ }^{\circ} \mathrm{C}$ wurde eine Lösung von $1.07 \mathrm{~g}(3.83 \mathrm{mmol}) 27$ in $15 \mathrm{~mL}$ Toluol innerhalb $1 \mathrm{~h}$ zugetropft. Nach $2 \mathrm{~h}$ Rühren wurde das Kältebad entfernt und die Reaktionsmischung für weitere $16 \mathrm{~h}$ bei RT gerührt. Die ockerfarbene Suspension wurde mit $30 \mathrm{~mL}$ Schwefelsäure (1 M) extrahiert und anschließend die saure Phase mit Natronlauge (1 M) auf einen pH-Wert von 10 eingestellt. Der dabei ausfallende braune Feststoff wurde abgetrennt, mit $15 \mathrm{~mL} n$-Hexan gewaschen und i. Vak. getrocknet. Durch Lagerung einer gesättigten Lösung in THF / $n$-Hexan (1: 1) wurden nach einigen Tagen Kristalle erhalten, die zur Bestimmung der Molekülstruktur geeignet waren.

Summenformel: $\quad \mathrm{C}_{24} \mathrm{H}_{17} \mathrm{~N}_{2} \mathrm{P}$

Ausbeute: $270 \mathrm{mg}(0.741 \mathrm{mmol}), 19 \%$
Molare Masse: $\quad 364.37 \mathrm{~g} / \mathrm{mol}$ Schmelzpunkt: n. b.

${ }^{1} \mathrm{H}$-NMR (500.130 MHz, CDCl$)_{3}$ ): $\delta 8.66-8.63\left(\mathrm{~m}, 3 \mathrm{H}, \mathrm{H}_{1,8,10}, \mathrm{An}\right), 8.60$ (ddd, ${ }^{3} \mathrm{~J}_{\mathrm{HH}}=$ $\left.4.82 \mathrm{~Hz},{ }^{4} \mathrm{~J}_{\mathrm{HH}}=1.73 \mathrm{~Hz},{ }^{5} \mathrm{~J}_{\mathrm{HH}}=0.97 \mathrm{~Hz}, 2 \mathrm{H}, \mathrm{H}_{\mathrm{a}}, \mathrm{Py}\right), 8.03\left(\mathrm{~d},{ }^{3} \mathrm{~J}_{\mathrm{HH}}=8.45 \mathrm{~Hz}, 2 \mathrm{H}\right.$, $\left.\mathrm{H}_{4,5}, \mathrm{An}\right), 7.52\left(\mathrm{~d},{ }^{3} \mathrm{~J}_{\mathrm{HH}}=7.93 \mathrm{~Hz}, 2 \mathrm{H}, \mathrm{H}_{\mathrm{d}}, \mathrm{Py}\right), 7.42-7.38\left(\mathrm{~m}, 4 \mathrm{H}, \mathrm{H}_{3,6}, \mathrm{An} / \mathrm{H}_{\mathrm{c}}, \mathrm{Py}\right)$, 7.29 (ddd, ${ }^{3} \mathrm{~J}_{\mathrm{HH}}=8.90 \mathrm{~Hz},{ }^{3} \mathrm{~J}_{\mathrm{HH}}=6.50 \mathrm{~Hz},{ }^{4} \mathrm{~J}_{\mathrm{HH}}=1.31 \mathrm{~Hz}, 2 \mathrm{H}, \mathrm{H}_{2,7}$, An), $7.04 \mathrm{ppm}$ (ddd, ${ }^{3} J_{\mathrm{HH}}=7.52 \mathrm{~Hz},{ }^{3} \mathrm{~J}_{\mathrm{HH}}=4.84 \mathrm{~Hz},{ }^{4} \mathrm{~J}_{\mathrm{HH}}=1.18 \mathrm{~Hz}, 2 \mathrm{H}, \mathrm{H}_{\mathrm{b}}, \mathrm{An}$ ).

${ }^{13} \mathrm{C}\left\{{ }^{1} \mathrm{H}\right\}$-NMR $\left(125.756 \mathrm{MHz}, \mathrm{CDCl}_{3}\right): \delta 163.2\left(\mathrm{~d},{ }^{1} \mathrm{~J}_{\mathrm{CP}}=2.57 \mathrm{~Hz}, 2 \mathrm{C}, \mathrm{C}_{i p s o}, \mathrm{Py}\right)$, $149.9\left(\mathrm{~d},{ }^{3} J_{\mathrm{CP}}=10.7 \mathrm{~Hz}, 2 \mathrm{C}, \mathrm{C}_{\mathrm{a}}, \mathrm{Py}\right), 136.9\left(\mathrm{~d},{ }^{2} J_{\mathrm{CP}}=13.1 \mathrm{~Hz}, 2 \mathrm{C}, \mathrm{C}_{8 \mathrm{a}, 9 \mathrm{a}}, A n\right)$, $135.3\left(\mathrm{~d},{ }^{3} J_{\mathrm{CP}}=2.17 \mathrm{~Hz}, 2 \mathrm{C}, \mathrm{C}_{\mathrm{c}}, \mathrm{Py}\right), 132.3\left(\mathrm{~d},{ }^{4} \mathrm{~J}_{\mathrm{CP}}=1.79 \mathrm{~Hz}, 1 \mathrm{C}, \mathrm{C}_{10}, \mathrm{An}\right), 132.0$ $\left(\mathrm{d},{ }^{3} J_{\mathrm{CP}}=4.94 \mathrm{~Hz}, 2 \mathrm{C}, \mathrm{C}_{4 \mathrm{a}, 10 \mathrm{a}}, \mathrm{An}\right), 130.4\left(\mathrm{~d},{ }^{1} \mathrm{~J}_{\mathrm{CP}}=109.1 \mathrm{~Hz}, 1 \mathrm{C}, \mathrm{C}_{9}, \mathrm{An}\right), 129.4(\mathrm{~s}$, 
2 C, $\left.\mathrm{C}_{4,5}, \mathrm{An}\right), 128.3\left(\mathrm{~d},{ }^{3} \mathrm{~J}_{\mathrm{CP}}=22.8 \mathrm{~Hz}, 2 \mathrm{C}, \mathrm{C}_{1,8}, \mathrm{An}\right), 126.6\left(\mathrm{~d},{ }^{2} \mathrm{~J}_{\mathrm{CP}}=18.4 \mathrm{~Hz}, 2 \mathrm{C}\right.$, $\left.\mathrm{C}_{\mathrm{d}}, \mathrm{Py}\right), 126.3\left(\mathrm{~d},{ }^{4} \mathrm{~J}_{\mathrm{CP}}=1.53 \mathrm{~Hz}, 2 \mathrm{C}, \mathrm{C}_{2,7}, \mathrm{An}\right), 125.0$ (s, $\left.2 \mathrm{C}, \mathrm{C}_{3,6}, \mathrm{An}\right), 121.5 \mathrm{ppm}$ (d, ${ }^{4} J_{\mathrm{CP}}=1.41 \mathrm{~Hz}, 2 \mathrm{C}, \mathrm{C}_{\mathrm{b}}, \mathrm{An}$ ).

${ }^{31} \mathrm{P}\left\{{ }^{1} \mathrm{H}\right\}-N M R\left(202.456 \mathrm{MHz}, \mathrm{CDCl}_{3}\right): \delta-24.8 \mathrm{ppm}(\mathrm{s})$.

El-MS (70 eV): m/z (\%): 364 (40) [M] ${ }^{+}, 285$ (100) [M - HPy] ${ }^{+}, 187$ (4) [PPy $]^{+}$.

Elementaranalyse (gef. (ber.) [\%]): C 72.04 (79.11), H 4.85 (4.70), N 6.84 (7.69).

\subsubsection{Bis[10-(diphenylthiophosphoryl)anthracen-9-yl]sulfid (29)}

$200 \mathrm{mg}$ (0.423 mmol) 3 (vermutlich mit Schwefel verunreinigt) und $150 \mathrm{mg}$ (0.847 mmol) Lithiumdi(2-pyridyl)amid wurden in $30 \mathrm{~mL}$ THF suspendiert und für $20 \mathrm{~d}$ bei RT gerührt. Die braune Reaktionsmischung wurde über Celite filtriert und das Filtrat auf etwa die Hälfte aufkonzentriert. Durch Lagerung der Lösung bei $4{ }^{\circ} \mathrm{C}$ bildeten sich gelbe, nadelförmige Kristalle von 29, die zur Bestimmung der Molekülstruktur geeignet waren.

Summenformel: $\mathrm{C}_{52} \mathrm{H}_{36} \mathrm{P}_{2} \mathrm{~S}_{3} \quad$ Molare Masse: $818.98 \mathrm{~g} / \mathrm{mol}$ Ausbeute: $\quad$ n. b. Schmelzpunkt: n. b.

${ }^{1} \mathrm{H}-\mathrm{NMR}\left(300.130 \mathrm{MHz}, \mathrm{CDCl}_{3}\right.$ ): $\delta 8.51\left(\mathrm{~d},{ }^{3} \mathrm{~J}_{\mathrm{HH}}=9.00 \mathrm{~Hz}, 4 \mathrm{H}, \mathrm{H}_{1,8}, \mathrm{An}\right.$ ), 8.04 (d, $\left.{ }^{3} J_{\mathrm{HH}}=9.00 \mathrm{~Hz}, 4 \mathrm{H}, \mathrm{H}_{4,5}, \mathrm{An}\right), 7.53-7.46\left(\mathrm{~m}, 8 \mathrm{H}, \mathrm{H}_{\text {ortho }}, \mathrm{Ph}\right), 7.29-7.18(\mathrm{~m}, 8 \mathrm{H}$, $\left.\mathrm{H}_{2,7}, \mathrm{An} / \mathrm{H}_{\text {para }}, \mathrm{Ph}\right), 7.11$ - 7.05 (m, $\left.8 \mathrm{H}, \mathrm{H}_{\text {meta }}, \mathrm{Ph}\right), 7.06$ - 6.98 ppm (m, $4 \mathrm{H}, \mathrm{H}_{3,6}$, An).

${ }^{31} \mathrm{P}\left\{{ }^{1} \mathrm{H}\right\}-N M R\left(121.495 \mathrm{MHz}, \mathrm{CDCl}_{3}\right): \delta 34.4 \mathrm{ppm}(\mathrm{s})$.

El-MS (70 eV): m/z (\%): 818 (5) [M] ${ }^{+}, 786(19)[\mathrm{M}-\mathrm{S}]^{+}, 633(100)\left[\mathrm{M}-\mathrm{PPh}_{2}\right]^{+}, 601$ (29) $\left[\mathrm{M}-\mathrm{Ph}_{2} \mathrm{PS}\right]^{+}, 447$ (52) [M - Ph $\left.{ }_{2} \mathrm{PS}-2 \mathrm{Ph}\right]^{+}, 217(61)\left[\mathrm{Ph}_{2} \mathrm{PS}\right]^{+}$.

\subsubsection{Natrium-[10-(diphenylthiophosphoryl)anthracen-9-yl]thiolat (30)}

$200 \mathrm{mg} \quad(0.423 \mathrm{mmol}) 3$ (vermutlich mit Schwefel verunreinigt) und $150 \mathrm{mg}$ (3.85 mmol) Natriumamid wurden in $20 \mathrm{~mL}$ THF suspendiert und für $5 \mathrm{~d}$ bei RT gerührt. Die tiefrote Reaktionsmischung wurde über Celite filtriert, das Filtrat auf etwa die Hälfte aufkonzentriert und mit $5 \mathrm{~mL} n$-Hexan überschichtet. Nach einigen Tagen Lagerung bei $6^{\circ} \mathrm{C}$ bildeten sich rote Kristalle, die für eine Röntgenstruktur- 
untersuchung geeignet waren. 30 wurde ausschließlich durch Bestimmung der Molekülstruktur charakterisiert.

Summenformel: $\quad \mathrm{C}_{42} \mathrm{H}_{50} \mathrm{NaO}_{4} \mathrm{PS}_{2}$

Molare Masse: $\quad 736.90 \mathrm{~g} / \mathrm{mol}$

\subsubsection{9-Brom-10-hydroxydiphenylmethyl-9,10-epidioxyanthracen} (31)

Eine Suspension von $1.00 \mathrm{~g}$ (2.98 mmol) 9,10-Dibromanthracen in $25 \mathrm{~mL}$ Diethylether wurde bei $-15^{\circ} \mathrm{C}$ mit $1.30 \mathrm{~mL}(3.03 \mathrm{mmol}){ }^{n} \mathrm{BuLi}$ in $n$-Hexan (2.33 M) versetzt. Nach $10 \mathrm{~min}$ wurde eine Lösung von $540 \mathrm{mg}$ (2.96 mmol) Benzophenon in $10 \mathrm{~mL}$ Diethylether zugetropft und nach weiteren 15 min wurde das Kältebad entfernt. Nach Erwärmen der Reaktionsmischung auf RT fiel ein blassgelber Niederschlag aus. Nach $16 \mathrm{~h}$ Rühren wurde mit $30 \mathrm{~mL}$ Wasser hydrolysiert, die Phasen getrennt und die wässrige mit $10 \mathrm{~mL}$ Diethylether gewaschen. Die vereinigten organischen Phasen wurden über Magnesiumsulfat getrocknet und anschließend das Lösungsmittel i. Vak. entfernt. Durch Lagerung einer Lösung des Rückstandes in Diethylether bei RT wurden nach einigen Tagen gelbe Kristalle von $\mathbf{3 1}$ erhalten, die zur Bestimmung der Molekülstruktur geeignet waren. Die Verbindung wurde ausschließlich durch eine Einkristall-Röntgenstrukturanalyse charakterisiert.

Summenformel: $\mathrm{C}_{27} \mathrm{H}_{19} \mathrm{BrO}_{3}$

Molare Masse: $\quad 471.33 \mathrm{~g} / \mathrm{mol}$

Ausbeute: $\quad 820 \mathrm{mg}(1.74 \mathrm{mmol}),(59 \%) \quad$ Schmelzpunkt: $\mathrm{n} . \mathrm{b}$.

\subsubsection{9 [9-Diphenylphosphoryl-10-diphenylhydroxyphosphoryl-9,10- dihydroanthracen]hydrogensulfat (32)}

Eine Lösung von $500 \mathrm{mg}(0.864 \mathrm{mmol}) 15$ (vermutlich mit PAnP verunreinigt) in $20 \mathrm{~mL}$ Dichlormethan wurde bei $-15^{\circ} \mathrm{C}$ mit $100 \mathrm{mg}(1.06 \mathrm{mmol}) \mathrm{H}_{2} \mathrm{O}_{2} \cdot\left(\mathrm{H}_{2} \mathrm{~N}\right)_{2} \mathrm{C}=\mathrm{O}$ versetzt. Nach 30 min wurde das Kältebad entfernt und nach weiteren 45 min die Reaktionsmischung mit Wasser gewaschen $(2 \times 20 \mathrm{~mL})$ und die organische Phase über Magnesiumsulfat getrocknet. Nach Entfernen des Lösungsmittels i. Vak. wurde 32 als oranger Schaum erhalten. Lagerung einer gesättigten Lösung in Toluol bei RT lieferte nach einigen Tagen Kristalle, die für eine röntgenkristallographische Strukturbestimmung geeignet waren. Die Verbindung wurde ausschließlich durch Bestimmung der Molekülstruktur charakterisiert.

Summenformel: $\mathrm{C}_{38} \mathrm{H}_{32} \mathrm{O}_{6} \mathrm{P}_{2} \mathrm{~S}$

Molare Masse: $\quad 678.64 \mathrm{~g} / \mathrm{mol}$ 


\section{KRISTALLOGRAPHISCHER TEIL}

\subsection{Kristallapplikation und Datensammlung}

Luftempfindliche Kristalle wurden mittels Schlenk-Technik ${ }^{[164-166]}$ im Argongegenstrom aus Kolben entnommen und auf einem Objektträger in perfluoriertes Polyetheröl gegeben, bei temperaturempfindlichen Kristallen wurde das Öl zusätzlich mit einer X-TEMP2-Kühleinheit gekühlt. ${ }^{[168,169]}$ Das Öl hat den Vorteil, dass alle bisher untersuchten Verbindungen darin unlöslich waren. So war gewährleistet, dass die Kristalle sich weder auflösten noch Lösungsmittelmoleküle verloren. Ein geeigneter Kristall hoher Qualität wurde mit Hilfe eines Polarisationsmikroskops ausgewählt und so auf der Spitze eines Glasfadens positioniert, dass ein Öltropfen den Kristall vollständig umschloss. ${ }^{[170]}$ Dieser wurde sofort im Stickstoff-Kaltgasstrom der Tieftemperaturanlage am Diffraktometer schockgefroren. Der Vorteil dieser Methode liegt darin, dass das Öl glasartig erstarrt und der Kristall während der Datensammlung auf dem Glasfaden fixiert bleibt.

\section{Bruker Diffraktometer:}

Die Verbindungen 2, 3, 11, 15, 19, 22 und 29 wurden unter Verwendung graphitmonochromatisierter Mo-K $\mathrm{K}_{\alpha}-$ Strahlung $(\lambda=71.073 \mathrm{pm})$ vermessen. Die Sammlung der Daten erfolgte auf einem Bruker D8 Goniometer mit einem SMART APEX I CCDFlächenzähler. In der zweiten Hälfte dieser Arbeit wurde das Diffraktometer mit einem SMART APEX II CCD-Flächenzähler bestückt und mit diesem Aufbau die Verbindungen 7, 8, 10, 12, 14, 17, 18, 20, 23, 25, 28, 30 - 32 gemessen.

5, 13 und 21 wurden mit einer Bruker TXS Drehanode vermessen. Diese war ausgestattet mit einem Dreikreis D8 Goniometer und einem SMART APEX II CCDFlächenzähler. Die Monochromatisierung der Strahlung erfolgte mittels INCOATEC Helios Spiegeln, die eine sehr intensive Mo-K $\mathrm{K}_{\alpha}$-Strahlung $(\lambda=71.073 \mathrm{pm})$ liefern.

Alle Kristalle wurden nach der Applikation mit Hilfe einer Videokamera vor Beginn der Datensammlung zentriert.

Die Sammlung der Daten erfolgte je nach verwendetem Detektor und Goniometer mit Hilfe der SMART ${ }^{[171]}$ oder APEX2 ${ }^{[172]}$ Programmpakete. Um die Kristallqualität bewerten zu können und einen ersten Wert für die Gitterkonstanten der Elementarzelle zu bekommen, wurden etwa 50 Bilder im $\omega$-scan-Modus bei $\varphi=0^{\circ}$ 
mit einer Schrittbreite von $0.3^{\circ}$ aufgenommen. Entsprechend der Ergebnisse dieses Prescans wurden für die nachfolgenden Datensammlungen passende Belichtungszeiten gewählt. Die Reflexe wurden im $\omega$-scan-Modus mit einer Schrittbreite von $0.3^{\circ}$ unter festem $\varphi$-Winkel detektiert.

Die Bestimmungen und Verfeinerungen der Elementarzellen wurden mit den SMART und APEX2 Programmpaketen durchgeführt. Die Datenintegration erfolgte mit SAINT ${ }^{[173,174]}$ unter Verwendung der 3d-profiling Methode nach Kabsch. ${ }^{[175]}$

Alle Datensätze mit Ausnahme von 11 und 16 wurden mittels SADABS ${ }^{[176,177]}$ absorptionskorrigiert und skaliert. Vor der Absorptionskorrektur wurde mit XPREP ${ }^{[178]}$ die Raumgruppe bestimmt, da diese bekannt sein muss, um mit SADABS eine empirische Modell-Funktion durch den Vergleich symmetrie-äquivalenter Reflexe zu verfeinern.

Die Daten wurden gemäß der ermittelten Symmetrie gemerged und die Dateien für die Strukturlösung und -verfeinerung mit XPREP erstellt.

\section{Stoe Diffraktometer:}

Die Verbindungen 4, 16 und 24 wurden mit einem Stoe IPDS II Diffraktometer mit einem Bildplatten-Detektor ("image plate") unter Verwendung graphitmonochromatisierter Mo- $\mathrm{K}_{\alpha}$-Strahlung $(\lambda=71.073 \mathrm{pm})$ gemessen.

Nach der Applikation wurde der Kristall mit Hilfe einer Videokamera vor Beginn der Datensammlung zentriert.

Alle Schritte von der Datensammlung bis zur Integration wurden mit dem X-AREA Programmpaket von Stoe \& Cie durchgeführt. ${ }^{[179]}$ Vor der Messung wurden 20 Bilder im $\omega$-scan-Modus bei $\varphi=0^{\circ}$ aufgenommen, um die Kristallqualität bewerten zu können, einen ersten Wert für die Gitterkonstanten der Elementarzelle zu bekommen und die optimale Belichtungszeit abschätzen zu können. Die Reflexe wurden im $\omega$ scan-Modus mit einer Schrittbreite von $1.0^{\circ}$ unter festem $\varphi$-Winkel detektiert. Die Strategie der Datensammlung wurde so gewählt, dass $100 \%$ der Reflexe bis zu einer Auflösung von $\sin \theta / \lambda=0.8 \AA$ mit einer Gesamtredundanz von mindestens 5 gesammelt wurden. 


\subsection{Strukturlösung und Verfeinerung}

Alle Strukturen wurden mit direkten Methoden gelöst (SHELXS). ${ }^{[180]}$ Die Strukturverfeinerungen erfolgten gegen $F^{2}$ mit SHELXL. ${ }^{[181,182]}$

Sofern nicht anders angegeben wurden die Wasserstoffatome nach dem Reitermodell geometrisch ideal positioniert und mit isotropen Auslenkungsparametern in die Verfeinerung einbezogen. Dabei wurden die $U_{\text {iso }}$ Werte für Wasserstoffatome in $\mathrm{CH}_{3}$-Gruppen auf das 1.5-fache, für alle anderen Wasserstoffatome auf das 1.2-fache der $U_{\text {eq }}$-Werte der dazugehörigen Kohlenstoffatome gesetzt. In allen Verfeinerungen wurde die Funktion $M\left(p_{i}, k\right)$ (GI. 9-1) über das Gewichtungsschema (GI. 9-2) minimiert. Die Werte $g 1$ und $g 2$ sind in den kristallographischen Tabellen aufgeführt.

GI. 9-1:

$$
M\left(p_{i}, k\right)=\sum_{\mathbf{H}} w_{\mathbf{H}}\left[k\left|F_{\text {obs }}(\mathbf{H})\right|^{2}-\left|F_{\text {calc }}(\mathbf{H})\right|^{2}\right]^{2}=\min
$$

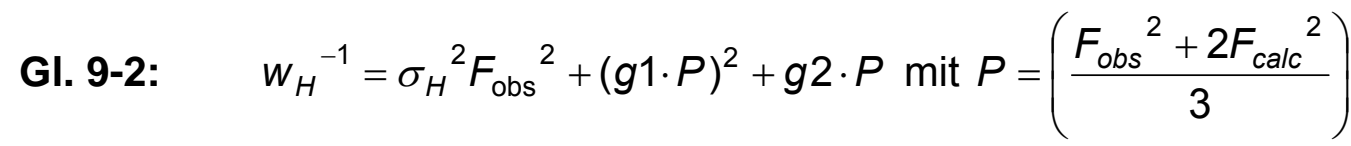

Um die Ergebnisse der Verfeinerungen bewerten zu können wurden die berechneten mit den beobachteten Strukturfaktoren verglichen. Gebräuchliche Gütekriterien sind die Residualwerte $R 1$ (GI. 9-3) und wR2 (GI. 9-4). Der wR2 ist aussagekräftiger, weil das Modell gegen $F^{2}$ verfeinert wird.

GI. 9-3: $\quad R 1=\frac{\sum_{H} w_{H}\left(\left|F_{\text {obs }}\right|-\left|F_{\text {calc }}\right|\right)}{\sum_{H} w_{H}\left|F_{\text {obs }}\right|} \quad$ GI. 9-4: $\quad w R 2=\frac{\sum_{H} w_{H}\left(\left|F_{\text {obs }}\right|^{2}-\left|F_{\text {calc }}\right|^{2}\right)^{2}}{\sum_{H} w_{H}\left|F_{\text {obs }}\right|^{4}}$

Zusätzlich wurde der GoF (goodness of fit, Gl. 9-5) berechnet, der Abweichungen von $F_{\text {calc }}$ gegenüber $F_{\text {obs }}$ sowie die Überbewertung von verfeinerten Parametern widerspiegelt.

GI. 9-5:

$$
\mathrm{GoF}=\sqrt{\frac{\sum_{H} w_{H}\left(\left|F_{\text {obs }}\right|-\left|F_{\text {calc }}\right|\right)^{2}}{N-n}}
$$

Die Residualdichten der Differenzfourieranalyse sollten möglichst niedrig sein und werden gemäß dem verwendeten Modell in den Bindungsregionen zwischen Atomen gefunden. Höhere Differenzdichten für stark streuende Atome sind akzeptabel, da sie vorwiegend aus Absorptionseffekten sowie dem begrenzten Auflösungsbereich während der Messung resultieren Die maximalen und minimalen Differenzelektronendichten sind in den kristallographischen Tabellen angegeben. 
Darüber hinaus zeigen die Größe, Orientierung und Elliptizität der anisotropen Auslenkungsellipsoide die Qualität des Modells. Im Idealfall sollten die ADPs senkrecht zu den Bindungen angeordnet sein, ähnliche Größen aufweisen und geringe Elliptizität zeigen. Die ADPs aller Atome der asymmetrischen Einheiten der Verbindungen sind in Kap. 9.3 und 9.4 gezeigt und stellen $50 \%$ der Aufenthaltswahrscheinlichkeit der Elektronendichte der Atome dar. Nicht frei verfeinerte Wasserstoffatome werden aus Gründen der Übersichtlichkeit nicht gezeigt. Alle Strukturbilder wurden mit dem Programm XSHELL erstellt. ${ }^{[183]}$

\section{Verfeinerung von Fehlordnungen:}

Strukturen mit Fehlordnungen wurden mit Hilfe von constraints und restraints aufgelöst und verfeinert. Ein constraint ist eine mathematische Operation, die Parameter auf einen exakten Wert setzt, beispielsweise die Berechnung der Wasserstoffpositionen nach geometrischen Gesichtspunkten.

Restraints sind zusätzliche chemische oder kristallographische Informationen über die Struktur einer Verbindung, die mit einer Standardabweichung versehen sind. Diese restraints gehen als zusätzliche Daten in die Verfeinerung ein. Die Standardabweichung hat dabei die Funktion einer Gewichtung.

Wenn in einer Struktur chemisch gleiche, aber kristallographisch unabhängige Strukturelemente vokommen, ist die Anwendung von Abstands-restraints sinnvoll. Hier können vor allem 1,2-Abstände (Bindungslängen) und 1,3-Abstände (Winkel) innerhalb einer Standardabweichung zu einem ähnlichen Wert verfeinert werden. Vor allem bei der Verfeinerung fehlgeordneter Positionen kann durch diese restraints eine erhebliche Verbesserung des Strukturmodells erfolgen, da über Mittelung äquivalenter Strukturfragmente chemische Informationen genutzt werden können.

Um auch fehlgeordnete Atompositionen mit Hilfe eines anisotropen Modells verfeinern zu können, sind häufig restraints nötig, die die ADPs betreffen. Mittels rigid bond restraints (DELU) werden die Komponenten der ADPs entlang der Bindungen innerhalb einer Standardabweichung angeglichen. Similarity restraints (SIMU) sind schwache restraints, welche die Auslenkungsparameter benachbarter Atome in einem bestimmten Radius einander annähern. ISOR restraints zwingen ADP's in eine eher sphärische, isotrope Form. ${ }^{[182]}$ 


\subsection{Verfeinerung der Verbindungen 2-5, 7-8, 10 - 25, $28-32$}

\subsection{1 $\mathrm{Br}\left(\mathrm{C}_{14} \mathrm{H}_{8}\right) \mathrm{P}(\mathrm{O}) \mathrm{Ph}_{2}(2), \mathrm{CCDC}: 610421$}

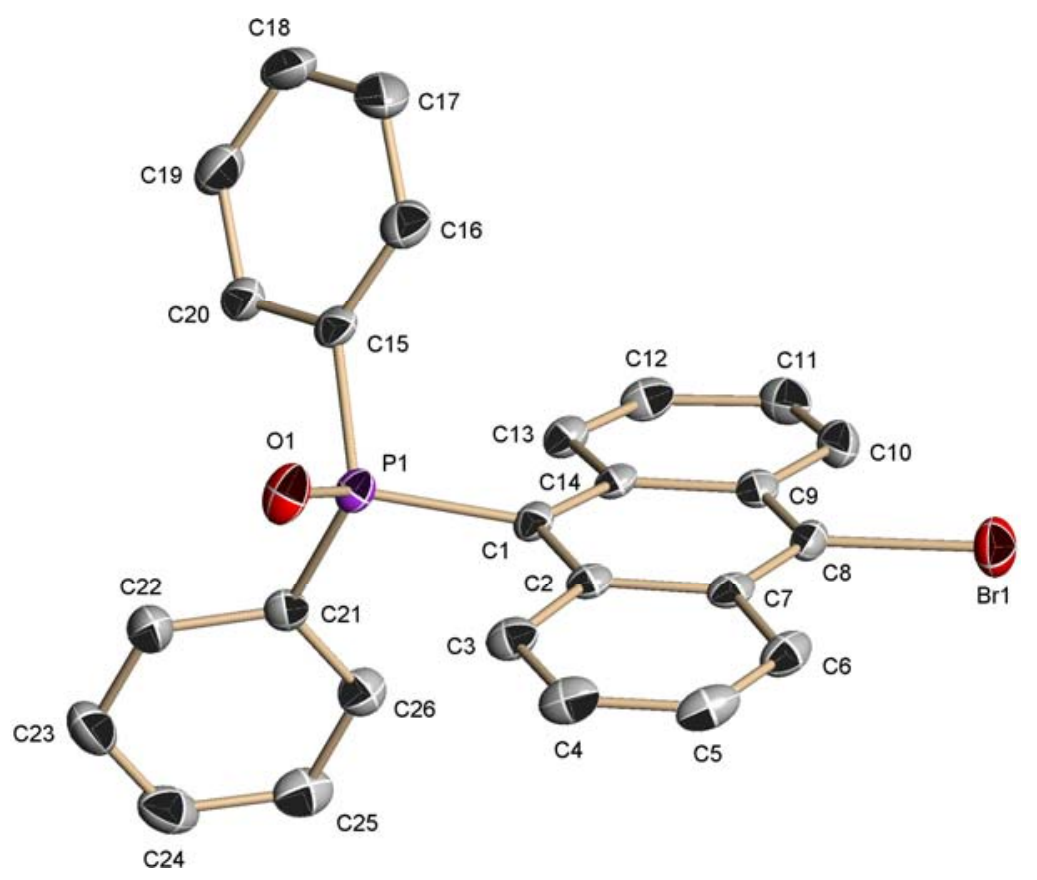

Abb. 9-1: Asymmetrische Einheit von $\operatorname{Br}\left(\mathrm{C}_{14} \mathrm{H}_{8}\right) \mathrm{P}(\mathrm{O}) \mathrm{Ph}_{2}$ (2).

Tab. 9-1: Kristallographische Daten für $\operatorname{Br}\left(\mathrm{C}_{14} \mathrm{H}_{8}\right) \mathrm{P}(\mathrm{O}) \mathrm{Ph}_{2}$ (2).

\begin{tabular}{|c|c|c|c|}
\hline Strukturcode & OPAnBr & $\rho_{\text {calc }} / \mathrm{Mg} \cdot \mathrm{m}^{-3}$ & 1.512 \\
\hline Summenformel & $\mathrm{C}_{26} \mathrm{H}_{18} \mathrm{BrOP}$ & $\mu / \mathrm{mm}^{-1}$ & 2.141 \\
\hline Molmasse $/ \mathrm{g} \cdot \mathrm{mol}^{-1}$ & 457.28 & $F(000)$ & 928 \\
\hline Kristallgröße / mm & $0.2 \times 0.15 \times 0.15$ & $\theta$-Bereich / ${ }^{\circ}$ & $2.01-26.39$ \\
\hline Kristallsystem & monoklin & Reflexe gesamt/unabhängig & 16414 / 4108 \\
\hline Raumgruppe & $P 2_{1} / n$ & Daten/Restraints/Parameter & 4108 / 0 / 262 \\
\hline $\mathrm{a} / \mathrm{pm}$ & $944.01(5)$ & $R 1[I>2 \sigma(I)]$ & 0.0381 \\
\hline $\mathrm{b} / \mathrm{pm}$ & $1682.77(9)$ & $w R 2$ (alle Daten) & 0.1050 \\
\hline $\mathrm{c} / \mathrm{pm}$ & $1265.86(7)$ & $g 1 / g 2$ & $0.0575 / 1.8977$ \\
\hline$\beta /{ }^{\circ}$ & $92.7740(10)$ & GoF & 1.097 \\
\hline $\mathrm{V} / \mathrm{nm}^{3}$ & $2.00853(19)$ & Differenzelektronendichte: & \\
\hline Z & 4 & $\max . / \min . /\left(10^{-6} \mathrm{e} \cdot \mathrm{pm}^{-3}\right)$ & $0.519 /-0.349$ \\
\hline Messtemperatur / K & $100(2)$ & max. / min. Transmission & $0.99 / 0.85$ \\
\hline
\end{tabular}




\subsection{2 $\mathrm{Br}\left(\mathrm{C}_{14} \mathrm{H}_{8}\right)\left(\mathrm{P}(\mathrm{S}) \mathrm{Ph}_{2}\right)(3), \mathrm{CCDC}: 610422$}
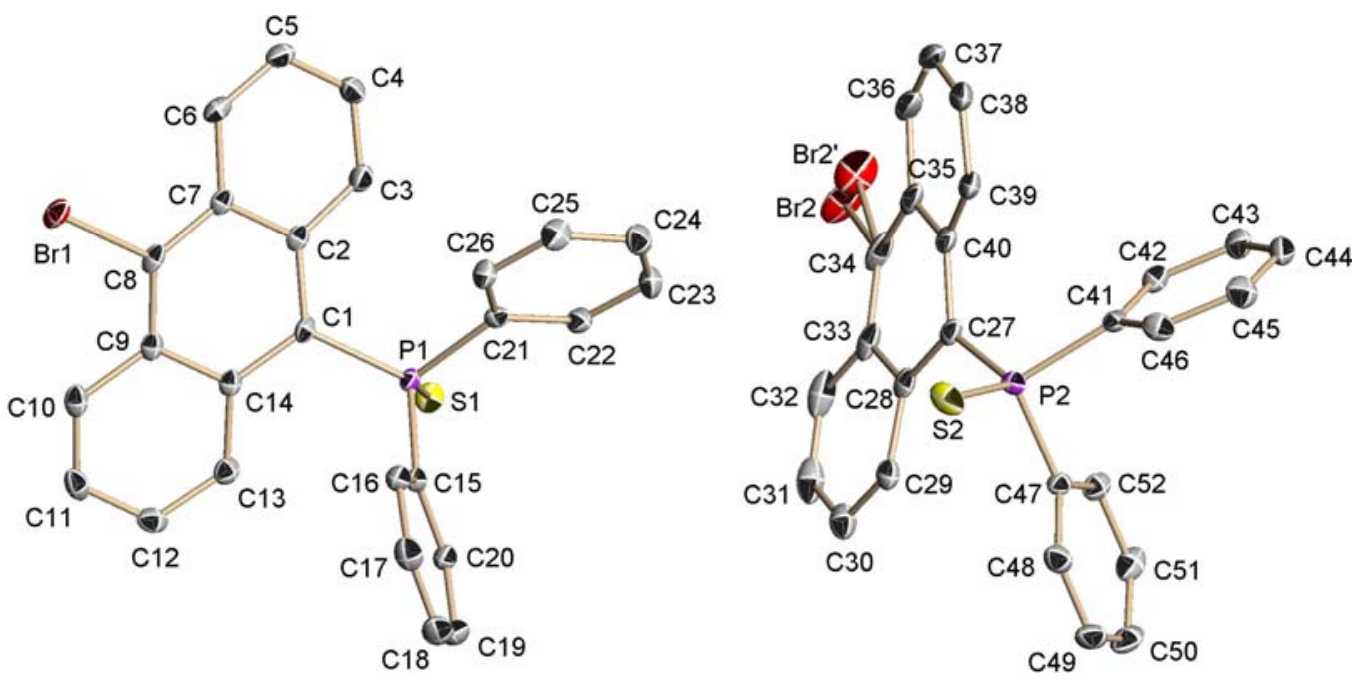

Abb. 9-2: Asymmetrische Einheit von $\operatorname{Br}\left(\mathrm{C}_{14} \mathrm{H}_{8}\right) \mathrm{P}(\mathrm{S}) \mathrm{Ph}_{2}(\mathbf{3})$.

In der asymmetrischen Einheit befinden sich zwei Moleküle, von denen eines ein zweifach fehlgeordnetes Bromatom aufweist. Mit den Besetzungsfaktoren 0.88 / 0.12 konnte eine anisotrope Verfeinerung durchgeführt werden.

Tab. 9-2: Kristallographische Daten für $\operatorname{Br}\left(\mathrm{C}_{14} \mathrm{H}_{8}\right) \mathrm{P}(\mathrm{S}) \mathrm{Ph}_{2}$ (3).

\begin{tabular}{|c|c|c|c|}
\hline Strukturcode & SPAnBr & $\rho_{\text {calc }} / \mathrm{Mg} \cdot \mathrm{m}^{-3}$ & 1.512 \\
\hline Summenformel & $\mathrm{C}_{26} \mathrm{H}_{18} \mathrm{BrPS}$ & $\mu / \mathrm{mm}^{-1}$ & 2.164 \\
\hline Molmasse $/ \mathrm{g} \cdot \mathrm{mol}^{-1}$ & 473.34 & $F(000)$ & 1920 \\
\hline Kristallgröße / mm & $0.3 \times 0.15 \times 0.05$ & $\theta$-Bereich / ${ }^{\circ}$ & $1.67-26.37$ \\
\hline Kristallsystem & monoklin & Reflexe gesamt/unabhängig & $51924 / 8512$ \\
\hline Raumgruppe & $P 2_{1} / n$ & Daten/Restraints/Parameter & $8512 / 0$ / 533 \\
\hline $\mathrm{a} / \mathrm{pm}$ & $1723.27(11)$ & $R 1[I>2 \sigma(I)]$ & 0.0391 \\
\hline $\mathrm{b} / \mathrm{pm}$ & $1392.49(9)$ & wR2 (alle Daten) & 0.0870 \\
\hline $\mathrm{c} / \mathrm{pm}$ & $1733.47(11)$ & $g 1 / g 2$ & $0.0365 / 4.4777$ \\
\hline$\beta /{ }^{\circ}$ & $90.4330(10)$ & GoF & 1.118 \\
\hline $\mathrm{V} / \mathrm{nm}^{3}$ & $4.1596(5)$ & Differenzelektronendichte: & \\
\hline Z & 8 & $\max . / \min . /\left(10^{-6} \mathrm{e} \cdot \mathrm{pm}^{-3}\right)$ & $0.565 /-0.269$ \\
\hline Messtemperatur / K & $100(2)$ & max. / min. Transmission & $0.99 / 0.67$ \\
\hline
\end{tabular}




\subsection{3 $\mathrm{Br}\left(\mathrm{C}_{14} \mathrm{H}_{8}\right) \mathrm{P}(\mathrm{Se}) \mathrm{Ph}_{2}(4), \mathrm{CCDC}: 610423$}

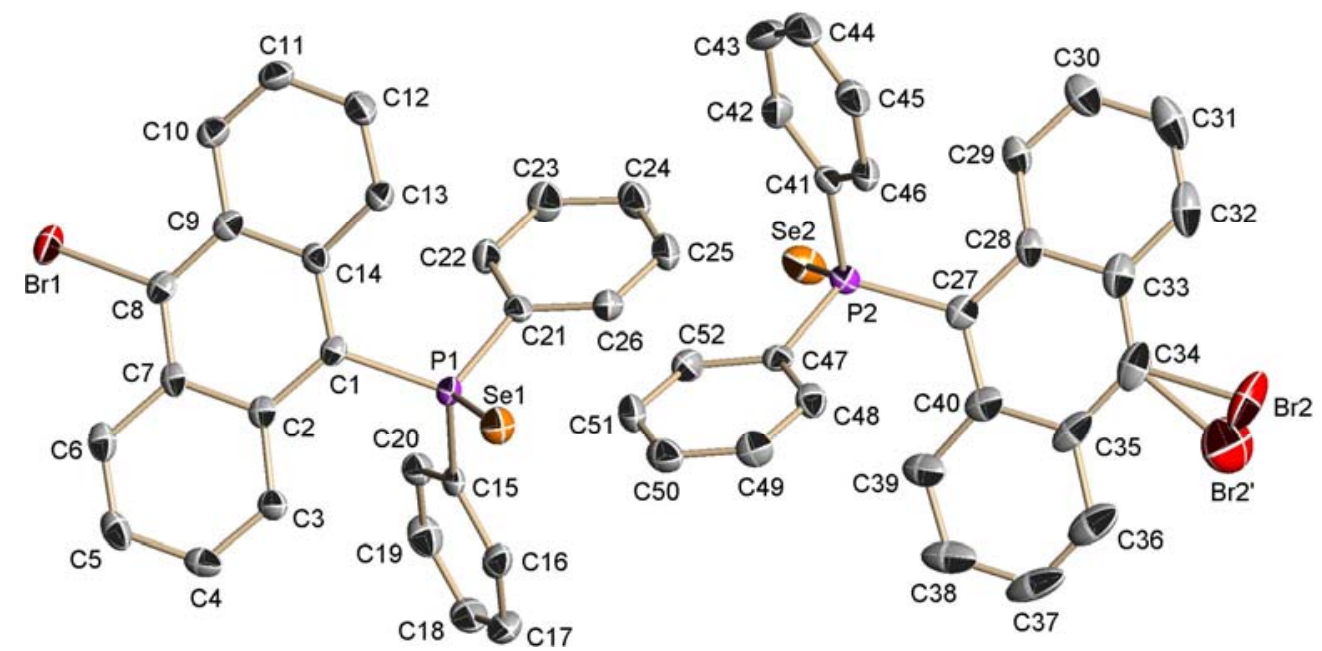

Abb. 9-3: Asymmetrische Einheit von $\operatorname{Br}\left(\mathrm{C}_{14} \mathrm{H}_{8}\right) \mathrm{P}(\mathrm{Se}) \mathrm{Ph}_{2}(4)$.

In der asymmetrischen Einheit befinden sich zwei Moleküle, von denen eines ein zweifach fehlgeordnetes Bromatom aufweist. Mit den Besetzungsfaktoren 0.90 / 0.10 konnte eine anisotrope Verfeinerung durchgeführt werden.

Tab. 9-3: Kristallographische Daten für $\mathrm{Br}\left(\mathrm{C}_{14} \mathrm{H}_{8}\right) \mathrm{P}(\mathrm{Se}) \mathrm{Ph}_{2}$ (4).

\begin{tabular}{|c|c|c|c|}
\hline Strukturcode & SePAnBr & $\rho_{\text {calc }} / \mathrm{Mg} \cdot \mathrm{m}^{-3}$ & 1.638 \\
\hline Summenformel & $\mathrm{C}_{26} \mathrm{H}_{18} \mathrm{BrPSe}$ & $\mu / \mathrm{mm}^{-1}$ & 3.759 \\
\hline Molmasse $/ \mathrm{g} \cdot \mathrm{mol}^{-1}$ & 520.24 & $F(000)$ & 2064 \\
\hline Kristallgröße / mm & $0.4 \times 0.2 \times 0.2$ & $\theta$-Bereich / ${ }^{\circ}$ & $1.65-24.77$ \\
\hline Kristallsystem & monoklin & Reflexe gesamt/unabhängig & $63532 / 7213$ \\
\hline Raumgruppe & $P 2_{1} / n$ & Daten/Restraints/Parameter & $7213 / 0 / 533$ \\
\hline $\mathrm{a} / \mathrm{pm}$ & $1717.1(3)$ & $R 1[I>2 \sigma(I)]$ & 0.0379 \\
\hline $\mathrm{b} / \mathrm{pm}$ & $1414.5(3)$ & $w R 2$ (alle Daten) & 0.0712 \\
\hline $\mathrm{c} / \mathrm{pm}$ & $1737.8(4)$ & $g 1 / g 2$ & $0.0339 / 0$ \\
\hline$\beta /{ }^{\circ}$ & $90.90(3)$ & GoF & 0.964 \\
\hline $\mathrm{V} / \mathrm{nm}^{3}$ & $4.2203(15)$ & Differenzelektronendichte: & \\
\hline Z & 8 & $\max . / \min . /\left(10^{-6} \mathrm{e} \cdot \mathrm{pm}^{-3}\right)$ & $0.348 /-0.516$ \\
\hline Messtemperatur / K & $133(2)$ & max. / min. Transmission & $0.6697 / 0.3966$ \\
\hline
\end{tabular}




\subsection{4 $\mathrm{Br}\left(\mathrm{C}_{14} \mathrm{H}_{8}\right) \mathrm{P}^{i} \mathrm{Pr}_{2}(5), \mathrm{CCDC}: 667523$}
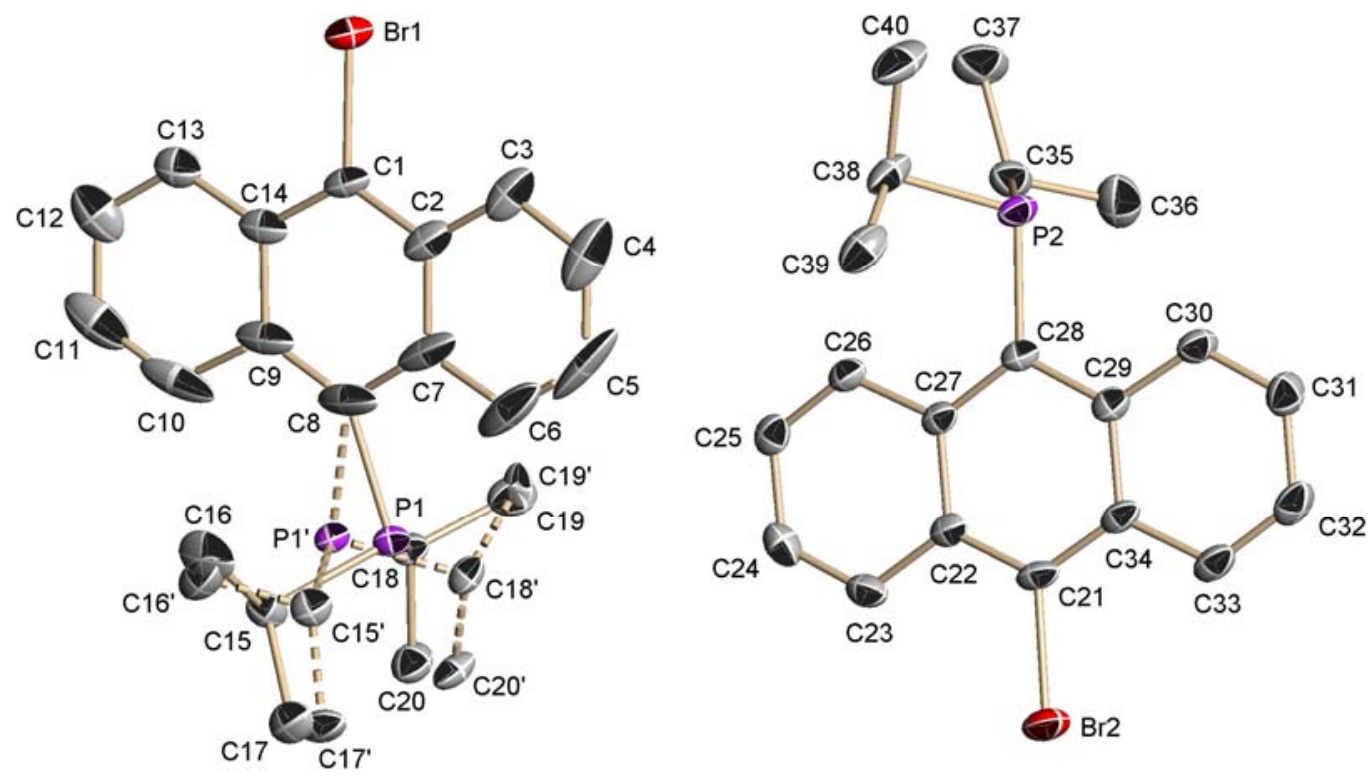

Abb. 9-4: Asymmetrische Einheit von $\operatorname{Br}\left(\mathrm{C}_{14} \mathrm{H}_{8}\right) \mathrm{P}^{\prime} \mathrm{Pr}_{2}$ (5).

In der asymmetrischen Einheit befinden sich zwei Moleküle, von denen eines eine zweifach fehlgeordnete ${ }^{i} \operatorname{Pr}_{2} \mathrm{P}$-Gruppe aufweist. Mit Hilfe von Abstands-restraints (SADI) konnte eine anisotrope Verfeinerung durchgeführt werden, die Besetzungsfaktoren verfeinerten zu 0.58 / 0.42 .

Tab. 9-4: Kristallographische Daten für $\operatorname{Br}\left(\mathrm{C}_{14} \mathrm{H}_{8}\right) \mathrm{P}^{i} \mathrm{Pr}_{2}$ (5).

\begin{tabular}{|c|c|c|c|}
\hline Strukturcode & Elli2 & $\rho_{\text {calc }} / \mathrm{Mg} \cdot \mathrm{m}^{-3}$ & 1.408 \\
\hline Summenformel & $\mathrm{C}_{20} \mathrm{H}_{22} \mathrm{BrP}$ & $\mu / \mathrm{mm}^{-1}$ & 2.421 \\
\hline Molmasse $/ \mathrm{g} \cdot \mathrm{mol}^{-1}$ & 373.26 & $F(000)$ & 1536 \\
\hline Kristallgröße / mm & $0.2 \times 0.2 \times 0.05$ & $\theta$-Bereich $/^{\circ}$ & $1.50-26.34$ \\
\hline Kristallsystem & monoklin & Reflexe gesamt/unabhängig & $20298 / 7113$ \\
\hline Raumgruppe & $P 2_{1} / n$ & Daten/Restraints/Parameter & $7113 / 75 / 473$ \\
\hline $\mathrm{a} / \mathrm{pm}$ & $833.27(7)$ & $R 1[I>2 \sigma(I)]$ & 0.0373 \\
\hline $\mathrm{b} / \mathrm{pm}$ & $1555.70(13)$ & $w R 2$ (alle Daten) & 0.0961 \\
\hline $\mathrm{c} / \mathrm{pm}$ & $2719.7(2)$ & $g 1 / g 2$ & $0.0475 / 2.5647$ \\
\hline$\beta /{ }^{\circ}$ & $93.059(2)$ & GoF & 1.064 \\
\hline $\mathrm{V} / \mathrm{nm}^{3}$ & $3.5206(5)$ & Differenzelektronendichte: & \\
\hline Z & 8 & $\max . / \min . /\left(10^{-6} \mathrm{e} \cdot \mathrm{pm}^{-3}\right)$ & $0.710 /-0.626$ \\
\hline Messtemperatur / K & $173(2)$ & max. / min. Transmission & $0.99 / 0.87$ \\
\hline
\end{tabular}




\subsection{5 $\mathrm{Br}\left(\mathrm{C}_{14} \mathrm{H}_{8}\right) \mathrm{P}(\mathrm{S})^{j} \mathrm{Pr}_{2}(7), \mathrm{CCDC}: 667524$}

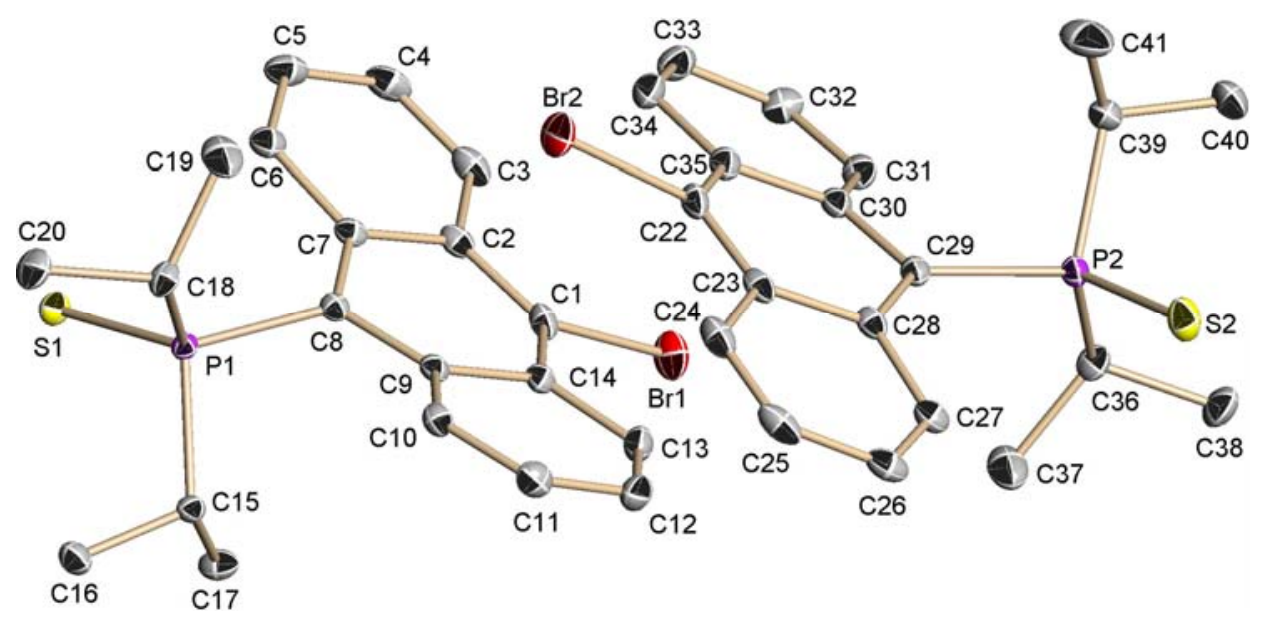

Abb. 9-5: Asymmetrische Einheit von $\operatorname{Br}\left(\mathrm{C}_{14} \mathrm{H}_{8}\right) \mathrm{P}(\mathrm{S})^{i} \mathrm{Pr}_{2}$ (7).

Tab. 9-5: Kristallographische Daten für $\operatorname{Br}\left(\mathrm{C}_{14} \mathrm{H}_{8}\right) \mathrm{P}(\mathrm{S})^{\prime} \mathrm{Pr}_{2}$ (7).

\begin{tabular}{|c|c|c|c|}
\hline Strukturcode & Hilde & $\rho_{\text {calc }} / \mathrm{Mg} \cdot \mathrm{m}^{-3}$ & 1.484 \\
\hline Summenformel & $\mathrm{C}_{20} \mathrm{H}_{22} \mathrm{BrPS}$ & $\mu / \mathrm{mm}^{-1}$ & 2.466 \\
\hline Molmasse $/ \mathrm{g} \cdot \mathrm{mol}^{-1}$ & 405.32 & $F(000)$ & 1664 \\
\hline Kristallgröße / mm & $0.4 \times 0.3 \times 0.1$ & $\theta$-Bereich / ${ }^{\circ}$ & $2.32-26.42$ \\
\hline Kristallsystem & monoklin & Reflexe gesamt/unabhängig & $65575 / 7452$ \\
\hline Raumgruppe & $P 2_{1} / c$ & Daten/Restraints/Parameter & $7452 / 0 / 419$ \\
\hline $\mathrm{a} / \mathrm{pm}$ & $2259.51(12)$ & $R 1[I>2 \sigma(I)]$ & 0.0232 \\
\hline $\mathrm{b} / \mathrm{pm}$ & $1105.27(6)$ & $w R 2$ (alle Daten) & 0.0634 \\
\hline $\mathrm{c} / \mathrm{pm}$ & $1454.79(8)$ & $g 1 / g 2$ & $0.0360 / 2.5749$ \\
\hline$\beta /{ }^{\circ}$ & $93.0230(10)$ & GoF & 1.034 \\
\hline $\mathrm{V} / \mathrm{nm}^{3}$ & $3.6281(3)$ & Differenzelektronendichte: & \\
\hline Z & 8 & $\max . / \min . /\left(10^{-6} \mathrm{e} \cdot \mathrm{pm}^{-3}\right)$ & $0.404 /-0.468$ \\
\hline Messtemperatur / K & $100(2)$ & max. / min. Transmission & $0.99 / 0.73$ \\
\hline
\end{tabular}




\subsection{6 $\mathrm{Br}\left(\mathrm{C}_{14} \mathrm{H}_{8}\right) \mathrm{P}(\mathrm{Se})^{i} \mathrm{Pr}_{2}$ (8), CCDC: 667525}

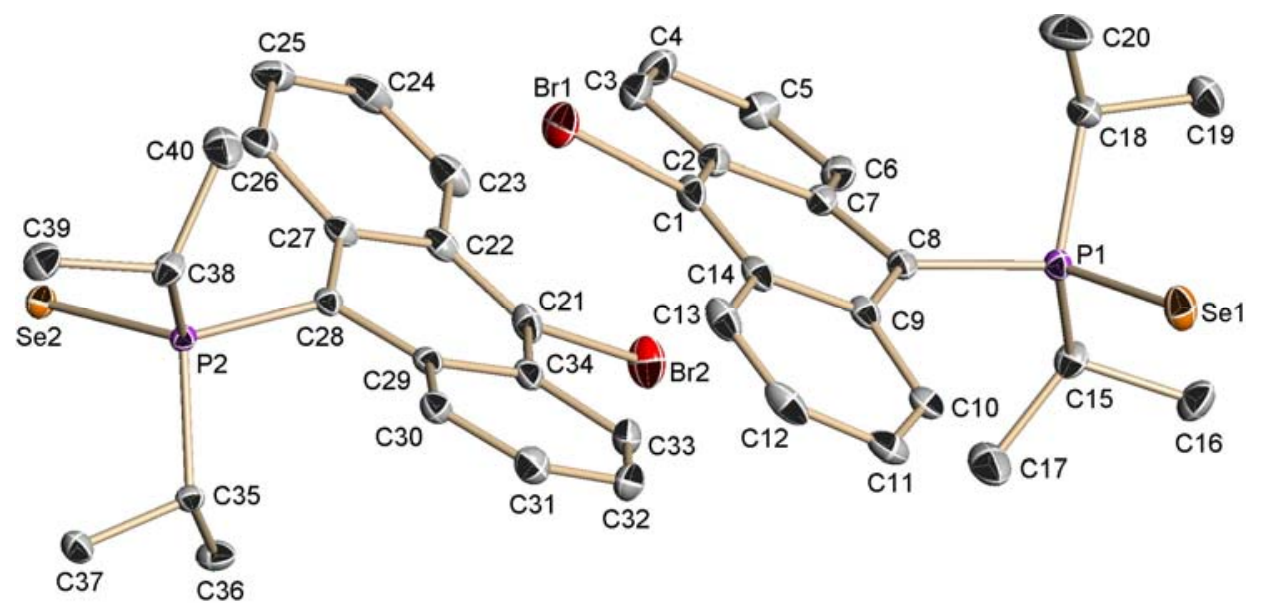

Abb. 9-6: Asymmetrische Einheit von $\operatorname{Br}\left(\mathrm{C}_{14} \mathrm{H}_{8}\right) \mathrm{P}(\mathrm{Se})^{j} \mathrm{Pr}_{2}$ (8).

Tab. 9-6: Kristallographische Daten für $\operatorname{Br}\left(\mathrm{C}_{14} \mathrm{H}_{8}\right) \mathrm{P}(\mathrm{Se})^{i} \mathrm{Pr}_{2}$ (8).

\begin{tabular}{|c|c|c|c|}
\hline Strukturcode & Singani & $\rho_{\text {calc }} / \mathrm{Mg} \cdot \mathrm{m}^{-3}$ & 1.631 \\
\hline Summenformel & $\mathrm{C}_{20} \mathrm{H}_{22} \mathrm{BrPSe}$ & $\mu / \mathrm{mm}^{-1}$ & 4.292 \\
\hline Molmasse $/ \mathrm{g} \cdot \mathrm{mol}^{-1}$ & 452.22 & $F(000)$ & 1808 \\
\hline Kristallgröße / mm & $0.4 \times 0.25 \times 0.2$ & $\theta$-Bereich $/^{\circ}$ & $2.03-26.41$ \\
\hline Kristallsystem & monoklin & Reflexe gesamt/unabhängig & $77880 / 7556$ \\
\hline Raumgruppe & $P 2_{1} / c$ & Daten/Restraints/Parameter & $7556 / 0$ / 419 \\
\hline $\mathrm{a} / \mathrm{pm}$ & $2261.34(14)$ & $R 1[I>2 \sigma(I)]$ & 0.0212 \\
\hline $\mathrm{b} / \mathrm{pm}$ & $1119.67(7)$ & $w R 2$ (alle Daten) & 0.0553 \\
\hline $\mathrm{c} / \mathrm{pm}$ & $1457.51(9)$ & $g 1 / g 2$ & $0.0242 / 4.2764$ \\
\hline$\beta /{ }^{\circ}$ & $93.4680(10)$ & GoF & 1.084 \\
\hline $\mathrm{V} / \mathrm{nm}^{3}$ & $3.6836(4)$ & Differenzelektronendichte: & \\
\hline Z & 8 & $\max . / \min . /\left(10^{-6} \mathrm{e} \cdot \mathrm{pm}^{-3}\right)$ & $0.416 /-0.699$ \\
\hline Messtemperatur / K & $100(2)$ & max. / min. Transmission & $0.99 / 0.65$ \\
\hline
\end{tabular}




\subsection{7 [2 $\left.\mathrm{C}_{6} \mathrm{H}_{6} @\left\{\left(\mathrm{Ph}_{2}(\mathrm{~S}) \mathrm{P}\right)_{2}\left(\mathrm{C}_{14} \mathrm{H}_{8}\right)\right\}\right](10)$}

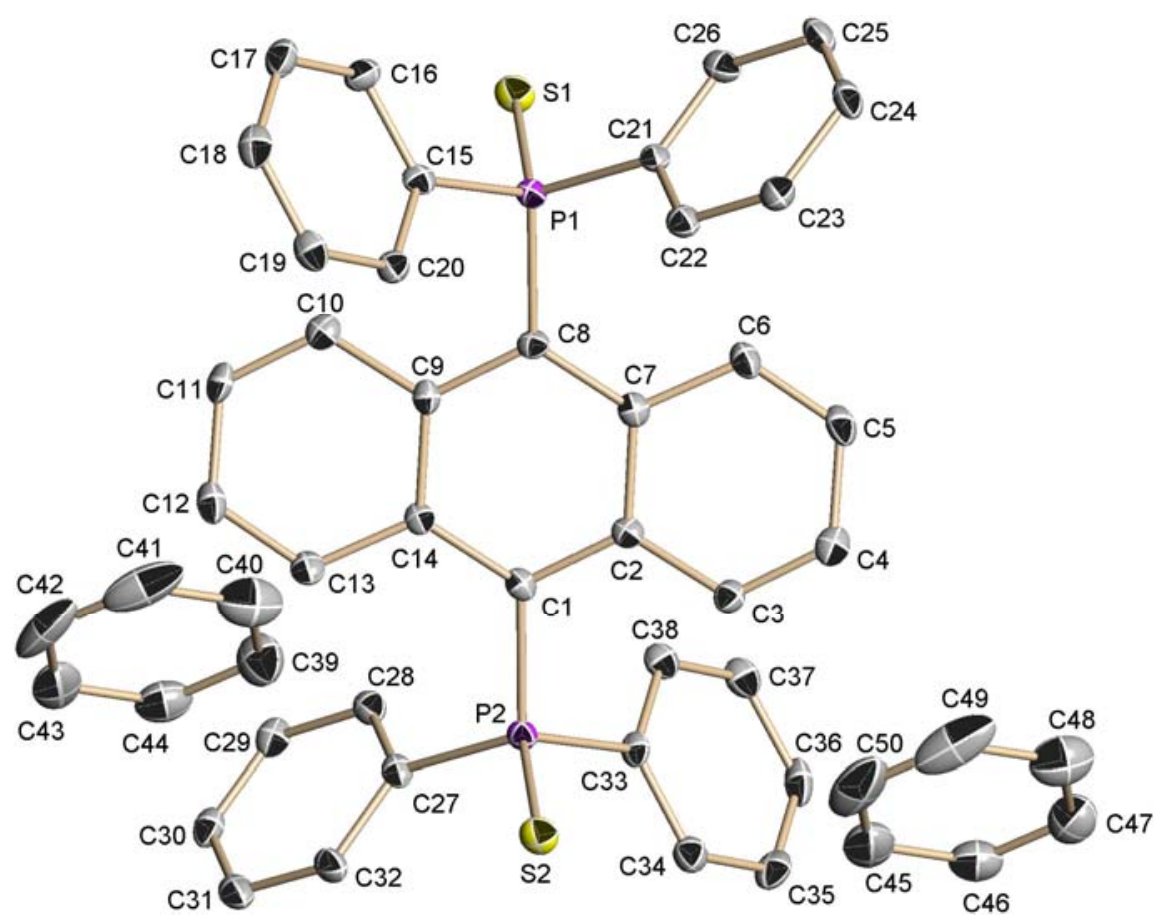

Abb. 9-7: Asymmetrische Einheit von $\left[2 \mathrm{C}_{6} \mathrm{H}_{6} @\left\{\left(\mathrm{Ph}_{2}(\mathrm{~S}) \mathrm{P}\right)_{2}\left(\mathrm{C}_{14} \mathrm{H}_{8}\right)\right\}\right](10)$.

Tab. 9-7: Kristallographische Daten für $\left[2 \mathrm{C}_{6} \mathrm{H}_{6} @\left\{\left(\mathrm{Ph}_{2}(\mathrm{~S}) \mathrm{P}\right)_{2}\left(\mathrm{C}_{14} \mathrm{H}_{8}\right)\right\}\right]$ (10).

\begin{tabular}{|ll|ll|}
\hline Strukturcode & Müller-Thurgau & Messtemperatur / K & $100(2)$ \\
Summenformel & $\mathrm{C}_{50} \mathrm{H}_{40} \mathrm{P}_{2} \mathrm{~S}_{2}$ & $\rho_{\text {calc }} / \mathrm{Mg} \cdot \mathrm{m}^{-3}$ & 1.297 \\
Molmasse / g $\cdot \mathrm{mol}^{-1}$ & 766.88 & $\mu / \mathrm{mm}^{-1}$ & 0.253 \\
Kristallgröße / mm & $0.3 \times 0.25 \times 0.2$ & $F(000)$ & 804 \\
Kristallsystem & triklin & $\theta-$ Bereich ${ }^{\circ}$ & $1.58-27.88$ \\
Raumgruppe & $P^{\overline{1}}$ & Reflexe gesamt/unabhängig & $26690 / 8818$ \\
$\mathrm{a} / \mathrm{pm}$ & $918.19(5)$ & Daten/Restraints/Parameter & $8818 / 0 / 463$ \\
$\mathrm{~b} / \mathrm{pm}$ & $1350.05(8)$ & $R 1[/>2 \sigma(I)]$ & 0.0330 \\
$\mathrm{c} / \mathrm{pm}$ & $1661.48(10)$ & $w R 2($ alle Daten $)$ & 0.1407 \\
$\alpha /{ }^{\circ}$ & $107.0040(10)$ & $g 1 / g 2$ & $0.0690 / 0.1370$ \\
$\beta /{ }^{\circ}$ & $90.8860(10)$ & GoF & 1.222 \\
$\gamma /{ }^{\circ}$ & $93.8300(10)$ & Differenzelektronendichte: & \\
$\mathrm{V} / \mathrm{nm}^{3}$ & $1.9638(2)$ & max. / min. / $\left(10^{-6} \mathrm{e} \cdot \mathrm{pm}^{-3}\right)$ & $0.433 /-0.318$ \\
$\mathrm{Z}$ & 2 & max. / min. Transmission & $0.99 / 0.92$ \\
\hline
\end{tabular}




\subsection{8 $\left[2 \mathrm{C}_{8} \mathrm{H}_{6} @\left\{\left(\mathrm{Ph}_{2}(\mathrm{~S}) \mathrm{P}\right)_{2}\left(\mathrm{C}_{14} \mathrm{H}_{8}\right)\right\}\right](11)$}

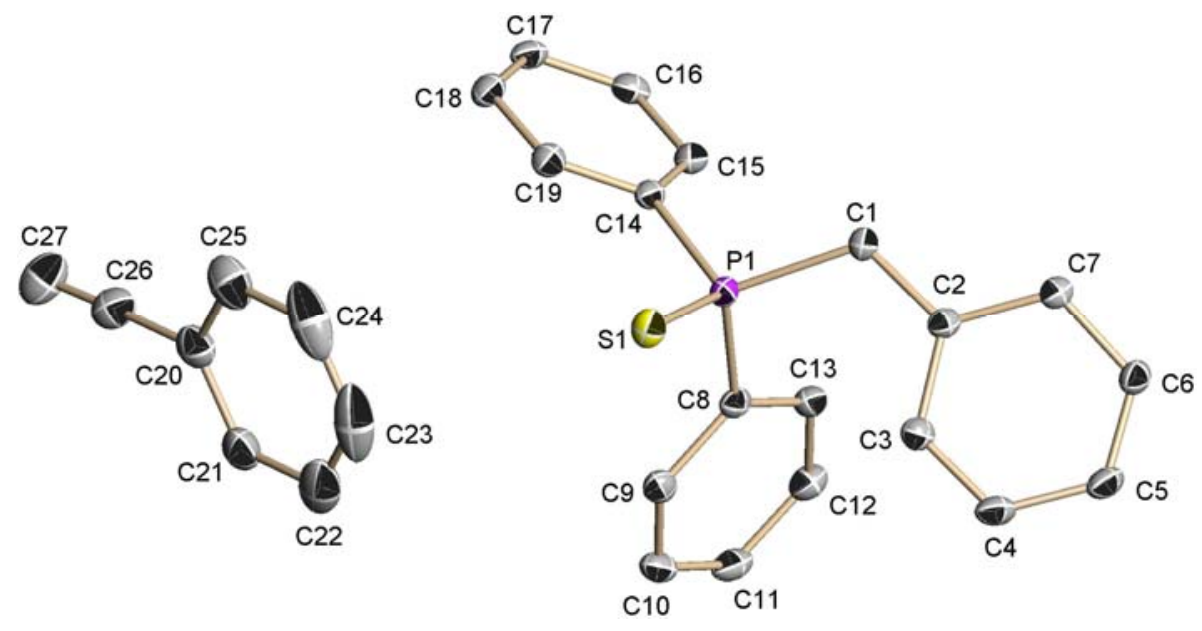

Abb. 9-8: Asymmetrische Einheit von $\left[2 \mathrm{C}_{8} \mathrm{H}_{6} @\left\{\left(\mathrm{Ph}_{2}(\mathrm{~S}) \mathrm{P}\right)_{2}\left(\mathrm{C}_{14} \mathrm{H}_{8}\right)\right\}\right](\mathbf{1 1})$.

Tab. 9-8: Kristallographische Daten für $\left[2 \mathrm{C}_{8} \mathrm{H}_{6} @\left\{\left(\mathrm{Ph}_{2}(\mathrm{~S}) \mathrm{P}\right)_{2}\left(\mathrm{C}_{14} \mathrm{H}_{8}\right)\right\}\right](\mathbf{1 1})$.

\begin{tabular}{|ll|ll|}
\hline Strukturcode & Phenylacetylen & Messtemperatur / K & $100(2)$ \\
Summenformel & $\mathrm{C}_{54} \mathrm{H}_{40} \mathrm{P}_{2} \mathrm{~S}_{2}$ & $\rho_{\text {calc }} / \mathrm{Mg} \cdot \mathrm{m}^{-3}$ & 1.269 \\
Molmasse / g $\cdot \mathrm{mol}^{-1}$ & 814.92 & $\mu / \mathrm{mm}^{-1}$ & 0.237 \\
Kristallgröße / mm & $0.25 \times 0.2 \times 0.15$ & $F(000)$ & 426 \\
Kristallsystem & triklin & $\theta-$ Bereich ${ }^{\circ}$ & $1.82-26.40$ \\
Raumgruppe & $P \overline{1}$ & Reflexe gesamt/unabhängig & $4269 / 4269$ \\
$\mathrm{a} / \mathrm{pm}$ & $974.6(3)$ & Daten/Restraints/Parameter & $4269 / 0 / 262$ \\
$\mathrm{~b} / \mathrm{pm}$ & $1015.1(3)$ & $R 1[/>2 \sigma(I)]$ & 0.0412 \\
$\mathrm{c} / \mathrm{pm}$ & $1196.6(3)$ & $w R 2($ alle Daten $)$ & 0.1103 \\
$\alpha /{ }^{\circ}$ & $72.340(4)$ & $g 1 / g 2$ & $0.0634 / 0.1968$ \\
$\beta / /^{\circ}$ & $74.660(4)$ & GoF & 1.047 \\
$\gamma /{ }^{\circ}$ & $74.847(4)$ & Differenzelektronendichte: & \\
$\mathrm{V} / \mathrm{nm}^{3}$ & $1.0664(5)$ & max. / min. / $\left(10^{-6} \mathrm{e} \cdot \mathrm{pm}^{-3}\right)$ & $0.726 /-0.311$ \\
$\mathrm{z}$ & 1 & max. / min. Transmission & ohne Abs.korr. \\
\hline
\end{tabular}




\subsection{9 [3 $\left.\mathrm{CDCl}_{3} @\left\{\left(\mathrm{Ph}_{2}(\mathrm{~S}) \mathrm{P}\right)_{2}\left(\mathrm{C}_{14} \mathrm{H}_{8}\right)\right\}\right](12)$}

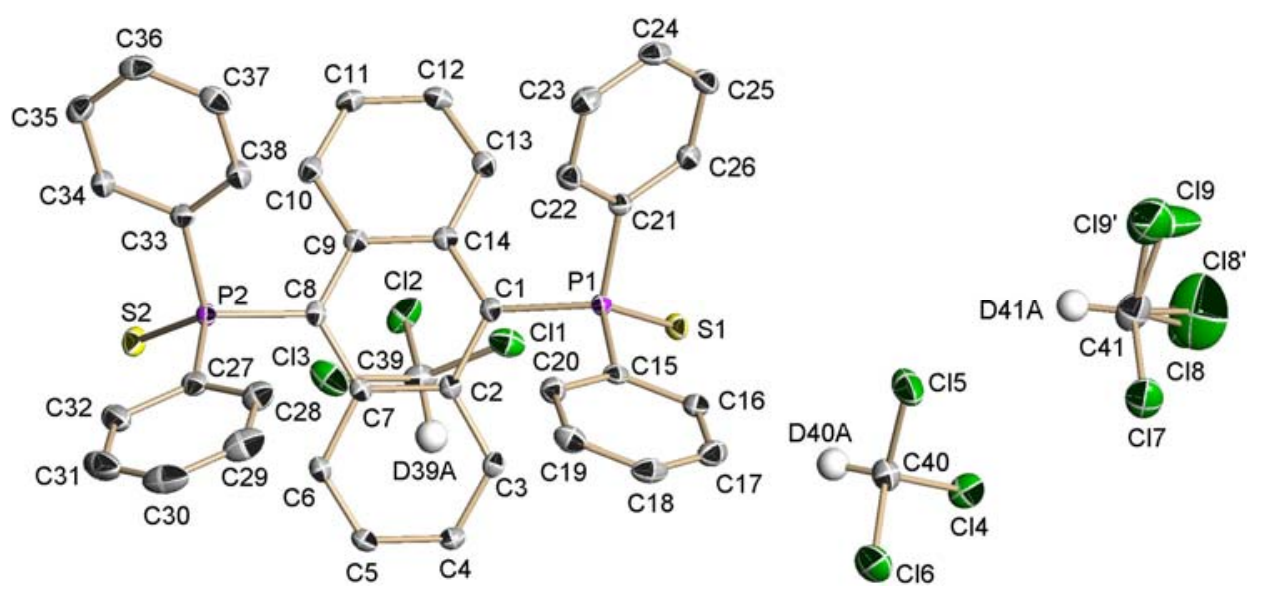

Abb. 9-9: Asymmetrische Einheit von $\left[3 \mathrm{CDCl}_{3} @\left\{\left(\mathrm{Ph}_{2}(\mathrm{~S}) \mathrm{P}\right)_{2}\left(\mathrm{C}_{14} \mathrm{H}_{8}\right)\right\}\right](\mathbf{1 2})$.

Die asymmetrische Einheit enthält neben einem Molekül SPAnPS drei Lösungsmittelmoleküle $\mathrm{CDCl}_{3}$, von denen eines zwei über zwei Positionen fehlgeordnete Chloratome aufweist. Ohne Hilfe von restraints konnte eine anisotrope Verfeinerung durchgeführt werden, die Besetzungsfaktoren verfeinerten zu 0.65 / 0.35.

Tab. 9-9: Kristallographische Daten für [3 $\left.\mathrm{CDCl}_{3} @\left\{\left(\mathrm{Ph}_{2}(\mathrm{~S}) \mathrm{P}\right)_{2}\left(\mathrm{C}_{14} \mathrm{H}_{8}\right)\right\}\right]$ (12).

\begin{tabular}{|ll|ll|}
\hline Strukturcode & Michel & Messtemperatur / K & $100(2)$ \\
Summenformel & $\mathrm{C}_{41} \mathrm{H}_{31} \mathrm{Cl}_{9} \mathrm{P}_{2} \mathrm{~S}_{2}$ & $\rho_{\text {calc }} / \mathrm{Mg} \cdot \mathrm{m}^{-3}$ & 1.490 \\
Molmasse $/ \mathrm{g} \cdot \mathrm{mol}^{-1}$ & 968.77 & $\mu / \mathrm{mm}^{-1}$ & 0.785 \\
Kristallgröße / mm & $0.2 \times 0.15 \times 0.1$ & $F(000)$ & 984 \\
Kristallsystem & triklin & $\theta-$ Bereich ${ }^{\circ}$ & $2.25-26.03$ \\
Raumgruppe & $P \overline{1}$ & Reflexe gesamt/unabhängig & $41071 / 8481$ \\
$\mathrm{a} / \mathrm{pm}$ & $978.46(11)$ & Daten/Restraints/Parameter & $8481 / 0 / 505$ \\
$\mathrm{~b} / \mathrm{pm}$ & $1228.44(13)$ & $R 1[/>2 \sigma(I)]$ & 0.0287 \\
$\mathrm{c} / \mathrm{pm}$ & $1869.9(2)$ & $w R 2($ alle Daten $)$ & 0.0698 \\
$\alpha /{ }^{\circ}$ & $102.8520(10)$ & $g 1 / g 2$ & $0.0295 / 1.3439$ \\
$\beta / /^{\circ}$ & $99.2540(10)$ & GoF & 1.036 \\
$\gamma /{ }^{\circ}$ & $91.3000(10)$ & Differenzelektronendichte: & \\
$\mathrm{V} / \mathrm{nm}^{3}$ & $2.1588(4)$ & max. / min. / (10 $\left.{ }^{-6} \mathrm{e} \cdot \mathrm{pm}^{-3}\right)$ & $0.421 /-0.316$ \\
$\mathrm{Z}$ & 2 & max. / min. Transmission & $0.99 / 0.92$ \\
\hline
\end{tabular}




\subsubsection{0 ( $\left.{ }^{i} \mathrm{Pr}_{2} \mathrm{P}\right)_{2}\left(\mathrm{C}_{14} \mathrm{H}_{8}\right)(13), \mathrm{CCDC}: 667526$}

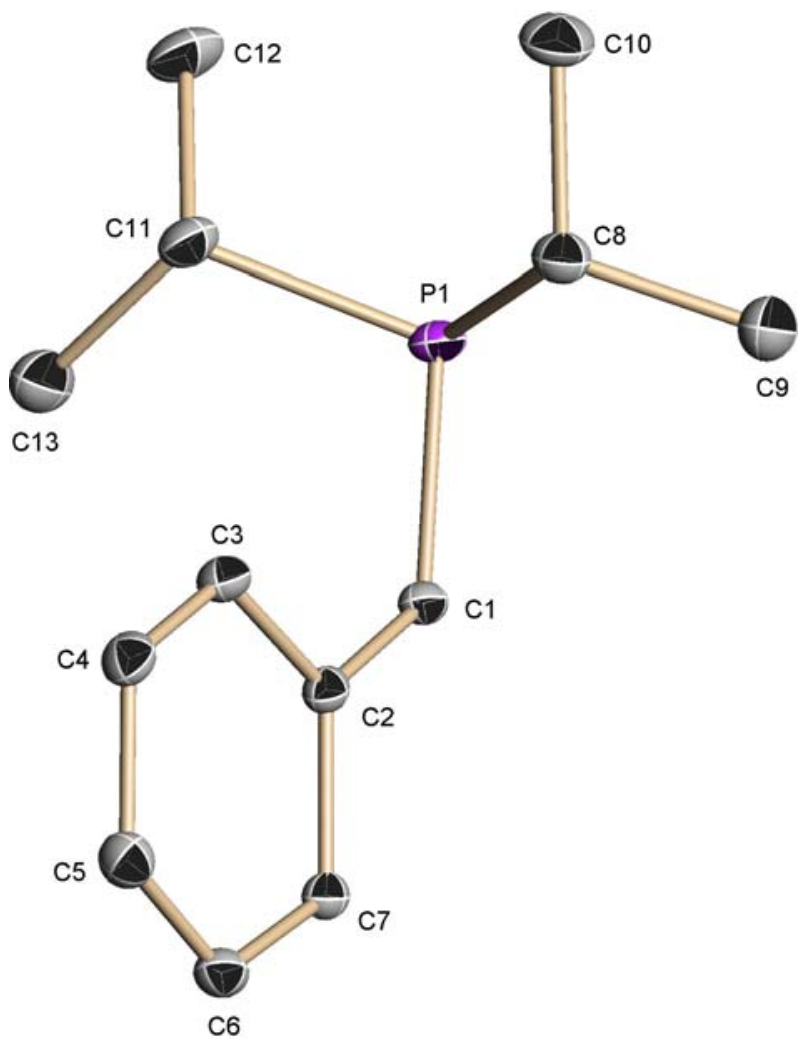

Abb. 9-10: Asymmetrische Einheit von $\left({ }^{i} \mathrm{Pr}_{2} \mathrm{P}\right)_{2}\left(\mathrm{C}_{14} \mathrm{H}_{8}\right)(13)$.

Tab. 9-10: Kristallographische Daten für $\left({ }^{i} \mathrm{Pr}_{2} \mathrm{P}\right)_{2}\left(\mathrm{C}_{14} \mathrm{H}_{8}\right)(\mathbf{1 3})$.

\begin{tabular}{|c|c|c|c|}
\hline Strukturcode & Frieda & $\rho_{\text {calc }} / \mathrm{Mg} \cdot \mathrm{m}^{-3}$ & 1.194 \\
\hline Summenformel & $\mathrm{C}_{26} \mathrm{H}_{36} \mathrm{P}_{2}$ & $\mu / \mathrm{mm}^{-1}$ & 0.200 \\
\hline Molmasse $/ \mathrm{g} \cdot \mathrm{mol}^{-1}$ & 410.49 & $F(000)$ & 444 \\
\hline Kristallgröße / mm & $0.3 \times 0.25 \times 0.08$ & $\theta$-Bereich $/^{\circ}$ & $1.77-27.13$ \\
\hline Kristallsystem & monoklin & Reflexe gesamt/unabhängig & 10878 / 2529 \\
\hline Raumgruppe & $P 2_{1} / C$ & Daten/Restraints/Parameter & $2529 / 0 / 131$ \\
\hline $\mathrm{a} / \mathrm{pm}$ & $1156.37(17)$ & $R 1[I>2 \sigma(I)]$ & 0.0352 \\
\hline $\mathrm{b} / \mathrm{pm}$ & $829.11(12)$ & $w R 2$ (alle Daten) & 0.0906 \\
\hline $\mathrm{c} / \mathrm{pm}$ & $1194.97(18)$ & $g 1 / g 2$ & $0.0413 / 0.6717$ \\
\hline$\beta /{ }^{\circ}$ & $94.953(2)$ & GoF & 1.049 \\
\hline $\mathrm{V} / \mathrm{nm}^{3}$ & $1.1414(3)$ & Differenzelektronendichte: & \\
\hline Z & 2 & $\max . / \min . /\left(10^{-6} \mathrm{e} \cdot \mathrm{pm}^{-3}\right)$ & $0.415 /-0.194$ \\
\hline Messtemperatur / K & $100(2)$ & max. / min. Transmission & $0.99 / 0.94$ \\
\hline
\end{tabular}




\subsubsection{1 ( $\left.{ }^{i} \operatorname{Pr}_{2}(\mathrm{~S}) \mathrm{P}\right)_{2}\left(\mathrm{C}_{14} \mathrm{H}_{8}\right)(14)$, CCDC: 667527}

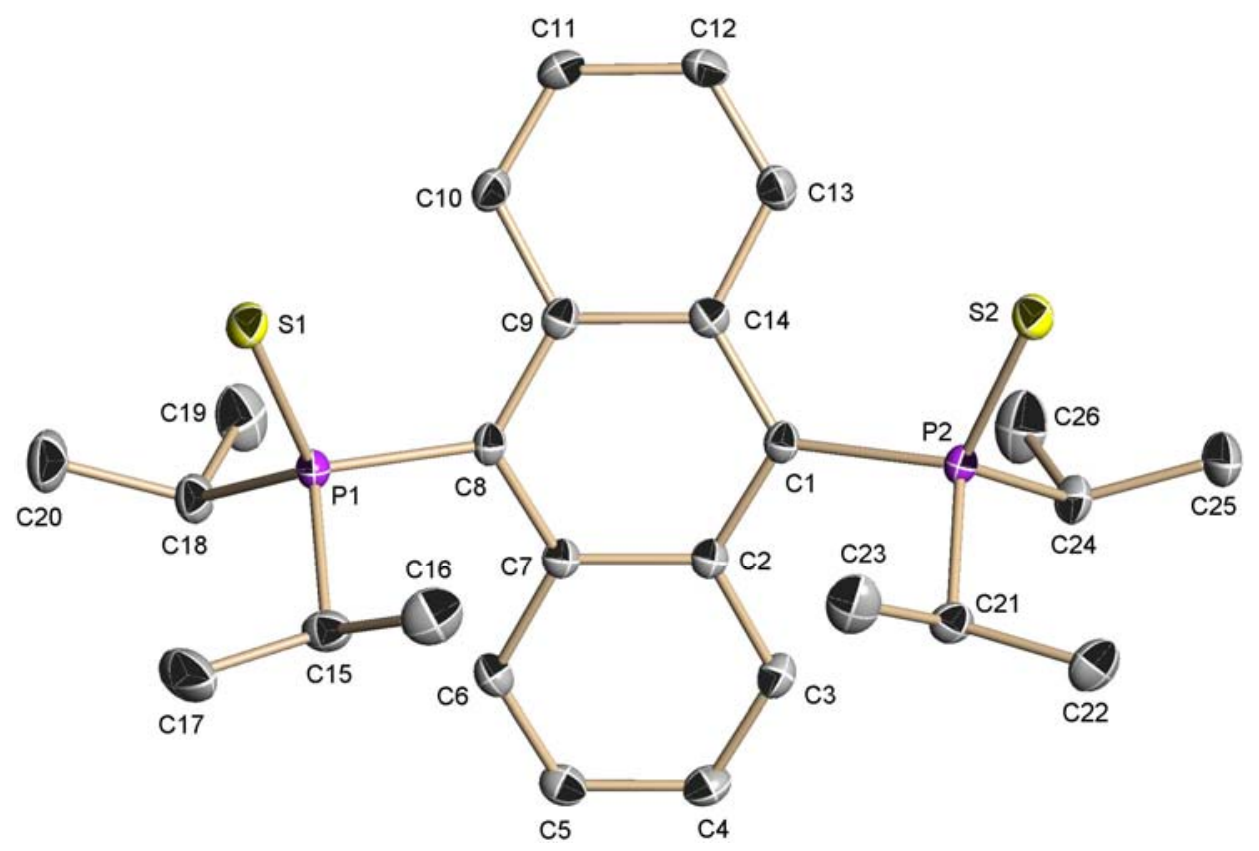

Abb. 9-11: Asymmetrische Einheit von $\left({ }^{i} \mathrm{Pr}_{2}(\mathrm{~S}) \mathrm{P}\right)_{2}\left(\mathrm{C}_{14} \mathrm{H}_{8}\right)($ 14).

Die Verbindung kristallisiert in der orthorhombischen, chiralen Raumgruppe $P 2_{1} 2_{1} 2_{1}$. Der Flack-Parameter ${ }^{[184,185]}$ verfeinerte zu -0.0067 mit einer Standardabweichung von 0.0496, die absolute Struktur ist somit korrekt bestimmt.

Tab. 9-11: Kristallographische Daten für $\left({ }^{\prime} \mathrm{Pr}_{2}(\mathrm{~S}) \mathrm{P}\right)_{2}\left(\mathrm{C}_{14} \mathrm{H}_{8}\right)(\mathbf{1 4})$.

\begin{tabular}{|ll|ll|}
\hline Strukturcode & Friedox & $\rho_{\text {calc }} / \mathrm{Mg} \cdot \mathrm{m}^{-3}$ & 1.259 \\
Summenformel & $\mathrm{C}_{26} \mathrm{H}_{36} \mathrm{P}_{2} \mathrm{~S}_{2}$ & $\mu / \mathrm{mm}^{-1}$ & 0.352 \\
Molmasse / g.mol ${ }^{-1}$ & 474.61 & $F(000)$ & 1016 \\
Kristallgröße / mm & $0.40 \times 0.20 \times 0.05$ & $\theta-$ Bereich / & $2.27-26.37$ \\
Kristallsystem & orthorhombisch & Reflexe gesamt/unabhängig & $34356 / 5114$ \\
Raumgruppe & $P 2{ }_{1} 2_{1} 2_{1}$ & Daten/Restraints/Parameter & $5114 / 0 / 271$ \\
a / pm & $733.33(12)$ & $R 1[/>2 \sigma(I)]$ & 0.0245 \\
b / pm & $1552.2(3)$ & $w R 2($ alle Daten $)$ & 0.0615 \\
c / pm & $2199.7(4)$ & $g 1 / g 2$ & $0.0344 / 0.6095$ \\
Flack x & $-0.0067(496)$ & GoF & 1.043 \\
V / nm ${ }^{3}$ & $2.5038(7)$ & Differenzelektronendichte: & \\
Z & 4 & max. / min. / $\left(10^{-6} \mathrm{e} \cdot \mathrm{pm}^{-3}\right)$ & $0.275 /-0.158$ \\
Messtemperatur / K & $153(2)$ & max. / min. Transmission & $0.99 / 0.90$ \\
\hline
\end{tabular}




\subsubsection{2 $\mathrm{Ph}_{2}(\mathrm{~S}) \mathrm{P}\left(\mathrm{C}_{14} \mathrm{H}_{8}\right) \mathrm{PPh}_{2}(15)$}

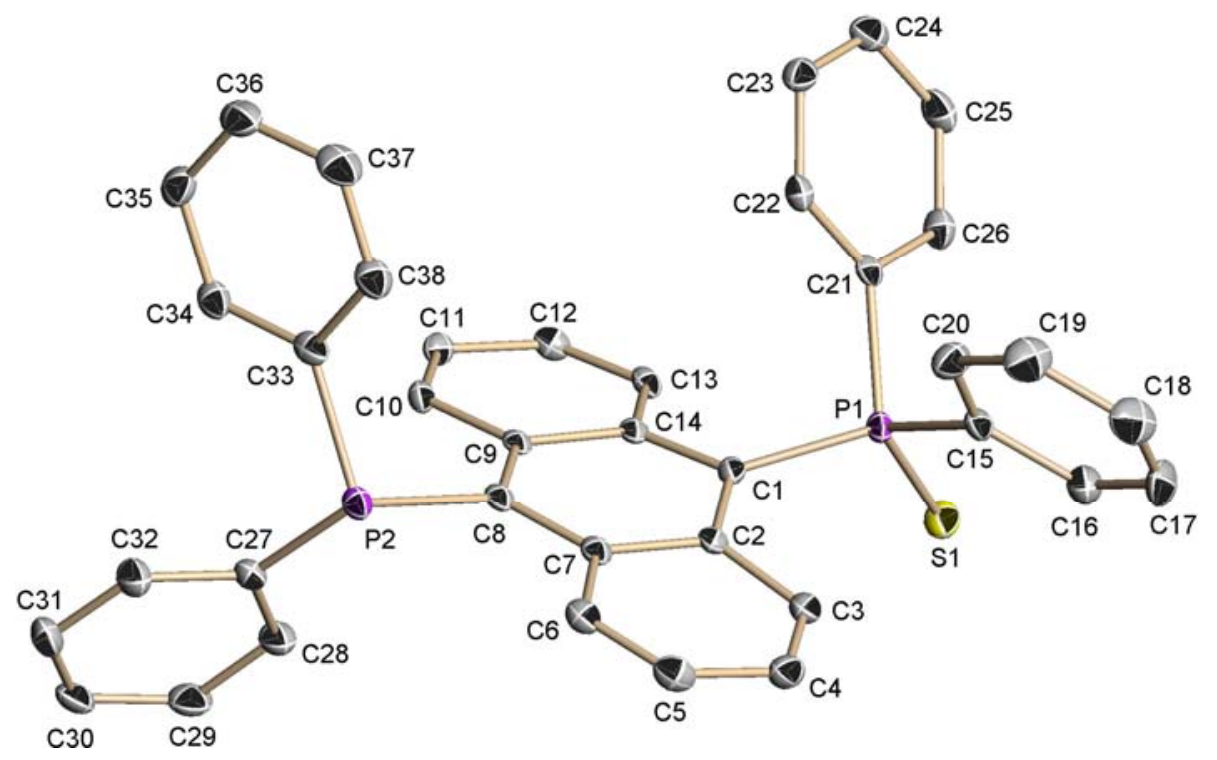

Abb. 9-12: Asymmetrische Einheit von $\mathrm{Ph}_{2}(\mathrm{~S}) \mathrm{P}\left(\mathrm{C}_{14} \mathrm{H}_{8}\right) \mathrm{PPh}_{2}$ (15).

Tab. 9-12: Kristallographische Daten für $\mathrm{Ph}_{2}(\mathrm{~S}) \mathrm{P}\left(\mathrm{C}_{14} \mathrm{H}_{8}\right) \mathrm{PPh}_{2}$ (15).

\begin{tabular}{|c|c|c|c|}
\hline Strukturcode & Jim Beam & $\mu / \mathrm{mm}^{-1}$ & 0.246 \\
\hline Summenformel & $\mathrm{C}_{38} \mathrm{H}_{28} \mathrm{P}_{2} \mathrm{~S}$ & $F(000)$ & 2416 \\
\hline Molmasse $/ \mathrm{g} \cdot \mathrm{mol}^{-1}$ & 578.60 & $\theta$-Bereich $/^{\circ}$ & $2.15-26.42$ \\
\hline Kristallgröße / mm & $0.3 \times 0.25 \times 0.2$ & Reflexe gesamt/unabhängig & 63755 / 6023 \\
\hline Kristallsystem & orthorhombisch & Daten/Restraints/Parameter & $6023 / 0 / 370$ \\
\hline Raumgruppe & Pbca & $R 1[I>2 \sigma(I)]$ & 0.0489 \\
\hline $\mathrm{a} / \mathrm{pm}$ & $1635.06(7)$ & $w R 2$ (alle Daten) & 0.1136 \\
\hline $\mathrm{b} / \mathrm{pm}$ & $1275.26(6)$ & $g 1 / g 2$ & $0.0530 / 3.7060$ \\
\hline $\mathrm{c} / \mathrm{pm}$ & 2819.96(13) & GoF & 1.107 \\
\hline $\mathrm{V} / \mathrm{nm}^{3}$ & $5.8800(5)$ & Differenzelektronendichte: & \\
\hline Z & 8 & $\max . / \min . /\left(10^{-6} \mathrm{e} \cdot \mathrm{pm}^{-3}\right)$ & $0.525 /-0.288$ \\
\hline Messtemperatur / K & $100(2)$ & max. / min. Transmission & $0.99 / 0.83$ \\
\hline$\rho_{\text {calc }} / \mathrm{Mg} \cdot \mathrm{m}^{-3}$ & 1.307 & & \\
\hline
\end{tabular}




\subsubsection{3 [2 $\left.\mathrm{C}_{7} \mathrm{H}_{8} @\left\{\mathrm{Ph}_{2}(\mathrm{Se}) \mathrm{P}\left(\mathrm{C}_{14} \mathrm{H}_{8}\right) \mathrm{P}(\mathrm{S}) \mathrm{Ph}_{2}\right\}\right](16)$}

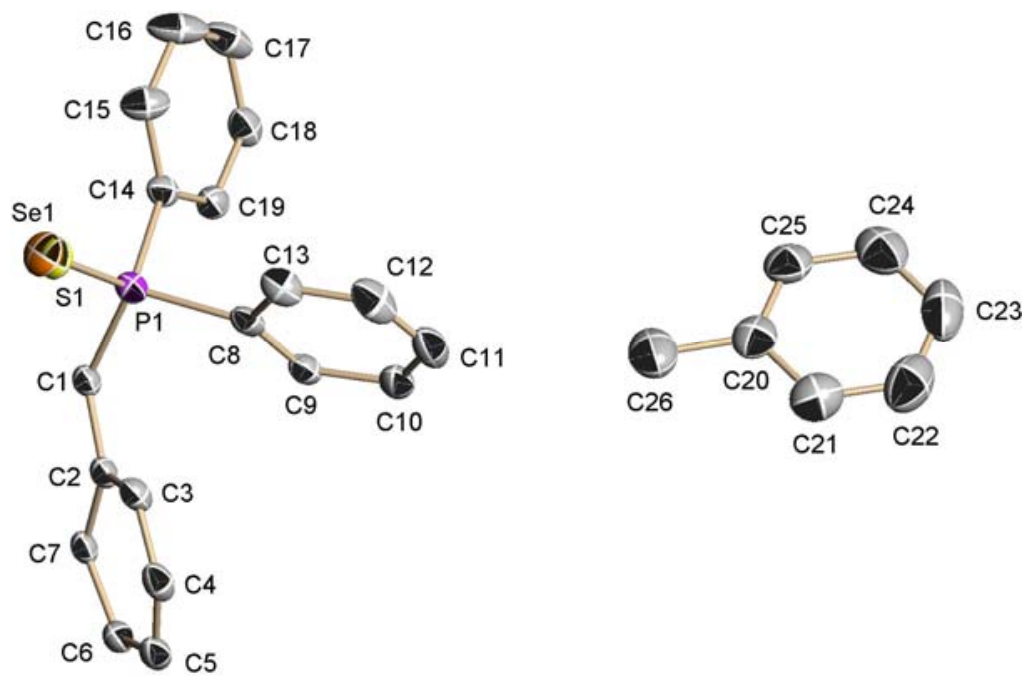

Abb. 9-13: Asymmetrische Einheit von $\left[2 \mathrm{C}_{7} \mathrm{H}_{8} @\left\{\mathrm{Ph}_{2}(\mathrm{Se}) \mathrm{P}\left(\mathrm{C}_{14} \mathrm{H}_{8}\right) \mathrm{P}(\mathrm{S}) \mathrm{Ph}_{2}\right\}\right](16)$.

Die Verbindung kristallisiert isostrukturell zu 9 in der monoklinen, zentrosymmetrischen Raumgruppe $P 2_{1} / n$, die Position des Chalkogens ist jeweils zur Hälfte mit Selen und Schwefel besetzt. Mit Hilfe von Abstands-restraints (DFIX) konnte eine anisotrope Verfeinerung durchgeführt werden.

Tab. 9-13: Kristallographische Daten für $\left[2 \mathrm{C}_{7} \mathrm{H}_{8} @\left\{\mathrm{Ph}_{2}(\mathrm{Se}) \mathrm{P}\left(\mathrm{C}_{14} \mathrm{H}_{8}\right) \mathrm{P}(\mathrm{S}) \mathrm{Ph}_{2}\right\}\right](16)$.

\begin{tabular}{|c|c|c|c|}
\hline Strukturcode & Goldkrone & $\rho_{\text {calc }} / \mathrm{Mg} \cdot \mathrm{m}^{-3}$ & 1.332 \\
\hline Summenformel & $\mathrm{C}_{52} \mathrm{H}_{44} \mathrm{P}_{2} \mathrm{SSe}$ & $\mu / \mathrm{mm}^{-1}$ & 1.057 \\
\hline Molmasse $/ \mathrm{g} \cdot \mathrm{mol}^{-1}$ & 841.83 & $F(000)$ & 872 \\
\hline Kristallgröße / mm & $0.2 \times 0.2 \times 0.2$ & $\theta$-Bereich / ${ }^{\circ}$ & $2.08-24.70$ \\
\hline Kristallsystem & monoklin & Reflexe gesamt/unabhängig & $20810 / 3562$ \\
\hline Raumgruppe & $P 2_{1} / n$ & Daten/Restraints/Parameter & 3562 / 2 / 263 \\
\hline $\mathrm{a} / \mathrm{pm}$ & $1086.8(2)$ & $R 1[I>2 \sigma(I)]$ & 0.0355 \\
\hline $\mathrm{b} / \mathrm{pm}$ & $1479.6(3)$ & $w R 2$ (alle Daten) & 0.0738 \\
\hline $\mathrm{c} / \mathrm{pm}$ & $1346.0(3)$ & $g 1 / g 2$ & $0.0364 / 0.0000$ \\
\hline$\beta /{ }^{\circ}$ & $104.12(3)$ & GoF & 0.962 \\
\hline $\mathrm{V} / \mathrm{nm}^{3}$ & $2.0989(7)$ & Differenzelektronendichte: & \\
\hline Z & 2 & $\max . / \min . /\left(10^{-6}\right.$ e.pm $\left.{ }^{-3}\right)$ & $0.246 /-0.224$ \\
\hline Messtemperatur / K & $133(2)$ & max. / min. Transmission & ohne Abs.korr. \\
\hline
\end{tabular}




\subsubsection{4 $\mathrm{Ph}_{2} \mathrm{P}\left(\mathrm{C}_{14} \mathrm{H}_{8}\right) \mathrm{P}^{i} \mathrm{Pr}_{2}(17)$}

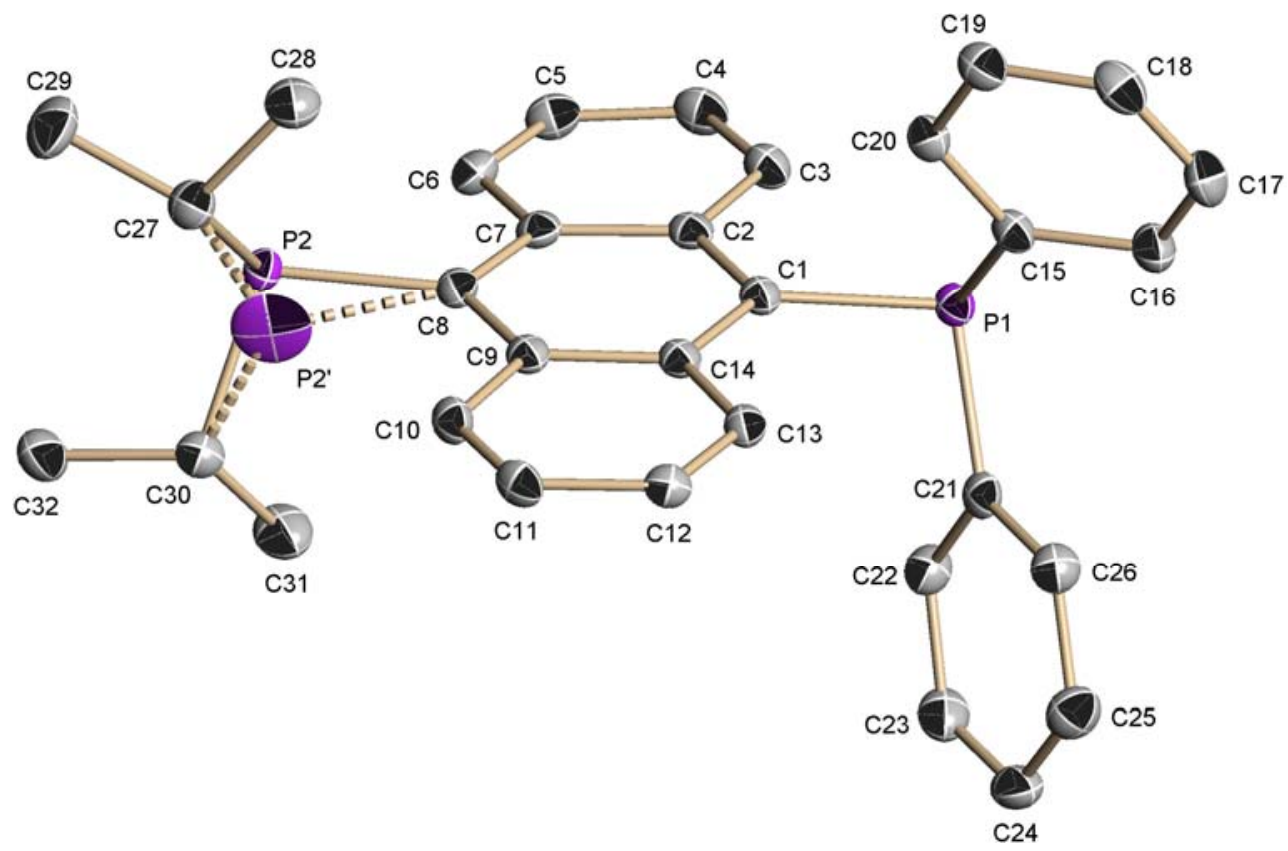

Abb. 9-14: Asymmetrische Einheit von $\mathrm{Ph}_{2} \mathrm{P}\left(\mathrm{C}_{14} \mathrm{H}_{8}\right) \mathrm{P}^{i} \mathrm{Pr}_{2}$ (17).

Die Beschreibung der Struktur ohne eine Fehlordnung von P2 weist eine Restelektronendichte von $0.83 \cdot 10^{-6} \mathrm{e} \cdot \mathrm{pm}^{-3}$ auf. Durch die Einführung von P2' mit einem Besetzungsfaktor von $3.5 \%$ sowie durch Verwendung von Abstands-restraints (DFIX) wird diese auf $0.36 \cdot 10^{-6} \mathrm{e} \cdot \mathrm{pm}^{-3}$ reduziert.

Tab. 9-14: Kristallographische Daten für $\mathrm{Ph}_{2} \mathrm{P}\left(\mathrm{C}_{14} \mathrm{H}_{8}\right) \mathrm{P}^{j} \mathrm{Pr}_{2}$ (17).

\begin{tabular}{|c|c|c|c|}
\hline Strukturcode & Cuba & $\rho_{\text {calc }} / \mathrm{Mg} \cdot \mathrm{m}^{-3}$ & 1.235 \\
\hline Summenformel & $\mathrm{C}_{32} \mathrm{H}_{32} \mathrm{P}_{2}$ & $\mu / \mathrm{mm}^{-1}$ & 0.188 \\
\hline Molmasse $/ \mathrm{g} \cdot \mathrm{mol}^{-1}$ & 478.52 & $F(000)$ & 1016 \\
\hline Kristallgröße / mm & $0.20 \times 0.20 \times 0.10$ & $\theta$-Bereich / ${ }^{\circ}$ & $2.25-26.43$ \\
\hline Kristallsystem & monoklin & Reflexe gesamt/unabhängig & 36909 / 5287 \\
\hline Raumgruppe & $P 2_{1} / c$ & Daten/Restraints/Parameter & $5287 / 1 / 320$ \\
\hline $\mathrm{a} / \mathrm{pm}$ & $1789.2(2)$ & $R 1[I>2 \sigma(I)]$ & 0.0335 \\
\hline $\mathrm{b} / \mathrm{pm}$ & $1051.58(12)$ & $w R 2$ (alle Daten) & 0.0868 \\
\hline $\mathrm{c} / \mathrm{pm}$ & $1378.80(16)$ & $g 1 / g 2$ & $0.0423 / 1.0384$ \\
\hline$\beta /{ }^{\circ}$ & $97.313(2)$ & GoF & 1.068 \\
\hline $\mathrm{V} / \mathrm{nm}^{3}$ & $2.5731(5)$ & Differenzelektronendichte: & \\
\hline Z & 4 & $\max . / \min . /\left(10^{-6} \mathrm{e} \cdot \mathrm{pm}^{-3}\right)$ & $0.358 /-0.243$ \\
\hline Messtemperatur / K & $100(2)$ & max. / min. Transmission & $0.99 / 0.95$ \\
\hline
\end{tabular}




\subsubsection{5 $\mathrm{Ph}_{2}(\mathrm{O}) \mathrm{P}\left(\mathrm{C}_{14} \mathrm{H}_{8}\right) \mathrm{P}(\mathrm{O})^{i} \mathrm{Pr}_{2}(18)$}

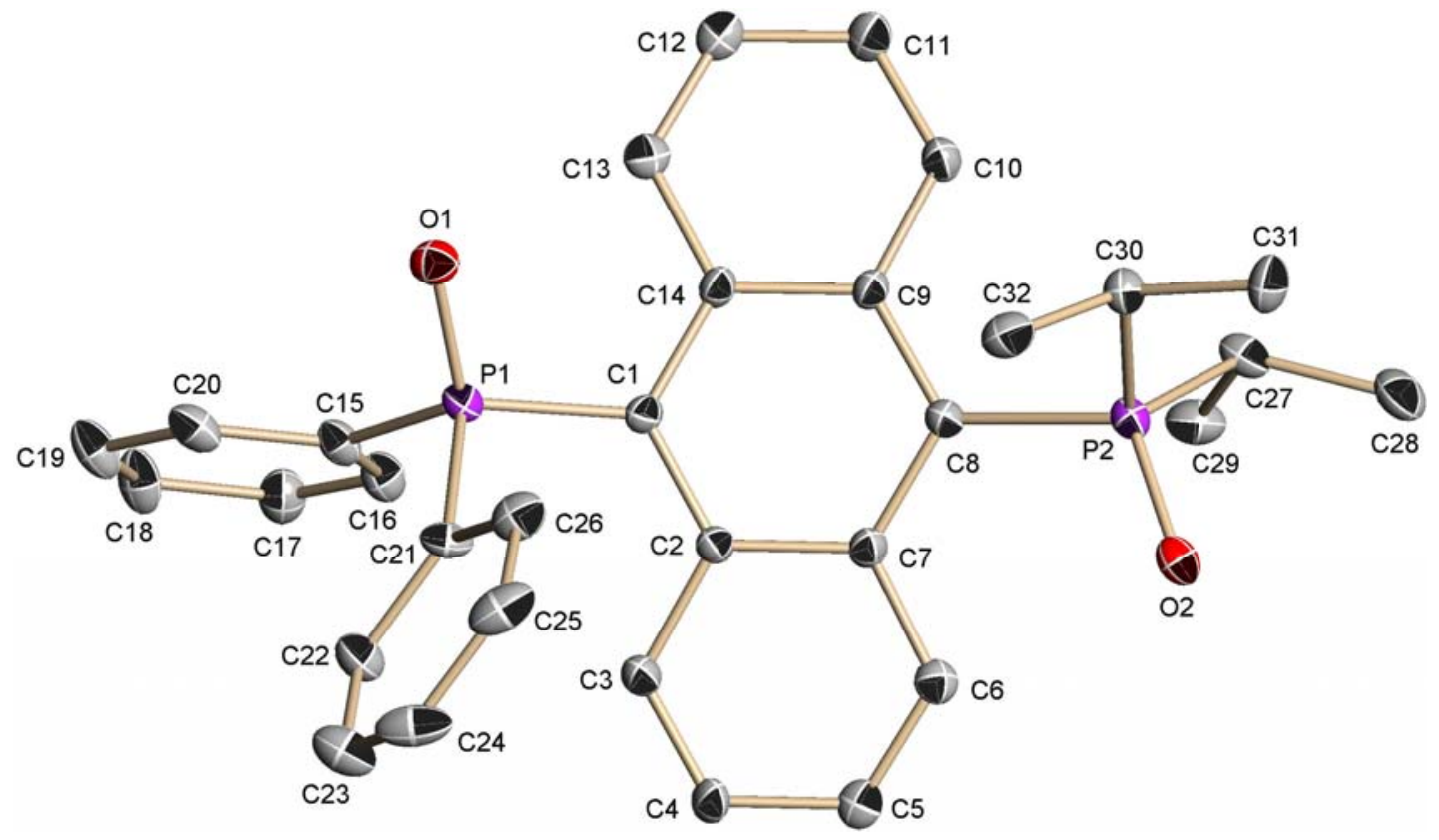

Abb. 9-15: Asymmetrische Einheit von $\mathrm{Ph}_{2}(\mathrm{O}) \mathrm{P}\left(\mathrm{C}_{14} \mathrm{H}_{8}\right) \mathrm{P}(\mathrm{O})^{i} \mathrm{Pr}_{2}$ (18).

Die Verbindung kristallisiert in der orthorhombischen, chiralen Raumgruppe $P 2{ }_{1}{ }_{2}{ }_{1}{ }_{1}$. Der Flack-Parameter ${ }^{[184,185]}$ verfeinerte zu -0.069 mit einer Standardabweichung von 0.070 , die absolute Struktur ist somit korrekt bestimmt.

Tab. 9-15: Kristallographische Daten für $\mathrm{Ph}_{2}(\mathrm{O}) \mathrm{P}\left(\mathrm{C}_{14} \mathrm{H}_{8}\right) \mathrm{P}(\mathrm{O})^{i} \mathrm{Pr}_{2}$ (18).

\begin{tabular}{|ll|ll|}
\hline Strukturcode & Pitu & $\rho_{\text {calc }} /{\mathrm{Mg} \cdot \mathrm{m}^{-3}}^{-3}$ & 1.284 \\
Summenformel & $\mathrm{C}_{32} \mathrm{H}_{32} \mathrm{O}_{2} \mathrm{P}_{2}$ & $\mu / \mathrm{mm}^{-1}$ & 0.193 \\
Molmasse / g $\cdot \mathrm{mol}^{-1}$ & 510.52 & $F(000)$ & 1080 \\
Kristallgröße / mm & $0.40 \times 0.40 \times 0.40$ & $\theta$-Bereich / & $2.01-26.02$ \\
Kristallsystem & orthorhombisch & Reflexe gesamt/unabhängig & $5210 / 5210$ \\
Raumgruppe & $P 2{ }_{1} 2_{1}{ }_{1}$ & Daten/Restraints/Parameter & $5210 / 0 / 329$ \\
a / pm & $1278.39(11)$ & $R 1[/>2 \sigma(I)]$ & 0.0370 \\
b / pm & $1336.61(12)$ & $w R 2($ alle Daten $)$ & 0.1011 \\
c / pm & $1545.27(14)$ & $g 1 / g 2$ & $0.0609 / 0.1584$ \\
Flack x & $-0.0690(703)$ & GoF & 1.070 \\
V / nm ${ }^{3}$ & $2.6404(4)$ & Differenzelektronendichte: & \\
Z & 4 & max. / min. / $\left(10^{-6} \mathrm{e} \cdot \mathrm{pm}^{-3}\right)$ & $0.293 /-0.276$ \\
Messtemperatur / K & $100(2)$ & max. / min. Transmission & $0.99 / 0.84$ \\
\hline
\end{tabular}




\subsubsection{6 $\left[\mathrm{Ph}_{2}(\mathrm{~S}) \mathrm{P}\left(\mathrm{C}_{14} \mathrm{H}_{8}\right) \mathrm{P}(\mathrm{S})^{i} \mathrm{Pr}_{2} \cdot 1.5 \mathrm{C}_{7} \mathrm{H}_{8}\right](19)$}

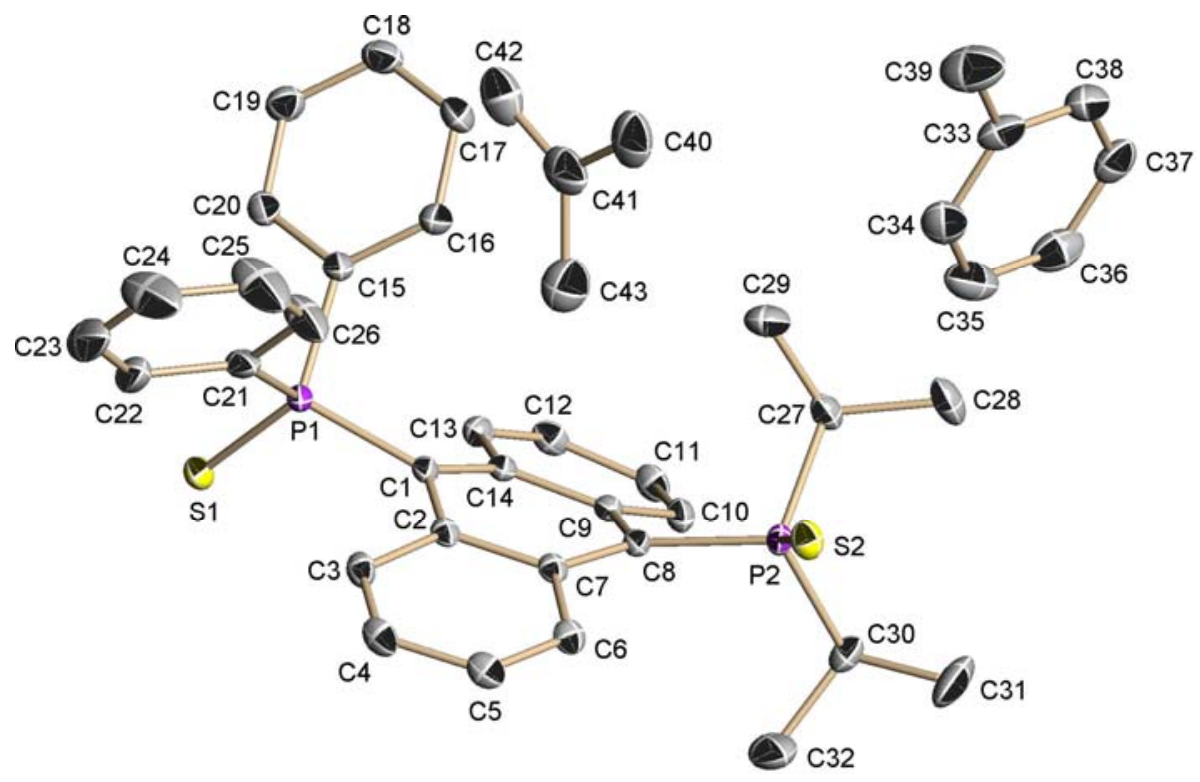

Abb. 9-16: Asymmetrische Einheit von $\left[\mathrm{Ph}_{2}(\mathrm{~S}) \mathrm{P}\left(\mathrm{C}_{14} \mathrm{H}_{8}\right) \mathrm{P}(\mathrm{S})^{i} \mathrm{Pr}_{2} \cdot 1.5 \mathrm{C}_{7} \mathrm{H}_{8}\right]$ (19).

Die asymmetrische Einheit enthält neben einem Molekül Diphosphorylanthracen noch 1.5 Toluolmoleküle. Der Rest des halben Toluolmoleküls wird durch ein Inversionszentrum im Mittelpunkt des Phenylringes erzeugt. Daher wurden die Besetzungsfaktoren der Methylgruppe sowie von $\mathrm{H} 41^{\prime}$ auf jeweils 0.50 gesetzt.

Tab. 9-16: Kristallographische Daten für $\left[\mathrm{Ph}_{2}(\mathrm{~S}) \mathrm{P}\left(\mathrm{C}_{14} \mathrm{H}_{8}\right) \mathrm{P}(\mathrm{S})^{i} \mathrm{Pr}_{2} \cdot 1.5 \mathrm{C}_{7} \mathrm{H}_{8}\right]$ (19).

\begin{tabular}{|c|c|c|c|}
\hline Strukturcode & Havana & $\rho_{\text {calc }} / \mathrm{Mg} \cdot \mathrm{m}^{-3}$ & 1.234 \\
\hline Summenformel & $\mathrm{C}_{42.5} \mathrm{H}_{44} \mathrm{P}_{2} \mathrm{~S}_{2}$ & $\mu / \mathrm{mm}^{-1}$ & 0.262 \\
\hline Molmasse $/ \mathrm{g} \cdot \mathrm{mol}^{-1}$ & 680.84 & $F(000)$ & 1442 \\
\hline Kristallgröße / mm & $0.30 \times 0.25 \times 0.20$ & $\theta$-Bereich $/^{\circ}$ & $2.00-26.40$ \\
\hline Kristallsystem & monoklin & Reflexe gesamt/unabhängig & $64355 / 7514$ \\
\hline Raumgruppe & $P 2_{1} / c$ & Daten/Restraints/Parameter & $7514 / 0$ / 430 \\
\hline $\mathrm{a} / \mathrm{pm}$ & $1304.05(7)$ & $R 1[I>2 \sigma(I)]$ & 0.0391 \\
\hline $\mathrm{b} / \mathrm{pm}$ & $925.46(5)$ & $w R 2$ (alle Daten) & 0.0991 \\
\hline $\mathrm{c} / \mathrm{pm}$ & $3039.76(17)$ & $g 1 / g 2$ & $0.0472 / 2.5673$ \\
\hline$\beta /{ }^{\circ}$ & $93.1330(10)$ & GoF & 1.044 \\
\hline $\mathrm{V} / \mathrm{nm}^{3}$ & $3.6630(3)$ & Differenzelektronendichte: & \\
\hline Z & 4 & $\max . / \mathrm{min} . /\left(10^{-6} \mathrm{e} \cdot \mathrm{pm}^{-3}\right)$ & $0.562 /-0.337$ \\
\hline Messtemperatur / K & $100(2)$ & max. / min. Transmission & $0.99 / 0.92$ \\
\hline
\end{tabular}




\subsubsection{7 $\left[\mathrm{Ph}_{2}(\mathrm{Se}) \mathrm{P}\left(\mathrm{C}_{14} \mathrm{H}_{8}\right) \mathrm{P}(\mathrm{Se})^{i} \mathrm{Pr}_{2} \cdot 1.5 \mathrm{C}_{7} \mathrm{H}_{8}\right](20)$}
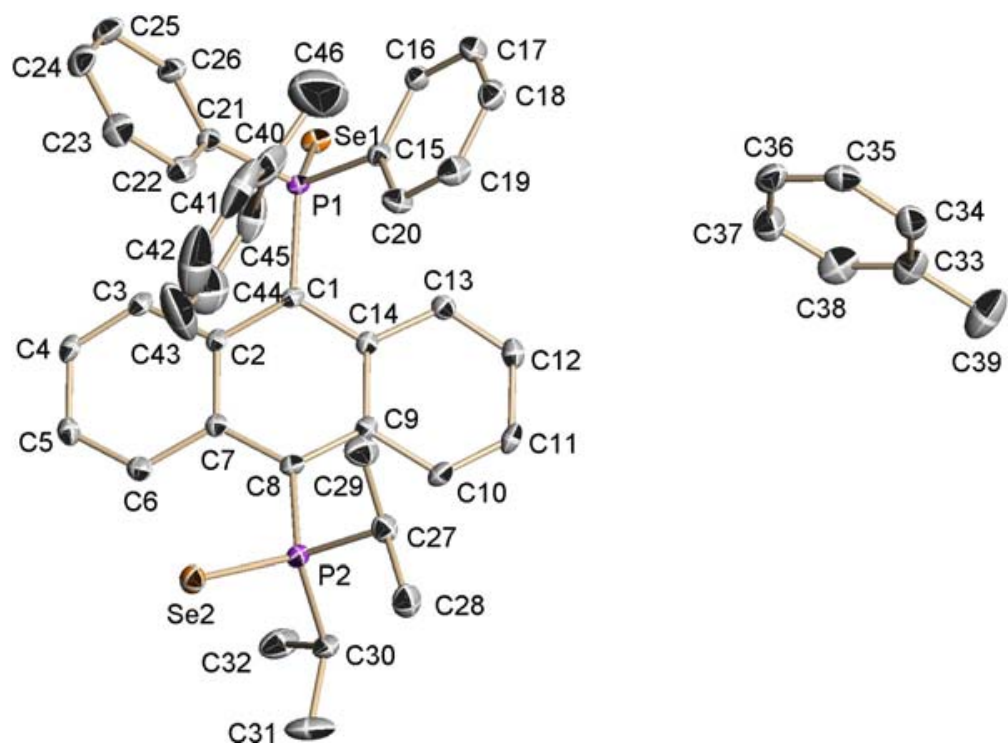

Abb. 9-17: Asymmetrische Einheit von $\left[\mathrm{Ph}_{2}(\mathrm{Se}) \mathrm{P}\left(\mathrm{C}_{14} \mathrm{H}_{8}\right) \mathrm{P}(\mathrm{Se})^{i} \mathrm{Pr}_{2} \cdot 1.5 \mathrm{C}_{7} \mathrm{H}_{8}\right](20)$.

In der asymmetrischen Einheit befinden sich neben einem Molekül Diphosphorylanthracen noch Toluolmoleküle, von denen sich eines (C40 bis C46) in der Nähe eines Inversionszentrums befindet und dadurch auf zwei Positionen fehlgeordnet ist (Besetzungsfaktoren: 0.50).

Tab. 9-17: Kristallographische Daten für $\left[\mathrm{Ph}_{2}(\mathrm{Se}) \mathrm{P}\left(\mathrm{C}_{14} \mathrm{H}_{8}\right) \mathrm{P}(\mathrm{Se})^{j} \mathrm{Pr}_{2} \cdot 1.5 \mathrm{C}_{7} \mathrm{H}_{8}\right](20)$.

\begin{tabular}{|c|c|c|c|}
\hline Strukturcode & Martini & $\rho_{\text {calc }} / \mathrm{Mg} \cdot \mathrm{m}^{-3}$ & 1.380 \\
\hline Summenformel & $\mathrm{C}_{42.5} \mathrm{H}_{44} \mathrm{P}_{2} \mathrm{Se}_{2}$ & $\mu / \mathrm{mm}^{-1}$ & 2.098 \\
\hline Molmasse $/ \mathrm{g} \cdot \mathrm{mol}^{-1}$ & 774.64 & $F(000)$ & 1588 \\
\hline Kristallgröße / mm & $0.40 \times 0.30 \times 0.20$ & $\theta$-Bereich / ${ }^{\circ}$ & $2.09-26.39$ \\
\hline Kristallsystem & monoklin & Reflexe gesamt/unabhängig & $39126 / 7617$ \\
\hline Raumgruppe & $P 2_{1} / c$ & Daten/Restraints/Parameter & $7617 / 57 / 440$ \\
\hline $\mathrm{a} / \mathrm{pm}$ & $1299.15(9)$ & $R 1[I>2 \sigma(I)]$ & 0.0316 \\
\hline $\mathrm{b} / \mathrm{pm}$ & $948.38(7)$ & $w R 2$ (alle Daten) & 0.0799 \\
\hline $\mathrm{c} / \mathrm{pm}$ & $3026.4(2)$ & $g 1 / g 2$ & $0.0390 / 4.1791$ \\
\hline$\beta /{ }^{\circ}$ & $91.0610(10)$ & GoF & 1.033 \\
\hline $\mathrm{V} / \mathrm{nm}^{3}$ & $3.7282(5)$ & Differenzelektronendichte: & \\
\hline Z & 4 & $\max . / \min . /\left(10^{-6} \mathrm{e} \cdot \mathrm{pm}^{-3}\right)$ & $0.600 /-0.496$ \\
\hline Messtemperatur / K & $100(2)$ & max. / min. Transmission & $0.99 / 0.71$ \\
\hline
\end{tabular}




\subsubsection{8 $\mathrm{Ph}_{2} \mathrm{P}\left(\mathrm{C}_{14} \mathrm{H}_{8}\right) \mathrm{PCy}_{2}(21)$}

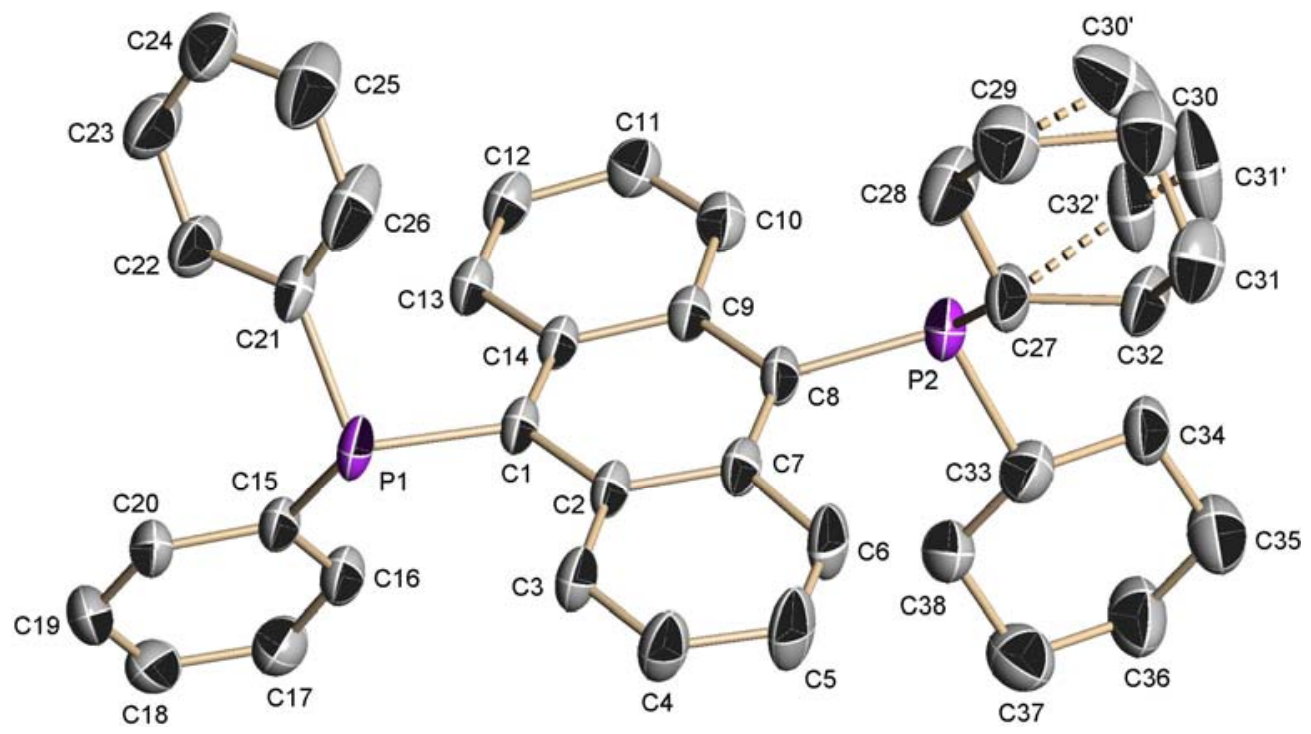

Abb. 9-18: Asymmetrische Einheit von $\mathrm{Ph}_{2} \mathrm{P}\left(\mathrm{C}_{14} \mathrm{H}_{8}\right) \mathrm{PCy}_{2}$ (21).

Ein Cyclohexylsubstituent weist drei über jeweils zwei Positionen fehlgeordnete $\mathrm{CH}_{2}$-Gruppen auf (siehe rechts). Mit Hilfe von Schwingungs-restraints (SIMU, DELU) konnte eine anisotrope Verfeinerung durchgeführt werden (Besetzungsfaktoren: 0.77 / 0.23).

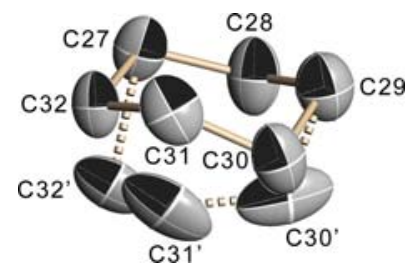

Tab. 9-18: Kristallgraphische Daten für $\mathrm{Ph}_{2} \mathrm{P}\left(\mathrm{C}_{14} \mathrm{H}_{8}\right) \mathrm{PCy}_{2}$ (21).

\begin{tabular}{|c|c|c|c|}
\hline Strukturcode & Wodka & $\rho_{\text {calc }} / \mathrm{Mg} \cdot \mathrm{m}^{-3}$ & 1.247 \\
\hline Summenformel & $\mathrm{C}_{38} \mathrm{H}_{40} \mathrm{P}_{2}$ & $\mu / \mathrm{mm}^{-1}$ & 0.172 \\
\hline Molmasse $/ \mathrm{g} \cdot \mathrm{mol}^{-1}$ & 558.64 & $F(000)$ & 1192 \\
\hline Kristallgröße / mm & $0.3 \times 0.2 \times 0.08$ & $\theta$-Bereich / ${ }^{\circ}$ & $1.36-26.37$ \\
\hline Kristallsystem & monoklin & Reflexe gesamt/unabhängig & 29095 / 6076 \\
\hline Raumgruppe & $P 2_{1} / n$ & Daten/Restraints/Parameter & 6076 / 32 / 379 \\
\hline $\mathrm{a} / \mathrm{pm}$ & $1830.3(2)$ & $R 1[I>2 \sigma(I)]$ & 0.0720 \\
\hline $\mathrm{b} / \mathrm{pm}$ & $909.04(11)$ & $w R 2$ (alle Daten) & 0.2043 \\
\hline $\mathrm{c} / \mathrm{pm}$ & $1874.0(2)$ & $g 1 / g 2$ & $0.0994 / 4.7450$ \\
\hline$\beta /{ }^{\circ}$ & $107.413(2)$ & GoF & 1.037 \\
\hline $\mathrm{V} / \mathrm{nm}^{3}$ & $2.9751(6)$ & Differenzelektronendichte: & \\
\hline Z & 4 & $\max . / \min . /\left(10^{-6}\right.$ e.pm $\left.{ }^{-3}\right)$ & $0.634 /-0.791$ \\
\hline Messtemperatur / K & $100(2)$ & max. / min. Transmission & $0.99 / 0.90$ \\
\hline
\end{tabular}




\subsubsection{9 $\left[\mathrm{Ph}_{2}(\mathrm{~S}) \mathrm{P}\left(\mathrm{C}_{14} \mathrm{H}_{8}\right) \mathrm{P}(\mathrm{S}) \mathrm{Cy}_{2} \cdot \mathrm{C}_{7} \mathrm{H}_{8}\right](22)$}

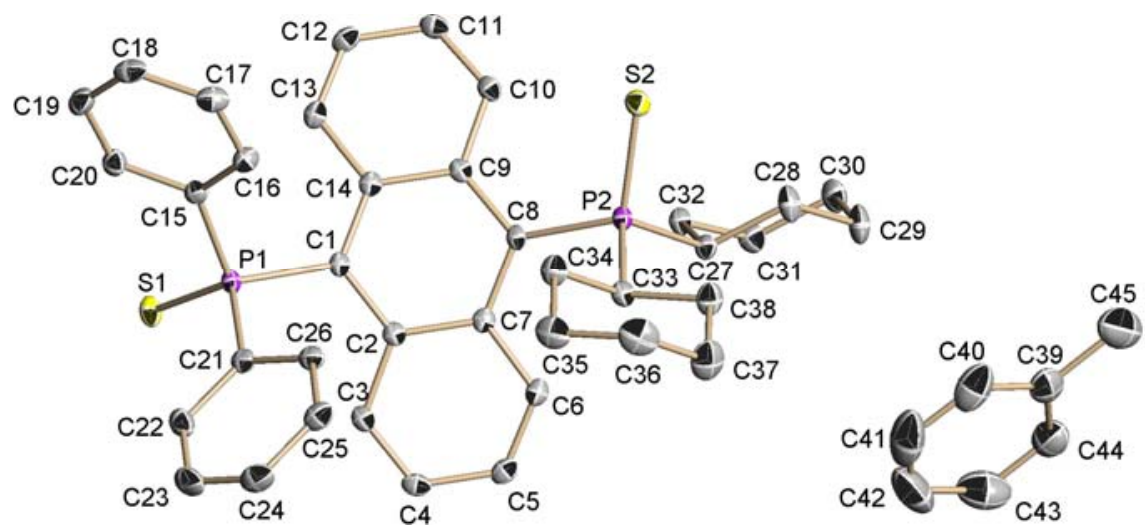

Abb. 9-19: Asymmetrische Einheit von $\left[\mathrm{Ph}_{2}(\mathrm{~S}) \mathrm{P}\left(\mathrm{C}_{14} \mathrm{H}_{8}\right) \mathrm{PCy}_{2} \cdot \mathrm{C}_{7} \mathrm{H}_{8}\right](22)$.

Tab. 9-19: Kristallographische Daten für $\left[\mathrm{Ph}_{2}(\mathrm{~S}) \mathrm{P}\left(\mathrm{C}_{14} \mathrm{H}_{8}\right) \mathrm{P}(\mathrm{S}) \mathrm{Cy}_{2} \cdot \mathrm{C}_{7} \mathrm{H}_{8}\right](22)$.

\begin{tabular}{|ll|ll|}
\hline Strukturcode & Bacardi & Messtemperatur / K & $100(2)$ \\
Summenformel & $\mathrm{C}_{45} \mathrm{H}_{48} \mathrm{P}_{2} \mathrm{~S}_{2}$ & $\rho_{\text {calc }} / \mathrm{Mg} \cdot \mathrm{m}^{-3}$ & 1.275 \\
Molmasse / g $\cdot \mathrm{mol}^{-1}$ & 714.89 & $\mu / \mathrm{mm}^{-1}$ & 0.261 \\
Kristallgröße / mm & $0.3 \times 0.25 \times 0.2$ & $F(000)$ & 760 \\
Kristallsystem & triklin & $\theta-$ Bereich ${ }^{\circ}$ & $2.23 / 26.39$ \\
Raumgruppe & $P \overline{1}$ & Reflexe gesamt/unabhängig & $39858 / 7594$ \\
$\mathrm{a} / \mathrm{pm}$ & $960.49(8)$ & Daten/Restraints/Parameter & $7594 / 0 / 443$ \\
$\mathrm{~b} / \mathrm{pm}$ & $1398.05(12)$ & $R 1[/>2 \sigma(I)]$ & 0.0400 \\
$\mathrm{c} / \mathrm{pm}$ & $1593.79(14)$ & $w R 2($ alle Daten $)$ & 0.0981 \\
$\alpha /{ }^{\circ}$ & $66.0840(10)$ & $g 1 / g 2$ & $0.1015 / 5.2023$ \\
$\beta / \circ$ & $73.1670(10)$ & GoF & 1.054 \\
$\gamma /{ }^{\circ}$ & $77.9340(10)$ & Differenzelektronendichte: & \\
$\mathrm{V} / \mathrm{nm}^{3}$ & $1.8623(3)$ & max. / min. / $\left(10^{-6} \mathrm{e} \cdot \mathrm{pm}^{-3}\right)$ & $0.635 /-0.411$ \\
$\mathrm{z}$ & 2 & max. / min. Transmission & $0.99 / 0.95$ \\
\hline
\end{tabular}




\subsubsection{0 $\left[\mathrm{Ph}_{2}(\mathrm{~S}) \mathrm{P}\left(\mathrm{C}_{14} \mathrm{H}_{8}\right)\left\{\mathrm{HNLi}(\text { thf })_{3}\right\} \cdot \mathrm{THF}\right](23)$}

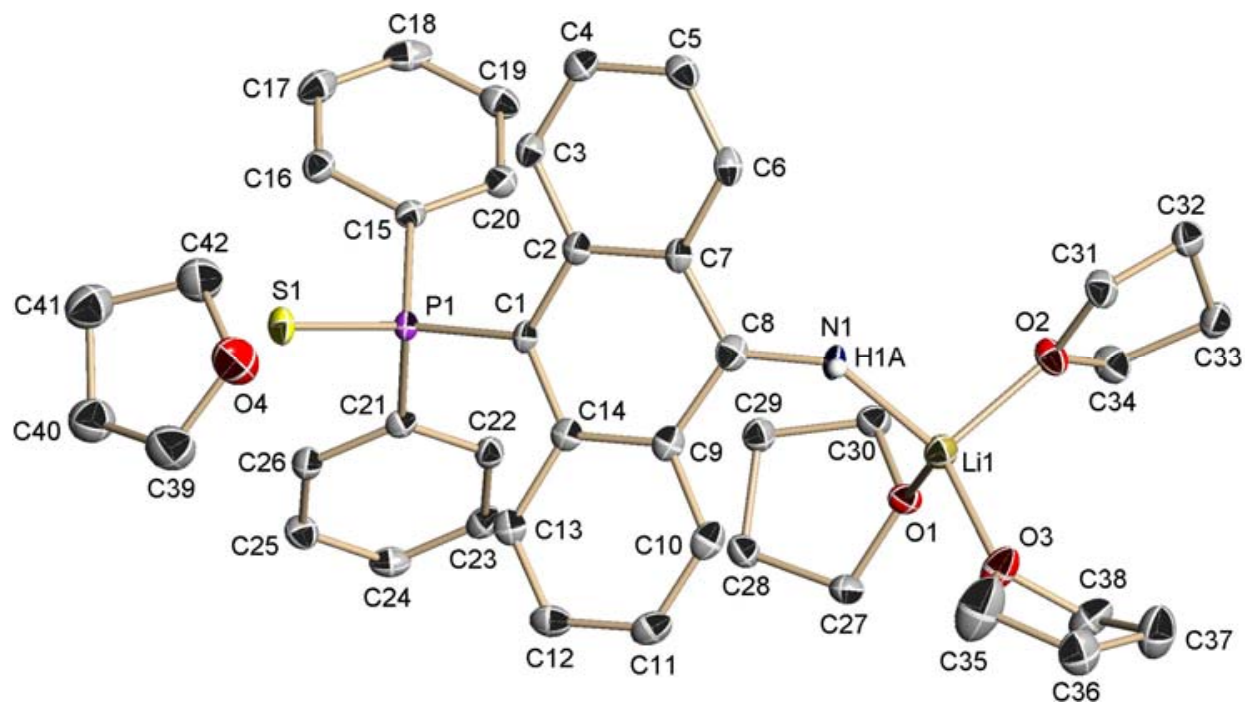

Abb. 9-20: Asymmetrische Einheit von $\left[\mathrm{Ph}_{2}(\mathrm{~S}) \mathrm{P}\left(\mathrm{C}_{14} \mathrm{H}_{8}\right)\left\{\mathrm{HNLi}(\text { thf })_{3}\right\} \cdot \mathrm{THF}\right](23)$.

Die asymmetrische Einheit enthält insgesamt vier THF-Moleküle, davon sind drei an das Lithiumatom koordiniert. Die Position des Wasserstoffatoms H1A konnte ohne Verwendung von restraints frei verfeinert werden, die N1-H1A-Bindungslänge beträgt $0.836 \mathrm{pm}$.

Tab. 9-20: Kristallographische Daten für $\left[\mathrm{Ph}_{2}(\mathrm{~S}) \mathrm{P}\left(\mathrm{C}_{14} \mathrm{H}_{8}\right)\left\{\mathrm{HNLi}(\text { thf })_{3}\right\} \cdot \mathrm{THF}\right](23)$.

\begin{tabular}{|c|c|c|c|}
\hline Strukturcode & Irish2 & $\rho_{\text {calc }} / \mathrm{Mg} \cdot \mathrm{m}^{-3}$ & 1.274 \\
\hline Summenformel & $\mathrm{C}_{42} \mathrm{H}_{51} \mathrm{LiNO}_{4} \mathrm{PS}$ & $\mu / \mathrm{mm}^{-1}$ & 0.175 \\
\hline Molmasse $/ \mathrm{g} \cdot \mathrm{mol}^{-1}$ & 703.81 & $F(000)$ & 1504 \\
\hline Kristallgröße / mm & $0.6 \times 0.4 \times 0.1$ & $\theta$-Bereich / ${ }^{\circ}$ & $2.07-26.37$ \\
\hline Kristallsystem & monoklin & Reflexe gesamt/unabhängig & $31840 / 7445$ \\
\hline Raumgruppe & $P 2_{1} / C$ & Daten/Restraints/Parameter & $7445 / 0 / 454$ \\
\hline $\mathrm{a} / \mathrm{pm}$ & $1556.4(2)$ & $R 1[I>2 \sigma(I)]$ & 0.0530 \\
\hline $\mathrm{b} / \mathrm{pm}$ & $957.70(12)$ & $w R 2$ (alle Daten) & 0.1284 \\
\hline $\mathrm{c} / \mathrm{pm}$ & $2463.9(3)$ & $g 1 / g 2$ & 0.0464 / 6.1294 \\
\hline$\beta /^{\circ}$ & $92.246(2)$ & GoF & 1.047 \\
\hline $\mathrm{V} / \mathrm{nm}^{3}$ & $3.6697(8)$ & Differenzelektronendichte: & \\
\hline Z & 4 & $\max . / \min . /\left(10^{-6} \mathrm{e} \cdot \mathrm{pm}^{-3}\right)$ & $0.95 /-0.559$ \\
\hline Messtemperatur / K & $100(2)$ & max. / min. Transmission & $0.99 / 0.80$ \\
\hline
\end{tabular}




\subsubsection{1 $\left[\mathrm{Ph}_{2}(\mathrm{~S}) \mathrm{P}\left(\mathrm{C}_{14} \mathrm{H}_{8}\right) \mathrm{NH}_{2} \cdot \mathrm{THF}\right](24)$}

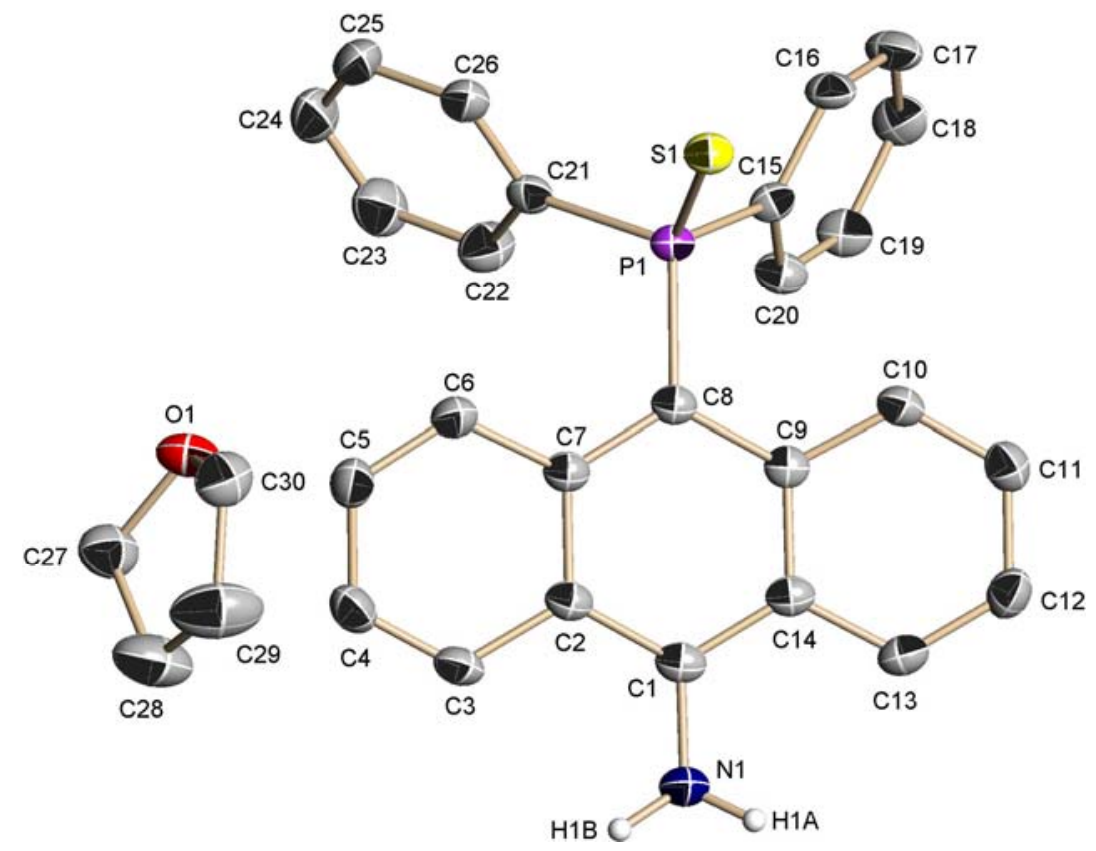

Abb. 9-21: Asymmetrische Einheit von $\left[\mathrm{Ph}_{2}(\mathrm{~S}) \mathrm{P}\left(\mathrm{C}_{14} \mathrm{H}_{8}\right) \mathrm{NH}_{2} \cdot \mathrm{THF}\right](24)$.

Die asymmetrische Einheit enthält neben einem Molekül Thiophosphorylanthracen noch ein nicht-koordinierendes THF-Molekül. Die Positionen der beiden AminWasserstoffatome H1A und H1B konnen mit Hilfe von Abstands-restraints (SADI) frei verfeinert werden.

Tab. 9-21: Kristallographische Daten für $\left[\mathrm{Ph}_{2}(\mathrm{~S}) \mathrm{P}\left(\mathrm{C}_{14} \mathrm{H}_{8}\right) \mathrm{NH}_{2} \cdot \mathrm{THF}\right]$ (24).

\begin{tabular}{|c|c|c|c|}
\hline Strukturcode & Asbach & $\rho_{\text {calc }} / \mathrm{Mg} \cdot \mathrm{m}^{-3}$ & 1.302 \\
\hline Summenformel & $\mathrm{C}_{30} \mathrm{H}_{28} \mathrm{NOPS}$ & $\mu / \mathrm{mm}^{-1}$ & 0.221 \\
\hline Molmasse $/ \mathrm{g} \cdot \mathrm{mol}^{-1}$ & 481.56 & $F(000)$ & 1016 \\
\hline Kristallgröße / mm & $0.2 \times 0.2 \times 0.2$ & $\theta$-Bereich / ${ }^{\circ}$ & $1.87-24.67$ \\
\hline Kristallsystem & monoklin & Reflexe gesamt/unabhängig & 38122 / 4143 \\
\hline Raumgruppe & $P 2_{1} / c$ & Daten/Restraints/Parameter & $4143 / 1 / 313$ \\
\hline $\mathrm{a} / \mathrm{pm}$ & $1106.1(2)$ & $R 1[I>2 \sigma(I)]$ & 0.0471 \\
\hline $\mathrm{b} / \mathrm{pm}$ & $1880.2(4)$ & $w R 2$ (alle Daten) & 0.0990 \\
\hline $\mathrm{c} / \mathrm{pm}$ & $1199.1(2)$ & $g 1 / g 2$ & $0.0504 / 0.0196$ \\
\hline$\beta /{ }^{\circ}$ & $99.83(3)$ & GoF & 1.029 \\
\hline $\mathrm{V} / \mathrm{nm}^{3}$ & $2.4571(9)$ & Differenzelektronendichte: & \\
\hline Z & 4 & $\max . / \min . /\left(10^{-6} \mathrm{e} \cdot \mathrm{pm}^{-3}\right)$ & $0.356 /-0.263$ \\
\hline Messtemperatur / K & $133(2)$ & max. / min. Transmission & ohne Abs.korr. \\
\hline
\end{tabular}




\subsubsection{2 $\operatorname{Br}\left(\mathrm{C}_{14} \mathrm{H}_{8}\right) \mathrm{P}\left(\mathrm{N}^{i} \mathrm{Pr}_{2}\right)_{2}(25)$}

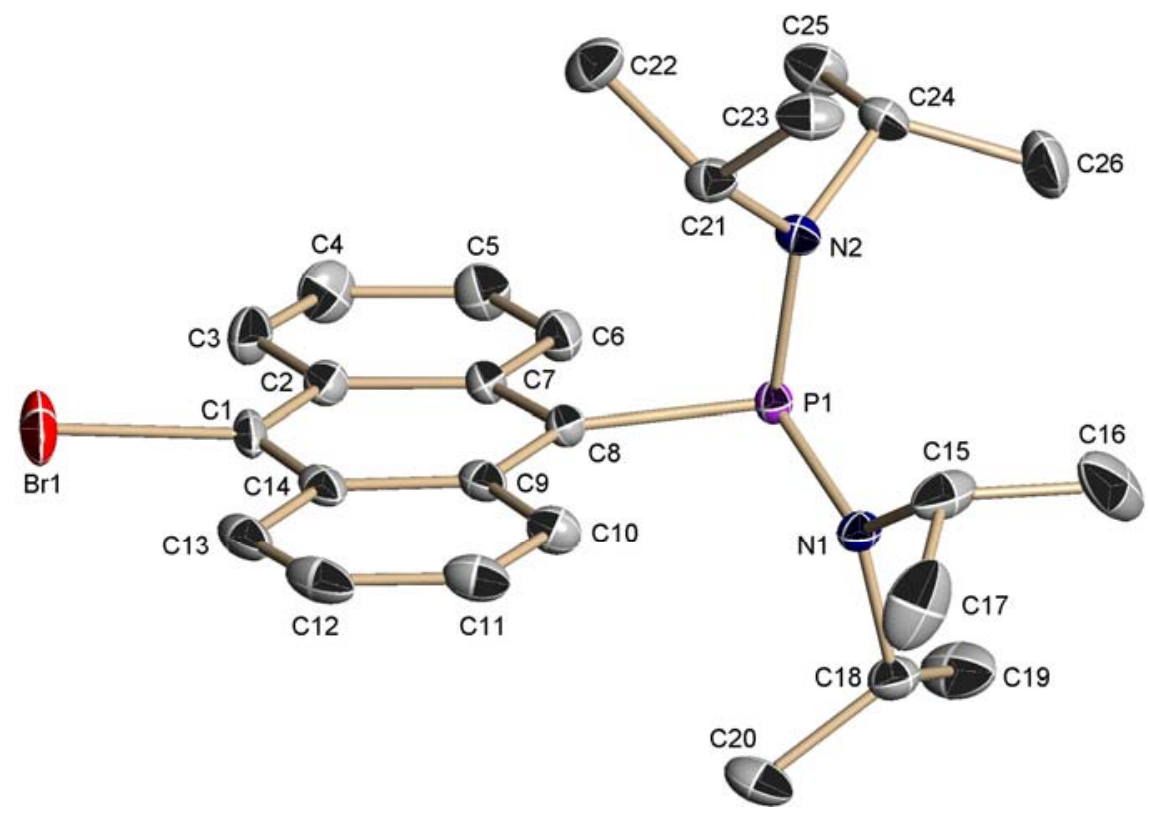

Abb. 9-22: Asymmetrische Einheit von $\operatorname{Br}\left(\mathrm{C}_{14} \mathrm{H}_{8}\right) \mathrm{P}\left(\mathrm{N}^{j} \mathrm{Pr}_{2}\right)_{2}$ (25).

Die Verbindung kristallisiert in der monoklinen, polaren Raumgruppe Cc. Der FlackParameter $^{[184,185]}$ verfeinerte $\mathrm{zu}-0.0056$ mit einer Standardabweichung von 0.0037 , die absolute Struktur ist somit korrekt bestimmt.

Tab. 9-22: Kristallographische Daten für $\operatorname{Br}\left(\mathrm{C}_{14} \mathrm{H}_{8}\right) \mathrm{P}\left(\mathrm{N}^{\prime} \mathrm{Pr}_{2}\right)_{2}$ (25).

\begin{tabular}{|c|c|c|c|}
\hline Strukturcode & Jägermeister & $\rho_{\text {calc }} / \mathrm{Mg} \cdot \mathrm{m}^{-3}$ & 1.331 \\
\hline Summenformel & $\mathrm{C}_{26} \mathrm{H}_{36} \mathrm{BrN}_{2} \mathrm{P}$ & $\mu / \mathrm{mm}^{-1}$ & 1.771 \\
\hline Molmasse $/ \mathrm{g} \cdot \mathrm{mol}^{-1}$ & 487.45 & $F(000)$ & 1024 \\
\hline Kristallgröße / mm & $0.35 \times 0.2 \times 0.1$ & $\theta$-Bereich / ${ }^{\circ}$ & $2.30-26.38$ \\
\hline Kristallsystem & monoklin & Reflexe gesamt/unabhängig & $18153 / 4948$ \\
\hline Raumgruppe & $\mathrm{Cc}$ & Daten/Restraints/Parameter & 4948 / 2 / 271 \\
\hline $\mathrm{a} / \mathrm{pm}$ & $1180.50(7)$ & $R 1[I>2 \sigma(I)]$ & 0.0208 \\
\hline $\mathrm{b} / \mathrm{pm}$ & 1517.39(9) & $w R 2$ (alle Daten) & 0.0552 \\
\hline $\mathrm{c} / \mathrm{pm}$ & $1466.64(8)$ & $g 1 / g 2$ & $0.0368 / 1.0084$ \\
\hline$\beta /{ }^{\circ}$ & $112.1560(10)$ & GoF & 1.039 \\
\hline Flack $\mathrm{x}$ & $-0.0056(37)$ & Differenzelektronendichte: & \\
\hline $\mathrm{V} / \mathrm{nm}^{3}$ & $2.4332(2)$ & $\max . / \min . /\left(10^{-6} \mathrm{e} \cdot \mathrm{pm}^{-3}\right)$ & $0.454 /-0.199$ \\
\hline Z & 4 & max. / min. Transmission & $0.99 / 0.69$ \\
\hline Messtemperatur / K & $100(2)$ & & \\
\hline
\end{tabular}




\subsubsection{3 $\mathrm{Py}_{2} \mathrm{P}\left(\mathrm{C}_{14} \mathrm{H}_{9}\right)(28)$}

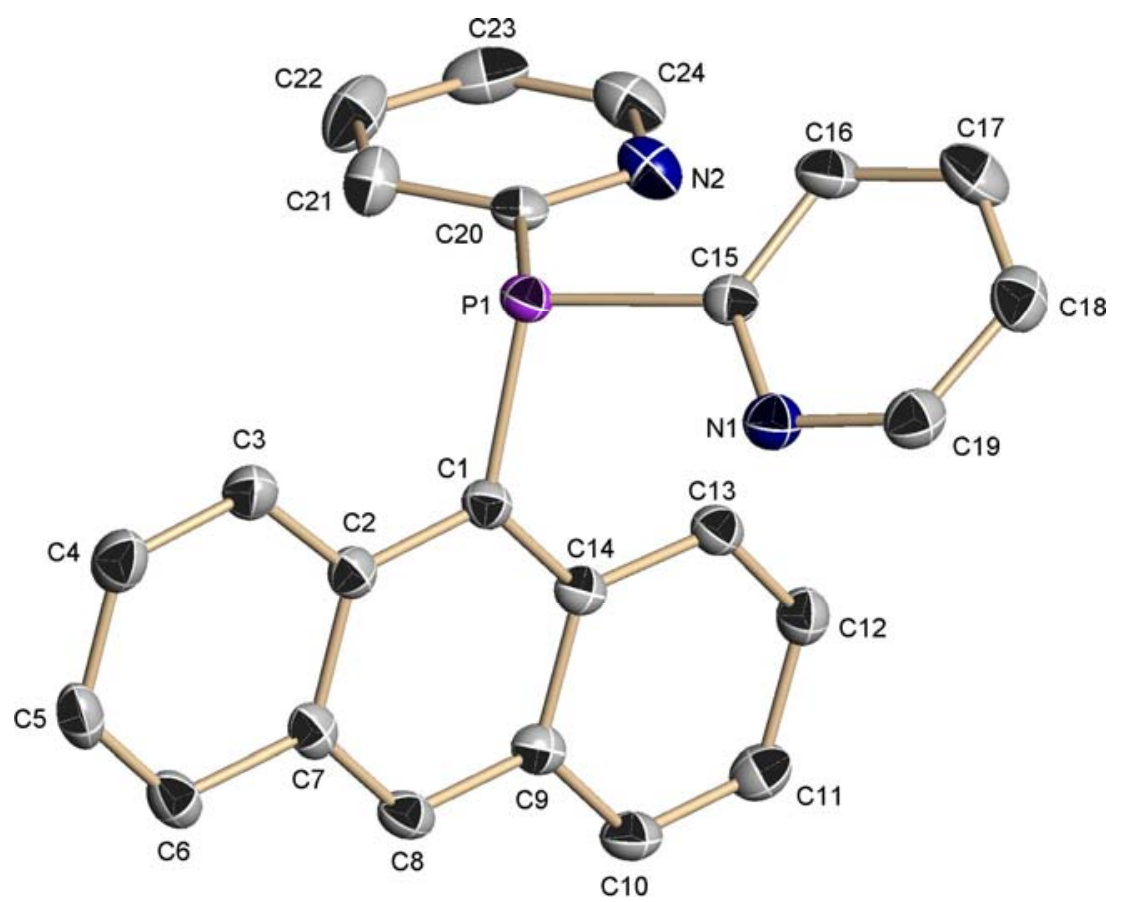

Abb. 9-23: Asymmetrische Einheit von $\mathrm{Py}_{2} \mathrm{P}\left(\mathrm{C}_{14} \mathrm{H}_{9}\right)(28)$.

Tab. 9-23: Kristallographische Daten für $\mathrm{Py}_{2} \mathrm{P}\left(\mathrm{C}_{14} \mathrm{H}_{9}\right)(28)$.

\begin{tabular}{|c|c|c|c|}
\hline Strukturcode & Averna & $\rho_{\text {calc }} / \mathrm{Mg} \cdot \mathrm{m}^{-3}$ & 1.386 \\
\hline Summenformel & $\mathrm{C}_{24} \mathrm{H}_{17} \mathrm{~N}_{2} \mathrm{P}$ & $\mu / \mathrm{mm}^{-1}$ & 0.169 \\
\hline Molmasse $/ \mathrm{g} \cdot \mathrm{mol}^{-1}$ & 364.37 & $F(000)$ & 760 \\
\hline Kristallgröße / mm & $0.30 \times 0.20 \times 0.20$ & $\theta$-Bereich / ${ }^{\circ}$ & $2.35-25.70$ \\
\hline Kristallsystem & monoklin & Reflexe gesamt/unabhängig & $12973 / 3308$ \\
\hline Raumgruppe & $P 2_{1} / n$ & Daten/Restraints/Parameter & $3308 / 0 / 244$ \\
\hline $\mathrm{a} / \mathrm{pm}$ & $1025.89(14)$ & $R 1[I>2 \sigma(I)]$ & 0.0406 \\
\hline $\mathrm{b} / \mathrm{pm}$ & $1684.4(2)$ & $w R 2$ (alle Daten) & 0.1086 \\
\hline $\mathrm{c} / \mathrm{pm}$ & $1028.47(15)$ & $g 1 / g 2$ & $0.0528 / 0.9700$ \\
\hline$\beta /{ }^{\circ}$ & $100.691(2)$ & GoF & 1.043 \\
\hline $\mathrm{V} / \mathrm{nm}^{3}$ & $1.7464(4)$ & Differenzelektronendichte: & \\
\hline Z & 4 & $\max . / \min . /\left(10^{-6}\right.$ e.pm $\left.{ }^{-3}\right)$ & $0.483 /-0.330$ \\
\hline Messtemperatur / K & $100(2)$ & max. / min. Transmission & $0.99 / 0.87$ \\
\hline
\end{tabular}




\subsubsection{4 $\left[\mathrm{S}\left\{\left(\mathrm{C}_{14} \mathrm{H}_{8}\right) \mathrm{P}(\mathrm{S}) \mathrm{Ph}_{2}\right\}_{2} \cdot 3 \mathrm{THF}\right](29)$}

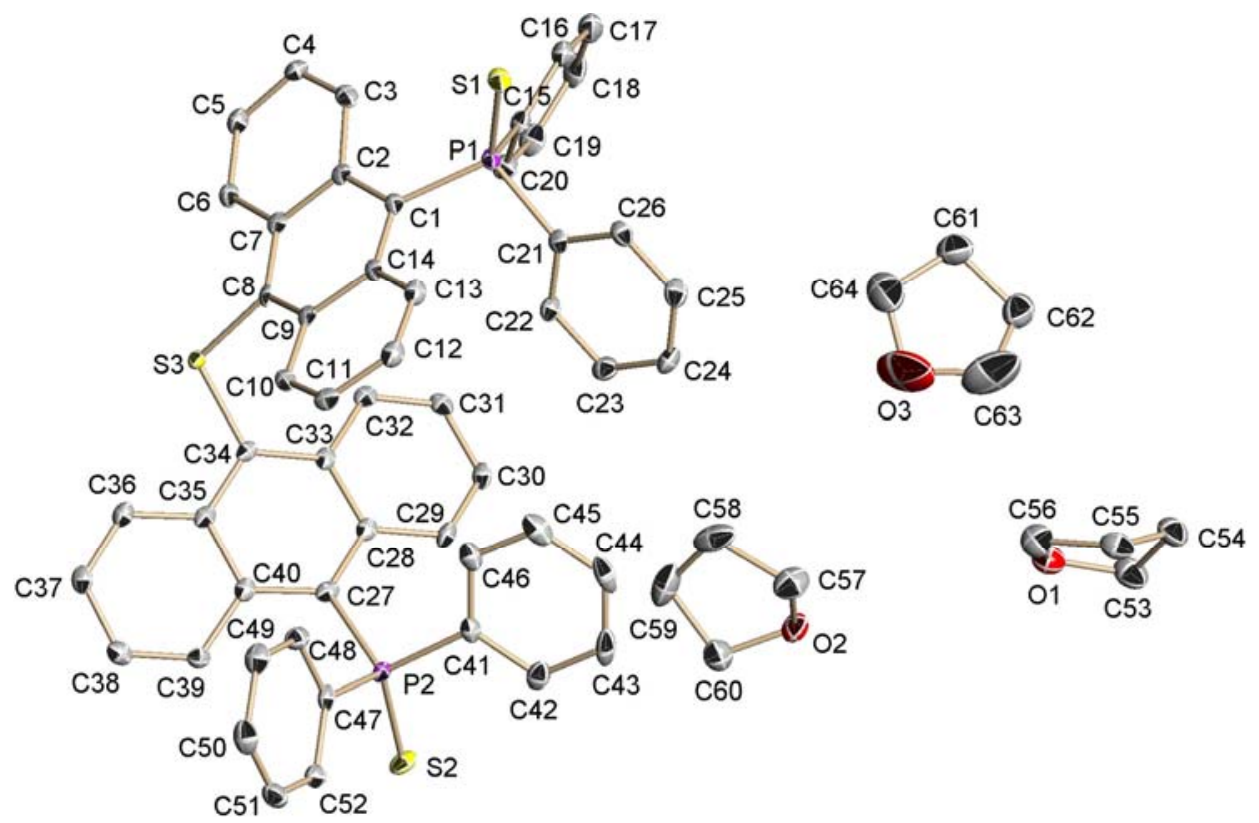

Abb. 9-24: Asymmetrische Einheit von $\left[\mathrm{S}\left\{\left(\mathrm{C}_{14} \mathrm{H}_{8}\right) \mathrm{P}(\mathrm{S}) \mathrm{Ph}_{2}\right\}_{2} \cdot 3 \mathrm{THF}\right](29)$.

Tab. 9-24: Kristallographische Daten für $\left[\mathrm{S}\left\{\left(\mathrm{C}_{14} \mathrm{H}_{8}\right) \mathrm{P}(\mathrm{S}) \mathrm{Ph}_{2}\right\}_{2} \cdot 3 \mathrm{THF}\right](29)$.

\begin{tabular}{|ll|ll|}
\hline Strukturcode & Doppelkorn & Messtemperatur / K & $100(2)$ \\
Summenformel & $\mathrm{C}_{64} \mathrm{H}_{60} \mathrm{O}_{3} \mathrm{P}_{2} \mathrm{~S}_{3}$ & $\rho_{\text {calc }} / \mathrm{Mg} \cdot \mathrm{m}^{-3}$ & 1.311 \\
Molmasse $/ \mathrm{g} \cdot \mathrm{mol}^{-1}$ & 1035.24 & $\mu / \mathrm{mm}^{-1}$ & 0.251 \\
Kristallgröße / mm & $0.3 \times 0.2 \times 0.03$ & $F(000)$ & 1092 \\
Kristallsystem & triklin & $\theta-$ Bereich ${ }^{\circ}$ & $1.95-26.13$ \\
Raumgruppe & $P \overline{1}$ & Reflexe gesamt/unabhängig & $52061 / 10435$ \\
$\mathrm{a} / \mathrm{pm}$ & $934.28(6)$ & Daten/Restraints/Parameter & $10435 / 0 / 634$ \\
$\mathrm{~b} / \mathrm{pm}$ & $1732.81(11)$ & $R 1[/>2 \sigma(I)]$ & 0.0732 \\
$\mathrm{c} / \mathrm{pm}$ & $1765.99(11)$ & $w R 2($ alle Daten $)$ & 0.1612 \\
$\alpha /{ }^{\circ}$ & $73.4690(10)$ & $g 1 / g 2$ & $0.0522 / 6.6967$ \\
$\beta / /^{\circ}$ & $77.5220(10)$ & GoF & 1.166 \\
$\gamma /{ }^{\circ}$ & $75.5740(10)$ & Differenzelektronendichte: & \\
$\mathrm{V} / \mathrm{nm}^{3}$ & $2.6218(3)$ & max. / min. / (10 $\left.{ }^{-6} \mathrm{e} \cdot \mathrm{pm}^{-3}\right)$ & $0.615 /-0.530$ \\
$\mathrm{Z}$ & 2 & max. / min. Transmission & $0.99 / 0.81$ \\
\hline
\end{tabular}




\subsubsection{5 [\{(thf) $\left.\left.{ }_{4} \mathrm{NaS}\right\}\left(\mathrm{C}_{14} \mathrm{H}_{8}\right) \mathrm{P}(\mathrm{S}) \mathrm{Ph}_{2}\right](30)$}

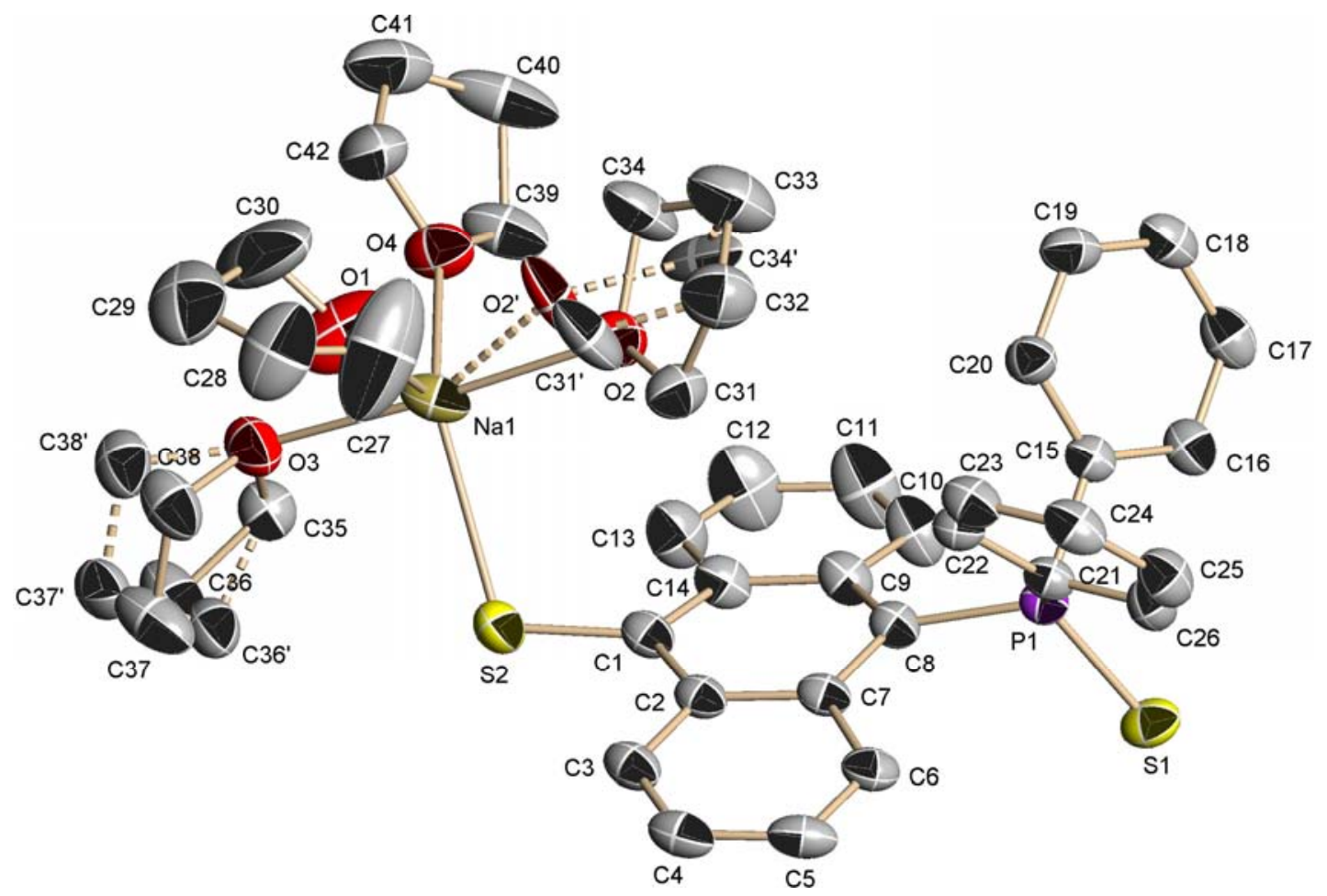

Abb. 9-25: Asymmetrische Einheit von $\left[\left\{(\text { thf })_{4} \mathrm{NaS}\right\}\left(\mathrm{C}_{14} \mathrm{H}_{8}\right) \mathrm{P}(\mathrm{S}) \mathrm{Ph}_{2}\right](\mathbf{3 0})$.

Unter Verwendung von Schwingungs-restraints (ISOR) konnte eine anisotrope Verfeinerung der fehlgeordneten THF-Atompositionen durchgeführt werden.

Tab. 9-25: Kristallographische Daten für $\left[\left\{(\text { thf })_{4} \mathrm{NaS}\right\}\left(\mathrm{C}_{14} \mathrm{H}_{8}\right) \mathrm{P}(\mathrm{S}) \mathrm{Ph}_{2}\right](\mathbf{3 0})$.

\begin{tabular}{|c|c|c|c|}
\hline Strukturcode & Ramazotti & $\rho_{\text {calc }} / \mathrm{Mg} \cdot \mathrm{m}^{-3}$ & 1.265 \\
\hline Summenformel & $\mathrm{C}_{42} \mathrm{H}_{50} \mathrm{NaO}_{4} \mathrm{PS}_{2}$ & $\mu / \mathrm{mm}^{-1}$ & 0.231 \\
\hline Molmasse $/ \mathrm{g} \cdot \mathrm{mol}^{-1}$ & 736.90 & $F(000)$ & 1568 \\
\hline Kristallgröße / mm & $0.60 \times 0.50 \times 0.30$ & $\theta$-Bereich $/^{\circ}$ & $2.07-27.10$ \\
\hline Kristallsystem & orthorhombisch & Reflexe gesamt/unabhängig & 64637 / 8523 \\
\hline Raumgruppe & $P_{n a 2}$ & Daten/Restraints/Parameter & $8523 / 19 / 508$ \\
\hline $\mathrm{a} / \mathrm{pm}$ & $2500.55(18)$ & $R 1[I>2 \sigma(I)]$ & 0.0524 \\
\hline $\mathrm{b} / \mathrm{pm}$ & $1603.08(12)$ & $w R 2$ (alle Daten) & 0.1440 \\
\hline $\mathrm{c} / \mathrm{pm}$ & $965.38(7)$ & $g 1 / g 2$ & $0.0850 / 2.5824$ \\
\hline Flack $\mathrm{x}$ & $0.0096(733)$ & GoF & 1.036 \\
\hline $\mathrm{V} / \mathrm{nm}^{3}$ & $3.8698(5)$ & Differenzelektronendichte: & \\
\hline Z & 4 & $\max . / \min . /\left(10^{-6} \mathrm{e} \cdot \mathrm{pm}^{-3}\right)$ & $0.982 /-0.464$ \\
\hline Messtemperatur / K & $143(2)$ & max. / min. Transmission & $0.99 / 0.92$ \\
\hline
\end{tabular}




\subsubsection{6 $\mathrm{Br}\left(\mathrm{C}_{14} \mathrm{H}_{8} \mathrm{O}_{2}\right) \mathrm{C}(\mathrm{OH}) \mathrm{Ph}_{2}(31)$}

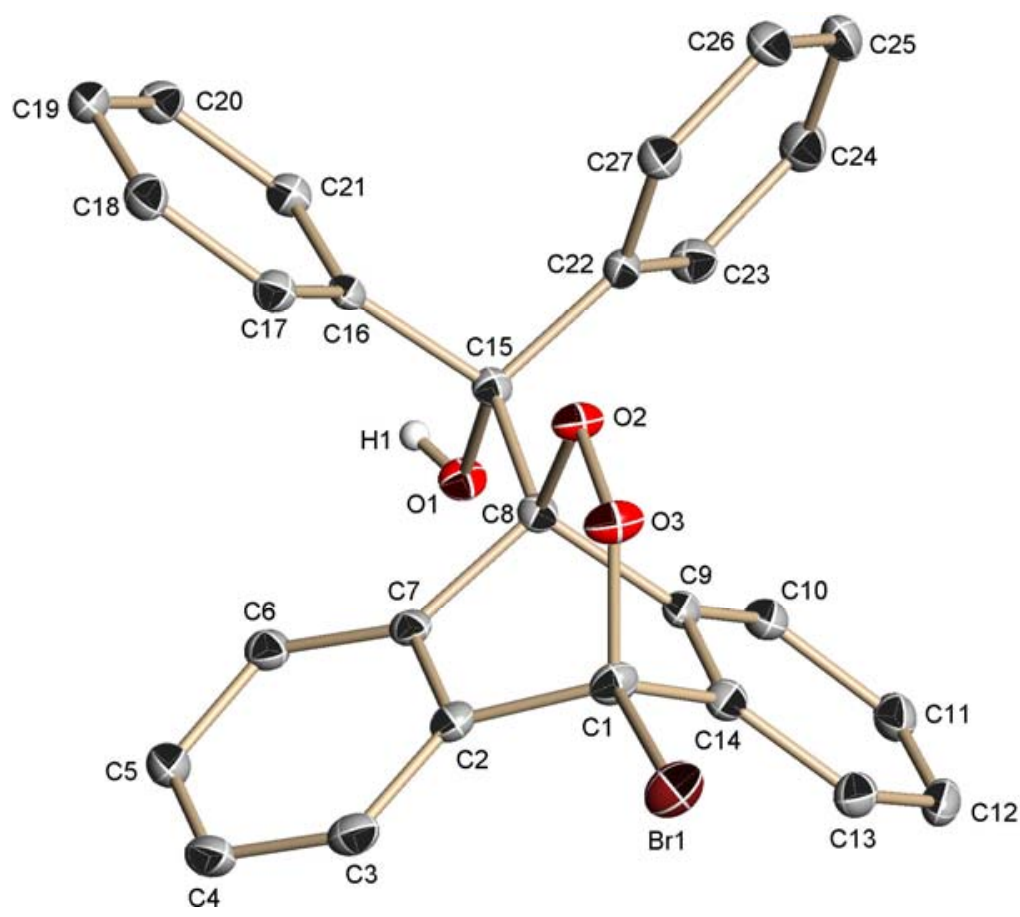

Abb. 9-26: Asymmetrische Einheit von $\mathrm{Br}\left(\mathrm{C}_{14} \mathrm{H}_{8} \mathrm{O}_{2}\right) \mathrm{C}(\mathrm{OH}) \mathrm{Ph}_{2}(31)$.

Die Position des Wasserstoffatoms der Hydroxylgruppe wurde frei verfeinert, die Bindungslänge $\mathrm{O} 1-\mathrm{H} 1$ beträgt $73.8 \mathrm{pm}$.

Tab. 9-26: Kristallographische Daten für $\mathrm{Br}\left(\mathrm{C}_{14} \mathrm{H}_{8} \mathrm{O}_{2}\right) \mathrm{C}(\mathrm{OH}) \mathrm{Ph}_{2}$ (31).

\begin{tabular}{|c|c|c|c|}
\hline Strukturcode & Küstennebel & $\rho_{\text {calc }} / \mathrm{Mg} \cdot \mathrm{m}^{-3}$ & 1.557 \\
\hline Summenformel & $\mathrm{C}_{27} \mathrm{H}_{19} \mathrm{BrO}_{3}$ & $\mu / \mathrm{mm}^{-1}$ & 2.073 \\
\hline Molmasse $/ \mathrm{g} \cdot \mathrm{mol}^{-1}$ & 471.33 & $F(000)$ & 960 \\
\hline Kristallgröße / mm & $0.4 \times 0.3 \times 0.1$ & $\theta$-Bereich $/^{\circ}$ & 2.03 to 27.10 \\
\hline Kristallsystem & monoklin & Reflexe gesamt/unabhängig & $63320 / 4429$ \\
\hline Raumgruppe & $P 2_{1} / c$ & Daten/Restraints/Parameter & 4429 / 0 / 284 \\
\hline $\mathrm{a} / \mathrm{pm}$ & $864.79(2)$ & $R 1[I>2 \sigma(I)]$ & 0.0272 \\
\hline $\mathrm{b} / \mathrm{pm}$ & $2003.70(4)$ & $w R 2$ (alle Daten) & 0.0765 \\
\hline $\mathrm{c} / \mathrm{pm}$ & $1212.77(2)$ & $g 1 / g 2$ & $0.0443 / 1.5686$ \\
\hline$\beta /^{\circ}$ & $106.9050(10)$ & GoF & 1.066 \\
\hline $\mathrm{V} / \mathrm{nm}^{3}$ & $2.01066(7)$ & Differenzelektronendichte: & \\
\hline Z & 4 & $\max . / \min . /\left(10^{-6} \mathrm{e} \cdot \mathrm{pm}^{-3}\right)$ & $0.443 /-0.554$ \\
\hline Messtemperatur / K & $100(2)$ & max. / min. Transmission & $0.99 / 0.75$ \\
\hline
\end{tabular}




\subsubsection{7 $\left[\mathrm{Ph}_{2}(\mathrm{O}) \mathrm{P}\left(\mathrm{C}_{14} \mathrm{H}_{10}\right) \mathrm{P}(\mathrm{OH}) \mathrm{Ph}_{2}\right]^{+}\left[\mathrm{HSO}_{4}\right]^{-}(32)$}

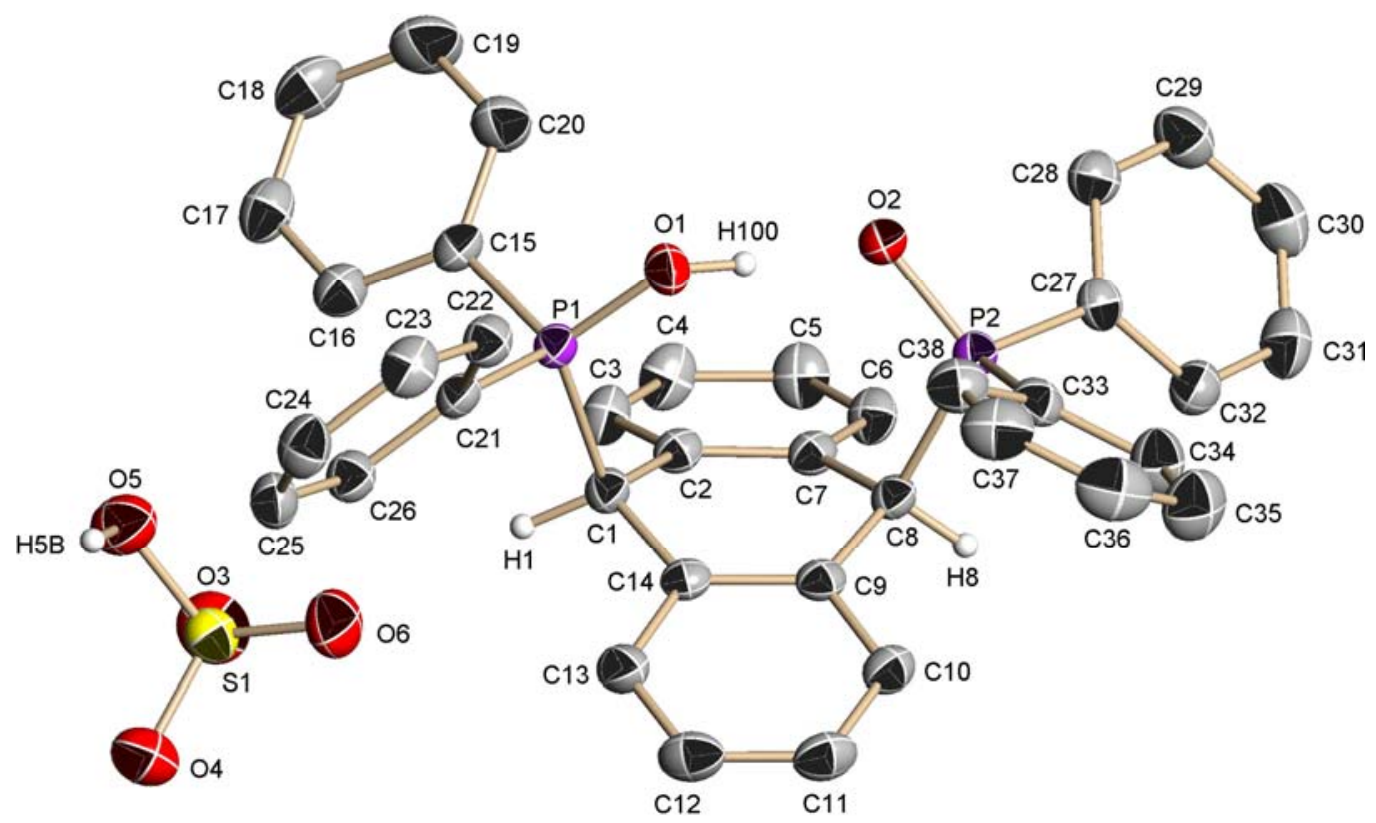

Abb. 9-27: Asymmetrische Einheit von $\left[\mathrm{Ph}_{2}(\mathrm{O}) \mathrm{P}\left(\mathrm{C}_{14} \mathrm{H}_{10}\right) \mathrm{P}(\mathrm{OH}) \mathrm{Ph}_{2}\right]^{+}\left[\mathrm{HSO}_{4}\right]^{-}$(32).

Die Positionen der vier in Abb. 9-27 abgebildeten Wasserstoffatome wurden frei verfeinert, die Bindungslängen betragen 98.2 (C1-H1), 93.9 (C8-H8), 87.4 (O1-H100) bzw. 96.9 pm (O5-H5B). Die beiden Sauerstoffatome $\mathrm{O} 1$ und $\mathrm{O} 2$ sind 238.6 pm voneinander entfernt.

Tab. 9-27: Kristallographische Daten für $\left[\mathrm{Ph}_{2}(\mathrm{O}) \mathrm{P}\left(\mathrm{C}_{14} \mathrm{H}_{10}\right) \mathrm{P}(\mathrm{OH}) \mathrm{Ph}_{2}\right]^{+}\left[\mathrm{HSO}_{4}\right]^{-}(32)$.

\begin{tabular}{|c|c|c|c|}
\hline Strukturcode & Mariacron & $\rho_{\text {calc }} / \mathrm{Mg} \cdot \mathrm{m}^{-3}$ & 1.387 \\
\hline Summenformel & $\mathrm{C}_{38} \mathrm{H}_{32} \mathrm{O}_{6} \mathrm{P}_{2} \mathrm{~S}$ & $\mu / \mathrm{mm}^{-1}$ & 0.247 \\
\hline Molmasse $/ \mathrm{g} \cdot \mathrm{mol}^{-1}$ & 678.64 & $F(000)$ & 1416 \\
\hline Kristallgröße / mm & $0.3 \times 0.2 \times 0.1$ & $\theta$-Bereich / ${ }^{\circ}$ & $1.59-26.41$ \\
\hline Kristallsystem & monoklin & Reflexe gesamt/unabhängig & 39342 / 6677 \\
\hline Raumgruppe & $P 2_{1} / C$ & Daten/Restraints/Parameter & $6677 / 0 / 436$ \\
\hline $\mathrm{a} / \mathrm{pm}$ & $983.73(5)$ & $R 1[I>2 \sigma(I)]$ & 0.0454 \\
\hline $\mathrm{b} / \mathrm{pm}$ & $1718.34(9)$ & $w R 2$ (alle Daten) & 0.1216 \\
\hline $\mathrm{c} / \mathrm{pm}$ & $1955.26(10)$ & $g 1 / g 2$ & $0.0624 / 1.8683$ \\
\hline$\beta /{ }^{\circ}$ & $100.5840(10)$ & GoF & 1.046 \\
\hline $\mathrm{V} / \mathrm{nm}^{3}$ & $3.2489(3)$ & Differenzelektronendichte: & \\
\hline Z & 4 & $\max . / \min . /\left(10^{-6} \mathrm{e} \cdot \mathrm{pm}^{-3}\right)$ & $0.437 /-0.319$ \\
\hline Messtemperatur / K & $173(2)$ & max. / min. Transmission & $0.99 / 0.89$ \\
\hline
\end{tabular}




\subsection{Kristallographische Servicemessungen}

Im Rahmen dieser Doktorarbeit wurden 24 Molekülstrukturen in Kooperation mit anderen Arbeitskreisen bestimmt (Tab. 9-28). Die Diskussion dieser Strukturen ist in Kap. 9.4 auf die kristallographischen Informationen beschränkt, da alle weiteren Angaben in den entsprechenden Veröffentlichungen zu finden sind bzw. sein werden.

Tab. 9-28: Übersicht über die während dieser Arbeit bestimmten Servicestrukturen.<smiles>O=[N+]([O-])[Pb](c1ccccn1)(c1cccc[nH+]1)[N+]([O-])([O-])[O-]</smiles>

S01<smiles>CCN1Cc2cc(C)cc3c2O[C@]1(CC)N(CC)[Al](N)N3C</smiles>

S04

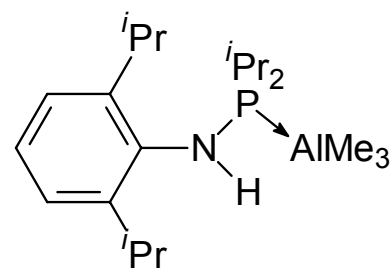

S07

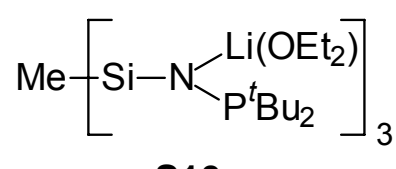

S10

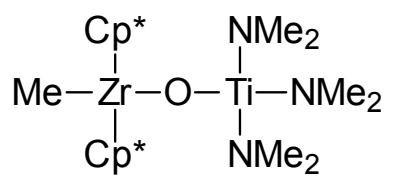

$\mathrm{S} 02$<smiles>CCCc1cccc(CCC)c1N(P)[Ge]</smiles>

S05

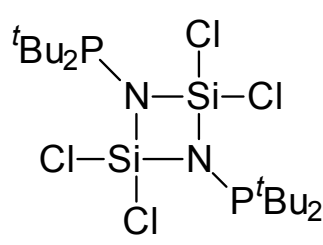

S08<smiles>CCCCP(C)(c1ccccc1)(c1ccccc1)N(C)C</smiles>

S11

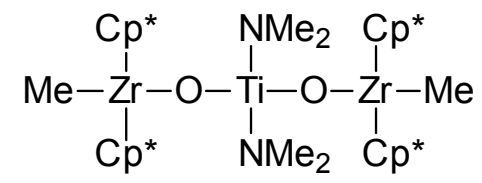

S03<smiles>CCCc1cccc(Br)c1P(N)c1c(CCC)cccc1CCC</smiles>

S06

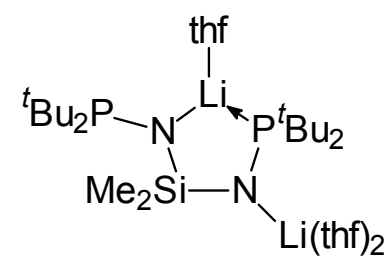

S09<smiles>CSN1[N+](C)(Cl)N([SiH3])P1(Cl)(Cl)c1ccccc1</smiles>

S12 


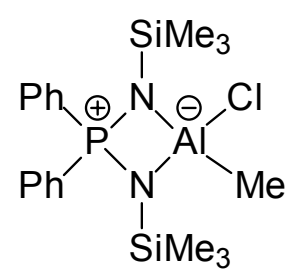

S13

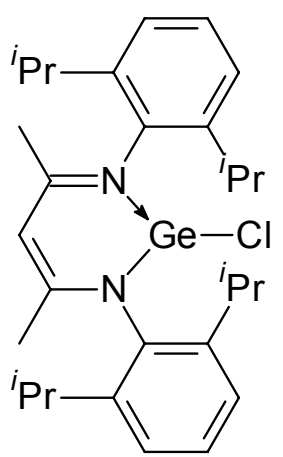

S16

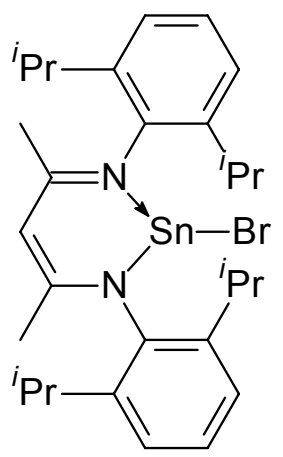

S19

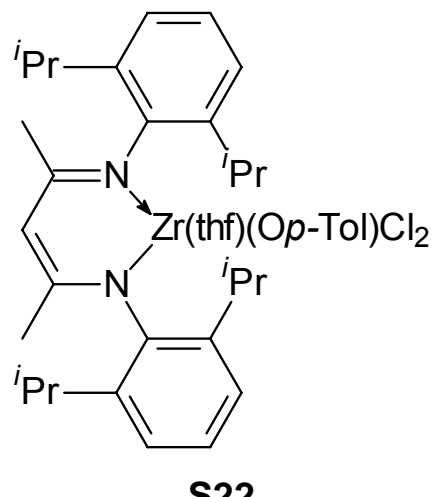

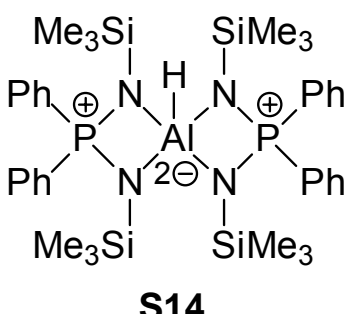

S14

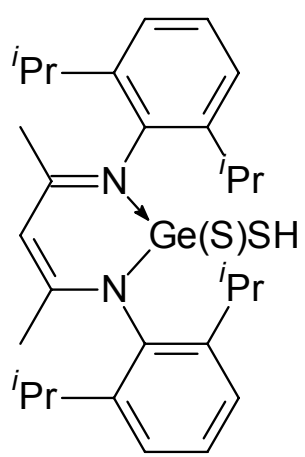

S17

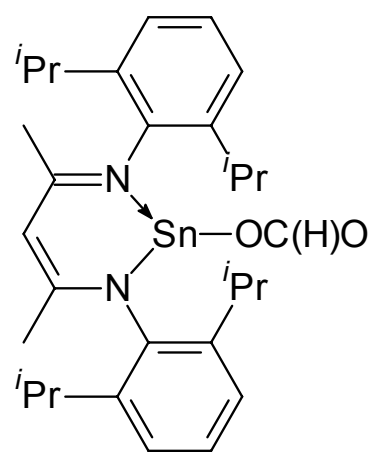

S20

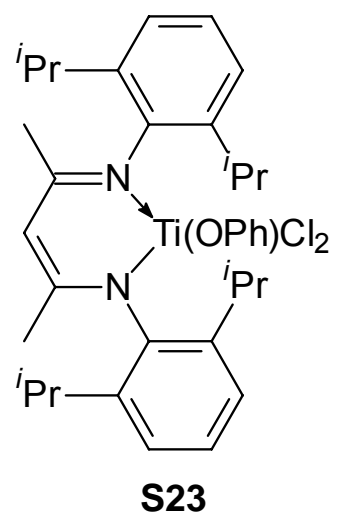

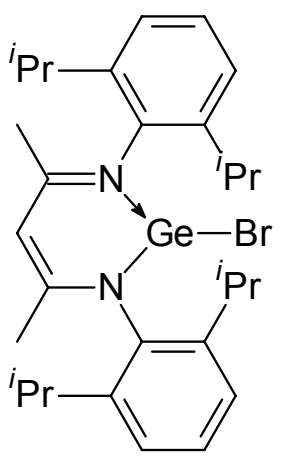

S15

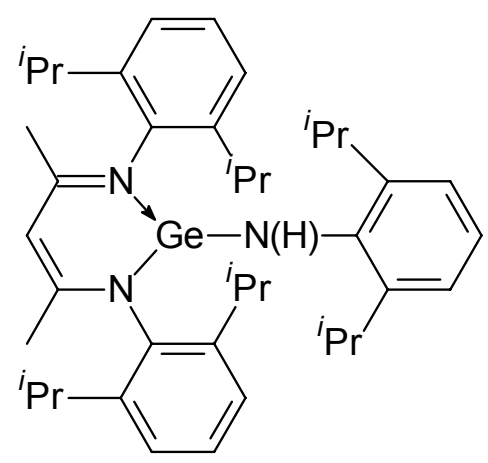

S18

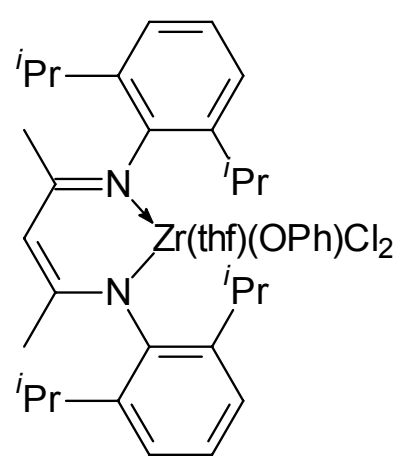

S21

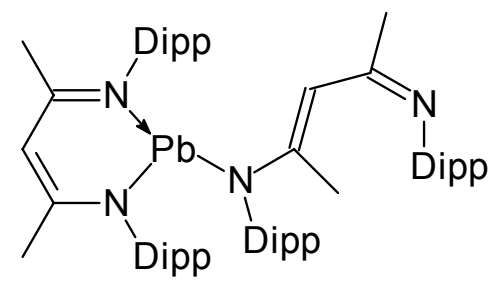

S24 


\subsection{1 [\{(OC) $\left.\left.)_{5} \mathrm{~W}\right\}_{2} \mathrm{PPy}(\mathrm{HPy})\right](\mathrm{S} 01)$}

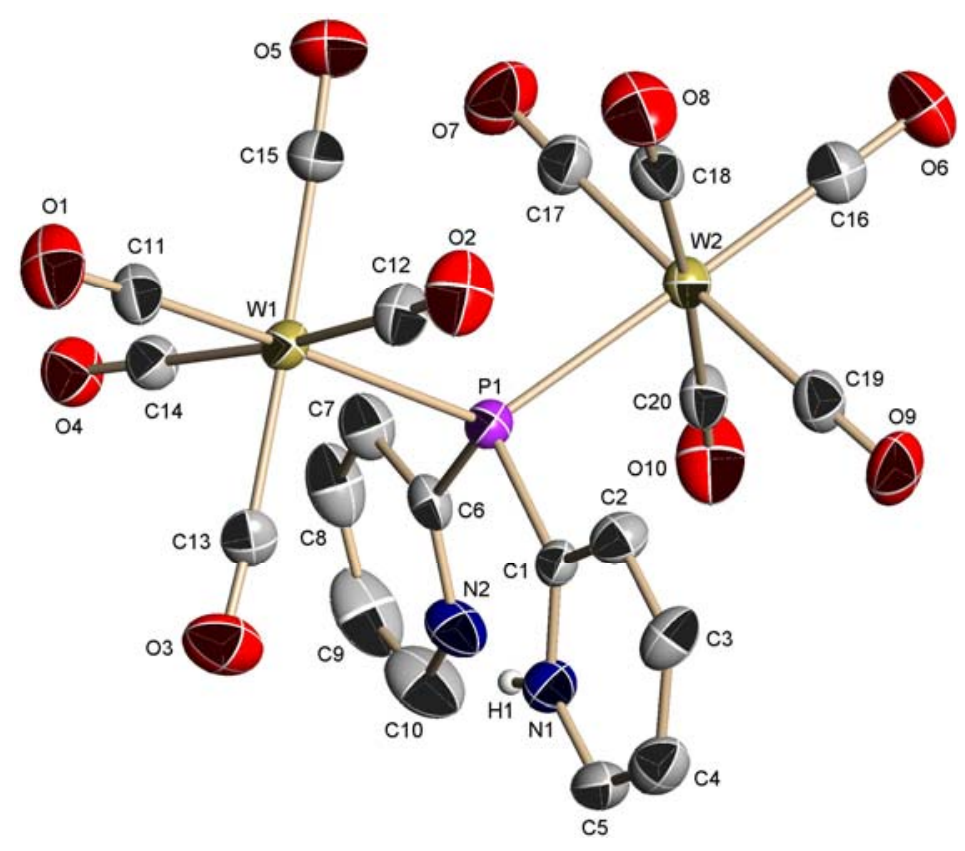

Abb. 9-28: Asymmetrische Einheit von $\left[\left\{(\mathrm{OC})_{5} \mathrm{~W}\right\}_{2} \mathrm{PPy}(\mathrm{HPy})\right](\mathrm{S} 01)$.

Tab. 9-29: Kristallographische Daten für $\left.\left[(\mathrm{OC})_{5} \mathrm{~W}\right\}_{2} \mathrm{PPy}(\mathrm{HPy})\right](\mathrm{S} 01)$.

\begin{tabular}{|c|c|c|c|}
\hline Strukturcode & Doppelwolle & Z & 2 \\
\hline Servicepartner & A. Oechsner & Messtemperatur / K & $173(2)$ \\
\hline CCDC-Nummer & 611064 & $\rho_{\text {calc }} / \mathrm{Mg} \cdot \mathrm{m}^{-3}$ & 2.314 \\
\hline Summenformel & $\mathrm{C}_{20} \mathrm{H}_{9} \mathrm{~N}_{2} \mathrm{O}_{10} \mathrm{PW}_{2}$ & $\mu / \mathrm{mm}^{-1}$ & 9.703 \\
\hline Molmasse $/ \mathrm{g} \cdot \mathrm{mol}^{-1}$ & 835.96 & $F(000)$ & 772 \\
\hline Kristallgröße / mm & $0.1 \times 0.05 \times 0.01$ & $\theta$-Bereich $/^{\circ}$ & $1.57-26.10$ \\
\hline Kristallsystem & triklin & Reflexe gesamt/unabhängig & 20962 / 4768 \\
\hline Raumgruppe & $P \overline{1}$ & Daten/Restraints/Parameter & $4768 / 0 / 320$ \\
\hline $\mathrm{a} / \mathrm{pm}$ & $936.60(14)$ & $R 1[I>2 \sigma(I)]$ & 0.0280 \\
\hline $\mathrm{b} / \mathrm{pm}$ & $1072.47(16)$ & $w R 2$ (alle Daten) & 0.0651 \\
\hline $\mathrm{c} / \mathrm{pm}$ & $1320.8(2)$ & $g 1 / g 2$ & $0.0311 / 1.1744$ \\
\hline$\alpha /^{\circ}$ & $79.317(3)$ & GoF & 1.040 \\
\hline$\beta /^{\circ}$ & $87.413(3)$ & Differenzelektronendichte: & \\
\hline$\gamma /{ }^{\circ}$ & $67.030(3)$ & $\max . / \min . /\left(10^{-6} \mathrm{e} \cdot \mathrm{pm}^{-3}\right)$ & $1.619 /-0.562$ \\
\hline $\mathrm{V} / \mathrm{nm}^{3}$ & $1.1998(3)$ & max. / min. Transmission & $0.99 / 0.59$ \\
\hline
\end{tabular}




\subsection{2 $\left[\mathrm{Cp}_{2}{ }_{2}(\mathrm{Me}) \mathrm{Zr}(\mu-\mathrm{O}) \mathrm{Ti}\left(\mathrm{NMe}_{2}\right)_{3}\right](\mathrm{S} 02)$}

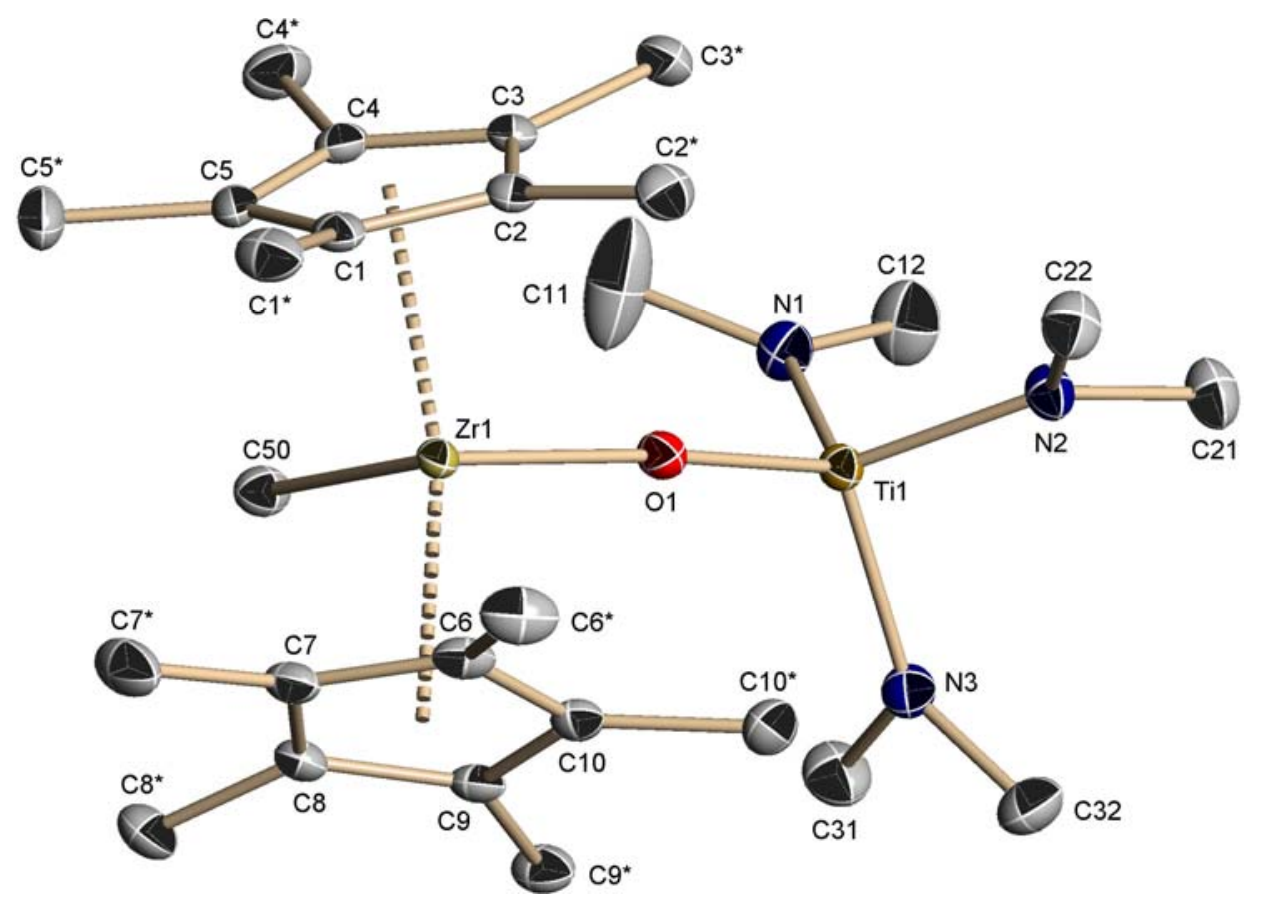

Abb. 9-29: Asymmetrische Einheit von $\left[\mathrm{Cp}_{2}{ }_{2}(\mathrm{Me}) \mathrm{Zr}(\mu-\mathrm{O}) \mathrm{Ti}\left(\mathrm{NMe}_{2}\right)_{3}\right](\mathbf{S 0 2})$.

Tab. 9-30: Kristallographische Daten für $\left[\mathrm{Cp}_{2}{ }_{2}(\mathrm{Me}) \mathrm{Zr}(\mu-\mathrm{O}) \mathrm{Ti}\left(\mathrm{NMe}_{2}\right)_{3}\right](\mathrm{S} 02)$.

\begin{tabular}{|c|c|c|c|}
\hline Strukturcode & R1230 & Z & 2 \\
\hline Servicepartner & S. Mandal & Messtemperatur / K & $100(2)$ \\
\hline CCDC-Nummer & $642715^{[186]}$ & $\rho_{\text {calc }} / \mathrm{Mg} \cdot \mathrm{m}^{-3}$ & 1.301 \\
\hline Summenformel & $\mathrm{C}_{27} \mathrm{H}_{51} \mathrm{~N}_{3} \mathrm{OTiZr}$ & $\mu / \mathrm{mm}^{-1}$ & 0.651 \\
\hline Molmasse $/ \mathrm{g} \cdot \mathrm{mol}^{-1}$ & 572.83 & $F(000)$ & 608 \\
\hline Kristallgröße / mm & $0.25 \times 0.20 \times 0.10$ & $\theta$-Bereich $/^{\circ}$ & $2.09-26.39$ \\
\hline Kristallsystem & triklin & Reflexe gesamt/unabhängig & $26990 / 5981$ \\
\hline Raumgruppe & $P \overline{1}$ & Daten/Restraints/Parameter & $5981 / 0 / 315$ \\
\hline $\mathrm{a} / \mathrm{pm}$ & $1066.44(5)$ & $R 1[I>2 \sigma(I)]$ & 0.0226 \\
\hline $\mathrm{b} / \mathrm{pm}$ & $1163.10(5)$ & $w R 2$ (alle Daten) & 0.0637 \\
\hline $\mathrm{c} / \mathrm{pm}$ & $1290.48(6)$ & $g 1 / g 2$ & $0.0368 / 0.4719$ \\
\hline$\alpha /^{\circ}$ & $89.4060(10)$ & GoF & 1.088 \\
\hline$\beta /^{\circ}$ & $89.3900(10)$ & Differenzelektronendichte: & \\
\hline$\gamma /{ }^{\circ}$ & $66.0210(10)$ & $\max . / \min . /\left(10^{-6} \mathrm{e} \cdot \mathrm{pm}^{-3}\right)$ & $0.609 /-0.503$ \\
\hline $\mathrm{V} / \mathrm{nm}^{3}$ & $1.46242(12)$ & max. / min. Transmission & $0.99 / 0.90$ \\
\hline
\end{tabular}




\subsection{3 $\left[\left\{\mathrm{Cp}_{2}(\mathrm{Me}) \mathrm{Zr}(\mu-\mathrm{O})\right\}_{2} \mathrm{Hf}\left(\mathrm{NMe}_{2}\right)_{2}\right](\mathrm{S} 03)$}

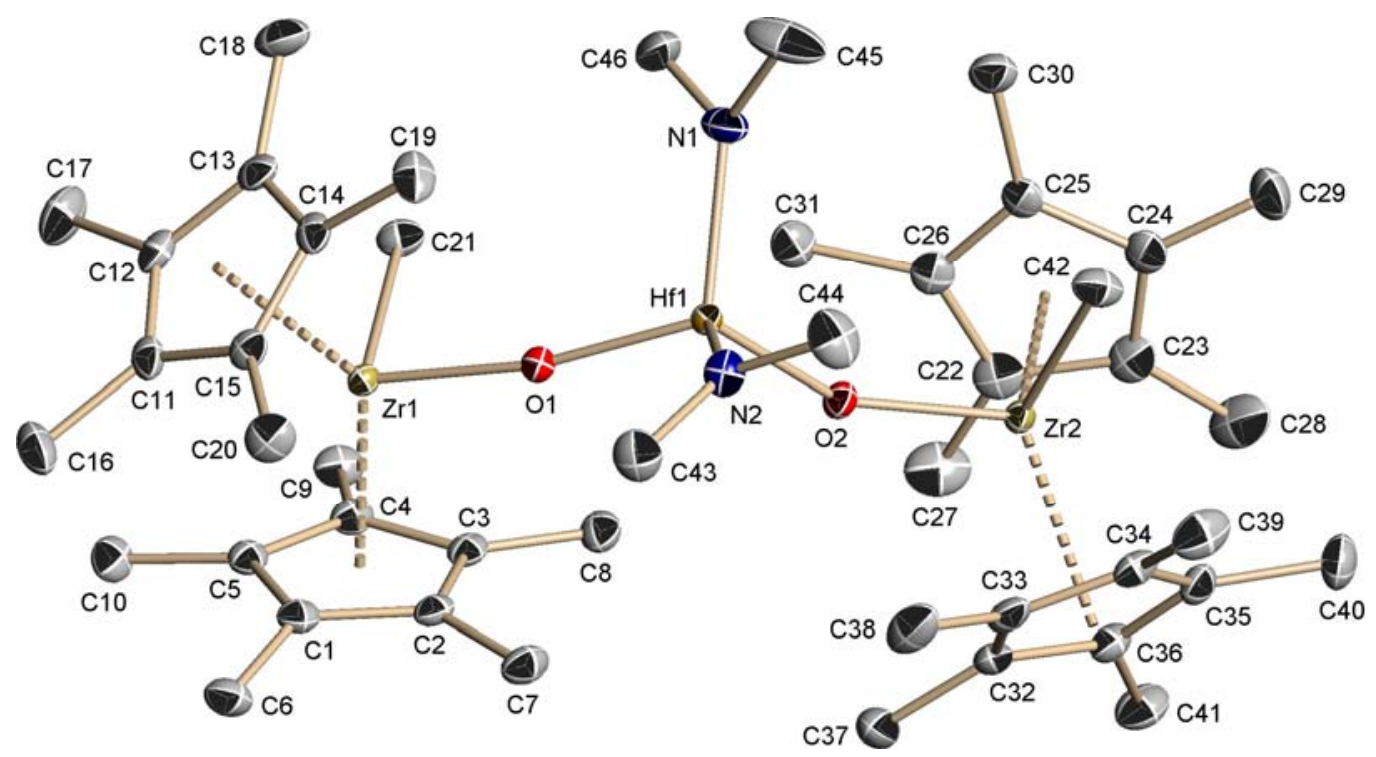

Abb. 9-30: Asymmetrische Einheit von $\left[\left\{\mathrm{Cp}_{2}{ }_{2}(\mathrm{Me}) \mathrm{Zr}(\mu-\mathrm{O})\right\}_{2} \mathrm{Hf}\left(\mathrm{NMe}_{2}\right)_{2}\right](\mathbf{S 0 3})$.

Tab. 9-31: Kristallographische Daten für $\left[\left\{\mathrm{Cp}_{2}{ }_{2}(\mathrm{Me}) \mathrm{Zr}(\mu-\mathrm{O})\right\}_{2} \mathrm{Hf}\left(\mathrm{NMe}_{2}\right)_{2}\right](\mathrm{S} 03)$.

\begin{tabular}{|c|c|c|c|}
\hline Strukturcode & SKM5 & Messtemperatur / K & $100(2)$ \\
\hline Servicepartner & S. Mandal & $\rho_{\text {calc }} / \mathrm{Mg} \cdot \mathrm{m}^{-3}$ & 1.514 \\
\hline CCDC-Nummer & $642716^{[186]}$ & $\mu / \mathrm{mm}^{-1}$ & 2.724 \\
\hline Summenformel & $\mathrm{C}_{46} \mathrm{H}_{78} \mathrm{HfN}_{2} \mathrm{O}_{2} \mathrm{Zr}_{2}$ & $F(000)$ & 2144 \\
\hline Molmasse $/ \mathrm{g} \cdot \mathrm{mol}^{-1}$ & 1052.03 & $\theta$-Bereich / ${ }^{\circ}$ & 1.54 to 26.37 \\
\hline Kristallgröße / mm & $0.4 \times 0.2 \times 0.15$ & Reflexe gesamt/unabhängig & 76389 / 9408 \\
\hline Kristallsystem & monoklin & Daten/Restraints/Parameter & $9408 / 0 / 478$ \\
\hline Raumgruppe & $P 2_{1} / n$ & $R 1[I>2 \sigma(I)]$ & 0.0220 \\
\hline $\mathrm{a} / \mathrm{pm}$ & $1483.18(8)$ & wR2 (alle Daten) & 0.0613 \\
\hline $\mathrm{b} / \mathrm{pm}$ & $1877.43(10)$ & $g 1 / g 2$ & $0.0312 / 10.4730$ \\
\hline $\mathrm{c} / \mathrm{pm}$ & $1775.62(9)$ & GoF & 1.037 \\
\hline$\beta /{ }^{\circ}$ & $111.0510(10)$ & Differenzelektronendichte: & \\
\hline $\mathrm{V} / \mathrm{nm}^{3}$ & $4.6144(4)$ & $\max . / \min . /\left(10^{-6} \mathrm{e} \cdot \mathrm{pm}^{-3}\right)$ & $0.821 /-0.705$ \\
\hline Z & 4 & max. / min. Transmission & $0.99 / 0.68$ \\
\hline
\end{tabular}




\subsection{4 $\left[\mathrm{Me}\left(\mathrm{C}_{6} \mathrm{H}_{2}\right)\left(\mu_{4}-\mathrm{O}\right) \mathrm{Li}\left\{\mathrm{CH}_{2} \mathrm{NEt}_{2} \mathrm{LiN}\left(\mathrm{SiMe}_{3}\right)_{2}\right\}_{2}\right](\mathrm{S} 04)$}
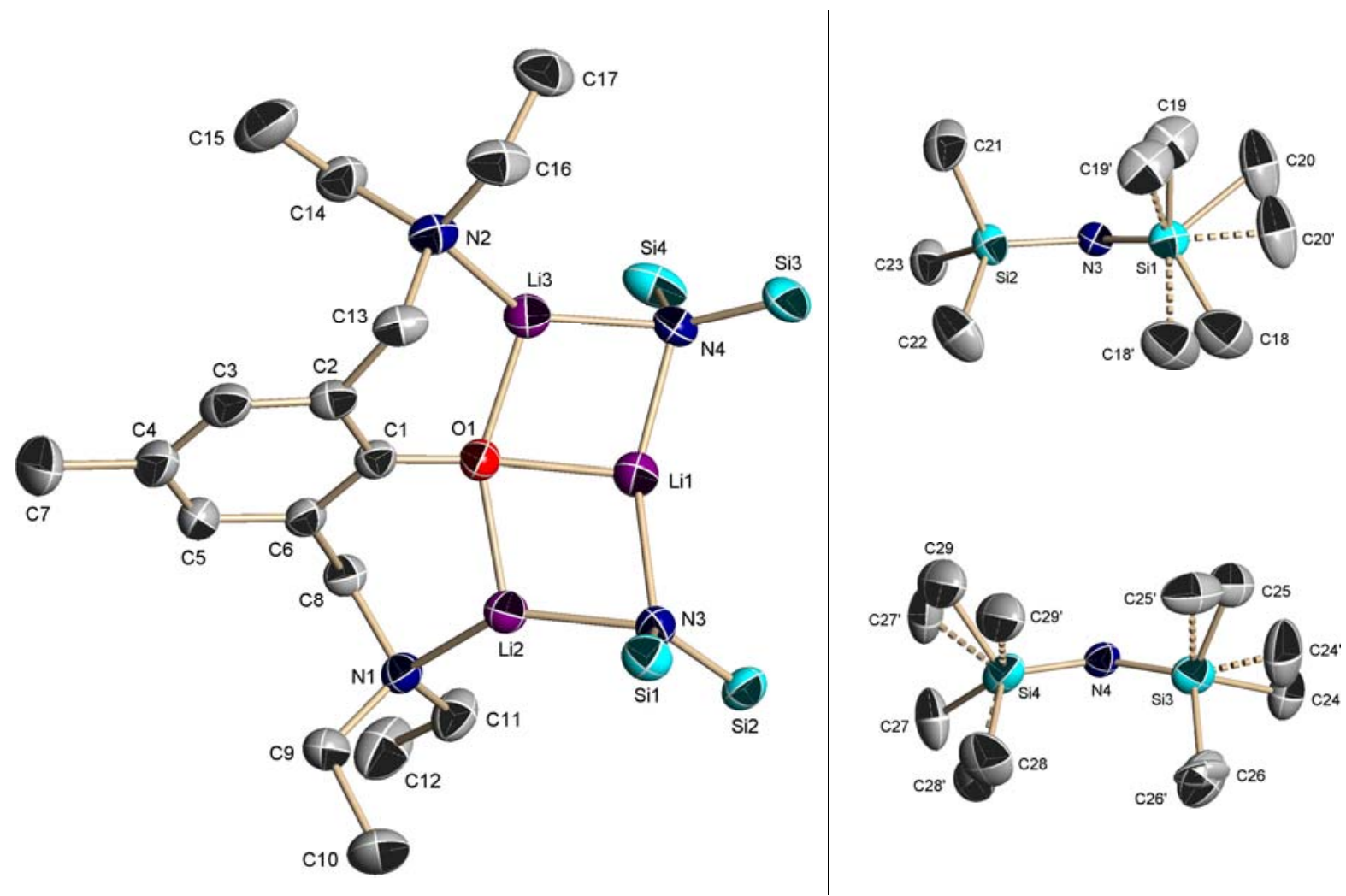

Abb. 9-31: Links: asymmetrische Einheit von $\left[\mathrm{Me}\left(\mathrm{C}_{6} \mathrm{H}_{2}\right)\left(\mu_{4}-\mathrm{O}\right) \mathrm{Li}\left\{\mathrm{CH}_{2} \mathrm{NEt}_{2} \mathrm{LiN}\left(\mathrm{SiMe}_{3}\right)_{2}\right\}_{2}\right]$ (SO4) ohne Darstellung der $\mathrm{SiMe}_{3}-$ Kohlenstoffatome; rechts: Fehlordnung der $\mathrm{SiMe}_{3}-$ Kohlenstoffatome, Besetzungsfaktoren: 0.24 (C18 - C20), 0.52 (C24 - C29).

Tab. 9-32: Kristallographische Daten für $\left[\mathrm{Me}\left(\mathrm{C}_{6} \mathrm{H}_{2}\right)\left(\mu_{4}-\mathrm{O}\right) \mathrm{Li}\left\{\mathrm{CH}_{2} \mathrm{NEt}_{2} \mathrm{LiN}\left(\mathrm{SiMe}_{3}\right)_{2}\right\}_{2}\right](\mathbf{S 0 4})$.

\begin{tabular}{|c|c|c|c|}
\hline Strukturcode & BiNNO & $\rho_{\text {calc }} / \mathrm{Mg} \cdot \mathrm{m}^{-3}$ & 1.023 \\
\hline Servicepartner & N. Bijan & $\mu / \mathrm{mm}^{-1}$ & 0.172 \\
\hline CCDC-Nummer & - & $F(000)$ & 2720 \\
\hline Summenformel & $\mathrm{C}_{29} \mathrm{H}_{65} \mathrm{Li}_{3} \mathrm{~N}_{4} \mathrm{OSi}_{4}$ & $\theta$-Bereich / ${ }^{\circ}$ & $2.09 / 27.12$ \\
\hline Molmasse $/ \mathrm{g} \cdot \mathrm{mol}^{-1}$ & 619.03 & Reflexe gesamt/unabhängig & $65956 / 8707$ \\
\hline Kristallgröße / mm & $0.5 \times 0.4 \times 0.2$ & Daten/Restraints/Parameter & $8707 / 0 / 457$ \\
\hline Kristallsystem & orthorhombisch & $R 1[I>2 \sigma(I)]$ & 0.0428 \\
\hline Raumgruppe & Pbca & $w R 2$ (alle Daten) & 0.1173 \\
\hline $\mathrm{a} / \mathrm{pm}$ & $1982.61(10)$ & $g 1 / g 2$ & $0.0566 / 2.9273$ \\
\hline $\mathrm{b} / \mathrm{pm}$ & $1953.22(10)$ & GoF & 1.052 \\
\hline $\mathrm{c} / \mathrm{pm}$ & 2074.99(11) & Differenzelektronendichte: & \\
\hline $\mathrm{V} / \mathrm{nm}^{3}$ & $8.0353(7)$ & $\max . / \min . /\left(10^{-6} \mathrm{e} \cdot \mathrm{pm}^{-3}\right)$ & $0.294 /-0.330$ \\
\hline Z & 8 & max. / min. Transmission & $0.99 / 0.85$ \\
\hline Messtemperatur / K & $173(2)$ & & \\
\hline
\end{tabular}




\subsection{5 [DippN(SiCl 3$\left.) \mathrm{P}^{i} \mathrm{Pr}_{2}\right](\mathrm{S} 05)$}
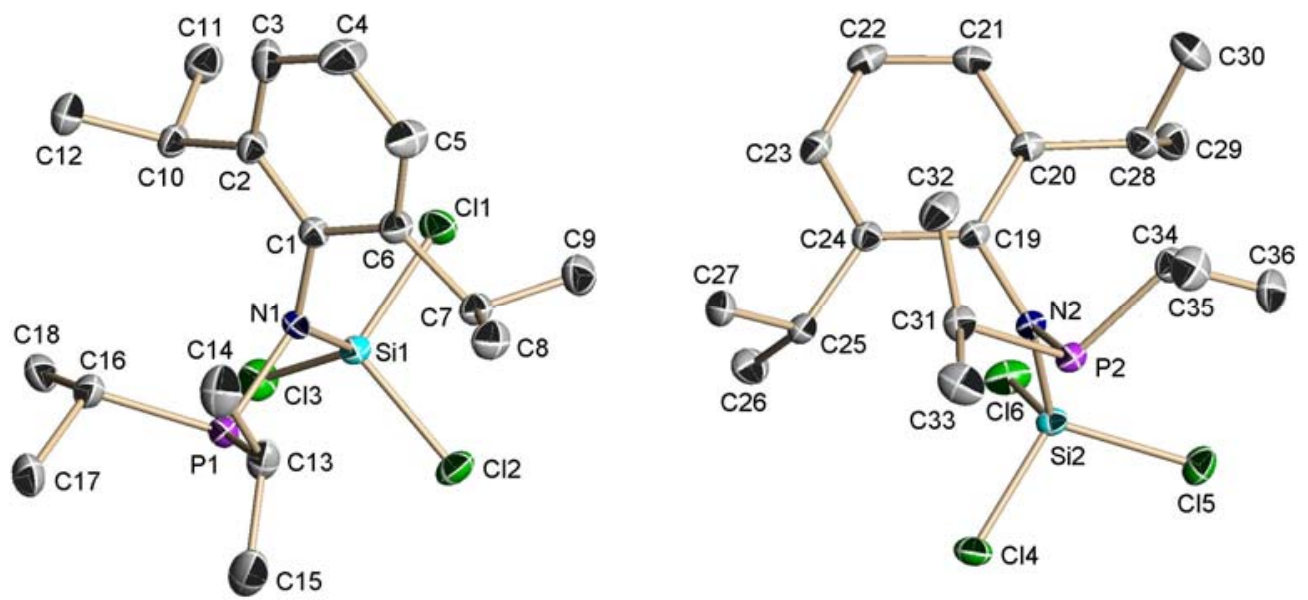

Abb. 9-32: Asymmetrische Einheit von [DippN( $\left.\left(\mathrm{SiCl}_{3}\right) \mathrm{P}^{i} \mathrm{Pr}_{2}\right]$ (S05).

Tab. 9-33: Kristallographische Daten für [DippN( $\left.\left(\mathrm{SiCl}_{3}\right) \mathrm{P}^{j} \mathrm{Pr}_{2}\right]$ (S05).

\begin{tabular}{|c|c|c|c|}
\hline Strukturcode & AJ6 & $\rho_{\text {calc }} / \mathrm{Mg} \cdot \mathrm{m}^{-3}$ & 1.258 \\
\hline Servicepartner & A. Jana & $\mu / \mathrm{mm}^{-1}$ & 0.533 \\
\hline CCDC-Nummer & - & $F(000)$ & 3616 \\
\hline Summenformel & $\mathrm{C}_{18} \mathrm{H}_{31} \mathrm{Cl}_{3} \mathrm{NPSi}$ & $\theta$-Bereich $/^{\circ}$ & $1.75 / 26.37$ \\
\hline Molmasse $/ \mathrm{g} \cdot \mathrm{mol}^{-1}$ & 426.85 & Reflexe gesamt/unabhängig & 47748 / 9210 \\
\hline Kristallgröße / mm & $0.45 \times 0.40 \times 0.35$ & Daten/Restraints/Parameter & $9210 / 0 / 449$ \\
\hline Kristallsystem & orthorhombisch & $R 1[I>2 \sigma(I)]$ & 0.0339 \\
\hline Raumgruppe & Pbca & $w R 2$ (alle Daten) & 0.1158 \\
\hline $\mathrm{a} / \mathrm{pm}$ & $1853.0(2)$ & $g 1 / g 2$ & $0.0650 / 1.7874$ \\
\hline $\mathrm{b} / \mathrm{pm}$ & $1631.68(19)$ & GoF & 1.193 \\
\hline $\mathrm{c} / \mathrm{pm}$ & $2980.9(3)$ & Differenzelektronendichte: & \\
\hline $\mathrm{V} / \mathrm{nm}^{3}$ & $9.0128(18)$ & $\max . / \min . /\left(10^{-6} \mathrm{e} \cdot \mathrm{pm}^{-3}\right)$ & $0.634 /-0.498$ \\
\hline Z & 16 & max. / min. Transmission & $0.99 / 0.82$ \\
\hline Messtemperatur / K & $100(2)$ & & \\
\hline
\end{tabular}




\subsection{6 $\left[\right.$ Tripp $\left._{2} \mathrm{PNH}_{2}\right](\mathrm{S} 06)$}

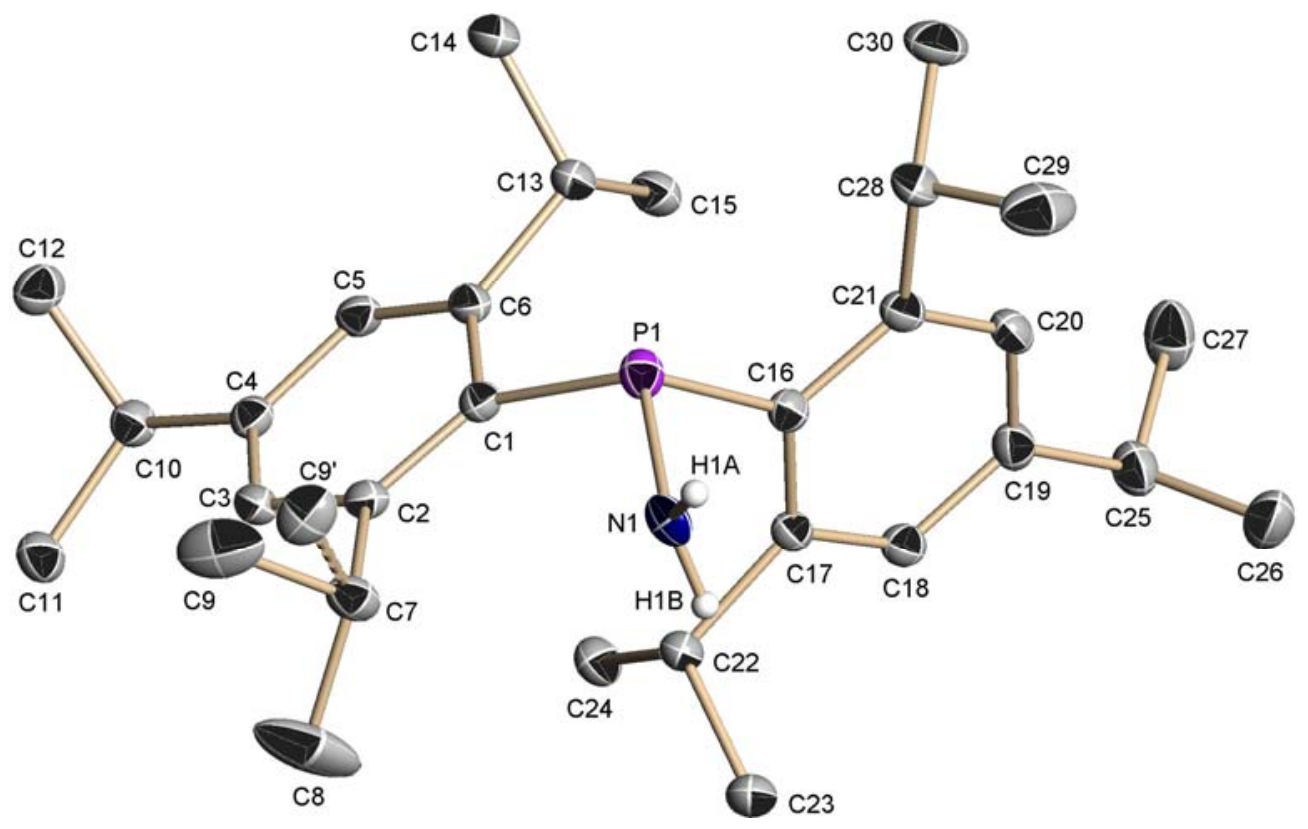

Abb. 9-33: Asymmetrische Einheit von $\left[\mathrm{Tripp}_{2} \mathrm{PNH}_{2}\right]$ (S06).

Die Verbindung weist eine über zwei Positionen fehlgeordnete Methylgruppe auf (C9 / C9'). Unter Verwendung von Abstands-restraints (SADI) konnte eine anisotrope Verfeinerung durchgeführt werden (Besetzungsfaktoren: 0.60 / 0.40).

Tab. 9-34: Kristallographische Daten für [Tripp $\left.{ }_{2} \mathrm{PNH}_{2}\right]$ (S06).

\begin{tabular}{|ll|ll|}
\hline Strukturcode & AJ9 & $Z$ & 2 \\
Servicepartner & A. Jana & Messtemperatur / K & $100(2)$ \\
CCDC-Nummer & 679028 & $\rho_{\text {calc } / \mathrm{Mg} \cdot \mathrm{m}^{-3}}$ & 1.067 \\
Summenformel & $\mathrm{C}_{30} \mathrm{H}_{48} \mathrm{NP}$ & $\mu / \mathrm{mm}^{-1}$ & 0.114 \\
Molmasse / g $\cdot \mathrm{mol}^{-1}$ & 453.66 & $F(000)$ & 500 \\
Kristallgröße / mm & $0.5 \times 0.45 \times 0.4$ & $\theta-$-Bereich / & 1.48 to 26.07 \\
Kristallsystem & triklin & Reflexe gesamt/unabhängig & $28805 / 5572$ \\
Raumgruppe & $P \overline{1}$ & Daten/Restraints/Parameter & $5572 / 4 / 318$ \\
$\mathrm{a} / \mathrm{pm}$ & $963.1(3)$ & $R 1[/>2 \sigma(I)]$ & 0.0466 \\
$\mathrm{~b} / \mathrm{pm}$ & $1077.9(3)$ & $w R 2($ alle Daten $)$ & 0.1281 \\
$\mathrm{c} / \mathrm{pm}$ & $1448.4(5)$ & $g 1 / \mathrm{g} 2$ & $0.0618 / 1.1681$ \\
$\alpha /{ }^{\circ}$ & $106.199(3)$ & GoF & 1.025 \\
$\beta / /^{\circ}$ & $95.848(4)$ & Differenzelektronendichte: & \\
$\gamma /{ }^{\circ}$ & $98.495(3)$ & max. / min. / (10 $\left.0^{-6} \mathrm{e} \cdot \mathrm{pm}^{-3}\right)$ & $0.593 /-0.476$ \\
$\mathrm{~V} / \mathrm{nm}^{3}$ & $1.4119(8)$ & max. / min. Transmission & $0.99 / 0.84$ \\
\hline
\end{tabular}




\subsection{7 [DippN(H) $\left.{ }^{i} \mathrm{Pr}_{2} \mathrm{P}-\mathrm{AlMe}_{3}\right](\mathrm{S} 07)$}

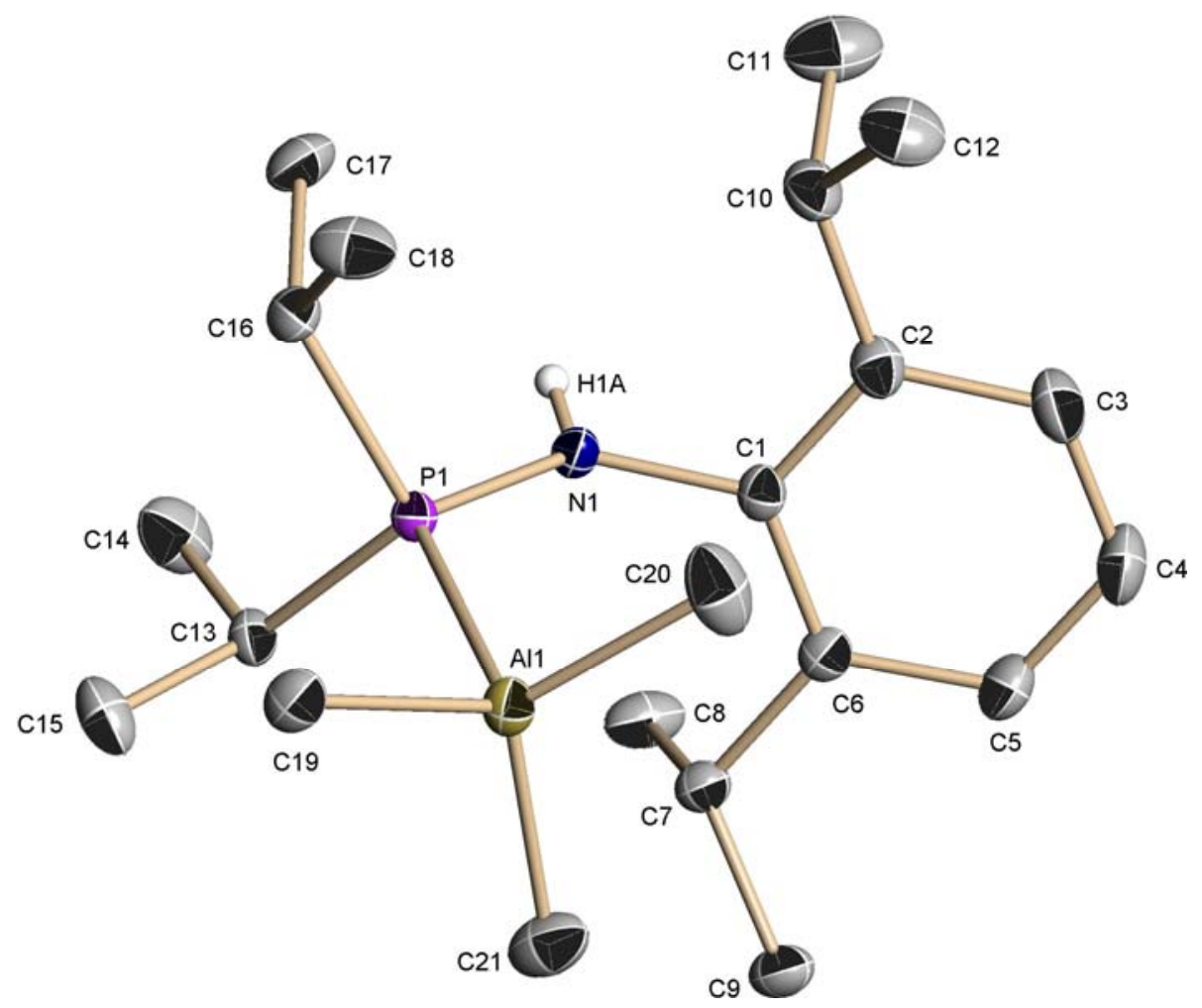

Abb. 9-34: Asymmetrische Einheit von [DippN(H) $\left.{ }^{i} \mathrm{Pr}_{2} \mathrm{P}-\mathrm{AlMe}_{3}\right]$ (S07).

Tab. 9-35: Kristallographische Daten für [DippN(H) $\left.{ }^{i} \mathrm{Pr}_{2} \mathrm{P}-\mathrm{AlMe}_{3}\right]$ (S07).

\begin{tabular}{|c|c|c|c|}
\hline Strukturcode & AJ15 & Messtemperatur / K & $100(2)$ \\
\hline Servicepartner & A. Jana & $\rho_{\text {calc }} / \mathrm{Mg} \cdot \mathrm{m}^{-3}$ & 1.055 \\
\hline CCDC-Nummer & - & $\mu / \mathrm{mm}^{-1}$ & 0.161 \\
\hline Summenformel & $\mathrm{C}_{21} \mathrm{H}_{41}$ AINP & $F(000)$ & 808 \\
\hline Molmasse $/ \mathrm{g} \cdot \mathrm{mol}^{-1}$ & 365.50 & $\theta$-Bereich / ${ }^{\circ}$ & $2.47-27.96$ \\
\hline Kristallgröße / mm & $0.2 \times 0.2 \times 0.1$ & Reflexe gesamt/unabhängig & 53652 / 5500 \\
\hline Kristallsystem & monoklin & Daten/Restraints/Parameter & $5500 / 0 / 231$ \\
\hline Raumgruppe & $P 2_{1} / c$ & $R 1[I>2 \sigma(I)]$ & 0.0321 \\
\hline $\mathrm{a} / \mathrm{pm}$ & $1735.3(2)$ & $w R 2$ (alle Daten) & 00877 \\
\hline $\mathrm{b} / \mathrm{pm}$ & $891.23(13)$ & $g 1 / g 2$ & $0.0410 / 0.8991$ \\
\hline $\mathrm{c} / \mathrm{pm}$ & $1568.6(2)$ & GoF & 1.061 \\
\hline$\beta /{ }^{\circ}$ & $108.438(2)$ & Differenzelektronendichte: & \\
\hline $\mathrm{V} / \mathrm{nm}^{3}$ & $2.3013(6)$ & $\max . / \min . /\left(10^{-6} \mathrm{e} \cdot \mathrm{pm}^{-3}\right)$ & $0.421 /-0.201$ \\
\hline Z & 4 & max. / min. Transmission & $0.99 / 0.80$ \\
\hline
\end{tabular}




\subsection{8 $\left[{ }^{t} \mathrm{Bu}_{2} \mathrm{PNSiCl}_{2}\right]_{2}(\mathrm{~S} 08)$}

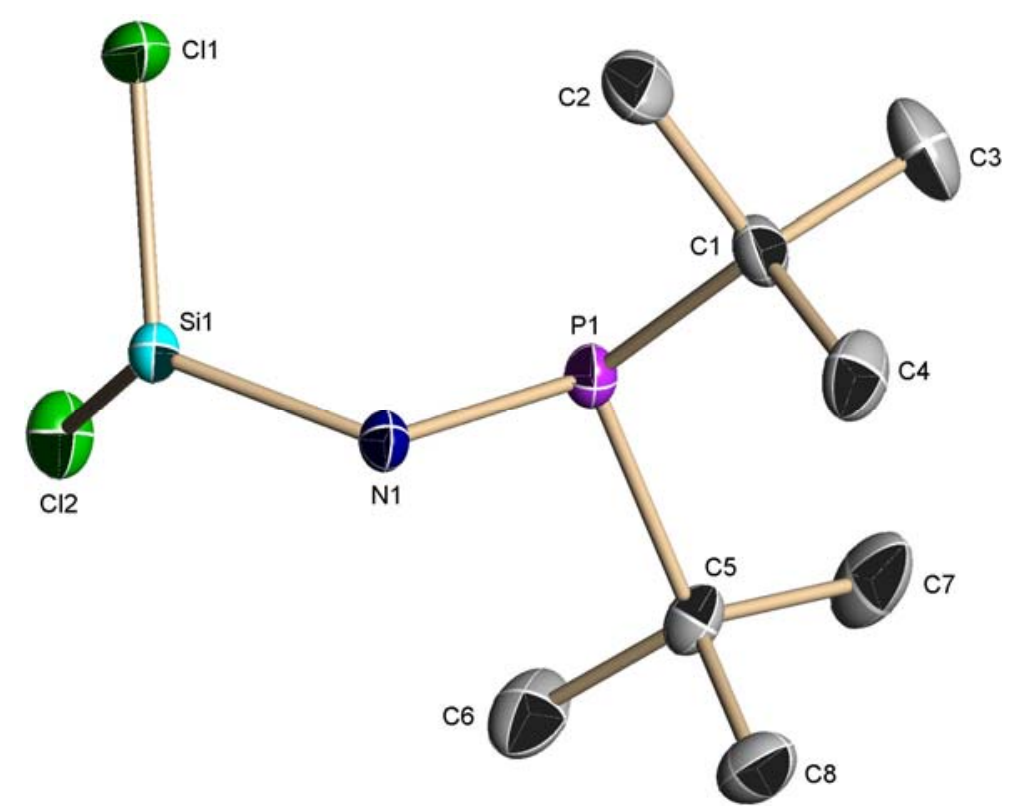

Abb. 9-35: Asymmetrische Einheit von $\left[{ }^{t} \mathrm{Bu}_{2} \mathrm{PNSiCl}_{2}\right]_{2}$ (S08).

Die asymmetrische Einheit enthält ein halbes Molekül des Dimers, die zweite Hälfte wird durch Anwendung eines Inversionszentrums erzeugt.

Tab. 9-36: Kristallographische Daten für $\left[{ }^{t} \mathrm{Bu}_{2} \mathrm{PNSiCl}_{2}\right]_{2}$ (S08).

\begin{tabular}{|c|c|c|c|}
\hline Strukturcode & AJ7 & Messtemperatur / K & $100(2)$ \\
\hline Servicepartner & A. Jana & $\rho_{\text {calc }} / \mathrm{Mg} \cdot \mathrm{m}^{-3}$ & 1.353 \\
\hline CCDC-Nummer & - & $\mu / \mathrm{mm}^{-1}$ & 0.694 \\
\hline Summenformel & $\mathrm{C}_{16} \mathrm{H}_{36} \mathrm{Cl}_{4} \mathrm{~N}_{2} \mathrm{P}_{2} \mathrm{Si}_{2}$ & $F(000)$ & 544 \\
\hline Molmasse $/ \mathrm{g} \cdot \mathrm{mol}^{-1}$ & 516.39 & $\theta$-Bereich / ${ }^{\circ}$ & $3.00-27.84$ \\
\hline Kristallgröße / mm & $0.35 \times 0.20 \times 0.15$ & Reflexe gesamt/unabhängig & 7032 / 2809 \\
\hline Kristallsystem & monoklin & Daten/Restraints/Parameter & $2809 / 0 / 124$ \\
\hline Raumgruppe & $P 2_{1} / n$ & $R 1[I>2 \sigma(I)]$ & 0.0330 \\
\hline $\mathrm{a} / \mathrm{pm}$ & $867.74(10)$ & $w R 2$ (alle Daten) & 0.0880 \\
\hline $\mathrm{b} / \mathrm{pm}$ & $1218.38(14)$ & $g 1 / g 2$ & $0.0473 / 0.6106$ \\
\hline $\mathrm{c} / \mathrm{pm}$ & $1274.11(14)$ & GoF & 1.045 \\
\hline$\beta /{ }^{\circ}$ & 109.7470(10) & Differenzelektronendichte: & \\
\hline $\mathrm{V} / \mathrm{nm}^{3}$ & $1.2678(2)$ & $\max . / \min . /\left(10^{-6} \mathrm{e} \cdot \mathrm{pm}^{-3}\right)$ & $0.841 /-0.281$ \\
\hline Z & 2 & max. / min. Transmission & $0.99 / 0.80$ \\
\hline
\end{tabular}




\subsection{9 $\left[\left({ }^{t} \mathrm{Bu}_{2} \mathrm{P}\right)_{2} \mathrm{~N}_{2} \mathrm{Li}_{2}(\text { thf })_{3} \mathrm{SiMe}_{2}\right](\mathrm{S} 09)$}
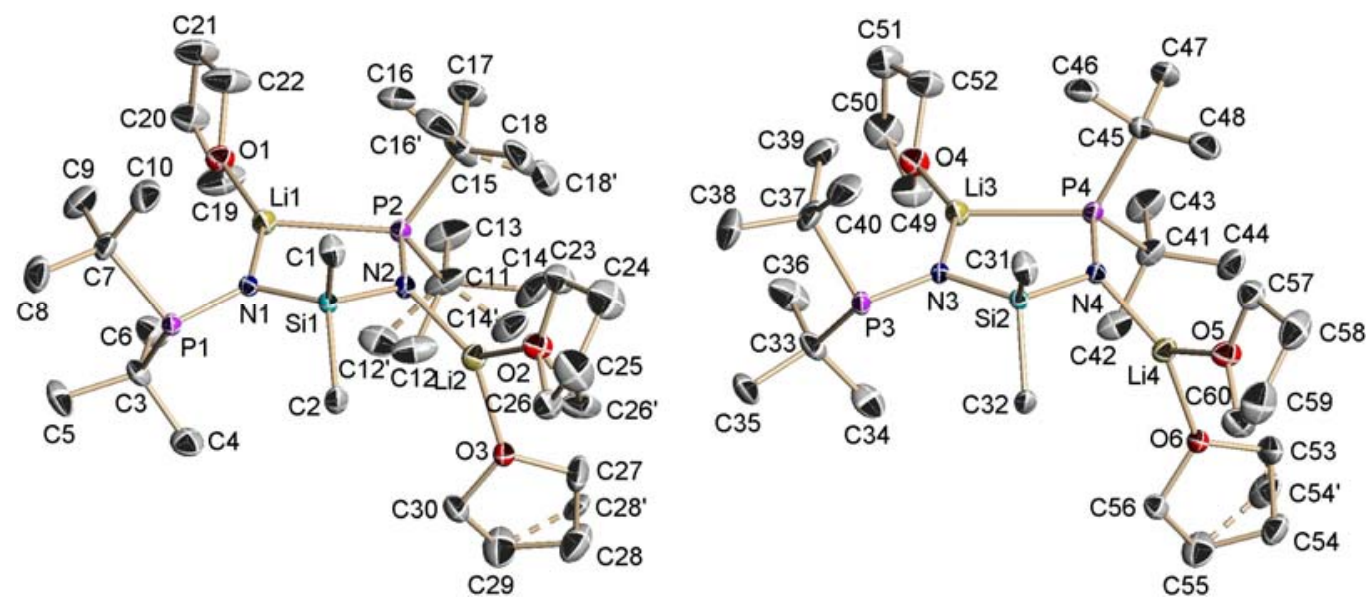

Abb. 9-36: Asymmetrische Einheit von $\left[\left({ }^{t} \mathrm{Bu}_{2} \mathrm{P}\right)_{2} \mathrm{~N}_{2} \mathrm{Li}_{2}(\text { thf })_{3} \mathrm{SiMe}_{2}\right]$ (S09).

Unter Verwendung eines Schwingungs-restraints (ISOR) konnte eine anisotrope Verfeinerung sämtlicher Atome durchgeführt werden. Die Besetzungsfaktoren verfeinerten wie folgt:

\begin{tabular}{|c|c|c|c|c|c|c|c|c|c|}
\hline C12, C14 & C12', C14' & C16, C18 & C16', C18' & $\mathrm{C} 25, \mathrm{C} 26$ & C25', C26' & C27-C29 & C27'-C29' & C53-C55 & C53'-C55' \\
\hline 0.44 & 0.56 & 0.40 & 0.60 & 0.80 & 0.20 & 0.85 & 0.15 & 0.75 & 0.25 \\
\hline
\end{tabular}

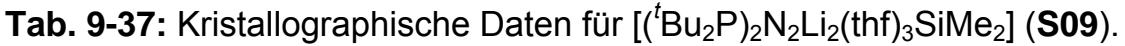

\begin{tabular}{|c|c|c|c|}
\hline Strukturcode & AJ4 & Z & 4 \\
\hline Servicepartner & A. Jana & Messtemperatur / K & $100(2)$ \\
\hline CCDC-Nummer & 679029 & $\rho_{\text {calc }} / \mathrm{Mg} \cdot \mathrm{m}^{-3}$ & 1.076 \\
\hline Summenformel & $\mathrm{C}_{30} \mathrm{H}_{66} \mathrm{Li}_{2} \mathrm{~N}_{2} \mathrm{O}_{3} \mathrm{P}_{2} \mathrm{Si}$ & $\mu / \mathrm{mm}^{-1}$ & 0.177 \\
\hline Molmasse $/ \mathrm{g} \cdot \mathrm{mol}^{-1}$ & 606.76 & $F(000)$ & 1336 \\
\hline Kristallgröße / mm & $0.4 \times 0.32 \times 0.3$ & $\theta$-Bereich / ${ }^{\circ}$ & $1.66 / 27.92$ \\
\hline Kristallsystem & triklin & Reflexe gesamt/unabhängig & $59281 / 16997$ \\
\hline Raumgruppe & $P \overline{1}$ & Daten/Restraints/Parameter & $16997 / 6 / 818$ \\
\hline $\mathrm{a} / \mathrm{pm}$ & $1175.83(6)$ & $R 1[I>2 \sigma(I)]$ & 0.0426 \\
\hline $\mathrm{b} / \mathrm{pm}$ & $1800.55(9)$ & wR2 (alle Daten) & 0.1197 \\
\hline $\mathrm{c} / \mathrm{pm}$ & $1932.88(10)$ & $g 1 / g 2$ & $0.0603 / 1.6032$ \\
\hline$\alpha /{ }^{\circ}$ & $87.7880(10)$ & GoF & 1.058 \\
\hline$\beta /{ }^{\circ}$ & $72.9230(10)$ & Differenzelektronendichte: & \\
\hline$\gamma /{ }^{\circ}$ & $73.4670(10)$ & $\max . /$ min. $/\left(10^{-6} \mathrm{e} \cdot \mathrm{pm}^{-3}\right)$ & $0.716 /-0.374$ \\
\hline $\mathrm{V} / \mathrm{nm}^{3}$ & $3.7455(3)$ & max. / min. Transmission & $0.99 / 0.94$ \\
\hline
\end{tabular}




\subsubsection{0 $\left[\mathrm{MeSi}\left\{\mathrm{NLi}\left(\mathrm{OEt}_{2}\right) \mathrm{P}^{\mathrm{t}} \mathrm{Bu}_{2}\right\}_{3}\right](\mathrm{S} 10)$}

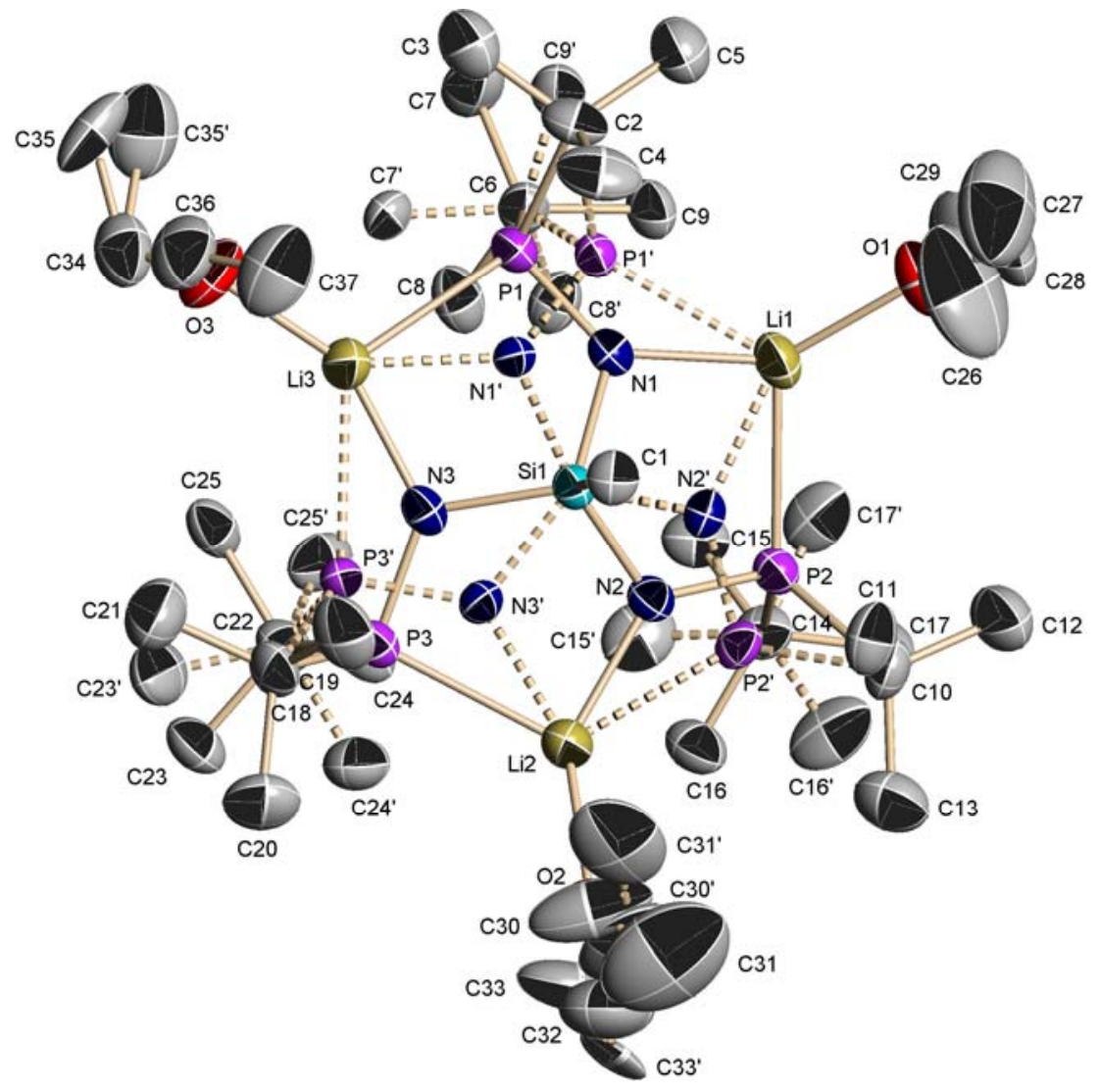

Abb. 9-37: Asymmetrische Einheit von $\left[\mathrm{MeSi}\left\{\mathrm{NLi}\left(\mathrm{OEt}_{2}\right) \mathrm{P}^{t} \mathrm{Bu}_{2}\right\}_{3}\right](\mathbf{S} 10)$.
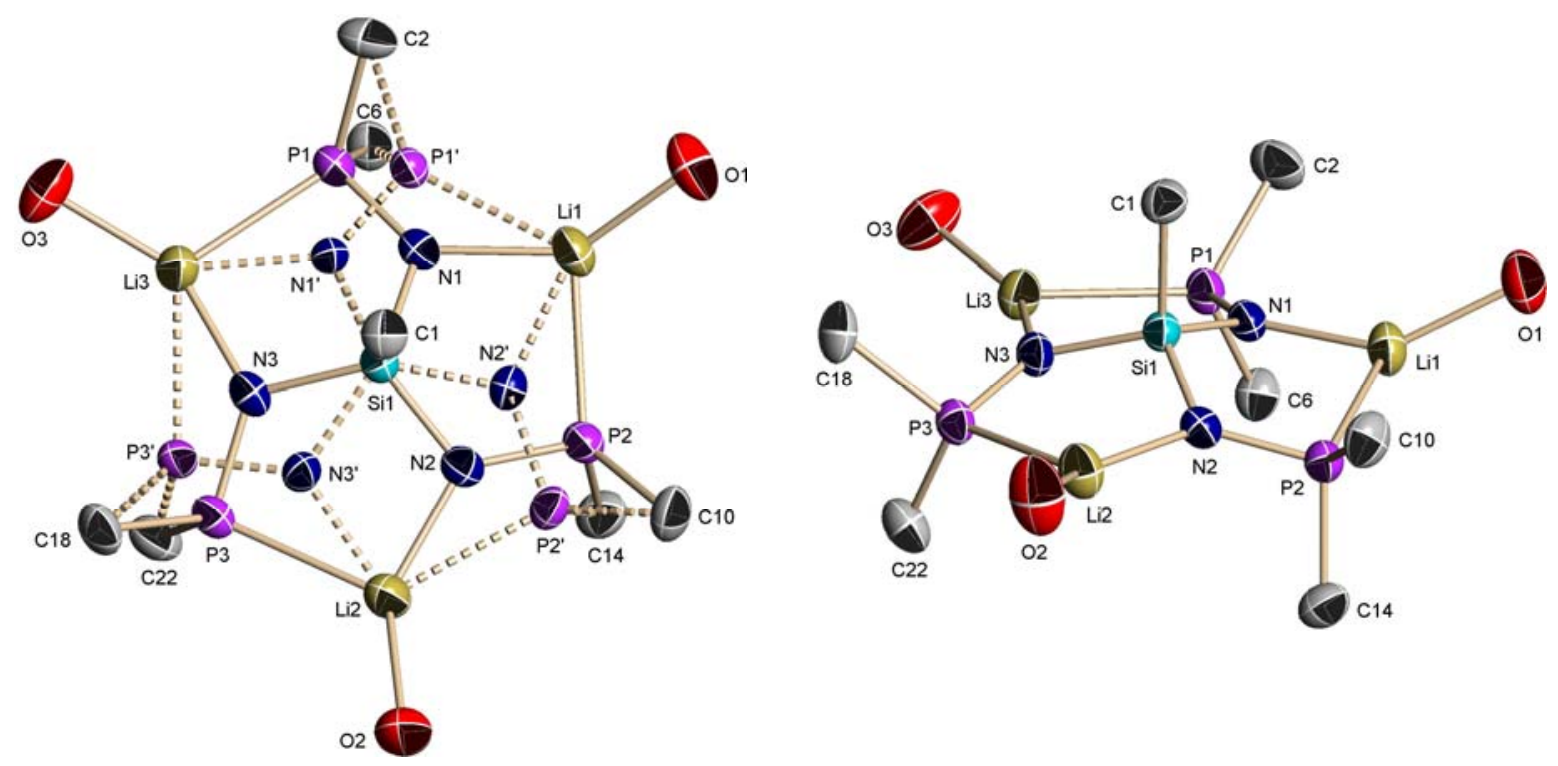

Abb. 9-38: Molekülstruktur von $\left[\mathrm{MeSi}\left\{\mathrm{NLi}\left(\text { thf }^{\mathrm{t}} \mathrm{PB}_{2}\right\}_{3}\right]\right.$ (S10),

$\mathrm{CH}_{3}\left({ }^{\mathrm{t}} \mathrm{Bu}\right)$ - und $\mathrm{OEt}_{2}-$ Kohlenstoffatome nicht dargestellt;

rechts ist außerdem nur jeweils eine Position der fehlgeordneten Atome dargestellt. 
Die Verbindung kristallisiert in der orthorhombischen, chiralen Raumgruppe $P 2_{1} 2_{1} 2_{1}$. Der Flack-Parameter ${ }^{[184,185]}$ verfeinerte zu 0.0072 mit einer Standardabweichung von 0.0972, die absolute Struktur ist somit korrekt bestimmt. Die Stickstoff- und Phosphoratome der $\mathrm{Si}(\mathrm{N}-\mathrm{P})_{3}$-Einheit sind jeweils auf zwei Positionen fehlgeordnet, die Besetzungsfaktoren verfeinerten zu 0.57 (N1, N2, N3, P1, P2, P3) und 0.43 (N1', N2', N3', P1', P2', P3'). Des Weiteren weist die Verbindung fehlgeordnete Methylund $\mathrm{OEt}_{2}-$ Gruppen auf. Mit Hilfe von Abstands- (SADI) und Schwingungs-restraints (SIMU, DELU) konnte eine anisotrope Verfeinerung sämtlicher Atome durchgeführt werden, die Besetzungsfaktoren verfeinerten wie folgt:

$\begin{array}{cccccccc}\mathrm{C} 7-\mathrm{C} 9, \mathrm{C} 15-\mathrm{C} 17, \mathrm{C} 23-\mathrm{C} 25 & \mathrm{C}^{\prime}-\mathrm{C}^{\prime}, \mathrm{C}^{\prime} 5^{\prime}-\mathrm{C} 17^{\prime}, \mathrm{C} 23^{\prime}-\mathrm{C} 25^{\prime} & \mathrm{C} 30, \mathrm{C} 31 & \mathrm{C} 30^{\prime}, \mathrm{C} 31^{\prime} & \mathrm{C} 33 & \mathrm{C} 33^{\prime} & \mathrm{C} 34, \mathrm{C} 35 & \mathrm{C} 34^{\prime}, \mathrm{C} 35^{\prime} \\ 0.57 & 0.43 & 0.39 & 0.61 & 0.81 & 0.19 & 0.45 & 0.55\end{array}$

Tab. 9-38: Kristallographische Daten für [MeSi $\left.\left\{\mathrm{NLi}\left(\mathrm{OEt}_{2}\right) \mathrm{P}^{t} \mathrm{Bu}_{2}\right\}_{3}\right]$ (S10).

\begin{tabular}{|c|c|c|c|}
\hline Strukturcode & Jana1-AJ3 & Messtemperatur / K & $100(2)$ \\
\hline Servicepartner & A. Jana & $\rho_{\text {calc }} / \mathrm{Mg} \cdot \mathrm{m}^{-3}$ & 1.038 \\
\hline CCDC-Nummer & 668449 & $\mu / \mathrm{mm}^{-1}$ & 0.179 \\
\hline Summenformel & $\mathrm{C}_{37} \mathrm{H}_{87} \mathrm{Li}_{3} \mathrm{~N}_{3} \mathrm{O}_{3} \mathrm{P}_{3} \mathrm{Si}$ & $F(000)$ & 1688 \\
\hline Molmasse $/ \mathrm{g} \cdot \mathrm{mol}^{-1}$ & 763.92 & $\theta$-Bereich / ${ }^{\circ}$ & $1.43-26.02$ \\
\hline Kristallgröße / mm & $0.4 \times 0.2 \times 0.2$ & Reflexe gesamt/unabhängig & 46209 / 9632 \\
\hline Kristallsystem & orthorhombisch & Daten/Restraints/Parameter & 9632 / 44 / 662 \\
\hline Raumgruppe & $P 2_{1} 2_{1} 2_{1}$ & $R 1[I>2 \sigma(I)]$ & 0.0492 \\
\hline $\mathrm{a} / \mathrm{pm}$ & $1113.71(7)$ & $w R 2$ (alle Daten) & 0.1573 \\
\hline $\mathrm{b} / \mathrm{pm}$ & $1700.11(10)$ & $g 1 / g 2$ & $0.1150 / 0.3242$ \\
\hline $\mathrm{c} / \mathrm{pm}$ & $2582.04(16)$ & GoF & 1.088 \\
\hline Flack $x$ & $0.0072(972)$ & Differenzelektronendichte: & \\
\hline $\mathrm{V} / \mathrm{nm}^{3}$ & $4.8889(5)$ & $\max . / \min . /\left(10^{-6} \mathrm{e} \cdot \mathrm{pm}^{-3}\right)$ & $0.503 /-0.607$ \\
\hline Z & 4 & max. / min. Transmission & $0.99 / 0.92$ \\
\hline
\end{tabular}




\subsubsection{1 $\left[\mathrm{Ph}_{2} \mathrm{P}\left\{\mathrm{N}(\mathrm{H}) \mathrm{SiMe}_{3}\right\}_{2}\right]^{+}\left[\mathrm{Bi}_{2} \mathrm{Cl}_{8}(\text { thf })_{2}\right]^{-}(\mathrm{S} 11)$}

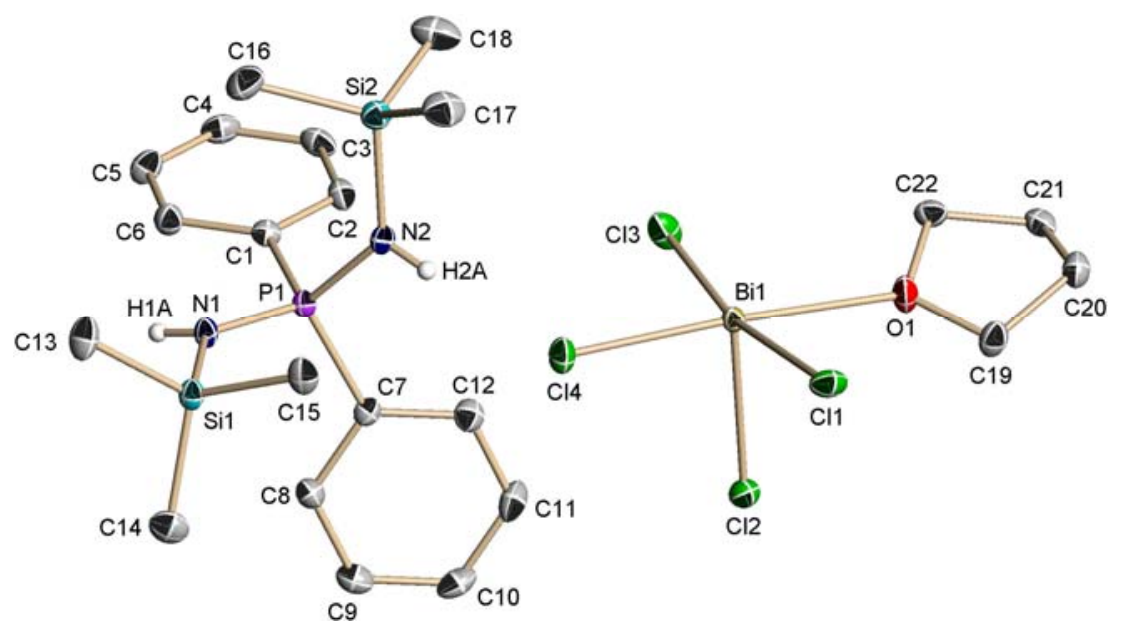

Abb. 9-39: Asymmetrische Einheit von $\left[\mathrm{Ph}_{2} \mathrm{P}\left\{\mathrm{N}(\mathrm{H}) \mathrm{SiMe}_{3}\right\}_{2}\right]^{+}\left[\mathrm{Bi}_{2} \mathrm{Cl}_{8}(\text { thf })_{2}\right]^{-}(\mathrm{S} 11)$.

Die asymmetrische Einheit enthält neben dem Phosphonium-Kation die Hälfte des bismuthaltigen Gegenions. Der Rest des Anions wird durch Anwendung eines Inversionszentrums erzeugt (rechts).

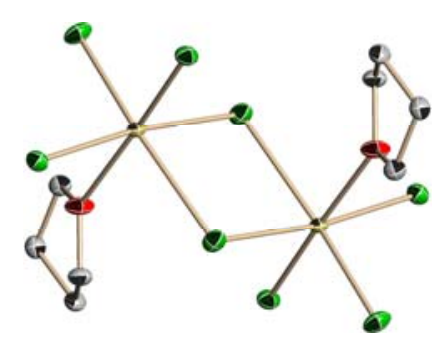

Tab. 9-39: Kristallographische Daten für $\left[\mathrm{Ph}_{2} \mathrm{P}\left\{\mathrm{N}(\mathrm{H}) \mathrm{SiMe}_{3}\right\}_{2}\right]^{+}\left[\mathrm{Bi}_{2} \mathrm{Cl}_{8}(\text { thf })_{2}\right]^{-}$(S11).

\begin{tabular}{|c|c|c|c|}
\hline Strukturcode & BN02 & Messtemperatur / K & $100(2)$ \\
\hline Servicepartner & N. Bijan & $\rho_{\text {calc }} / \mathrm{Mg} \cdot \mathrm{m}^{-3}$ & 1.664 \\
\hline CCDC-Nummer & - & $\mu / \mathrm{mm}^{-1}$ & 6.117 \\
\hline Summenformel & $\mathrm{C}_{22} \mathrm{H}_{38} \mathrm{BiCl}_{4} \mathrm{~N}_{2} \mathrm{OPSi}_{2}$ & $F(000)$ & 1544 \\
\hline Molmasse $/ \mathrm{g} \cdot \mathrm{mol}^{-1}$ & 784.47 & $\theta$-Bereich $/^{\circ}$ & $2.32-27.89$ \\
\hline Kristallgröße / mm & $0.15 \times 0.10 \times 0.10$ & Reflexe gesamt/unabhängig & $33581 / 7250$ \\
\hline Kristallsystem & monoklin & Daten/Restraints/Parameter & $7250 / 0 / 306$ \\
\hline Raumgruppe & $P 2_{1} / n$ & $R 1[I>2 \sigma(I)]$ & 0.0187 \\
\hline $\mathrm{a} / \mathrm{pm}$ & $1020.48(5)$ & $w R 2$ (alle Daten) & 0.0382 \\
\hline $\mathrm{b} / \mathrm{pm}$ & $1748.49(9)$ & $g 1 / g 2$ & $0.0163 / 1.6415$ \\
\hline $\mathrm{c} / \mathrm{pm}$ & $1761.40(9)$ & GoF & 1.024 \\
\hline$\beta /^{\circ}$ & $94.8160(10)$ & Differenzelektronendichte: & \\
\hline $\mathrm{V} / \mathrm{nm}^{3}$ & $3.1318(3)$ & $\max . / \min . /\left(10^{-6} \mathrm{e} \cdot \mathrm{pm}^{-3}\right)$ & $0.547 /-0.417$ \\
\hline Z & 4 & max. / min. Transmission & $0.99 / 0.81$ \\
\hline
\end{tabular}




\subsubsection{2 $\left[\mathrm{Ph}_{2} \mathrm{P}\left(\mu-\mathrm{NSiMe}_{3}\right)_{2} \mathrm{AlCl}_{2}\right](\mathrm{S} 12)$}
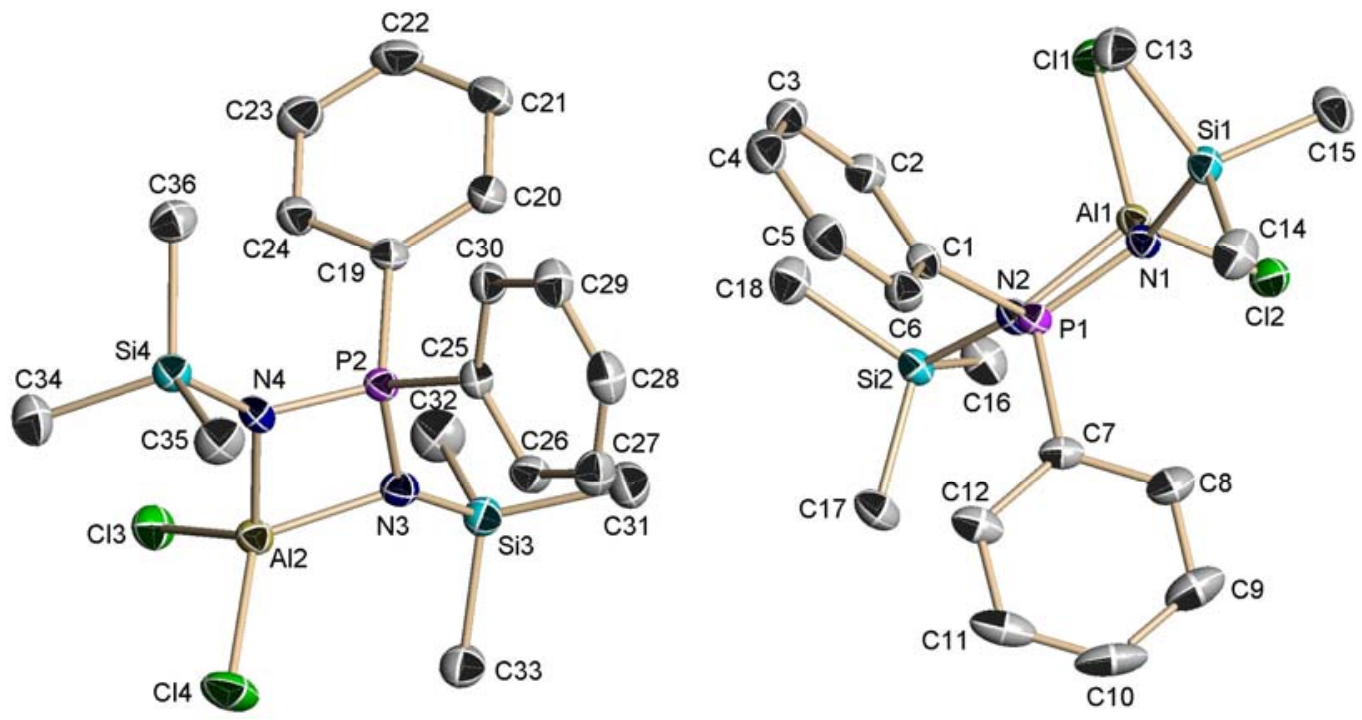

Abb. 9-40: Asymmetrische Einheit von $\left[\mathrm{Ph}_{2} \mathrm{P}\left(\mu-\mathrm{NSiMe}_{3}\right)_{2} \mathrm{AlCl}_{2}\right]$ (S12).

Tab. 9-40: Kristallographische Daten für $\left[\mathrm{Ph}_{2} \mathrm{P}\left(\mu-\mathrm{NSiMe}_{3}\right)_{2} \mathrm{AlCl}_{2}\right]$ (S12).

\begin{tabular}{|c|c|c|c|}
\hline Strukturcode & BN05 & Z & 4 \\
\hline Servicepartner & N. Bijan & Messtemperatur / K & $100(2)$ \\
\hline CCDC-Nummer & 681331 & $\rho_{\text {calc }} / \mathrm{Mg} \cdot \mathrm{m}^{-3}$ & 1.248 \\
\hline Summenformel & $\mathrm{C}_{18} \mathrm{H}_{28} \mathrm{AlCl}_{2} \mathrm{~N}_{2} \mathrm{PSi}_{2}$ & $\mu / \mathrm{mm}^{-1}$ & 0.473 \\
\hline Molmasse $/ \mathrm{g} \cdot \mathrm{mol}^{-1}$ & 457.45 & $F(000)$ & 960 \\
\hline Kristallgröße / mm & $0.2 \times 0.2 \times 0.15$ & $\theta$-Bereich $/^{\circ}$ & $2.68-26.36$ \\
\hline Kristallsystem & triklin & Reflexe gesamt/unabhängig & $53683 / 9866$ \\
\hline Raumgruppe & $P \overline{1}$ & Daten/Restraints/Parameter & 9866 / 0 / 481 \\
\hline $\mathrm{a} / \mathrm{pm}$ & $984.45(7)$ & $R 1[I>2 \sigma(I)]$ & 0.0341 \\
\hline $\mathrm{b} / \mathrm{pm}$ & $1012.46(7)$ & $w R 2$ (alle Daten) & 0.0771 \\
\hline $\mathrm{c} / \mathrm{pm}$ & $2809.0(2)$ & $g 1 / g 2$ & $0.0252 / 2.0663$ \\
\hline$\alpha /^{\circ}$ & $81.2820(10)$ & GoF & 1.057 \\
\hline$\beta /{ }^{\circ}$ & $89.0870(10)$ & Differenzelektronendichte: & \\
\hline$\gamma /{ }^{\circ}$ & $61.8080(10)$ & $\max . / \min . /\left(10^{-6} \mathrm{e} \cdot \mathrm{pm}^{-3}\right)$ & $0.660 /-0.326$ \\
\hline $\mathrm{V} / \mathrm{nm}^{3}$ & $2.4342(3)$ & max. / min. Transmission & $0.99 / 0.90$ \\
\hline
\end{tabular}




\subsubsection{3 $\left[\mathrm{Ph}_{2} \mathrm{P}\left(\mu-\mathrm{NSiMe}_{3}\right)_{2} \mathrm{Al}(\mathrm{Cl}) \mathrm{Me} \cdot 0.5 \mathrm{C}_{7} \mathrm{H}_{8}\right](\mathrm{S} 13)$}

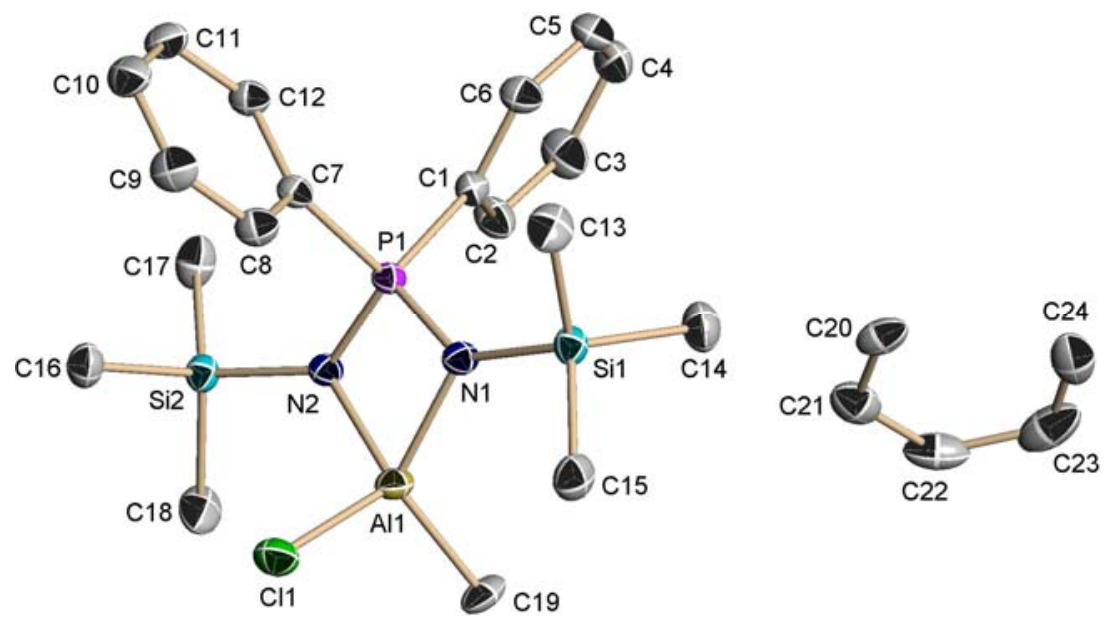

Abb. 9-41: Asymmetrische Einheit von $\left[\mathrm{Ph}{ }_{2} \mathrm{P}\left(\mu-\mathrm{NSiMe}_{3}\right)_{2} \mathrm{Al}(\mathrm{Cl}) \mathrm{Me} \cdot 0.5 \mathrm{C}_{7} \mathrm{H}_{8}\right]$ (S13).

Die asymmetrische Einheit enthält neben einem Molekül des Aluminiumkomplexes noch die Hälfte eines fehlgeordneten Toluolmoleküls. Es konnte eine anisotrope Verfeinerung durchgeführt werden, indem die Besetzungsfaktoren von C20, C22 und C24 jeweils auf 0.5 gesetzt wurden (Abb. rechts: Toluolmolekül nach Anwendung des Inversionszentrums).

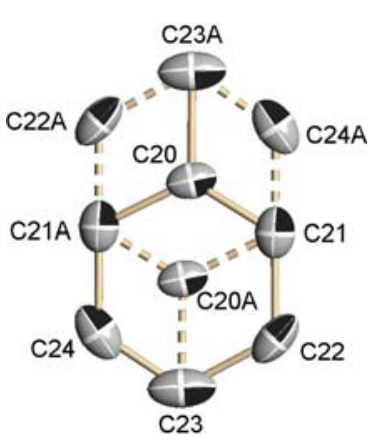

Tab. 9-41: Kristallographische Daten für $\left[\mathrm{Ph}_{2} \mathrm{P}\left(\mu-\mathrm{NSiMe}_{3}\right)_{2} \mathrm{Al}(\mathrm{Cl}) \mathrm{Me} \cdot 0.5 \mathrm{C}_{7} \mathrm{H}_{8}\right](\mathrm{S} 13)$.

\begin{tabular}{|c|c|c|c|}
\hline Strukturcode & BN06 & Messtemperatur / K & $100(2)$ \\
\hline Servicepartner & N. Bijan & $\rho_{\text {calc }} / \mathrm{Mg} \cdot \mathrm{m}^{-3}$ & 1.171 \\
\hline CCDC-Nummer & 681332 & $\mu / \mathrm{mm}^{-1}$ & 0.329 \\
\hline Summenformel & $\mathrm{C}_{22.50} \mathrm{H}_{35} \mathrm{AlCIN}_{2} \mathrm{PSi}_{2}$ & $F(000)$ & 1028 \\
\hline Molmasse $/ \mathrm{g} \cdot \mathrm{mol}^{-1}$ & 483.11 & $\theta$-Bereich ${ }^{\circ}$ & $1.74-26.05$ \\
\hline Kristallgröße / mm & $0.15 \times 0.15 \times 0.1$ & Reflexe gesamt/unabhängig & $52109 / 5405$ \\
\hline Kristallsystem & monoklin & Daten/Restraints/Parameter & $5405 / 0 / 288$ \\
\hline Raumgruppe & $P 2_{1} / c$ & $R 1[I>2 \sigma(I)]$ & 0.0298 \\
\hline $\mathrm{a} / \mathrm{pm}$ & $1086.67(10)$ & $w R 2$ (alle Daten) & 0.0818 \\
\hline $\mathrm{b} / \mathrm{pm}$ & $1080.34(10)$ & $g 1 / g 2$ & $0.0417 / 1.4381$ \\
\hline $\mathrm{c} / \mathrm{pm}$ & $2391.5(2)$ & GoF & 1.068 \\
\hline$\beta /{ }^{\circ}$ & $102.5070(10)$ & Differenzelektronendichte: & \\
\hline $\mathrm{V} / \mathrm{nm}^{3}$ & $2.7409(4)$ & $\max . / \min . /\left(10^{-6} \mathrm{e} \cdot \mathrm{pm}^{-3}\right)$ & $0.409 /-0.364$ \\
\hline Z & 4 & max. / min. Transmission & $0.99 / 0.90$ \\
\hline
\end{tabular}




\subsubsection{4 $\left[\left\{\mathrm{Ph}_{2} \mathrm{P}\left(\mu-\mathrm{NSiMe}_{3}\right)_{2}\right\}_{2} \mathrm{AlH} \cdot \mathrm{THF}\right](\mathrm{S} 14)$}
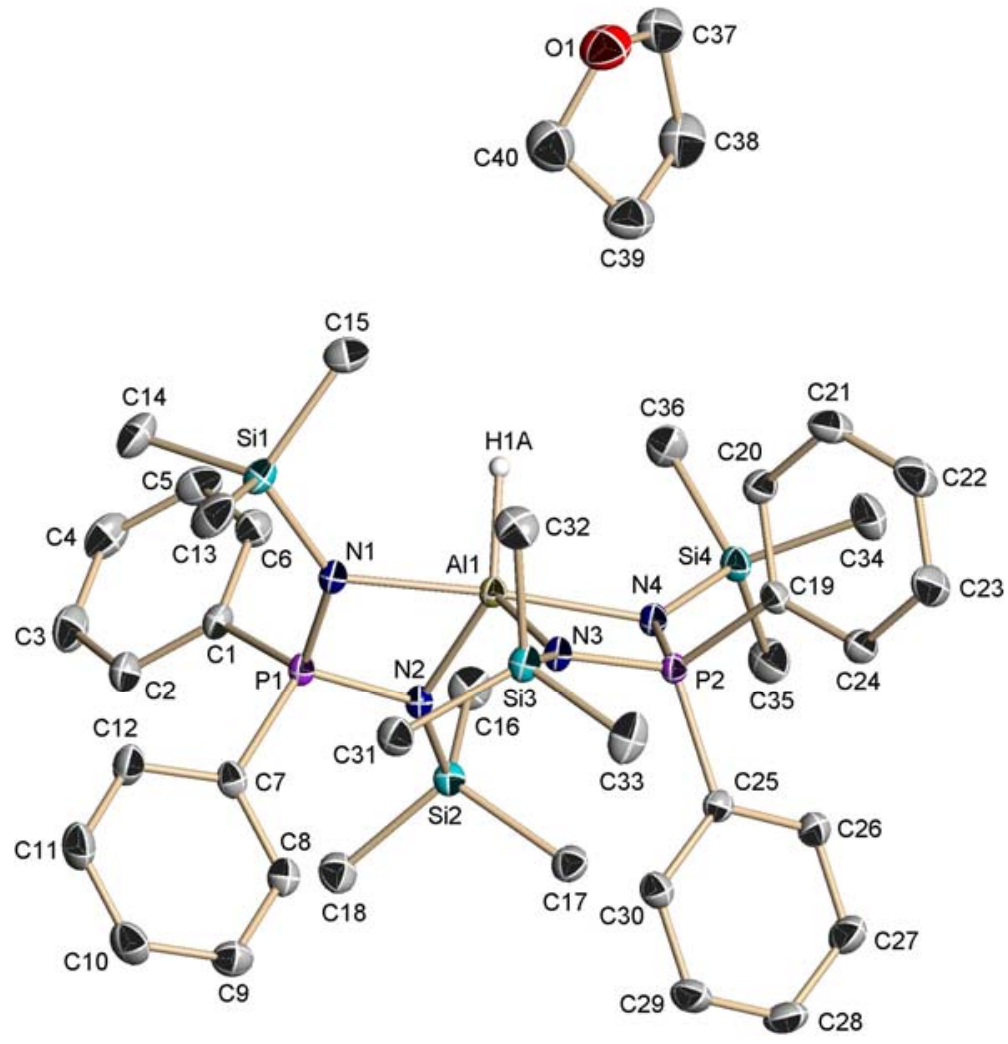

9-42: Asymmetrische Einheit von $\left[\left\{\mathrm{Ph}_{2} \mathrm{P}\left(\mu-\mathrm{NSiMe}_{3}\right)_{2}\right\}_{2} \mathrm{AlH} \cdot \mathrm{THF}\right](\mathbf{S 1 4})$.

Tab. 9-42: Kristallographische Daten für $\left[\left\{\mathrm{Ph}_{2} \mathrm{P}(\mu-\mathrm{NSiMe})_{2}\right\}_{2} \mathrm{AlH} \cdot \mathrm{THF}\right](\mathrm{S} 14)$.

\begin{tabular}{|c|c|c|c|}
\hline Strukturcode & BN07 & Messtemperatur / K & $100(2)$ \\
\hline Servicepartner & N. Bijan & $\rho_{\text {calc }} / \mathrm{Mg} \cdot \mathrm{m}^{-3}$ & 1.161 \\
\hline CCDC-Nummer & 681333 & $\mu / \mathrm{mm}^{-1}$ & 0.248 \\
\hline Summenformel & $\mathrm{C}_{40} \mathrm{H}_{65} \mathrm{AIN}_{4} \mathrm{OP}_{2} \mathrm{Si}_{4}$ & $F(000)$ & 1760 \\
\hline Molmasse $/ \mathrm{g} \cdot \mathrm{mol}^{-1}$ & 819.24 & $\theta$-Bereich / ${ }^{\circ}$ & $1.50-26.74$ \\
\hline Kristallgröße / mm & $0.3 \times 0.25 \times 0.2$ & Reflexe gesamt/unabhängig & 41456 / 9929 \\
\hline Kristallsystem & monoklin & Daten/Restraints/Parameter & 9929 / 0 / 484 \\
\hline Raumgruppe & $P 2_{1} / n$ & $R 1[I>2 \sigma(I)]$ & 0.0318 \\
\hline $\mathrm{a} / \mathrm{pm}$ & $1276.03(12)$ & $w R 2$ (alle Daten) & 0.0842 \\
\hline $\mathrm{b} / \mathrm{pm}$ & $1902.26(18)$ & $g 1 / g 2$ & $0.0400 / 2.1930$ \\
\hline $\mathrm{c} / \mathrm{pm}$ & $1941.2(2)$ & GoF & 1.047 \\
\hline$\beta /^{\circ}$ & $96.103(2)$ & Differenzelektronendichte: & \\
\hline $\mathrm{V} / \mathrm{nm}^{3}$ & $4.6853(8)$ & $\max . / \min . /\left(10^{-6} \mathrm{e} \cdot \mathrm{pm}^{-3}\right)$ & $0.441 /-0.254$ \\
\hline Z & 4 & max. / min. Transmission & $0.99 / 0.89$ \\
\hline
\end{tabular}




\subsubsection{5 $\left[\mathrm{CH}\{\mu-\mathrm{C}(\mathrm{Me}) \mathrm{N}(\mathrm{Dipp})\}_{2} \mathrm{GeBr}\right](\mathrm{S} 15)$}

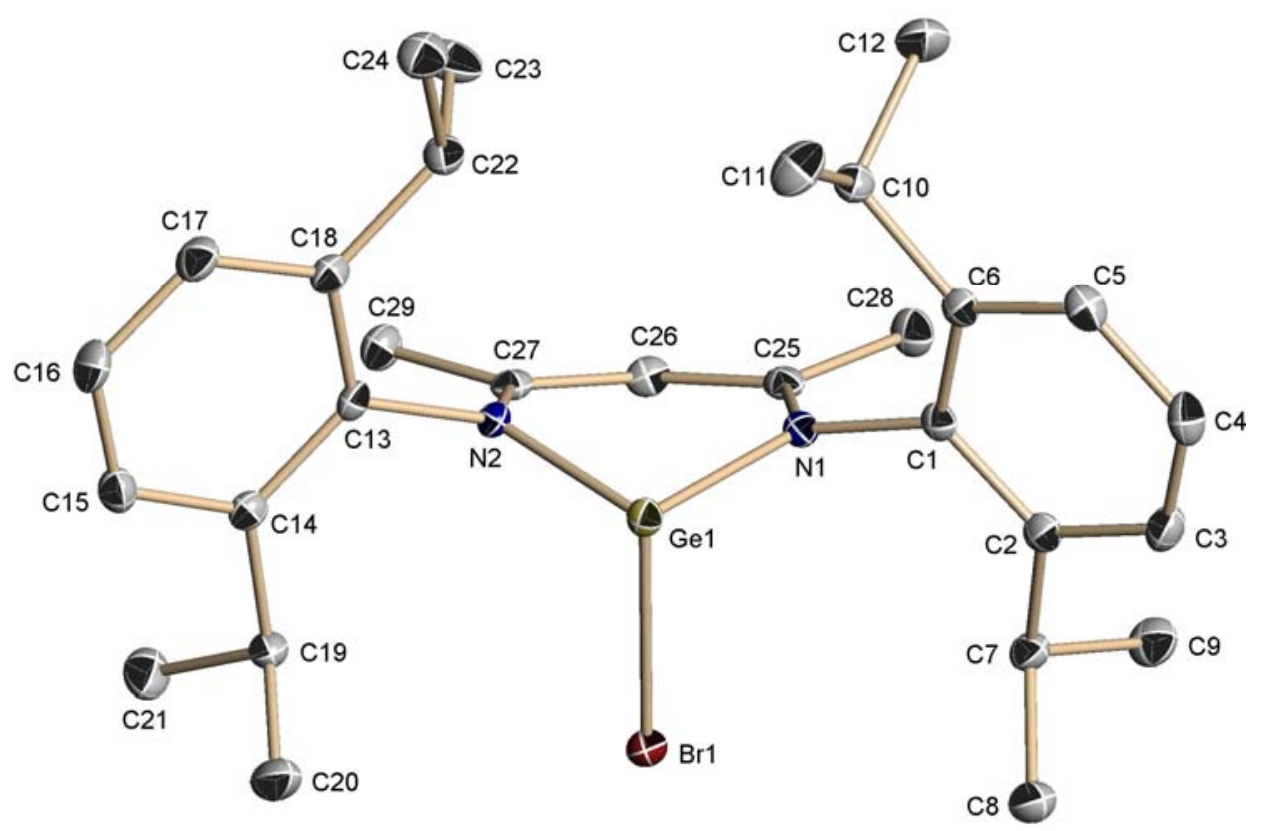

Abb. 9-43: Asymmetrische Einheit von $\left[\mathrm{CH}\{\mu-\mathrm{C}(\mathrm{Me}) \mathrm{N}(\mathrm{Dipp})\}_{2} \mathrm{GeBr}\right]$ (S15).

Tab. 9-43: Kristallographische Daten für $\left[\mathrm{CH}\{\mu-\mathrm{C}(\mathrm{Me}) \mathrm{N}(\mathrm{Dipp})\}_{2} \mathrm{GeBr}\right]$ (S15).

\begin{tabular}{|c|c|c|c|}
\hline Strukturcode & AJ17 & Z & 2 \\
\hline Servicepartner & A. Jana & Messtemperatur / K & $100(2)$ \\
\hline CCDC-Nummer & - & $\rho_{\text {calc }} / \mathrm{Mg} \cdot \mathrm{m}^{-3}$ & 1.388 \\
\hline Summenformel & $\mathrm{C}_{29} \mathrm{H}_{41} \mathrm{BrGeN}_{2}$ & $\mu / \mathrm{mm}^{-1}$ & 2.606 \\
\hline Molmasse $/ \mathrm{g} \cdot \mathrm{mol}^{-1}$ & 570.14 & $F(000)$ & 592 \\
\hline Kristallgröße / mm & $0.3 \times 0.3 \times 0.1$ & $\theta$-Bereich / ${ }^{\circ}$ & $1.76-26.37$ \\
\hline Kristallsystem & triklin & Reflexe gesamt/unabhängig & $32510 / 5574$ \\
\hline Raumgruppe & $P \overline{1}$ & Daten/Restraints/Parameter & 5574 / 0 / 308 \\
\hline $\mathrm{a} / \mathrm{pm}$ & $1026.07(7)$ & $R 1[I>2 \sigma(I)]$ & 0.0213 \\
\hline $\mathrm{b} / \mathrm{pm}$ & 1221.77(9) & $w R 2$ (alle Daten) & 0.0557 \\
\hline $\mathrm{c} / \mathrm{pm}$ & $1223.14(9)$ & $g 1 / g 2$ & $0.0251 / 1.2861$ \\
\hline$\alpha /^{\circ}$ & $88.5760(10)$ & GoF & 1.046 \\
\hline$\beta /{ }^{\circ}$ & $69.8670(10)$ & Differenzelektronendichte: & \\
\hline$\gamma /{ }^{\circ}$ & $72.0860(10)$ & $\max . / \min . /\left(10^{-6} \mathrm{e} \cdot \mathrm{pm}^{-3}\right)$ & $0.424 /-0.287$ \\
\hline $\mathrm{V} / \mathrm{nm}^{3}$ & $1.36422(17)$ & max. / min. Transmission & $0.99 / 0.83$ \\
\hline
\end{tabular}




\subsubsection{6 [CH $\left.\{\mu-\mathrm{C}(\mathrm{Me}) \mathrm{N}(\mathrm{Dipp})\}_{2} \mathrm{GeCl}\right](\mathrm{S} 16)$}

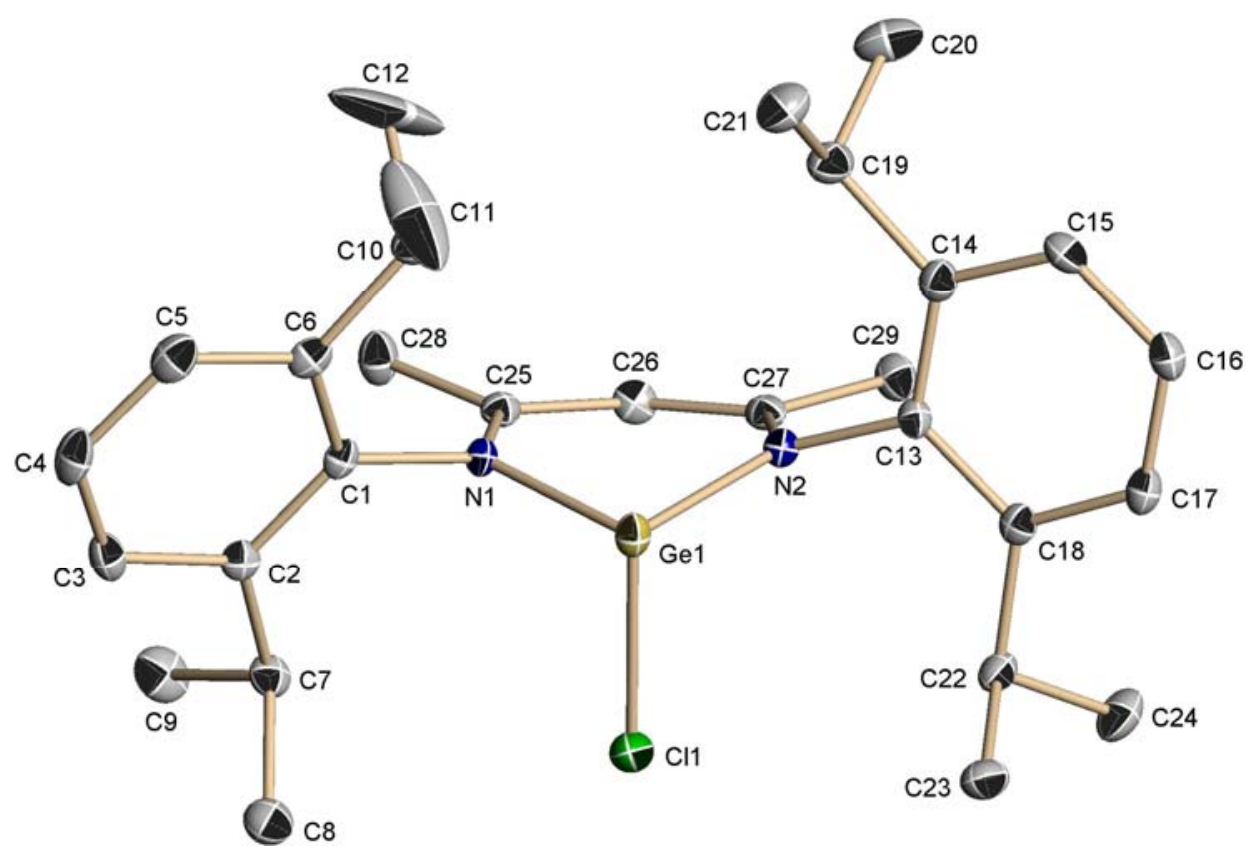

Abb. 9-44: Asymmetrische Einheit von $\left[\mathrm{CH}\{\mu-\mathrm{C}(\mathrm{Me}) \mathrm{N}(\mathrm{Dipp})\}_{2} \mathrm{GeCl}\right]$ (S16).

Tab. 9-44: Asymmetrische Einheit von $\left[\mathrm{CH}\{\mu-\mathrm{C}(\mathrm{Me}) \mathrm{N}(\mathrm{Dipp})\}_{2} \mathrm{GeCl}\right]$ (S16).

\begin{tabular}{|c|c|c|c|}
\hline Strukturcode & AJ22 & Messtemperatur / K & $100(2)$ \\
\hline Servicepartner & A. Jana & $\rho_{\text {calc }} / \mathrm{Mg} \cdot \mathrm{m}^{-3}$ & 1.250 \\
\hline CCDC-Nummer & - & $\mu / \mathrm{mm}^{-1}$ & 1.211 \\
\hline Summenformel & $\mathrm{C}_{29} \mathrm{H}_{41} \mathrm{ClGeN}_{2}$ & $F(000)$ & 1112 \\
\hline Molmasse $/ \mathrm{g} \cdot \mathrm{mol}^{-1}$ & 525.68 & $\theta$-Bereich / ${ }^{\circ}$ & $2.00-28.02$ \\
\hline Kristallgröße / mm & $0.3 \times 0.2 \times 0.15$ & Reflexe gesamt/unabhängig & 12002 / 6087 \\
\hline Kristallsystem & monoklin & Daten/Restraints/Parameter & $6087 / 0 / 308$ \\
\hline Raumgruppe & $P 2_{1} / c$ & $R 1[I>2 \sigma(I)]$ & 0.0436 \\
\hline $\mathrm{a} / \mathrm{pm}$ & $1712.5(6)$ & $w R 2$ (alle Daten) & 0.1176 \\
\hline $\mathrm{b} / \mathrm{pm}$ & $1314.8(5)$ & $g 1 / g 2$ & $0.0554 / 0.0000$ \\
\hline $\mathrm{c} / \mathrm{pm}$ & $1311.4(4)$ & GoF & 1.062 \\
\hline$\beta /{ }^{\circ}$ & $108.950(5)$ & Differenzelektronendichte: & \\
\hline $\mathrm{V} / \mathrm{nm}^{3}$ & $2.7927(17)$ & $\max . / \min . /\left(10^{-6} \mathrm{e} \cdot \mathrm{pm}^{-3}\right)$ & $0.617 /-0.439$ \\
\hline Z & 4 & max. / min. Transmission & $0.99 / 0.52$ \\
\hline
\end{tabular}




\subsubsection{7 $\left[\mathrm{CH}\{\mu-\mathrm{C}(\mathrm{Me}) \mathrm{N}(\mathrm{Dipp})\}_{2} \mathrm{Ge}(\mathrm{S}) \mathrm{SH} \cdot \mathrm{C}_{7} \mathrm{H}_{8}\right](\mathrm{S} 17)$}
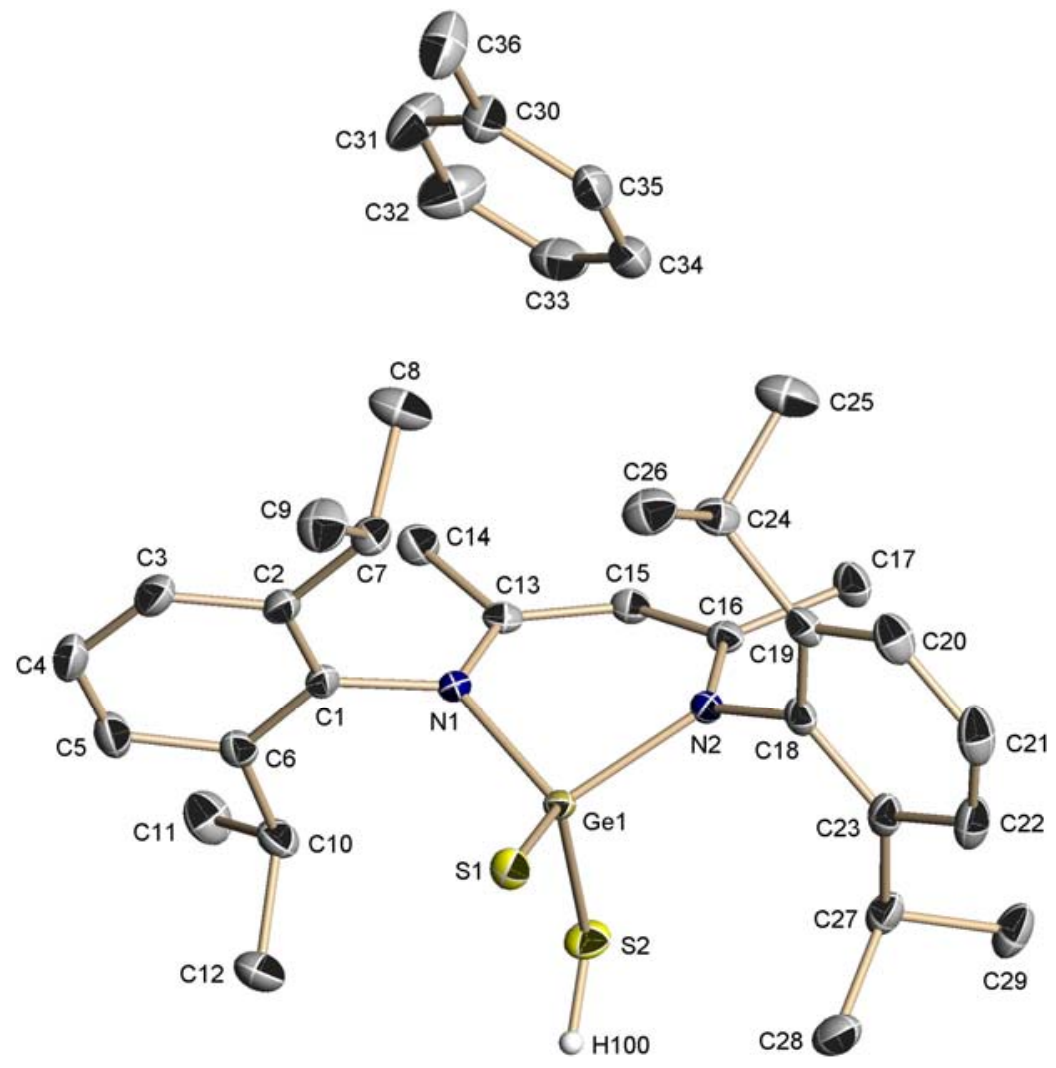

Abb. 9-45: Asymmetrische Einheit von $\left[\mathrm{CH}\{\mu-\mathrm{C}(\mathrm{Me}) \mathrm{N}(\mathrm{Dipp})\}_{2} \mathrm{Ge}(\mathrm{S}) \mathrm{SH} \cdot \mathrm{C}_{7} \mathrm{H}_{8}\right]$ (S17).

Tab. 9-45: Kristallographische Daten für $\left[\mathrm{CH}\{\mu-\mathrm{C}(\mathrm{Me}) \mathrm{N}(\mathrm{Dipp})\}_{2} \mathrm{Ge}(\mathrm{S}) \mathrm{SH} \cdot \mathrm{C}_{7} \mathrm{H}_{8}\right](\mathrm{S} 17)$.

\begin{tabular}{|c|c|c|c|}
\hline Strukturcode & AJ12 & Messtemperatur / K & $100(2)$ \\
\hline Servicepartner & A. Jana & $\rho_{\text {calc }} / \mathrm{Mg} \cdot \mathrm{m}^{-3}$ & 1.242 \\
\hline CCDC-Nummer & 667925 & $\mu / \mathrm{mm}^{-1}$ & 1.031 \\
\hline Summenformel & $\mathrm{C}_{36} \mathrm{H}_{50} \mathrm{GeN}_{2} \mathrm{~S}_{2}$ & $F(000)$ & 1376 \\
\hline Molmasse $/ \mathrm{g} \cdot \mathrm{mol}^{-1}$ & 647.49 & $\theta$-Bereich $/^{\circ}$ & $1.75-27.90$ \\
\hline Kristallgröße / mm & $0.2 \times 0.2 \times 0.1$ & Reflexe gesamt/unabhängig & 65843 / 8257 \\
\hline Kristallsystem & monoklin & Daten/Restraints/Parameter & $8257 / 0 / 377$ \\
\hline Raumgruppe & $P 2_{1} / n$ & $R 1[I>2 \sigma(I)]$ & 0.0238 \\
\hline $\mathrm{a} / \mathrm{pm}$ & $1274.26(10)$ & $w R 2$ (alle Daten) & 0.0642 \\
\hline $\mathrm{b} / \mathrm{pm}$ & $1565.50(12)$ & $g 1 / g 2$ & $0.0290 / 2.1773$ \\
\hline $\mathrm{c} / \mathrm{pm}$ & $1809.31(14)$ & GoF & 1.056 \\
\hline$\beta /{ }^{\circ}$ & $106.3350(10)$ & Differenzelektronendichte: & \\
\hline $\mathrm{V} / \mathrm{nm}^{3}$ & $3.4636(5)$ & $\max . / \min . /\left(10^{-6} \mathrm{e} \cdot \mathrm{pm}^{-3}\right)$ & $0.520 /-0.519$ \\
\hline Z & 4 & max. / min. Transmission & $0.99 / 0.89$ \\
\hline
\end{tabular}




\subsubsection{8 $\left[\mathrm{CH}\{\mu-\mathrm{C}(\mathrm{Me}) \mathrm{N}(\mathrm{Dipp})\}_{2} \mathrm{GeN}(\mathrm{H}) \mathrm{Dipp}\right](\mathrm{S} 18)$}

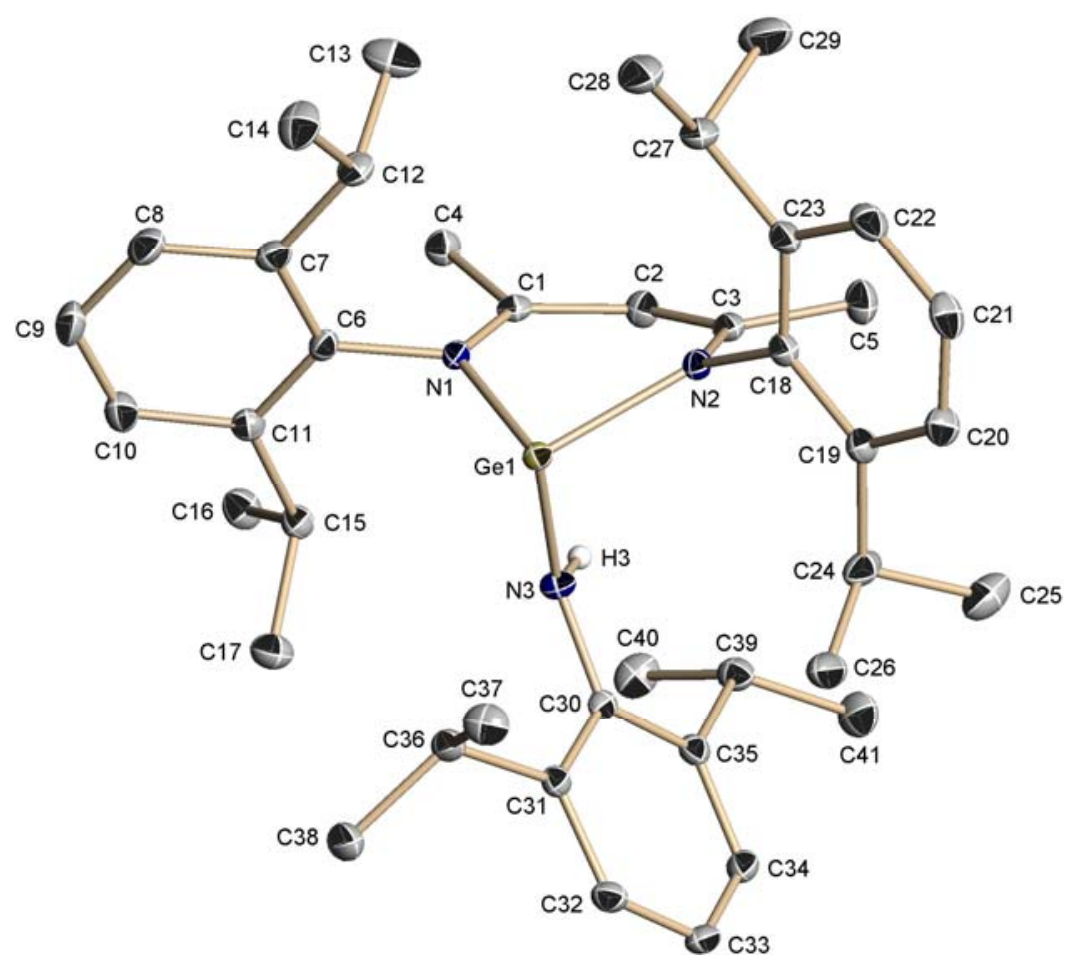

Abb. 9-46: Asymmetrische Einheit von $\left[\mathrm{CH}\{\mu-\mathrm{C}(\mathrm{Me}) \mathrm{N}(\mathrm{Dipp})\}_{2} \mathrm{GeN}(\mathrm{H}) \operatorname{Dipp}\right]$ (S18).

Tab. 9-46: Kristallographische Daten für $\left[\mathrm{CH}\{\mu-\mathrm{C}(\mathrm{Me}) \mathrm{N}(\operatorname{Dipp})\}_{2} \mathrm{GeN}(\mathrm{H}) \operatorname{Dipp}\right]$ (S18).

\begin{tabular}{|ll|ll|}
\hline Strukturcode & AJ25 & Z & 2 \\
Servicepartner & A. Jana & Messtemperatur / K & $100(2)$ \\
CCDC-Nummer & - & $\rho_{\text {calc }} / \mathrm{Mg} \cdot \mathrm{m}^{-3}$ & 1.203 \\
Summenformel & $\mathrm{C}_{41} \mathrm{H}_{59} \mathrm{GeN}_{3}$ & $\mu / \mathrm{mm}^{-1}$ & 0.864 \\
Molmasse $/ \mathrm{g} \cdot \mathrm{mol}^{-1}$ & 666.50 & $F(000)$ & 716 \\
Kristallgröße / mm & $0.25 \times 0.2 \times 0.05$ & $\theta-$ Bereich $/{ }^{\circ}$ & $1.86-27.17$ \\
Kristallsystem & triklin & Reflexe gesamt/unabhängig & $25667 / 8108$ \\
Raumgruppe & $P \overline{1}$ & Daten/Restraints/Parameter & $8108 / 0 / 423$ \\
$\mathrm{a} / \mathrm{pm}$ & $906.01(10)$ & $R 1[/>2 \sigma(I)]$ & 0.0276 \\
$\mathrm{~b} / \mathrm{pm}$ & $1132.19(13)$ & $w R 2($ alle Daten $)$ & 0.0676 \\
$\mathrm{c} / \mathrm{pm}$ & $1897.4(2)$ & $g 1 / g 2$ & $0.0314 / 0.9274$ \\
$\alpha /{ }^{\circ}$ & $100.494(2)$ & GoF & 1.057 \\
$\beta /{ }^{\circ}$ & $101.773(2)$ & Differenzelektronendichte: & \\
$\gamma /{ }^{\circ}$ & $98.346(2)$ & max. / min. / $\left(10^{-6} \mathrm{e} \cdot \mathrm{pm}^{-3}\right)$ & $0.477 /-0.297$ \\
$\mathrm{~V} / \mathrm{nm}^{3}$ & $1.8396(4)$ & max. / min. Transmission & $0.75 / 0.67$ \\
\hline
\end{tabular}




\subsubsection{9 $\left[\mathrm{CH}\{\mu-\mathrm{C}(\mathrm{Me}) \mathrm{N}(\mathrm{Dipp})\}_{2} \mathrm{SnBr}\right](\mathrm{S} 19)$}

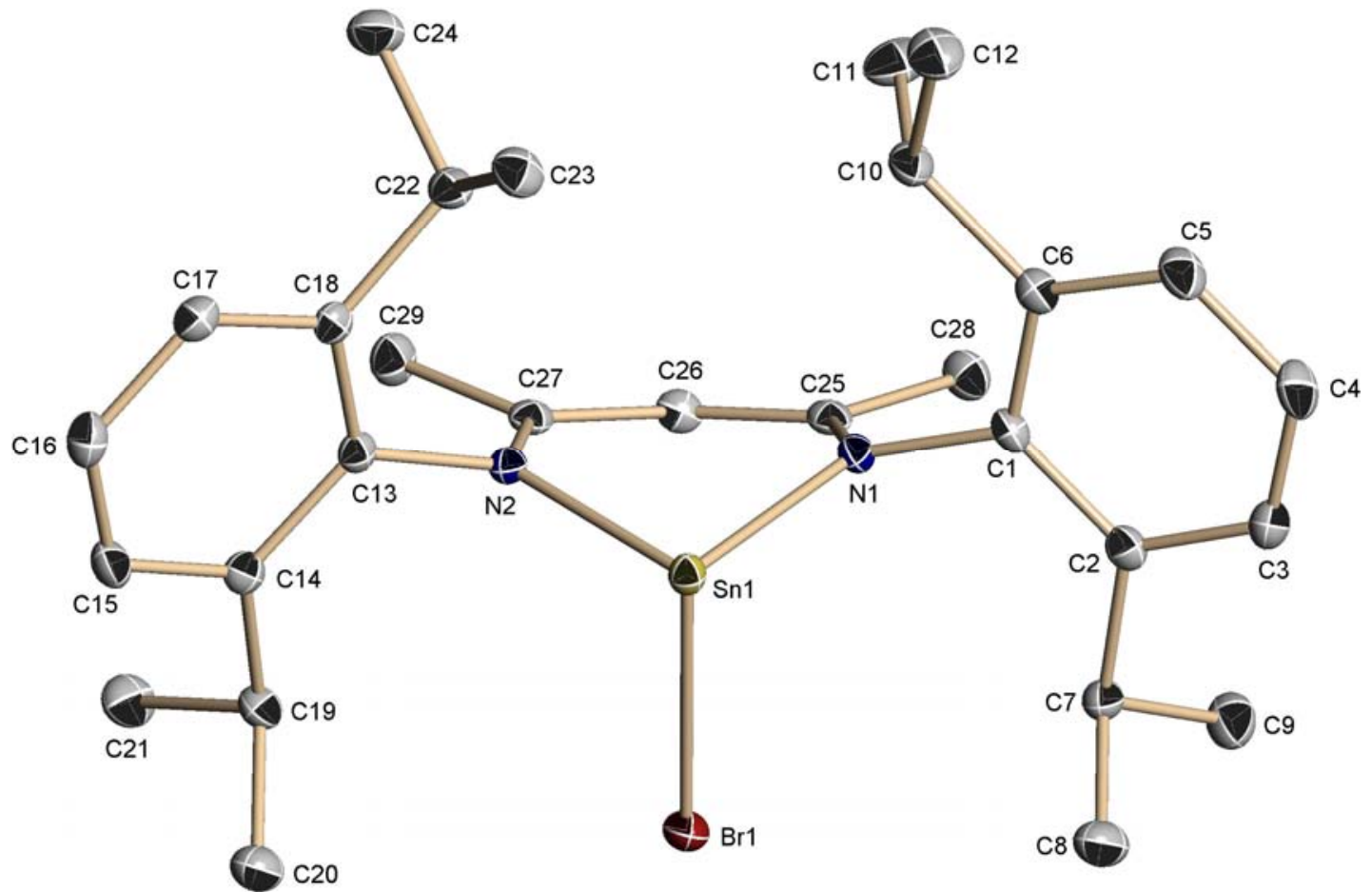

Abb. 9-47: Asymmetrische Einheit von [CH $\left.\{\mu-\mathrm{C}(\mathrm{Me}) \mathrm{N}(\mathrm{Dipp})\}_{2} \mathrm{SnBr}\right]$ (S19).

Tab. 9-47: Kristallographische Daten für $\left[\mathrm{CH}\{\mu-\mathrm{C}(\mathrm{Me}) \mathrm{N}(\mathrm{Dipp})\}_{2} \mathrm{SnBr}\right](\mathbf{S} 19)$.

\begin{tabular}{|c|c|c|c|}
\hline Strukturcode & AJ14 & Z & 2 \\
\hline Servicepartner & A. Jana & Messtemperatur / K & $100(2)$ \\
\hline CCDC-Nummer & - & $\rho_{\text {calc }} / \mathrm{Mg} \cdot \mathrm{m}^{-3}$ & 1.479 \\
\hline Summenformel & $\mathrm{C}_{29} \mathrm{H}_{41} \mathrm{BrN}_{2} \mathrm{Sn}$ & $\mu / \mathrm{mm}^{-1}$ & 2.387 \\
\hline Molmasse $/ \mathrm{g} \cdot \mathrm{mol}^{-1}$ & 616.24 & $F(000)$ & 628 \\
\hline Kristallgröße / mm & $0.3 \times 0.3 \times 0.2$ & $\theta$-Bereich / ${ }^{\circ}$ & $1.74-26.37$ \\
\hline Kristallsystem & triklin & Reflexe gesamt/unabhängig & $16293 / 5617$ \\
\hline Raumgruppe & $P \overline{1}$ & Daten/Restraints/Parameter & $5617 / 0 / 308$ \\
\hline $\mathrm{a} / \mathrm{pm}$ & $1034.47(8)$ & $R 1[I>2 \sigma(I)]$ & 0.0170 \\
\hline $\mathrm{b} / \mathrm{pm}$ & $1206.01(9)$ & $w R 2$ (alle Daten) & 0.0405 \\
\hline $\mathrm{c} / \mathrm{pm}$ & $1236.28(9)$ & $g 1 / g 2$ & $0.0165 / 1.0565$ \\
\hline$\alpha /^{\circ}$ & $89.9690(10)$ & GoF & 1.036 \\
\hline$\beta /{ }^{\circ}$ & $72.4870(10)$ & Differenzelektronendichte: & \\
\hline$\gamma /{ }^{\circ}$ & $71.0940(10)$ & $\max . / \min . /\left(10^{-6} \mathrm{e} \cdot \mathrm{pm}^{-3}\right)$ & $0.363 /-0.286$ \\
\hline $\mathrm{V} / \mathrm{nm}^{3}$ & $1.38346(18)$ & max. / min. Transmission & $0.99 / 0.74$ \\
\hline
\end{tabular}




\subsubsection{0 $\left[\mathrm{CH}\{\mu-\mathrm{C}(\mathrm{Me}) \mathrm{N}(\mathrm{Dipp})\}_{2} \mathrm{SnOC}(\mathrm{H}) \mathrm{O}\right](\mathrm{S} 20)$}

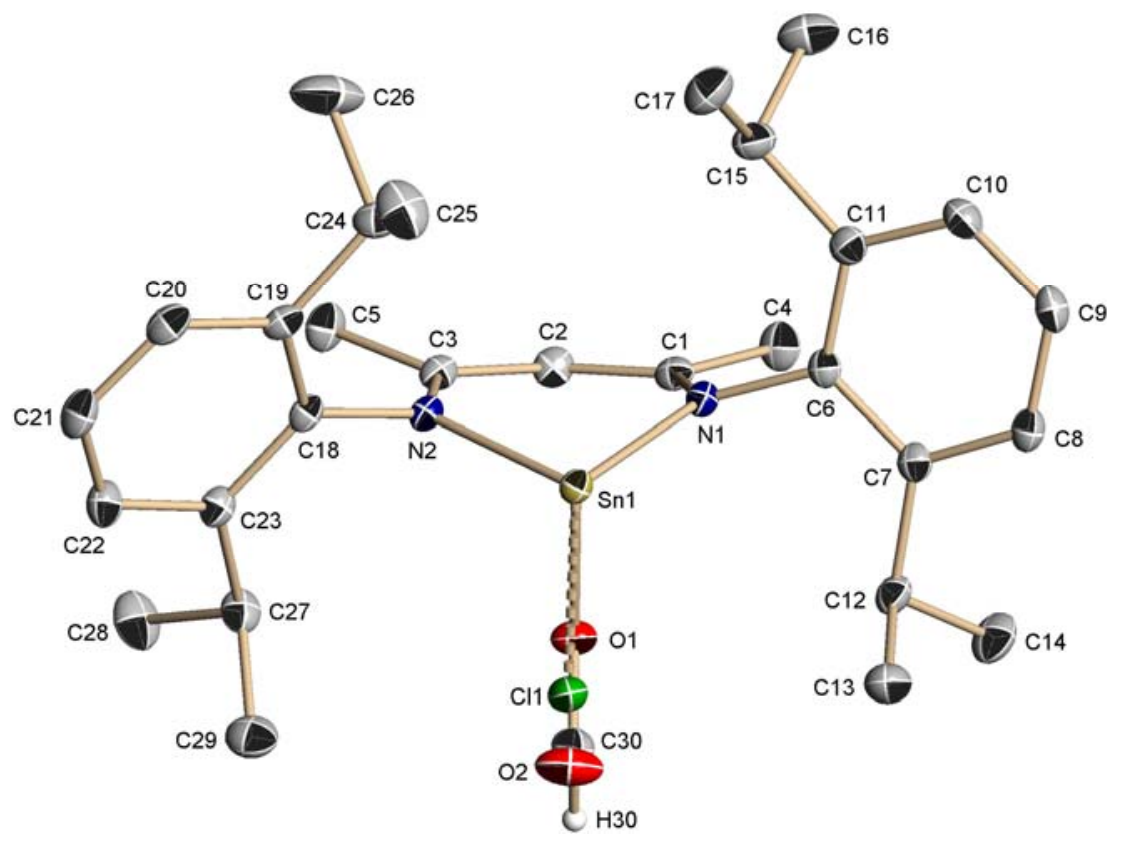

Abb. 9-48: Asymmetrische Einheit von $\left[\mathrm{CH}\{\mu-\mathrm{C}(\mathrm{Me}) \mathrm{N}(\mathrm{Dipp})\}_{2} \mathrm{SnOC}(\mathrm{H}) \mathrm{O}\right](\mathbf{S 2 0})$.

Die Molekülstruktur weist eine Fehlordnung der $\mathrm{OC}(\mathrm{H}) \mathrm{O}-$ Gruppe und einem Chloratom auf, die Besetzungsfaktoren verfeinerten zu $0.75(\mathrm{OCHO})$ und $0.25(\mathrm{Cl})$.

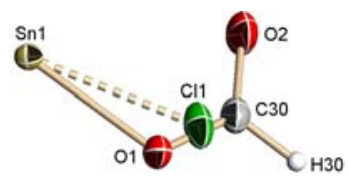

Tab. 9-48: Kristallographische Daten für $\left[\mathrm{CH}\{\mu-\mathrm{C}(\mathrm{Me}) \mathrm{N}(\operatorname{Dipp})\}_{2} \mathrm{SnOC}(\mathrm{H}) \mathrm{O}\right](\mathbf{S 2 0})$.

\begin{tabular}{|c|c|c|c|}
\hline Strukturcode & AJ23 & Messtemperatur / K & $100(2)$ \\
\hline Servicepartner & A. Jana & $\rho_{\text {calc }} / \mathrm{Mg} \cdot \mathrm{m}^{-3}$ & 1.332 \\
\hline CCDC-Nummer & - & $\mu / \mathrm{mm}^{-1}$ & 0.935 \\
\hline Summenformel & $\mathrm{C}_{29.75} \mathrm{H}_{41.75} \mathrm{Cl}_{0.25} \mathrm{~N}_{2} \mathrm{O}_{1.50} \mathrm{Sn}$ & $F(000)$ & 1199 \\
\hline Molmasse $/ \mathrm{g} \cdot \mathrm{mol}^{-1}$ & 578.95 & $\theta$-Bereich / ${ }^{\circ}$ & $1.99-27.90$ \\
\hline Kristallgröße / mm & $0.3 \times 0.25 \times 0.15$ & Reflexe gesamt/unabhängig & 49424 / 6851 \\
\hline Kristallsystem & monoklin & Daten/Restraints/Parameter & $6851 / 0 / 338$ \\
\hline Raumgruppe & $P 2_{1} / c$ & $R 1[I>2 \sigma(I)]$ & 0.0183 \\
\hline $\mathrm{a} / \mathrm{pm}$ & $1706.90(15)$ & $w R 2$ (alle Daten) & 0.0465 \\
\hline $\mathrm{b} / \mathrm{pm}$ & $1308.81(11)$ & $g 1 / g 2$ & $0.0190 / 2.0550$ \\
\hline $\mathrm{c} / \mathrm{pm}$ & 1341.68(12) & GoF & 1.066 \\
\hline$\beta /{ }^{\circ}$ & $105.9190(10)$ & Differenzelektronendichte: & \\
\hline $\mathrm{V} / \mathrm{nm}^{3}$ & $2.8824(4)$ & $\max . / \min . /\left(10^{-6} \mathrm{e} \cdot \mathrm{pm}^{-3}\right)$ & $0.639 /-0.417$ \\
\hline Z & 4 & max. / min. Transmission & $0.75 / 0.68$ \\
\hline
\end{tabular}




\subsubsection{1 $\left[\mathrm{CH}\{\mu-\mathrm{C}(\mathrm{Me}) \mathrm{N}(\mathrm{Dipp})\}_{2} \mathrm{Zr}(\mathrm{thf})(\mathrm{OPh}) \mathrm{Cl}_{2}\right](\mathrm{S} 21)$}

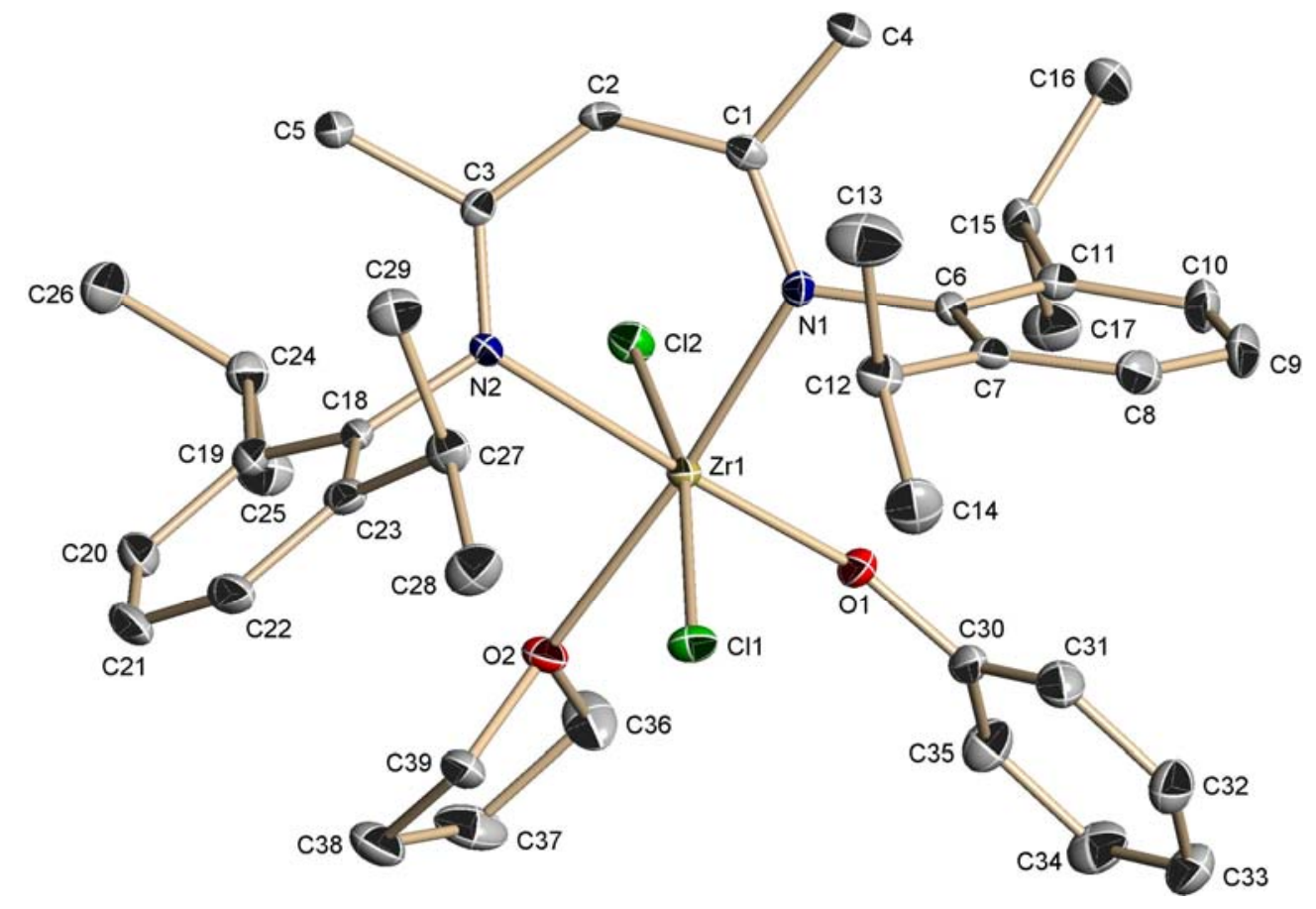

Abb. 9-49: Asymmetrische Einheit von $\left[\mathrm{CH}\{\mu-\mathrm{C}(\mathrm{Me}) \mathrm{N}(\mathrm{Dipp})\}_{2} \mathrm{Zr}(\right.$ thf $\left.)(\mathrm{OPh}) \mathrm{Cl}_{2}\right](\mathrm{S} 21)$.

Tab. 9-49: Kristallographische Daten für $\left[\mathrm{CH}\{\mu-\mathrm{C}(\mathrm{Me}) \mathrm{N}(\mathrm{Dipp})\}_{2} \mathrm{Zr}(\mathrm{thf})(\mathrm{OPh}) \mathrm{Cl}_{2}\right](\mathbf{S} 21)$.

\begin{tabular}{|c|c|c|c|}
\hline Strukturcode & BN09 & $\rho_{\text {calc }} / \mathrm{Mg} \cdot \mathrm{m}^{-3}$ & 1.335 \\
\hline Servicepartner & N. Bijan & $\mu / \mathrm{mm}^{-1}$ & 0.476 \\
\hline CCDC-Nummer & - & $F(000)$ & 3136 \\
\hline Summenformel & $\mathrm{C}_{39} \mathrm{H}_{54} \mathrm{Cl}_{2} \mathrm{~N}_{2} \mathrm{O}_{2} \mathrm{Zr}$ & $\theta$-Bereich / ${ }^{\circ}$ & $1.81-26.46$ \\
\hline Molmasse $/ \mathrm{g} \cdot \mathrm{mol}^{-1}$ & 744.96 & Reflexe gesamt/unabhängig & 86686 / 7584 \\
\hline Kristallgröße / mm & $0.3 \times 0.2 \times 0.15$ & Daten/Restraints/Parameter & $7584 / 0 / 425$ \\
\hline Kristallsystem & orthorhombisch & $R 1[I>2 \sigma(I)]$ & 0.0353 \\
\hline Raumgruppe & Pbca & $w R 2$ (alle Daten) & 0.0905 \\
\hline $\mathrm{a} / \mathrm{pm}$ & 1915.7(0) & $g 1 / g 2$ & $0.0422 / 3.0684$ \\
\hline $\mathrm{b} / \mathrm{pm}$ & $1915.7(5)$ & GoF & 1.076 \\
\hline $\mathrm{c} / \mathrm{pm}$ & $2020.2(5)$ & Differenzelektronendichte: & \\
\hline $\mathrm{V} / \mathrm{nm}^{3}$ & $7.41(1)$ & $\max . / \min . /\left(10^{-6} \mathrm{e} \cdot \mathrm{pm}^{-3}\right)$ & $0.418 /-0.727$ \\
\hline Z & 8 & max. / min. Transmission & $0.99 / 0.66$ \\
\hline Messtemperatur / K & $100(2)$ & & \\
\hline
\end{tabular}




\subsubsection{2 $\left[\mathrm{CH}\{\mu-\mathrm{C}(\mathrm{Me}) \mathrm{N}(\mathrm{Dipp})\}_{2} \mathrm{Zr}(\right.$ thf $\left.)(\mathrm{Op}-\mathrm{Tol}) \mathrm{Cl}_{2}\right](\mathrm{S} 22)$}

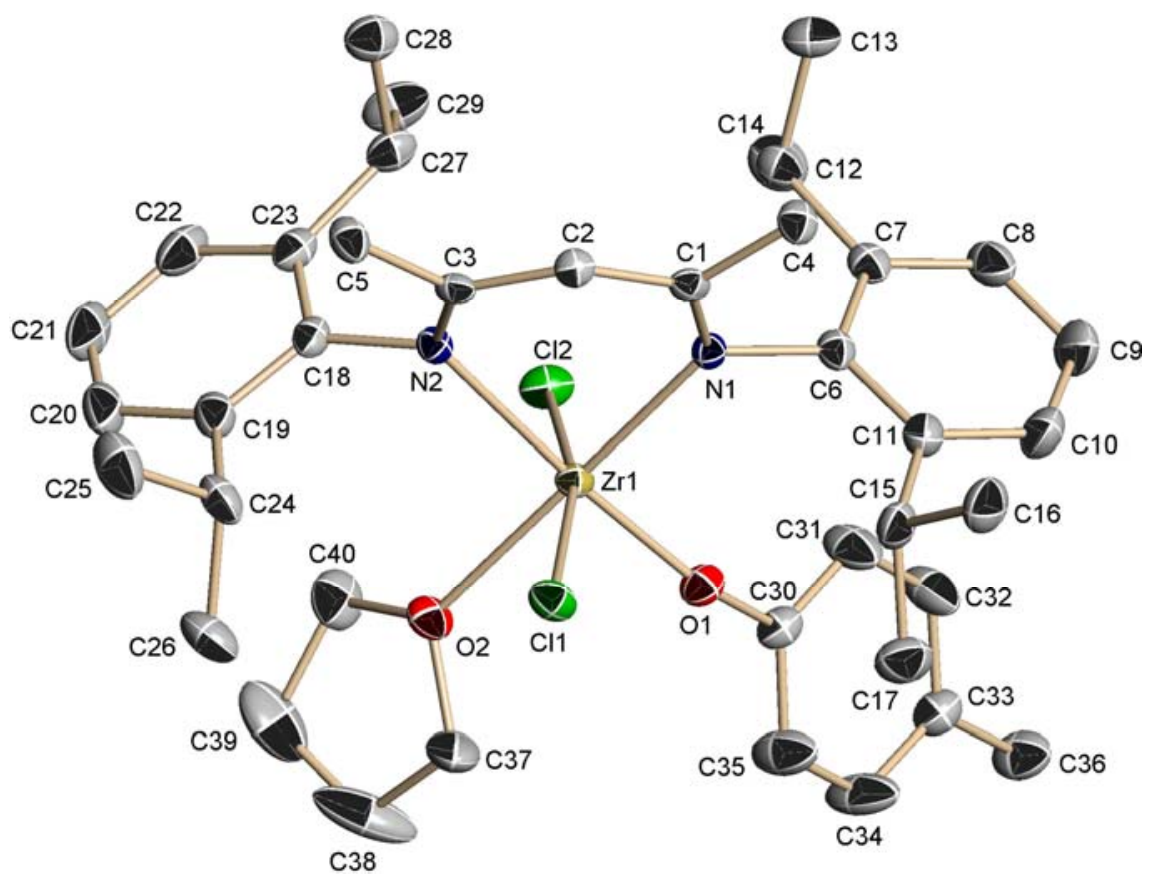

Abb. 9-50: Asymmetrische Einheit von $\left[\mathrm{CH}\{\mu-\mathrm{C}(\mathrm{Me}) \mathrm{N}(\mathrm{Dipp})\}_{2} \mathrm{Zr}(\right.$ thf $\left.)(\mathrm{Op}-\mathrm{Tol}) \mathrm{Cl}_{2}\right]$ (S22).

Tab. 9-50: Kristallographische Daten für $\left[\mathrm{CH}\{\mu-\mathrm{C}(\mathrm{Me}) \mathrm{N}(\mathrm{Dipp})\}_{2} \mathrm{Zr}(\right.$ thf $\left.)(\mathrm{O} p-\mathrm{Tol}) \mathrm{Cl}_{2}\right](\mathbf{S 2 2})$.

\begin{tabular}{|c|c|c|c|}
\hline Strukturcode & BZ2 & $\rho_{\text {calc }} / \mathrm{Mg} \cdot \mathrm{m}^{-3}$ & 1.284 \\
\hline Servicepartner & N. Bijan & $\mu / \mathrm{mm}^{-1}$ & 0.451 \\
\hline CCDC-Nummer & - & $F(000)$ & 3200 \\
\hline Summenformel & $\mathrm{C}_{40} \mathrm{H}_{56} \mathrm{Cl}_{2} \mathrm{~N}_{2} \mathrm{O}_{2} \mathrm{Zr}$ & $\theta$-Bereich $/^{\circ}$ & $2.09-26.37$ \\
\hline Molmasse $/ \mathrm{g} \cdot \mathrm{mol}^{-1}$ & 758.99 & Reflexe gesamt/unabhängig & $43581 / 8017$ \\
\hline Kristallgröße / mm & $0.20 \times 0.20 \times 0.20$ & Daten/Restraints/Parameter & 8017 / 0 / 435 \\
\hline Kristallsystem & orthorhombisch & $R 1[I>2 \sigma(I)]$ & 0.0343 \\
\hline Raumgruppe & Pbca & $w R 2$ (alle Daten) & 0.0830 \\
\hline $\mathrm{a} / \mathrm{pm}$ & $1885.14(19)$ & $g 1 / g 2$ & $0.0345 / 4.5450$ \\
\hline $\mathrm{b} / \mathrm{pm}$ & $1950.3(2)$ & GoF & 1.062 \\
\hline $\mathrm{c} / \mathrm{pm}$ & $2136.0(2)$ & Differenzelektronendichte: & \\
\hline $\mathrm{V} / \mathrm{nm}^{3}$ & $7.8533(14)$ & $\max . / \min . /\left(10^{-6} \mathrm{e} \cdot \mathrm{pm}^{-3}\right)$ & $0.434 /-0.329$ \\
\hline Z & 8 & max. / min. Transmission & $0.99 / 0.75$ \\
\hline Messtemperatur / K & $100(2)$ & & \\
\hline
\end{tabular}




\subsubsection{3 $\left[\mathrm{CH}\{\mu-\mathrm{C}(\mathrm{Me}) \mathrm{N}(\mathrm{Dipp})\}_{2} \mathrm{Ti}(\mathrm{OPh}) \mathrm{Cl}_{2}\right](\mathrm{S} 23)$}

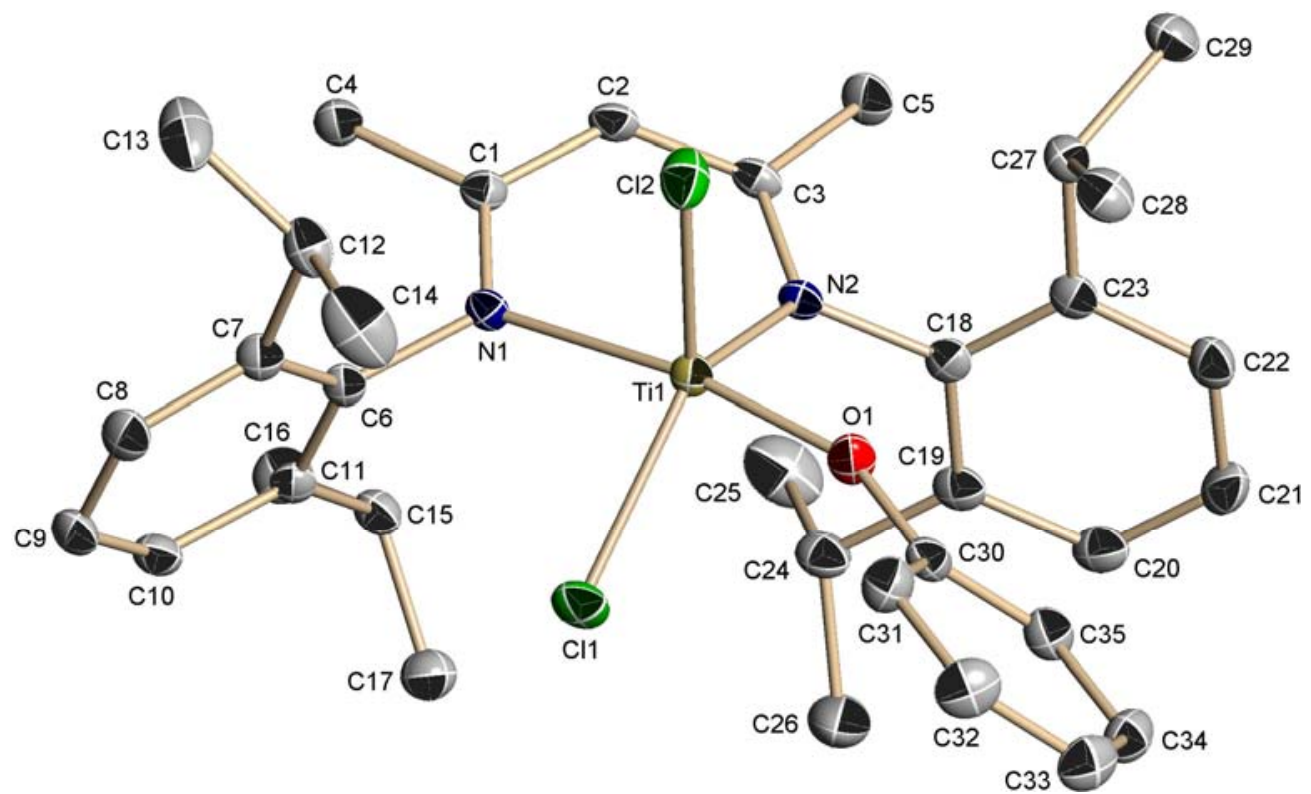

Abb. 9-51: Asymmetrische Einheit von $\left[\mathrm{CH}\{\mu-\mathrm{C}(\mathrm{Me}) \mathrm{N}(\mathrm{Dipp})\}_{2} \mathrm{Ti}(\mathrm{OPh}) \mathrm{Cl}_{2}\right]$ (S23).

Tab. 9-51: Kristallographische Daten für $\left[\mathrm{CH}\{\mu-\mathrm{C}(\mathrm{Me}) \mathrm{N}(\mathrm{Dipp})\}_{2} \mathrm{Ti}(\mathrm{OPh}) \mathrm{Cl}_{2}\right]$ (S23).

\begin{tabular}{|c|c|c|c|}
\hline Strukturcode & BT1 & Messtemperatur / K & $100(2)$ \\
\hline Servicepartner & N. Bijan & $\rho_{\text {calc }} / \mathrm{Mg} \cdot \mathrm{m}^{-3}$ & 1.239 \\
\hline CCDC-Nummer & - & $\mu / \mathrm{mm}^{-1}$ & 0.441 \\
\hline Summenformel & $\mathrm{C}_{35} \mathrm{H}_{46} \mathrm{Cl}_{2} \mathrm{~N}_{2} \mathrm{OTi}$ & $F(000)$ & 1336 \\
\hline Molmasse $/ \mathrm{g} \cdot \mathrm{mol}^{-1}$ & 629.54 & $\theta$-Bereich / ${ }^{\circ}$ & $2.09-26.37$ \\
\hline Kristallgröße / mm & $0.2 \times 0.15 \times 0.1$ & Reflexe gesamt/unabhängig & 50157 / 6904 \\
\hline Kristallsystem & monoklin & Daten/Restraints/Parameter & 6904 / 0 / 380 \\
\hline Raumgruppe & $P 2_{1} / n$ & $R 1[I>2 \sigma(I)]$ & 0.0388 \\
\hline $\mathrm{a} / \mathrm{pm}$ & $1214.46(15)$ & $w R 2$ (alle Daten) & 0.1032 \\
\hline $\mathrm{b} / \mathrm{pm}$ & $2210.7(3)$ & $g 1 / g 2$ & $0.0511 / 2.2225$ \\
\hline $\mathrm{c} / \mathrm{pm}$ & $1389.35(17)$ & GoF & 1.086 \\
\hline$\beta /{ }^{\circ}$ & $115.188(2)$ & Differenzelektronendichte: & \\
\hline $\mathrm{V} / \mathrm{nm}^{3}$ & $3.3754(7)$ & $\max . / \min . /\left(10^{-6} \mathrm{e} \cdot \mathrm{pm}^{-3}\right)$ & $0.628 /-0.527$ \\
\hline Z & 4 & max. / min. Transmission & $0.99 / 0.59$ \\
\hline
\end{tabular}




\subsubsection{4 [(CH $\left.\left.\{\mu-\mathrm{C}(\mathrm{Me}) \mathrm{N}(\mathrm{Dipp})\}_{2}\right)_{2} \mathrm{~Pb} \cdot \mathrm{C}_{6} \mathrm{H}_{14}\right](\mathrm{S} 24)$}
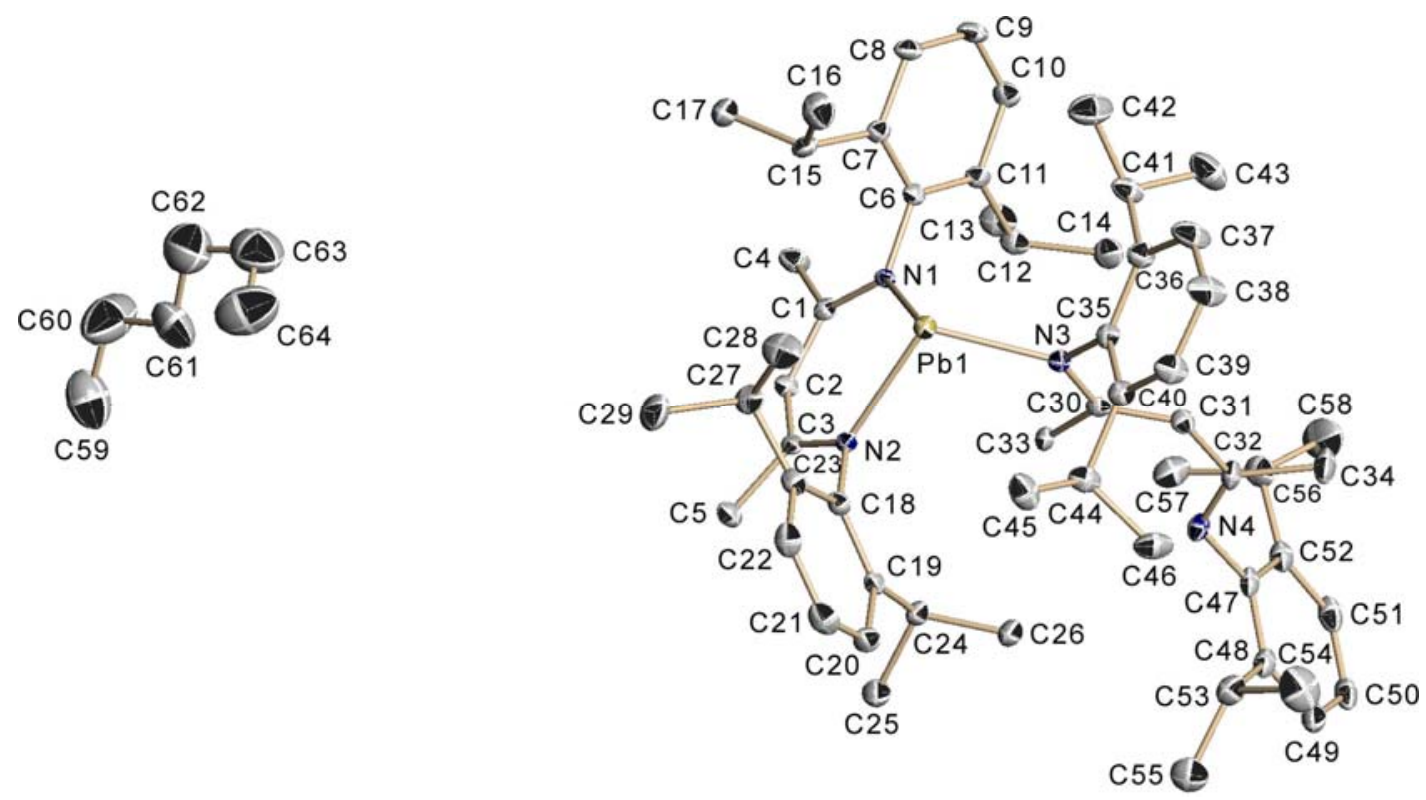

Abb. 9-52: Asymmetrische Einheit von $\left[\left(\mathrm{CH}\{\mu-\mathrm{C}(\mathrm{Me}) \mathrm{N}(\mathrm{Dipp})\}_{2}\right)_{2} \mathrm{~Pb} \cdot \mathrm{C}_{6} \mathrm{H}_{14}\right](\mathbf{S 2 4})$.

Die Verbindung kristallisiert in der orthorhombischen, chiralen Raumgruppe $P 2_{1} 2_{1} 2_{1}$. Der Flack-Parameter ${ }^{[184,185]}$ verfeinerte zu -0.0100 mit einer Standardabweichung von 0.0032, die absolute Struktur wurde somit korrekt bestimmt.

Tab. 9-52: Kristallographische Daten für $\left[\left(\mathrm{CH}\{\mu-\mathrm{C}(\mathrm{Me}) \mathrm{N}(\mathrm{Dipp})\}_{2}\right)_{2} \mathrm{~Pb} \cdot \mathrm{C}_{6} \mathrm{H}_{14}\right]$ (S24).

\begin{tabular}{|c|c|c|c|}
\hline Strukturcode & AJ24 & Messtemperatur / K & $100(2)$ \\
\hline Servicepartner & A. Jana & $\rho_{\text {calc }} / \mathrm{Mg} \cdot \mathrm{m}^{-3}$ & 1.240 \\
\hline CCDC-Nummer & - & $\mu / \mathrm{mm}^{-1}$ & 2.829 \\
\hline Summenformel & $\mathrm{C}_{64} \mathrm{H}_{96} \mathrm{~N}_{4} \mathrm{~Pb}$ & $F(000)$ & 2360 \\
\hline Molmasse $/ \mathrm{g} \cdot \mathrm{mol}^{-1}$ & 1128.64 & $\theta$-Bereich $/^{\circ}$ & $1.49-27.51$ \\
\hline Kristallgröße / mm & $0.3 \times 0.25 \times 0.2$ & Reflexe gesamt/unabhängig & $79870 / 13862$ \\
\hline Kristallsystem & orthorhombisch & Daten/Restraints/Parameter & $13862 / 0 / 644$ \\
\hline Raumgruppe & $P 2{ }_{1}{ }_{1} 2_{1}$ & $R 1[I>2 \sigma(I)]$ & 0.0240 \\
\hline $\mathrm{a} / \mathrm{pm}$ & $1561.45(13)$ & $w R 2$ (alle Daten) & 0.0618 \\
\hline $\mathrm{b} / \mathrm{pm}$ & $1711.34(14)$ & $g 1 / g 2$ & $0.0347 / 5.3645$ \\
\hline $\mathrm{c} / \mathrm{pm}$ & $2263.20(19)$ & GoF & 1.086 \\
\hline Flack $\mathrm{x}$ & $-0.0100(32)$ & Differenzelektronendichte: & \\
\hline $\mathrm{V} / \mathrm{nm}^{3}$ & $6.0477(9)$ & $\max . / \min . /\left(10^{-6} \mathrm{e} \cdot \mathrm{pm}^{-3}\right)$ & $1.918 /-0.635$ \\
\hline Z & 4 & max. / min. Transmission & $0.75 / 0.66$ \\
\hline
\end{tabular}




\section{LITERATURVERZEICHNIS}

[1] H. Binelli, Alchimia Nova, das ist die güldene Kunst, Frankfurt a. M., 1603, S. 3 ff.

[2] J. C. Elsholz, De Phosphoris quatuor observatio, Berlin, 1676.

[3] E. Aubertin, L. Boblique, Bull. Soc. Chim. Paris 1868, 2, 335.

[4] R. J. Meyer, E. H. E. Pietsch, Gmelins Handbuch der Anorganischen Chemie, Systemnummer 16: Phosphor; Teil A, 8. Aufl., Verlag Chemie, Weinheim/Bergstr., 1965.

[5] F. Krafft, Angew. Chem. 1969, 81, 634; Angew. Chem. Int. Ed. Engl. 1969, 8, 660.

[6] A. F. Holleman, E. Wiberg, N. Wiberg, Lehrbuch der Anorganischen Chemie, 102. Aufl., W. de Gruyter, Berlin, New York, 2007, S. $743 \mathrm{ff}$.

[7] M. J. Dumas, Ann. Chim. Phys. 1832, 50, 182.

[8] J. Dumas, Liebigs Ann. Chem. 1833, 5, 5.

[9] A. Laurent, Ann. Chim. Phys. 1837, 66, 149.

[10] C. Wiegand, Angew. Chem. 1948, 60, 127.

[11] A. Laurent, Liebigs Ann. Chem. 1840, 34, 287.

[12] J. Fritzsche, Liebigs Ann. Chem. 1859, 109, 247.

[13] J. Fritzsche, J. Prakt. Chem. 1867, 101, 333.

[14] J. Fritzsche, J. Prakt. Chem. 1869, 106, 274.

[15] C. Linebarger, Am. Chem. J. 1892, 14, 597.

[16] T. Anderson, Liebigs Ann. Chem. 1862, 122, 294.

[17] H. Limpricht, Liebigs Ann. Chem. 1866, 139, 303.

[18] C. Graebe, C. Liebermann, Liebigs Ann. Chem. 1871, 160, 121.

[19] R. Anschütz, F. Eltzbacher, Ber. Dtsch. Chem. Ges. 1883, 16, 623.

[20] E. Clar, Polycyclic Hydrocarbons, Vol. 1, Springer, Berlin, Göttingen, Heidelberg, 1964, S. $288 \mathrm{ff}$.

[21] P. Garrigues, M. Lamotte, Polycyclic Aromatic Compounds: Synthesis, Properties, Analytical Measurements, Occurence and Biological Effects, Proceedings of the 13th International Symposium on Polynuclear Aromatic Hydrocarbons, Gordon and Breach, Bordeaux, Frankreich, 1991.

[22] D. O. Cowan, R. L. Drisko, Elements of Organic Photochemistry, Plenum, New York, London, 1976.

[23] H. Bouas-Laurent, A. Castellan, J.-P. Desvergne, Pure Appl. Chem. 1980, 52, 2633.

[24] B. M. Mikhailov, Izv. Akad. Nauk SSSR, Ser. Khim. 1948, 4, 420.

[25] B. M. Mikhailov, N. F. Kucherova, Dokl. Akad. Nauk SSSR 1950, 74, 501. 
[26] B. M. Mikhailov, N. F. Kucherova, Zh. Obshch. Khim. 1952, 22, 792; J. Gen. Chem. USSR $1952,22,855$.

[27] K. Akasaka, T. Suzuki, H. Ohrui, H. Meguro, Anal. Lett. 1987, 20, 731.

[28] L. Heuer, D. Schomburg, R. Schmutzler, Chem. Ber. 1989, 122, 1473.

[29] J. Wesemann, P. G. Jones, D. Schomburg, L. Heuer, R. Schmutzler, Chem. Ber. 1992, 125, 2187.

[30] T. G. Meyer, P. G. Jones, R. Schmutzler, Z. Naturforsch. 1993, 48b, 875.

[31] A. Karacar, H. Thönnessen, P. G. Jones, R. Bartsch, R. Schmutzler, Heteroat. Chem. 1997, 8, 539.

[32] A. Karacar, M. Freytag, H. Thönnessen, J. Omelanczuk, P. G. Jones, R. Bartsch, R. Schmutzler, Z. Anorg. Allg. Chem. 2000, 626, 2361.

[33] A. Karacar, M. Freytag, H. Thönnessen, J. Omelanczuk, P. G. Jones, R. Bartsch, R. Schmutzler, Heteroat. Chem. 2001, 12, 102.

[34] A. Karacar, M. Freytag, H. Thönnessen, P. G. Jones, R. Bartsch, R. Schmutzler, J. Organomet. Chem. 2002, 643-644, 68.

[35] J. Omelanczuk, A. Karacar, M. Freytag, P. G. Jones, R. Bartsch, M. Mikolajczyk, R. Schmutzler, Inorg. Chim. Acta 2003, 350, 583.

[36] F. Yang, P. E. Fanwick, C. P. Kubiak, Organometallics 1999, 18, 4222.

[37] F. Yang, P. E. Fanwick, C. P. Kubiak, Inorg. Chem. 2002, 41, 4805.

[38] J. H. K. Yip, J. Prabhavathy, Angew. Chem. 2001, 113, 2217; Angew. Chem. Int. Ed. 2001, 40, 2159.

[39] K. Zhang, J. Prabhavathy, J. H. K. Yip, L. L. Koh, G. K. Tan, J. J. Vittal, J. Am. Chem. Soc. 2003, 125, 8452.

[40] J.-L. Chen, L.-Y. Zhang, L.-X. Shi, H.-Y. Ye, Z.-N. Chen, Inorg. Chim. Acta 2005, 358, 859.

[41] R. Lin, J. H. K. Yip, K. Zhang, L. L. Koh, K.-Y. Wong, K. P. Ho, J. Am. Chem. Soc. 2004, 126, 15852.

[42] R. Lin, J. H. K. Yip, Inorg. Chem. 2006, 45, 4423.

[43] J. Hu, R. Lin, J. H. K. Yip, K.-Y. Wong, D.-L. Ma, J. J. Vittal, Organometallics 2007, 26, 6533.

[44] M. W. Haenel, D. Jakubik, C. Krüger, P. Betz, Chem. Ber. 1991, 124, 333.

[45] M. W. Haenel, S. Oevers, J. Bruckmann, J. Kuhnigk, C. Krüger, Synlett 1998, 301.

[46] M. W. Haenel, S. Oevers, K. Angermund, W. C. Kaska, H.-J. Fan, M. B. Hall, Angew. Chem. 2001, 113, 3708; Angew. Chem. Int. Ed. 2001, 40, 3596.

[47] M. Yamashita, K. Watanabe, Y. Yamamoto, K.-y. Akiba, Chem. Lett. 2001, 30, 1104.

[48] P. Kilian, A. M. Z. Slawin, Dalton Trans. 2007, 3289.

[49] N. Solin, J. Kjellgren, K. J. Szabó, J. Am. Chem. Soc. 2004, 126, 7026. 
[50] K. A. Petrov, S. V. Agafonov, V. P. Pokatun, V. M. Chizov, Zh. Obshch. Khim. 1987, 57, 299.

[51] J. Keller, C. Schlierf, C. Nolte, P. Mayer, B. F. Straub, Synthesis 2006, 354.

[52] R. H. Altiparmakian, R. S. W. Braithwaite, J. Chem. Soc. C 1967, 1818.

[53] T. C. Tempesti, A. B. Pierini, M. T. Baumgartner, J. Org. Chem. 2005, 70, 6508.

[54] S. Mirsadeghi, G. K. B. Prasad, N. Whittaker, D. R. Thakker, J. Org. Chem. 1989, 54, 3091.

[55] F. Lang, D. Zewge, I. N. Houpis, R. P. Volante, Tetrahedron Lett. 2001, 42, 3251.

[56] J. P. Wolfe, S. Wagaw, S. L. Buchwald, J. Am. Chem. Soc. 1996, 118, 7215.

[57] J. F. Hartwig, Angew. Chem. 1998, 110, 2154; Angew. Chem. Int. Ed. 1998, 37, 2046.

[58] B. H. Yang, S. L. Buchwald, J. Organomet. Chem. 1999, 576, 125.

[59] D. Chakraborti, L. Colis, R. Schneider, A. K. Basu, Org. Lett. 2003, 5, 2861.

[60] A. Sanyal, Q. Yuan, J. K. Snyder, Tetrahedron Lett. 2005, 46, 2475.

[61] W. Cui, R. N. Loeppky, Tetrahedron 2001, 57, 2953.

[62] A. Togni, C. Breutel, A. Schnyder, F. Spindler, H. Landert, A. Tijani, J. Am. Chem. Soc. 1994, 116,4062 .

[63] Q. Shen, J. F. Hartwig, J. Am. Chem. Soc. 2006, 128, 10028.

[64] R. C. Ropp, Luminescence and the Solid State, 2. Aufl., Elsevier, Amsterdam, 2004.

[65] M. Hissler, P. W. Dyer, R. Reau, Top. Curr. Chem. 2005, 250, 127.

[66] J. E. Anthony, Chem. Rev. 2006, 106, 5028.

[67] B. M. Krasovickij, B. M. Bolotin, Organic Luminescent Materials, VCH, Weinheim, 1988.

[68] H. Haken, H. C. Wolf, Molekülphysik und Quantenchemie, 5. Aufl., Springer, Berlin, Heidelberg, New York, 2006.

[69] M. Klessinger, J. Michl, Lichtabsorption und Photochemie organischer Moleküle, VCH, Weinheim, New York, 1990.

[70] A. W. Czarnik, Fluorescent Chemosensors for lon and Molecule Recognition, ACS Symp. Ser. 538, Am. Chem. Soc., Washington (DC), 1992.

[71] R. Martínez-Máñez, F. Sancenón, Chem. Rev. 2003, 103, 4419.

[72] B. Witulski, M. Weber, U. Bergsträsser, J.-P. Desvergne, D. M. Bassani, H. Bouas-Laurent, Org. Lett. 2001, 3, 1467.

[73] M. Montalti, L. Prodi, N. Zaccheroni, J. Fluoresc. 2000, 10, 71.

[74] L. Zang, R. Liu, M. W. Holman, K. T. Nguyen, D. M. Adams, J. Am. Chem. Soc. 2002, 124, 10640.

[75] S. Alves, F. Pina, M. T. Albelda, E. García-España, C. Soriano, S. V. Luis, Eur. J. Inorg. Chem. 2001, 405.

[76] T. Gunnlaugsson, J. P. Leonard, Chem. Commun. 2005, 3114.

[77] P. D. Beer, P. A. Gale, Angew. Chem. 2001, 113, 502; Angew. Chem. Int. Ed. 2001, 40, 2556. 
[78] M. Albrecht, M. Lutz, A. L. Spek, G. v. Koten, Nature 2000, 406, 970.

[79] J. F. Callan, A. P. de Silva, D. C. Magri, Tetrahedron 2005, 61, 8551.

[80] R. A. Bissell, A. P. de Silva, H. Q. N. Gunaratne, P. L. M. Lynch, G. E. M. Maguire, C. P. McCoy, K. R. A. S. Sandanayake in Fluorescent PET (Photoinduced Electron Transfer) Sensors in Top. in Curr. Chem.: Photoinduced Electron Transfer V, 168 (Hrsg.: J. Mattay), Springer, Berlin, Heidelberg, New York, London, Paris, Tokyo, Hong Kong, Barcelona, Budapest, 1993, S. $224 \mathrm{ff}$.

[81] Y. Ooyama, T. Nakamura, K. Yoshida, New J. Chem. 2005, 29, 447.

[82] M. Nishio, CrystEngComm 2004, 6, 130.

[83] K. Yoshida, Y. Ooyama, H. Miyazaki, K. Watanabe, J. Chem. Soc., Perkin Trans. 2 2002, 700.

[84] Y. Ooyama, S. Yoshikawa, S. Watanabe, K. Yoshida, Org. Biomol. Chem. 2006, 4, 3406.

[85] Y. Ooyama, T. Okamoto, T. Yamaguchi, T. Suzuki, A. Hayashi, K. Yoshida, Chem. Eur. J. 2006, $12,7827$.

[86] H. Langhals, T. Potrawa, H. Nöth, G. Linti, Angew. Chem. 1989, 101, 497; Angew. Chem. Int. Ed. Engl. 1989, 28, 478.

[87] E. Horiguchi, S. Matsumoto, K. Funabiki, M. Matsui, Bull. Chem. Soc. Jpn. 2005, 78, 1167.

[88] Y. Mizobe, I. Hiromichi, I. Hisaki, M. Miyata, Y. Hasegawa, N. Tohnai, Chem. Commun. 2006, 2126.

[89] G. Zhang, G. Yang, S. Wang, Q. Chen, J.-S. Ma, Chem. Eur. J. 2007, 13, 3630.

[90] Y. Mizobe, N. Tohnai, M. Miyata, Y. Hasegawa, Chem. Commun. 2005, 1839.

[91] B.-K. An, S.-K. Kwon, S.-D. Jung, S. Y. Park, J. Am. Chem. Soc. 2002, 124, 14410.

[92] K. Yoshida, Y. Ooyama, S. Tanikawa, K. Watanabe, J. Chem. Soc., Perkin Trans. 2 2002, 708.

[93] J. L. Scott, T. Yamada, K. Tanaka, New J. Chem. 2004, 28, 447.

[94] J. L. Scott, T. Yamada, K. Tanaka, Bull. Chem. Soc. Jpn. 2004, 77, 1697.

[95] G.-q. Zhang, G.-q. Yang, L.-y. Yang, Q.-q. Chen, J.-S. Ma, Eur. J. Inorg. Chem. 2005, 1919.

[96] Y. Ooyama, K. Yoshida, New J. Chem. 2005, 29, 1204.

[97] S. Das, P. K. Bharadwaj, Inorg. Chem. 2006, 45, 5257.

[98] Y. Mizobe, M. Miyata, I. Hisaki, Y. Hasegawa, N. Tohnai, Org. Lett. 2006, 8, 4295.

[99] E. Cariati, J. Bourassa, P. C. Ford, Chem. Commun. 1998, 1623.

[100] E. Cariati, X. Bu, P. C. Ford, Chem. Mater. 2000, 12, 3385.

[101] W. Lu, M. C. W. Chan, N. Zhu, C.-M. Che, Z. He, K.-Y. Wong, Chem. Eur. J. 2003, 9, 6155.

[102] C. E. Buss, K. R. Mann, J. Am. Chem. Soc. 2002, 124, 1031.

[103] J. C. Vickery, M. M. Olmstead, E. Y. Fung, A. L. Balch, Angew. Chem. 1997, 109, 1227; Angew. Chem. Int. Ed. Engl. 1997, 36, 1179. 
[104] M. A. Mansour, W. B. Connick, R. J. Lachicotte, H. J. Gysling, R. Eisenberg, J. Am. Chem. Soc. 1998, 120, 1329.

[105] E. J. Fernández, J. López-de-Luzuriaga, M. Monge, M. E. Olmos, J. Pérez, A. Laguna, A. A. Mohamed, J. P. Fackler Jr., J. Am. Chem. Soc. 2003, 125, 2022.

[106] C. A. Daws, C. L. Exstrom, J. R. Sowa Jr., K. R. Mann, Chem. Mater. 1997, 9, 363.

[107] S. M. Drew, D. E. Janzen, C. E. Buss, D. I. MacEwan, K. M. Dublin, K. R. Mann, J. Am. Chem. Soc. 2001, 123, 8414 .

[108] J.-S. Yang, T. M. Swager, J. Am. Chem. Soc. 1998, 120, 5321.

[109] A. W. Czarnik, Nature 1998, 394, 417.

[110] J.-S. Yang, T. M. Swager, J. Am. Chem. Soc. 1998, 120, 11864.

[111] S. Content, W. C. Trogler, M. J. Sailor, Chem. Eur. J. 2000, 6, 2205.

[112] Z. Fei, N. Kocher, C. J. Mohrschladt, H. Ihmels, D. Stalke, Angew. Chem. 2003, 115, 807; Angew. Chem. Int. Ed. 2003, 42, 783.

[113] G. Schwab, Diplomarbeit, Würzburg, 2004.

[114] G. Schwab, D. Stern, D. Leusser, D. Stalke, Z. Naturforsch. 2007, 62b, 711.

[115] A. Herrbach, A. Marinetti, O. Baudoin, D. Guénard, F. Guéritte, J. Org. Chem. 2003, 68, 4897.

[116] T. J. Geldbach, P. S. Pregosin, A. Albinati, Organometallics 2003, 22, 1443.

[117] H. Lehmkuhl, K. Mehler, R. Benn, A. Rufinska, G. Schroth, C. Krüger, Chem. Ber. 1984, 117, 389.

[118] R. Mason, Acta Crystallogr. 1964, 17, 547.

[119] J. Trotter, Acta Crystallogr., Sect. C: Cryst. Struct. Commun. 1986, 42, 862.

[120] J. C. Bellows, E. D. Stevens, P. N. Prasad, Acta Crystallogr., Sect. B: Struct. Sci. 1978, 34, 3256.

[121] R. H. Laitinen, V. Heikkinen, M. Haukka, A. M. P. Koskinen, J. Pursiainen, J. Organomet. Chem. 2000, 598, 235.

[122] T. E. Müller, J. C. Green, D. M. P. Mingos, C. M. McPartlin, C. Whittingham, D. J. Williams, T. M. Woodroffe, J. Organomet. Chem. 1998, 551, 313.

[123] R. F. De Ketelaere, G. P. Van der Kelen, Z. Eeckhaut, Phosphorus Relat. Gr. V Elem. 1974, 5, 43.

[124] A. L. Spek, Acta Crystallogr., Sect. C: Cryst. Struct. Commun. 1987, 43, 1233.

[125] D. van Allen, D. Venkataraman, J. Org. Chem. 2003, 68, 4590.

[126] J. Heinicke, R. Kadyrov, M. K. Kindermann, M. Kloss, A. Fischer, P. G. Jones, Chem. Ber. 1996, 129, 1061.

[127] P. W. Codding, K. A. Kerr, Acta Crystallogr., Sect. B: Struct. Sci. 1978, 34, 3785

[128] P. W. Codding, K. A. Kerr, Acta Crystallogr., Sect. B: Struct. Sci. 1979, 35, 1261. 
[129] G. Schwab, D. Stern, D. Stalke, J. Org. Chem. 2008, eingereicht.

[130] M. Hesse, H. Meier, B. Zeeh, Spektroskopische Methoden in der organischen Chemie, 5. Aufl., G. Thieme, Stuttgart, New York, 1995.

[131] A. Bondi, J. Phys. Chem. 1964, 68, 441.

[132] M. Veith, S. Weidner, K. Kunze, D. Käfer, J. Hans, V. Huch, Coord. Chem. Rev. 1994, 137, 297.

[133] F. Baier, Z. Fei, H. Gornitzka, A. Murso, S. Neufeld, M. Pfeiffer, I. Rüdenauer, A. Steiner, T. Stey, D. Stalke, J. Organomet. Chem. 2002, 661, 111.

[134] D. Atwood, D. Rutherford, Chem. Commun. 1996, 1251.

[135] D. Rutherford, D. A. Atwood, J. Am. Chem. Soc. 1996, 118, 11535.

[136] G. V. Gridunova, V. N. Petrov, Y. T. Struchkov, I. G. II'lina, O. V. Mikhalev, Crystallogr. Rep. $1990,35,54$

[137] H. Nakazawa, Y. Yamaguchi, K. Miyoshi, Organometallics 1996, 15, 1337.

[138] J. M. Duff, B. L. Shaw, J. Chem. Soc., Dalton Trans. 1972, 2219.

[139] S. A. Reiter, S. D. Nogai, K. Karaghiosoff, H. Schmidbaur, J. Am. Chem. Soc. 2004, 126, 15833.

[140] T. Sasamori, A. Tsurusaki, N. Nagahora, K. Matsuda, Y. Kanemitsu, Y. Watanabe, Y. Furukawa, N. Tokitoh, Chem. Lett. 2006, 35, 1382.

[141] R. H. Laitinen, H. Riihimäki, M. Haukka, S. Jääskeläinen, T. A. Pakkanen, J. Pursiainen, Eur. J. Inorg. Chem. 1999, 1253.

[142] Y. Uchida, K. Onoue, N. Tada, F. Nagao, H. Kozawa, S. Oae, Heteroat. Chem. 1990, 1, 295.

[143] P. L. Arnold, L. S. Natrajan, J. J. Hall, S. J. Bird, C. Wilson, J. Organomet. Chem. 2002, 647, 205.

[144] K. Ruhlandt-Senge, P. P. Power, Bull. Soc. Chim. Fr. 1992, 129, 594.

[145] N. Bertel, H. W. Roesky, F. T. Edelmann, M. Noltemeyer, H.-G. Schmidt, Z. Anorg. Allg. Chem. $1990,586,7$

[146] U. Englich, S. Chadwick, K. Ruhlandt-Senge, Inorg. Chem. 1998, 37, 283.

[147] M. Niemeyer, P. P. Power, Inorg. Chem. 1996, 35, 7264.

[148] C. J. Brown, M. Ehrenberg, Acta Crystallogr., Sect. C: Cryst. Struct. Commun. 1984, 40, 1059.

[149] A. Usman, H.-K. Fun, Y. Li, J.-H. Xu, Acta Crystallogr., Sect. C: Cryst. Struct. Commun. 2003, 59,0308 .

[150] S.-H. Chien, M.-F. Cheng, K.-C. Lau, W.-K. Li, J. Phys. Chem. A 2005, 109, 7509.

[151] N. Nardello, J.-M. Aubry, P. Johnston, I. Bulduk, A. H. M. de Vries, P. L. Alsters, Synlett 2005, 17, 2667.

[152] J.-M. Aubry, J. Am. Chem. Soc. 1985, 107, 5844.

[153] H. Kotani, K. Ohkubo, S. Fukuzumi, J. Am. Chem. Soc. 2004, 126, 15999. 
[154] E. Zadok, S. Rubinraut, F. Frolow, Y. Mazur, J. Am. Chem. Soc. 1985, 107, 2489.

[155] M. J. Fuchter, B. M. Hoffman, A. G. M. Barrett, J. Org. Chem. 2006, 71, 724.

[156] D. G. Ho, R. Gao, J. Celaje, H.-Y. Chung, M. Selke, Science 2003, 302, 259.

[157] G. Bartoli, M. Bosco, R. D. Pozzo, P. Sgarabotto, J. Chem. Soc., Perkin Trans. 2 1982, 929.

[158] G. L. Squadrito, F. R. Fronczek, S. F. Watkins, D. F. Church, W. A. Pryor, J. Org. Chem. 1990, $55,4322$.

[159] N.-u.-d. Ahmad, R. J. Goddard, I. K. Hatton, J. A. K. Howard, N. J. Lewis, J. MacMillan, J. Chem. Soc., Perkin Trans. 1 1985, 1859.

[160] P. W. Dyer, J. Fawcett, M. J. Hanton, R. D. W. Kemmitt, R. Padda, N. Singh, Dalton Trans. 2003, 104.

[161] J. A. Casares, P. Espinet, R. Hernando, G. Iturbe, F. Villafane, D. D. Ellis, A. G. Orpen, Inorg. Chem. 1997, 36, 44.

[162] J. A. Casares, S. Coco, P. Espinet, Y.-S. Lin, Organometallics 1995, 14, 3058.

[163] J. A. Casares, P. Espinet, J. M. Martín-Alvarez, V. Santos, Inorg. Chem. 2004, 43, 189.

[164] W. Schlenk, A. Thal, Ber. Dtsch. Chem. Ges. 1913, 46, 2840.

[165] W. Schlenk in Die Methoden der Organischen Chemie (Hrsg.: J. Houben), 2. Aufl., G. Thieme, Leipzig, 1924, S. $720 \mathrm{ff}$.

[166] T. T. Tidwell, Angew. Chem. 2001, 113, 343; Angew. Chem. Int. Ed. 2001, 40, 331.

[167] P. G. Chantrell, C. A. Pearce, C. R. Toyer, R. Twaits, J. Appl. Chem. 1964, 14, 563.

[168] T. Kottke, D. Stalke, J. Appl. Crystallogr. 1993, 26, 615.

[169] D. Stalke, Chem. Soc. Rev. 1998, 27, 171.

[170] H. Hope, Acta Crystallogr., Sect. B: Struct. Sci. 1988, 44, 22.

[171] G. M. Sheldrick, SMART-NT V5.6, Bruker AXS Inst. Inc., Madison (WI, USA), 2000.

[172] Bruker APEX v2.1-0, Bruker AXS Inst. Inc., Madison (WI, USA), 2007.

[173] SAINT v7.34A in Bruker APEX v2.1-0, Bruker AXS Inst. Inc., Madison (WI, USA), 2005.

[174] G. M. Sheldrick, SAINT-NT in SAINTPLUS v6/6.0, Bruker AXS Inst. Inc., Madison (WI, USA), 2000.

[175] W. Kabsch, J. Appl. Crystallogr. 1988, 21, 916.

[176] G. M. Sheldrick, SADABS 2.0 in SAINTPLUS v6/6.0, Bruker AXS Inst. Inc., Madison (WI, USA), 2000.

[177] G. M. Sheldrick, SADABS 2004/1, Göttingen, 2004.

[178] G. M. Sheldrick, XPREP in SHELXTL v6.12, Bruker AXS Inst. Inc., Madison (WI, USA), 2000.

[179] X-AREA, Stoe \& Cie GmbH, Darmstadt, 2002.

[180] G. M. Sheldrick, SHELXS in SHELXTL v6.12, Bruker AXS Inst. Inc., Madison (WI, USA), 2000.

[181] G. M. Sheldrick, SHELXL in SHELXTL v6.12, Bruker AXS Inst. Inc., Madison (WI, USA), 2000. 
[182] G. M. Sheldrick, Acta Crystallogr., Sect. A: Found. Crystallogr. 2008, 64, 112.

[183] XSHELL v4.02, Bruker AXS Inst. Inc., Madison (WI, USA), 2000.

[184] H. D. Flack, Acta Crystallogr., Sect. A: Found. Crystallogr. 1983, 39, 876.

[185] G. Bernardinelli, H. D. Flack, Acta Crystallogr., Sect. A: Found. Crystallogr. 1985, 41, 500.

[186] S. K. Mandal, P. M. Gurubasavaraj, H. W. Roesky, G. Schwab, D. Stalke, R. B. Oswald, V. Dolle, Inorg. Chem. 2007, 46, 10158. 



\section{Publikationen und PosterbeitrÄge}

[1.] Fluoreszenz von Wirt/Gast-Komplexen der (Di-)Phosphinylanthracene Gerald Schwab, Diplomarbeit, Würzburg 2004.

[2.] 9,10-Bis(diphenylthiophosphanyl)anthracene as a Solid-State Fluorescent Chemosensor

Gerald Schwab, Dirk Leusser, Kathrin Meindl, Dietmar Stalke, Poster auf dem IV European Charge Density Meeting in Brandenburg, 2006.

[3.] Experimental Charge Density and Theoretical Studies on $\mathrm{Me}_{2} \mathrm{Al}(\mu-\mathrm{Py})_{2} \mathrm{P}-$ Evidence for P-C Single Bonding

Kathrin C. Götz, Julian Henn, Dirk Leusser, Andreas Oechsner, Gerald Schwab, Bernd Engels, Dietmar Stalke, Poster auf dem IV European Charge Density Meeting in Brandenburg, 2006.

[4.] Syntheses and Structures of 9-Bromo-10-diphenylphosphanylanthracene and its Oxidation Products

Gerald Schwab, Daniel Stern, Dirk Leusser, Dietmar Stalke, Z. Naturforsch., B: Chem. Sci. 2007, 62b, 711.

[5.] Festkörperfluoreszenz von Phosphanylanthracenen

Daniel Stern, Gerald Schwab, Dietmar Stalke, Poster auf dem Jahresforum der GdCh in Ulm, 2007.

[6.] Synthese und Strukturen neuer picolinsubstituierter Phosphane Christian Kling, Holger Ott, Gerald Schwab, Dietmar Stalke, Poster auf dem Jahresforum der GdCh in UIm, 2007.

[7.] Oxygen-Bridged Hybrid Metallocene-Nonmetallocene Polymetallic Catalysts of Group 4 Metals for Bimodal Activity in Olefin Polymerization: Synthesis, Characterization, and Theoretical Investigation

Swadhin K. Mandal, Prabhuodeyara M. Gurubasavaraj, Herbert W. Roesky, Gerald Schwab, Dietmar Stalke, Rainer B. Oswald, Volker Dolle, Inorg. Chem. 2007, 46, 10158.

[8.] Structural and Variable Temperature NMR Studies of 9-Diisopropylphosphanylanthracenes and 9,10-Bis(diisopropylphosphanyl)anthracenes and their Oxidation Products

Gerald Schwab, Daniel Stern, Dietmar Stalke, J. Org. Chem. 2008, im Druck. 
[9.] Heteroaromatic Substituted Phosphoranes with Enhanced Hemilabile Character

Christian Kling, Holger Ott, Gerald Schwab, Dietmar Stalke, Organometallics 2008, zur Publikation angenommen.

[10.] Functionalization of an Aminophosphine: Synthesis and X-ray Crystal Structure of Novel Dilithium and Trilithium Complexes Containing Silicon Caped Hetero-Nuclear $\mathrm{SiN}_{2}$ PLi Five-Membered Rings

Anukul Jana, Gerald Schwab, Herbert W. Roesky, Dietmar Stalke, Inorg. Chem. 2008, eingereicht.

[11.] A Germanium Analogue of a Dithiocarboxylic Acid Anukul Jana, Gerald Schwab, Herbert W. Roesky, Dietmar Stalke, in Bearbeitung.

[12.] Synthesis and Structural Characterization of Chloride, Hydride and Methylchloride Aluminium Complexes Based on Iminophosphonamide Ligand

Nekoueishahraki Bijan, Prabhuodeyara M. Gurubasavaraj, Herbert W. Roesky, Gerald Schwab, Dietmar Stalke, in Bearbeitung. 


\section{CuRriculum VItAE}

\section{Persönliche Daten}

Name: $\quad$ Gerald Harald Schwab

Geburtsdatum: 24.05.1979

Geburtsort: Würzburg

Familienstand: ledig

\section{Schulische Ausbildung}

1985 - $1989 \quad$ Arthur-Knorr-Grundschule in Altertheim

1989 - 1998 Deutschhaus-Gymnasium in Würzburg,

Abschluss: Allgemeine Hochschulreife

Wehrdienst

1998 - 1999 Grundwehrdienst in Wildflecken und Veitshöchheim

\section{Studium}

1999 - $2004 \quad$ Studium der Chemie (Diplom) an der Bayerischen JuliusMaximilians-Universität Würzburg

12/2003 - 09/2004 Diplomarbeit im Arbeitskreis von Prof. Dr. D. Stalke am Institut für Anorganische Chemie zum Thema: "Fluoreszenz von Wirt/Gast-Komplexen der (Di-)Phosphinylanthracene"

$10 / 2004$ Abschluss des Studiums der Chemie als Dipl.-Chem. Univ.

10/2004 - 03/2005 Promotion im Arbeitskreis von Prof. Dr. D. Stalke am Institut für Anorganische Chemie der Universität Würzburg zum Thema "Die Strukturabhängigkeit der Festkörperfluoreszenz bei Phosphanylanthracenen"

seit 04/2005 Fortsetzung der Promotion im Arbeitskreis von Prof. Dr. D. Stalke an der Georg-August-Universität Göttingen seit 04/2005 wissenschaftlicher Mitarbeiter am Institut für Anorganische Chemie der Georg-August-Universität Göttingen 